\title{
Ride Comfort in Commercial Aircraft During Formation Flight Using Conventional Flight Control
}

by

Evert Frederick Trollip

16080572

Thesis presented in partial fulfilment of the requirements for the degree of Master in Electrical \& Electronic Engineering at the University of Stellenbosch

The financial assistance of the National Research Foundation (NRF) towards this research is hereby acknowledged. Opinions expressed and conclusions arrived at, are those of the author and are not necessarily to be attributed to the NRF.

Supervisor: Mr. J.A.A. Engelbrecht 


\section{Declaration}

By submitting this thesis electronically, I declare that the entirety of the work contained therein is my own, original work, that I am the sole author thereof (save to the extent explicitly otherwise stated), that reproduction and publication thereof by Stellenbosch University will not infringe any third party rights and that I have not previously in its entirety or in part submitted it for obtaining any qualification.

Date:

March 2016

Copyright (C) 2016 Stellenbosch University All rights reserved. 


\section{Abstract}

Global market forecasts and rapid air transport growth are making the aeronautical industry become aware of the necessity to develop high capacity long-range and fuel-efficient aircraft while maintaining high levels of passenger comfort. With the large interest in automated formation flight and improvements in automated flight control, it is the aim of this research to evaluate passenger ride comfort in commercial aircraft during formation flight.

The main objective of this thesis is to take into account the aircraft dynamics, atmospheric turbulence and the effect of a flight control system when evaluating the ride comfort of passengers. To achieve this objective, a detailed literature study into current and past projects was performed, focusing on formation flight, passenger comfort evaluation and improvements in passenger comfort with efficient control systems design. Following the literature study, a theoretical Boeing B747 aircraft model was built and tested in MATLAB and Simulink. Subsequently, a conventional model of the fly-by-wire architecture used in modern transport aircraft was designed, implemented, and verified in simulation.

To model the formation flight effects, the induced forces and moments on the follower aircraft due to the trailing vortices of the leader were derived in a previous study by Bizinos at the University of Cape Town. These induced loads, expressed as aerodynamic coefficients, were used in conjunction with the conventional aerodynamic model to produce an extended aerodynamic model for formation flight. The conventional fly-by-wire control architecture used by modern transport aircraft has been extended for formation flight of two or more aircraft, with the focus on two aircraft in right echelon formation. A full non-linear simulation with realistic turbulence was produced to verify the working of the extended controllers and to ensure the follower maintains its path behind the leader aircraft.

In formation flight, the passenger comfort is influenced by disturbance loads due to the turbulence, as well as by compensatory control inputs produced by the autopilot controllers. The accelerations of passengers in a seated position were determined by considering the passenger's seated position with respect to the mass centre, and the forces and moments at and around the mass centre respectively. These accelerations are weighted according to their frequency to determine the comfort levels in accordance with the International Organisation for Standardisation (ISO 2631-1).

Simulation results of the accelerations experienced at different locations in the trailing aircraft of a formation showed that very little difference in comfort can be expected between the different formation flight aircraft under the same flight conditions. It was concluded for both 
aircraft that a seating location at the front of the aircraft is more comfortable than one at the back of the aircraft. During light and moderate turbulence, the overall acceleration magnitudes remained within the not uncomfortable region suggested by the ISO 2631-1 standard. The percentage of ill passengers in isolated and formation flight is very low, making motion sickness incidence during formation flight almost no different to motion sickness during isolated flight. 


\section{Opsomming}

Wêreldwye markvooruitskattings en snelle lugvervoergroei maak tans die lugvaartbedryf bewus van die noodsaaklikheid van hoëkapasiteit-, brandstofdoeltreffende langafstandvliegtuie wat ook hoë vlakke van passasiersgemak handhaaf. Gegewe die groot belangstelling in geoutomatiseerde formasievlug en die verbeteringe in geoutomatiseerde vlugbeheer, is dit die doel van hierdie navorsing om passasiersgemak in burgerlugvaart in formasievlug te evalueer.

Die hoofdoelstelling van hierdie tesis is om die vliegtuigdinamiek, turbulensie en die effek van 'n vlugbeheerstelsel in ag te neem wanneer die evaluering van passasiersgemak gedoen word. Om hierdie doelstelling te bereik, is 'n gedetailleerde literatuurstudie oor huidige en vorige projekte onderneem, met die fokus op formasievlug, die evaluering van passasiersgemak, en verbeteringe in passasiersgemak deur doeltreffende beheerstelselontwerp. Ná die literatuurstudie is 'n teoretiese Boeing B747-vliegtuigmodel gebou en in MATLAB en Simulink getoets. Vervolgens is 'n konvensionele model van die elektroniese (fly-by-wire) argitektuur wat in moderne vervoervliegtuie gebruik word, ontwerp, geïmplementeer, en in simulasie geverifieer.

Om die effek van formasievlug te modelleer, is die geïnduseerde kragte en momente op die volgervliegtuig wat aan die volgwerwels van die leiervliegtuig te wyte is, afgelei in 'n vorige studie deur Bizinos aan die Universiteit van Kaapstad. Hierdie geïnduseerde kragte, uitgedruk as aërodinamiese koëffisiënte, is tesame met die konvensionele aërodinamiese model gebruik om 'n uitgebreide aërodinamiese model vir formasievlug te skep. Die konvensionele elektroniese (flyby-wire) beheerargitektuur wat moderne vervoervliegtuie gebruik, is uitgebrei vir formasievlugte met twee of meer vliegtuie, met die fokus op twee vliegtuie in regter-echelon formasie. 'n Volle nie-lineêre simulasie met realistiese turbulensie is geskep om die werking van die uitgebreide beheerders te verifieer, en te verseker dat die volgervliegtuig sy roete agter die leiervliegtuig volhou.

In formasievlug word passasiersgemak beïnvloed deur steuringslaste as gevolg van turbulensie, asook deur kompensatoriese beheerinsette wat deur die outoloodsbeheerders geproduseer word. Die versnellings van die passasiers in 'n sittende posisie is bepaal deur te kyk na die passasier se sittende posisie met betrekking tot onderskeidelik die massamiddelpunt en die kragte en momente by en rondom die massamiddelpunt. Hierdie versnellings is geweeg volgens hul frekwensie om die gemaksvlakke ingevolge die Internasionale Standaardeorganisasie (ISO 2631-1) te bepaal.

Die simulasieresultate van die versnellings wat by verskillende liggings in die volgervliegtuig van 'n formasie ervaar is, het getoon dat weinig gemaksverskil verwag kan word tussen die verskil- 
lende formasievliegtuie onder dieselfde vlugtoestande. Die gevolgtrekking vir albei vliegtuie was dat 'n sitplekligging voor in die vliegtuig meer gemaklik is as een agter in die vliegtuig. Tydens ligte en matige turbulensie het die algehele versnellingsgroottes gebly binne die nie ongemaklik nie-gebied (not uncomfortable) soos voorgestel deur die ISO 2631-1-standaard. Die persentasie siek passasiers in geïsoleerde en formasievlug is baie laag, wat beteken die voorkoms van bewegingsiekte (motion sickness) tydens formasievlug verskil byna glad nie van die voorkoms daarvan tydens geïsoleerde vlug nie. 


\section{Acknowledgements}

This project would not have been possible if it wasn't for the people who supported me along the way. In all honesty, there are a countless number of people I would like to thank. Specifc thanks goes out to the following people:

- Japie Engelbrecht (soon Dr. Engelbrecht), I can’t thank you enough for the support, advice, and jokes that you have shared with me. It has been a pleasure to have you as a supervisor.

- Gerrie van Wyk, my lab buddy, thanks for the countless discussions, help when I needed it and the long hours spent together working on fixing simulation bugs.

- The staff of the Electronic Systems Laboratory (ESL) and all the people who keep the place running, I have been very fortunate to do my postgraduate studies here.

- Everyone who studied or worked in the ESL through 2014 to 2015, for all the support, friendships and good stories.

- Manrich van Greunen and Daniël Schoonwinkel, for the coffee mornings in the MIH Media Lab, the right way to start every day.

- The National Research Foundation as well as the National Aerospace Centre of South Africa, for providing funding for the project.

- All formation flight research collaborators, and special thanks to Jordan Adams from the University of Cape Town. The information and chat sessions were invaluable.

- ESL Engineers Cornelus le Roux, Chris Fourie, Wiaan Beeton and Nico Alberts.

- Dr. Layla Cassim, thesis writing workshop facilitator, for her valuable presentations, tips and advice for writing and adding finishing touches to my thesis.

- Jolette Roodt, for proof-reading and editing of my thesis.

- My family, for the support, love and care you have always given to me. 


\section{Dedications}

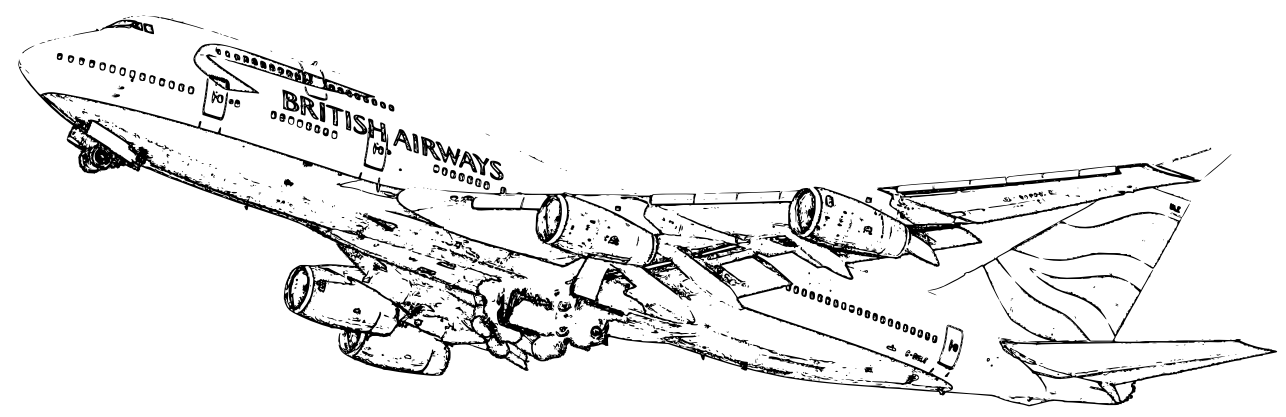

Hierdie tesis word opgedra aan my familie en vriende 


\section{Contents}

Declaration i

Abstract

Opsomming iv

\begin{tabular}{|ll}
\hline Acknowledgements & vi
\end{tabular}

Dedications vii

Contents viii

List of Figures $\quad$ xiii

List of Tables $\quad$ xviii

Nomenclature $\quad x x$

1 Introduction 1

1.1 Project Description . . . . . . . . . . . . . . . . . . . . . . 3

1.2 Problem Statement . . . . . . . . . . . . . . . . . . . . . . . . . . . . . . . . . . . . . .

$1.2 .1 \quad$ Research Question . . . . . . . . . . . . . . . . . . . . . . . . . . . . . . . . . . . . . .

1.2 .2 Research Objectives . . . . . . . . . . . . . . . . . . 4

1.3 Expected Results and Significance $\ldots \ldots \ldots \ldots$. . . . . . . . . . . . . . 4

1.4 Thesis Outline . . . . . . . . . . . . . . . . . . . . . . 5

\begin{tabular}{lll}
\hline 2 & Literature Review & $\mathbf{7}$
\end{tabular}

2.1 Formation Flight . . . . . . . . . . . . . . . . . . . . . . . . 7

$2.1 .1 \quad$ Drag Savings $\ldots \ldots \ldots \ldots \ldots$. . . . . . . . . . . . . . . 7

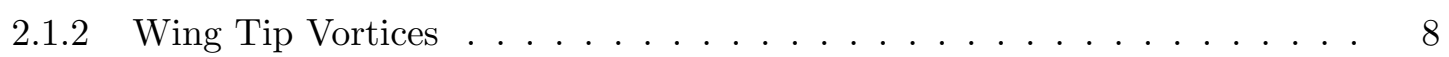

$2.1 .3 \quad$ Close and Extended Formation Flight . . . . . . . . . . . . . . . . . 9

2.1 .4 Formation Types $\ldots \ldots \ldots \ldots \ldots \ldots$

2.2 Passenger Ride Comfort $\ldots \ldots \ldots \ldots \ldots \ldots$

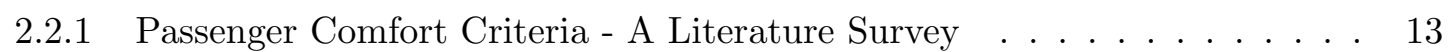

$2.2 .2 \quad$ Flight Control Laws and Passenger Comfort Improvement . . . . . . . . . . 14 
2.3 Collaborative Internal Research $\ldots \ldots \ldots$. . . . . . . . . . . . . . . . . . . . . . . 19

2.3.1 Passenger Comfort during Formation Flight within Atmospheric Turbulence 20

$2.3 .2 \quad$ Automatic Control of Commercial Airliners in Formation Flight . . . . . 23

\begin{tabular}{lll}
\hline 3 & Mathematical Modelling & $\mathbf{2 7}$
\end{tabular}

3.1 Axis Systems and Notation $\ldots \ldots \ldots \ldots \ldots \ldots \ldots$. . . . . . . . . . . . . . . . . . . . . . .

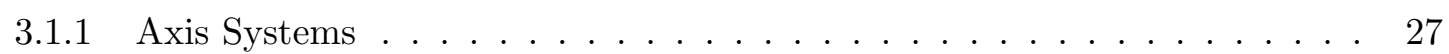

3.1 .2 Aircraft Notation . . . . . . . . . . . . . . . . . . . . . 29

3.2 Development of Formation Flight Model for Simulation . . . . . . . . . . . . . . 31

3.3 Conventional Model . . . . . . . . . . . . . . . . . . . . . 33

3.3 .1 Six Degrees of Freedom Equations of Motion . . . . . . . . . . . . . . 33

3.3 .2 Attitude and Position Dynamics $\ldots \ldots \ldots \ldots \ldots$. . . . . . . . . . . . . . . . . . . . . . 35

3.3 .3 Forces and Moments . . . . . . . . . . . . . . . . . . . . 36

3.4 Aircraft Separation . . . . . . . . . . . . . . . . . . . . . . . 41

$3.4 .1 \quad$ Geometric Separation . . . . . . . . . . . . . . . . . . . . . 41

3.4 .2 Effective Separation . . . . . . . . . . . . . . . . . . . . . . . . . . . . . . . . . 43

3.5 Formation-Extended Model . . . . . . . . . . . . . . . . . . . . . . . . . . 46

$3.5 .1 \quad$ Wake Interaction Effects during Formation Flight . . . . . . . . . . . . . . . . . . 48

3.5 .2 Feasible Geometric Separations for Formation Flight . . . . . . . . . . . . 49

$3.5 .3 \quad$ Full Aerodynamic Model for Formation Flight . . . . . . . . . . . . . . . . . . . . . 53

3.6 Modelling the Effects of Atmospheric Turbulence . . . . . . . . . . . . . . . . . . 54

3.7 Modelling the Vibrations . . . . . . . . . . . . . . . . . . 54

$3.7 .1 \quad$ Axis Systems of Seated Passenger . . . . . . . . . . . . . . . 54

3.7 .2 Vibration Measurement . . . . . . . . . . . . . . . . . 55

3.8 A Fully Integrated Dynamic Formation Flight Simulation Model . . . . . . . . . 57

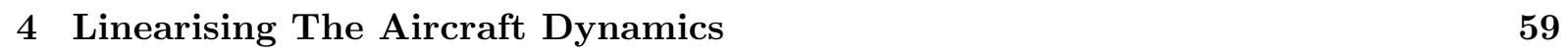

4.1 Small Disturbance Theory . . . . . . . . . . . . . . . . . . . . . . . . 59

4.2 Defining and Calculating the Trim Condition Variables . . . . . . . . . . . . . . . . 60

4.2 .1 The Straight and Level Flight Trim Condition . . . . . . . . . . . . . . . 60

4.2 .2 Calculated Trim Values . . . . . . . . . . . . . . . . . . . . . 62

4.3 Linearising About Trim $\ldots \ldots \ldots \ldots$. . . . . . . . . . . . . . 63

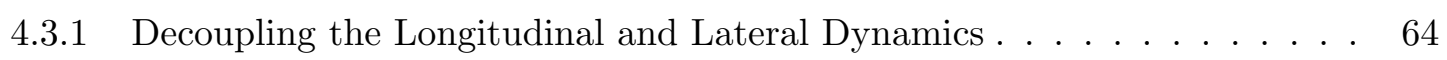

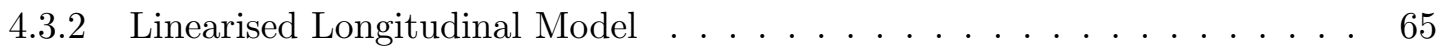

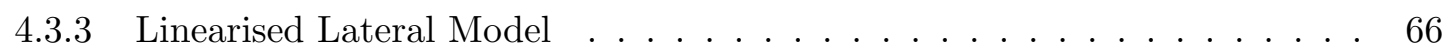

4.4 A Linear Analysis $\ldots \ldots \ldots$. . . . . . . . . . . . . . . . . . . . 67

$4.4 .1 \quad$ Longitudinal Modes of Motion . . . . . . . . . . . . . . . . . . . . . . . . . . 68

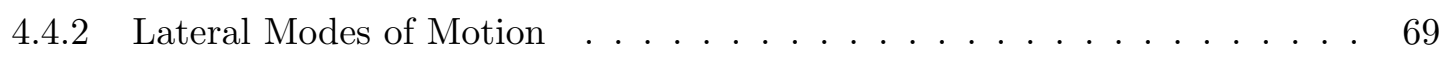

4.4 .3 Response to Controls . . . . . . . . . . . . . . . . . . . . . . . . . . . . . . . . .

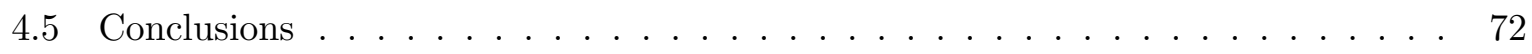

\begin{tabular}{|lll}
5 & Conventional Flight Control Systems Design & 75
\end{tabular} 
5.1 Fly-by-wire in Commercial Aircraft $\ldots \ldots \ldots \ldots$. . . . . . . . . . . 75

5.1 .1 Objectives and Constraints $\ldots \ldots \ldots \ldots \ldots$

5.1 .2 Control Law Structure . . . . . . . . . . . . . . . . . . . 77

$5.1 .3 \quad$ Fly-by-wire System Integration $\ldots \ldots \ldots$. . . . . . . . . . . . . . . . . . . . . . .

5.2 Conventional Fly-by-Wire Flight Control Architecture . . . . . . . . . . . . . 80

$5.2 .1 \quad$ Conventional Fly-by-Wire Flight Controllers: Normal Laws . . . . . . . . 80

5.2 .2 Conventional Guidance Laws . . . . . . . . . . . . . . . . . 81

5.3 Longitudinal Control Systems Design . . . . . . . . . . . . . . . . . . . . . . . . . . . . . . . . .

5.3 .1 DQ Law: A Normal Load Factor Controller . . . . . . . . . . . . . . . . . . . . . 82

5.3 .2 Altitude-Hold Guidance: FPA or CR Controller . . . . . . . . . . . . . . 93

5.3 .3 Autothrust: An Airspeed Controller . . . . . . . . . . . . . . . . . 98

5.3 .4 Altitude-Hold Guidance: Altitude Controller . . . . . . . . . . . . . . . . 104

5.4 Lateral Control Systems Design . . . . . . . . . . . . . . . . . . . . . . . . . . . . .

5.4 .1 DP and DR Law: A Roll and Sideslip Angle Controller . . . . . . . . . . 110

5.4 .2 Cross-Track Error Guidance: A Lateral Guidance Controller .. . . . . . 118

5.5 Conventional Flight Control Simulations . . . . . . . . . . . . . . . . . . . . . . . . . 126

$5.5 .1 \quad$ Longitudinal Controller Performance . . . . . . . . . . . . . . . . 126

5.5 .2 Lateral Controller Performance . . . . . . . . . . . . . . . . . . . 129

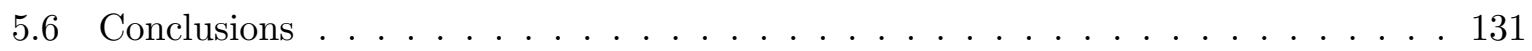

$6 \quad$ Extended Formation Flight Control Systems Design 132

6.1 Extended Formation Flight Control Architecture . . . . . . . . . . . . . . . . 132

6.1 .1 Formation-Extended Guidance Laws . . . . . . . . . . . . . . . . . 133

6.2 Longitudinal Formation Guidance $\ldots \ldots \ldots$. . . . . . . . . . . 133

6.2 .1 Axial Guidance Controller . . . . . . . . . . . . . . . . . . . 133

6.2 .2 Vertical Guidance Controller . . . . . . . . . . . . . . . . . 137

6.3 Lateral Formation Guidance . . . . . . . . . . . . . . . . . . . . . . . . . . . 142

6.3 .1 Lateral Guidance Controller . . . . . . . . . . . . . . . . . . . . . . . . . . . . . . . . . . . . 142

6.4 Extended Formation Flight Simulations ． . . . . . . . . . . . . . . . . 143

6.4 .1 Performance of Formation Guidance Laws . . . . . . . . . . . . . . . . . 144

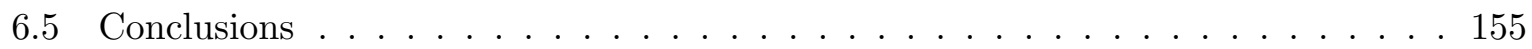

\begin{tabular}{lll}
\hline & Passenger Comfort & 156
\end{tabular}

7.1 Rigid Body Aircraft with Seat Transmissibility . . . . . . . . . . . . . 156

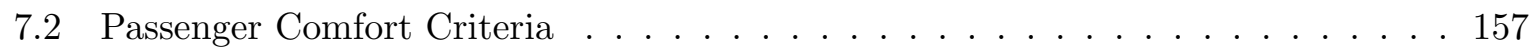

7.2 .1 Vibration Evaluation Method . . . . . . . . . . . . . . . . . . . 158

7.2 .2 Effect of Vibrations on Levels of Comfort . . . . . . . . . . . . . . . 161

7.2 .3 Effect of Vibrations on the Incidence of Motion Sickness . . . . . . . . . . 162

7.3 Development of Simulations for Ride Comfort Evaluation . . . . . . . . . . . . 163

7.3 .1 Simulation Resolution . . . . . . . . . . . . . . . . . . . . . . . . . . . . . . . . . 163

7.3 .2 Seating Locations. . . . . . . . . . . . . . . . . . . . . . . 164

7.3.3 Feasible Geometric Separations for Passenger Comfort Evaluation . . . . 165 
7.4 Results and Discussion $\ldots \ldots \ldots \ldots$. . . . . . . . . . . . . . 166

7.4 .1 Measured Acceleration Data . . . . . . . . . . . . . . . . . 166

$7.4 .2 \quad$ Power Spectral Densities . . . . . . . . . . . . . . . . . . . 167

$7.4 .3 \quad$ Weighted Power Spectral Densities . . . . . . . . . . . . . . . . . . 174

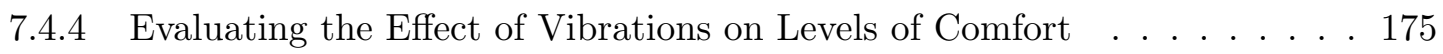

7.4.5 Evaluating the Effect of Vibrations on Motion Sickness Incidence . . . . . 178

7.5 Conclusions $\ldots \ldots \ldots \ldots \ldots \ldots$

8 Conclusions, Recommendations and Future Work $\quad 180$

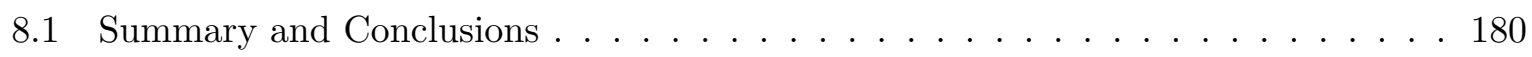

8.2 Recommendations and Future Work . . . . . . . . . . . . . . . . . . . . 183

\begin{tabular}{lr}
\hline Appendices & 186
\end{tabular}

\begin{tabular}{|lr}
\hline A Conventional Aerodynamic Model & 187
\end{tabular}

\begin{tabular}{ll}
\hline B Conventional Linear Model Partial Derivatives & 191
\end{tabular}

B.1 Longitudinal Partial Derivatives . . . . . . . . . . . . . . . . . . . . . . . . . . . . . . . . . 191

B.1.1 Translational Acceleration Derivatives . . . . . . . . . . . . . . . . 192

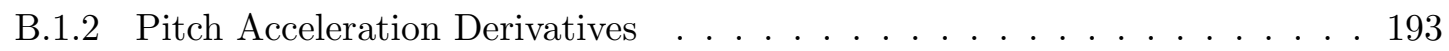

B.1.3 Pitch Attitude Rate Derivatives . . . . . . . . . . . . . . . . . . . . 193

B.2 Lateral Partial Derivatives . . . . . . . . . . . . . . . . . . . . . . . 193

B.2.1 Translational Acceleration Derivatives . . . . . . . . . . . . . . . . 194

B.2.2 Roll Acceleration Derivatives . . . . . . . . . . . . . . . . . . 194

B.2.3 Yaw Acceleration Derivatives . . . . . . . . . . . . . . . . . . 195

B.2.4 Roll Attitude Rate Derivatives . . . . . . . . . . . . . . . . . . 195

\begin{tabular}{lll}
\hline C Linear Incremental Normal Load Factor Partial Derivatives & 196
\end{tabular}

\begin{tabular}{|l|l|}
\hline D Conventional Aircraft Response Transfer Functions & 197
\end{tabular}

\begin{tabular}{|lll}
\hline E Conventional Flight Control Simulation Results & 199
\end{tabular}

\begin{tabular}{lll}
\hline F & Wake Interaction Model & 205
\end{tabular}

F.1 Influence Parameters . . . . . . . . . . . . . . . . . . . . . . . . . . . 205

F.2 Wake Model Inaccuracies . . . . . . . . . . . . . . . . . . 207

F.3 Feasible Regions . . . . . . . . . . . . . . . . . . . . . . . . . 209

F.4 Conclusion $\ldots \ldots \ldots \ldots \ldots \ldots$

G Extended Formation Flight Simulations 210

G.1 Geometric Separation Tracking Performance Simulations . . . . . . . . . . . . . . 210

G.2 Formation-Hold Performance Simulations . . . . . . . . . . . . . . . . . . 215

\begin{tabular}{ll}
\hline H Von Kármán Atmospheric Turbulence & 228
\end{tabular} 
$\begin{array}{lll}I & \text { Spectral Density Estimation } & 231\end{array}$

I.1 The Welch Method . . . . . . . . . . . . . . . . . . . . . . . . . . . . 231

I.1.1 FFT and Windowing . . . . . . . . . . . . . . . . . . 231

I.1.2 Normalised Power Spectral Density . . . . . . . . . . . . . . . . . . . . . . . . 232

$[.2$ Implementation of the Welch Method in MATLAB . . . . . . . . . . . . . . . . . . . . . . . . . . . . .

I.2.1 Frequency Resolution and Length of the DFT . . . . . . . . . . . . . . . . . . . . . . . . . . . . . . . . . . . . . .

I.2.2 Window Function . . . . . . . . . . . . . . . . . . 235

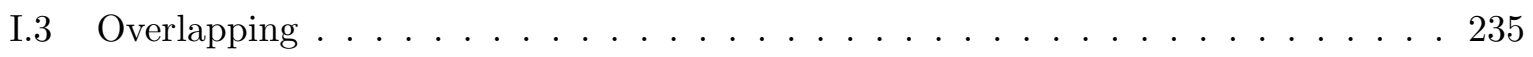

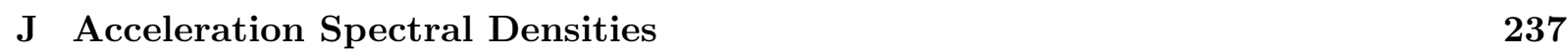

\begin{tabular}{ll}
\hline K Comfort and Motion Sickness & 249
\end{tabular}

\begin{tabular}{lr}
\hline Bibliography & 253
\end{tabular} 


\section{List of Figures}

$2.1 \quad$ Vertical component of velocity induced behind the aircraft . . . . . . . . . . . . 8

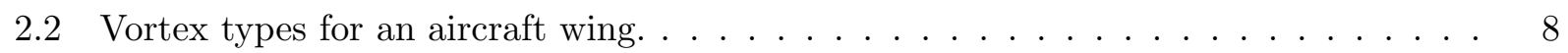

2.3 Close formation flying of an Airbus A380 and two Airbus A350 aircraft. . . . . . . . 9

$2.4 \quad$ A horseshoe vortex caused by a uniform lift distribution over an aircraft's wing . . . 11

2.5 The three common types of three-aircraft formations . . . . . . . . . . . . 11

$2.6 \quad$ Formation induced drag reduction as the number of aircraft in the formation increases. 12

2.7 Frequency distribution with the classic and integrated approach to control law design 16

2.8 Frequency weighting filter used for motion sickness sensitivity . . . . . . . . . . . . 18

2.9 Percentage of Ill Passengers in lateral and vertical turbulence . . . . . . . . . . . . . 19

2.10 Comfort improvement in vertical turbulence $\ldots \ldots \ldots \ldots \ldots$

2.11 Horseshoe vortices in right echelon formation . . . . . . . . . . . . . . 20

2.12 Trailing vortices of leader aircraft $\ldots \ldots \ldots \ldots \ldots \ldots \ldots$

2.13 Side and top view of formation in vertical and lateral turbulence . . . . . . . . . . 21

2.14 Overall RMS acceleration magnitudes in a follower aircraft found by Bizinos. . . . . 22

2.15 Mathematical model as used by Büchner for a follower aircraft . . . . . . . . . . . . 24

2.16 A comparison of the aileron deflection and throttle trim settings . . . . . . . . . 25

2.17 Trim values in sandwhich and outer regions over varying vertical separation . . . . . 25

3.1 North-East-Down axis system. $\ldots \ldots \ldots \ldots \ldots \ldots \ldots \ldots$

3.2 B747-100 Aircraft body axis system. . . . . . . . . . . . . . . . . . . 28

$3.3 \quad$ Wind, Stability and Body axes relationship. . . . . . . . . . . . . . . . . . . . . . 29

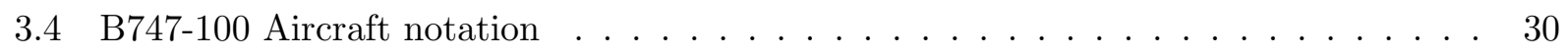

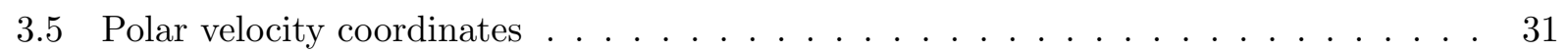

3.6 Proposed framework for the formation flight simulation model. . . . . . . . . . . . 32

3.7 Conventional aircraft model used by an isolated aircraft $\ldots \ldots \ldots \ldots$. . . . . . . 33

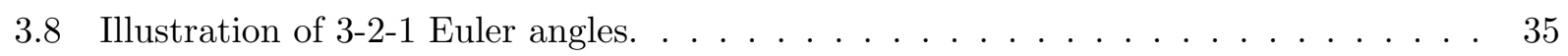

3.9 Top-view of geometric aircraft separation. . . . . . . . . . . . . . . . . . . . 42

3.10 Top-view of geometric aircraft separation. . . . . . . . . . . . . . . . . . 43

3.12 Top-view separation scenario during formation flight with a lateral gust. . . . . . . . 44

3.13 Side-view separation scenario during formation flight with a lateral gust. . . . . . . . 45

3.14 Extended aerodynamic aircraft model used by the follower aircraft. . . . . . . . . . . 47

3.15 Formation flight induced aerodynamic coefficients. . . . . . . . . . . . . 50 
3.16 Trim deflection angles of the right inboard aileron over various lateral separations. 51

3.17 Induced lift and roll coefficients comparison . . . . . . . . . . . . . . . . . . . . . 51

3.18 Trim throttle settings in conventional isolated flight and formation flight. . . . . . . 52

3.19 Axis systems for a passenger in the seated position . . . . . . . . . . . . . . . . . 55

$4.1 \quad$ Forces and moments at straight and level flight trim. . . . . . . . . . . . . . . . . . . 61

4.2 Conventional isolated linear model modes of motion. . . . . . . . . . . . . . . . . . . . 68

4.3 Longitudinal response to an elevator step input. . . . . . . . . . . . . . . . . . . . . 71

4.4 Lateral response to an aileron step input. . . . . . . . . . . . . . . . . . . . . . . . . . 73

4.5 Lateral response to an rudder step input. . . . . . . . . . . . . . . . . . . . . . . . . . 74

$4.6 \quad$ Closer look at the roll rate response to rudder deflection. . . . . . . . . . . . . . . . . 74

5.1 General structure of control laws . . . . . . . . . . . . . . . . . . . . . . . . . . 76

5.2 Longitudinal control law structure . . . . . . . . . . . . . . . . . . . . . . . . . . . . 77

5.3 Lateral control law structure . . . . . . . . . . . . . . . . . . . . . . . . . 78

5.4 General structure of the Airbus fly-by-wire system . . . . . . . . . . . . . . . . . . . 79

5.5 A representative architecture of conventional fly-by-wire flight control . . . . . . . . 80

$5.6 \quad$ Overview of the altitude-hold guidance law strategy. . . . . . . . . . . . . . . . . . . 81

$5.7 \quad$ Full-order vs reduced-order longitudinal model . . . . . . . . . . . . . . . . . . . . . 83

5.8 Illustration of the normal load factor during straight and level flight. . . . . . . . . . 85

5.9 Illustration of total normal load factor during a longitudinal manoeuvre. . . . . . . . 85

5.10 Aircraft illustrated as a point mass during a steady, coordinated turn. . . . . . . . . 86

5.11 DQ law control architecture. . . . . . . . . . . . . . . . . . . . . . 89

5.12 Short-period dynamic requirements for Category A flight phases of MIL-STD-1797A. 90

5.13 DQ law closed-loop step response, actuator response and pole placement . . . . . . . 91

5.14 Closed-loop poles after adding the DQ law controller. . . . . . . . . . . . . . . . . 92

5.15 Flight path angle controller architecture. . . . . . . . . . . . . . . . . . . . . . . . 93

5.16 Root locus of full-order FPA controller . . . . . . . . . . . . . . . . . . . . . . . . . . 94

5.17 Reduced-order flight path angle controller architecture. . . . . . . . . . . . . . . . . 95

5.18 Root locus of reduced-order FPA controller . . . . . . . . . . . . . . . . . . . . . . . 95

5.19 Unit step response of the non-linear FPA controller in the A330 aircraft model. . . . 95

5.20 Closed-loop step response and pole placement of reduced-order FPA controller . . . 96

5.21 Closed-loop step response and pole placement of full-order FPA controller . . . . . . 97

5.22 Closed-loop poles after adding the FPA controller. . . . . . . . . . . . . . . . . . . . 98

5.23 Full-order longitudinal step response results for a $1^{\circ}$ FPA command . . . . . . . . . 99

5.24 Autothrust controller architecture. . . . . . . . . . . . . . . . . . . . 99

5.25 Root locus of autothrust controller . . . . . . . . . . . . . . . . . . . . . . . . . 100

5.26 Evaluation of the A330 non-linear model response to commanded airspeeds. . . . . . 100

5.27 Root locus of the autothrust controller . . . . . . . . . . . . . . . . . . . 101

5.28 Closed-loop step response and control signal for airspeed steps . . . . . . . . . . . . 102

5.29 Actuator response to a commanded airspeed of $1 \mathrm{~m} / \mathrm{s}$. . . . . . . . . . . . . . . . 103

5.30 Closed-loop poles after adding the autothrust controller. . . . . . . . . . . . . . . . . 104 
5.31 Altitude controller architecture. . . . . . . . . . . . . . . . . . 105

5.32 Root locus of altitude controller $\ldots \ldots \ldots \ldots \ldots$. . . . . . . . . . . . . . . . . . . . . . .

5.33 Altitude response of A330 non-linear model. . . . . . . . . . . . . . . . 106

5.34 Root locus and unit step response of altitude controller $\ldots \ldots$. . . . . . . . 107

5.35 Full-order longitudinal step response results for a $15 \mathrm{~m}$ altitude command. . . . . . . 108

5.36 Closed-loop poles after adding the altitude controller. . . . . . . . . . . . . . . . . . . . . . . . . . 11

5.37 Full architecture of the DP and DR Law . . . . . . . . . . . . . . . . . . . . . 111

5.38 DP and DR architecture with sideslip output and rudder turn coordination . . . . 112

5.39 Non-linear roll and sideslip response in the A330 aircraft model . . . . . . . . . . . 114

5.40 Closed-loop poles after full-state feedback using an LQR optimisation algorithm . . 114

5.41 Roll response without turn coordination . . . . . . . . . . . . . . . . . 115

5.42 Bode plot of the reduced-order RCC. . . . . . . . . . . . . . . . . . 115

5.43 Roll response with rudder turn coordination $\ldots \ldots \ldots$. . . . . . . . . . 116

5.44 Sideslip response with a natural aircraft response in roll angle . . . . . . . . . . . . . . . . . . . . . . . 11

5.45 Closed-loop poles after adding the DP and DR law. . . . . . . . . . . . . . 119

5.46 Illustration of cross-track error and in-track distance . . . . . . . . . . . . . . . 120

5.47 Full architecture of the cross-track error controller. . . . . . . . . . . . . . . . . . . 121

5.48 Root locus of derivative feedback loop $\ldots \ldots \ldots \ldots \ldots \ldots$

5.49 Root locus of proportional control loop . . . . . . . . . . . . . . . . 123

5.50 Root locus and unit step response of derivative loop controller . . . . . . . . . . 124

5.51 Root locus and unit step response of cross-track controller . . . . . . . . . . . . . 124

5.52 Cross-track error response of the full-order linear aircraft model . . . . . . . . . . 125

5.53 Closed-loop poles after adding the cross-track error guidance controller. . . . . . . . 126

5.54 Linear and non-linear altitude step response $\ldots \ldots \ldots \ldots$. . . . . . . . . . . . . . . . . . . . . . . 127

5.55 Linear and non-linear airspeed step response $\ldots \ldots \ldots \ldots . \ldots \ldots$

5.56 Unexpected response in linear model for an airspeed step command . . . . . . . . . 129

5.57 Lateral aircraft response with only the DPDR law active . . . . . . . . . . . . . 130

5.58 Lateral tracking response for a $5 \mathrm{~m}$ step command. . . . . . . . . . . . . . . 131

6.1 Overview of the extended formation flight control architecture. . . . . . . . . . . 133

6.2 Architectural change for the axial separation controller . . . . . . . . . . . . . . . 133

6.3 Longitudinal/axial separation controller architecture. . . . . . . . . . . . . . . . . . 134

6.4 Root locus of full-order axial controller . . . . . . . . . . . . . . . . . . . . 135

6.5 Root locus and unit step response of axial separation guidance controller . . . . 136

6.6 Full-order axial step response results $\ldots \ldots \ldots \ldots$. . . . . . . . . . . . . 136

6.7 Closed-loop poles after adding the axial separation controller. . . . . . . . . . . . . 137

6.8 Architectural change for the vertical separation controller . . . . . . . . . . . . . . 138

6.9 Vertical separation controller architecture . . . . . . . . . . . . . . . . . . 138

6.10 Root locus of vertical separation controller $\ldots \ldots \ldots$. . . . . . . . . . . . . 139

6.11 Root locus and unit step response of vertical separation controller . . . . . . . . . 140

6.12 Unit step response results for a commanded vertical separation . . . . . . . . . . . . 141 
6.13 Closed-loop poles after adding the vertical separation controller . . . . . . . . . . . 141

6.14 Architectural change for the lateral separation controller . . . . . . . . . . . . . . 142

6.15 Lateral separation controller architecture. . . . . . . . . . . . . . . . . 143

6.16 Lateral response results in light turbulence for a commanded lateral separation . . . 145

6.17 Revised lateral separation controller architecture. . . . . . . . . . . . . . . 146

6.18 Lateral response results in light turbulence after adding a limited integrator . . . . . 146

6.19 Position response of aircraft during formation flight in moderate turbulence . . . . . 147

6.20 Longitudinal and lateral response results during formation flight in light turbulence 149

6.21 Geometric separations during formation flight in varying levels of turbulence intensity 151

6.22 Formation-hold performance during formation flight in light turbulence . . . . . . . 152

6.23 Aircraft response in light turbulence and decreasing lateral separation . . . . . . . 153

6.24 Formation-hold error of follower aircraft in moderate and severe turbulence . . . . . 154

7.1 Vertical and lateral transmissibility ratios. . . . . . . . . . . . . . . . . . . 157

7.2 Axis systems for a passenger in the seated position (repeated) . . . . . . . . . . . . 157

7.3 Basic Evaluation Method for vibration evaluation. . . . . . . . . . . . . . . . . 158

$7.4 \quad$ Frequency weighting curves for principal and additional weightings. . . . . . . . . 160

7.5 Seating locations in the Boeing 747-100 international all-economy arrangement . . . 164

7.6 Measured time series feet acceleration data in a leader aircraft. . . . . . . . . . . . 167

7.7 Comparative data indicating the range of peak accelerations during cruise. . . . . 167

7.8 Linear feet and seat-surface acceleration spectra near the CG. . . . . . . . . . . . . . . . . . . . . . . . . . .

7.9 Leader acceleration spectra near the CG $\ldots \ldots \ldots \ldots$. . . . . . . . . . . . . . . . . . . . . 169

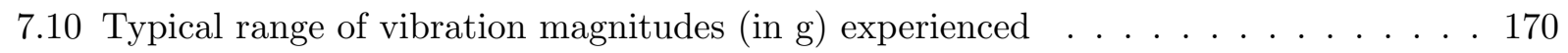

7.11 Linear and rotational acceleration spectra in formation . . . . . . . . . . . . 170

7.12 Linear and rotational acceleration spectra at a seating location A near the CG . . . 171

7.13 Linear passenger acceleration spectra at the seat-surface in a follower aircraft . . . . 173

7.14 Weighted spectral density estimations of accelerations for the frequency range $0.5-50 \mathrm{~Hz} 174$

7.15 Weighted spectral density estimation of accelerations for the frequency range $0.1-0.5 \mathrm{~Hz} 175$

7.16 Levels of comfort in a leader and follower along the aircraft fuselage. . . . . . . . 176

7.17 Comfort in light and moderate turbulence comparison plot. . . . . . . . . . . . . 177

7.18 Percentage of ill passengers in the vertical and lateral axes of a leader and follower . 178

8.1 Illustration of symmetric and anti-symmetric elastic modes . . . . . . . . . . . 184

A.1 Aerodynamic sign conventions (stability axis) $\ldots \ldots \ldots \ldots \ldots$. . . . . . . . 188

E.1 Longitudinal response results for a $5 \mathrm{~m}$ altitude step command . . . . . . . . . . . . 200

E.2 $\quad$ Longitudinal response results for a $5 \mathrm{~m} / \mathrm{s}$ airspeed step command . . . . . . . . . . . 201

E.3 Lateral response results for a $5^{\circ}$ roll reference command . . . . . . . . . . . . . . . 202

E.4 Lateral response results for a $1^{\circ}$ sideslip reference command . . . . . . . . . . . . . . 203

E.5 Lateral response results for a 5 m cross-track error step command . . . . . . . . . . . 204

F.1 Formation-induced aerodynamic coefficients as a function of lateral separation. . . . 207 
F.2 The approximated method versus numerical integration over an elliptical wing . . . 208 F.3 Induced lift and roll coefficients comparison . . . . . . . . . . . . . . . . . 209

G.1 Position response of aircraft during formation flight in varying levels of turbulence . 211 G.2 Longitudinal and lateral response during formation in light turbulence . . . . . . . . 212

G.3 Longitudinal and lateral response during formation in moderate turbulence . . . . . 213 G.4 Longitudinal and lateral response during formation in severe turbulence . . . . . . . 214 G.5 Position response of aircraft during formation flight in varying levels of turbulence . 216 G.6 $\quad$ Formation-hold performance at $\eta=1.5$ wingspans during formation flight . . . . . . 217 G.7 Position response of aircraft during formation flight in varying levels of turbulence . 218 G.8 $\quad$ Formation-hold performance at $\eta=1.4$ wingspans during formation flight . . . . . . 219 G.9 Position response of aircraft during formation flight in varying levels of turbulence . 220 G.10 Formation-hold performance at $\eta=1.3$ wingspans during formation flight . . . . . . 221 G.11 Position response of aircraft during formation flight in varying levels of turbulence . 222 G.12 Formation-hold performance at $\eta=1.2$ wingspans during formation flight . . . . . . 223 G.13 Position response of aircraft during formation flight in varying levels of turbulence . 224 G.14 Formation-hold performance at $\eta=1.1$ wingspans during formation flight . . . . . . 225 G.15 Position response of aircraft during formation flight in varying levels of turbulence . 226 G.16 Formation-hold performance at $\eta=1.0$ wingspans during formation flight . . . . . . 227

H.1 Lookup table used for turbulence intensities. . . . . . . . . . . . . . . . . . . 230

I.1 A comparison between an arbitrary length Hanning and Hamming window . . . . . 235

K.1 Levels of comfort in a leader and follower along the aircraft fuselage. . . . . . . . . . 250

K.2 Percentage of ill passengers in a leader and follower aircraft during light turbulence. 251 K.3 Percentage of ill passengers in a leader and follower aircraft during moderate turbulence.252 


\section{List of Tables}

3.1 Standard aircraft notation used in the inertial axis. . . . . . . . . . . . . . . . . 30

3.2 B747-100 Aircraft notation used in the body axis. . . . . . . . . . . . . . . 30

3.3 Geometric separations used in the extended non-linear formation flight simulations . 49

4.1 Modes of motion for the linear isolated conventional aircraft. . . . . . . . . . . . . 68

4.2 Longitudinal characteristics $\ldots \ldots \ldots \ldots \ldots \ldots$

4.3 Lateral characteristics $\ldots \ldots \ldots \ldots \ldots \ldots$

5.1 Comparison of altitude response specifications. . . . . . . . . . . . . . 127

5.2 Comparison of airspeed response specifications. . . . . . . . . . . . . . . . 128

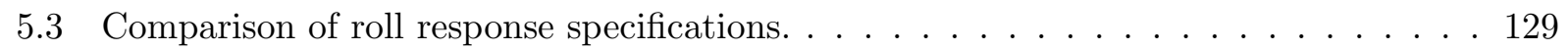

5.4 Comparison of sideslip response specifications. . . . . . . . . . . . . . 130

5.5 Comparison of cross-track error tracking response specifications. . . . . . . . . . . . 131

6.1 Simulation settings used to evaluate the formation guidance performance. . . . . . . 144

6.2 Simulation settings used to evaluate the formation guidance performance. . . . . . . 147

6.3 Summary of formation-hold performance . . . . . . . . . . . . . . . . 154

7.1 Standard notation used in the seated passenger axis. . . . . . . . . . . . . 158

7.2 Frequency weightings used for comfort evaluation. . . . . . . . . . . . . . . . 159

7.3 Approximate indications of likely reactions to various magnitudes of the total vibration 162

7.4 Seating location coordinates from the CG in an international all-economy B747-100. 165

7.5 Summary of feasible formation flight regions for passenger comfort evaluation . . . . 165

A.1 Summary of aerodynamic equation variables at the linearisation point. . . . . . . . . 187

A.2 Summary of non-dimensional aerodynamic coefficients at the linearisation point. . . 187

A.3 Summary of the longitudinal and lateral aerodynamic stability and control derivatives. 189

D.1 Longitudinal and lateral coefficients of the denominator in the response transfer function . . . . . . . . . . . . . . . . . . . . . . . . . . . 198

D.2 Longitudinal and lateral coefficients of the numerator in the response transfer function 198

G.1 Reference guide for separation tracking performance simulations . . . . . . . . . . 210

G.2 Reference guide for formation-hold performance simulations . . . . . . . . . . . . . . 215 
H.1 MIL-STD-1797A spectral component functions and forming filter transfer functions 229

H.2 Turbulence severities . . . . . . . . . . . . . . . . . . . . 230

I.1 Correction factors for three different window types $\ldots \ldots \ldots \ldots$. . . . . . . 232

J.1 $\quad$ Reference guide for linear and rotational acceleration spectra estimations _ . . . . . 237

K.1 Reference guide for case 1 vibrational comfort and PIP results . . . . . . . . . . . . 249 


\section{Nomenclature}

\section{Acronyms}

6DOF Six Degrees of Freedom

ADIRUs Air Data and Inertial Reference Units

ALT Alternative Mode

APC Aircraft Pilot Coupling

CG Centre of Gravity

CIT Comfort In Turbulence

DCM Direction Cosine Matrix

DFT Discrete Fourier Transform

DGPS Differential Global Positioning System

DIR Direct Mode

ELACs Elevator and Aileron Computers

ESL Electronic Systems Laboratory

FBW Fly-by-wire

FCPCs Flight Control Primary Computers

FCS Flight Control System

FCSCs Flight Control Secondary Computers

FFT $\quad$ Fast Fourier Transform

FRL Fuselage Reference Line

GPS Global Positioning System

ISO The International Organisation for Standardisation 
LQR Linear-quadratic Regulator

MSDV Motion Sickness Dose Value

NAC National Aerospace Centre

NED North-East-Down

PI Proportional-Integral

PIP Percentage of Ill Passengers

RCC Rudder Coordination Controller

RMS Root Mean Square

SECs Spoiler and Elevator Computers

SISO Single-Input-Single-Output

SUN Stellenbosch University

UCT University of Cape Town

\section{Aerodynamic}

$C_{D} \quad$ Aerodynamic drag coefficient

$C_{L} \quad$ Aerodynamic lift coefficient

$C_{l} \quad$ Aerodynamic roll coefficient

$C_{m} \quad$ Aerodynamic pitch coefficient

$C_{n} \quad$ Aerodynamic yaw coefficient

$C_{X} \quad$ Aerodynamic axial force coefficient

$C_{Y} \quad$ Aerodynamic side force coefficient

$C_{Z} \quad$ Aerodynamic normal force coefficient

\section{Physical}

$\begin{array}{ll}\bar{c} & \text { Mean aerodynamic chord } \\ b & \text { Wingspan of the aircraft } \\ g & \text { Gravitational acceleration } \\ I_{x x}, I_{y y}, I_{z z} & \text { Moments of inertia about the } X_{B}, Y_{B}, Z_{B} \text { body axes } \\ L_{\text {Track }} & \text { Ground track length }\end{array}$


Aircraft mass

$S$

Wing surface area

\section{Atmospheric}

$\begin{array}{ll}\rho & \text { Air density } \\ q & \text { Dynamic pressure } \\ v_{g} & \text { Lateral turbulent velocity } \\ w_{g} & \text { Vertical turbulent velocity }\end{array}$

\section{Vectors and Matrices}

$\boldsymbol{A} \quad$ Continuous system matrix

$\boldsymbol{B} \quad$ Continuous input matrix

$C \quad$ Continuous input matrix

$\boldsymbol{u}$ Control input vector

$\boldsymbol{x} \quad$ System state vector

$\dot{\omega} \quad$ Angular acceleration of the aircraft about the CG

$\omega \quad$ Angular velocity of the aircraft about the CG

$\mathbf{a}_{p} \quad$ Linear acceleration at a point $p$

$\mathbf{a}_{r} \quad$ Passenger rotational acceleration

$\mathbf{a}_{c g} \quad$ Aircraft acceleration at the centre of gravity

$\mathbf{a}_{r e l} \quad$ The acceleration of point $p$ measured relative to a rotating axes at the CG

Q State weighting matrix

R Control weighting matrix

$\mathbf{r}_{p / c g} \quad$ Relative displacement vector between the point $p$ and the centre of gravity

$\mathbf{v}_{\text {rel }} \quad$ The velocity of point $p$ measured relative to rotating axes at the CG

$\vec{i}, \vec{j}, \vec{k} \quad$ Inertial axes unit vectors

$\vec{i}_{B}, \vec{j}_{B}, \vec{k}_{B} \quad$ Body axes unit vectors

$\vec{i}_{s}, \vec{j}_{s}, \vec{k}_{s} \quad$ Separation axes unit vectors

\section{Co-ordinate Vectors}


$\bar{V}, \alpha, \beta \quad$ Polar velocity magnitude, angle of attack and angle of sideslip

$\delta_{A}, \delta_{E}, \delta_{R} \quad$ Aileron, elevator and rudder control surface deflection respectively

$\phi, \theta, \psi \quad$ Roll, pitch and yaw angle respectively

$L, M, N \quad$ Roll, pitch and yaw moment respectively

$N, E, D \quad$ North, east and down position coordinates respectively

$P, Q, R \quad$ Roll, pitch and yaw rate respectively

$U, V, W \quad$ Axial, lateral and normal velocity respectively

$X, Y, Z \quad$ Axial, lateral and normal force respectively

\section{Formation}

$\bar{V}_{\text {Formation }}$ The average formation flight airspeed

$\Delta x \quad$ Geometric axial separation

$\Delta y \quad$ Geometric lateral separation

$\Delta z \quad$ Geometric vertical separation

$\eta \quad$ Geometric lateral separation, normalised to aircraft wingspan

$\eta_{\text {eff }} \quad$ Effective lateral separation, normalised to aircraft wingspan

$\eta_{I, \text { eff }} \quad$ Instantaneous effective lateral separation, normalised to aircraft wingspan

$\tau_{d} \quad$ Time delay between two aircraft in formation

$\xi \quad$ Geometric longitudinal separation, normalised to aircraft wingspan

$\zeta \quad$ Geometric vertical separation, normalised to aircraft wingspan

$\zeta_{\text {eff }} \quad$ Effective vertical separation, normalised to aircraft wingspan

$\zeta_{I, e f f} \quad$ Instantaneous effective vertical separation, normalised to aircraft wingspan

\section{Angles}

$\psi_{a z} \quad$ Flight path heading angle

$\psi_{\text {Track }} \quad$ Track heading angle

$\theta_{g} \quad$ Induced change in separation angle due to lateral or vertical gust disturbance

$\theta_{s} \quad$ Geometric lateral and vertical separation angle

$\theta_{\text {eff }} \quad$ Effective angle of separation due to lateral or vertical gust disturbance 


\section{Control}

\begin{tabular}{|c|c|}
\hline$\Delta$ & Pertubation \\
\hline$\delta_{n_{z}}$ & Incremental normal load factor in the body axis \\
\hline$\dot{h}$ & Climb rate \\
\hline$\gamma$ & Flight path angle \\
\hline $\mathbf{K}_{F F}$ & Feed-forward gain \\
\hline $\mathbf{K}_{F S F}$ & Full-state feedback gain \\
\hline$K_{P}, K_{I}, K_{D}$ & Proportional, Integral and Derivative control gains \\
\hline$K_{\gamma}, K_{\dot{h}}, K_{h}$ & Longitudinal controller gains \\
\hline$K_{\phi_{D P}}, K_{\beta_{D P}}$ & DP Law Feed-forward gains \\
\hline$K_{\phi_{D R}}, K_{\beta_{D R}}$ & DR Law Feed-forward gains \\
\hline$K_{\xi}, K_{\zeta}$ & Formation-specific controller gains \\
\hline$n_{z}$ & Total inertial normal load factor \\
\hline$n_{z_{B, \text { Nominal }}}$ & Nominal normal load factor in the aircraft body axis \\
\hline$n_{z_{B}}$ & Total normal load factor in the aircraft body axis \\
\hline$p, z$ & Poles and zeros \\
\hline$t_{s_{2} \%}$ & $2 \%$ settling time \\
\hline$v$ & Disturbance signal \\
\hline$w_{n}, \zeta$ & Natural frequency and damping ratio \\
\hline ALT & Altitude \\
\hline AS & Axial Separation \\
\hline AT & Autothrust \\
\hline $\mathrm{CR}$ & Climb Rate \\
\hline $\mathrm{CT}$ & Cross Track \\
\hline CTR & Cross Track Rate \\
\hline $\mathrm{FF}$ & Feed-Forward \\
\hline FPA & Flight Path Angle \\
\hline
\end{tabular}


FSF Full-state Feedback

H Heading

VS Vertical Separation

\section{List Of Symbols}

a Translational or rotational root-mean-square (RMS) vibrational acceleration

$H(s) \quad$ Filter transfer function

$k \quad$ Weighting multiplication constant or weighting factor

$K_{m} \quad$ Motion sickness susceptibility or ill constant

$P_{y y} \quad$ Power spectral density function

W $\quad$ Frequency weighting

\section{Subscripts}

$B$

Body axis

dest Destination

F $\quad$ Formation flight

$f \quad$ Frequency-weighted value at the feet

I Inertial axis

$k \quad$ Follower number in formation

$l p \quad$ Linearisation point

$p \quad$ Arbitrary point in fuselage

$r \quad$ Reduced-order

Ref Reference

$S \quad$ Stability axis

$s \quad$ Frequency-weighted value at the seat-surface

src Source

ss Steady-state

T Trim

$v \quad$ Total frequency-weighted value 
$W$

$w$

\section{Superscripts}

A

G

$T$
Wind axis

Frequency-weighted value

Aerodynamic

Gravitational

Thrust 


\section{Chapter 1}

\section{Introduction}

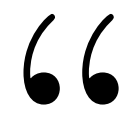

We follow the story about the geese staying in formation.

They fly together, and we ride together. - Christie Cichra

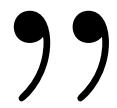

Formation flight of commercial passenger airliners is being considered as an option for increasing the fuel efficiency of large scale air transport [1] 2]. However, the ride comfort of passengers in trailing aircraft of a formation flight convoy is a concern. In the presence of disturbances caused by the trailing vortex of the leader aircraft and the compensatory inputs of the pilot and control laws, questions are being raised about the levels of comfort in a trailing aircraft. In a study by Bizinos [3], the author evaluated the levels of comfort experienced in formation flight during atmospheric turbulence. Investigating various formation separations, static simulations were performed in nominal cruising conditions assuming an ideal control law where disturbance loads are only caused by the presence of turbulence and the trailing vortex. A clear increase in acceleration magnitudes was found for longitudinal, vertical, roll and yaw acceleration magnitudes. Significant increases in discomfort were found in a follower aircraft flying close to the trailing vortex of a leader - especially for seats longitudinally displaced from the aircraft centre of gravity. A shortcoming in this study, however, was the practical simulation of dynamic aircraft motion.

A previous study by Büchner [4] investigated the dynamic simulation of formation flight with automatic flight control, but did not evaluate the ride comfort in the aircraft. The author revealed two formation flight regions where the aircraft could be trimmed with conventional straight and level flight trim settings, and still achieve drag savings relative to the conventional isolated aircraft. These were called the "sandwich" and "outer" regions. The sandwich region is tightly bounded between two un-trimmable regions at approximately 0.7 wingspans lateral separation. The outer region lies at lateral separations of larger than 1.1 wingspans. However, these regions were not at the optimum drag savings position. The author discovered that the follower aircraft could not be trimmed with conventional trim settings at the optimum position due to large rolling moments and aileron saturation. Although successfully demonstrating flight control in formation flight, Büchner worked with limited information regarding the fly-by-wire 
flight control architecture used in modern-day transport aircraft.

In order to evaluate and possibly improve passenger comfort in formation flight, we first require quantitative metrics for passenger comfort. The concept of comfort is a subjective one, making it difficult to define and measure levels of comfort. As Branton [5] suggested in a research contribution to the design of the passenger environment, comfort is only really defined in terms of its absence, arguing that it is only possible to measure levels of discomfort. Moreover, the subject of passenger comfort has become extremely important with the introduction of new forms of transportation, such as formation flying. Kirk et al. [6] recommend that in addition to the comfort experienced by a person, consideration should be given to the passenger's satisfaction. Satisfaction, as shown by Richards et al. [7], is highly dependent on the levels of comfort experienced. In an attempt to fit the transporting environment to the passenger as comfortably as possible, the ergonomics of passenger comfort can be divided into three categories: ride comfort, local comfort and organisational comfort [6]. This thesis focuses on the first of these categories, namely ride comfort in commercial aircraft during extended formation flight.

The primary contribution of this thesis lies in the evaluation of ride comfort in commercial transport aircraft during formation flight. The ride comfort experienced by passengers in the follower aircraft of a two-ship right echelon extended formation will be evaluated with the flight dynamics and the effects of the compensatory flight control inputs taken into account. This thesis also aims to improve upon the control architecture of Büchner [4, and in so doing, obtain realistic aircraft responses during formation flight. With limited access to Airbus proprietary information, an Airbus A330 aircraft fly-by-wire architecture is used to model representative flyby-wire flight control systems in this study. The associated comfort levels in formation should then be more likely to represent the ride comfort experienced during formation flight. Only if the discomfort in a follower aircraft during formation increases significantly, will the flight control laws be modified to filter the disturbances caused by the wake of the leading aircraft to improve the ride comfort of passengers.

The accelerations of passengers in a seated position will be determined by considering the passenger's seated position with respect to the mass centre, and the forces and moments at and around the mass centre respectively. The linear feet accelerations will be combined with linear and rotational seat-surface accelerations and weighted according to their frequency to determine the comfort levels in accordance with the International Organisation for Standardisation (ISO 2631-1). The linear seat-surface accelerations will be obtained indirectly from the feet accelerations with transmissibility filters.

The rest of this chapter provides the context and motivation for the research. It starts by giving some context to the current study, which is a research collaboration, followed by a formulation of the research problem statement, given in terms of research questions and objectives. Next, the expected results and significance of this research are discussed. The chapter ends with an outline of the thesis. 


\section{$1.1 \quad$ Project Description}

This project was one of many formation flight projects funded and performed with the assistance of the National Aerospace Centre (NAC) of South Africa, providing support for the development of aerospace programmes at universities and institutes in South Africa. The NAC agreed to provide support for a skills development initiative that would further existing competence in the South African aerospace sector as well as increase public access to aerospace research and development. It is in the interest of aircraft manufacturers, to evaluate the benefit of formation flight coupling interactions between lead and trailing airliners, reduce drag and improve fuel efficiency in commercial airliners. Although the efficiency of aircraft transportation will likely improve during formation flight, the benefits of formation flight would ultimately reduce emissions through reduced fuel consumption and will reduce the environmental impact, moving towards a more cost-effective and sustainable future.

The first study performed in formation flight research was done by Bizinos [3] at the University of Cape Town (UCT), with the aim of investigating levels of passenger comfort during formation flight within atmospheric turbulence. In this study, a wake model was developed to include the formation flight effects as induced aerodynamic coefficients, superimposed on the conventional aerodynamic coefficients. The results of this study showed that the presence of a leading aircraft requires a set of flight control laws to maintain separation distances, particularly as the aircraft moves closer to optimum separation.

This led to another study, performed by Büchner [4] at Stellenbosch University (SUN), who investigated automatic flight control of modern-day commercial transport aircraft during formation flight. This work served as the first contribution in the field of flight control systems during formation flight, with the aim of evaluating the stability and performance of newly designed flight control systems for formation flight requirements. The results of this study showed that formation flight control is possible at various lateral separations and during varying levels of turbulence intensities. Little change to the conventional fly-by-wire flight control architecture was required for lateral separations close to the optimum, supported by an eigenvalue analysis.

In parallel with the current study, various other research is currently being done under the same research collaboration. Lacking aeroelastic effects in the study by Bizinos [3], Biden at UCT is currently investigating the effect of wing aeroelasticity on passenger ride comfort. Sanders [8] at UCT investigated the effects of atmospheric turbulence on fuel consumption in extended formation flight and showed that the actual fuel saving in formation flight is strongly influenced by the flight control activities. Van Wyk at SUN is currently investigating the design of optimum-seeking flight control algorithms for passenger transport aircraft that use feedback control to optimise the fuel consumption during formation flight. Adams at UCT is examining the safe operation of airliners in controlled formation flight by the modelling and simulation of multiple aircraft in formation flight.

Following the foundational work of Bizinos and Büchner, the need for the current study was established. 


\section{$1.2 \quad$ Problem Statement}

The problem statement is formulated as a research question and listed research objectives, given next.

\subsubsection{Research Question}

How is passenger ride comfort in the trailing aircraft affected by the trailing vortices of the leader aircraft, taking into account the flight dynamics of both aircraft, the effects of the flight control system and the effects of atmospheric turbulence? If passenger ride comfort in the trailing aircraft decreases, how can a flight control system be used to optimise the passenger ride comfort in formation flight?

\subsubsection{Research Objectives}

The research objectives are outlined as follows:

1. To gain an understanding of commercial aircraft fly-by-wire flight control laws, formationhold strategies and vibration evaluation methods for evaluating levels of comfort.

2. To implement a representative conventional Boeing 747-100 aircraft model, which shall be used by the leader aircraft in a dynamic formation flight simulation.

3. To model, design, implement and verify the working of a representative conventional flyby-wire flight control architecture with outer-loop guidance laws.

4. To develop a formation-extended Boeing 747-100 aircraft model, that will include the aerodynamic effects of trailing vortices of the leader on the trailing airliner, and will be used by the follower aircraft in a dynamic formation flight simulation.

5. To design and implement a set of outer-loop guidance laws for a follower aircraft to maintain acceptable formation-hold performance.

6. To perform extended flight simulations with a developed formation flight model that incorporates all of the above.

7. To model the vibrations experienced by seated passengers at different locations in the leader and follower aircraft.

8. To assess passenger comfort with suitable comfort criteria, and by evaluating the effect of vibrations on levels of comfort and motion sickness incidence.

\subsection{Expected Results and Significance}

In the current study a B747-100 aircraft model with an Airbus fly-by-wire flight control architecture is being developed and used. The aircraft model was chosen to be the B747-100 due 
to its public availability in the open domain [9] [10] but also because the formation flight interaction model used in the current study was derived for the B747-100. The fly-by-wire flight control design was based on the Airbus A330, the latest available information on the current fly-by-wire, and used to evaluate the stability and performance of a representative fly-by-wire flight control system. The significance of using a dynamic B747-100 aircraft simulation model with a representative fly-by-wire flight control structure is expected to be beneficial for a few reasons.

After evaluating the effects of vibrations on the levels of comfort during formation flight, it is expected that passenger comfort in a trailing aircraft will naturally be less than the comfort in a leader aircraft under the same flight conditions and at the same seating locations. Lacking a dynamic simulation and assuming ideal flight control laws that maintain perfect separation, unrealistic separations were considered by Bizinos [3], where large induced forces and moments raise questions about actuator saturation and structural loadings. It is expected that the dynamic simulation in the current study will reveal realistic formation flight separations that are possible with current representative fly-by-wire flight control.

The study by Büchner [4] made the first attempt to outline the dangers of flying too close to the trailing vortices. The author successfully demonstrated flight control in formation flight, but worked with limited information regarding the fly-by-wire flight control architecture. The current study will seek to obtain a more representative model of the fly-by-wire flight control used in modern-day transport aircraft by working with an A330 aircraft model, available under the research collaboration, but with limited proprietary information. It is expected that the new flight control model will produce realistic aircraft responses, and the vibrations experienced by a seated passenger would be closer to what is really experienced.

A paper by Kubica et al. at Aerospatiale Matra Airbus [11] reported that experience in the design of flight control laws for aircraft such as the A320 and A330/340, showed that flight mechanic modes are located at frequencies below $1 \mathrm{~Hz}$, the region affecting motion sickness. Although it is important to evaluate the effect of vibrations on levels of comfort in this current study, further consideration is given to the incidence of motion sickness during formation flight. It is expected that motion sickness incidence will increase in a trailing aircraft during formation flight, where the chances of having ill passengers in a follower will be higher than the chances of having ill passengers in a leader aircraft under the same flight conditions and at the same seating locations.

\subsection{Thesis Outline}

Chapter 2 provides a detailed literature review of formation flight and passenger ride comfort. This offers insight into formation flight benefits and limitations as well as vibration evaluation methods to determine overall ride comfort. This chapter also provides a detailed overview of the collaborative internal research between Stellenbosch University and the University of Cape Town.

Chapter 3 provides the required mathematical modelling to describe an aircraft model for 
B747-100 aircraft in isolated and formation flight. It also discusses atmospheric turbulence generation and the method used to measure vibrations at an arbitrary location in rigid body airliners. This crucial chapter produces the foundational framework where realistic formation flight simulations are performed.

Chapter 4 outlines the linearisation of an aircraft model about a straight and level flight trim condition. A linear dynamics analysis is performed to provide insight into the aircraft's natural modes of motion and static stability. This level of insight is fundamental for a proper control systems design in the next chapters.

Chapter 5 represents a significant part of this thesis, presenting a representative model for the conventional fly-by-wire flight control architecture used in modern transport aircraft. The structure of the conventional control laws used in the Airbus A320/330 aircraft are used to design, implement and verify a representative control architecture with similar aircraft responses. Implementation of the conventional fly-by-wire flight control systems, as well as the guidance laws in simulation, are followed by an evaluation and discussion of the most important results.

Chapter 6 presents the design of three formation-specific guidance controllers, another significant requirement for evaluating passenger comfort in formation flight. These controllers maintain the desired geometric axial, vertical and lateral separations. Extended simulations are performed at different levels of turbulence intensity and geometric lateral separations. A discussion of the guidance laws and formation-hold performance is given at the end of this chapter.

Chapter 7 uses the developed formation flight model with conventional and formationextended flight control systems in dynamic simulations to produce and measure typical vibrations during formation flight. Passenger comfort criteria used to evaluate the ride comfort in airliners is defined, post-simulation settings are described, and a discussion of the measured vibrations, associated comfort levels and motion sickness incidence is given.

Finally, Chapter 8 concludes the thesis by providing an overview of the achieved objectives, a summary of the simulation results, a review of the discovered limitations and recommendations for future work. 


\title{
Chapter 2
}

\section{Literature Review}

\author{
66 \\ The work of the individual still remains the spark that moves \\ mankind ahead even more than teamwork. - Igor Sikorsky

In order to pursue the challenges presented in this project, it is necessary to gain an understanding into formation flight and passenger comfort. In this chapter, a detailed literature review of formation flight is given, offering insight into formation flight benefits, formation types and limitations of formation flight. A detailed literature review of passenger ride comfort is then given, offering insight into vibration evaluation methods used to determine overall ride comfort. This chapter ends with a detailed overview of the collaborative internal research between Stellenbosch University and the University of Cape Town.

\subsection{Formation Flight}

Formation flight is defined as the flight of two or more aircraft under the command of a leading aircraft. In this section, the fundamentals of formation flight will be covered in detail, looking at aspects such as drag savings, vortex types, formation separation, close versus extended formation flight, and types of formation.

\subsubsection{Drag Savings}

The potential for drag savings and reduced fuel consumption in long-range flights is the main reason why much research has been focused on exploiting the benefits of formation flight in passenger aircraft. The manner in which drag savings are achieved in formation flight can be understood by considering Figure 2.1, which illustrates an example of the induced vertical velocity field behind an aircraft. Lift is generated as the body of the aircraft moves through the air by inducing downward momentum. The downward flow of air spirals around the edge of the wake, which creates regions of upwash and downwash. Drag savings are realised by a second aircraft when flying in the region of upwash, effectively increasing the angle of attack 
and achieving additional lift which would not have been achieved in isolated flight. Wing tip vortices are formed because of the pressure differential between the bottom and top surfaces of a wing. [1] [3]
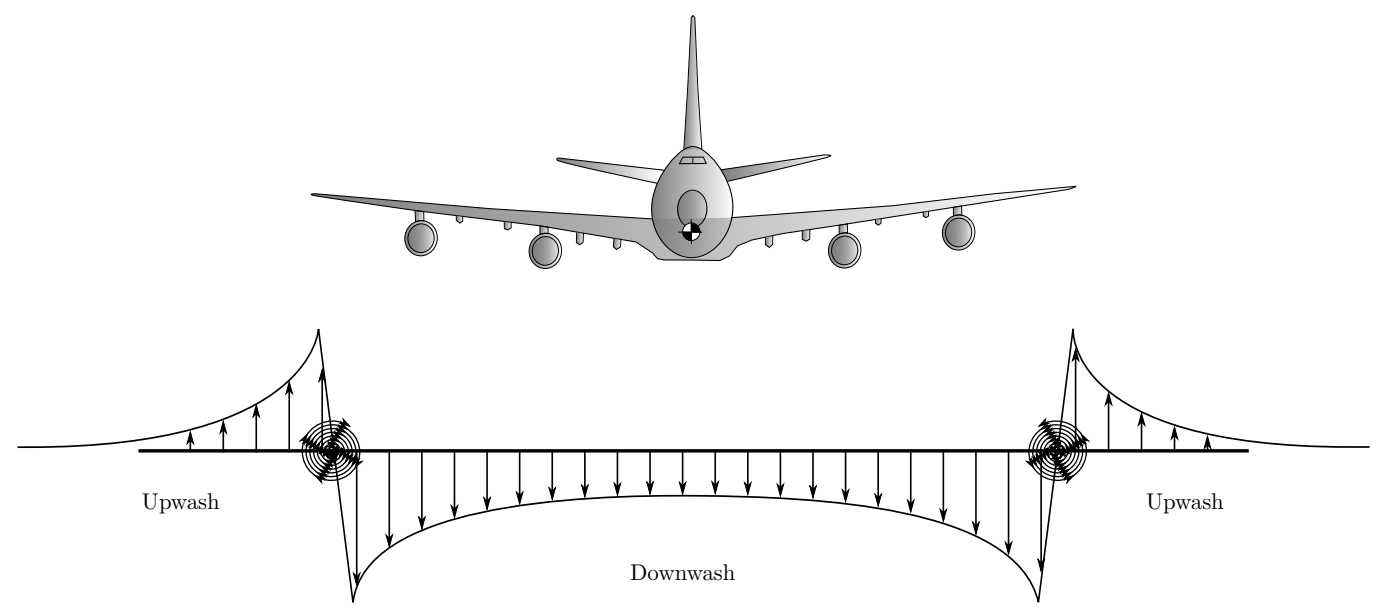

Figure 2.1: Vertical component of velocity induced behind the aircraft (not to scale and adapted from Ning [1]).

\subsubsection{Wing Tip Vortices}

The development of wing tip vortices is well documented in the literature [12] 13] 14] [15]. Figure 2.2 shows the flow of air over a fully spanned wing, neglecting the fuselage.

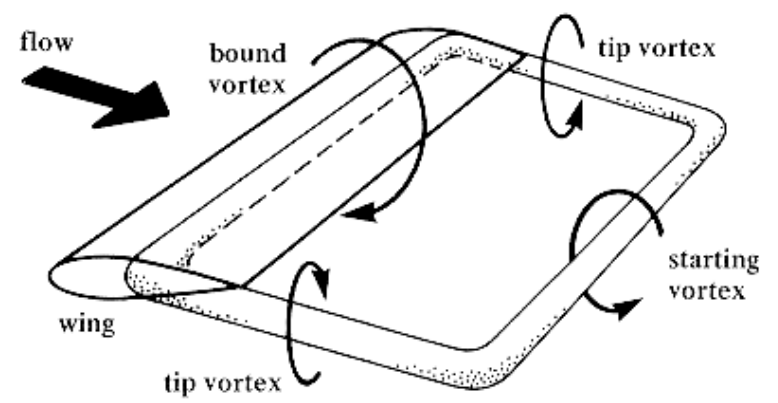

Figure 2.2: Simplified illustration of air flow over a wing, showing the vortex types for an aircraft wing (from Wegener [16]).

While a strong bound vortex is formed around the wing due to the pressure differential between the bottom and top surfaces of a wing, a spanwise flow is generated towards the wing tip on the bottom surface and towards the fuselage on the top surface. This process develops a vortex sheet [12]. Directly behind the trailing edge of the wing, a region known as the near-wake field, the flow field is characterised by the joining of various concentrated starting vortices downstream of all wing surface discontinuities, and the rapid upward motion of the tip vortices [3] [13] [14]. Within approximately 10 wingspans downstream of the wing trailing edge [17] 18, the vortex sheet begins to roll up and two distinct, counter-rotating vortices are 
formed. This region is known as the extended near-wake field, limited between approximately 10 and 12 wingspans [13]. The next region is termed the far-wake field and stretches from 10 to 100 wingspans. In this region, the counter-rotating vortices descend in the atmosphere without undergoing any significant changes [3]. Immediately following the far-wake field, the final stage of the vortex structure is entered and the vortex rapidly starts to decay [19].

Formation flight can take place in either the near-wake, extended near-wake or the far-wake field and is what differentiates close from extended formation flight.

\subsubsection{Close and Extended Formation Flight}

The streamwise separation between aircraft in formation flight defines whether trailing aircraft are in close or extended formation flight [1]. However, several implications should be considered before choosing the separation distance for formation flight.

\section{Savings Versus Safety}

The benefits of formation flight have long ago been recognised by many researchers, scientists and aerodynamicists [20] 21]. Interest was shown in formation flight due to its potential to reduce fuel consumption in long-range flights. Since its recognition, various studies have been undertaken to investigate the effects and advantages of formation flight. Some literature studies have focused on close formation flight, illustrated in Figure 2.3, where aircraft are separated by merely a few wingspans [22] 23] 24] [25]. Showing good aerodynamic and control benefits, close formation flight minimises the induced drag of an aircraft and should greatly improve fuel efficiency in commercial aviation. However, this type of close proximity between commercial aircraft poses an unacceptably high risk of collision in many applications. Formation flight in the current study takes advantage of extended formation flying at separations of up to tens of wingspans [1] [26] [27], where the persistent cruise wakes are still beneficial, but the safety is significantly improved.

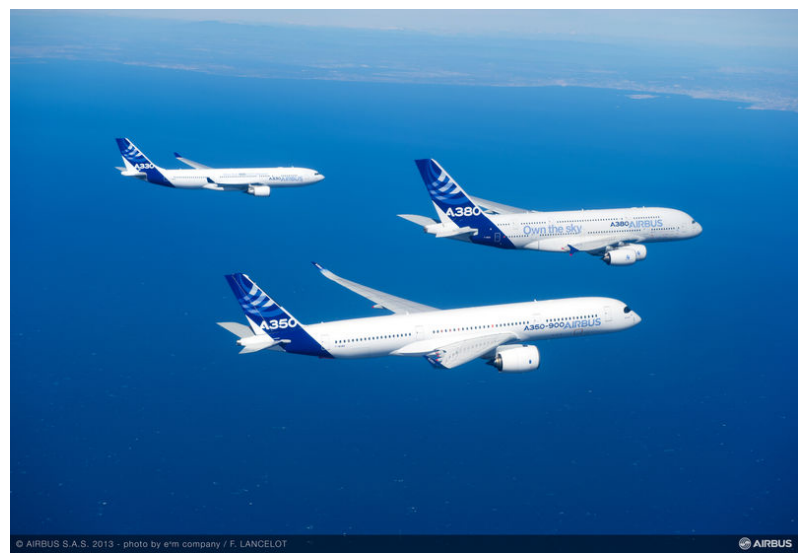

Figure 2.3: Close formation flying of an Airbus A380 and two Airbus A350 aircraft. 
Wind tunnel tests for formation flight have shown that a substantial decrease in drag can be obtained in formation flight depending on the configuration. Blake and Gingras [24] measured a reduction of up to $25 \%$ in close formation flight. According to a study of extended formation flight by Ning [1] [26], for streamwise spacings of around 10 to 40 spans with low to moderately low atmospheric turbulence, a two-aircraft formation is capable of achieving a maximum drag reduction of approximately $30 \%$, while a three-aircraft formation showed maximum reductions of around 40\%. Later, in an inviscid analysis of extended formation flight, Kless et al. [27] analysed a two-body echelon formation and found that simulations indicated peak induced drag savings for a trailing aircraft of up to $54 \%$ in subsonic flow and $35 \%$ in transonic flow. Bower et al. 22 performed a case study in order to quantify the amount of fuel burn reductions achievable during formation flight. Using the flight data of five FedEx formation flights between two reasonably distanced countries, fuel savings of $4 \%$ for tip-to-tip gaps of about $10 \%$ of the wingspan were achievable. Fuel savings of up to $11.5 \%$ were achievable for tip-to-tip overlaps of about $10 \%$ of the wingspan. Other wind-tunnel tests have shown that a decrease in drag of between $10 \%$ and $30 \%$ is obtainable during formation flight depending on the configuration [22] 23] 225].

In spite of its many benefits, formation flight induces large aerodynamic forces and moments due to trailing wake vortices; resulting in dangerous handling characteristics. This is commonly referred to as the "Vortex Problem". Research that analysed wake vortices and developed experimental systems and alleviation devices to cope with the vortex problem is well documented 28]. As the trailing aircraft moves from close to extended formation flight, wake development and modelling become very important.

\section{Wake Interaction Models}

In close formation flight, the wake is still in the first stage of development, and flat wake models prove to have reasonable approximation of the formation interaction effects [1]. However, as the aircraft moves to tens of wingspans of streamwise separation, the wake moves into the next two stages, where the influences of atmospheric turbulence and viscous decay play an important role in determining the interaction effects [1]. Having chosen to limit formation flight in this study to tens of wingspans of streamwise separations, such that the aircraft remains in the extended near-wake field, a suitable wake model is required.

Many formation flight studies have considered the single horseshoe vortex method to evaluate the benefits of formation flight [20] 21] [29] [30] [31]. Ning et al. [26] suggested considering formation flight of commercial aircraft at axial separations of 10 to 40 wingspans, and a more recent study by Bizinos [3] limited the axial separation to 10 wingspans. This study considers the same axial separation, limited to 10 wingspans. The trailing vortex is considered to be fully rolled up at this separation [13], and the basic single horseshoe vortex has been shown to have reasonable agreement with flight data [22] [32].

The horseshoe vortex model is a simplified model to represent the vortex system of a wing, illustrated in Figure 2.4, and is used to model the interference effects of a lead on a following aircraft. The wing vorticity is modelled by a bound vortex of constant circulation and travels 
with the wing and two trailing wing tip vortices. For this reason, it has a shape vaguely reminiscent of a horseshoe. [33] [34]

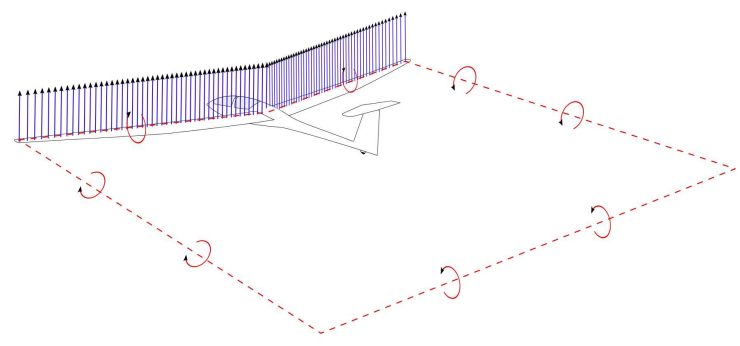

Figure 2.4: A horseshoe vortex caused by a uniform lift distribution over an aircraft's wing (used with permission of author Olivier Cleynen).

Choosing to pursue formation flight at extended separations, with a suitable and sufficient interaction model, the best formation type and number of aircraft in formation must also be considered.

\subsubsection{Formation Types}

There are three well-known possibilities for arranging three or more aircraft in formation [1], illustrated in Figure 2.5, they are the V, Echelon and Inverted-V formations. There are various factors that contribute to which type of formation is optimal for a given set of conditions and desired application. These factors include the weight of the aircraft, fuel consumption and formation trip distance [1].

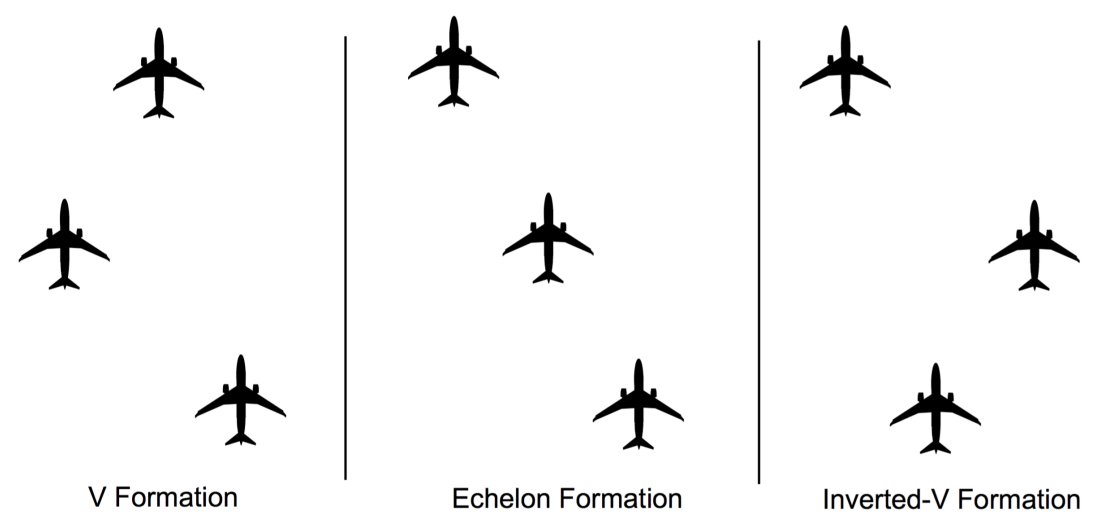

Figure 2.5: The three common types of three-aircraft formations (from Ning [1]).

In a study by Ning [1, a metric to quantify the drag of all aircraft in formation, relative to the drag of all aircraft out of formation (in isolated flight) is defined. This metric is called the drag fraction $\left(D_{i}\right)$, and helps to evaluate the impact of formation as a whole and not for a particular aircraft. Using the drag fraction, the benefits of adding more aircraft to a formation 
can be estimated. With some assumptions, the lower bound for the induced drag fraction of the formation is derived as follows,

$$
\frac{\left(D_{i}\right)_{\text {formation }}}{\left(D_{i}\right)_{1}+\ldots+\left(D_{i}\right)_{n}}=\frac{1}{n}
$$

where $n$ is the number of aircraft, $\left(D_{i}\right)_{n}$ is the minimum induced drag for the $n^{\text {th }}$ aircraft and $\left(D_{i}\right)_{\text {formation }}$ is the minimum induced drag of the formation as a whole. Illustrated in Figure 2.6 is the theoretical lower bound of the induced drag fraction for a formation lined up with tip-to-tip lateral separation.

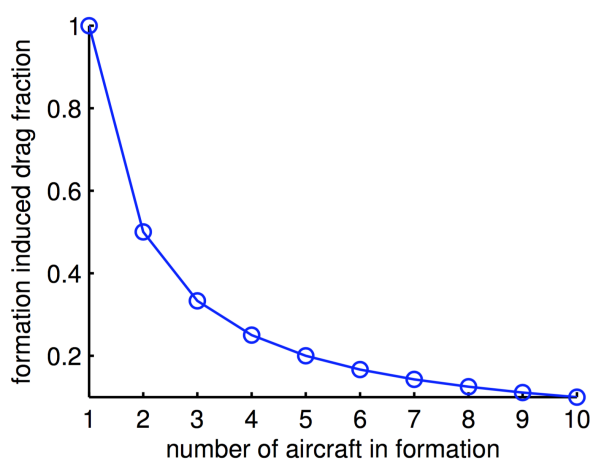

Figure 2.6: Formation induced drag reduction as the number of aircraft in the formation increases (from Ning [1]).

This figure highlights that the greatest drag savings should be achieved in changing from solo flight to a two-aircraft formation; as the number of aircraft in formation increases, the overall drag benefit decreases quickly. It is thus clear that a large number of aircraft in formation is not beneficial for fuel savings. This does not mean that three-aircraft formations do not provide sufficient drag savings; the benefit is still significant. Limiting the number of aircraft to two in the current study, the trailing aircraft will be placed on the right-hand side of the leader aircraft (referred to as a right echelon formation in the rest of this thesis) and will be limited to a streamwise separation of 10 wingspans.

Formation flight also leads to questions about the increased acceleration magnitudes and the associated passenger ride comfort levels experienced in the trailing aircraft of a formation flight convoy. Longitudinal, vertical, roll and yaw acceleration magnitudes have been shown to particularly increase due to formation flight, with roll acceleration magnitudes showing the most significant increase [3] [28] [35].

\subsection{Passenger Ride Comfort}

Oborne [36] concludes that ride comfort may be considered in two ways: as a reaction to the entire trip from the time of departure to the time of arrival, or to a number of different aspects of the environment (motion, seating, noise, lighting, leg room etc.). Choosing to evaluate comfort 
during formation flight by considering the latter, the scope of this study is limited to the effect of motion vibrations on levels of comfort, defined by suitable comfort criteria. This choice led to a need for a literature survey of the different comfort criteria studies, focusing on different techniques of comfort evaluation. Henceforth, the terms passenger comfort and ride comfort will be used interchangebly.

\subsubsection{Passenger Comfort Criteria - A Literature Survey}

A number of ride quality comfort criteria are in existence. Various literature studies summarise most of the important comfort criteria defined and, in some cases, are then used to evaluate the ride comfort of a passenger transportation system [36] 37] [38] [39] [40]. The investigation into the various comfort criteria were important to understand how the need for more quantative guidance on the effects of vibration on the human body was desired; ultimately leading to a standard widely used today and in the current study.

Janeway [41] performed an analytical study on available experimental data that documented human tolerance to vertical sinusoidal vibrations. The objective of this study was to derive various safe limits of vibration intensity for comfort. Three frequency ranges were considered and a constant maximum value in each range was proposed: (1) a low frequency range (1 to $6 \mathrm{~Hz}$ ) with a maximum acceleration rate of change limit; (2) a middle frequency range (6 to $20 \mathrm{~Hz}$ ) with a maximum acceleration limit; and lastly (3) a high frequency range (20 to $60 \mathrm{~Hz}$ ) with a maximum velocity limit. The proposed idea was that if the RMS amplitude of a sinusoidal component did not exceed the maximum limits in a certain frequency range, the ride should be comfortable.

Lee et al. 42 proposed a method to analytically determine human response to vibration by using a parameter called the "absorbed power". This concept related the ride quality to a single number: the total power absorbed by a passenger due to vibrations during the ride. The total absorbed power was the sum of the average vertical, side-to-side and fore-and-aft absorbed power. The average absorbed power due to vertical, side-to-side and fore-and-aft accelerations was computed as a weighted integral of the acceleration spectral densities. A similar method of analytically determining the human response to vibration was proposed in a study by Butkunas [43]. In this study, a ride index was obtained by measuring the seat-surface accelerations with transducers and calculating a RMS value from the weighted acceleration to indicate the level of comfort. The weighting function was represented by a generalised transfer function representing acceleration input to comfort index output. These two studies developed comfort criteria assuming a functional relationship between vibration magnitudes and human perception of vibration.

Stevens [44] attempted to outline that a general psychophysical law exists which related subjective magnitudes (such as levels of comfort) to stimulus magnitudes (such as vibration magnitudes). Furthermore, provided that stimulus ratios of the same type produce equal subjective ratios, the direct assessment of subjective magnitude (such as a direct indication of the level of comfort) was simply a relation to the magnitude of the stimulus. Hence, Stevens developed a psychophysical law as a first-order approximation: an indication of ride sensation 
was proportional to the stimulus raised to a power $n$. Many different experiments have been conducted to determine different suitable $n$ values for whole-body vibration stimuli [45] [46].

Jacobson et al. [47] extended a general method for determining passenger satisfaction with ride quality to commuter-type aircraft. The aim of the study was to provide a method for current and future air vehicles to predict, assess and re-design for acceptable passenger satisfaction to ride quality. A forcing function was defined as an input to a vehicle transfer function. Aerodynamics and mass properties were used to characterise the vehicle transfer function for an aircraft. The estimated motion spectra of the aircraft was then obtained as power spectral density acceleration spectra in the frequency domain, and integrated to obtain RMS vertical and horizontal accelerations. A subjective transfer function previously developed was used to relate the subjective comfort response to the RMS vertical and horizontal accelerations, producing a comfort rating $C$. The comfort rating was restricted to values between 2 (comfortable) and 5 (very uncomfortable). Lastly, since the vertical and lateral accelerations were described by a joint probability distribution function derived from flight data, a probability of exceeding a given comfort level $C^{\prime}$ was provided.

Numerous other studies were gathered from a Ride Quality Symposium, jointly supported by the National Aeronautics and Space Administration (NASA) and U.S. Department of Transportation agencies. Studies were grouped into categories including, but not limited to, experimental ride quality studies and ride quality modeling and criteria [48] 40, 50] [51] [52]. Since these studies were done, the awareness of the complexity of human physiological and behavioural responses to vibration has continuously increased. The lack of clear, universally recognised and accepted criteria to assess human responses to vibrations made it desirable to give more quantitative guidance on the effects of vibration on health and comfort, perception and motion sickness incidence.

Gathering years of contributions to defining suitable comfort criteria, the International Organisation of Standardisation (ISO) 2631-1 [53] became a widely accepted standard to evaluate comfort. It aims to provide guidance methods with which to evaluate the effect of whole-body vibrations on the human body and is primarily concerned with quantifying whole-body vibrations in relation to human health, comfort, perception and motion sickness. Recognised as the predominant influencing factor, vibrations are to be measured at various points on the human body and jointly used to determine an in toto effect on passenger comfort.

\subsubsection{Flight Control Laws and Passenger Comfort Improvement}

With the development of more and more high capacity long-range aircraft that are characterised by flexible structures, an increase in the interaction between control laws and structural dynamics modes exists which challenges modern flight control system designs [11] [54] [55]. This leads to new technological challenges during aircraft design phases. Kubica et al. [11] [55] from Aerospatiale in Toulouse, France, mention two control techniques that could be used to cope with this problem:

1. A passive control design, which consists of filtering the flexible modes in order to avoid 
coupling with the control laws.

2. An active control design, which consists of controlling the first flexible modes.

In order to confidently evaluate the passenger ride comfort, both in isolated and formation flight, the structure of the flight control laws should be as representative as possible to modern transport aircraft. Although the scope of developing a fully accurate, active control design, is too large for the current study, it is necessary to gain an idea of what a full control design entails. The study by Kubica et al. [11] [55] is used in the current study as the basis of a typical design process, used for flight control laws, ride comfort evaluation and improvement.

\section{New Flight Control Laws for Large Capacity Aircraft (1998)}

Traditionally, for aircraft before the Airbus A340, the passive approach was a sufficient means whereby the problems introduced by aeroservoelastic coupling were solved by filtering [55. This was to prevent the flight control laws from exciting the flexible modes. However, for future transport aircraft it will become difficult to reduce structural vibrations without reducing flight control law bandwidth [55]. For this reason, a new active control approach was necessary, which would provide good handling qualities and high levels of passenger comfort in spite of the flexibility introduced by new aircraft. In his paper titled "New Flight Control Laws for Large Capacity Aircraft", Kubica [55] presents the main stages of an experimentation on an Airbus A340-300 to test new flight control laws. This aircraft was the most flexible aircraft at the time of the tests, making it a suitable candidate.

Figure 2.7 shows the generalised frequency distribution when using the classic (passive) or the integrated (active) approach. With the classical approach, a damping function called Comfort In Turbulence (CIT) was used to attenuate the fuselage response to turbulent conditions. This was done by increasing the damping of the fuselage modes. It was argued that the flight control law bandwidth was much less than the fuselage mode, ensuring no degradation of the flying qualities obtained by a specific set of flight control laws. Kubica made the remark that to filter the control laws without decreasing performance and to design these laws and structural mode functions independently is a difficult task. For this reason an integrated approach, whereby the rigid and flexible modes are controlled, was developed.

This control approach, which is referred to as active control, provides numerous advantages. It allows increased damping of structural modes, which leaves room for comfort improvement. It provides the possibility to reduce loads in turbulence and to avoid coupling between the flight control laws and flexible modes of the aircraft. Notch filters can exist to protect the structural modes of the aircraft. These filters will have an effect on the frequency response, and thus the passenger comfort, but the effect is minimal. The active approach also extends the control law bandwidth, making it possible to improve the handling qualities and to avoid Aircraft Pilot Coupling (APC) by minimising the dephasing in the flight control loop. APC events are unintentional, undesirable aircraft motions that stem from the interactions between the aircraft and pilot. It is interesting to note that the general philosophy of the Airbus Flight Control 
System (FCS) is maintained in the transition between the classical and integrated approach of flight control system design.

nie melding gemaak van notch filters wat kan bestaan om strukture modusse te beskerm nie. Dit het 'n effek op die freq resp. Min effekmaar ek dink hymoet dit stel.
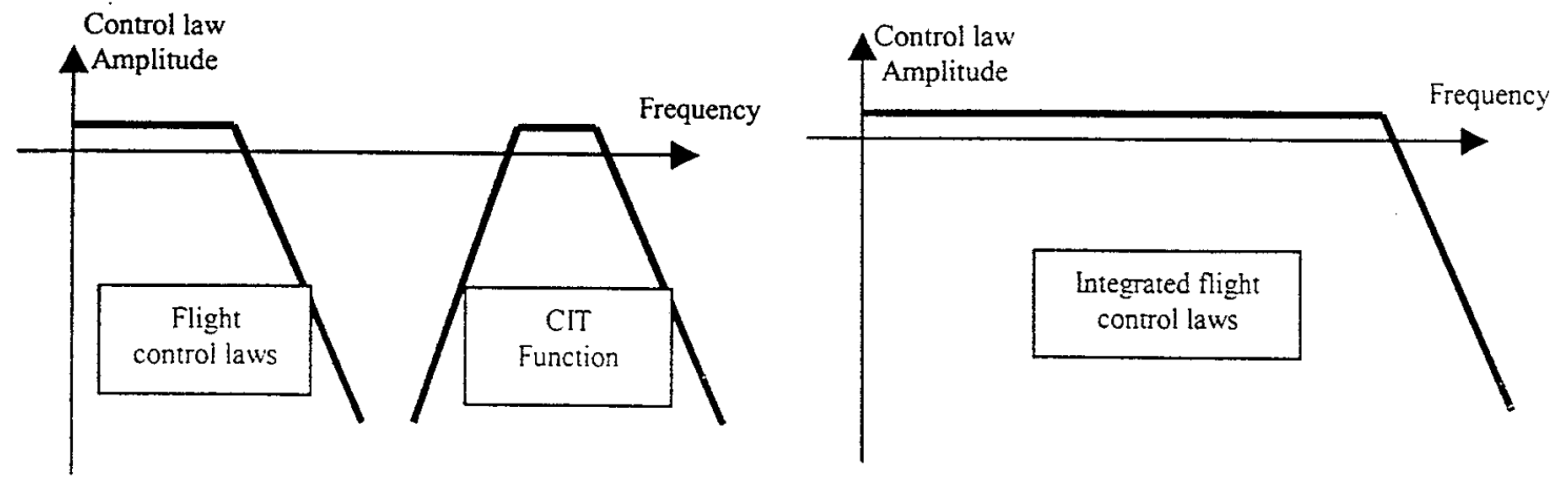

Figure 2.7: Frequency distribution with classic (left) and integrated (right) approach to control law design (from Kubica [55]).

To test the validity of the concept of active control in aircraft, a set of flight tests was planned. These flight tests were to set the methodology for similar tests of active control law in future aircraft. Kubica [55] explains the main stages of an experiment on an Airbus A340-300 to test the new flight control laws.

In the design phase, a complete set of new flight control laws was designed on the whole flight envelope with specific handling and structural flexibility specifications. These new flight control laws were used on a complete aircraft model, which included flight mechanics and aeroelasticity. In order to actively control the fuselage modes, specific placement of feedback accelerometers at the rear of the aircraft was performed. A pitch rate gyro was placed at the centre of gravity, while all other information for the flight control laws were provided by the Air Data and Inertial Reference Units (ADIRUs) at the front of the fuselage. Actuator characteristics were taken into account and the computation time between sensor acquisition and control law emission was selected as $10 \mathrm{~ms}$, in order to guarantee good robustness properties.

The control laws were then defined in the Aerospatiale graphic language, ready for validation. The validation process included assessment of the control laws and aircraft response characteristics in a non-linear environment. Simulations were representative of pilot inputs and the handling qualities were evaluated. Correct functioning of the new control laws in the whole system was also verified. System integration with the Flight Control Primary Computer (FCPC) was then done. The usual A340 laws were still in place, with the new control laws in parallel. This allowed for a comparison of the behaviour between the different control laws. After a complete validation of the A340 simulator, the overall flight control system behaviour was good and ready for the flight test.

The flight test was performed towards the end of 1996, on an A340-300 aircraft. The new control laws showed excellent stability margins. For different aircraft configurations and ma- 
noeuvres, the pilots found the aircraft very precise and easy to fly. The response times of the control laws were better than before. Considering the effects of the control laws on the flexible modes, a reduction in fuselage acceleration levels were obtained for the lateral and vertical axes. The new control laws were showed to be very robust.

Kubica [55] concluded this successful experiment by stating that active control of modern flexible aircraft through the current fly-by-wire system provides high levels of handling qualities and comfort.

\section{Passenger Comfort Improvement by Integrated Control Law Design (1999)}

In another study, Kubica and Madelaine [11] pointed out that although the active control approach was more convenient in terms of handling qualities, it was more difficult to make comparisons given the subjectivity related to passenger comfort. The authors investigated how the comfort criteria based on the ISO 2631-1 standard could be applied to large capacity transport aircraft for passenger comfort evaluation. These criteria had to take into account the rigid-body and elastic dynamic aircraft responses. Another objective of the study was to investigate how these criteria could be used in order to effectively design the control laws of the aircraft.

In terms of comfort criteria, the paper focuses on vibrational comfort, which is known to predominantly influence passenger comfort. The authors mentioned that particular attention should be paid to frequencies below $1 \mathrm{~Hz}$, where flight mechanics modes are located and which can influence passenger comfort. Following this, comfort evaluation is divided by the authors into two frequency bands closely corresponding to the ISO 2631-1 standard: [53] [11]

1. A "very low frequency" range below $1 \mathrm{~Hz}$ and known as the motion sickness phenomenon.

2. A "low frequency" range above $1 \mathrm{~Hz}$ and known as vibrating comfort.

Comfort criteria in the "very low frequency" range In this frequency range the ISO standard is based on the vertical acceleration felt by a human passenger. Kubica and Madelaine [11] considered a frequency weighting filter $\left(W_{f}\right)$ in order to represent sensitivity to motion sickness. This filter is presented in Figure 2.8 as a band-pass filter centred at $0.16 \mathrm{~Hz}$, which is considered the critical frequency for motion sickness. The authors followed the ISO standard by computing a Percentage of Ill Passengers (PIP), giving the percentage of people who may vomit during the motion period [11] [53].

Comfort criteria in the "low frequency" range In this frequency range, the ISO standard is based on the measurement of the lateral or vertical acceleration felt by a human passenger at one point. When the comfort is affected by vibrations at several points, the overall vibration is computed by Kubica and Madelaine [1] from the root mean square (RMS) value of the global vibrations at different measurement points on the passenger seat. The ISO standard proposes that, for a seated person, three points should be considered to evaluate the discomfort felt by a person: vibrations at the supporting seat-surface, at the feet and at the back of the seat. It 


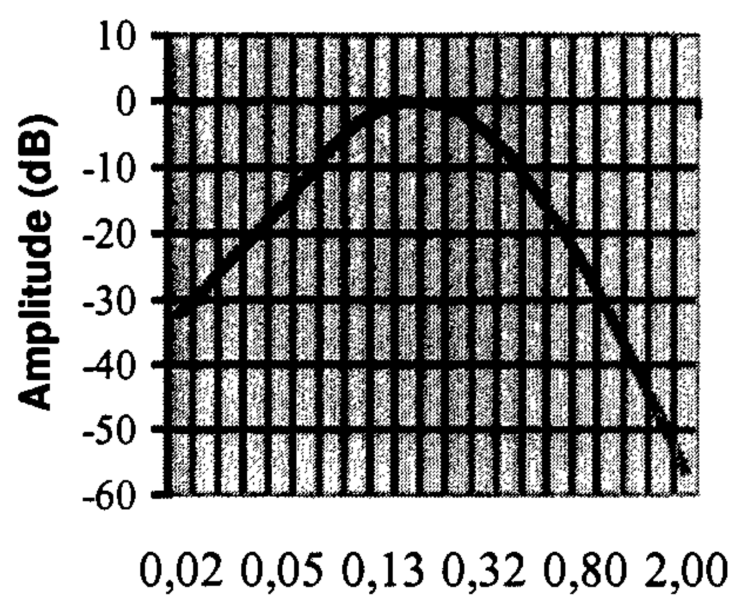

Figure 2.8: Frequency weighting filter used for motion sickness sensitivity (from Kubica and Madelaine [11]).

is interesting to note, however, that the authors mentioned that for civil aircraft, the rotational vibrations as well as the vibrations transmitted by the back of the seat could be neglected, which left only the vertical and lateral accelerations at the supporting seat-surface and at the feet to be taken into account [11. Using the total frequency-weighted RMS acceleration, approximate indications of the likely comfort levels were obtained with the ISO standard.

Simulation Results A simulated large capacity aircraft was used in order to evaluate passenger comfort at various places from the front of the fuselage to the back of the fuselage. Different scenarios were considered, including manoeuvres and turbulence, for different control configurations.

Concerning the very low frequency range, PIP in manoeuvres was found to be negligible for any type of control configuration and locations along the fuselage, recording less than $0.1 \%$. Concerning turbulence scenarios, the results are shown in Figure 2.9. The PIP was small for any configuration $(<0.9 \%$ for the lateral axis and $<3 \%$ for the vertical axis), implying that an aircraft would be a comfortable choice of transport. For simulations of 3 minutes in strong turbulence and any control law configuration, an increase in the predicted PIP level for further aft seating positions were revealed.

Concerning the low frequency range, Figure 2.10 shows the comfort along the fuselage for realistic vertical turbulence. The authors were of the opinion that acceleration due to lateral turbulence was far less critical than that due to vertical turbulence. Results showed that the aircraft was considered not uncomfortable almost all along the fuselage, and a little uncomfortable only at the front and back of the fuselage. The active control law improved passenger comfort only at the front and back of the fuselage, with a maximum improvement of $10 \%$. This was successfully checked by passengers during laboratory tests with a vibrating seat. [1]

Conclusion Kubica and Madelaine [11] conluded that an active control approach in controlling the first flexible modes allowed improvement in both the "low" frequency and "very low" 


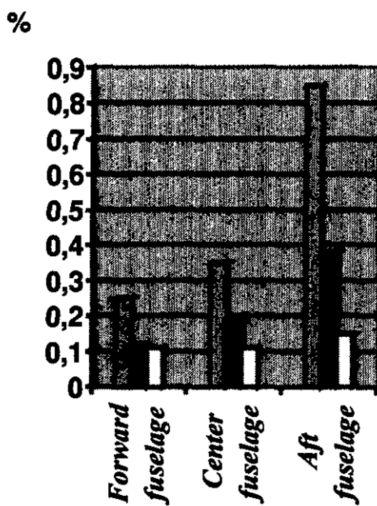

(a) PIP in lateral turbulence

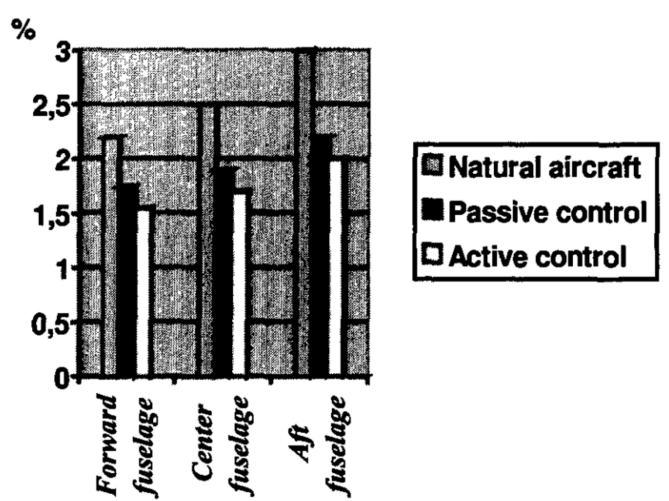

(b) PIP in vertical turbulence

Figure 2.9: Percentage of Ill Passengers (PIP) in lateral and vertical turbulence (from Kubica and Madelaine [11]).

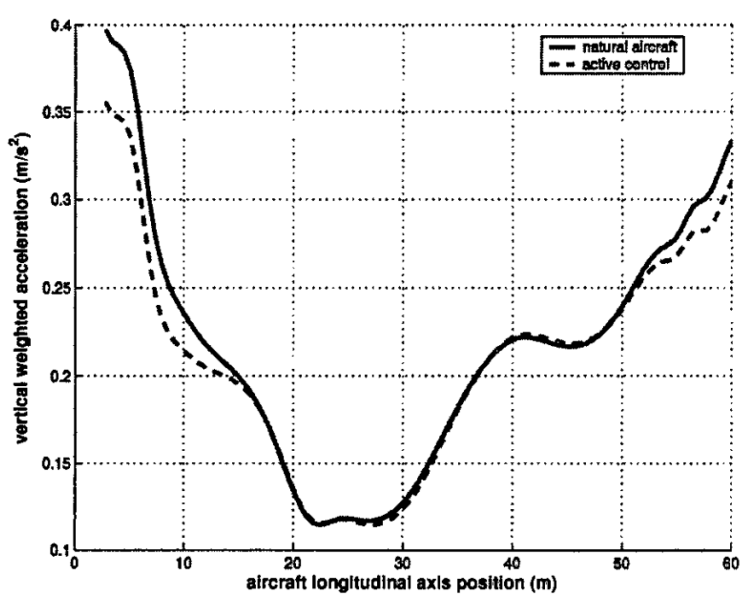

Figure 2.10: Comfort improvement in vertical turbulence (from Kubica and Madelaine [11]).

frequency comfort levels. This result meant that an integrated design which actively controls both rigid and flexible modes was preferable for comfort improvement.

\subsection{Collaborative Internal Research}

In this section, internal research performed in collaboration with Stellenbosch University and the University of Cape Town, will be detailed. Two foundational research studies, performed by Bizinos at the University of Cape Town [3] and Büchner in the Electronic Systems Laboratory (ESL) at Stellenbosch University [4], are considered the main contributors to the development of the current study. 


\subsubsection{Passenger Comfort during Formation Flight within Atmospheric Turbulence}

Bizinos [3] did a study with the objective to determine the levels of comfort experienced by a passenger on board a trailing aircraft during formation flight in atmospheric turbulence. In this study, the irregular motion of an aircraft flying through a disturbed atmosphere was simulated by considering only the effect of turbulence on the aerodynamic loads on the aircraft. The author considered a static simulation and did not implement and use the flight control system of the aircraft during the study. Both aircraft were trimmed at a selected flight condition such that the control surfaces of both aircraft result in a straight and level flight equilibrium.

Bizinos considered two identical aircraft as illustrated in Figure 2.11, flying straight and level at a constant speed and in a right echelon formation. The main wing of the leading aircraft was represented by a single horsehoe vortex, while the trailing aircraft was approximated by a single horsehoe vortex on the wing, tailplane and tailfin. The induced forces and moments on the follower aircraft due to the trailing vortices of the leader were derived as induced loads converted to aerodynamic coefficients. The Burnham Hallock profile was used to approximate the influence of the lead aircraft on the trailing aircraft as influence factors, dependent only on the formation geometry.

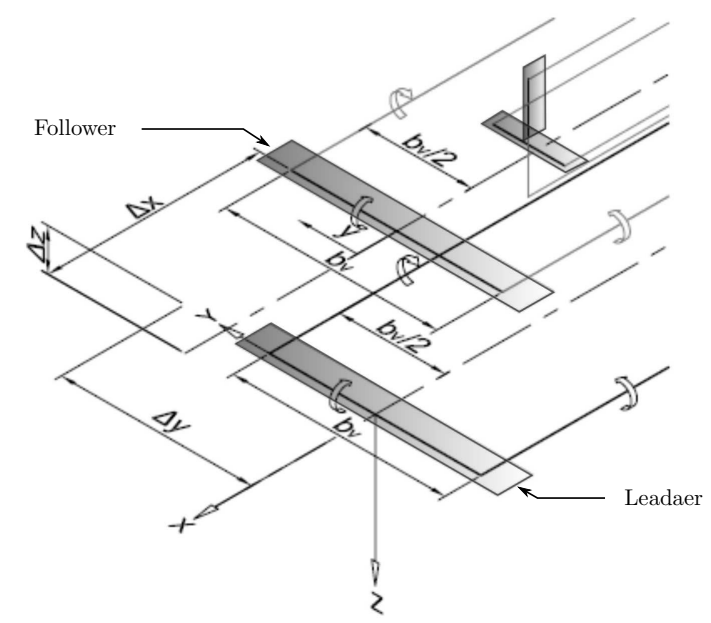

Figure 2.11: Horseshoe vortices in right echelon formation. The main wing of the leading aircraft is represented by a single horsehoe vortex, while the trailing aircraft is approximated by a single horsehoe vortex on the wing, tailplane and tailfin (Adapted from Bizinos [3]).

Bizinos pointed out that the atmospheric turbulence model plays an important role in generating the induced forces and moments. He used the von Kármán turbulence model to model the atmospheric turbulence as stationary, homogeneous, isotropic, Gaussian turbulence. Some assumptions were made by the author to simplify the modelling but still obtain realistic effects of atmospheric turbulence on aircraft in formation. The first of these assumptions was to consider the aircraft as two points negligibly smaller than the wavelengths of all significant spectral components. Furthermore, the lateral and vertical separations were considered small enough to not be included in the modelling of the effects of atmospheric turbulence. The author also 
assumed that the gust velocities experienced by the lead aircraft remain frozen until the trailing aircraft reached the same streamwise location. This allowed the one-dimensional correlations and spectral densities given in the literature to be applied. Bizinos decided to fix the longitudinal separation to 10 wingspans to limit the divergence of this assumption.

Within atmospheric turbulence, the disturbed horseshoe vortices alter the geometric separation between the aircraft, which will have an effect on the resulting induced aerodynamic loads caused by the trailing vortices. The author assumed that the lead aircraft's trailing vortices both shift with the lateral and vertical gust velocities by the same amounts, thereby maintaining the relative spacing between the two trailing vortices, illustrated in Figure 2.12 .

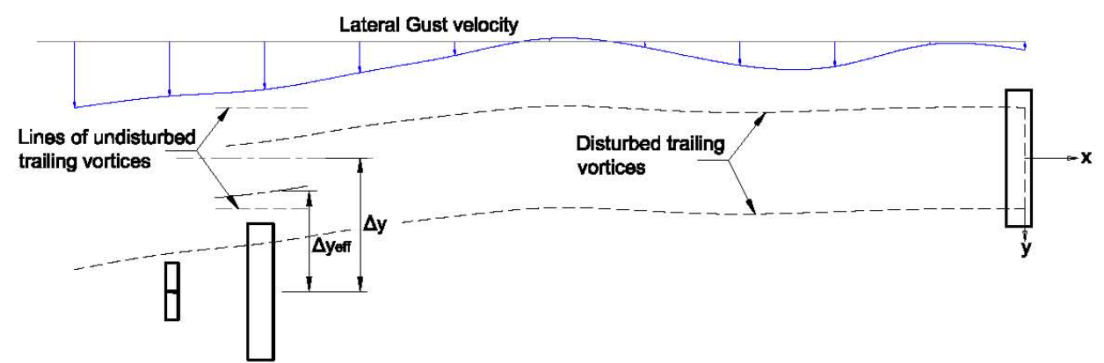

Figure 2.12: Trailing vortices of leader aircraft moving in an ideal fashion during formation flight and in atmospheric turbulence. The trailing vortices are assumed to shift with the lateral and vertical gust velocities (from Bizinos [3]).

At any point, the direction of the trailing vortex was assumed to be parallel to the local instantaneous velocity with respect to the air. Furthermore, the author assumed that the lead aircraft would maintain its altitude by satisfying an ideal control law. A lateral component of turbulent velocity changes the geometric lateral separation to an effective lateral separation and, similarly, in the case of the turbulent vertical velocity, the geometric vertical separation changes to an effective vertical separation. The effective separation $\Delta y_{\text {eff }}$ and $\Delta z_{\text {eff }}$ is illustrated in Figure 2.13a for lateral turbulence and Figure 2.13b for vertical turbulence.

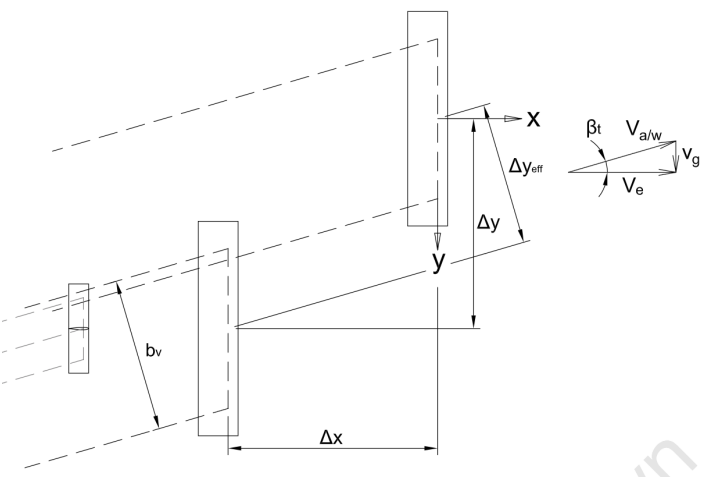

(a) Top view with lateral turbulence

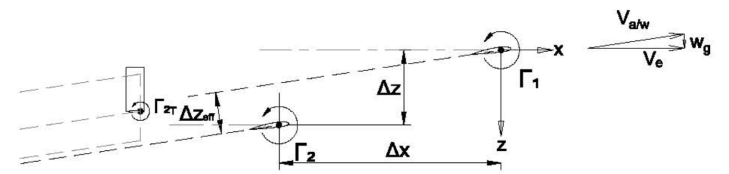

(b) Side view with vertical turbulence

Figure 2.13: Side and top view of formation in vertical and lateral turbulence (from Bizinos [3]). 
Bizinos considered passenger comfort as frequency-dependent [53], implying that the accelerations and respective frequencies of the passenger are required. Concerning a seated passenger, the accelerations were determined by considering the passenger's seated position with respect to the mass centre and the forces and moments at and around the mass centre respectively. The accelerations were weighted according to their frequency in order to determine the comfort levels via comparison to ISO 2631-1 [53]. Monte Carlo simulations were performed with different levels of turbulence intensity.

The simulation results predicted a large increase in discomfort due to formation flight, and also showed that passenger comfort proved to be most dependent on lateral separation, variation in turbulence intensity and passenger seat displacement from the mass centre. Figure 2.14 illustrates the overall vibration values in a follower aircraft for $\eta=0.6$ to 1.2 wingspans at two seating locations: one at the back of the aircraft (Figure 2.14a) and one at the front of the aircraft (Figure 2.14b). The straight dashed lines indicate the level of comfort in an isolated aircraft flying in the same flight conditions. The straight light gray lines indicate the regions of comfort as suggested by the ISO 2631-1 standard.

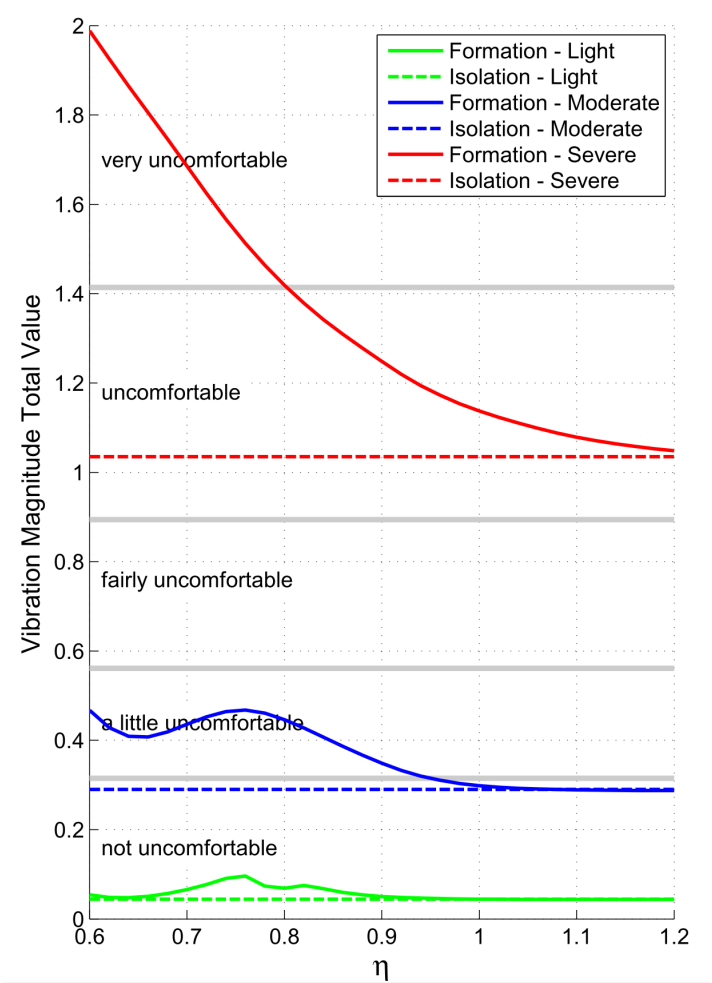

(a) Seating location D (at the back of the aircraft).

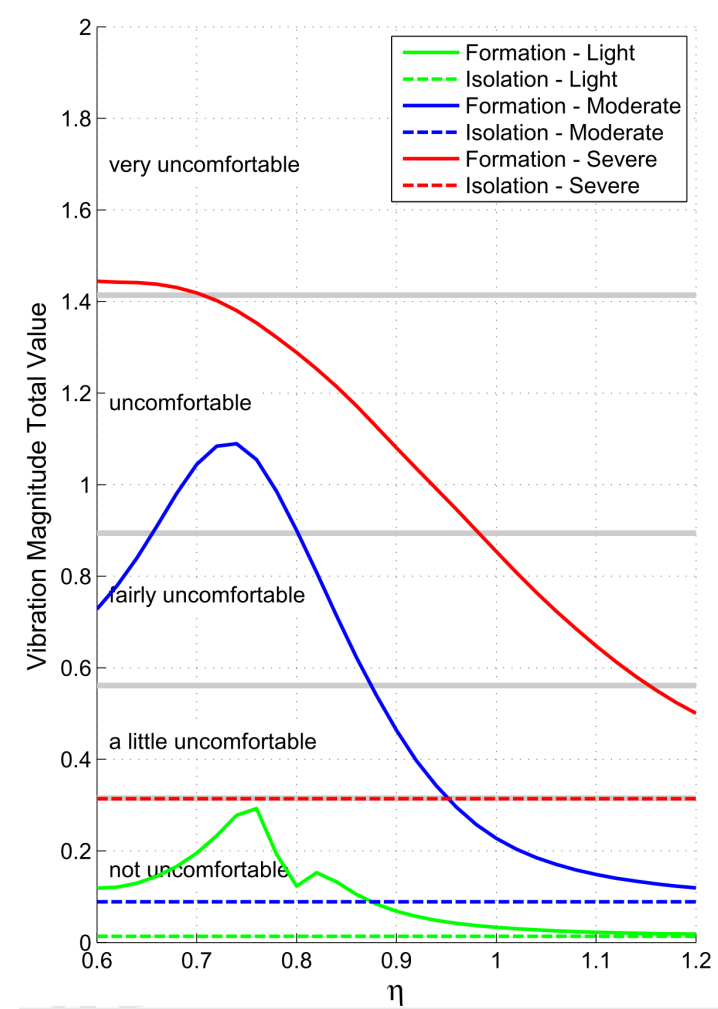

(b) Seating location F (at the front of the aircraft).

Figure 2.14: Overall RMS acceleration magnitudes $\left(\mathrm{m} / \mathrm{s}^{2}\right)$ in a follower aircraft at two seating locations: one at the back of the aircraft (D) and one at the front of the aircraft (F) (from Bizinos [3]).

At lateral separations of 1 wingspan or larger, the least discomfort was predicted. In all cases of turbulence, the peaks of discomfort were found to be at a lateral separation of 0.7 to 0.8 
wingspans. For severe turbulence, a monotonic increase in discomfort was found for decreasing lateral separation. It was also found that seat locations closer to the lead aircraft (being at the front and left-hand side of the aircraft for a right echelon formation) produced higher vibration values than those at the rear and right-hand side of the fuselage, implying more discomfort at the front of the aircraft.

To conclude, it was the objective of this study to measure and evaluate the comfort levels felt by a passenger, focusing on the follower aircraft in a two-aircraft right echelon formation. At various turbulent intensities, the discomfort levels increased all along the fuselage with decreasing lateral separation, with the most discomfort shown to be at the front of the fuselage. This study also highlighted that formation flight during severe turbulence is unadvisable in terms of passenger comfort, and that formation in light and moderate turbulence at one wingspan would be the most suitable. The evaluation of passenger comfort was very limited due to a static formation model being used. The author recommended that a more practical formation flight control algorithm should be implemented with a dynamically integrated mathematical model, simulating the aircraft motion more realistically over time. Furthermore, the author suggested that the inclusion of aeroelastic effects in the model would increase the fidelity of the simulation.

\subsubsection{Automatic Control of Commercial Airliners in Formation Flight}

Büchner presented work on automatic flight control of modern-day commercial transport aircraft. This work was the first contribution to this collaborative research in the field of flight control systems during formation flight, setting the foundation for further studies in formation flight for commercial aircraft, such as optimal flight control for passenger comfort and fuel consumption.

A mathematical model of an isolated aircraft was implemented and extended for a formation flight aircraft, and Figure 2.15illustrates the final model for a follower in formation flight. A six degrees of freedom (6DOF) equations of motion block, describing the kinetics and kinematics, was developed and implemented. A forces and moments model block describing the engine, gravitational and conventional aerodynamic forces and moments was added. The control inputs and feedback of the current aircraft states were used to produce the forces and moments as desired.

The additional forces and moments due to vortex interactions were encapsulated in an aerodynamic interaction model superimposed onto the conventional aerodynamics model. The interaction model used in the study by Büchner was developed by Bizinos and Redelinghuys from the University of Cape Town [3] [56] and was described in the previous section. The same results were reproduced. Büchner mentioned two difficulties posed by the interaction model. Firstly, the linearisation of the interactions would not be easy, given the highly non-linear nature of influence factors, and a sufficient linear model is vital for the flight control systems design. A further difficulty, mentions Büchner, was the point of optimum lift benefit and corresponding drag reduction, which was found in the same region as the peak in induced rolling moment. Due to actuator saturation with conventional trimming, the flight control system would not able to 


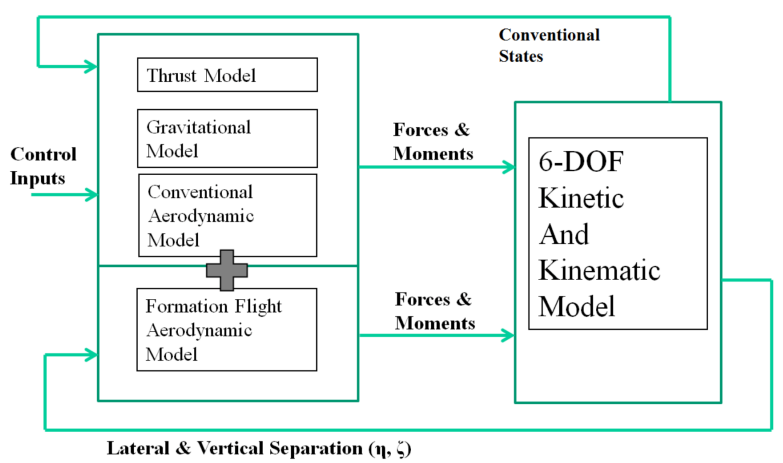

Figure 2.15: Overview of mathematical model architecture used by Büchner for a follower aircraft in formation flight (from Büchner [4]).

fully control the aircraft in this region, making formation flight not possible at the optimum position.

Feasible regions for formation flight were defined as those regions that adhered to certain requirements: unsaturated actuators and a throttle reduction such that all control surfaces and thrust levels remain within the limitations of the aircraft. An extensive trim analysis was performed over ranges of lateral and vertical separation for straight and level flight to determine the feasible regions. Figure 2.16 illustrates two feasible regions: a "sandwich" region at approximatly 0.7 wingspans of lateral separation, and an "outer" region at lateral separations of larger than 1.1 wingspans. The sandwich region showed the most potential in terms of reduced fuel consumption, but proved to be an impractical and risky region to fly in - especially during atmospheric conditions. The outer region showed a reduced throttle saving, but was considerably less than the sandwich region savings. However, the outer region was classified as a safer region for the aircraft. It was concluded that the outer region was the most practically feasible region of the two, and is argued to be a region that gives the follower aircraft enough room to maintain formation in the presence of atmospheric turbulence and disturbances, and to still have a savings benefit compared to the leader aircraft.

The author did not consider non-vertical separations and motivated this decision by evaluating the throttle reduction and aileron deflection in the feasible regions over various vertical separations. The results are illustrated in Figure 2.17, showing that the peak throttle reduction was obtained at zero vertical separation with feasible aileron deflections. Furthermore, while the aileron deflections were less at non-zero vertical separations, the throttle setting became more than the conventional aircraft after approximately 0.4 wingspans, making it no longer feasible.

The proper design of a flight control system to maintain formation required a representative linear state space model of the aircraft. A linear analysis of the aircraft in formation showed that the follower was naturally unstable, reiterating the need for a flight control system. Furthermore, the dynamics in the sandwich region changed more than the dynamics in the outer region, which were more constant. The design of longitudinal and lateral conventional controllers were tested in both linear and non-linear simulations, showing good results and matching between the linear and non-linear models. Evaluation of the conventional controllers in formation flight revealed that certain changes to the outer loop architecture were required for a follower aircraft. The 


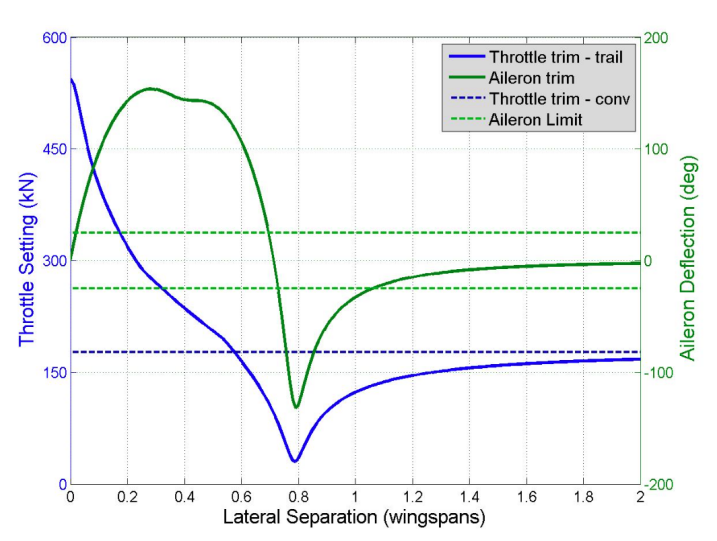

(a) Conventional (dotted lines) vs. formation (solid lines)

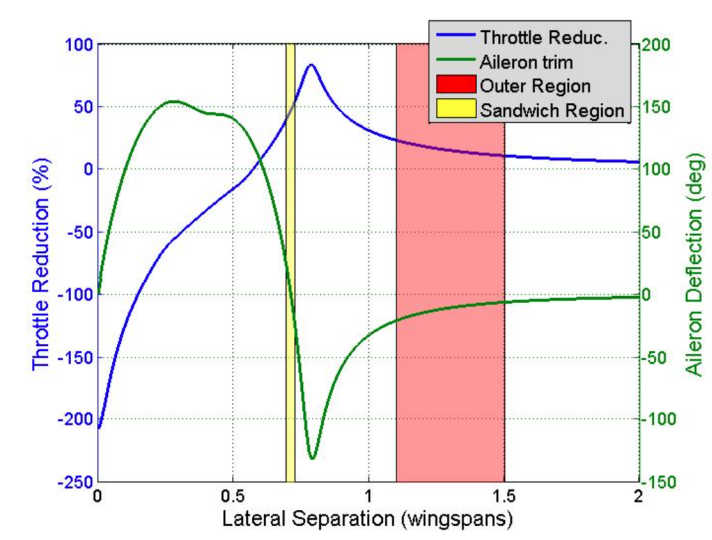

(b) Feasible regions

Figure 2.16: A comparison of the aileron deflection and throttle trim settings over lateral separation and zero vertical separation. Two feasible regions exist, termed as the "sandwich" and "outer" trim regions (from Büchner [4]).

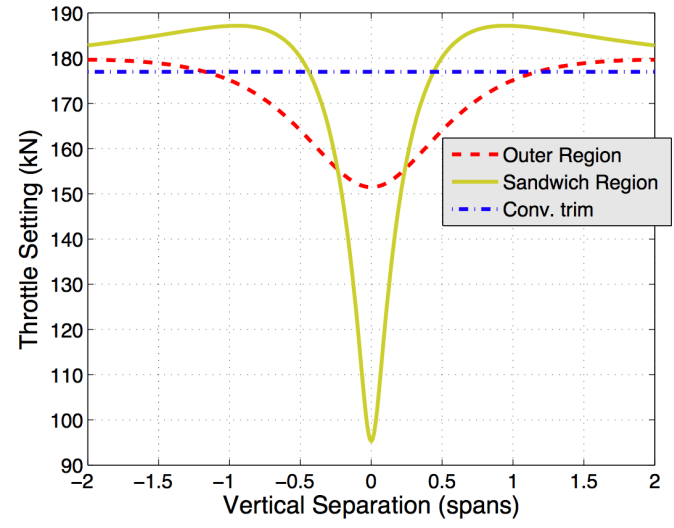

(a) Throttle setting comparison

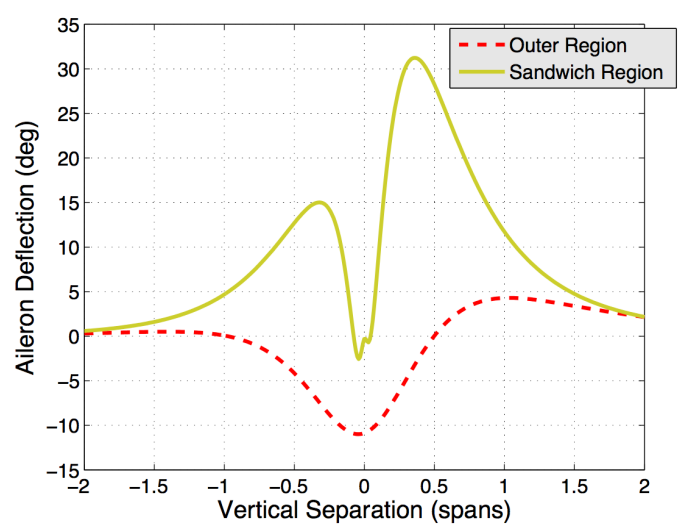

(b) Aileron deflection comparison

Figure 2.17: Trim values for throttle setting and aileron deflections in the sandwich and outer regions over vertical separations (from Büchner [4]).

inner-loop fly-by-wire representative controllers were left unchanged.

Further considerations were suggested by the author, such as lateral motion limiters and a state machine controller to use for entering and exiting the wake vortices. A robustness analysis performed on the follower aircraft in formation revealed that the designed and extended formation controllers were sufficient in the outer region, eliminating lateral and vertical separation disturbances in reasonable time. The author also performed a set of extended non-linear simulations to evaluate the tracking performance, the engine and actuator performance and the overall control system performance in various atmospheric turbulent conditions. It was shown that the follower aircraft consistently maintained a reduced throttle setting in formation flight, but with greater dynamic throttling required compared to the leader. 
To conclude, Büchner performed this study with the objective of determining whether formation flight is realistically viable for commercial passenger aircraft. Various scenarios were investigated and two feasible regions were discoverd: a "sandwich" region with a higher drag savings benefit but which is less safe, and an "outer" region with a lower benefit but which is safer. An integrated mathematical model was developed in simulation to describe the dynamics of the aircraft in formation, and a flight control system was designed and implemented for the formation aircraft. Simulation results showed good performance and the author successfully demonstrated flight control in formation flight, but worked with limited information regarding the fly-by-wire flight control architecture.

However, although the follower was able to successfully maintain the separation between itself and the leader aircraft in turbulent conditions, many practical aspects were not investigated in the Büchner study. In particular, the author suggested that further analysis is required into passenger comfort and fuel consumption. This would determine whether formation flight is practically viable for implementation on passenger aircraft. Additionally, it would be desirable to investigate the need for designing and evaluating optimal passenger comfort and fuel consumption controllers. 


\section{Chapter 3}

\section{Mathematical Modelling}

In this chapter, the foundational framework to describe an aircraft model, be it an isolated conventional model or a formation flight capable model, is detailed. The chapter starts by looking at the axis systems and aircraft notation, followed by an outline of the development of a formation flight model for simulation. Next the conventional isolated aircraft model is defined and extended for formation flight aircraft to produce an extended formation flight model. The atmospheric turbulence model is introduced, which plays an important role in generating realistic forces and moments on an aircraft. Following this, the aircraft separation variables are defined into two categories: the geometric separation variables, which will be used by the flight control architecture of a follower aircraft to maintain the desired separations during formation, and the effective separation variables, which are used in the aerodynamic model of the follower aircraft. Next, the measurement of translational and rotational vibrations experienced by a seated passenger is outlined. The chapter ends with an overview of the fully integrated formation flight simulation model.

\subsection{Axis Systems and Notation}

In the development of a conventional and formation flight extended aircraft model for simulation, a number of axis systems and corresponding aircraft notation is needed. In this section, the inertial, body, stability and wind axes as well as the standard notation are defined. These definitions are used in close accordance with the Boeing 747-100 aircraft model and follow closely from a modelling data document written by authors Hanke and Nordwall [10].

\subsubsection{Axis Systems}

\section{Inertial Axes}

In order to obtain the aircraft equations of motion with the help of Newton's second law of motion, the use of an inertial reference frame is necessary. The standard North-East-Down (NED) axis system, used in most aerospace problems and illustrated in Figure 3.1, can be considered as an inertial axis system. This axis system assumes a non-rotating and flat earth 
surface, and is typically used for short-range flight applications [57]. For the motion about straight and level flight, as in this thesis, it is adequate to assume flight above a flat earth [58].

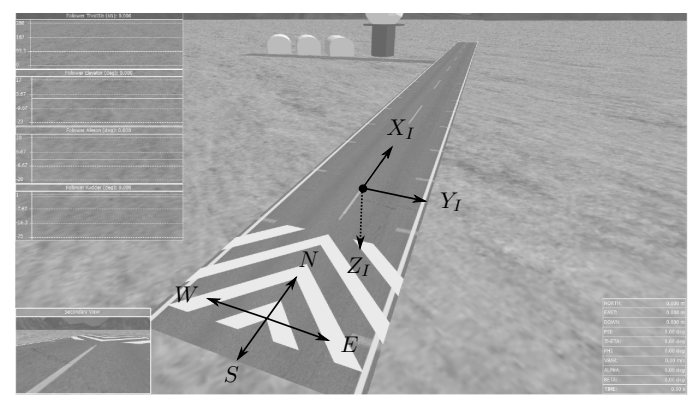

Figure 3.1: North-East-Down axis system.

The origin of the right-handed, orthogonal axis system is chosen to coincide with any arbitrary fixed point on the Earth's surface, such as the start of a runway . The $x$-axis points in the north direction, the $y$-axis points in the east direction and the $z$-axis points in the down direction.

\section{Body Axes}

The body axes, illustrated in Figure 3.2, are fixed to the aircraft and the origin coincides with the centre of mass of the aircraft. The $x$-axis lies in the plane of symmetry and points along the Fuselage Reference Line (FRL). The $y$-axis is perpendicular to the plane of symmetry and points in the direction of the right starboard wing. Completing the right-handed orthogonal axis system is the $z$-axis, pointing downwards relative to the FRL.

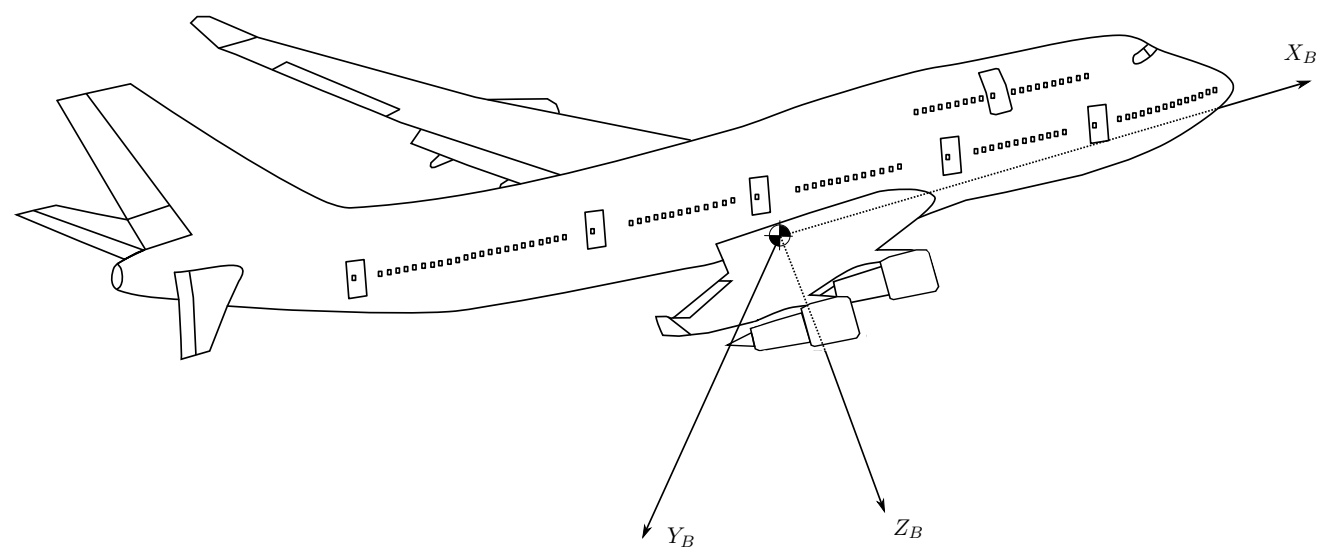

Figure 3.2: B747-100 Aircraft body axis system.

\section{The Stability and Wind Axes}

The stability and wind axes are similar to the body axes in that the origin of both axes coincides with the centre of mass. Figure 3.3 illustrates the relationship between the body, stability and wind axes. The data used for the aerodynamic stability and control derivatives for this study are obtained from a document compiled by Heffley and Jewel [9], who drew on the work of authors 
Hanke and Nordwall [10]. The data contained in these documents have been computed about the stability axes and are used in the current study.

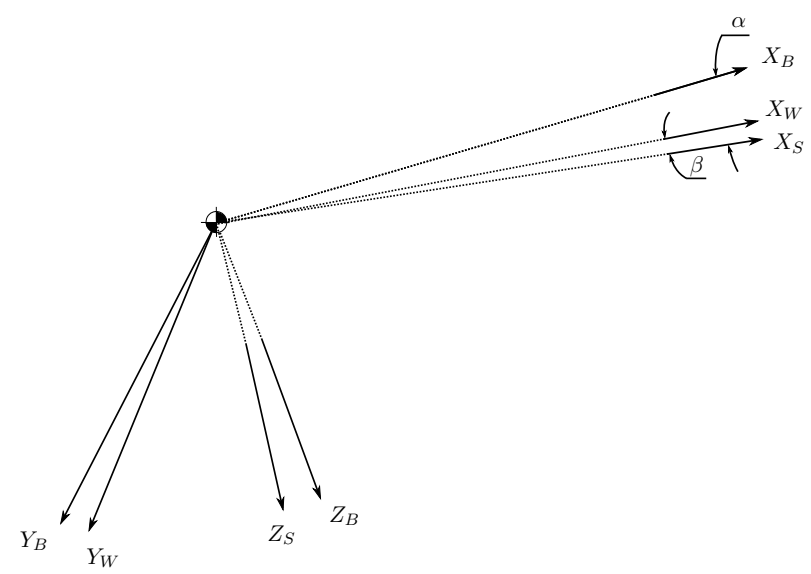

Figure 3.3: Wind, Stability and Body axes relationship.

Starting in the body axis, the stability axis is obtained by rotating the body axis in the negative direction ${ }^{1}$ about the $y$-axis through $\alpha_{F R L}$, the FRL angle of attack 2 . The wind axis is then obtained by rotating the stability axis about the $z$-axis towards the velocity vector by the sideslip angle $\beta$. In the wind axes, the $x$-axis coincides with the relative wind vector, which is also the direction of the velocity vector.

\subsubsection{Aircraft Notation}

Considering the inertial axis as shown in Figure 3.1, the notation in Table 3.1 is used. Considering the body axis as illustrated in Figure 3.2, the notation described in Table 3.2 is used. Figure 3.4 illustrates the notation and sign conventions used in the body axis system. It is assumed that the gravitational field is uniform and that the centre of mass and centre of gravity (CG) are at the same point.

It is common to define the actuator deflections as positive (negative) when a corresponding negative (positive) moment is caused by the deflection [57]. In the current study, positive and negative actuator deflections are defined as follows:

1. Right aileron upwards deflection as seen by the pilot (wheel or stick deflected to the right), which produces a positive rolling moment, is defined as positive aileron deflection 3

2. Elevator upwards deflection (wheel or stick pulled towards pilot), which produces a positive pitching moment, is defined as a negative elevator deflection.

3. Right rudder deflection (right pedal forward, left pedal backwards), which produces a positive yawing moment, is defined as a negative rudder deflection.

\footnotetext{
${ }^{1}$ The negative direction here means in the opposite sense to the positive direction of the right-hand rule.

${ }^{2}$ From here on the angle of attack $\alpha$ will be assumed to be along the FRL unless explicitly otherwise stated

${ }^{3}$ Note that the convention used for positive aileron deflection is different than usual.
} 
Table 3.1: Standard aircraft notation used in the inertial axis.

\begin{tabular}{ll}
\hline Symbol & Description \\
\hline$N, E, D$ & Coordinates of the position vector \\
$\phi, \theta, \psi$ & Euler $3-2-1$ attitude parameters \\
\hline
\end{tabular}

Table 3.2: B747-100 Aircraft notation used in the body axis.

\begin{tabular}{ll}
\hline Symbol & Description \\
\hline$X, Y, Z$ & Coordinates of the force vector (Axial, lateral and normal forces) \\
$L, M, N$ & Coordinates of the moment vector (Roll, pitch and yaw moments) \\
$U, V, W$ & Coordinates of the linear velocity vector (Axial, lateral and normal velocities) \\
$P, Q, R$ & Coordinates of the angular velocity vector (Roll, pitch and yaw rates) \\
$\delta_{A}, \delta_{E}, \delta_{R}$ & Aileron, elevator and rudder control surface deflections \\
\hline
\end{tabular}

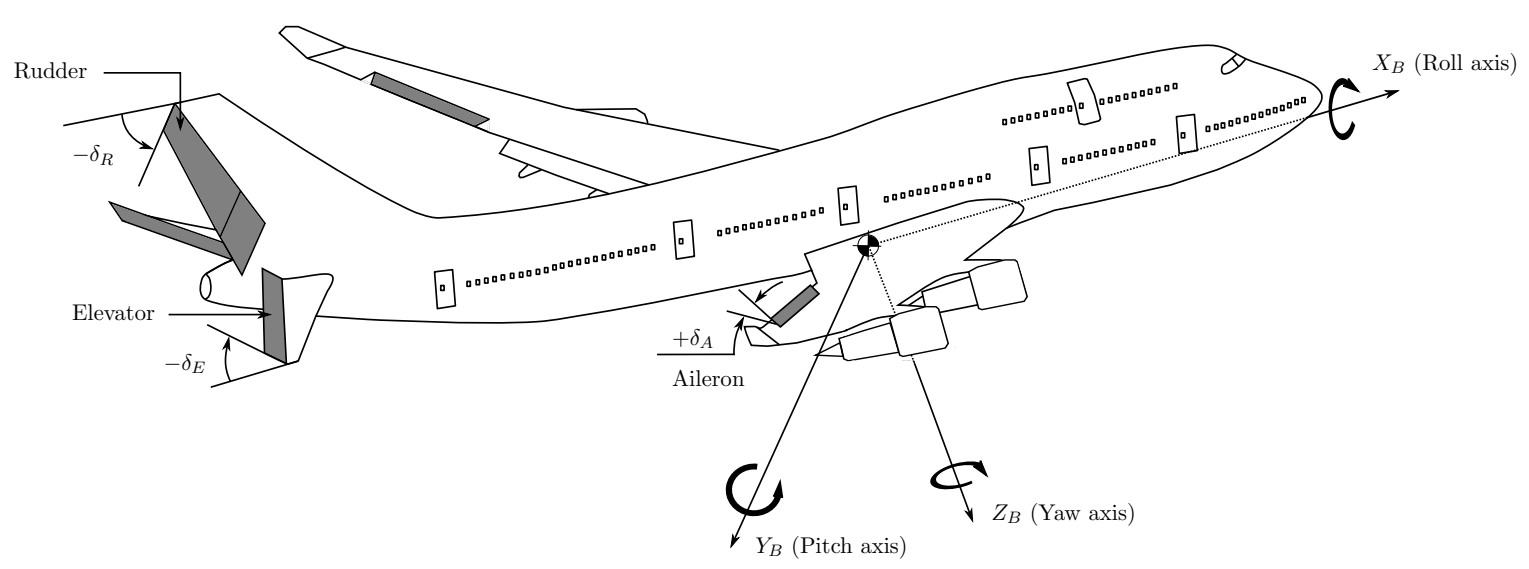

Figure 3.4: B747-100 Aircraft notation

The cartesian coordinates of the linear airspeed velocity vector are generally expressed in polar form, considering Figure 3.5, as follows,

$$
\begin{aligned}
\bar{V} & =\sqrt{U^{2}+V^{2}+W^{2}} \\
\alpha & =\arctan \left(\frac{W}{U}\right) \\
\beta & =\arcsin \left(\frac{V}{\bar{V}}\right)
\end{aligned}
$$


with the inverse relationships defined as follows,

$$
\begin{aligned}
U & =\bar{V} \cos (\alpha) \cos (\beta) \\
V & =\bar{V} \sin (\beta) \\
W & =\bar{V} \sin (\alpha) \cos (\beta)
\end{aligned}
$$

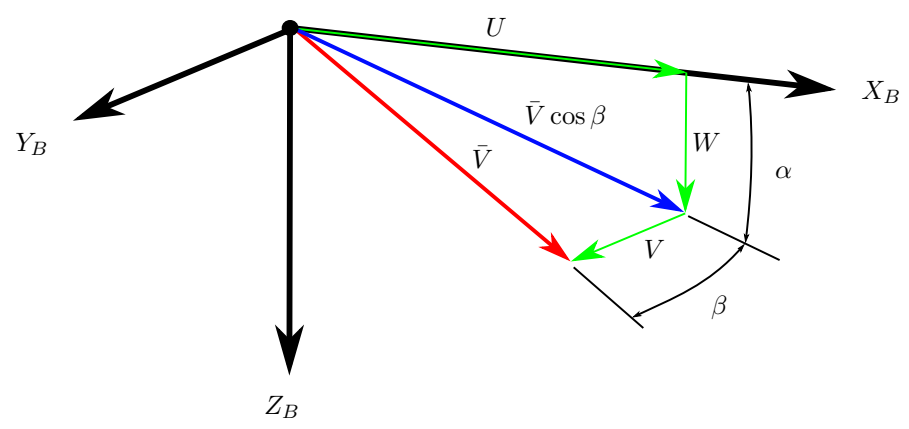

Figure 3.5: Polar velocity coordinates (adapted from Peddle et al. [57]).

\subsection{Development of Formation Flight Model for Simulation}

Figure 3.6 illustrates the proposed framework for the formation flight simulation model that will be used in this study. The Boeing B747-100 aircraft model has been used for a wide variety of engineering research studies and also by Bizinos [3] and Büchner [4]. Due to the public availability of the aircraft data documents [10] [], it is also used in this simulation model to represent both the leader and follower aircraft during formation flight.

Considering the leader and follower aircraft subsystems, each subsystem should include: pilot or autopilot commands that feed either directly to the actuators or through the flight control laws; a dynamic aircraft model; and a set of sampled measurement sensors, with a sufficiently high sampling rate. Two unique subsystems are included in the follower subsystem, one to measure the aircraft separation variables and another to measure the vibrations and evaluate the associated levels of comfort.

Each part of the simulation model, except for the pilot/autopilot model and the flight control architecture, will be covered in the following sections. The pilot/autopilot model is a straightforward subsystem that provides longitudinal and lateral-directional reference commands, coded into the simulation model. The flight control architecture will be designed, implemented and verified in Chapters 5 and 6 for conventional isolated and extended formation flight respectively. 

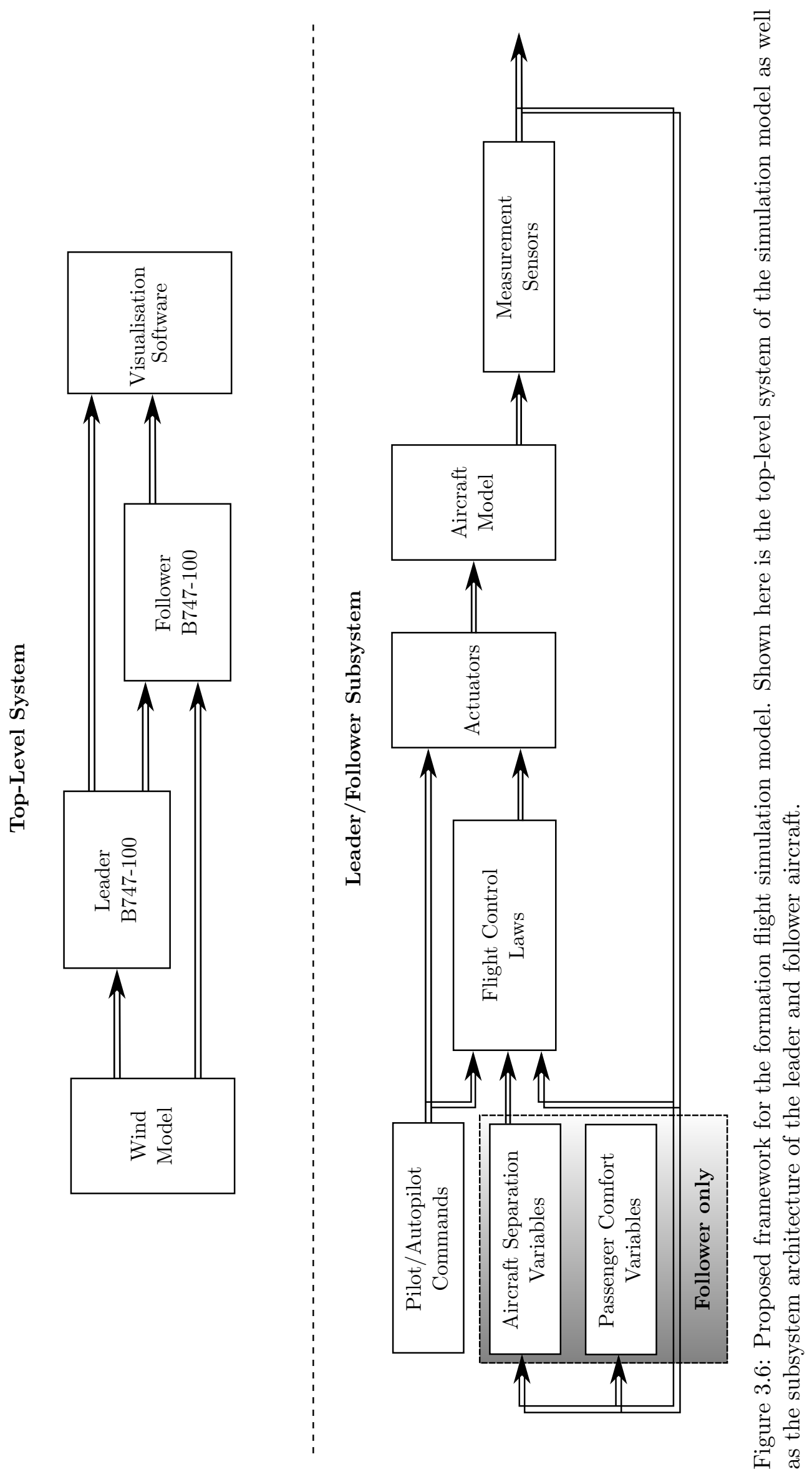


\subsection{Conventional Model}

In this section, the conventional aircraft model used by the leader aircraft, as illustrated in Figure 3.7, is defined.

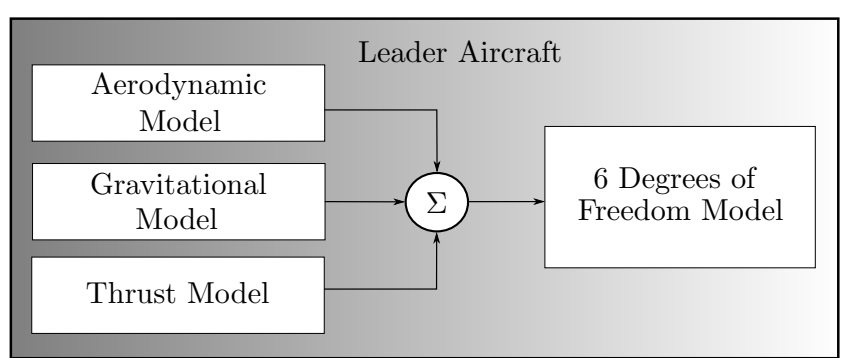

Figure 3.7: Conventional aircraft model used by an isolated aircraft. This model will be used in the leader aircraft of a formation flight simulation.

\subsubsection{Six Degrees of Freedom Equations of Motion}

The foundation for the study of aircraft flight dynamics is the proper and sufficient description of the aircraft equations of motion. Various models which describe the motion of the aircraft can be developed from simple to complex equations that completely describe the aircraft. For advanced applications, it is often necessary to use the fully descriptive non-linear form of the equations, which can be retained using computer simulation techniques. However, for the scope of this study and for the control system design purposes later in this thesis, an aircraft is modelled as a six degrees of freedom (6DOF) rigid body. Various literature sources quantify the development of the simplified 6DOF equations of motion for an B747-100 aircraft [59] 60] 61].

The equations of motion can be derived from Newton's second law of motion with respect to inertial space [59] 60] 62],

$$
\begin{aligned}
\mathbf{F}_{B} & =\left.\frac{d}{d t}\left(m \mathbf{V}_{B}\right)\right|_{I} \\
\mathbf{M}_{B} & =\left.\frac{d}{d t}\left(\mathbf{I}_{B} \omega_{B}\right)\right|_{I}
\end{aligned}
$$

where $m$ is the aircraft mass, $\mathbf{V}_{B}$ is the linear velocity in the body axes, $\omega_{B}$ is the angular velocity in the body axes, and $\mathbf{I}_{B}$ is the moment of inertia of the aircraft in body axes,

$$
\mathbf{I}_{B}=\left[\begin{array}{lll}
I_{x x} & I_{x y} & I_{x z} \\
I_{x y} & I_{y y} & I_{y z} \\
I_{x z} & I_{y z} & I_{z z}
\end{array}\right]
$$

The body axis system rotates with an angular velocity $\omega_{B}$ with respect to inertial space. To obtain the time derivatives in Equations (3.3a) and (3.3b) with respect to the body axes, the 
equation of Coriolis [59, 60, 62] is used to relate the inertial time derivative to the body time derivative,

$$
\begin{aligned}
\mathbf{F}_{B} & =\left.\frac{d}{d t}\left(m \mathbf{V}_{B}\right)\right|_{B}+\omega_{B} \times\left(m \mathbf{V}_{B}\right) \\
\mathbf{M}_{B} & =\left.\frac{d}{d t}\left(\mathbf{I}_{B} \omega_{B}\right)\right|_{B}+\omega_{B} \times\left(\mathbf{I}_{B} \omega_{B}\right)
\end{aligned}
$$

The vector equations for a general six degrees of freedom motion model can be displayed in scalar form as a set of six non-linear, coupled differential equations -3 translational and 3 rotational degrees of freedom equations,

$$
\begin{aligned}
X & =m(\dot{U}-V R+W Q) \\
Y & =m(\dot{V}+U R-W P) \\
Z & =m(\dot{W}-U Q+V P) \\
L & =\dot{P} I_{x x}+Q R\left(I_{z z}-I_{y y}\right) \\
M & =\dot{Q} I_{y y}+P R\left(I_{x x}-I_{z z}\right) \\
N & =\dot{R} I_{z z}+P Q\left(I_{y y}-I_{x x}\right)
\end{aligned}
$$

where $I_{x x}, I_{y y}$ and $I_{z z}$ are the moments of inertia about the $X_{B}, Y_{B}, Z_{B}$ body axes. These differential equations assume [59] 60] 61] 62]:

- That the $X Y$-plane in the body axis of the aircraft is symmetric with the origin of the body axis at the CG of the aircraft. The products of inertia, $I_{x y}$ and $I_{y z}$ are thus zero.

- The product of inertia $I_{x z}$ is considered negligibly small.

- A rigid body aircraft with constant mass.

- The Earth's rotation rate can be neglected over the time scales of the dynamics.

- Small perturbations from equilibrium.

- Quasi-steady flow - the airflow around the aircraft changes instantaneously when disturbed from equilibrium.

The author acknowledges that for simulation models of large aircraft such as the B747-100 aircraft, the off axis terms of the moment of inertia matrix should not actually be ignored in the simulation. They have been ignored in this study for both the linearisation and simulations to simplify the math complexity. 


\subsubsection{Attitude and Position Dynamics}

Kinematics is the branch of mechanics concerned with the motion of the aircraft in inertial space without reference to the forces and moments in Equation 3.6. These motion variables include the linear and angular velocities, as well as the attitude and position motion variables. To this end, it is necessary to describe the orientation of the body axis system with respect to the inertial axis before the kinematic equations can be included in the conventional aircraft model.

In the current study, the position and orientation of the aircraft are given relative to the Earth-fixed NED axis system. For commercial passenger airliners that travel for long times and over long distances, the rotation of the NED axis system as a function of changes in latitude and longitude should also be modelled. However, to minimise the mathematical complexity, it is assumed that the latitude and longitude will not change significantly over the time scales and distances travelled in the formation flight simulations later on.

\section{Euler 3-2-1 Attitude Parameterisation}

The attitude of the body axes relative to the inertial space is accurately represented by the popular three Euler attitude parameters: $\phi, \theta, \psi$. These angles are used in a predefined order to describe the rotation between the body and inertial axes. The sequence of rotation depends on the application, with the most common sequence used in aviation being the 3-2-1 sequence, illustrated in Figure 3.8. This sequence starts with the inertial and body axis systems aligned, and then moves the body axis system through the following set of ordered rotations:

(3) A positive rotation through the heading (azimuth) angle $\psi$

(2) A positive rotation through the pitch (elevation) angle $\theta$

(1) A positive rotation through the roll (bank) angle $\phi$
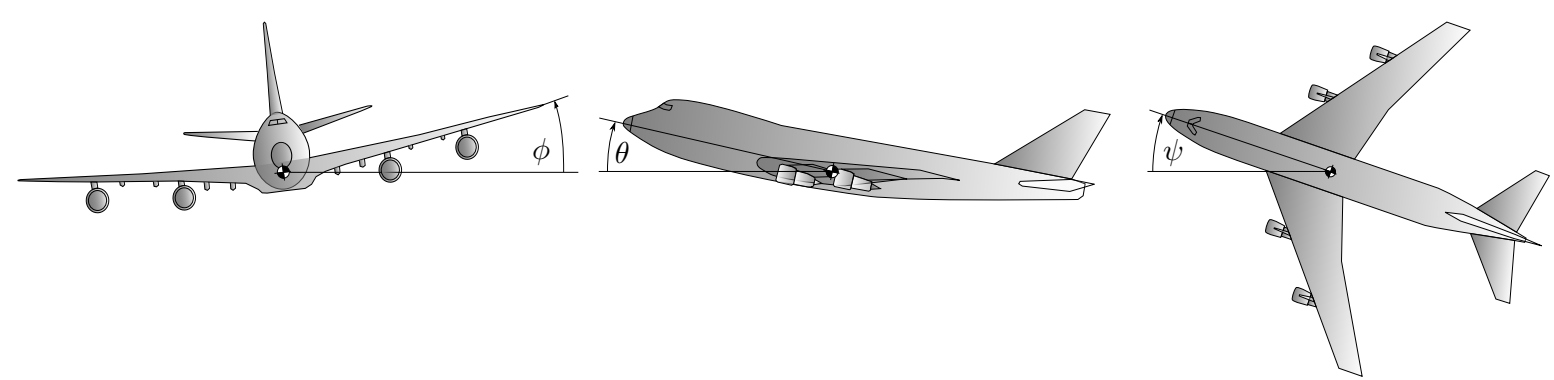

Figure 3.8: Illustration of 3-2-1 Euler angles.

More complex methods, such as Quaternions or Direction Cosines, could also be used to parameterise the attitude. These methods have the advantage over the Euler approach in the sense that there are no singularities present. For a 3-2-1 Euler parameterisation, a singularity occurs at $90^{\circ}$ about the $Y_{I}$ axis. Fortunately, in commercial transport aircraft flight conditions, this pitch angle is never achieved and the singularity is thus never realised. 


\section{Attitude Dynamics}

The equation describing how the roll, pitch and yaw rates $(P, Q, R)$ relate to the time rate of changes of the roll, pitch and yaw Euler angles is written as, 60]

$$
\left[\begin{array}{c}
\dot{\phi} \\
\dot{\theta} \\
\dot{\psi}
\end{array}\right]=\left[\begin{array}{ccc}
1 & \sin \phi \tan \theta & \cos \phi \tan \theta \\
0 & \cos \phi & -\sin \phi \\
0 & \sin \phi \sec \theta & \cos \phi \sec \theta
\end{array}\right]\left[\begin{array}{l}
P \\
Q \\
R
\end{array}\right]
$$

\section{Transformation Matrix}

Before the position dynamics, namely how the position vector changes over time as a function of the aircraft's velocity, can be obtained, a transformation matrix is required to convert vectors between two axis systems. The direction cosine matrix (DCM), denoted by $\mathbf{T}$, does this for right-handed orthogonal systems, and is defined as,

$$
\mathbf{T}=\left[\begin{array}{ccc}
\cos \phi \cos \theta & \sin \phi \cos \theta & -\sin \theta \\
\cos \phi \sin \theta \sin \phi-\sin \phi \cos \phi & \sin \psi \sin \theta \sin \phi+\cos \psi \cos \phi & \cos \theta \sin \phi \\
\cos \phi \sin \theta \cos \phi+\sin \psi \sin \phi & \sin \psi \sin \theta \cos \phi-\cos \psi \sin \phi & \cos \theta \cos \phi
\end{array}\right]
$$

Due to the orthonormality of the transformation matrix, the inverse of the DCM is simply the transpose,

$$
\mathbf{T}^{-1}=\mathbf{T}^{T}
$$

\section{Position Dynamics}

The kinematic relationship between the inertial position and the linear velocity vector in the body axis is described with the inverse (or transpose) of the DCM transformation matrix,

$$
\left[\begin{array}{c}
\dot{N} \\
\dot{E} \\
\dot{D}
\end{array}\right]=\mathbf{T}^{T}\left[\begin{array}{c}
U \\
V \\
W
\end{array}\right]
$$

\subsubsection{Forces and Moments}

Different mathematical models are used to describe the forces and moments that act on an aircraft as a function of its current state. For the conventional aircraft in this thesis, there are three categories which generate the most significant forces and moments: aerodynamic, thrust 
and gravitational. The force and moment equations of motion can be expanded as a sum of the different forces and moments as follows,

$$
\begin{aligned}
X & =X^{A}+X^{T}+X^{G} \\
Y & =Y^{A}+Y^{T}+Y^{G} \\
Z & =Z^{A}+Z^{T}+Z^{G} \\
L & =L^{A}+L^{T}+L^{G} \\
M & =M^{A}+M^{T}+M^{G} \\
N & =N^{A}+N^{T}+N^{G}
\end{aligned}
$$

where the superscripts $A, T$ and $G$ denote aerodynamic, thrust and gravitational respectively. The following subsections will address each of these categories in more detail.

\section{Aerodynamic Model}

The aerodynamics of an airframe, the $\mathrm{CG}$ as well as the control surfaces of an aircraft are considered to make the greatest contribution to the stability and control characteristics of an aircraft. For this reason, it is a fundamental model in the process of describing the forces and moments of an aircraft. The aerodynamic model is incorporated into the forces and moments model of the aircraft in the form of non-dimensional aerodynamic coefficients. They describe the aerodynamic properties of the Boeing airframe and contain various stability and control derivatives as a function of aerodynamic parameters.

The aerodynamic forces and moments are proportional to the dynamic pressure experienced by the aircraft [63]. If the dynamic pressure is defined as,

$$
q=\frac{1}{2} \rho \bar{V}^{2}
$$

where $\rho$ is the air density, the aerodynamic force and moment co-ordinates are expanded as follows,

$$
\begin{aligned}
X^{A} & =q S C_{X} \\
Y^{A} & =q S C_{Y} \\
Z^{A} & =q S C_{Z} \\
L^{A} & =q S b C_{l} \\
M^{A} & =q S \bar{c} C_{m} \\
N^{A} & =q S b C_{n}
\end{aligned}
$$

where $S$ is the wing area, $b$ is the wingspan, $\bar{c}$ is the mean aerodynamic chord and $C($.$) are$ the non-dimensional aerodynamic force and moment coefficients. The aircraft aerodynamic coefficients are specified as functions of the aerodynamic angles $(\alpha$ and $\beta$ ), the Mach number 
$(M)$ and altitude $(h)$, as well as the control surface deflections [64]. The dependence of an aerodynamic coefficient can thus be written as,

$$
C_{(.)}=f\left(\alpha, \beta, M, h, \delta_{E}, \delta_{A}, \delta_{R}\right)
$$

So far, then, the coefficients in Equation (3.14) are static coefficients obtained from measurements on a stationary model of the Boeing in a wind tunnel test (or using other methods such as system identification methods or computational fluid dynamics). However, it is also necessary to describe the change in these coefficients when an aircraft makes a manoeuvre, which will require differential terms in the aerodynamic model. For an aircraft that makes manoeuvres which significantly change its orientation, Equation (3.14) will exhibit a complicated functional dependence that would have to be modelled as a look-up table in a computer simulation. However, if the aerodynamic model is linearised about a straight and level flight equilibrium point, with small perturbations from this condition, the functional dependence of Equation (3.14) turns out to be much less complex, and any coefficient can be broken down into a sum of simpler linear terms.

To motivate why a high level of complexity is unnecessary, it is assumed in the straight and level flight envelope of the aircraft that any manoeuvres are slow enough that the flow field around the aircraft is quasi-steady. This type of flow assumes that the airflow around the aircraft changes instantaneously when the aircraft is disturbed from an equilibrium [59]. Stated differently by Stevens and Lewis [64, the flow field around the aircraft is able to adjust in step with the slow manoeuvres. Using this assumption allows the aerodynamic forces and moments to be modelled as terms linearly proportional to the angular rates, control deflections and other parameters that produce them. These terms are the aerodynamic stability and control derivatives. All derivatives with respect to the rates of change of velocities and of each control surface are neglected and omitted in this thesis.

Although the assumption of quasi-steady flow makes the problem of modelling aircraft manoeuvres much simpler, it is well known from the literature [59] that for high Mach numbers $(M \geq 0.8)$, the actual performance of the aircraft will not be well predicted by the theoretical results of the quasi-steady flow assumption. Although an improved aerodynamic interaction model would represent a more complete picture of the aerodynamic effects, it will be assumed that the divergence of the aircraft performance between the actual and theoretical results is small at a straight and level flight condition.

Using the available data for the Boeing 747 [10] [9, as well as data from previous studies that used these sources to build the definition of the aerodynamic model [3] [4], the various non-dimensional aerodynamic coefficients are expanded in the stability axes $\left(C_{(.)_{S}}\right)$ around a linearisation point (see Equations $3.15 \mathrm{a}$ ) to $3.15 \mathrm{~h})$ ). The linearisation point, denoted by the subscript $l p$, is chosen from the set of flight conditions in Heffley and Jewel [9], which provides the values of the aerodynamic coefficients as well as the aerodynamic derivatives at the linearisation point (see Appendix A for more details). 


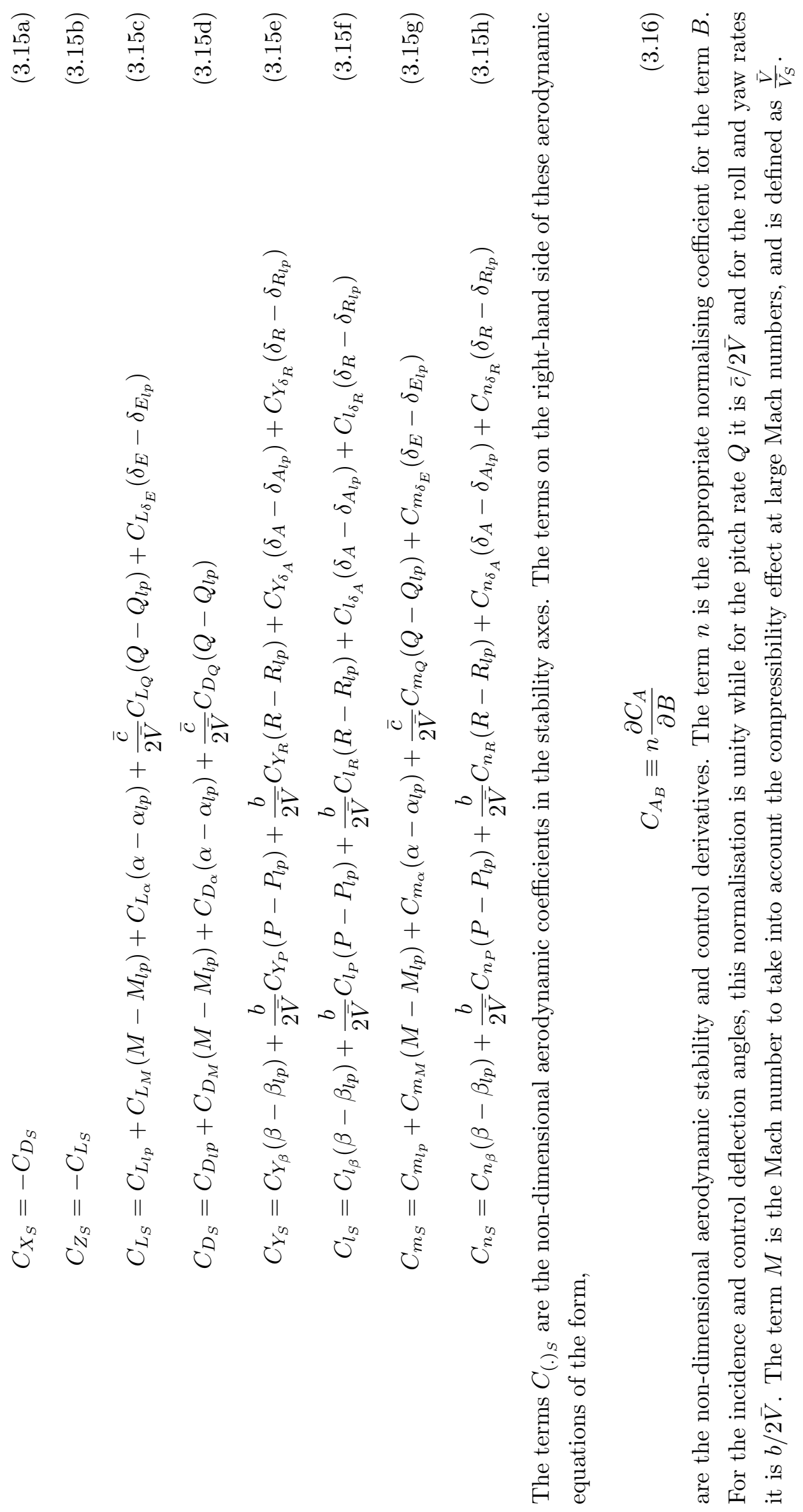


A transformation is required to obtain the coefficients in the same axis system in which the equations of motion are evaluated, namely the body axes. Since accurate simulation results are desirable wherever possible without increasing the complexity of the task at hand too much, all coefficients in the stability axes are transformed to the body axes. The transformation is also necessary due to the large magnitude difference between the lift and drag coefficients, arising from typical aircraft lift to drag ratios. This transformation, as shown in Equation 3.17, involves a rotation about the $Y$-axis through the angle of attack $\alpha$,

$$
\begin{aligned}
C_{X} & =C_{X_{S}} \cos \alpha-C_{Z_{S}} \sin \alpha \\
C_{Y} & =C_{Y_{S}} \\
C_{Z} & =C_{X_{S}} \sin \alpha+C_{Z_{S}} \cos \alpha \\
C_{l} & =C_{l_{S}} \cos \alpha-C_{n_{S}} \sin \alpha \\
C_{m} & =C_{m_{S}} \\
C_{n} & =C_{l_{S}} \sin \alpha+C_{n_{S}} \cos \alpha
\end{aligned}
$$

\section{Gravitational Model}

In the inertial axis, the weight of the aircraft is a force in the down direction, quantifying the gravitational force of the aircraft,

$$
\mathbf{F}_{I}^{G}=\left[\begin{array}{c}
0 \\
0 \\
m g
\end{array}\right]
$$

This coordinate vector is transformed to the body axes coordinates using the transformation matrix in Equation 3.8 to yield the transformed gravitational force vector,

$$
\left[\begin{array}{c}
X^{G} \\
Y^{G} \\
Z^{G}
\end{array}\right]=\mathbf{T}\left[\begin{array}{c}
0 \\
0 \\
m g
\end{array}\right]=\left[\begin{array}{c}
-\sin \theta \\
\cos \theta \sin \phi \\
\cos \theta \cos \phi
\end{array}\right] m g
$$

Since a uniform gravitational field is assumed, the CG coincides with the centre of mass, and the gravitational force will produce no moments on the aircraft,

$$
L^{G}=M^{G}=N^{G}=0
$$

\section{Thrust Model}

Considering that conventional aircraft would predominantly fly at a straight and level flight condition, experiencing minimal perturbations from trim, it is assumed that a simplistic firstorder engine model would suffice for the thrust model of the aircraft. The first order lag model is given as follows, 


$$
\dot{T}=-\frac{1}{\tau} T+\frac{1}{\tau} T_{c}
$$

where $T$ is the thrust magnitude in response to a commanded thrust $T_{c}$ and $\tau$ is the engine lag time constant. A further assumption made is that the thrust vector lies predominantly along the $X_{B}$-axis, resulting in the following force and moment vectors,

$$
\begin{aligned}
& X^{T}=T, Y^{T}=Z^{T}=0 \\
& L^{T}=M^{T}=N^{T}=0
\end{aligned}
$$

The jet engines of typical passenger transport airliners are slightly rotated upwards relative to the body axis and inwards toward the aircraft fuselage. The engines thus dont actually produce a force through the mass centre. Although the assumption of having a thrust vector predominantly about the $X_{B}$-axis might sound too simple, it is argued that without dynamic throttling this assumption is not too inaccurate. Ideally, without dynamic throttling, the y-axis components of the engine forces as well as any rolling and yawing moments should cancel each other out. The resulting effect of the slightly offset engines will then only be a small component of engine thrust in the $Z_{B}$-axis, and a small pitching moment. It is assumed that the flight control system will be able to counter these effects, and maintain formation flight without impacting passenger ride comfort.

\subsection{Aircraft Separation}

In a formation flight simulation, the relative positioning of the aircraft is required. The geometric separation of the aircraft is required in the formation guidance laws to maintain a desired axial, vertical and lateral separation during formation flight. The effective separation, which includes the turbulence effects as well as the time delay between the aircraft, is required by the extended formation aerodynamic model to include the induced forces and moments due to the trailing vortex of the leader. In this section, the geometric and effective aircraft separation variables are derived in detail.

\subsubsection{Geometric Separation}

Figure 3.9 illustrates a top-view of two aircraft in right echelon formation. The geometric longitudinal $\Delta x$ and lateral $\Delta y$ separations are shown in Figure 3.9. A new axis is defined at the centre of gravity of the leading aircraft, obtained by rotating the inertial $X_{I}$ and $Y_{I^{-} \text {-axes }}$ through the flight path heading angle $\psi_{a z}$ of the aircraft. This axis, called the separation axis, will be used to determine the relative geometric separation between a leader and a follower aircraft.

Considering Figure 3.9, the flight path heading angle of the leader is calculated as follows, 


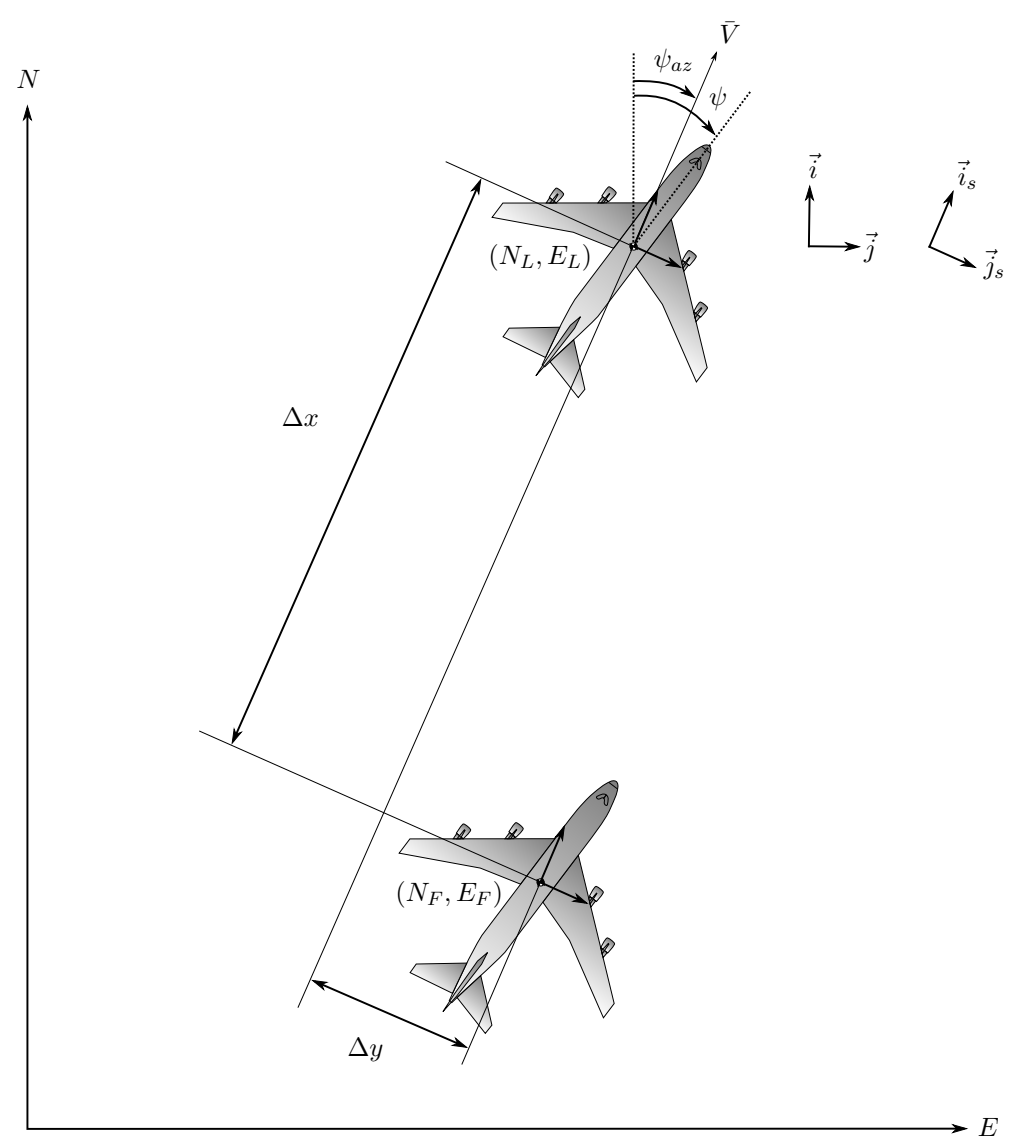

Figure 3.9: Top-view of geometric aircraft separation.

$$
\tan \psi_{a z}=\frac{\dot{E}_{L}}{\dot{N}_{L}}
$$

To obtain the geometric distances $\Delta x$ and $\Delta y$, the follower aircraft's position is transformed from the NED axis to the separation axis using a translation and rotation transformation,

$$
\left[\begin{array}{c}
\Delta x \\
\Delta y
\end{array}\right]=\left[\begin{array}{cc}
\cos \psi_{a z} & \sin \psi_{a z} \\
-\sin \psi_{a z} & \cos \psi_{a z}
\end{array}\right]\left[\begin{array}{c}
N_{F}-N_{L} \\
E_{F}-E_{L}
\end{array}\right]
$$

The geometric vertical separation is illustrated in Figure 3.10 and is somewhat more straightforward to calculate than the longitudinal and lateral geometric separations. Considering the side-view of the geometric aircraft separation,

$$
\Delta z=D_{F}-D_{L}
$$

In formation flight studies, it is common to normalise the geometric separations to wingspan. 


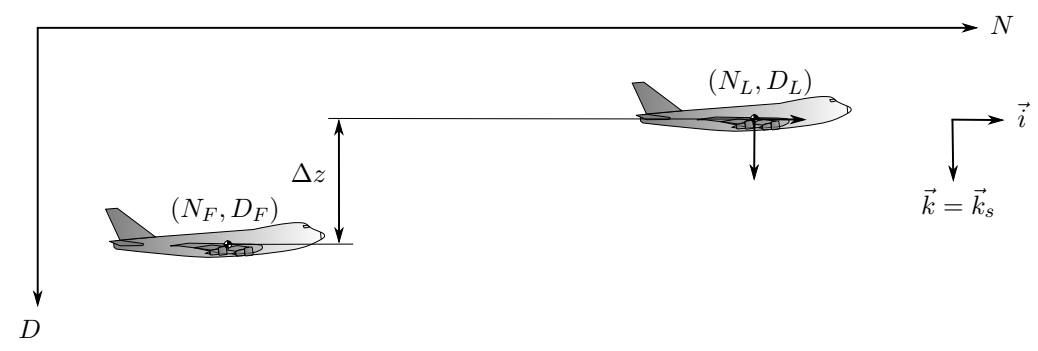

Figure 3.10: Top-view of geometric aircraft separation.

The normalised separations are defined as follows,

$$
\begin{aligned}
& \xi=\frac{\Delta x}{b} \\
& \eta=\frac{\Delta y}{b} \\
& \zeta=\frac{\Delta z}{b}
\end{aligned}
$$

where $\xi$ is the normalised axial separation, $\eta$ is the normalised lateral separation and $\zeta$ is the normalised vertical separation.

\subsubsection{Effective Separation}

The effective separation is a prediction of the vortex position relative to the follower after taking into account the turbulent effects on the trailing vortex, and the travelling time of the vortex from the leader to the follower. Only the lateral and vertical separations of the vortex are considered, and it is assumed the longitudinal separation will not change from the geometric longitudinal separation. An instantaneous effective separation will be defined as an intermediate value to obtain the effective separation, and describes the change in the geometric separation due to turbulent conditions, but does not yet take into account the time delay between the two aircraft. The instantaneous separation is delayed by a time $\tau_{d}$ seconds, dependent on the formation geometry, to obtain the effective separation between the aircraft.

In the following sections, the instantaneous lateral $\Delta y_{I, \text { eff }}$ and vertical $\Delta z_{I, \text { eff }}$ separations are defined, normalised to wingspan, and used as intermediate values to obtain the normalised lateral $\eta_{\text {eff }}$ and vertical $\zeta_{\text {eff }}$ effective separations.

\section{Instantaneous Lateral Separation}

To illustrate how this is obtained, consider the scenario given in Figure 3.11. The geometric lateral separation angle $\theta_{s}$ is used to give the following relationship,

$$
\sin \theta_{s}=\frac{\Delta y}{\sqrt{\Delta x^{2}+\Delta y^{2}}}
$$

It is also convenient to re-write the separation angle $\theta_{s}$ as follows, 


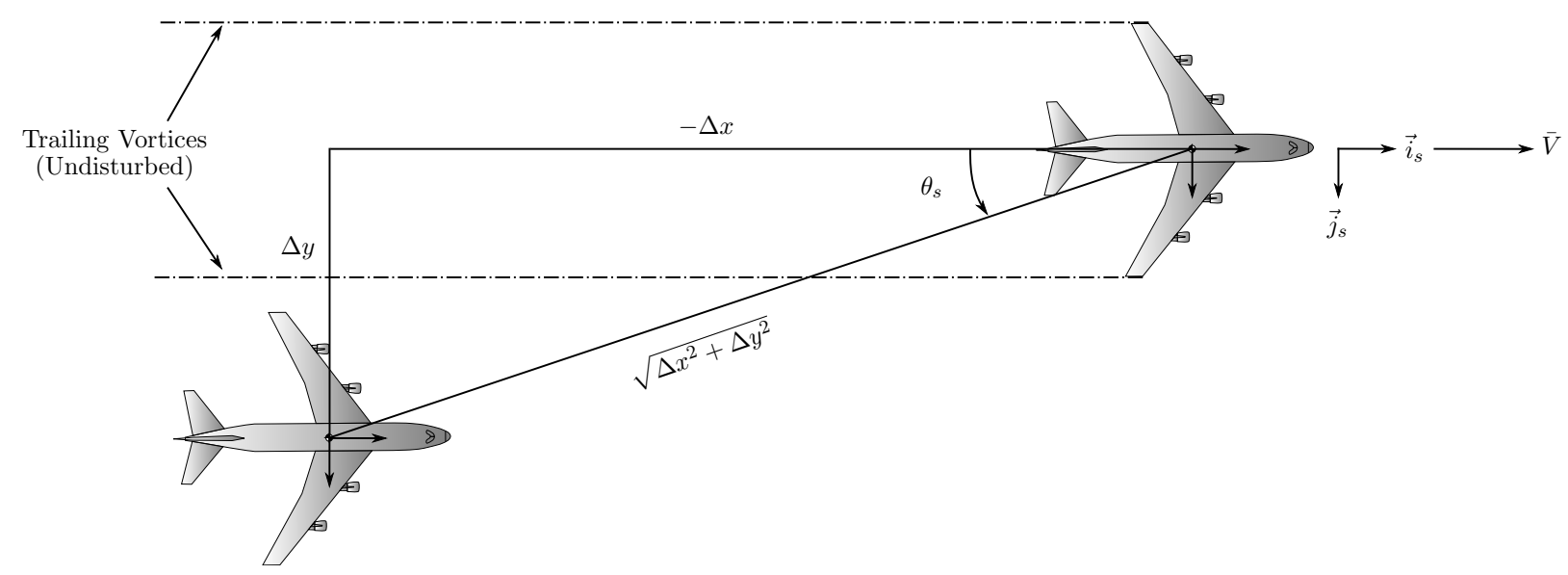

Figure 3.11: Top-view separation scenario during formation flight in non-turbulent conditions.

$$
\theta_{s}=\tan ^{-1}\left(\frac{\Delta y}{-\Delta x}\right)
$$

Through the substitution of Equations 3.27 and 3.28 , the following equation is derived for the geometric lateral separation after some rearrangement,

$$
\Delta y=\sqrt{\Delta x^{2}+\Delta y^{2}} \sin \left[\tan ^{-1}\left(\frac{\Delta y}{-\Delta x}\right)\right]
$$

Now consider a lateral gust velocity $v_{g}$ added to the system, as illustrated in Figure 3.12 . The vortices of the leader aircraft are now disturbed by a gust angle $\theta_{g}$ and move away from trailing vortices in non-turbulent conditions. To a good approximation, we have the following result,

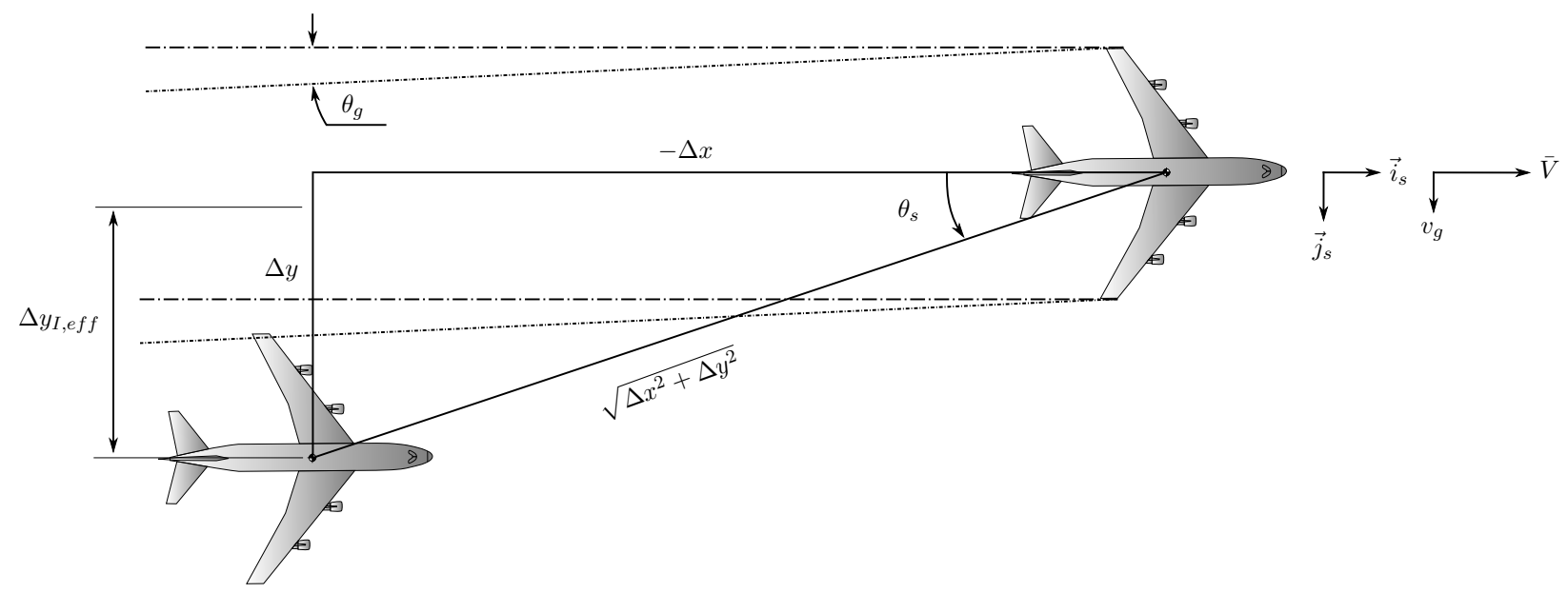

Figure 3.12: Top-view separation scenario during formation flight with a lateral gust $\left(v_{g}\right)$.

$$
\Delta y_{I, e f f}=\sqrt{\Delta x^{2}+\Delta y^{2}} \sin \theta_{e f f}
$$

where,

$$
\theta_{\text {eff }}=\theta_{s}-\theta_{g}
$$


is the new effective angle of separation due to the lateral gust disturbance. The induced change in separation angle due to the lateral gust $v_{g}$ is calculated from Figure 3.12 as follows,

$$
\theta_{g}=\tan ^{-1}\left(\frac{v_{g}}{-\bar{V}}\right) \approx-\frac{v_{g}}{\bar{V}}
$$

assuming that the aircraft velocity $\bar{V}$ is much larger than the lateral gust velocities. Substitution of Equations (3.28), (3.30), (3.31) and (3.32) yields the final instantaneous effective lateral separation between a leader and follower,

$$
\Delta y_{I, e f f} \approx \sqrt{\Delta x^{2}+\Delta y^{2}} \sin \left[\tan ^{-1}\left(\frac{\Delta y}{-\Delta x}\right)+\frac{v_{g}}{\bar{V}}\right]
$$

Intuitively, as the lateral gust velocity $v_{g}$ tends to zero, the instantaneous effective separation becomes the same as the geometric lateral separation.

\section{Instantaneous Vertical Separation}

The same approach is followed to obtain the instantaneous effective vertical separation between a leader and a follower. Consider Figure 3.13, which illustrates another separation scenario during formation flight, this time with a vertical gust $w_{g}$ added.

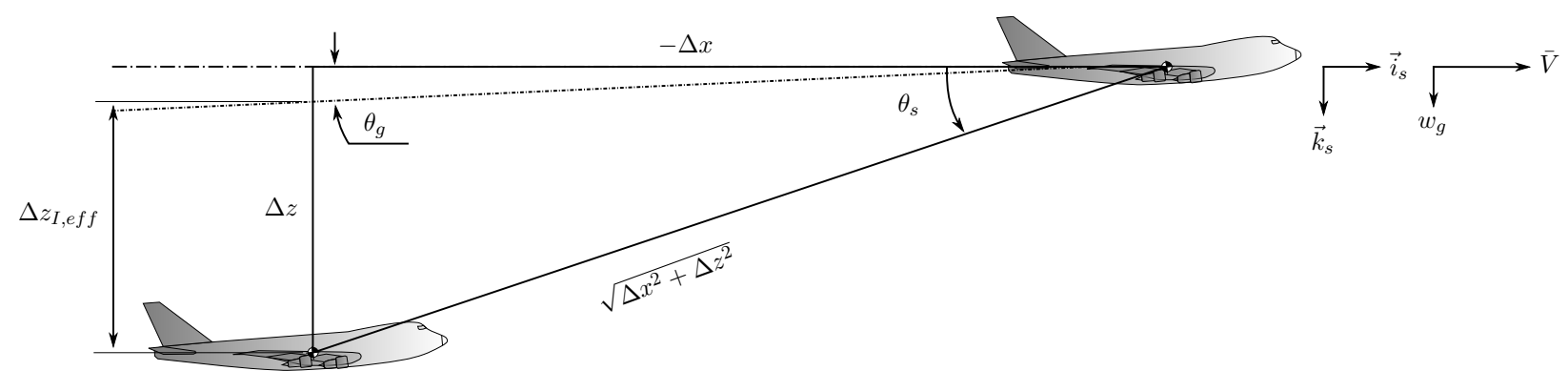

Figure 3.13: Side-view separation scenario during formation flight with a vertical gust $\left(w_{g}\right)$.

The separation angle $\theta_{s}$ is now defined as

$$
\theta_{s}=\tan ^{-1}\left(\frac{\Delta z}{-\Delta x}\right)
$$

and the effective vertical separation is approximated as

$$
\Delta z_{I, e f f}=\sqrt{\Delta x^{2}+\Delta z^{2}} \sin \theta_{e f f}
$$

where $\theta_{\text {eff }}$ is the new effective angle of separation due to the vertical gust disturbance - calculated as in Equation (3.31). The induced change in separation angle due to the vertical gust $w_{g}$ is found as follows,

$$
\theta_{g}=\tan ^{-1}\left(\frac{w_{g}}{-\bar{V}}\right) \approx-\frac{w_{g}}{\bar{V}}
$$


assuming that the aircraft velocity $\bar{V}$ is much larger than the vertical gust velocities. Substitution of Equations (3.34), 3.35), 3.31) and (3.36) yields the final instantaneous effective vertical separation between a leader and a follower,

$$
\Delta z_{I, e f f} \approx \sqrt{\Delta x^{2}+\Delta z^{2}} \sin \left[\tan ^{-1}\left(\frac{\Delta z}{-\Delta x}\right)+\frac{w_{g}}{\bar{V}}\right]
$$

Again, notice that intuitively, as the vertical gust velocity $w_{g}$ tends to zero, the instantaneous effective separation becomes the same as the geometric vertical separation. Normalising the results of Equation (3.33) and (3.37) with wingspans gives the following final two equations to determine the normalised lateral and vertical instantaneous effective separations,

$$
\begin{aligned}
& \eta_{I, \text { eff }}=\sqrt{\xi^{2}+\eta^{2}} \sin \left[\tan ^{-1}\left(\frac{\eta}{-\xi}\right)+\frac{v_{g}}{\bar{V}}\right] \\
& \zeta_{I, \text { eff }}=\sqrt{\xi^{2}+\zeta^{2}} \sin \left[\tan ^{-1}\left(\frac{\zeta}{-\xi}\right)+\frac{w_{g}}{\bar{V}}\right]
\end{aligned}
$$

\section{Instantaneous to Effective Separations}

Finally, the instantaneous effective separations in Equation (3.38a and (3.38b are delayed to obtain the delayed effective, or simply the effective separation, as follows,

$$
\begin{aligned}
& \eta_{e f f}=\eta_{I, e f f}\left(t-\tau_{d}\right) \\
& \zeta_{e f f}=\zeta_{I, e f f}\left(t-\tau_{d}\right)
\end{aligned}
$$

where,

$$
\tau_{d}=\frac{|\xi b|}{\bar{V}_{\text {Formation }}}
$$

and $\tau_{d}$ is the time delay between two aircraft in formation, $\xi b$ is the distance between aircraft in formation, and $\bar{V}_{\text {Formation }}$ is the average formation flight airspeed. Equation 3.40 only applies to steady flight.

\subsection{Formation-Extended Model}

Figure 3.14 illustrates the aircraft model used by the follower aircraft during formation flight. It is similar to the conventional model, with the addition of the formation flight interaction effects to the isolated aircraft aerodynamics. A wake model was developed by Bizinos [3] and is also used by Büchner [4]. The same model will be used in the current study, and is illustrated in Figure 3.14 .

The B747-100 aircraft includes mach effects (and thus compressibility effects), but the formation flight interaction model doesn't. To argue the validity of the current interaction model, the best defence is to make comparisons with the literature as far as possible. Work done by 


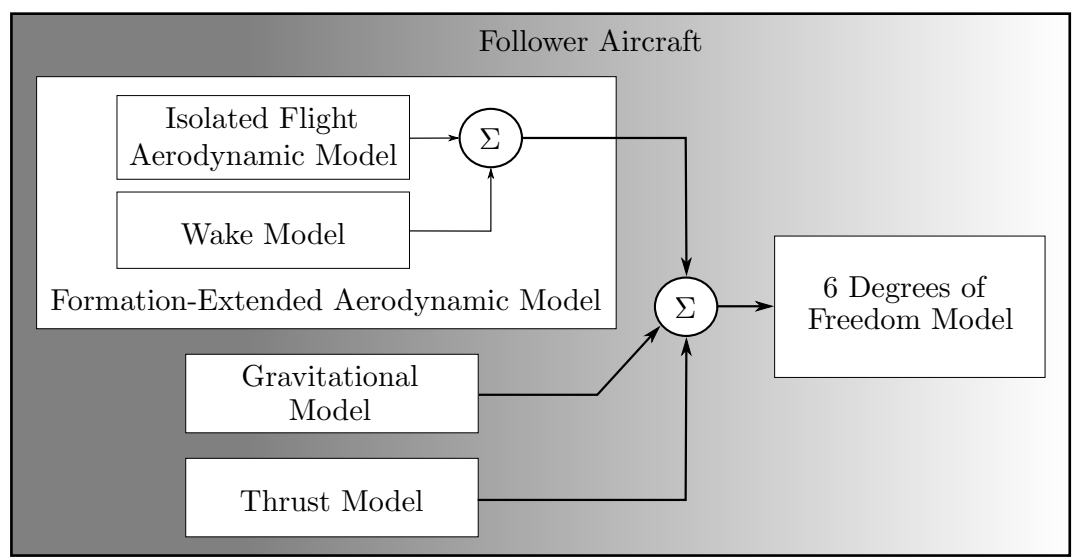

Figure 3.14: Extended aerodynamic aircraft model used by the follower aircraft.

author Ning et al. [26] considered an incompressible analysis, assuming it was sufficient to show many of the trends. Considering work done by author Blake et al. [22] 24] [25] using vortex lattice methods and the results of F18 flight tests, it is clear that the trends produced by the current interaction model are appropriate in the "outer" region.

It is acknowledged that the detailed physics are not being modelled, and that for practical application many of the compressible effects cannot be ignored. Things such as buffet, flutter, flow separation, and noise would then need to be considered. Some of the work done by Ning et al. 26] shows the percentage losses to compressibility effects are in the order of $10 \%$. However, a lot depends on the flight condition, the trim variables that result and where and how the control surfaces are deflected. It is assumed in the rest of this thesis that the trends in the current interaction model for the "outer" region are sufficient enough for the control designs later on in the study.

The presence of the leading aircraft in a formation aircraft scenario can be modelled as changes in the conventional aerodynamic coefficients. It is conceded that the interaction model does not contain any rate terms, and thus the aerodynamic coefficients representing the wake forces and moments are only functions of the separation distances. However, the follower aircraft in formation still has a conventional aerodynamic model to describe the changes in the aerodynamic coefficients given any changes in the aircrafts own states or control surfaces. This will take into account rate effects, but not formation flight rate effects.

It is further conceded that the linearised aircraft model in Chapter 4 does not explicitly contain the state of the leader aircraft, and it is derived and written only in terms of the states of the follower aircraft. The linearised model therefore assumes the leader is maintaining straight and level flight at a constant altitude, and would therefore not reveal the coupling between the leader and the follower aircraft. However, the state of the leader aircraft does couple to the state of the follower aircraft in the simulation model. The azimuth angle of the leader aircraft is used by the follower aircraft to compute the separation and observe any changes in the flight path heading angle of the leader aircraft. Thus, if the leader aircraft moves, while the follower aircraft keeps flying straight, the effective separations change, and there would therefore be an affect on the follower aircraft due to a change in the state of the leader aircraft. 
To conclude, although a linearised model of the formation flight model is not obtained in the current study, which includes both the states of the leader and the states of the follower aircraft, it is argued that the flight control system designed later on in Chapter 5 for isolated flight using the linearised model for isolated flight, was tested in simulation using a full non-linear simulation model that does represent the coupling effects between the leader and the follower.

In the next sections, the wake interaction effects during formation flight are defined as additional induced aerodynamic coefficients $\left(C_{()_{F}}\right)$, the feasible geometric separations for formation flight are investigated, and the full formation-extended aerodynamic model equations are defined as a sum of the isolated aerodynamic coefficients $\left(C_{(.)_{S}}\right)$ and the formation-induced aerodynamic coefficients $\left(C_{(.)_{F}}\right)$.

\subsubsection{Wake Interaction Effects during Formation Flight}

The induced downwash at a particular position on the wing bound vortex closely approximates the aerodynamic loads acting on the wing of the trailing aircraft. In formation flight, the downwash induced is often negative, and via the Kutta-Joukowski theorem, causes an increased angle of attack, increased lift and decreased induced drag. Integration along the bound vortex span $b_{v}$ produces induced load expressions for incremental lift, drag, rolling moment and yawing moment. A similar approach was used for the tailfin. [3]

The induced loads were then converted to aerodynamic coefficients, which revealed that these coefficients are proportional to certain dimensionless parameters, known as influence factors. It was also revealed that the coefficients only depend on the formation geometry. These influence parameters are $\sigma$ (influencing lift, side force, drag and yawing moment) and $\tau$ (influencing rolling and yawing moment). Assumptions made by the authors include: (1) the induced lift, drag and rolling moment are caused by the wing, (2) the induced side force is caused by the tailfin, (3) the induced yawing moment is caused by both the wing and the tailfin, (4) and the induced pitching moment is estimated by considering the change in downwash at the tailplane due to the formation-induced downwash at the wing. A full derivation of these influence factors and coefficients that result can be found in the work done by the original author [3].

Used in this current study, the functions that describe the induced forces and moment coefficients are reproduced from the work of Bizinos [3] as follows,

$$
\begin{aligned}
C_{L_{F}}(\eta, \zeta) & =\frac{-c_{l_{\alpha}} C_{L, j}}{2 \pi^{2} \mathrm{AR}} \sigma_{j k} & C_{m_{F}}(\eta, \zeta) & =C_{L_{F}}\left(h-h_{0}\right)-V_{T a i l} C_{L_{\omega_{h} F}}\left(1-\frac{d \epsilon}{d \alpha}\right) \\
C_{D_{F}}(\eta, \zeta) & =\frac{2 C_{L, k} C_{L, j}}{\pi^{3} \mathrm{AR}} \sigma_{j k} & C_{L_{\omega_{h} F}}(\eta, \zeta) & =\frac{-2 a_{1} C_{L, j}}{\pi^{3} \mathrm{AR} \eta_{h}} \sigma_{j k \omega_{h}} \\
C_{Y_{F}}(\eta, \zeta) & =\frac{S_{t}}{S} \frac{2 C_{L, j}}{\pi \mathrm{AR} \zeta_{t}} \sigma_{j k t} & C_{n_{F}}(\eta, \zeta) & =\frac{2 C_{L, k} C_{L, j}}{\pi^{3} \mathrm{AR}} \tau_{j k}-V_{t} \frac{2 C_{L, j}}{\pi \mathrm{AR} \zeta_{t}} \sigma_{j k t} \\
C_{l_{F}}(\eta, \zeta) & =\frac{c_{l_{\alpha}} C_{L, j}}{2 \pi^{2} \mathrm{AR}} \tau_{j k} & &
\end{aligned}
$$


where the terms $C_{(.)_{F}}$ represent the formation flight effects as additional induced aerodynamic coefficients and $(\eta, \zeta)$ are the effective lateral and vertical separations respectively - defined in Section 3.4. The influence parameters in Equation (3.41) are listed in Appendix F as Equations F.1a to (F.1d). Figure 3.15 shows the resulting induced aerodynamic coefficients due to formation flight at an effective vertical separation of zero and various effective lateral separations, selected as such prior to an investigation of the feasible geometric separations during formation flight. A discussion of this is provided next.

\subsubsection{Feasible Geometric Separations for Formation Flight}

A literature study and analysis was performed in an attempt to determine the most relevant aircraft separation variables required to explore formation flight. The lateral, vertical and longitudinal separation variables are investigated. These variables are illustrated in Figure 3.9 and 3.10, with the normalised values given in Equations (3.26a), 3.26b) and (3.26c). The choice of aircraft separations for investigation was limited by control surface deflections and realistic and desirable fuel savings.

Table 3.3 summarises the geometric separations investigated in the current study. The vertical and longitudinal separations are fixed at 0 and -10 wingspans respectively, while the lateral separation can take on any value from one to one and a half wingspans. In the following sections, a detailed overview is provided to motivate the choice of each separation listed in Table 3.3 .

Table 3.3: Geometric separations used in the extended non-linear formation flight simulations as well as the allowable variations for each variable. The values listed in this table are normalised to wingspan.

\begin{tabular}{lcc}
\hline Separation & Wingspan(s) & Allowable Variation \\
\hline Lateral & $1.0 \leqslant \eta \leqslant 1.5$ & \pm 0.1 \\
Vertical & $\zeta=0$ & \pm 0.1 \\
Axial & $\xi=-10$ & \pm 1 \\
\hline
\end{tabular}

\section{Lateral Separation}

The lateral separation $(\eta)$ is the most influential parameter regarding optimal formation flight. It was derived from several literature studies [2] 22] [24] 26] 227] 65] that the optimum lies close to a tip-to-tip separation and possibly moves in by up to $10 \%$ wing overlap. This would mean the optimum lies in a region of 0.9 to 1.1 wingspan lateral separation.

The first restriction in terms of the lateral separation is the aileron saturation, illustrated in Figure 3.16 to be occuring at approximately 0.95 wingspans and a zero vertical separation with the wake model used in this study. Consideration of various other wake interaction models in the different literature sources [2] 22, 24] [26] 27, 65] leads to reasons to believe that this wake model is inaccurate for lateral separations closer than one (tip-to-tip separation). 

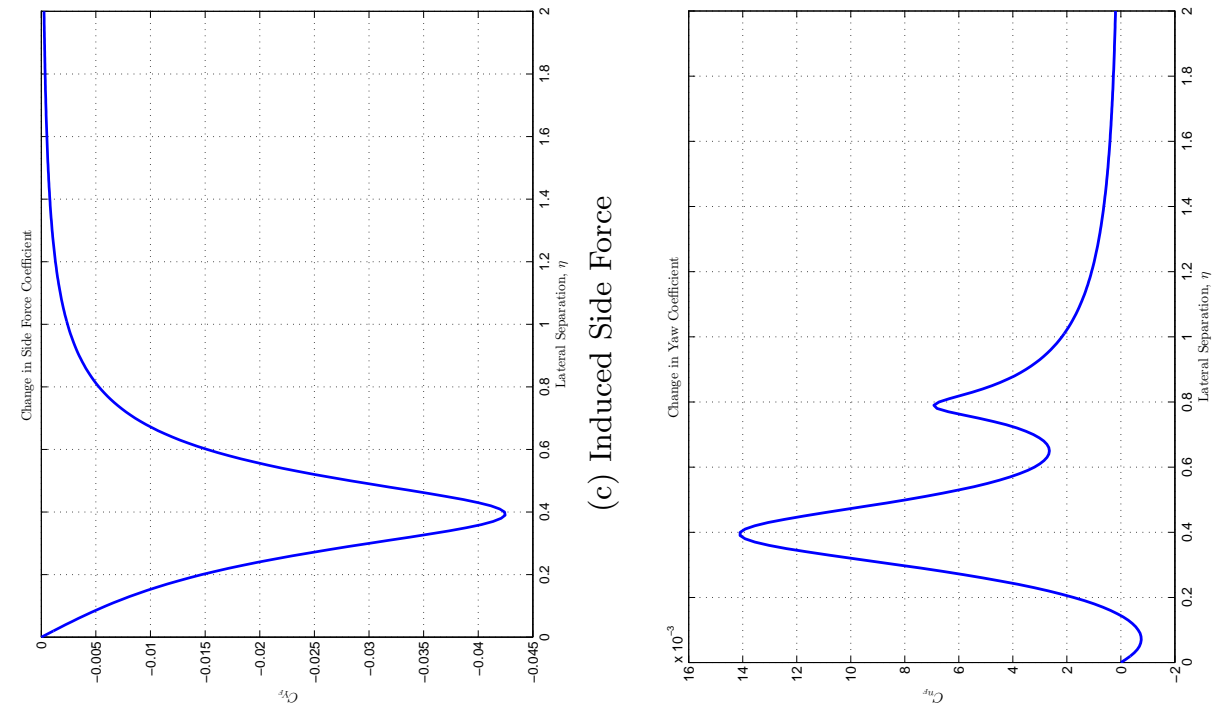

苞 $\quad 0$
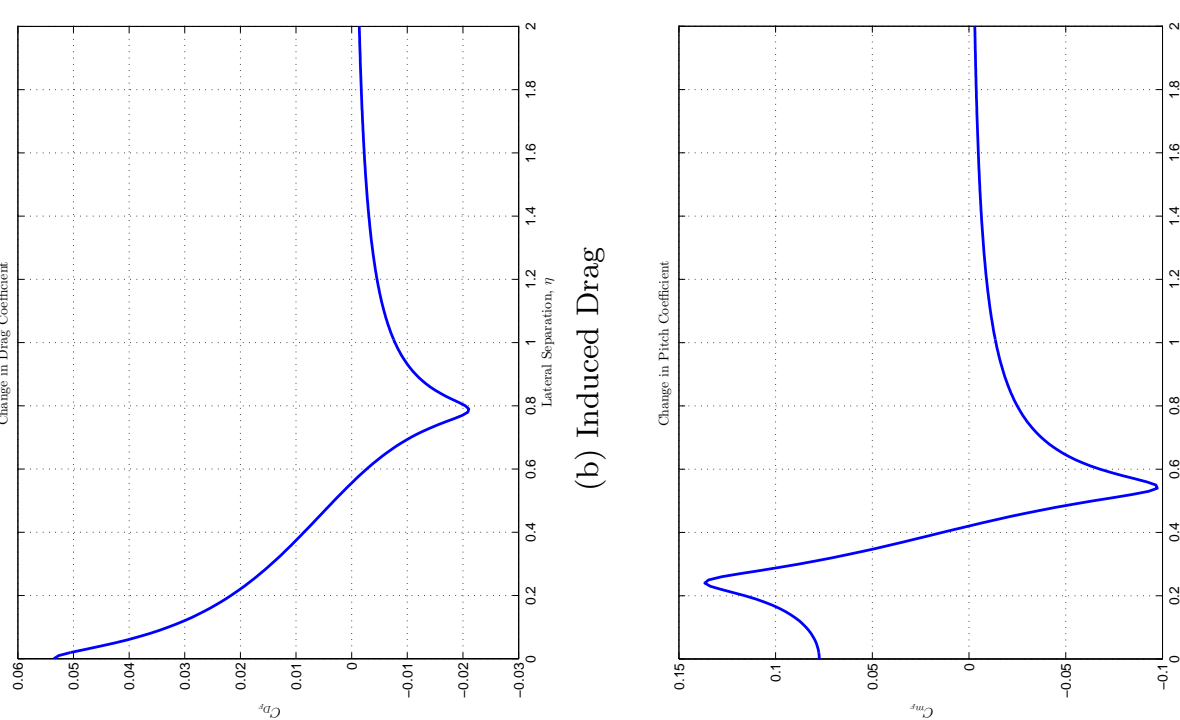

$\sum_{\infty}^{\infty} \underset{\sigma}{\sigma}$

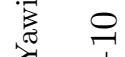

ठ

Ð

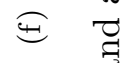

.

莡
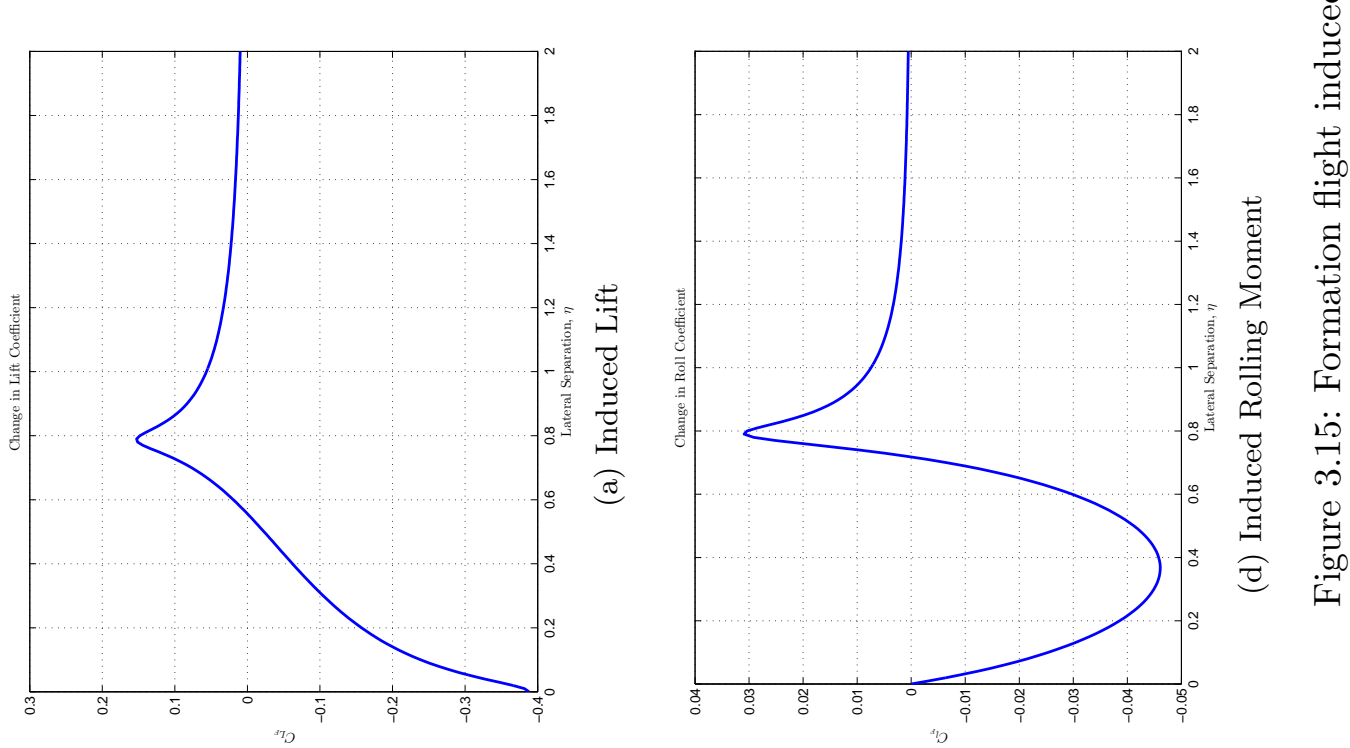


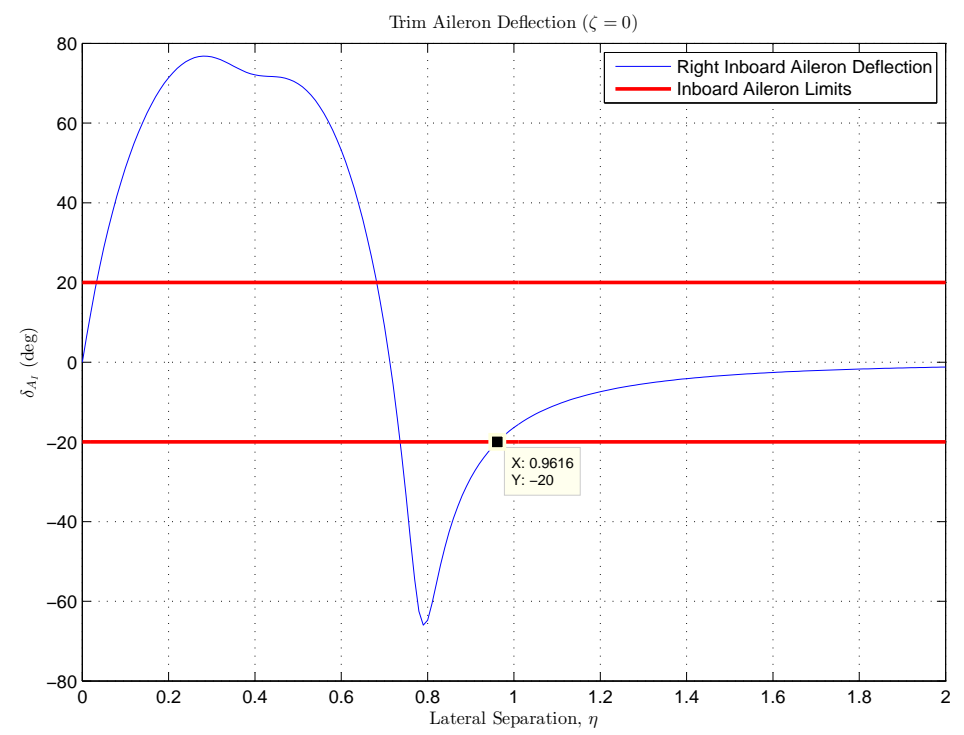

Figure 3.16: Trim deflection angles of the right inboard aileron over lateral separations of $\eta=0$ to $\eta=2$ wingspans. The vertical and longitudinal separations are restricted to zero and -10 wingspans respectively.

Figure 3.17, as found by Bizinos [3], illustrates the comparison of the horseshoe vortex model, reffered to as the approximate method, numerical integration over an elliptical wing as well as flight test data, experimental data and data from vortex lattice methods. Although an improved wake interaction model would allow a more complete investigation of the wake interaction problem, the model is not expected to result in interaction forces significantly larger or smaller than those predicted by the current model at wingspans larger than one. The unrealistic peaks of the current model will be in close agreement to the forces predicted at 0.9 wingspans. It has thus been decided to keep the lateral separation limited to no closer than one wingspan. (See Appendix $\mathrm{F}$ for more details).

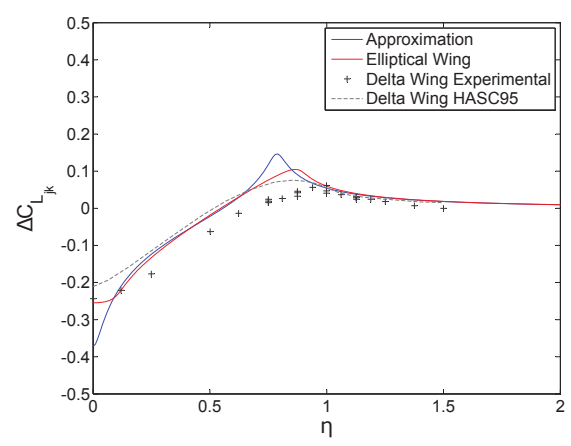

(a) $\Delta C_{L}$

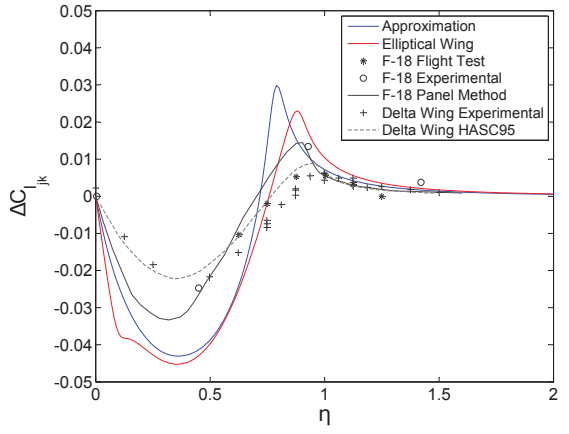

(b) $\Delta \mathrm{C}_{1}$

Figure 3.17: Induced lift and roll coefficients comparison after using the approximate method, numerical integration over an elliptical wing together with flight test data, experimental data and data from vortex lattice methods (from Bizinos [3]). 


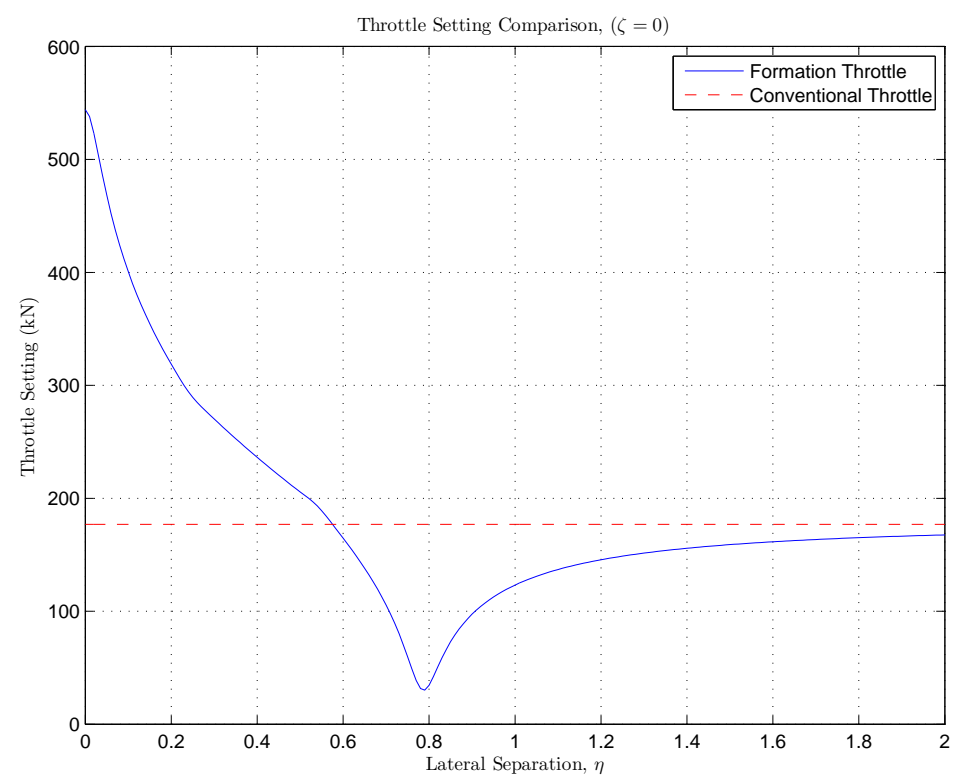

Figure 3.18: Trim throttle settings in conventional isolated flight and formation flight at lateral separations between $\eta=0$ and 2 wingspans.

Figure 3.18 illustrates the trim throttle settings required in conventional flight, compared to the changing throttle setting in a follower aircraft. In general, a lower throttle setting should mean a lower fuel flow rate, which should translate into more fuel savings. At a lateral separation of $\eta=2$ wingspans, the throttle setting of the follower aircraft is just less than that of the conventional aircraft. An upper limit is then arbitrarily chosen as $\eta=1.5$ wingspans, only to avoid computational overload when simulations are considered later.

In a study by G. Bower et al. [2, fuel savings of up to $13 \%$ were achievable. This study also showed that for a two-aircraft echelon formation, the maximum fuel savings changed by $6 \%$ for a lateral separation variation of 0.2 wingspans. To minimise the fluctuations in fuel savings and in an attempt to maximise the amount of fuel savings, the variation at any lateral separation larger than one wingspan should probably be limited to 0.1 wingspans, left and right.

\section{Vertical Separation}

Blake et al. 222] 24] considered zero vertical separation between two Lockhead tail-less aircraft, showing optimal benefit at this separation. Ning et al. [26] conducted an analysis of the aerodynamic performance of extended formation flight of commercially sized aircraft, and was closely followed by a related study by authors Kless et al. [27. These authors showed that for lateral separations near tip-to-tip separation, optimum drag benefits seemed achievable between \pm 0.1 wingspans of vertical separation. Bower et al. 2] considered a vertical wing tip gap of 0.01 wingspans in a route optimisation study for an optimal load distribution. Enough evidence suggests that the optimal vertical separation $\zeta$ is near zero wingspans.

There is no evident aileron saturation occuring in the outer region for any separation value (see Figure 2.17). However, regarding the throttle setting as illustrated in Figure 3.18 , it would be desirable to maintain the formation throttle setting lower than the conventional throttle 
setting during isolated flight. Figure 2.17 shows that the optimum vertical separation for formation flight where the throttle setting is at a minimum is zero wingspans. However, a vertical separation greater than -1 and less than 1 wingspan will still ensure lower throttle settings in formation than in isolated flight. Considering that it is desirable to reduce the fuel burn as much as possible during formation flight, the vertical separation is limited to zero wingspans and the variation is restricted with the same bounds as the lateral separation - that is, 0.1 wingspans up and down from the desired separation.

\section{Longitudinal or Axial Separation}

Lastly, and probably least importantly, there are no restrictions posed on the axial separation $(\xi)$ regarding control surface deflections or throttle usage. A study by Ning et al. [26] suggested 1040 wingspans being probable for formation flight. At a longitudinal separation of 10 wingspans, the basic horseshoe vortex model used in the current wake model derivation has been shown to have reasonable agreement with experiments and flight data [22] 24] [32].

The effective separation between the aircraft is derived in the current study on the assumption that the vortices move identically, and the relative spacing between the vortices is always maintained. This corresponds closely to a recent study by Bizinos [3]. Furthermore, the gust velocities produced by a von Kármán turbulence model are assumed to remain frozen for the time that it takes the follower aircraft to cover the current longitudinal separation. To limit the divergence of this assumption, the longitudinal separation is limited to around 10 wingspans with a variation of \pm 1 wingspan.

\subsubsection{Full Aerodynamic Model for Formation Flight}

By rewriting the full aerodynamic coefficients for a conventional aircraft in isolated flight to include the formation flight effects, the aerodynamic model for the induced forces and moments as experienced by a trailing aircraft can be obtained. From Equation 3.15, the non-dimensional aerodynamic coefficients are extended for formation flight and expanded in the stability axis,

$$
\begin{aligned}
C_{X_{S_{k}}} & =-C_{D_{S_{k}}} \\
C_{Z_{S_{k}}} & =-C_{L_{S_{k}}} \\
C_{L_{S_{k}}} & =C_{L_{S}}+C_{L_{F}}(\eta, \zeta) \\
C_{D_{S_{k}}} & =C_{D_{S}}+C_{D_{F}}(\eta, \zeta) \\
C_{Y_{S_{k}}} & =C_{Y_{S}}+C_{Y_{F}}(\eta, \zeta) \\
C_{l_{S_{k}}} & =C_{l_{S}}+C_{l_{F}}(\eta, \zeta) \\
C_{m_{S_{k}}} & =C_{m_{S}}+C_{m_{F}}(\eta, \zeta) \\
C_{n_{S_{k}}} & =C_{n_{S}}+C_{n_{F}}(\eta, \zeta)
\end{aligned}
$$

where the terms $C_{(.)_{S}}$ are the non-dimensional aerodynamic coefficients (the subscript $S$ denotes the stability axis), $C_{(.)_{F}}(\eta, \zeta)$ represent the formation flight effects as additional induced aero- 
dynamic coefficients (the subscript $F$ denotes formation flight), and $k$ represents the follower number in formation as any positive integer $\mathcal{Z}^{+}$. In a two-aircraft formation scenario, $k=1$ represents the follower. The transformation of Equations 3.42a to $3.42 \mathrm{~h}$ in the same manner as Equation 3.17 gives the full extended aerodynamic coefficients for formation flight,

$$
\begin{aligned}
C_{X_{k}} & =C_{X_{S_{k}}} \cos \alpha-C_{Z_{S_{k}}} \sin \alpha \\
C_{Y_{k}} & =C_{Y_{S_{k}}} \\
C_{Z_{k}} & =C_{X_{S_{k}}} \sin \alpha+C_{Z_{S_{k}}} \cos \alpha \\
C_{l_{k}} & =C_{l_{S_{k}}} \cos \alpha-C_{n_{S_{k}}} \sin \alpha \\
C_{m_{k}} & =C_{m_{S_{k}}} \\
C_{n_{k}} & =C_{l_{S_{k}}} \sin \alpha+C_{n_{S_{k}}} \cos \alpha
\end{aligned}
$$

\subsection{Modelling the Effects of Atmospheric Turbulence}

The atmospheric turbulence model plays an important role in generating forces and moments that are true to what could be experienced in a real aircraft flight scenario. The US Department of Defense military handbook MIL-STD-1797A recommends the use of a von Kármán form to generate continuous gusts and is the preferred model over the Dryden turbulence model.

The von Kármán turbulence model generates atmospheric turbulence as stationary, homogeneous, isotropic, Gaussian turbulence and its implementation is explained in Appendix $\mathrm{H}$. This turbulence is generated by passing unit variance band-limited white noise through appropriate forming filters, and follows the mathematical representation in the MIL-STD-1797A handbook. The implemented filter forms are shown in Table H.1.

\subsection{Modelling the Vibrations}

The International Organisation of Standardisation (ISO) aims to provide guidance methods with which to evaluate the effect of whole-body vibrations on the human body. It is primarily concerned with quantifying whole-body vibrations in relation to human health, comfort, perception and motion sickness. [53]

In the following sections, the axis systems of a seated passenger is defined, and the measurement of linear and rotational acceleration in the current study is outlined.

\subsubsection{Axis Systems of Seated Passenger}

Figure 3.19 illustrates the different axis systems for a passenger in the seated position. Three sets of othogonal axes are situated with the origin of the axis system at different points on the 
human body: the feet, the supporting seat-surface and the seat-back. The $x y$-axis lies parallel to the $X_{B} Y_{B}$-axis plane of the aircraft. Completing the right-handed orthogonal axis system is the $z$-axis, pointing upwards relative to the point under consideration.

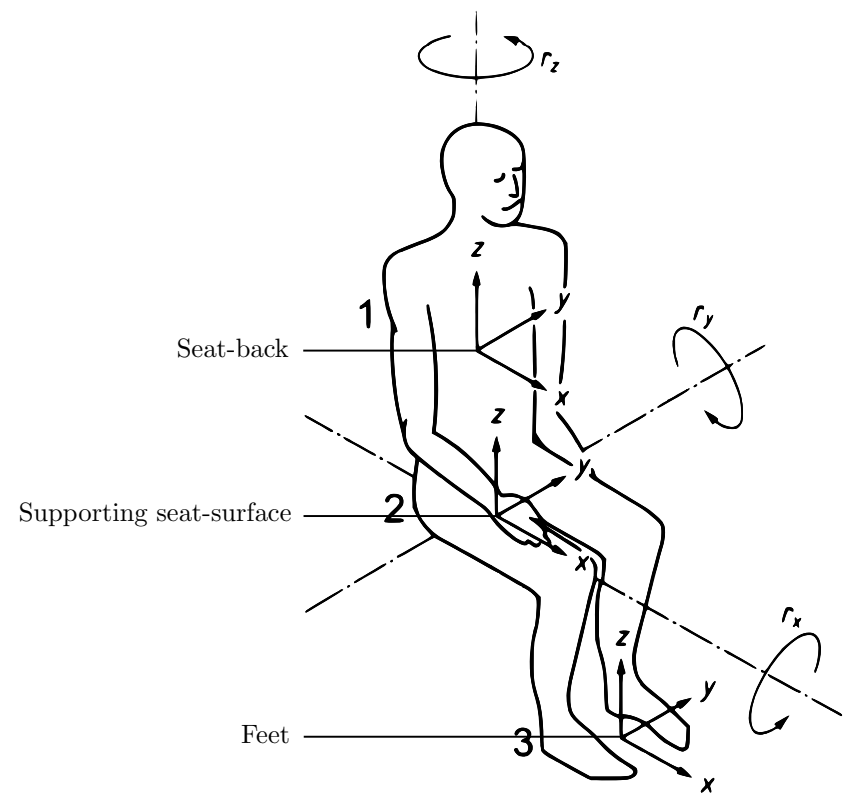

Figure 3.19: Axis systems for a passenger in the seated position. Three sets of othogonal axes are situated with the origin of the axis system at different points on the human body: the feet, the supporting seat-surface and the seat-back (Modified from ISO 2631-1 [53]).

\subsubsection{Vibration Measurement}

The primary quantity of vibration is considered as acceleration [53]. For a seated passenger, as illustrated in Figure 3.19, the vibrations felt by the human body occur at different points. In a study conducted by Kubica and Madelaine [11] in Toulouse, France, the authors presented comfort criteria based on the ISO 2631-1 standard and reported on how to apply these to largecapacity civil aircraft for passenger comfort evaluation. The authors were of the opinion that the rotational vibrations and the vibrations transmitted by the back of the seat are of negligible effect [11. This meant that the authors regarded only the vertical and lateral linear accelerations at the supporting seat-surface and feet as necessary to be considered for the evaluation of passenger comfort in civil aircraft [11].

A more recent study by Bizinos [3] revealed that there are increased roll and yaw acceleration magnitudes during formation flight. The roll acceleration magnitudes seemed to show the most significant increase. As such, it might not be a valid assumption to ignore the rotational vibrations when evaluating passenger comfort in a formation flying commercial aircraft.

It is the aim of this thesis to evaluate passenger comfort based on measured linear and rotational vibrations at the feet and seat-surface by using a rigid body assumption. A theoretical seat model will be considered in the current study, that filters the accelerations at the feet to 
obtain the accelerations at the supporting seat-surface. The ISO 2631-1 standard considers the likely reactions to overall vibration over a frequency range of,

1. $0.5 \mathrm{~Hz} \leqslant f \leqslant 80 \mathrm{~Hz}$ for health, comfort and perception evaluation

2. $0.1 \mathrm{~Hz} \leqslant f<0.5 \mathrm{~Hz}$ for motion sickness evaluation

\section{Linear Accelerations}

The linear vibrational acceleration experienced at any point in an aircraft can be determined from the general vector expression for the absolute acceleration of a passenger in terms of its acceleration $\mathbf{a}_{r e l}$ - measured relative to a moving coordinate system at the CG rotating with an angular velocity $\omega$ and an angular acceleration $\dot{\omega}[66$,

$$
\mathbf{a}_{p}=\mathbf{a}_{c g}+\dot{\omega} \times \mathbf{r}_{p / c g}+\omega \times\left(\omega \times \mathbf{r}_{p / c g}\right)+2 \omega \times \mathbf{v}_{r e l}+\mathbf{a}_{r e l}
$$

where

$\mathbf{a}_{p}$ is the linear acceleration at point $p$

$\mathbf{a}_{c g}$ is the absolute aircraft acceleration at the CG

$\dot{\omega}$ is the angular acceleration of the aircraft about the CG

$\omega$ is the angular velocity of the aircraft about the CG

$\mathbf{r}_{p / c g}$ is the relative displacement vector between point $p$ and the CG

$\mathbf{v}_{\text {rel }}$ is the velocity of point $p$ measured relative to rotating axes at the CG

$\mathbf{a}_{r e l}$ is the acceleration of point $p$ measured relative to a rotating axes at the CG

It is assumed that the axes at the CG are rigidly attached to the body, and thus $\mathbf{v}_{r e l}$ and $\mathbf{a}_{r e l}$ are both zero [66]. So then, for an arbitrary point $p$, the linear acceleration is obtained in the current study as follows,

$$
\mathbf{a}_{p}=\mathbf{a}_{c g}+\dot{\omega} \times \mathbf{r}_{p / c g}+\omega \times\left(\omega \times \mathbf{r}_{p / c g}\right)
$$

\section{Rotational Accelerations}

It is known that the rotation of a rigid body is described by its angular motion [66]. It is also known that all lines connecting different points in a rigid body to some fixed reference point, such as the CG of the aircraft, have the same angular displacement, angular velocity and angular acceleration [66]. It follows then that the rotational vibrations experienced by a seated passenger are simply the angular accelerations,

$$
\mathbf{a}_{r}=\dot{\omega}
$$

where $\mathbf{a}_{r}$ is the passenger rotational acceleration. 


\subsection{A Fully Integrated Dynamic Formation Flight Simulation Model}

To conclude the mathematical modelling, Figure 3.20 outlines the integrated simulation model for two aircraft in formation flight. This model is divided into three sections: starting with the aircraft models, the conventional leader consists of a 6DOF model. This model describes the equations of motion of the aircraft given the forces and moments that act on it; these are in turn described by the forces and moments model block. The follower has the same aircraft model as the leader, with only one change: an extended aerodynamic model to incorporate the effects of formation flight - the influence that the trailing vortices of the leader aircraft have on the follower's aerodynamics. The influence was modelled as influence factors, dependent only on the formation geometry.

The aircraft models in Figure 3.20 are enclosed by the flight control architecture. The flight control architecture for both aircraft consists of a set of inner-loop conventional fly-by-wire flight controllers followed by isolated or formation-specific guidance laws. The conventional guidance laws will need to be sufficient to maintain straight and level flight, as well as perform longitudinal and lateral manoeuvres. A set of waypoints will be used to determine the track for the leader aircraft. Regarding the follower aircraft, the guidance laws will need to maintain a desired geometric separation between the follower and the leader aircraft.

To increase the fidelity of the simulation, a von Kármán turbulence block is added in Figure 3.20. This block plays an important role in generating realistic induced forces and moments. Conforming to the MIL-F-8785C \& MIL-HDBK-1797 military standards, it models the atmospheric turbulence as stationary, homogeneous, isotropic, Gaussian turbulence.

A pilot or autopilot block is used to model the reference commands that will be given to the leader based on the desired or required path that the aircraft is to fly. The aircraft separation variables illustrated in Figure 3.20 consist of the geometric separation, required by the formation guidance laws, and the effective separation, required by the aerodynamic model of the follower aircraft.

Once full formation flight capability is active, the comfort levels along the fuselage are measured and evaluated with the passenger comfort block in Figure 3.20. For the evaluation of passenger comfort, the primary cause of discomfort is considered to be vibrations at a seating location, measured as acceleration.

In this chapter, everything required to set up the simulation model was covered, except for the flight control architecture. In the next two chapters, the models for a representative conventional fly-by-wire flight control architecture as well as an extended formation flight control architecture are proposed, designed, implemented and verified in simulation. 


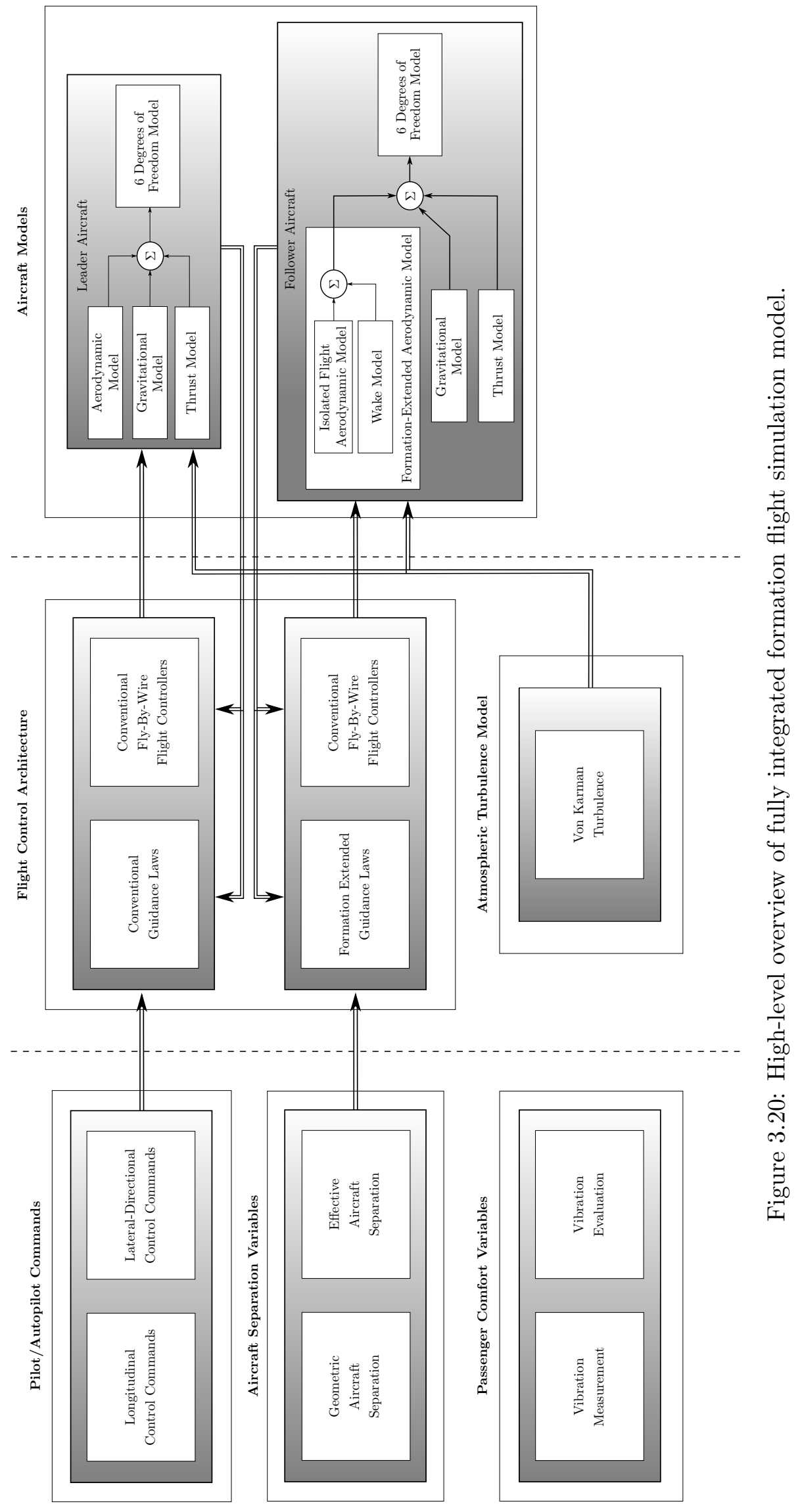




\section{Chapter 4}

\section{Linearising The Aircraft Dynamics}

In order to analyse the aircraft dynamics and design a suitable set of flight control laws for both isolated and formation flight, we require a linearised aircraft model. In this chapter, the nonlinear 6DOF equations of motion are used to derive and develop a linear aircraft model for the B747-100 in isolated flight. A linear aircraft model is derived by linearising the nonlinear, timevariant aircraft model in isolated flight about a nominal flight condition (called the linearisation point), assuming a rigid body, constant mass, and a flat, non-rotating Earth. In this thesis, symmetric aerodynamics and mass distribution and a straight and level flight trajectory are assumed, which are common assumptions found in much of the literature on obtaining traditional aircraft models [33] [58] 60] 67] 68] 69] [70].

The linearised model for a follower aircraft in formation flight was not developed and used in this study to design the flight control laws for the follower aircraft. Büchner [4] showed with an eigenvalue analysis that the dynamics of the linearised follower aircraft do not change significantly in the "outer region". It is also the aim of the current study to use representative and current fly-by-wire flight control, designed by flight engineers for current aircraft, in formation flight. As such, the same inner-loop flight controllers used on the leader aircraft will be used in the follower aircraft. Only a redesign of the outer-loop controllers using the isolated linear model, that encapsulates the inner-loop control systems and dynamics of an isolated aircraft, is used in Chapter 6.

This chapter starts with some assumptions that were made in order to linearise an isolated aircraft model about a straight and level flight trim condition. Following the definition and calculation of the straight and level flight trim condition variables, the aircraft is linearised about the trim condition and a linear dynamics analysis is performed to provide insight into the aircraft's natural modes of motion and static stability. This level of insight is fundamental to a proper control systems design in the next chapters.

\subsection{Small Disturbance Theory}

The small disturbance theory [60] is based on the absolute aircraft states being replaced by a constant trim value plus a perturbation or disturbance from the trim state. All the variables in 
the equations of motion are then rewritten as follows,

$$
X=X_{T}+\Delta x
$$

where $X$ is the absolute state, $X_{T}$ is the trim state and $\Delta x$ is the perturbation. In a straight and level trim flight condition, the small disturbance theory provides adequate practical results 60] based on the following assumptions:

- Small-angle approximation

- Products of small perturbations are negligibly small

Since the perturbations are assumed to be small, the angle of attack $(\alpha)$ and angle of sideslip $(\beta)$ can be approximated as being linearly related to the normal and lateral velocity perturbations,

$$
\begin{aligned}
& \alpha=\tan ^{-1}\left(\frac{\Delta w}{U_{T}+\Delta u}\right) \approx \frac{\Delta w}{U_{T}} \\
& \beta=\sin ^{-1}\left(\frac{\Delta w}{\sqrt{\left(U_{T}+\Delta u\right)^{2}+\Delta v^{2}+\Delta w^{2}}}\right) \approx \frac{\Delta v}{U_{T}}
\end{aligned}
$$

The aircraft can now be linearised about a trim condition using the small disturbance technique. To proceed with the linearisation process, the trim condition variables first need to be defined.

\subsection{Defining and Calculating the Trim Condition Variables}

The trim states define the equilibrium state about which the dynamics can be linearised. This will allow the analysis and design tools of the field of linear systems theory to be applied. Since the goal of this study is to evaluate passenger ride comfort in formation flight, it will be assumed that the aircraft does not experience large deviations from the trim state. The trim state for straight and level flight in the cruise phase will be used as the equilibrium state about which the aircraft is linearised.

The objective of obtaining a straight and level flight trim condition is to bring all forces and moments acting on an aircraft into a state of equilibrium. This is the condition where axial, normal and side forces, as well as the roll, pitch and yaw moments are all zero. Provided that the aircraft is stable, when initialised with these trim states it will stay in equilibrium until it is disturbed by pilot control inputs or external influences.

\subsubsection{The Straight and Level Flight Trim Condition}

Figure 4.1 illustrates the forces and moments acting on the aircraft at a straight and level flight condition. During this equilibrium condition, all forces and moments acting on the aircraft must be exactly zero. Furthermore, the symmetry of a typical airframe about its XZ-plane results in symmetric aerodynamic properties that make all lateral motion and control variables exactly zero, 


$$
\left[\begin{array}{llllll}
V & P & R & \Phi & \delta_{A} & \delta_{R}
\end{array}\right]_{T}=0
$$

The trim problem now becomes a $2 \mathrm{D}$ problem, requiring the solution of only the longitudinal states and control settings,

$$
\left[\begin{array}{llllll}
\bar{V} & \alpha & Q & \Theta & \delta_{E} & T
\end{array}\right]_{T}
$$

The trim dynamic pressure is calculated using,

$$
q_{T}=\frac{1}{2} \rho \bar{V}_{T}^{2}
$$

and at straight and level flight trim the pitch rate must be zero $\left(Q_{T}=0\right)$ and the following relationship exists,

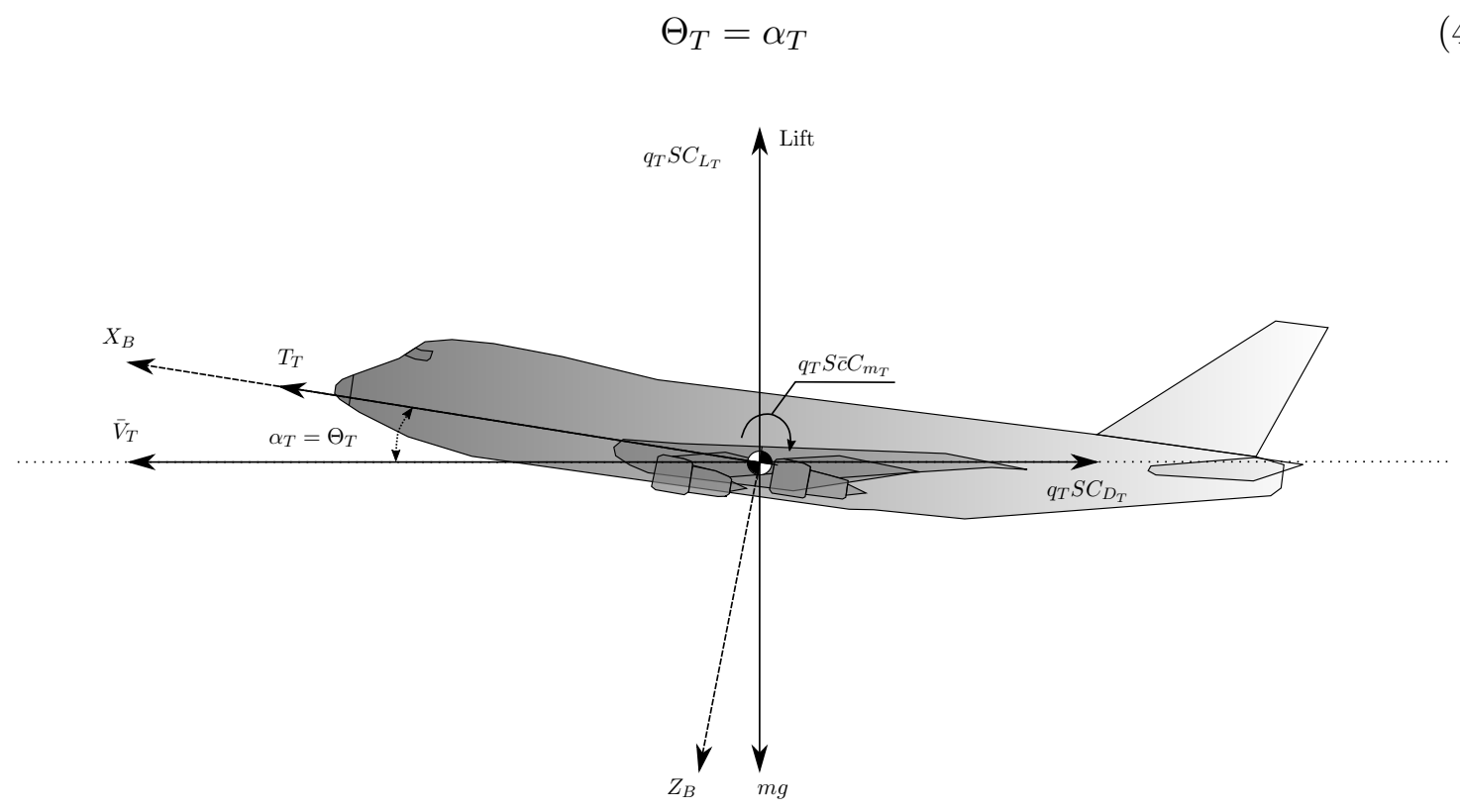

Figure 4.1: Forces and moments at straight and level flight trim.

At this point, the trim problem has been reduced to the solution of three variables, namely the angle of attack, elevator deflection and thrust setting at trim, $\left[\begin{array}{lll}\alpha & \delta_{E} & T\end{array}\right]_{T}$. Considering the trim force and moment diagram in Figure 4.1. Newton's law can be applied to balance all forces along the $x$ - and $z$-axes and the pitching moment about the centre of mass, generating three trim equations,

$$
\begin{aligned}
& \left(-q_{T} S C_{D_{T}} \cos \alpha_{T}+q_{T} S C_{L_{T}} \sin \alpha_{T}\right)+T_{T}-m g \sin \Theta_{T}=0 \\
& \left(-q_{T} S C_{L_{T}} \cos \alpha_{T}-q_{T} S C_{D_{T}} \sin \alpha_{T}\right)+m g \cos \Theta_{T}=0 \\
& q_{T} S \bar{c} C_{m_{T}}=0
\end{aligned}
$$


These equations can be solved simultaneously to solve for the three remaining variables. However, without simplifying assumptions, the solution requires the use of iterative numerical methods. In the current study, the following assumptions are made:

- The trim angle of attack is small

- The lift is an order of magnitude greater than the drag at trim

These assumptions simplify Equation 4.7b, allowing a closed-form solution for the trim variables $\alpha_{T}$ and $\delta_{E_{T}}$ to exist. Rewriting Equation $4.7 \mathrm{~b}$ together with Equation $4.7 \mathrm{c}$,

$$
\begin{aligned}
& -q_{T} S C_{L_{T}}+m g=0 \\
& q_{T} S \bar{c} C_{m_{T}}=0
\end{aligned}
$$

and substituting for the lift and pitching moment coefficients from Equations (3.15) after transformation to the body axis of the aircraft as in Equation (3.17) yields,

$$
\left[\begin{array}{c}
C_{L_{l p}}+C_{L_{M}}\left(M_{T}-M_{l p}\right) \\
C_{m_{l p}}+C_{m_{M}}\left(M_{T}-M_{l p}\right)
\end{array}\right]+\left[\begin{array}{cc}
C_{L_{\alpha}} & C_{L_{\delta_{E}}} \\
C_{m_{\alpha}} & C_{m_{\delta_{E}}}
\end{array}\right]\left[\begin{array}{c}
\left(\alpha_{T}-\alpha_{l p}\right) \\
\delta_{E_{T}}
\end{array}\right]=\left[\begin{array}{c}
\frac{m g}{q_{T} S} \\
0
\end{array}\right]
$$

Rearranging so that the trim variables $\alpha_{T}$ and $\delta_{E_{T}}$ become the subject of the equation yields,

$$
\left[\begin{array}{c}
\left(\alpha_{T}-\alpha_{l p}\right) \\
\delta_{E_{T}}
\end{array}\right]=\left[\begin{array}{cc}
C_{L_{\alpha}} & C_{L_{\delta_{E}}} \\
C_{m_{\alpha}} & C_{m_{\delta_{E}}}
\end{array}\right]^{-1}\left[\begin{array}{c}
\frac{m g}{q_{T} S}-C_{L_{l p}}-C_{L_{M}}\left(M_{T}-M_{l p}\right) \\
-C_{m_{l p}}-C_{m_{M}}\left(M_{T}-M_{l p}\right)
\end{array}\right]
$$

Finally, the resulting trim variables $\alpha_{T}$ and $\delta_{E_{T}}$ can be substituted back into the remaining trim equation 4.7 a to solve the trim thrust,

$$
T_{T}=q_{T} S C_{D_{T}} \cos \alpha_{T}-\left(m g-q_{T} S C_{L_{T}}\right) \sin \alpha_{T}
$$

It is noted that the method being used to calculate the trim condition variables is an approximate method, and that it is possible to calculate the trim more accurately using a non-linear solver that iteratively solves Equations $4.7 \mathrm{a}$ to $4.7 \mathrm{c}$, the set of non-linear trim equations. It is important to emphasise that the scope of this study is limited to straight and level flight, with no significant deviations from this equilibrium condition. As such, the non-linear solvers will not be considered more useful than the above approximate method, which has shown to be sufficient to obtain a linear aircraft model [4] [59] 60] 62], and matches the non-linear aircraft dynamics with reasonable accuracy.

\subsubsection{Calculated Trim Values}

The selected flight data has been extracted from a NASA Contractor report by Heffley and Jewell [9]. The same flight data was used by Bizinos [3]. The selected flight condition is chosen at an altitude of $40000 \mathrm{ft}$ and Mach number of $0.8 \mathrm{M}(236 \mathrm{~m} / \mathrm{s})$. 


\subsection{Linearising About Trim}

The dynamics of the aircraft were derived as a set of differential equations that models the dynamics of a rigid body aircraft for a set of forces and moments acting on it. These dynamics can be displayed in a more concise non-linear state space form,

$$
\dot{\mathbf{x}}=\mathbf{f}(\mathbf{x}, \mathbf{u})
$$

where $\mathbf{f}$ is a non-linear vector function representing the respective dynamic equations. The absolute states and controls are,

$$
\begin{aligned}
\mathbf{x} & =\left[\begin{array}{llllllll}
U & V & W & P & Q & R & \Phi & \Theta
\end{array}\right]^{T} \\
\mathbf{u} & =\left[\begin{array}{llll}
\delta_{A} & \delta_{E} & \delta_{R} & T
\end{array}\right]^{T}
\end{aligned}
$$

Using the small disturbance theory, each state and control can be written as the sum of a trim value and a perturbation about trim,

$$
\begin{aligned}
& \mathbf{x}=\mathbf{x}_{T}+\Delta \mathbf{x} \\
& \mathbf{u}=\mathbf{u}_{T}+\Delta \mathbf{u}
\end{aligned}
$$

where the perturbation states and controls are,

$$
\begin{aligned}
\boldsymbol{\Delta} \mathbf{x} & =\left[\begin{array}{llllllll}
u & v & w & p & q & r & \phi & \theta
\end{array}\right]^{T} \\
\boldsymbol{\Delta} \mathbf{u} & =\left[\begin{array}{lllll}
\delta_{a} & \delta_{e} & \delta_{r} & \Delta T
\end{array}\right]^{T}
\end{aligned}
$$

Expanding the non-linear vector function of Equation 4.12 as a Taylor series about the trim condition yields,

$$
\dot{\boldsymbol{x}}_{T}+\Delta \dot{\boldsymbol{x}}=\mathbf{f}\left(\boldsymbol{x}_{T}+\Delta \boldsymbol{x}, \mathbf{u}_{T}+\Delta \mathbf{u}\right)=\mathbf{f}\left(\boldsymbol{x}_{T}, \mathbf{u}_{T}\right)+\left.\frac{\partial \mathbf{f}}{\partial \boldsymbol{x}}\right|_{T} \Delta \boldsymbol{x}+\left.\frac{\partial \mathbf{f}}{\partial \mathbf{u}}\right|_{T} \Delta \mathbf{u}+\mathcal{O}
$$

where $\mathcal{O}$ represents higher-order terms. Assuming that the perturbations from the trim states are small, the higher-order terms in the Taylor series expansion are regarded as negligibly small. Furthermore, at equilibrium the time rate of change of the states is zero,

$$
\dot{\boldsymbol{x}}_{T}=\mathbf{f}\left(\boldsymbol{x}_{T}, \mathbf{u}_{T}\right)=0
$$

The dynamics are then approximated by the linearised disturbances about trim,

$$
\Delta \dot{\boldsymbol{x}} \approx \mathbf{A}_{T} \Delta \boldsymbol{x}+\mathbf{B}_{T} \Delta \mathbf{u}
$$


where,

$$
\begin{aligned}
& \mathbf{A}_{T}=\left.\frac{\partial \mathbf{f}}{\partial \boldsymbol{x}}\right|_{T} \\
& \mathbf{B}_{T}=\left.\frac{\partial \mathbf{f}}{\partial \mathbf{u}}\right|_{T}
\end{aligned}
$$

and the linearisation problem reduces to determining the vector partial derivatives of the state space matrices in Equations 4.19a), an 8 by 8 matrix, and 4.19b) an 8 by 4 matrix. However, by making some proper assumptions, the six equations of motion can be divided into two sets of three equations each. This can be linearised to obtain a decoupled longitudinal and lateral model with lower-order state space matrices.

\subsubsection{Decoupling the Longitudinal and Lateral Dynamics}

Writing the perturbation state and control vectors in Equation 4.15 as follows,

$$
\begin{aligned}
& \boldsymbol{\Delta} \mathbf{x}=\left[\begin{array}{ll}
\Delta \mathbf{x}_{\text {Long }} & \boldsymbol{\Delta} \mathbf{x}_{\text {Lat }}
\end{array}\right]^{T} \\
& \boldsymbol{\Delta} \mathbf{u}=\left[\begin{array}{ll}
\Delta \mathbf{u}_{\text {Long }} & \boldsymbol{\Delta} \mathbf{u}_{\text {Lat }}
\end{array}\right]^{T}
\end{aligned}
$$

where,

$$
\begin{aligned}
\boldsymbol{\Delta}_{\text {Long }} & =\left[\begin{array}{llll}
u & w & q & \theta
\end{array}\right]^{T} \\
\boldsymbol{\Delta}_{\text {Lat }} & =\left[\begin{array}{llll}
v & p & r & \phi
\end{array}\right]^{T} \\
\boldsymbol{\Delta} \mathbf{u}_{\text {Long }} & =\left[\begin{array}{ll}
\delta_{e} & \Delta T
\end{array}\right]^{T} \\
\mathbf{\Delta u}_{\text {Lat }} & =\left[\begin{array}{ll}
\delta_{a} & \delta_{r}
\end{array}\right]^{T}
\end{aligned}
$$

allows the Equation (4.18) to be rewritten as follows,

$$
\left[\begin{array}{c}
\Delta \dot{\mathbf{x}}_{\text {Long }} \\
\boldsymbol{\Delta} \dot{\mathbf{x}}_{\text {Lat }}
\end{array}\right]=\left[\begin{array}{ll}
\boldsymbol{A}_{T_{11}} & \boldsymbol{A}_{T_{12}} \\
\boldsymbol{A}_{T_{21}} & \boldsymbol{A}_{T_{22}}
\end{array}\right]\left[\begin{array}{c}
\boldsymbol{\Delta} \mathbf{x}_{\text {Long }} \\
\boldsymbol{\Delta} \mathbf{x}_{\text {Lat }}
\end{array}\right]+\left[\begin{array}{ll}
\boldsymbol{B}_{T_{11}} & \boldsymbol{B}_{T_{12}} \\
\boldsymbol{B}_{T_{21}} & \boldsymbol{B}_{T_{22}}
\end{array}\right]\left[\begin{array}{c}
\boldsymbol{\Delta} \mathbf{u}_{L o n g} \\
\boldsymbol{\Delta} \mathbf{u}_{L a t}
\end{array}\right]
$$

The first assumption can be made by considering that the aircraft is at a straight and level unaccelerated flight trim condition. A disturbance by the deflection of elevator will cause a pitching moment about the body y-axis $Y_{B}$, causing a rotation about this axis. This will eventually also cause a change in $F_{X_{B}}$ and $F_{Z_{B}}$. Recalling that at this flight condition and due to the symmetry of the aircraft about its XZ-plane, an elevator deflection does not cause a rolling or yawing moment or any change in the side force $F_{Y_{B}}$. This makes the following terms in the dynamics of Equation 4.22 zero, 


$$
\begin{aligned}
& \boldsymbol{A}_{T_{21}}=\mathbf{0} \\
& \boldsymbol{B}_{T_{21}}=\mathbf{0}
\end{aligned}
$$

Upon inspection of the three longitudinal equations, a lateral change in $V, P$ or $R$ will cause a change in the longitudinal dynamics. However, the second assumption stems from the small disturbance theory. If the perturbations are small and the products of small perturbations are negligibly small, then to a good approximation, the following terms in the dynamics of Equation 4.22 are also zero,

$$
\begin{aligned}
& \boldsymbol{A}_{T_{12}} \approx \mathbf{0} \\
& \boldsymbol{B}_{T_{12}} \approx \mathbf{0}
\end{aligned}
$$

These two assumptions allow the dynamics of Equation 4.22 to be decoupled into longitudinal and lateral dynamics,

$$
\begin{aligned}
\Delta \dot{\mathbf{x}}_{\text {Long }} & =\boldsymbol{A}_{\text {long }} \Delta \mathbf{x}_{\text {Long }}+\boldsymbol{B}_{\text {long }} \Delta \mathbf{u}_{\text {Long }} \\
\Delta \dot{\mathbf{x}}_{\text {Lat }} & =\boldsymbol{A}_{\text {lat }} \Delta \mathbf{x}_{\text {Lat }}+\boldsymbol{B}_{\text {lat }} \Delta \mathbf{u}_{\text {Lat }}
\end{aligned}
$$

where,

$$
\begin{aligned}
\boldsymbol{A}_{\text {long }} & =\boldsymbol{A}_{T_{11}} \\
\boldsymbol{B}_{\text {long }} & =\boldsymbol{B}_{T_{11}} \\
\boldsymbol{A}_{\text {lat }} & =\boldsymbol{A}_{T_{22}} \\
\boldsymbol{B}_{\text {lat }} & =\boldsymbol{B}_{T_{22}}
\end{aligned}
$$

and it is now necessary to determine the vector partial derivatives of the decoupled state space matrices. From this point onwards, the delta symbol $(\Delta)$ will be dropped from the equations for the sake of readability.

\subsubsection{Linearised Longitudinal Model}

The longitudinal dynamics of Equation 4.25a are expanded as partial derivatives to obtain the linearised longitudinal model,

$$
\left[\begin{array}{c}
\dot{u} \\
\dot{w} \\
\dot{q} \\
\dot{\theta}
\end{array}\right]=\left[\begin{array}{llll}
\frac{\partial \dot{U}}{\partial U} & \frac{\partial \dot{U}}{\partial W} & \frac{\partial \dot{U}}{\partial Q} & \frac{\partial \dot{U}}{\partial \Theta} \\
\frac{\partial \dot{W}}{\partial U} & \frac{\partial \dot{W}}{\partial W} & \frac{\partial \dot{W}}{\partial Q} & \frac{\partial \dot{W}}{\partial \Theta} \\
\frac{\partial \dot{Q}}{\partial U} & \frac{\partial \dot{Q}}{\partial W} & \frac{\partial \dot{Q}}{\partial Q} & \frac{\partial \dot{Q}}{\partial \Theta} \\
\frac{\partial \dot{\Theta}}{\partial U} & \frac{\partial \dot{\Theta}}{\partial W} & \frac{\partial \dot{\Theta}}{\partial Q} & \frac{\partial \dot{\Theta}}{\partial \Theta}
\end{array}\right]\left[\begin{array}{c}
u \\
w \\
q \\
\theta
\end{array}\right]+\left[\begin{array}{cc}
\frac{\partial \dot{U}}{\partial \delta_{E}} & \frac{\partial \dot{U}}{\partial \Delta T} \\
\frac{\partial \dot{W}}{\partial \delta_{E}} & \frac{\partial \dot{W}}{\partial \Delta T} \\
\frac{\partial \dot{Q}}{\partial \delta_{E}} & \frac{\partial \dot{Q}}{\partial \Delta T} \\
\frac{\partial \dot{\Theta}}{\partial \delta_{E}} & \frac{\partial \dot{\Theta}}{\partial \Delta T}
\end{array}\right]\left[\begin{array}{c}
\delta_{e} \\
\Delta T
\end{array}\right]
$$

In most studies of flight dynamics, it is most common to work with the velocity magnitude $(\bar{v})$ and angle of attack $(\alpha)$ perturbations in the state vector [58] 60]. Recalling from the small disturbance theory that for small perturbations the angle of attack is linearly related to the normal velocity perturbations, 


$$
\alpha \approx \frac{\Delta w}{U_{T}}
$$

Furthermore, given that the aircraft is flying at a straight and level flight condition, to a good approximation,

$$
\begin{aligned}
\bar{V} & =\sqrt{U^{2}+V^{2}+W^{2}} \approx \sqrt{U^{2}}=U \\
\therefore \bar{V}_{T} & =U_{T}
\end{aligned}
$$

and with these approximations, the following changes can be made to the state space model of Equation (4.27),

$$
\begin{aligned}
\dot{u} & \approx \dot{\bar{v}} \\
\dot{w} & \approx \dot{\alpha} \bar{V}_{T}
\end{aligned}
$$

to give the following linearised longitudinal state space model,

$$
\left[\begin{array}{c}
\dot{\bar{v}} \\
\dot{\alpha} \\
\dot{q} \\
\dot{\theta}
\end{array}\right]=\left[\begin{array}{cccc}
\frac{\partial \dot{U}}{\partial U} & \bar{V}_{T} \frac{\partial \dot{U}}{\partial W} & \frac{\partial \dot{U}}{\partial Q} & \frac{\partial \dot{U}}{\partial \Theta} \\
\frac{1}{\bar{V}_{T}} \frac{\partial \dot{W}}{\partial U} & \frac{\partial \dot{W}}{\partial W} & \frac{1}{V_{T}} \frac{\partial \dot{W}}{\partial Q} & \frac{1}{V_{T}} \frac{\partial \dot{W}}{\partial \Theta} \\
\frac{\partial \dot{Q}}{\partial U} & \bar{V}_{T} \frac{\partial \dot{Q}}{\partial W} & \frac{\partial \dot{Q}}{\partial Q} & \frac{\partial \dot{Q}}{\partial \Theta} \\
\frac{\partial \dot{\Theta}}{\partial U} & \bar{V}_{T} \frac{\partial \dot{\Theta}}{\partial W} & \frac{\partial \dot{\Theta}}{\partial Q} & \frac{\partial \dot{\Theta}}{\partial \Theta}
\end{array}\right]\left[\begin{array}{c}
\bar{v} \\
\alpha \\
q \\
\theta
\end{array}\right]+\left[\begin{array}{cc}
\frac{\partial \dot{U}}{\partial \delta_{E}} & \frac{\partial \dot{U}}{\partial \Delta T} \\
\frac{1}{V_{T}} \frac{\partial \dot{W}}{\partial \delta_{E}} & \frac{1}{V_{T}} \frac{\partial \dot{W}}{\partial \Delta T} \\
\frac{\partial \dot{Q}}{\partial \delta_{E}} & \frac{\partial \dot{Q}}{\partial \Delta T} \\
\frac{\partial \dot{\Theta}}{\partial \delta_{E}} & \frac{\partial \dot{\Theta}}{\partial \Delta T}
\end{array}\right]\left[\begin{array}{c}
\delta_{e} \\
\Delta T
\end{array}\right]
$$

The partial derivatives for the linear longitudinal model were calculated by beginning with the longitudinal equations of motion, performing the partial derivatives and evaluating the result at all longitudinal trim condition variables. This lengthy derivation process is not shown here and the final elements of the state space matrices $\boldsymbol{A}_{\text {long }}$ and $\boldsymbol{B}_{\text {long }}$ are listed in Appendix B.

\subsubsection{Linearised Lateral Model}

The lateral dynamics of Equation 4.25b are expanded as partial derivatives to obtain the linearised lateral model,

$$
\left[\begin{array}{c}
\dot{v} \\
\dot{p} \\
\dot{r} \\
\dot{\phi}
\end{array}\right]=\left[\begin{array}{llll}
\frac{\partial \dot{V}}{\partial V} & \frac{\partial \dot{V}}{\partial P} & \frac{\partial \dot{V}}{\partial R} & \frac{\partial \dot{V}}{\partial \Phi} \\
\frac{\partial \dot{P}}{\partial V} & \frac{\partial \dot{P}}{\partial P} & \frac{\partial \dot{P}}{\partial R} & \frac{\partial \dot{P}}{\partial \Phi} \\
\frac{\partial \dot{R}}{\partial V} & \frac{\partial \dot{R}}{\partial P} & \frac{\partial \dot{R}}{\partial R} & \frac{\partial \dot{R}}{\partial \Phi} \\
\frac{\partial \dot{\Phi}}{\partial V} & \frac{\partial \dot{\Phi}}{\partial P} & \frac{\partial \dot{\Phi}}{\partial R} & \frac{\partial \dot{\Phi}}{\partial \Phi}
\end{array}\right]\left[\begin{array}{c}
v \\
p \\
r \\
\phi
\end{array}\right]+\left[\begin{array}{cc}
\frac{\partial \dot{V}}{\partial \delta_{A}} & \frac{\partial \dot{V}}{\partial \delta_{R}} \\
\frac{\partial \dot{P}}{\partial \delta_{A}} & \frac{\partial \dot{P}}{\partial \delta_{R}} \\
\frac{\partial \dot{R}}{\partial \delta_{A}} & \frac{\partial \dot{R}}{\partial \delta_{R}} \\
\frac{\partial \dot{\Phi}}{\partial \delta_{A}} & \frac{\partial \dot{\Phi}}{\partial \delta_{R}}
\end{array}\right]\left[\begin{array}{c}
\delta_{a} \\
\delta_{r}
\end{array}\right]
$$

As with the longitudinal model, it is common to use the angle of sideslip $(\beta)$ perturbation in the lateral state vector [58] 60]. It follows from the small disturbance theory that for small perturbations, the sideslip angle is linearly related to the lateral velocity perturbations,

$$
\beta \approx \frac{v}{U_{T}}
$$


and together with the approximation of Equation 4.29 , the following change can be made to the state space model of Equation (4.32,

$$
\dot{v}=\bar{V}_{T} \dot{\beta}
$$

to give the following linearised lateral state space model,

$$
\left[\begin{array}{c}
\dot{\beta} \\
\dot{p} \\
\dot{r} \\
\dot{\phi}
\end{array}\right]=\left[\begin{array}{cccc}
\frac{\partial \dot{V}}{\partial V} & \frac{1}{V_{T}} \frac{\partial \dot{V}}{\partial P} & \frac{1}{V_{T}} \frac{\partial \dot{V}}{\partial R} & \frac{1}{V_{T}} \frac{\partial \dot{V}}{\partial \Phi} \\
\bar{V}_{T} \frac{\partial \dot{P}}{\partial V} & \frac{\partial \dot{P}}{\partial P} & \frac{\partial \dot{P}}{\partial R} & \frac{\partial \dot{P}}{\partial \Phi} \\
\bar{V}_{T} \frac{\partial \dot{R}}{\partial V} & \frac{\partial \dot{R}}{\partial P} & \frac{\partial \dot{R}}{\partial R} & \frac{\partial \dot{R}}{\partial \Phi} \\
\bar{V}_{T} \frac{\partial \dot{\Phi}}{\partial V} & \frac{\partial \dot{\Phi}}{\partial P} & \frac{\partial \dot{\Phi}}{\partial R} & \frac{\partial \dot{\Phi}}{\partial \Phi}
\end{array}\right]\left[\begin{array}{l}
\beta \\
p \\
r \\
\phi
\end{array}\right]+\left[\begin{array}{cc}
\frac{1}{V_{T}} \frac{\partial \dot{V}}{\partial \delta_{A}} & \frac{1}{V_{T}} \frac{\partial \dot{V}}{\partial \delta_{R}} \\
\frac{\partial \dot{P}}{\partial \delta_{A}} & \frac{\partial \dot{P}}{\partial \delta_{R}} \\
\frac{\partial \dot{R}}{\partial \delta_{A}} & \frac{\partial \dot{R}}{\partial \delta_{R}} \\
\frac{\partial \dot{\Phi}}{\partial \delta_{A}} & \frac{\partial \dot{\Phi}}{\partial \delta_{R}}
\end{array}\right]\left[\begin{array}{c}
\delta_{a} \\
\delta_{r}
\end{array}\right]
$$

The partial derivatives for the linear lateral model were calculated by beginning with the lateral equations of motion, performing the partial derivatives and evaluating the result at the trim condition variables. Once again, this lengthy derivation process is not shown, but the final elements of the state space matrices $\boldsymbol{A}_{\text {lat }}$ and $\boldsymbol{B}_{\text {lat }}$ are listed in Appendix B.

The non-linear isolated conventional aircraft model is now linearised and decoupled about a straight and level flight trim condition. This process has resulted in linear longitudinal and lateral dynamics. The next section focuses on the analysis and verification of these dynamics, to gain insight into the aircrafts natural modes of motion, and to look at the response of the model to longitudinal control inputs $\boldsymbol{u}_{\text {long }}$ and lateral control inputs $\boldsymbol{u}_{\text {lat }}$.

\subsection{A Linear Analysis}

The dynamic response of a linear system is governed by its poles; the eigenvalues of the system state matrix $\mathbf{A}$. The poles of the longitudinal and lateral systems can be obtained from the system state matrices $\mathbf{A}_{\text {long }}$ and $\mathbf{A}_{\text {lat }}$ respectively as the eigenvalues of each matrix. The eigenvalues of a matrix $\mathbf{A}$ are obtained by solving the following determinant,

$$
|s \mathbf{I}-\mathbf{A}|=0
$$

Table 4.1 lists the poles for the linear isolated conventional aircraft. Figures $4.2 \mathrm{a}$ and $4.2 \mathrm{~b}$ provide pole plots of the longitudinal and lateral poles respectively. The linearisation results were verified against Etkin et al. [60] as well as against Büchner [4], who used the same aircraft flight data from Heffley and Jewel [9] in the same flight condition - cruising at approximately $40000 \mathrm{ft}$ and at a Mach number of 0.8 . Considering the results in Table 4.1 and Figures $4.2 \mathrm{a}$ and $4.2 \mathrm{~b}$, the first conclusion drawn is a stable conventional isolated aircraft at a straight and level flight condition. 
Table 4.1: Modes of motion for the linear isolated conventional aircraft.

\begin{tabular}{llll}
\hline \multicolumn{4}{c}{ Longitudinal Modes } \\
\hline Mode & Calculated Value & Etkin \& Reid [60] & Büchner [4] \\
\hline Phugoid & $-0.0034 \pm j 0.0701$ & $-0.003289 \pm j 0.06723$ & $-0.0019 \pm j 0.0707$ \\
Short-period & $-0.3241 \pm j 0.9114$ & $-0.3719 \pm j 0.8875$ & $-0.324 \pm j 0.911$ \\
\hline
\end{tabular}

Lateral Modes

\begin{tabular}{llll}
\hline Mode & Calculated Value & Etkin \& Reid [60] & Büchner [4] \\
\hline Dutch roll & $-0.0431 \pm j 0.9778$ & $-0.033011 \pm j 0.94655$ & $-0.0705 \pm j 1.006$ \\
Roll & -0.5421 & -0.56248 & -0.506 \\
Spiral & -0.0106 & -0.0072973 & -0.0106 \\
\hline
\end{tabular}

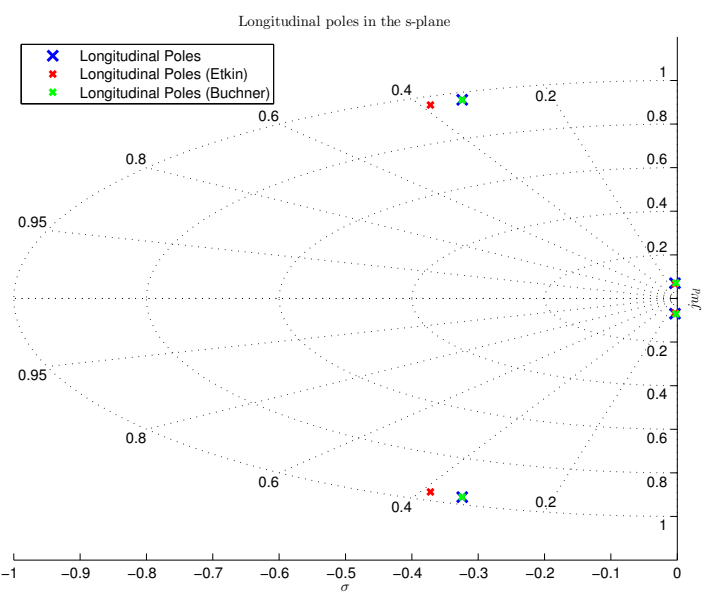

(a) Longitudinal poles in the s-plane

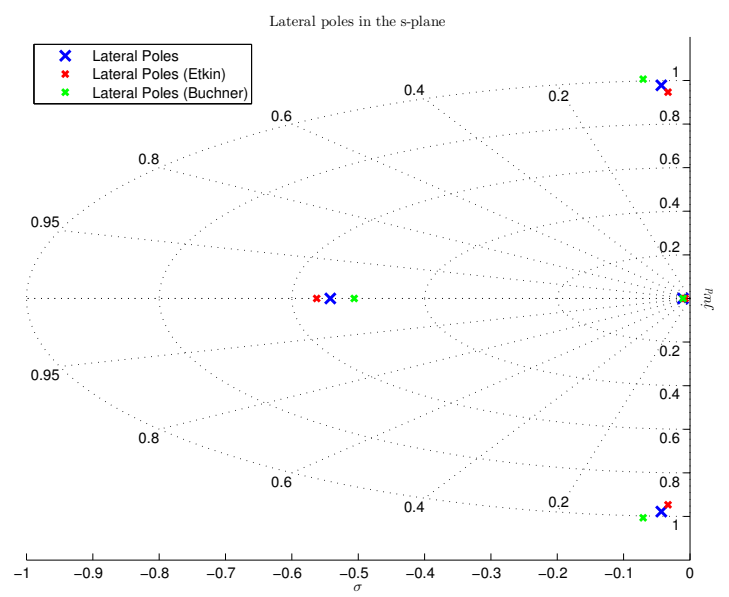

(b) Lateral poles in the s-plane

Figure 4.2: Conventional isolated linear model modes of motion.

\subsubsection{Longitudinal Modes of Motion}

The longitudinal poles illustrated in Figure 4.2a show two complex pole pairs. These modes of motion are commonly referred to as the short-period and phugoid modes.

The higher frequency complex pole pair in Figure $4.2 \mathrm{a}$ is referred to as the short-period mode. The approximate damping and natural frequency of this pole pair are shown in Table 4.2. The calculated damping is relatively small, indicating that some overshoot will be present in this mode. This mode describes the aircraft's tendency to realign itself with the velocity vector when disturbed. Its motion can be described as a torsional mass-spring-damper system. The pitch disturbance from trim equilibrium causes the "spring" to produce a pitching moment, and the damping (often lower than desired) is represented by a viscous damper, stabilising the response. [58]

The lower frequency pole pair in Figure 4.2a is referred to as the phugoid mode. The approximate damping and natural frequency of this pole pair are shown in Table 4.2. The calculated results show that the phugoid mode has poor damping, and a long time constant can be expected in the response. This mode can be seen as a kinematic mode of motion, 
which describes the exchange of potential and kinetic energy when the aircraft is disturbed from trimmed flight. Gradually, the effects of drag will cause the motion to damp out. [58] 60]

Table 4.2: Longitudinal characteristics

\begin{tabular}{lcc}
\hline Characteristic & Short-Period Mode & Phugoid Mode \\
\hline$w_{n}(\mathrm{rad} / \mathrm{s})$ & 0.967 & 0.07 \\
$\zeta$ & 0.335 & 0.048 \\
\hline
\end{tabular}

\subsubsection{Lateral Modes of Motion}

The lateral poles illustrated in Figure $4.2 \mathrm{~b}$ show two real poles and a complex pole pair. These modes of motion are commonly referred to (from highest to lowest natural frequency) as the roll, dutch roll and spiral modes.

The higher frequency real pole in Figure $4.2 \mathrm{~b}$ is referred to as the roll mode. The approximate natural frequency of this real pole is shown in Table 4.3. This mode describes the roll rate dynamics of an aircraft. If an aircraft experiences a positive rolling moment disturbance, it will, according to Newton's law, roll with an angular acceleration. The roll rate will thus initially start to grow. With the introduction of a roll angle, small incidence angles at the starboard and port wing develop, which introduces differential lift. This differential lift will provide a natural roll damping, quantified by $C_{l_{P}}$, and will eventually counter the rolling moment disturbance, restoring the aircraft to a constant roll rate. The very fast dynamics associated with this real pole, illustrated clearly in Figure $4.2 \mathrm{~b}$, make the aircraft appear to always operate at constant roll rates when disturbed about its roll axis. It is thus expected that aileron commands should almost immediately set up a constant roll rate on the aircraft. [57] [58]

The complex pole pair in Figure $4.2 \mathrm{~b}$ is referred to as the dutch roll mode. The approximate damping and natural frequency of this complex pole pair are indicated in Table 4.3. It is noted that this mode of motion is of a similar natural frequency to the short-period mode but very poorly naturally damped. The dutch roll mode is referred to as the directional equivalent of the short-period mode, as it describes the way in which the aircraft aligns itself with oncoming airflow when disturbed with a sideslip angle. The motion of this mode is described as a damped oscillation in yaw, coupling into roll and, to a lesser extent, into sideslip. [57] [58]

To understand the complex interaction between all three lateral-directional degrees of freedom, first consider an aircraft with a small sideslip perturbation from the trim condition, actuated by the rudders. The fin will create a restoring yaw moment, quantified by $C_{n_{\beta}}$, to move the nose of the aircraft back into the wind. It is then expected that the aircraft will return to a stable trim condition, and that the associated yaw rate $C_{n_{R}}$ will provide damping during the yawing motion. However, a rolling moment is also present in this motion, while the wing is constantly moved backwards and forwards through the air, introducing a differential velocity experienced by the wings. The differential velocity at the wings will introduce differential lift and drag. The differential lift introduces roll rate perturbations, while the differential drag 
introduces further yaw rate damping. The combination of rolling and yawing oscillations gives rise to traced ellipses when viewing the aircraft from behind. [57] [58]

Table 4.3: Lateral characteristics

\begin{tabular}{lccc}
\hline Characteristic & Roll Mode & Dutch Roll Mode & Spiral Mode \\
\hline$w_{n}(\mathrm{rad} / \mathrm{s})$ & 0.542 & 0.979 & 0.011 \\
$\zeta$ & 1 & 0.044 & 1 \\
\hline
\end{tabular}

The lower frequency real pole in Figure $4.2 \mathrm{~b}$ is referred to as the spiral mode. The approximate natural frequency of this real pole is shown in Table 4.3 . The spiral mode is mostly a kinematic mode of motion, describing the tendency of the aircraft to restore itself (or diverge if unstable) from wings-level flight when laterally disturbed in roll angle. The characteristics of this mode are very dependent on the lateral static stability (the dihedral effect) and the directional stability (the fin effect) of the aircraft. It is noted that for this aircraft, the mode has a very low natural frequency, but unlike for many aircraft, is a stable mode. [57] [58]

\subsubsection{Response to Controls}

To proceed with proper control system design in the next chapter, it is first necessary to evaluate the response dynamics of the linearised aircraft with the full-order non-linear model. The response transfer functions of the longitudinal and lateral system are obtained from any general state space system as follows,

$$
\mathbf{G}(s)=\frac{\mathbf{Y}(\mathbf{s})}{\mathbf{U}(s)}=\mathbf{C}(s \mathbf{I}-\boldsymbol{A})^{-1} \boldsymbol{B}
$$

These transfer functions completely describe the linear dynamic response to a control input in the plane of symmetry [58]. All transfer functions are conveniently written with individual numerator terms and a common denominator,

$$
G_{u}^{x}(s)=\frac{N_{u}^{x}(s)}{D_{u}(s)}
$$

where $u$ is the control input, $x$ is the state variable for the specific transfer function $G(s)$, and the terms $N$ and $D$ represent the numerator and denominator terms respectively.

\section{Longitudinal Dynamics}

Concerning the longitudinal dynamics, it is common to evaluate the longitudinal response to an elevator input where the thrust is held constant. The four transfer functions obtained in the solution of Equation 4.31, where the thrust is held constant $(\Delta T=0)$, are listed in Appendix $\mathrm{D}$. The longitudinal response to a positive $5^{\circ}$ elevator step input at 10 seconds is shown in Figure 4.3. The linear and non-linear model responses match very well during the initial part of the response, and the responses eventually diverge due to dynamic longitudinal and lateral coupling in the non-linear model, while in the linear model they are uncoupled. 
The short-period and phugoid modes of motion can clearly be depicted in the response the first 10s after the step input showing the fast short-period mode and the phugoid response being illustrated by the slow response between 20 and 200s. From this response, it is derived that a positive elevator deflection will cause the aircraft to pitch down, resulting in a negative pitch angle and pitch rate response. This coincides with the sign conventions of the literature available on the Boeing 747 [10] 9].

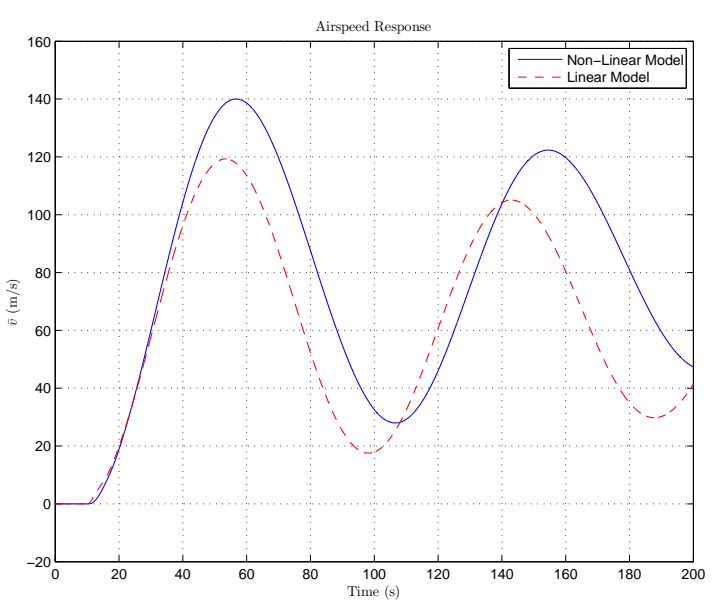

(a) Airspeed response

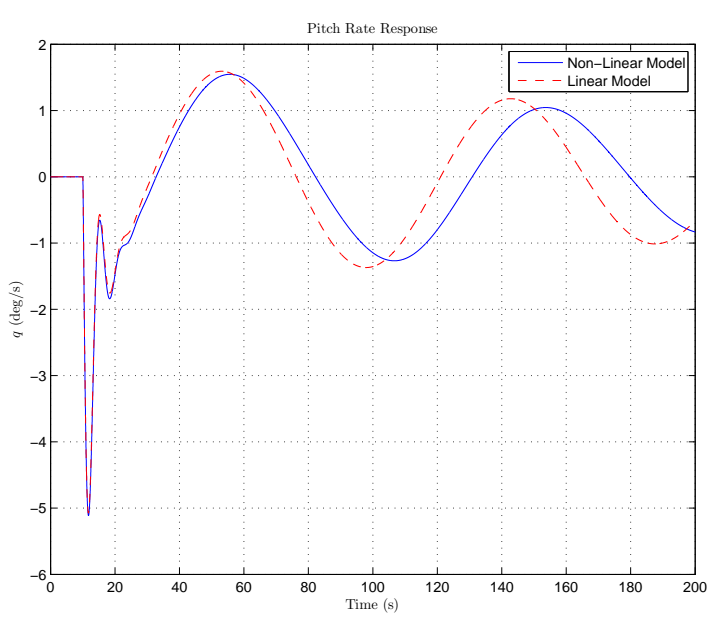

(c) Pitch rate response

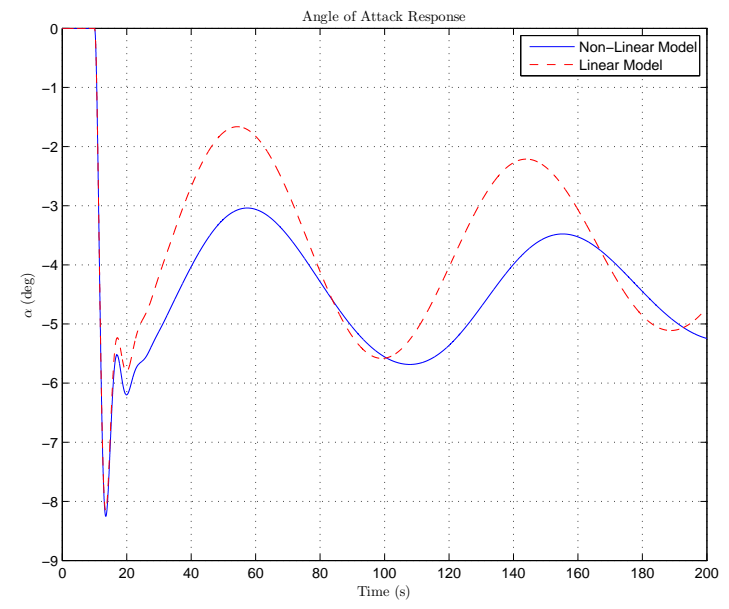

(b) Angle of attack response

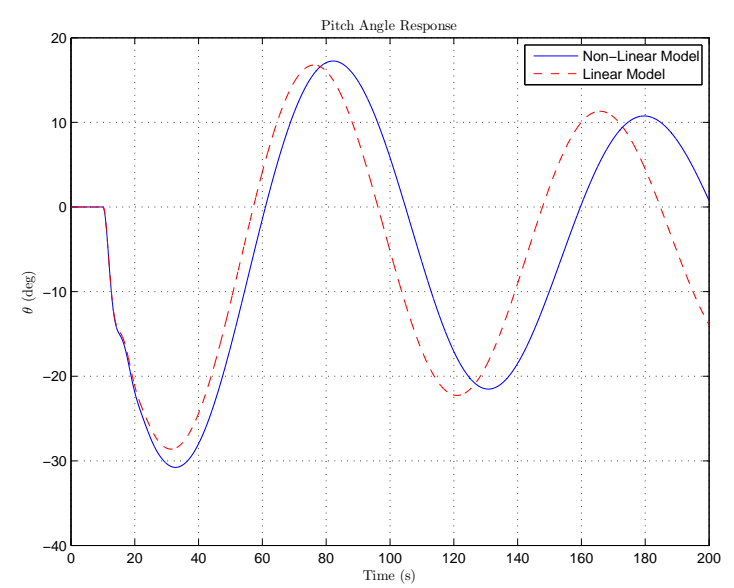

(d) Pitch angle response

Figure 4.3: Longitudinal response to a $5^{\circ}$ elevator step input at $10 \mathrm{~s}$.

\section{Lateral-directional Dynamics}

The solution of Equation 4.35 produces eight transfer functions describing the motion of the aircraft in response to an aileron and rudder input. These transfer functions are listed in Appendix $\mathrm{D}$. The lateral response to a positive $5^{\circ}$ aileron and $1^{\circ}$ rudder step input at 10 seconds is shown in Figures 4.4 and 4.5 respectively. Similar to the longitudinal dynamics, the linear and non-linear model responses match well during the initial part of the response, and eventually 
diverge due to uncoupled dynamics in the linear model. In both responses, it is clear that the response is dominated by the oscillatory dutch roll mode. Furthermore, it can be deduced that the aircraft is stable, since all responses appear as asymptotically convergent characteristics.

An interesting phenomenon in the response is observed on inspection of the transfer functions $G_{u}^{x}(s)$ after factorisation into the following form,

$$
G_{u}^{x}(s)=\frac{\left(s-z_{1}\right)\left(s-z_{2}\right) \ldots\left(s-z_{m}\right)}{\left(s-\lambda_{1}\right)\left(s-\lambda_{2}\right) \ldots\left(s-\lambda_{n}\right)}
$$

where $\lambda_{i}$ are the characteristic roots (poles), $z_{i}$ are the zeros of $G_{u}^{x}(s)$ and $m$ and $n$ are the number of poles and zeros respectively. Some of the transfer functions reveal by factorisation that they contain zeros in the right half plane or, stated differently, non-minimum phase numerator terms. The effect of these non-minimum phase terms seems insignificantly small on inspection of the response in Figure 4.4 and 4.5, with one exception - the roll rate response $p$ to rudder deflection $\delta_{r}$. Closer inspection shows in Figure 4.6 that the roll rate response exhibits a sign reversal; this is the manifestation of the non-minimum phase effect. It is referred to as adverse roll in response to rudder.

A positive rudder step input will cause the aircraft to turn to the left, which is a negative roll rate response. Once the turn is established, a negative yaw and yaw rate result, together with a negative roll and roll rate. When the rudder is positively deflected, a side force is generated on the fin of the aircraft, which in turn generates a negative yawing moment. This then causes the aircraft to turn to the left. However, the side force on the fin acts at a distance above the roll axis, which also generates a rolling moment. This rolling moment causes the aircraft to roll in the opposite direction as that caused by the yawing moment. The aircraft responds quicker in roll, and rolls in the wrong direction, since inertia in roll is lower than inertia in yaw. As the yawing motion becomes established, the rolling moment eventually overcomes the adverse rolling moment and the aircraft will then roll in the correct sense.

A similar characteristic is present in the response to the aileron deflections due to nonminimum phase zeros, and is referred to as adverse yaw. This effect is caused by the differential drag effects associated with the aileron deflections, causing a yawing motion in the wrong direction. Inspection of the transfer function after consulting Appendix $\mathrm{D}$ indicates that it is present in the aircraft but cannot be seen in the lateral response to an aileron deflection - it is insignificantly small at the chosen trim flight condition.

\subsection{Conclusions}

In this chapter, the small disturbance theory was used to calculate the straight and level trim condition variables. The conventional isolated aircraft was then successfully linearised around this trim condition, decoupled and represented as a final state space model form. The partial derivatives for each state space model were derived and summarised in Appendix B, A linear analysis was then performed to validate the accuracy of the linearised model against the fullorder non-linear model in simulation. Strong similarity to different literature sources was found 


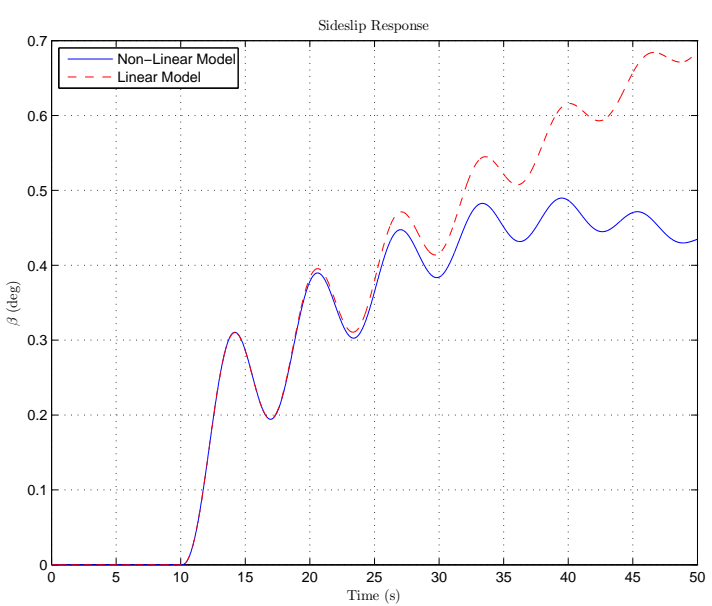

(a) Sideslip response

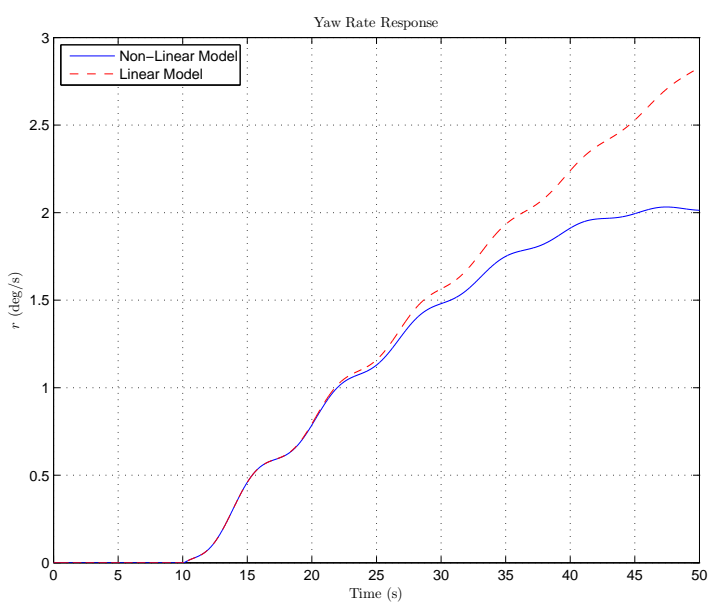

(c) Yaw rate response

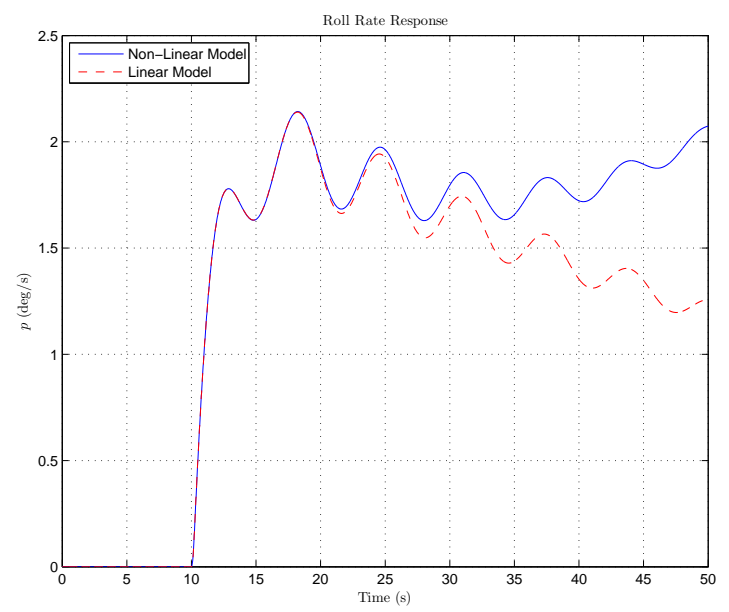

(b) Roll rate response

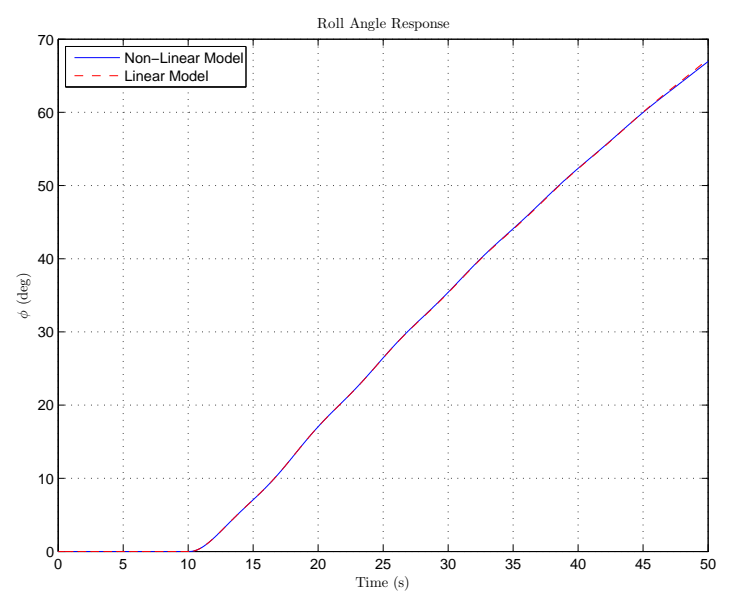

(d) Roll angle response

Figure 4.4: Lateral response to a $5^{\circ}$ aileron step input at $10 \mathrm{~s}$.

for the longitudinal and lateral modes of motion [4] 60]. A study was also performed on how the aircraft responds to actuation of the primary controls, namely elevator, aileron, rudder and throttle. In the response to the controls, it is assumed that there is no wind. The responses of the non-linear and linearised model matched well during the initial part of the response, and the responses eventually diverged due to coupled longitudinal and lateral dynamics in the non-linear model, while in the linear model they are uncoupled. The responses also agreed with the sign conventions used in this study [10] [9].

In the next chapter, the linearised aircraft model will be used to design the flight control systems for a conventional isolated aircraft, and will be extended for an aircraft in formation flight later on. 


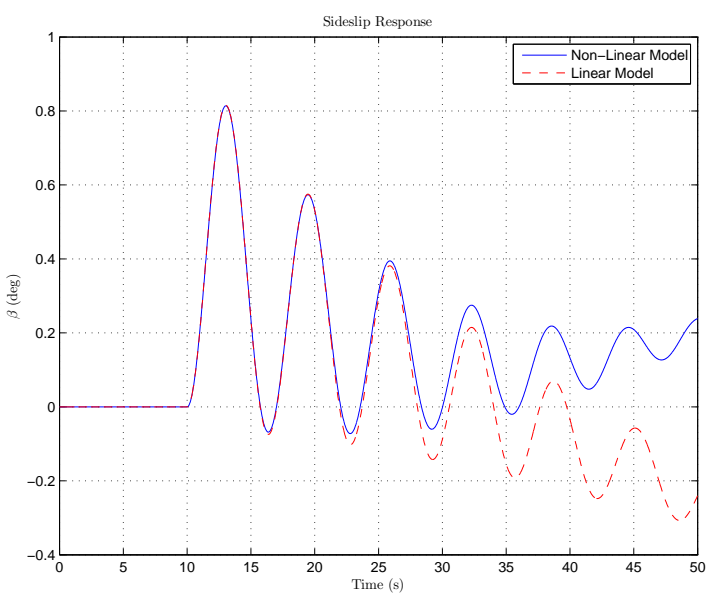

(a) Sideslip response

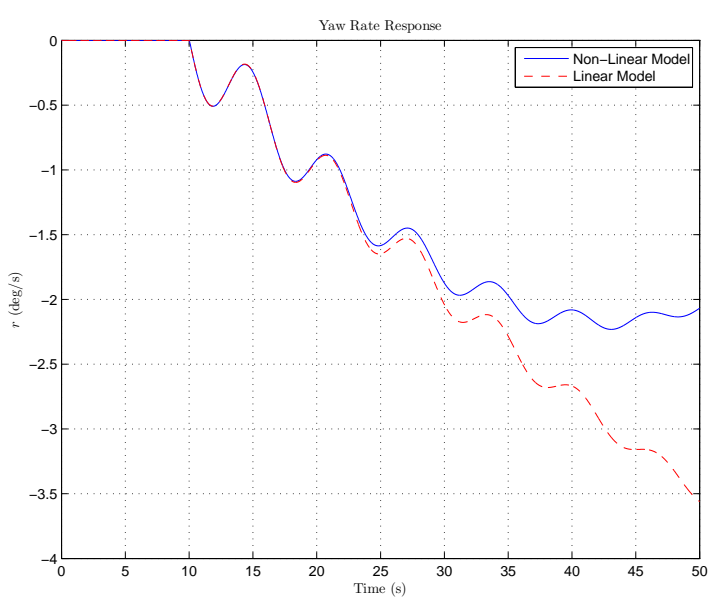

(c) Yaw rate response

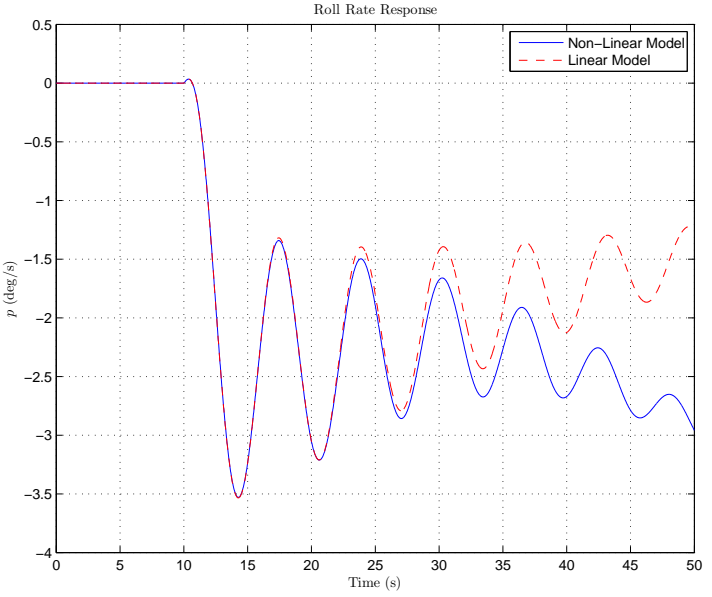

(b) Roll rate response

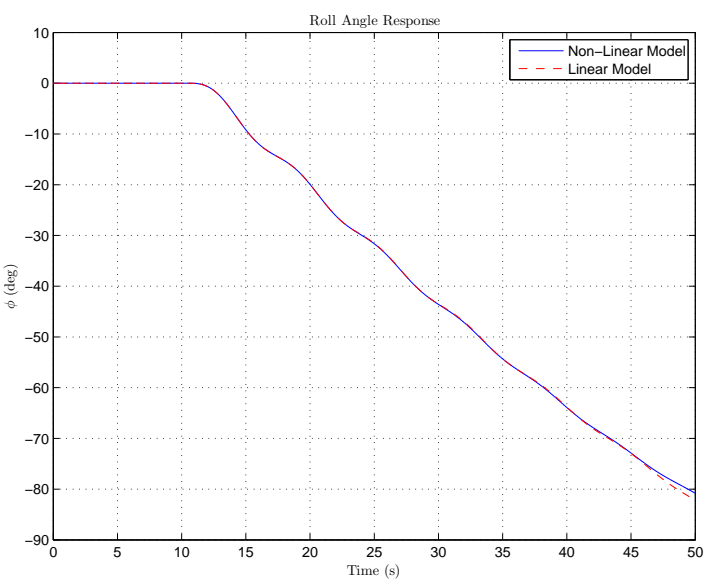

(d) Roll angle response

Figure 4.5: Lateral response to a $1^{\circ}$ rudder step input at $10 \mathrm{~s}$.

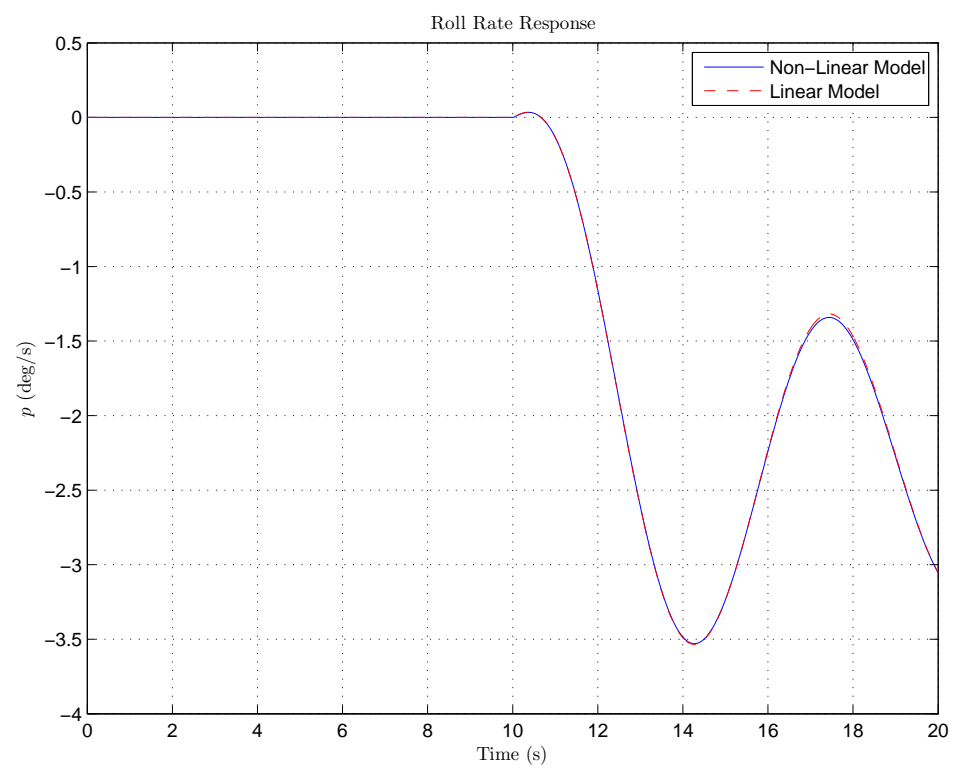

Figure 4.6: Closer look at the roll rate response to a $1^{\circ}$ rudder deflection. 


\section{Chapter 5}

\section{Conventional Flight Control Systems Design}

The formation flight control laws will be an extension of the conventional flight control laws used for an aircraft in isolated flight. A model of the conventional fly-by-wire control laws that are typically used on commercial passenger airliners is required to produce realistic aircraft responses. Büchner [4] presented the first research on automatic control of formation flight for commercial aircraft. As part of the authors research project, Büchner implemented a flight control system for passenger transport aircraft based on the available information to the author, at the time, on fly-by-wire systems used on commercial passenger airliners.

For this study, a more representative model of the fly-by-wire flight control system than the one used by Büchner [4] is desired. To accomplish this, the fly-by-wire architecture was reverse engineered from an A330 Airbus simulation model, and was applied to the Boeing aircraft model in such a way that its response matches the response of an Airbus A330 aircraft. The design processes for the various flight control laws were also reverse engineered based on a combination of information obtained from literature in the open domain, and at Airbus. The control laws were then designed to make the closed-loop responses of the Boeing aircraft match the closed-loop responses of a typical Airbus aircraft.

This chapter starts with a background to fly-by-wire flight control systems architecture in modern transport aircraft. A representative conventional fly-by-wire architecture is presented, after which the longitudinal and lateral-directional flight control laws are designed and analysed. The conventional control laws used in the Airbus A320/330 aircraft have been used to design, implement and verify a representative control architecture with similar aircraft responses [71] [72]. Implementation of the conventional fly-by-wire flight control systems as well as the guidance laws in simulation is followed by an evaluation and discussion of the most important results.

\subsection{Fly-by-wire in Commercial Aircraft}

Fly-by-wire (FBW) is used in modern aircraft transport and consists of a control system architecture with an electronic interface. Electronic signals convert the movement of the flight 
controls and are transmitted by wires to the on-board flight computers. Each actuator is then moved as determined by the Flight Control System (FCS). Automatic signals are also sent to the flight computers to perform functions without the pilot's input. This can help stabilise the aircraft, preventing unsafe operation of the aircraft. The first generation of aircraft using fly-bywire flight control systems was used at the start of the 1980's and included the Airbus A310. 73]

In the 1994 International Journal of Control, Favre [71 gives an overview of the characteristics of the Airbus FBW control laws, systems, certification and development methods. This was published after the Airbus A320 was certified and entered service in 1988, being the first example of a second generation of civil electrical flight control aircraft [73]. A new standard of fly-by-wire was defined in the flight control law and system integration fields [71].

\subsubsection{Objectives and Constraints}

The general control law is illustrated in Figure 5.1. Favre [71] explains that the general objective with the integration of flight controls with a fly-by-wire system is to improve the natural flying qualities of the aircraft. With on-board flight computers, anemometric and inertial information, as well as any information describing the aircraft state, is easily accesible. This makes it possible to design flight control laws corresponding to simple control objectives.

Longitudinal control is achieved through vertical load factor commands, while lateral control is achieved through roll rate, sideslip and bank angle objectives. With aircraft control that satisfies these objectives, the pilot workload is significantly reduced. The fly-by-wire system acts as the inner-loop autopilot system, while the pilot represents the outer-loop control to manage the vertical load factor, roll rate, sideslip and bank angle objectives. [71]

Favre [71] goes on to mention some practical considerations in the fly-by-wire control system. The first consideration is sensor failures. Sufficient redundancy must be available in case of a faulty source. The physical limitations of the control surfaces and actuators are also an important consideration - it is possible that the servo-controls can be asked for more than they are capable

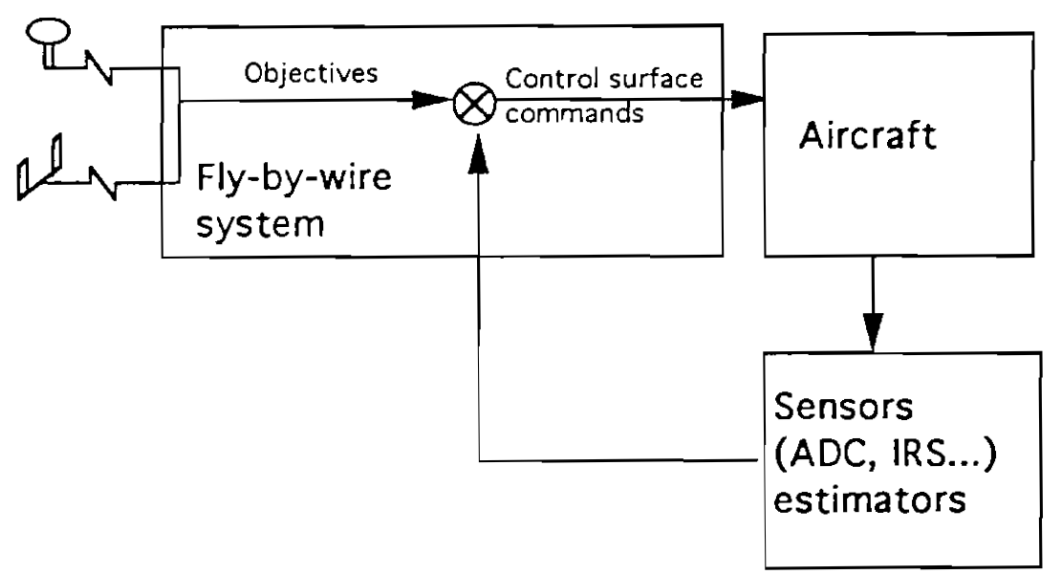

Figure 5.1: General structure of the control laws used in a fly-by-wire architecture (from Favre [71]). 
of. Passenger comfort, flutter and loadings are also important considerations which result from the control law interaction with the aircraft structure. Finally, human factors must be taken into consideration. This means that the general aircraft behaviour must be in accordance with the visualisations and sensations expected by pilots.

\subsubsection{Control Law Structure}

In a fly-by-wire system such as the one illustrated in Figure 5.1, each type of computer is intergrated with different control law configurations [71]. The longitudinal and lateral laws consists of 3 different modes, namely (1) the Normal mode, where full control and protection functions are active, (2) the Alternative (ALT) mode, where flight envelope protection and gust alleviation functions are lost, and (3) the Direct (DIR) mode, entered when severe failures occur and direct surface control is activated with rudimentary feedback [72]. The current study will focus on the first of these three modes, namely the Normal mode. The control laws in the normal mode (also referred to as the Normal laws from now on) implement aircraft feedback and are highly dependent on the full availability of the sensor measurements.

\section{Longitudinal Law}

The longitudinal control law structure in the Normal mode is illustrated in Figure 5.2. The architecture represents a vertical load factor controller with pitch rate and load factor feedbacks and integral control. The short-period mode is controlled with the feedback, ensuring that shortterm stability and tracking precision are achieved with the integral control. It incorporates load factor limiting, includes the auto-trim function and allows steady turns with sidesticks of up to $33^{\circ}$ roll angle, measured from the neutral stick position.

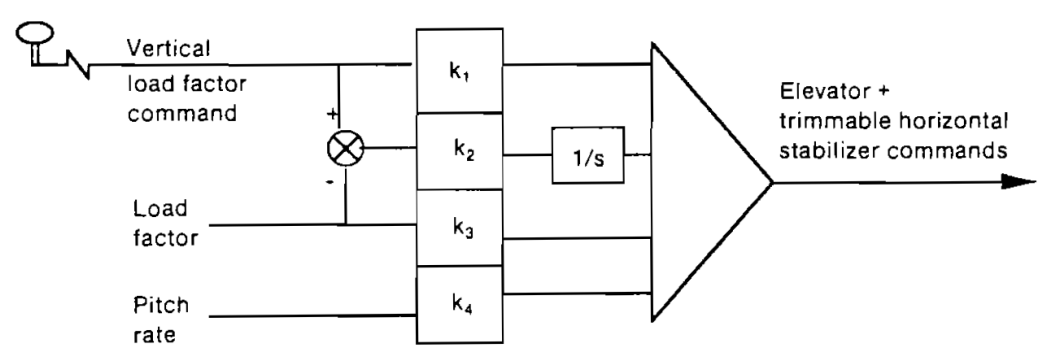

Figure 5.2: Longitudinal control law structure (from Favre [71]).

Favre [71] explains that the controlled modes are selected so that they are close to the natural aircraft modes in order to minimise the control surface activity and the difference between normal and degraded (direct) laws. With this objective, the static stability of the aircraft is almost neutral, and the phugoid mode is transformed into a highly damped mode. The following protections are activated:

- Angle of attack protection at low speeds 
- High-speed protections

- Pitch rate protection

A homogeneous law is then obtained for longitudinal control, which ensures that the aircraft behaviour is independent of the flight conditions and the centre of gravity locations of the aircraft. This homogeneity is achieved by tabulating control gains as a function of the computed airspeed, high-lift configuration and the centre of gravity location.

\section{Lateral Law}

The lateral control law structure is illustrated in Figure 5.3 . Favre 71 first explains the stability specifications and then moves on to the pilot control objectives. Concerning the stability objectives, it was desired to increase the dutch roll damping coefficient beyond 0.6, without significantly exciting the aircraft lateral modes. This kept the roll mode unchanged and increased the spiral mode stability. As with the longitudinal law, the controlled modes are selected so that they are close to the natural aircraft modes in order to minimise the control surface activity and the difference between normal and degraded (direct) laws.

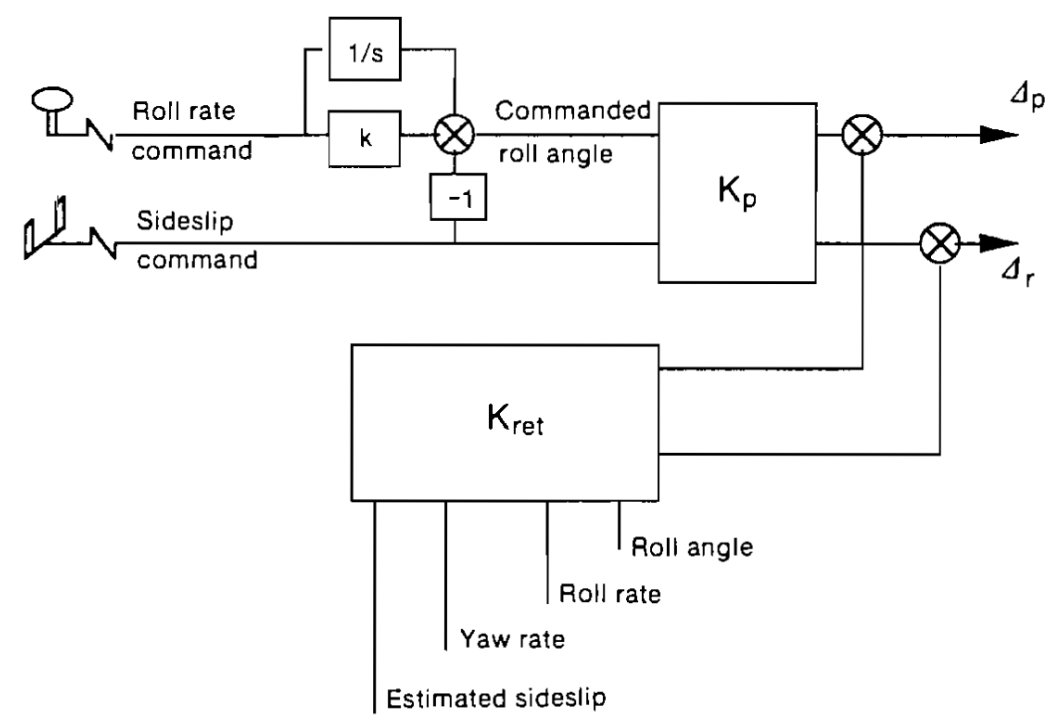

Figure 5.3: Lateral control law structure (from Favre [71]).

Concerning the pilot control management objectives, sidestick inputs are translated into roll rate commands. This is done at constant zero sideslip to provide automatic turn co-ordination and reduce pilot workload. Furthermore, the bank angle is kept constant in turns with the sidestick at a neutral position. Finally, the rudder pedals command a combination of sideslip and roll angle to imitate the natural behaviour of the aircraft, similar to what a pilot would expect from an aircraft without fly-by-wire.

Considering the lateral control law architecture in Figure 5.3, the stability objectives are achieved through the estimated sideslip, roll rate, yaw rate and roll angle feedbacks. The 
gain matrix $K_{\text {ret }}$, dependent on the flight condition, is computed from the stability and roll angle/sideslip decoupling objectives. The pilot control objectives are achieved through a roll rate command that is integrated and converted into a roll angle command. The rudder pedals provide a roll angle and sideslip command. Finally, a pre-command matrix $K_{p}$ is designed to achieve the commanded sideslip and roll angle in steady state without perturbations. [71]

\subsubsection{Fly-by-wire System Integration}

\section{General Architecture}

The classic Airbus fly-by-wire system architecture used in the second generation of civil electrical flight control aircraft, namely the A320, is illustrated in Figure 5.4. It consists of a set of five full-authority digital computers used to control the pitch, roll and yaw axes. It also has a mechanical back-up on the trimmable horizontal stabiliser and on the rudder. All five computers are simultaneously active, and perform control law computation as a function of pilot inputs and individual actuator control. This fly-by-wire system incorporates sufficient redundancy so that if one of the computers fails, nominal performance and safety levels are still achieveable. Furthermore, it would still be possible to fly the aircraft safely with only one active computer. 71 .

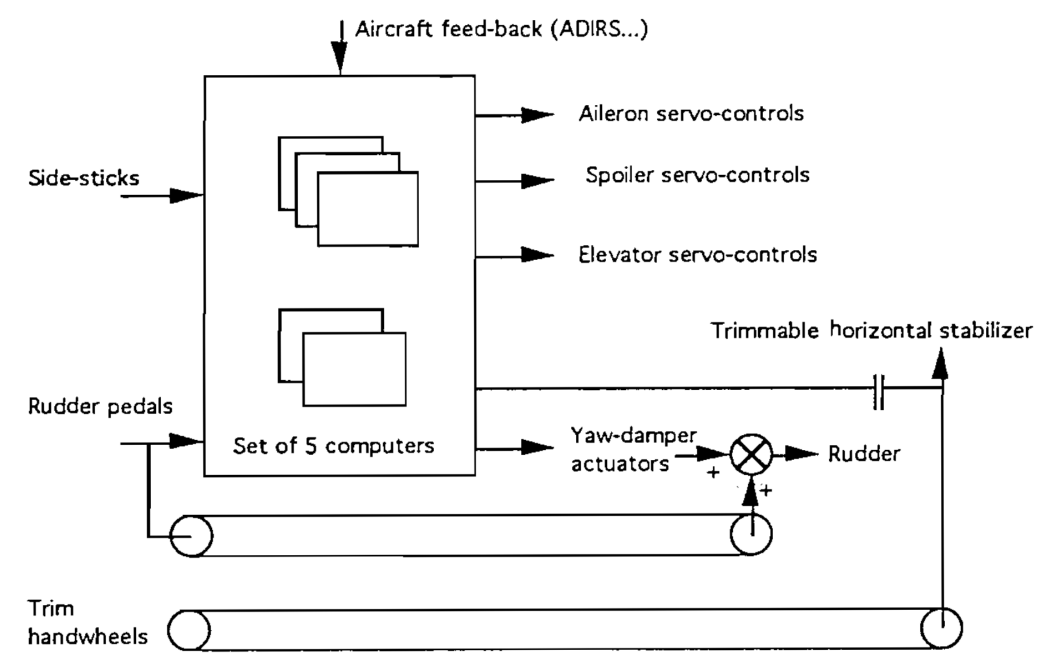

Figure 5.4: General structure of the Airbus fly-by-wire system (from Favre [71]).

Favre [71] explains that because control surface runaway could affect aircraft safety, each of the five on-board computers is divided into two physically separate channels: the first a control channel, and the second a monitor channel. The second channel permanently monitors the control channel (hence the name). If there is any disagreement between the control and monitor channels of a computer due to a failure in the system, the computer with the next highest priority takes control. It is also fundamental that the five computers are of different natures to avoid common mode failures and the total loss of the electrical flight control system. Two types of computers exist in the fly-by-wire systems: 
- In the A320/321: Two elevator and aileron computers (ELACs) and three spoiler and elevator computers (SECs)

- In the A330/340: Three flight control primary computers (FCPCs) and two flight control secondary computers (FCSCs)

\subsection{Conventional Fly-by-Wire Flight Control Architecture}

Figure 5.5 illustrates a proposed model of the flight control architecture used in modern-day passenger transport aircraft. The architecture consists of an inner-loop set of fly-by-wire flight controllers in the Normal law configuration, namely the DQ (direct pitch control), DP (direct roll control) and DR (direct yaw control) laws, directly commanding the actuators based on multiple reference inputs. The Normal control law configuration provides stability augmentations while allowing pilots to control the aircraft motion [72]. An autothrust controller is also added to command an engine thrust based on a reference airspeed. Commanding the fly-by-wire flight control is a set of conventional guidance laws used to guide the aircraft along a desired flight path, namely the altitude-hold and cross-track error guidance laws.

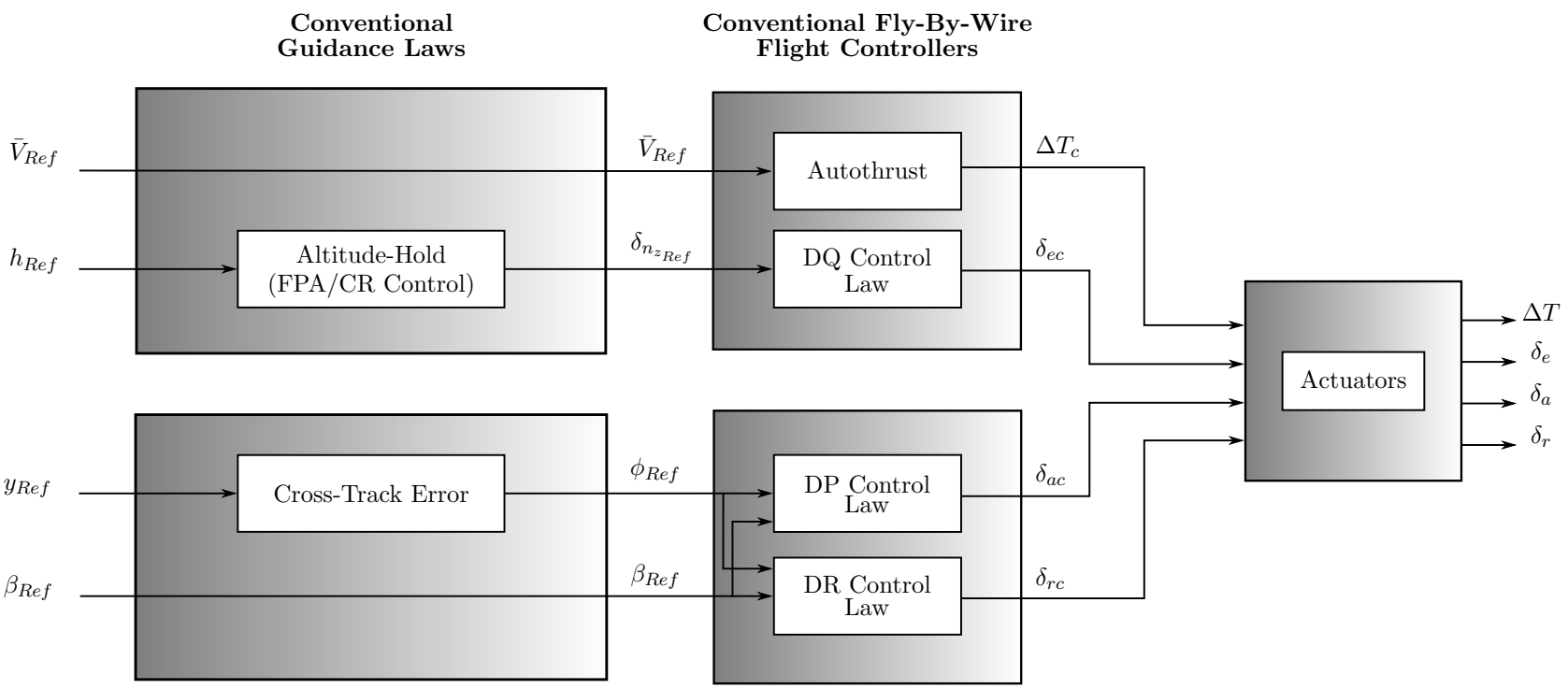

Figure 5.5: A representative architecture of conventional fly-by-wire flight control used in modern-day passenger transport aircraft.

\subsubsection{Conventional Fly-by-Wire Flight Controllers: Normal Laws}

For the longitudinal motion, the Normal law allow the pilots to control the vertical load factor $n_{z}$ of the aircraft by issuing a normal load factor or $C^{*}$ commands, which is a blended command of load factor and pitch rate [72]. After the $C^{*}$ parameter was accepted and used as a handling qualities metric, a control law based on the parameter evolved. The control approach can be explained by considering how the $C^{*}$ parameter evolved: At low velocities, the pitch rate cues 
dominate and the flight path of the aircraft can thus be controlled by controlling the pitch rate. On the other hand, at higher velocities, the normal load factor (or normal acceleration) cues dominate the pitch rate cues, and the flight path of the aircraft can be controlled through control of the normal acceleration and hence angle of attack. [74]

The DQ control law uses full-state feedback with integral control to achieve a desired normal load factor by commanding elevator deflections. The full longitudinal state vector is fed back, and one integrator state is added for the load factor. This is to ensure that the normal load factor commands are followed with zero steady-state error. An autothrust controller with a classic proportional-integral (PI) architecture is also implemented which commands engine thrust to maintain a reference airspeed.

For the lateral motion of the aircraft, the normal mode allows pilots to control the roll $\phi$ and sideslip angle $\beta$ of the aircraft [72]. The DP and DR law controllers were designed with a three-phase approach. The first phase entailed a full-state feedback design to place the poles and obtain a set of desired specifications. Following the pole placement, a rudder turn co-ordination controller was added, which computes the amount of rudder required to produce a sideslip angle that minimises the adverse yaw effect of the aircraft. In the third and final phase of the project, the controllers were designed to produce a natural response for a commanded sideslip by allowing a slight roll angle when commanding sideslip.

\subsubsection{Conventional Guidance Laws}

Following the inner-loop fly-by-wire flight control architecture, a set of outer-loop guidance laws are required to control the aircraft along a desired flight path. Due to limited proprietary information regarding flight control systems used by Airbus, the guidance law architecture presented here is based on what the author of the current study believes is representative of that used in the Airbus model.

For longitudinal guidance control, the altitude-hold guidance law was designed with the objective of maintaining a reference altitude $h_{\text {Ref }}$ by using flight path angle (FPA) or climb rate (CR) specific control, illustrated in Figure 5.6. The FPA control is commonly used to control the altitude at take-off and landing flight conditions, whereas the CR control is typically used during nominal cruising conditions. For this study, then, the CR specific altitude control will be considered. The complete design of the altitude-hold guidance law consisted of a double loop closure design: a CR controller followed by an altitude controller.

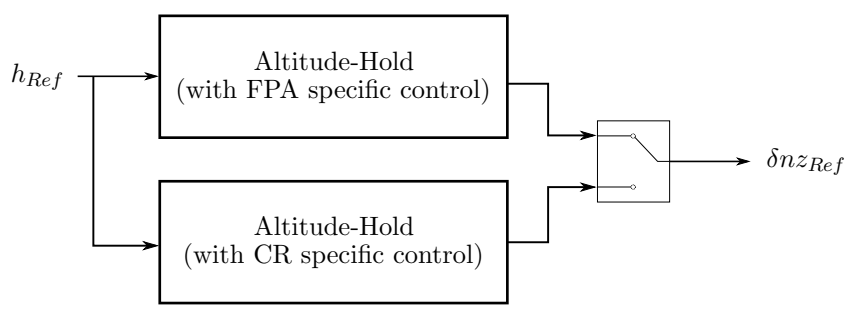

Figure 5.6: Overview of the altitude-hold guidance law strategy. 
For lateral-directional guidance, a cross-track error controller is designed to navigate the aircraft along a flight path plan consisting of a set of waypoints, defined as north and east position coordinates,

$$
W P=\left[\begin{array}{cc}
N_{1} & E_{1} \\
N_{2} & E_{2} \\
\cdot & \cdot \\
\cdot & \cdot \\
N_{n} & E_{n}
\end{array}\right]
$$

where $W P$ is the waypoint vector, $N$ is the north coordinate, $E$ is the east coordinate and $n$ is the number of waypoints. The set of waypoints forms a series of straight-line segments.

\subsection{Longitudinal Control Systems Design}

In this section, the longitudinal controllers depicted in Figure 5.5, namely the DQ law, the autothrust controller and the altitude-hold mode guidance law, will be designed and verified in simulation. The linearised longitudinal state space model in Equation (4.25a will be used with the following notation,

$$
\dot{\boldsymbol{x}}=\boldsymbol{A x}+\boldsymbol{B u}
$$

where,

$$
\begin{aligned}
\boldsymbol{x} & =\left[\begin{array}{llll}
\bar{v} & \alpha & q & \theta
\end{array}\right]^{T} \\
\boldsymbol{u} & =\left[\begin{array}{ll}
\delta_{e} & \Delta T
\end{array}\right]^{T} \\
\boldsymbol{A} & =\boldsymbol{A}_{\text {long }} \\
\boldsymbol{B} & =\boldsymbol{B}_{\text {long }}=\left[\begin{array}{ll}
\mathbf{B}_{\delta_{e}} & \mathbf{B}_{\Delta T}
\end{array}\right]
\end{aligned}
$$

\subsubsection{DQ Law: A Normal Load Factor Controller}

In order to design the normal load factor controller, a reduced-order longitudinal state space model was obtained. The definition of the normal load factor is given in the next subsections.

\section{Reduced-Order Model}

In the linear model derivation and analysis of the aircraft, the full-order state space model was used to obtain an exact solution of the longitudinal and lateral equations of motion. This in turn provided an accurate description of the stability and response characteristics of the aircraft. In the design process, the aircraft handling qualities are determined by the dynamics of the transient response to controls [58]. However, using the exact solution of the equations of motion can have two major disadvantages. Firstly, it requires the use of computers and complex 
programs if a manual solution is undesired. Secondly, it is difficult to establish the relationships between the stability and aerodynamic characteristics when using the full-order solution of the equations of motion.

With a reduced-order model, the above-mentioned complexities can be eliminated in the design process. For the longitudinal dynamics, it is well known that the short-term behaviour of the system is dominated by the higher frequency short-period mode and is thus of the greatest interest. It is common practice and convenient to suppress or omit the phugoid behaviour to obtain a reduced-order model derived from the full-order equations of motion. In the design of the controllers for the longitudinal dynamics, a reduced-order model gives the best visibility to the dominant motion drivers, and allows for better determination of good handling qualities. 58 .

The short-period pitching oscillation can thus be considered an oscillation in which the principal variables are the pitch rate $q$ and the angle of attack $\alpha$, with the speed remaining constant. The longitudinal state equation in Equation (5.2) can be simplified to a new reducedorder longitudinal state equation as follows,

$$
\begin{aligned}
\dot{\boldsymbol{x}}_{r} & =\mathbf{A}_{r} \boldsymbol{x}_{r}+\mathbf{B}_{r} u \\
{\left[\begin{array}{c}
\dot{\alpha} \\
\dot{q}
\end{array}\right] } & =\left[\begin{array}{cc}
\frac{\partial \dot{W}}{\partial W} & \frac{1}{V_{T}} \frac{\partial \dot{W}}{\partial Q} \\
\bar{V}_{T} \frac{\partial \dot{Q}}{\partial W} & \frac{\partial \dot{Q}}{\partial Q}
\end{array}\right]\left[\begin{array}{l}
\alpha \\
q
\end{array}\right]+\left[\begin{array}{c}
\frac{1}{V_{T}} \frac{\partial \dot{W}}{\partial \delta_{E}} \\
\frac{\partial \dot{Q}}{\partial \delta_{E}}
\end{array}\right] \delta_{e}
\end{aligned}
$$

Figure 5.7 shows the comparison between the full-order and the reduced-order model for a unit elevator step command.
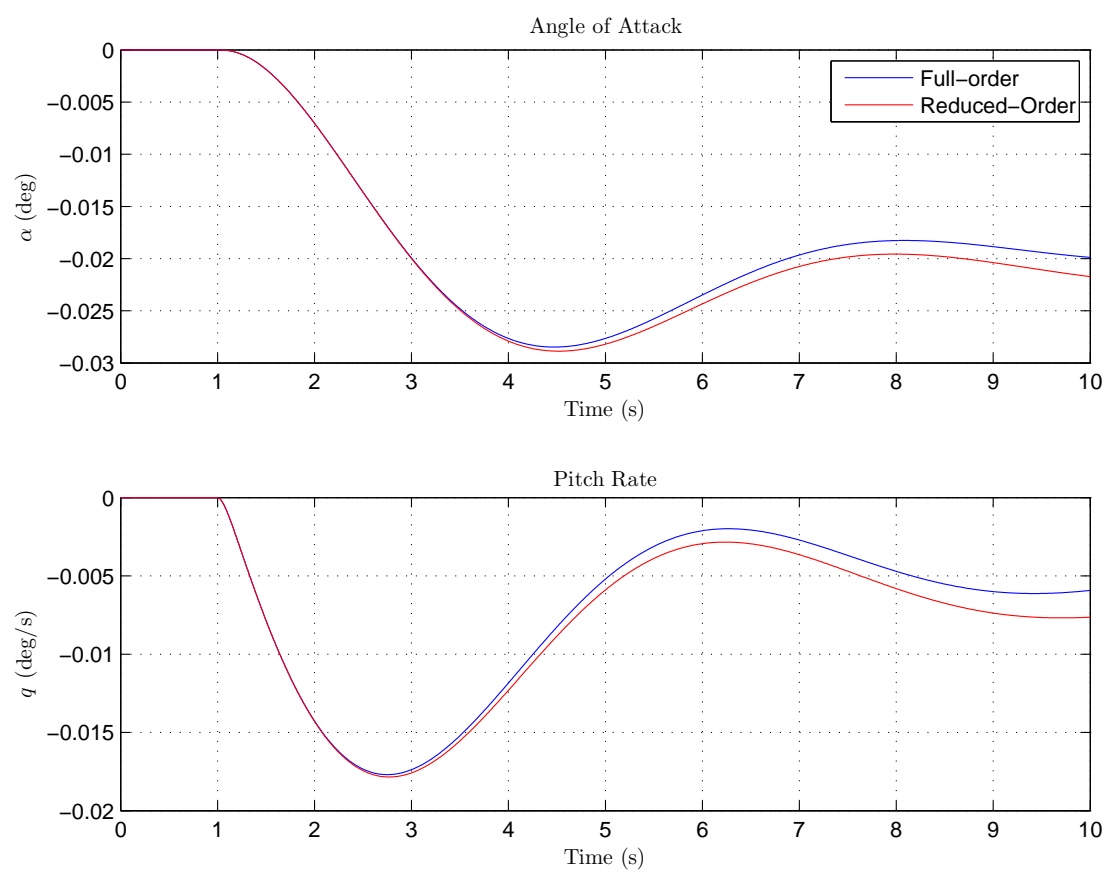

Figure 5.7: Comparison between the full-order and the reduced-order longitudinal model responses to a unit elevator step command. 


\section{Normal Load Factor}

The normal load factor quantifies the total aerodynamic lift necessary to maintain a manoeuvre or desired flight path as a ratio of the lift to its weight. It is an important structural design parameter, indicating the amount of load that the structure of the aircraft can bear. The normal load factor is also an important manoeuvring and performance limit, leaving the flight control designer to make sure that the load factor is well within the aircraft's limits during isolated and formation flight.

The total inertial normal load factor is defined as the ratio of the aircraft lift over the weight,

$$
n_{z}=\frac{L}{W}
$$

Transforming the lift to the body axes coordinates using the DCM transformation matrix of Equation (3.8) yields the normal load factor in the body axis,

$$
n_{z_{B}}=\frac{-Z_{B}^{A}}{m g}=\frac{-q S C_{Z}}{m g}
$$

Expanding the $Z_{B}$-axis non-dimensional aerodynamic coefficient,

$$
\begin{aligned}
C_{Z} & =C_{X_{S}} \sin \alpha+C_{Z_{S}} \cos \alpha \\
& =-C_{D} \sin \alpha-C_{L} \cos \alpha
\end{aligned}
$$

and assuming the angle of attack is small enough that, together with the assumption that the lift is an order of magnitude greater than the drag at the nominal flight condition,

$$
C_{Z} \approx-C_{L}
$$

and the total normal load factor in the body axis is given as,

$$
n_{z_{B}}=\frac{q S C_{L}}{m g}
$$

To keep the aircraft at straight and level flight as in Figure 5.8, the total lift in the body axis must always be enough to counter the weight of the aircraft. The normal load factor at straight and level flight is thus unity $(1 \mathrm{~g})$.

When the aircraft maintains a constant climb rate or flight path angle, the total normal load factor is also the nominal normal load factor,

$$
n_{z_{B, \text { Nominal }}}=n_{z_{B}}
$$

Now consider a change in the flight path angle, induced by a longitudinal manoeuvre and illustrated in Figure 5.9. During the manoeuvre, the aircraft has a total load factor that is now slightly more than the nominal normal load factor. This change is caused by an incremental normal load factor $\delta_{n_{z}}$ due to the manoeuvre, 


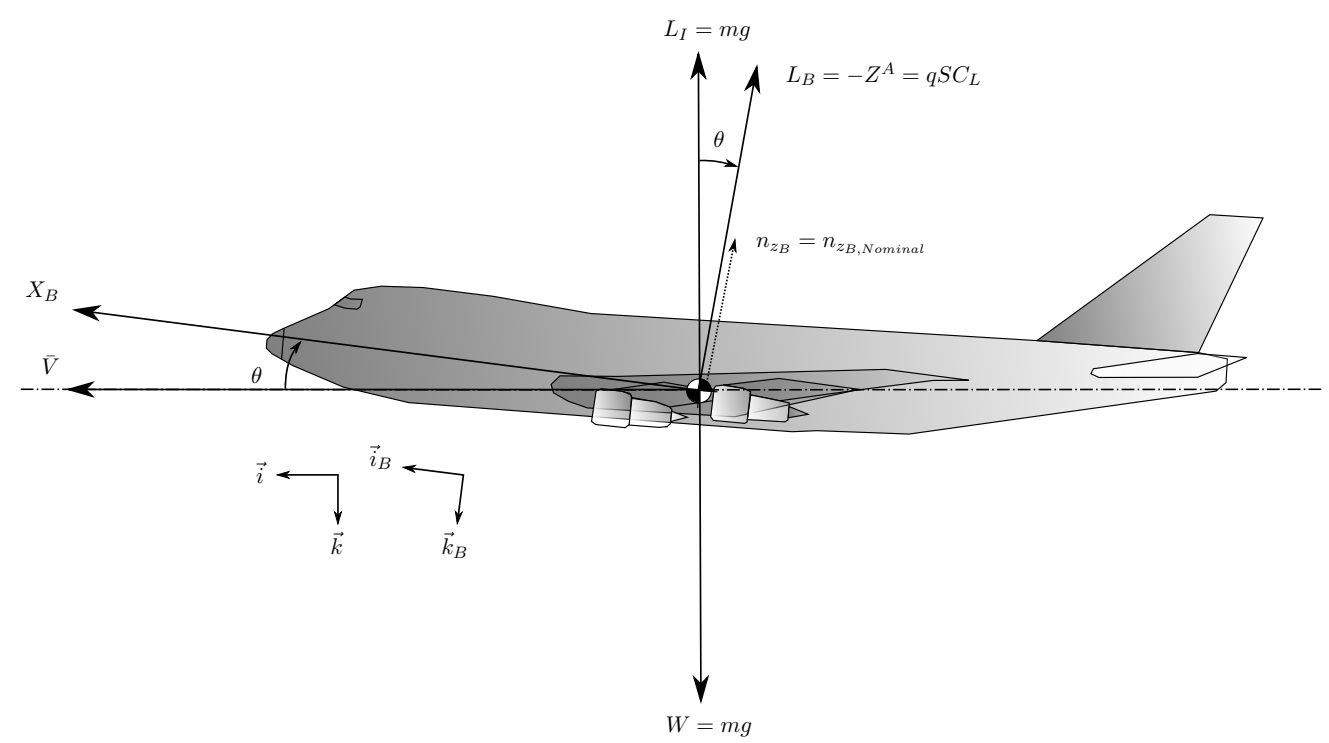

Figure 5.8: Illustration of the normal load factor during straight and level flight.

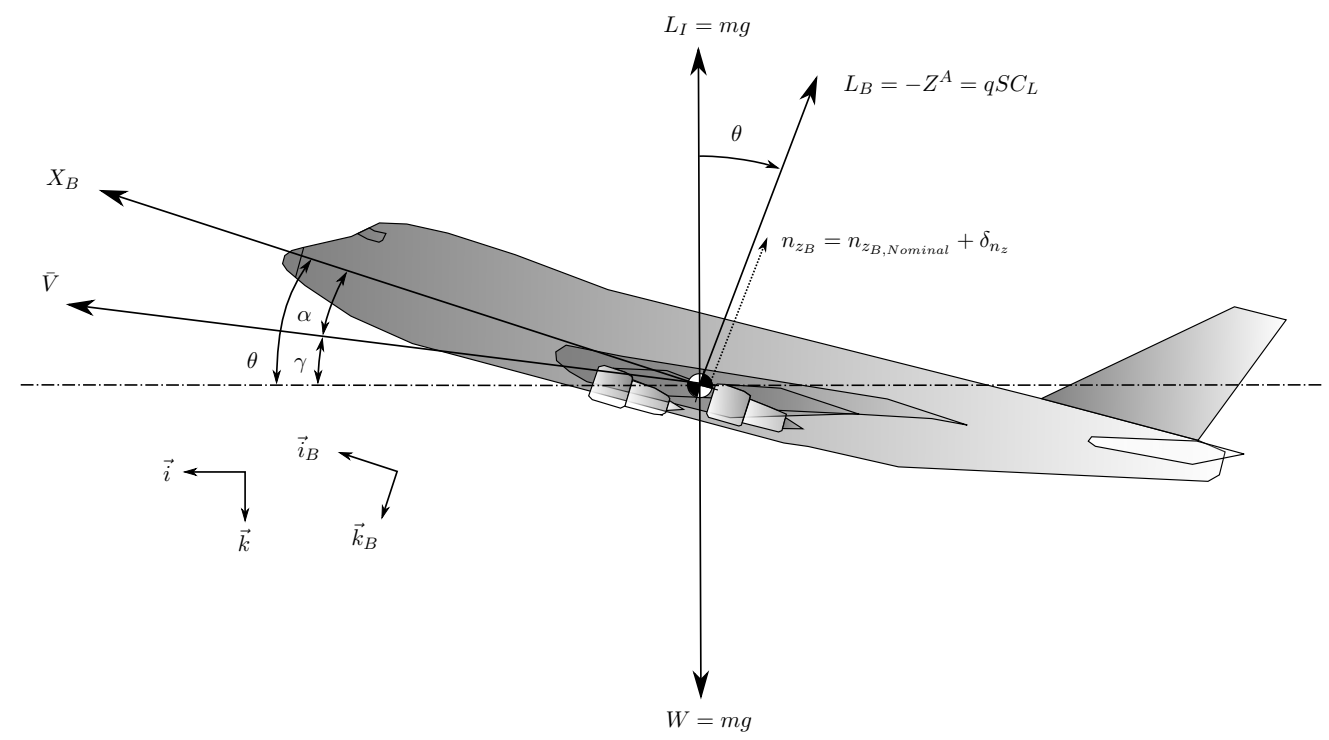

Figure 5.9: Illustration of total normal load factor during a longitudinal manoeuvre.

$$
n_{z_{B, \text { Nominal }}}+\delta_{n_{z}}=n_{z_{B}}
$$

The design of the DQ law requires the linearised incremental load factor $\delta_{n_{z}}$, and to linearise this around the trim point, the nominal load factor is first derived using point-mass kinematics.

\section{Nominal Normal Load Factor}

To obtain the nominal normal load factor in the body axis, consider the aircraft position as a point mass, as illustrated in Figure 5.10. Flying at any arbitrary heading angle $\psi$, the heading axis is defined as the inertial axis rotated around the $Z_{I}$-axis by the heading angle $\psi$. Viewing the aircraft from behind and at an arbitrary bank angle $\phi$, the corresponding force vector in the heading axis required to counter the gravitational force and maintain altitude is given by, 


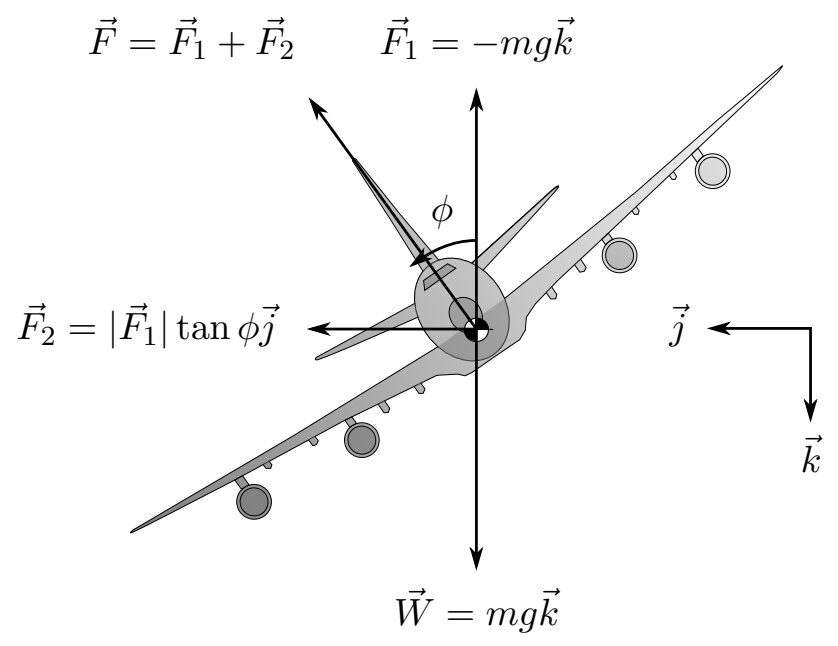

Figure 5.10: Aircraft illustrated as a point mass during a steady, coordinated turn.

$$
\begin{aligned}
\vec{F} & =\vec{F}_{1}+\vec{F}_{2} \\
& =\left[\begin{array}{c}
0 \\
m g \tan \phi \\
-m g
\end{array}\right]\left[\begin{array}{l}
\vec{i} \\
\vec{j} \\
\vec{k}
\end{array}\right]
\end{aligned}
$$

Transforming this force co-ordinate vector from the heading axis to the body axes co-ordinates will allow the extraction of the equivalent nominal vertical force in the body axis required to maintain altitude during a turn. This vertical force in the body axes is used to obtain the nominal normal load factor.

Using the DCM transformation matrix in Equation (3.8), the force vector in the heading axis is rotated through a pitch and roll angle while keeping the heading angle constant,

$$
\begin{aligned}
\vec{F}_{B} & =\left.\mathbf{T}\right|_{\theta, \phi, \psi=0} \vec{F} \\
{\left[\begin{array}{c}
F_{X} \\
F_{Y} \\
F_{Z}
\end{array}\right]_{B} } & =\left[\begin{array}{c}
\sin \theta \\
\sin \phi-\cos \theta \sin \phi \\
-\frac{\sin ^{2} \phi}{\cos \phi}-\cos \theta \cos \phi
\end{array}\right] m g
\end{aligned}
$$

and the nominal vertical force in the body axis is,

$$
F_{Z_{B, \text { Nominal }}}=\frac{-m g \sin ^{2} \phi}{\cos \phi}-m g \cos \theta \cos \phi
$$

Finally, then, the nominal normal load factor in the body axis is determined as,

$$
\begin{aligned}
n_{z_{B, \text { Nominal }}} & =\frac{-F_{Z_{B, \text { Nominal }}}}{m g} \\
& =\frac{\sin ^{2} \phi}{\cos \phi}+\cos \theta \cos \phi
\end{aligned}
$$




\section{Linearised Incremental Normal Load Factor}

Equations (5.9) and (5.15) describe the non-linear dynamics of the total and nominal normal load factor respectively. Using these in Equation (5.11), the non-linear incremental normal load factor is obtained as,

$$
\begin{aligned}
\delta_{n_{z}} & =n_{z_{B}}-n_{z_{B, \text { Nominal }}} \\
& =\frac{q S C_{L}}{m g}-\frac{\sin ^{2} \phi}{\cos \phi}-\cos \theta \cos \phi
\end{aligned}
$$

It is now necessary to linearise the incremental normal load factor equation about the trim point defined in Chapter 3 so that it can be used in the linear model of the aircraft. The incremental normal load factor in Equation (5.16) can be displayed more concisely in the non-linear state space form as a non-linear function,

$$
\begin{aligned}
\delta_{n_{z}} & =f(\boldsymbol{x}, u, v) \\
\boldsymbol{x} & =\left[\begin{array}{llll}
\bar{V} & \alpha & Q & \theta
\end{array}\right]^{T} \\
u & =\delta_{E} \\
v & =\phi
\end{aligned}
$$

where $\boldsymbol{x}$ is the longitudinal state vector, $u$ is the control input variable, and $v$ is the lateral roll angle. In Chapter 4, the linear aircraft model was decoupled into a longitudinal and lateral model. For the design of the longitudinal DQ law controller, the incremental normal load factor will only be described by the longitudinal states. The contribution of the roll angle to the normal load factor will be treated as a disturbance signal. Expanding the non-linear load factor equation in a Taylor series expansion about the trim condition yields an $n^{\text {th }}$-order polynomial function for $\delta_{n_{z}}$ as a function of the perturbations about trim,

$$
\begin{aligned}
\delta_{n_{z}} & =\delta_{n_{z_{T}}}+\Delta \delta_{n_{z}} \\
& =f\left(\boldsymbol{x}_{T}, u_{T}, v_{T}\right)+\left.\frac{\partial f}{\partial \boldsymbol{x}}\right|_{T}\left(\boldsymbol{x}-\boldsymbol{x}_{T}\right)+\left.\frac{\partial f}{\partial u}\right|_{T}\left(u-u_{T}\right)+\left.\frac{\partial f}{\partial v}\right|_{T}\left(v-v_{T}\right)+\mathcal{O}
\end{aligned}
$$

where $\mathcal{O}$ represents higher-order terms. Assuming that the deviations about trim are small, the higher-order terms in the Taylor expansion can be ignored, and the incremental normal load factor can be approximated as a first-order polynomial,

$$
\Delta \delta_{n_{z}} \approx \mathbf{C}_{\delta n_{z}} \Delta \boldsymbol{x}+D_{\delta n_{z}} \Delta u+N_{\delta n_{z}} \Delta v
$$


where,

$$
\begin{aligned}
\mathbf{C}_{\delta n_{z}} & =\left.\frac{\partial f}{\partial \boldsymbol{x}}\right|_{T}=\left[\begin{array}{llll}
\frac{\partial \delta_{n_{z}}}{\partial V} & \frac{\partial \delta_{n_{z}}}{\partial \alpha} & \frac{\partial \delta_{n_{z}}}{\partial Q} & \frac{\partial \delta_{n_{z}}}{\partial \theta}
\end{array}\right]_{T} \\
D_{\delta n_{z}}=\left.\frac{\partial f}{\partial u}\right|_{T} & =\left.\frac{\partial \delta_{n_{z}}}{\partial \delta_{E}}\right|_{T} \\
N_{\delta n_{z}} & =\left.\frac{\partial f}{\partial v}\right|_{T}=\left.\frac{\partial \delta_{n_{z}}}{\partial \phi}\right|_{T}
\end{aligned}
$$

All of the partial derivatives in $\mathbf{C}_{\delta n_{z}}$ and those of $D_{\delta n_{z}}$ and $N_{\delta n_{z}}$ can be calculated and evaluated at trim to obtain the linearised incremental normal load factor. The partial derivatives of Equations $5.20 \mathrm{a}$ and $5.20 \mathrm{~b}$ ) are listed in Appendix C.

The incremental load factor can now be used in the design of a normal load factor controller.

\section{Design}

A controller capable of regulating the normal load factor through the incremental normal load factor will be designed using the reduced-order model, as in Equation (5.4). The architecture of this DQ control law is shown in the block diagram of Figure 5.11. The plant for the design is the reduced-order longitudinal dynamics,

$$
\begin{aligned}
\dot{\boldsymbol{x}}_{r} & =\mathbf{A}_{r} \boldsymbol{x}_{r}+\mathbf{B}_{r} \delta_{e} \\
\delta_{n_{z}} & =\mathbf{C}_{\delta n_{z}} \boldsymbol{x}_{r}+D_{\delta n_{z}} \delta_{e}
\end{aligned}
$$

where the output matrices,

$$
\begin{aligned}
\mathbf{C}_{\delta n_{z}} & =\left[\begin{array}{ll}
\frac{\partial \delta_{n_{z}}}{\partial \alpha} & \frac{\partial \delta_{n_{z}}}{\partial Q}
\end{array}\right] \\
D_{\delta n_{z}} & =\frac{\partial \delta_{n_{z}}}{\partial \delta_{E}}
\end{aligned}
$$

are used to extract the incremental load factor $\delta_{n_{z}}$ from the reduced-order state vector $\boldsymbol{x}_{r}$. The incremental normal load factor output is fed into an integrator to obtain the incremental normal load factor error integrated over time, denoted as a new error state $x_{I_{D Q}}$. The reduced-order longitudinal state vector $\boldsymbol{x}_{r}$ is then augmented with this integrated error state and used for full-state feedback. While augmenting the system with an integrator eliminates the static error of the incremental normal load factor output, it produces undesired integrator dynamics. This includes a very long and normally unwanted settling time due to its dominance over the phugoid mode. Pole-zero cancellations, a technique achieved with a feed-forward gain $K_{F F_{D Q}}$ from the reference input to the plant input before full-state feedback, is used to remove the integrator dynamics. 


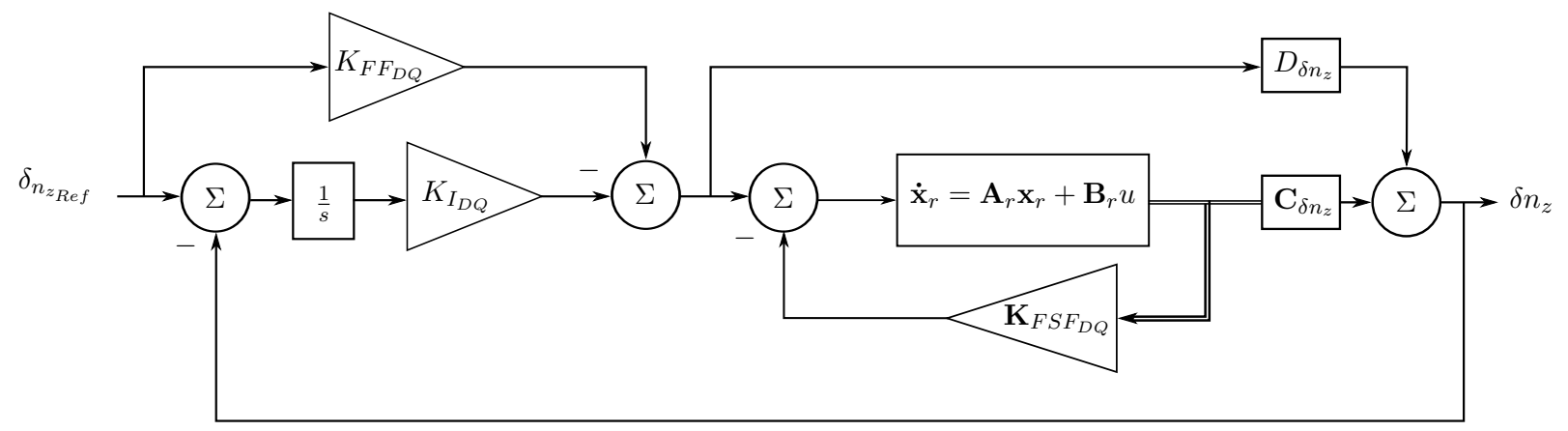

Figure 5.11: DQ law control architecture.

For the purposes of the controller design, it will be assumed that an estimator which estimates the reduced-order state vector from the available sensor measurements represented by the output vector can be implemented. Typically a pitch rate gyro and a vertical accelerometer are used to make these measurements.

The integrator dynamics can be written as,

$$
\begin{aligned}
\dot{x}_{I_{D Q}} & =\delta_{n_{z_{R e f}}}-\delta_{n_{z}} \\
& =\delta_{n_{z_{R e f}}}-\left(\mathbf{C}_{\delta_{n_{z}}} \boldsymbol{x}_{r}+D_{\delta_{n_{z}}} \delta_{e}\right)
\end{aligned}
$$

where

$$
x_{I_{D Q}}=\int_{0}^{t}\left(\delta_{n_{z_{R e f}}}-\delta_{n_{z}}\right) d t
$$

The state space model of the reduced-order longitudinal dynamics augmented with the integrator state is then given by,

$$
\left[\begin{array}{c}
\dot{\boldsymbol{x}}_{r} \\
\dot{x}_{I_{D Q}}
\end{array}\right]=\left[\begin{array}{cc}
\mathbf{A}_{r} & \mathbf{0}_{2 \times 1} \\
-\mathbf{C}_{\delta_{n_{z}}} & 0
\end{array}\right]\left[\begin{array}{c}
\boldsymbol{x}_{r} \\
x_{I_{D Q}}
\end{array}\right]+\left[\begin{array}{c}
\mathbf{B}_{r_{\delta_{e}}} \\
-D_{\delta_{n_{z}}}
\end{array}\right] \delta_{e}+\left[\begin{array}{c}
\mathbf{0}_{2 \times 1} \\
1
\end{array}\right] \delta_{n_{z_{R e f}}}
$$

The control law is derived as follows,

$$
\begin{aligned}
u=\delta_{e} & =-\mathbf{K}_{D Q}\left[\begin{array}{c}
\boldsymbol{x}_{r} \\
x_{I_{D Q}}
\end{array}\right]+K_{F F_{D Q}} \delta_{n_{z_{R e f}}} \\
& =-\left[\begin{array}{ll}
\mathbf{K}_{F S F_{D Q}} & K_{I_{D Q}}
\end{array}\right]\left[\begin{array}{c}
\boldsymbol{x}_{r} \\
x_{I_{D Q}}
\end{array}\right]+K_{F F_{D Q}} \delta_{n_{z_{R e f}}}
\end{aligned}
$$

where $\mathbf{K}_{F S F_{D Q}}=\left[\begin{array}{ll}k_{\alpha} & k_{q}\end{array}\right]$.

The DQ control law gain $\mathbf{K}_{D Q}$ is calculated using a pole placement design technique. The feed-forward control gain $K_{F F_{D Q}}$ can be accurately calculated to eliminate the integrator dynamics. Since the integral gain $K_{i_{D Q}}$ as well as integrator pole will be known through the pole placement technique, the feed-forward gain is calculated as, 


$$
K_{F F_{D Q}}=\frac{K_{I_{D Q}}}{z_{i}}
$$

where $z_{i}$ is chosen to be at the integrator pole position.

\section{Specifications}

In the DQ law controller, angle of attack and pitch rate feedback can be used to specify the desired closed-loop short-period dynamics of the aircraft. Feedback is required to achieve engineering performance specifications over a range of flight conditions. These flight conditions should consider different loading conditions, different altitudes, Mach numbers and different aircraft separations in formation flight. The excitation from gusts and wake turbulence should also be considered. It is assumed though that the aircraft does not diverge far off from the desired cruise condition at straight and level flight. Feedback is thus only calculated to achieve engineering specifications for a straight and level flight cruise condition.

The approximate damping and natural frequency of the short-period mode for the unaugmented aircraft are reproduced here as (see Table 4.2),

$$
\begin{aligned}
\zeta_{s p} & =0.335 \\
w_{n_{s p}} & =0.967
\end{aligned}
$$

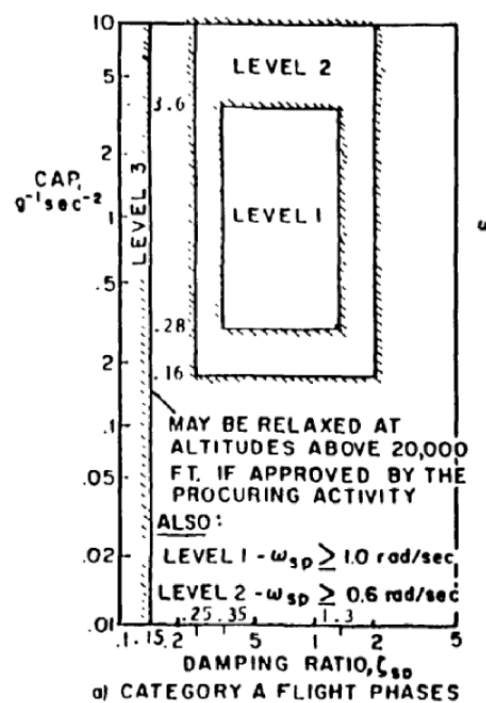

a) CATEGORY A FLIGHT PHASES

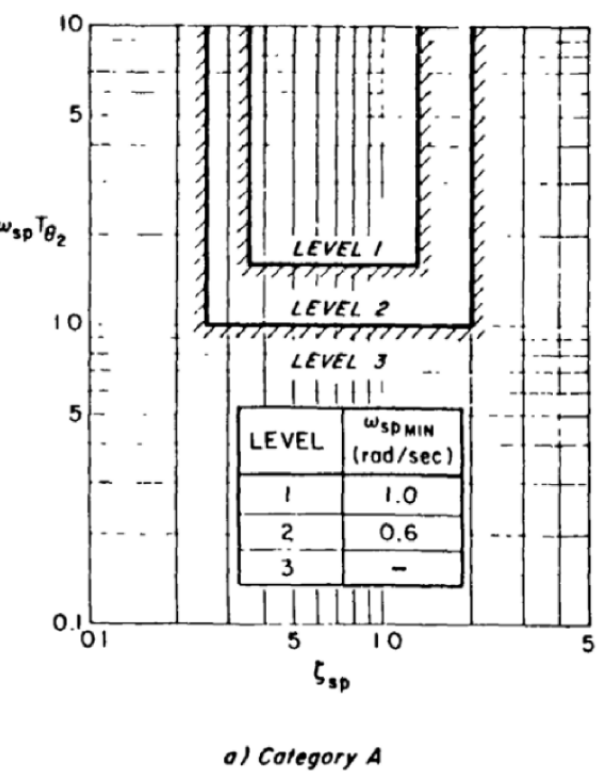

Figure 5.12: Short-period dynamic requirements for Category A flight phases of MIL-STD$1797 \mathrm{~A}$.

Figure 5.12 shows the short-period dynamic requirements for Category A flight phases of MILSTD-1797A. Using these requirements as a guide, an ideal well-damped value for the short-period damping ratio $\zeta_{s p}$ is chosen as, 


$$
\zeta_{s p}=0.9
$$

and for Level 1 flight, it is required that the natural frequency $w_{s p} \geq 1 \mathrm{rad} / \mathrm{s}$. The natural frequency is chosen here as,

$$
w_{s p}=1 \mathrm{rad} / \mathrm{s}
$$

\section{Results}

Figure 5.13 shows the closed-loop unit step response for the incremental load factor as well as the pole placement results. The response exhibits a zero steady-state error. The feed-forward gain from the pole-zero cancellation technique improved the speed of the response by cancelling the integrator dynamics. Overall, the response is well-damped with a settling time of approximately 5 seconds. This translates to a natural frequency of approximately $1 \mathrm{rad} / \mathrm{s}$, as was desired.

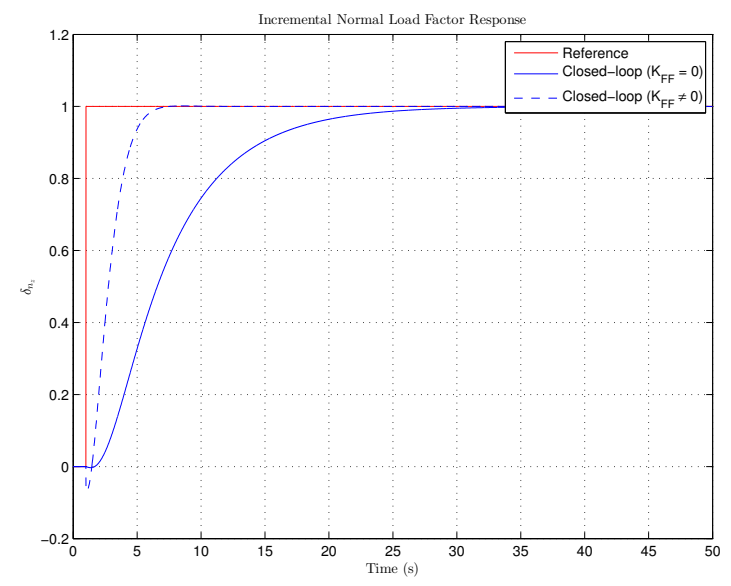

(a) Closed-loop step response

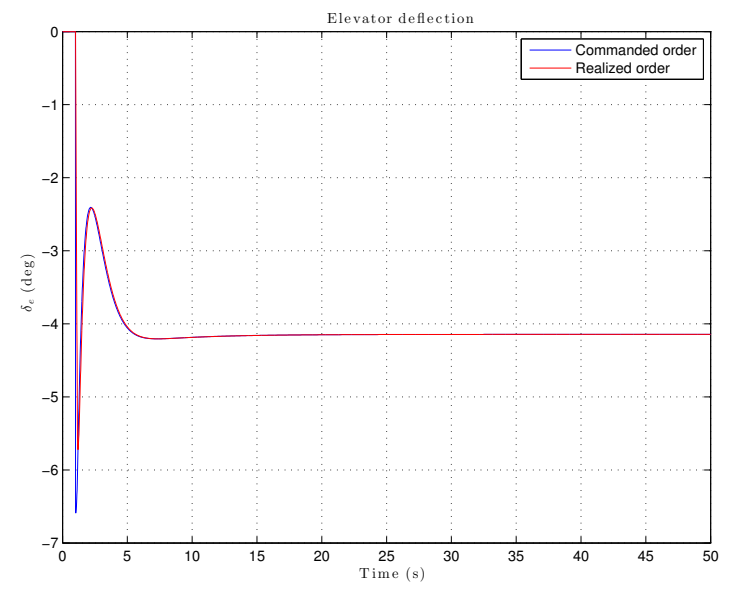

(b) Actuator response

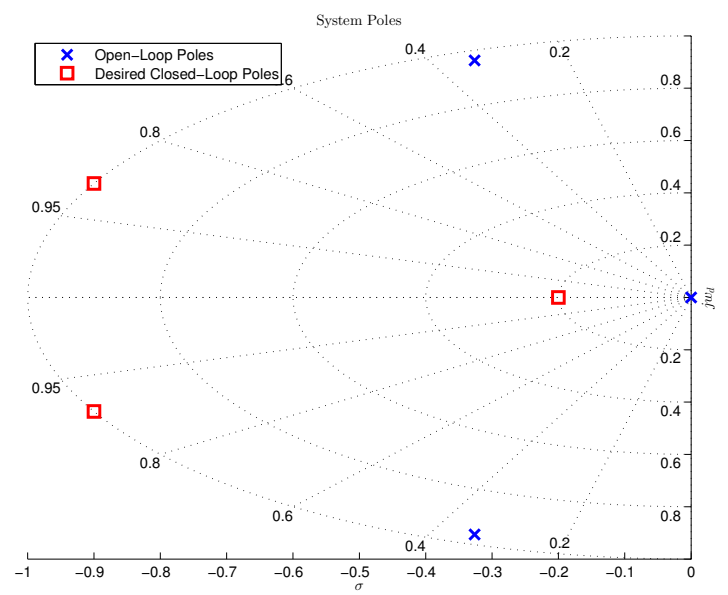

(c) Pole-placement

Figure 5.13: Results of the DQ law controller showing the closed-loop unit step response for the incremental load factor, the elevator deflection and the pole placement results. 


\section{Closed-Loop Model}

Once an acceptable gain matrix $\mathbf{K}_{D Q}$ and feed-forward control gain $K_{F F_{D Q}}$ have been found, the closed-loop system that encapsulates the full-order longitudinal dynamics with the designed DQ law controller can be expressed in state space form as,

$$
\begin{aligned}
\dot{\boldsymbol{x}}_{D Q} & =\mathbf{A}_{D Q} \boldsymbol{x}_{D Q}+\mathbf{B}_{D Q} \boldsymbol{u} \\
{\left[\begin{array}{c}
\dot{\boldsymbol{x}} \\
\dot{x}_{I_{D Q}}
\end{array}\right] } & =\left[\overline{\mathbf{A}}-\overline{\mathbf{B}} \mathbf{K}_{D Q}\right]\left[\begin{array}{c}
\boldsymbol{x} \\
x_{I_{D Q}}
\end{array}\right]+\left[\begin{array}{cc}
\mathbf{B}_{\delta_{e}} K_{F F_{D Q}} & \mathbf{B}_{\Delta T} \\
-D_{\delta n_{z}} K_{F F_{D Q}}+1 & 0
\end{array}\right]\left[\begin{array}{c}
\delta_{n_{z_{R e f}}} \\
\Delta T
\end{array}\right]
\end{aligned}
$$

where,

$$
\begin{aligned}
\overline{\mathbf{A}} & =\left[\begin{array}{cc}
\mathbf{A} & \mathbf{0}_{4 \times 1} \\
-\mathbf{C}_{\delta_{n_{z}}} & 0
\end{array}\right] \\
\overline{\mathbf{B}} & =\left[\begin{array}{c}
\mathbf{B}_{\delta_{e}} \\
-D_{\delta n_{z}}
\end{array}\right] \\
\mathbf{K}_{D Q} & =-\left[\begin{array}{lllll}
0 & k_{\alpha} & k_{q} & 0 & K_{I_{D Q}}
\end{array}\right] \\
K_{F F_{D Q}} & =\frac{K_{I_{D Q}}}{z_{i}}
\end{aligned}
$$

This closed-loop model will serve as the open-loop plant for the next controller. Figure 5.14 illustrates the new closed-loop poles after full-state feedback with integral control. Note that the phugoid mode poles have moved from a stable pole pair to an unstable real pole in the right half plane. This was not accounted for in this design, since the DQ law was concerned with the short-period stability of the aircraft, whereas the next feedback loop will rectify this stability by moving it back into the left half plane.

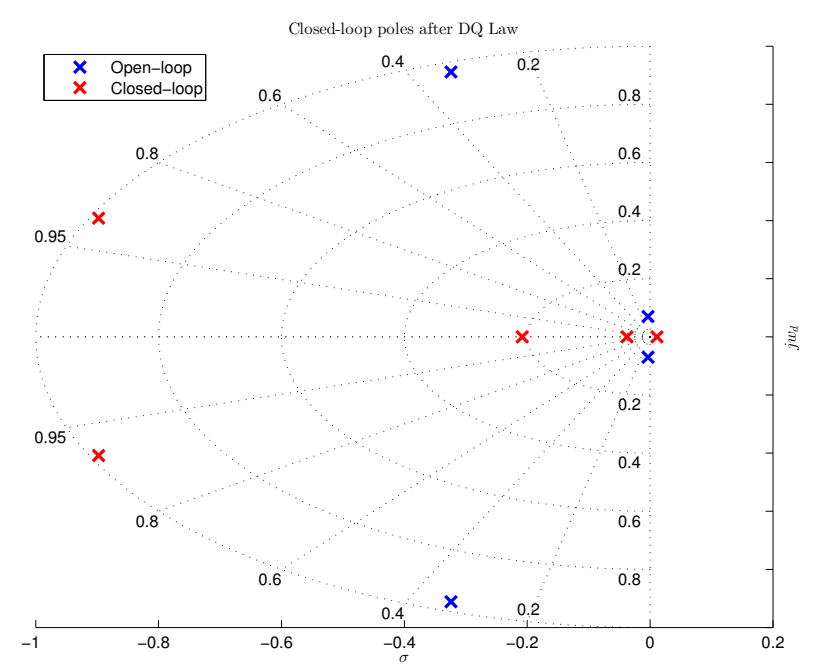

Figure 5.14: Closed-loop poles after adding the DQ law controller. 


\subsubsection{Altitude-Hold Guidance: FPA or CR Controller}

Following the DQ law inner-loop controller, the flight path angle (FPA) or climb rate (CR) controller of the altitude-hold guidance law is to be designed. It will be shown in the design process that the FPA and CR controllers are designed in the same manner, with the final gain only changing by a constant factor: the airspeed of the aircraft.

\section{Design}

The control architecture of the FPA controller is shown in the block diagram of Figure 5.15 . The plant is the DQ law with the longitudinal dynamics of the aircraft in state space form represented by Equation (5.31),

$$
\begin{aligned}
\dot{\boldsymbol{x}}_{D Q} & =\mathbf{A}_{D Q} \boldsymbol{x}_{D Q}+\mathbf{B}_{D Q} \boldsymbol{u} \\
y & =\gamma=\mathbf{C}_{\gamma} \boldsymbol{x}_{D Q}
\end{aligned}
$$

where,

$$
\boldsymbol{u}=\left[\begin{array}{c}
\delta_{n_{z_{R e f}}} \\
\Delta T
\end{array}\right]
$$

and the output matrix,

$$
\mathbf{C}_{\gamma}=\left[\begin{array}{lllll}
0 & -1 & 0 & 1 & 0
\end{array}\right]
$$

extracts the flight path angle from the DQ law state vector $\boldsymbol{x}_{D Q}$.

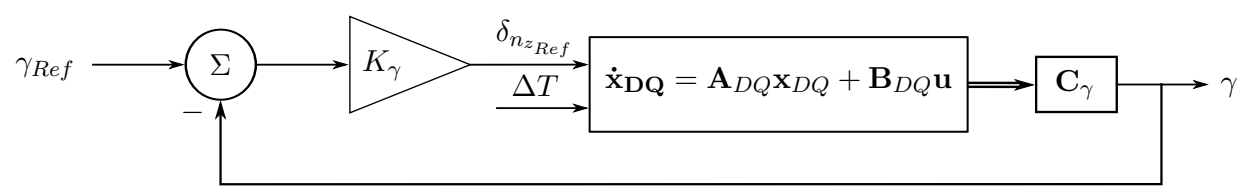

Figure 5.15: Flight path angle controller architecture.

The FPA controller is a classic proportional controller that generates an incremental normal load factor $\delta_{n_{z_{R e f}}}$ command proportional to the FPA error. The design consists of determining an appropriate value for the proportional feedback gain $K_{\gamma}$. If the design of a climb rate controller is desired, the plant will be the same as the flight path angle controller, except that the output matrix will extract the climb rate from the DQ law state vector $\boldsymbol{x}_{D Q}$ with,

$$
\mathbf{C}_{\dot{h}}=\left[\begin{array}{lllll}
0 & -\bar{V}_{T} & 0 & \bar{V}_{T} & 0
\end{array}\right]
$$

Continuing with the FPA controller design, the transfer function from the reference input $\delta_{n_{z_{R e f}}}$ to the FPA output $\gamma$ is obtained from the state space model through

$$
\frac{\gamma(s)}{\delta_{n_{z_{R e f}}}}=\mathbf{C}_{\gamma}\left(s I-\mathbf{A}_{D Q}\right)^{-1} \mathbf{B}_{\delta_{n_{z e f}}}
$$


where $\mathbf{B}_{\delta_{n_{z}} f}$ is the first column of $\mathbf{B}_{D Q}$. Figure 5.16 shows the root locus of the FPA controller with respect to the feedback gain $K_{\gamma}$ when the full-order plant as in Equation (5.33) is used.

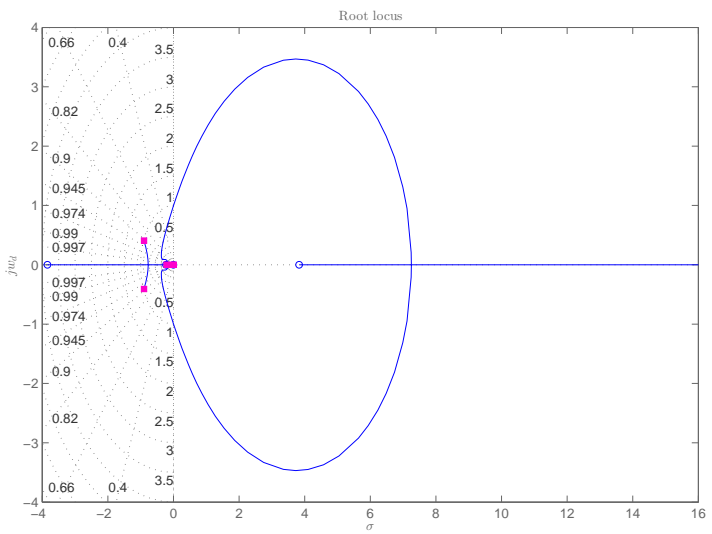

(a) Full view

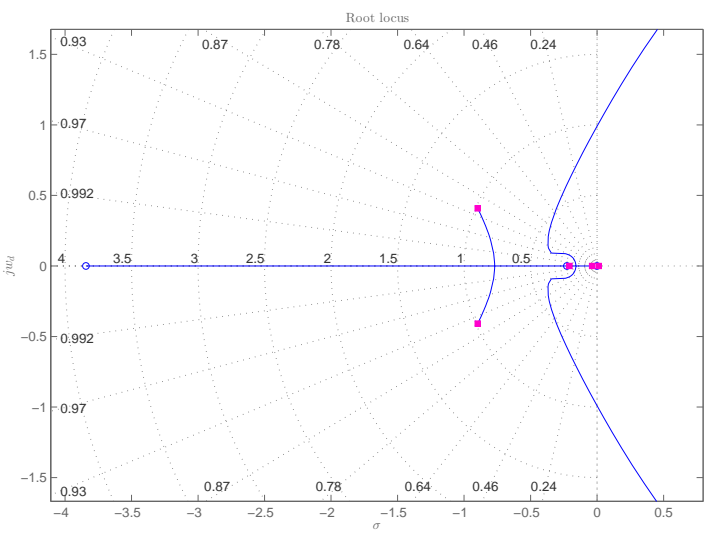

(b) Zoomed-in view

Figure 5.16: Root locus of full-order FPA controller for an arbitrary proportional feedback gain $K_{\gamma}$.

When the DQ law closed-loop model was discussed in the previous section, it was illustrated in Figure 5.14 that there exists an unstable real pole in the right half plane. This unstable pole makes it hard to design for the dominant response of the FPA controller that is desired. So, although this pole is important, it was decided to use a reduced-order model to design the FPA controller. The designed controller gain is evaluated in the full-order model to ensure the full-order system has a stable response.

Considering that the incremental normal load factor response to a commanded incremental normal load factor $\delta_{n_{z_{R e f}}}$ exhibits a dominant second-order response, the FPA rate is simply

$$
\dot{\gamma}=\frac{\delta_{n_{z}} g}{\bar{V}_{T}}
$$

and the FPA is obtained by integration of the FPA rate. This reduced-order FPA controller architecture is illustrated in Figure 5.17, and the root locus with respect to the feedback gain is illustrated in Figure 5.18, Notice that if a climb rate controller design is preferred, the vertical acceleration is approximated as,

$$
\ddot{h} \approx-a_{z_{B}}=\delta_{n_{z}} g
$$

which can be related to the FPA rate by the airspeed,

$$
\ddot{h}=\dot{\gamma} \bar{V}_{T}
$$




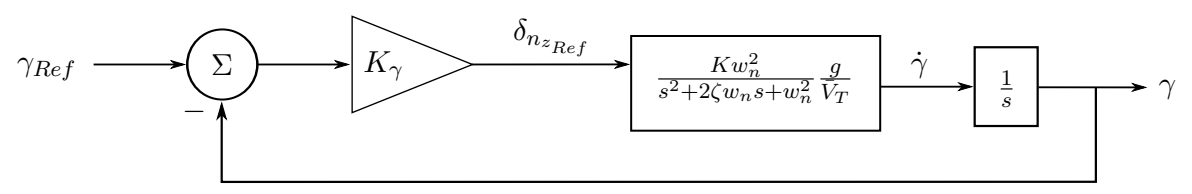

Figure 5.17: Reduced-order flight path angle controller architecture.

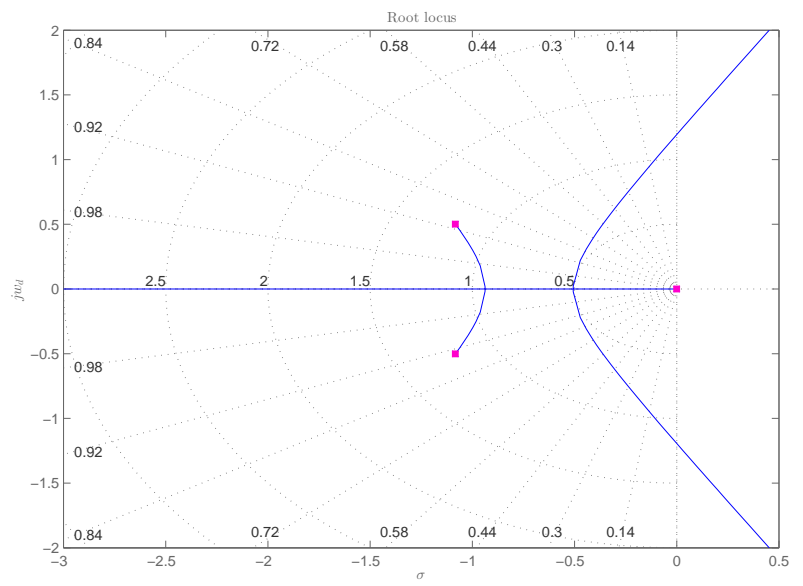

Figure 5.18: Root locus of reduced-order FPA controller for an arbitrary $K_{\gamma}$.

\section{Specifications}

The specifications for the FPA controller were chosen by using the A330 Simulink model in the same flight condition and evaluating the FPA response, as illustrated in Figure 5.19. Using the unit step response to an FPA angle change in the non-linear A330 model as a guideline, the following specifications were selected: the overshoot must be less than $5 \%$, and a $2 \%$ settling time of approximately 15 seconds is desired.

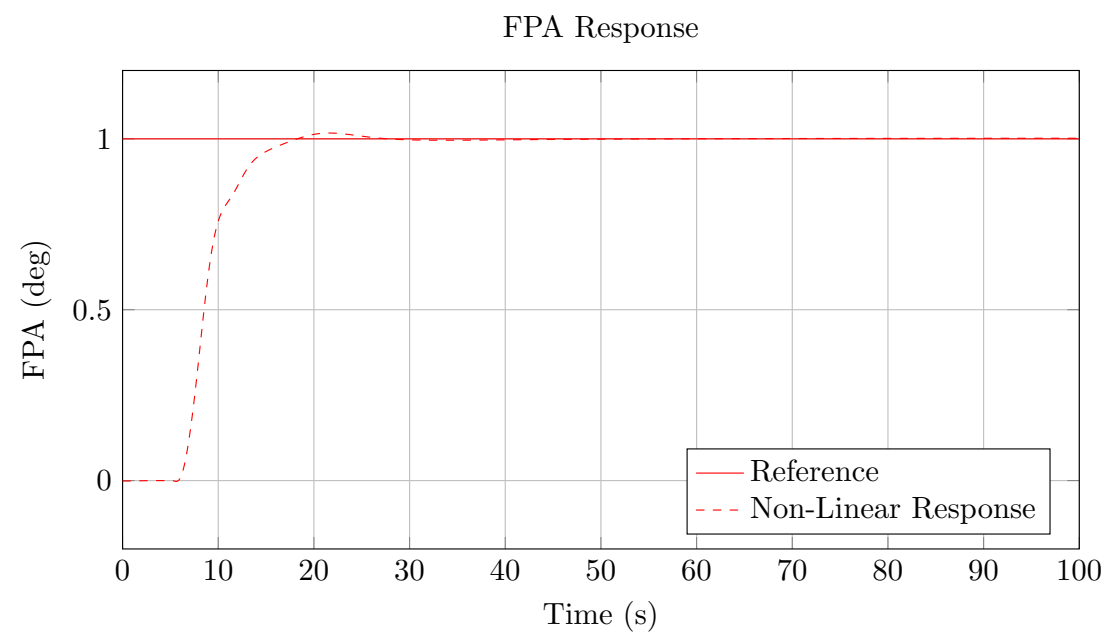

Figure 5.19: Unit step response of the non-linear FPA controller in the A330 aircraft model. 


\section{Results}

Figure 5.20 shows the closed-loop step response of the reduced-order FPA controller, as well as the root locus of the reduced-order FPA controller for a chosen gain $K_{\gamma}$ that reasonably achieves the desired specifications. The response exhibits a zero steady-state error with an overshoot of approximately $10 \%$ - slightly more than was desired. The $2 \%$ settling time was just over 13 seconds, but remained less than 15 seconds.

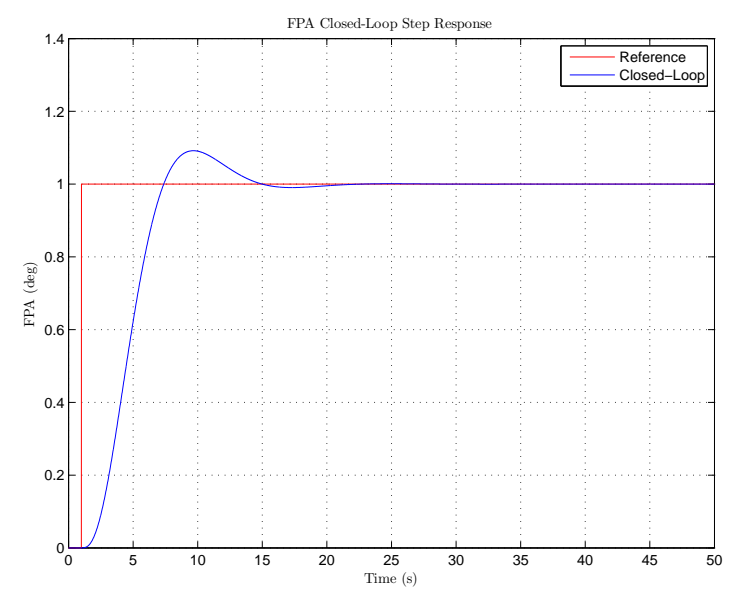

(a) Closed-loop step response

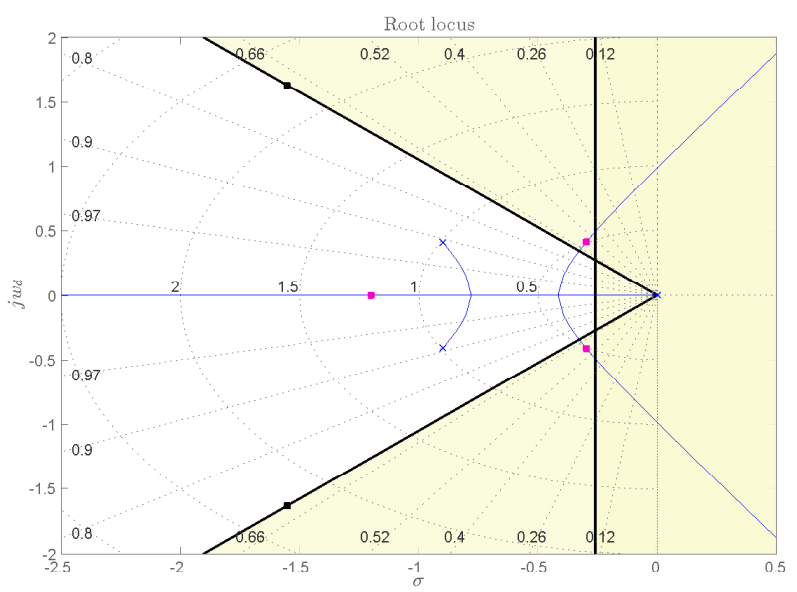

(b) Poles after proportional feedback

Figure 5.20: Closed-loop step response of the reduced-order FPA controller as well as the root locus of the reduced-order FPA controller for a chosen gain $K_{\gamma}$ that reasonably achieves the desired specifications.

The design of the reduced-order controller was verified with the full-order architecture. The closed-loop step response of the full-order FPA controller and the root locus after proportional feedback can be seen in Figure 5.21. The response of the incremental load factor and the aircraft actuator response, the elevator deflection, is also shown. 


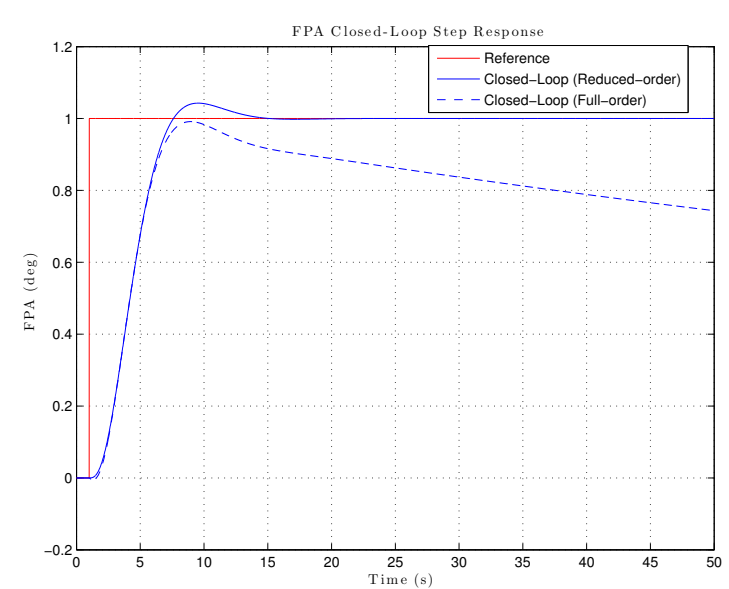

(a) Closed-loop step response

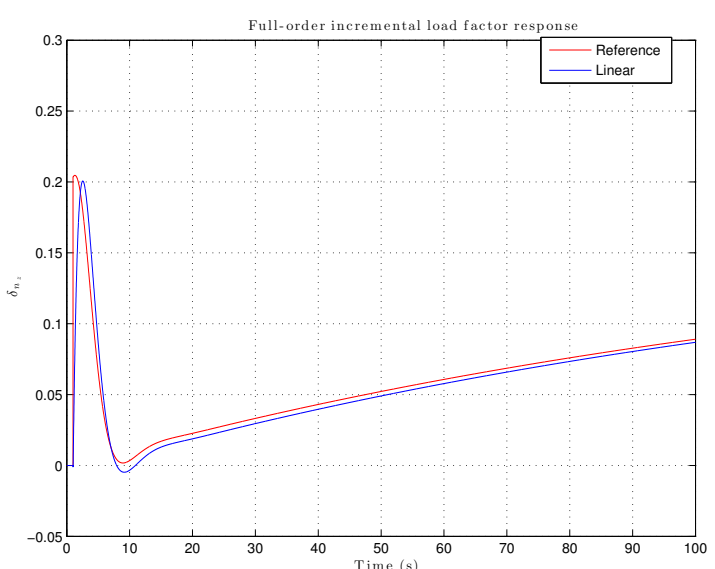

(c) Incremental load factor response

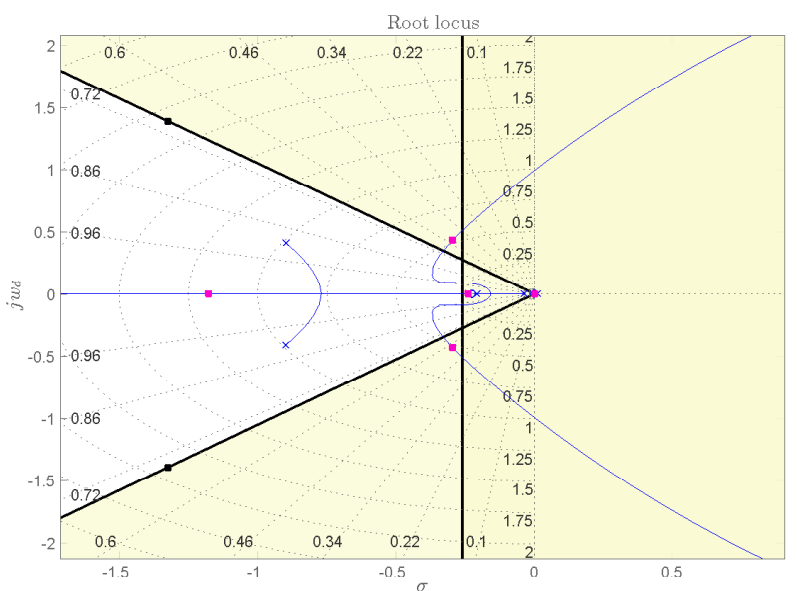

(b) Poles after proportional feedback

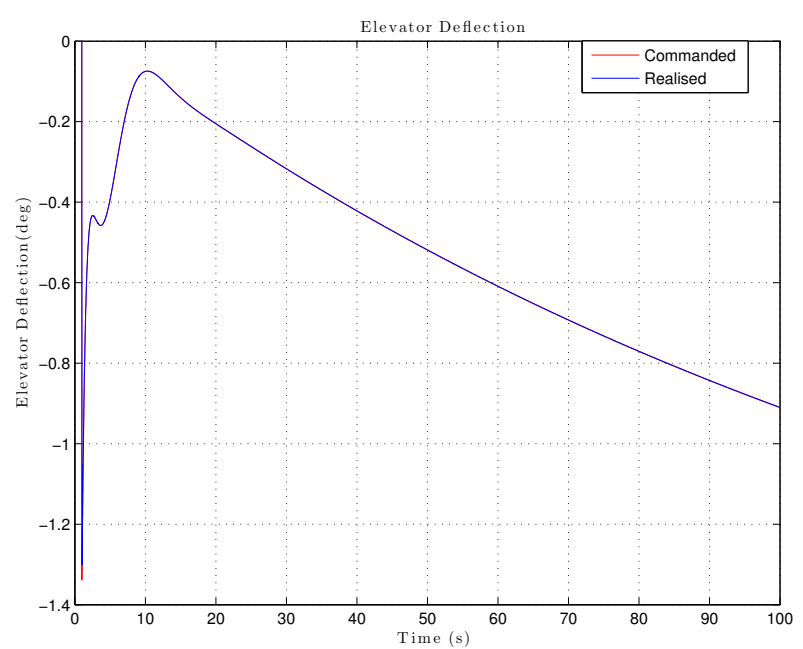

(d) Elevator deflection

Figure 5.21: The closed-loop step response of the full-order FPA controller and the root locus after proportional feedback. The incremental load factor and elevator response is also shown.

\section{Closed-Loop Model}

Once an acceptable proportional feedback gain $K_{\gamma}$ has been found, the closed-loop system that encapsulates the full-order longitudinal dynamics, the DQ law and the designed FPA controller can be expressed in state space form as,

$$
\begin{aligned}
& \dot{\boldsymbol{x}}_{F P A}=\mathbf{A}_{F P A} \boldsymbol{x}_{F P A}+\mathbf{B}_{F P A} \boldsymbol{u}
\end{aligned}
$$

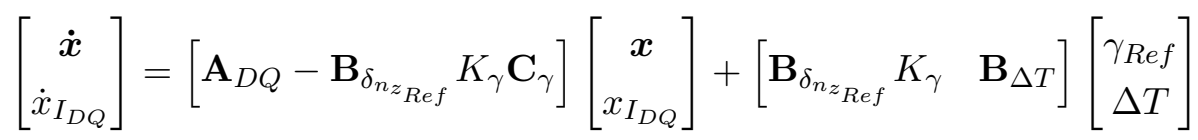

This closed-loop model will serve as the open-loop plant for the next controller. Figure 5.22 illustrates the new closed-loop poles after proportional FPA feedback. Note that the unstable real pole in the right half plane after the DQ law controller design has now moved to the stable left half plane. 


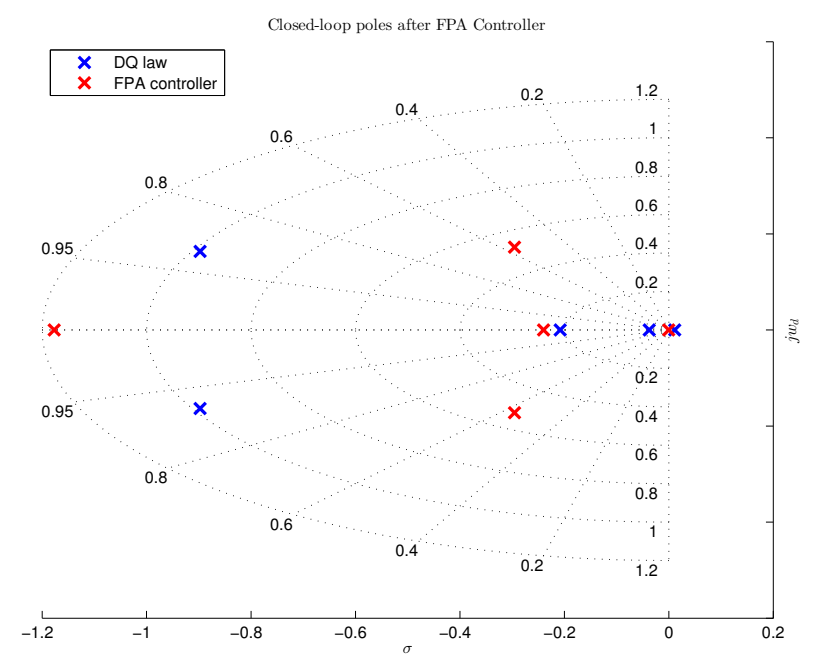

Figure 5.22: Closed-loop poles after adding the FPA controller.

\subsubsection{Autothrust: An Airspeed Controller}

With the DQ law inner-loop controller and an FPA or CR guidance controller in place, the aircraft requires an airspeed controller to maintain a reference airspeed during a longitudinal manoeuvre. Figure 5.23 shows the step response results for the full-order longitudinal model with an inner-loop DQ law controller and an FPA guidance controller. The altitude increases as expected, and the FPA controller initially tries to track the reference angle, as shown in Figure $5.23 \mathrm{~b}$. However, without an airspeed controller, the airspeed continuously decreases, as seen in Figure 5.23c and the FPA grows larger while the incremental normal load factor continues to decrease until the aircraft stalls. In this section, an autothrust controller is designed to maintain a desired airspeed such that the aircraft does not enter a stall condition and continues to hold the commanded flight path angle.

\section{Design}

The control architecture of the autothrust controller is shown in the block diagram of Figure 5.24. The plant is the FPA controller with the DQ law and the longitudinal dynamics of the aircraft in state space form, represented by Equation (5.41),

$$
\begin{aligned}
\dot{\boldsymbol{x}}_{F P A} & =\mathbf{A}_{F P A} \boldsymbol{x}_{F P A}+\mathbf{B}_{F P A} \boldsymbol{u} \\
y & =\bar{v}=\mathbf{C}_{\bar{v}} \boldsymbol{x}_{F P A}
\end{aligned}
$$

where,

$$
\boldsymbol{u}=\left[\begin{array}{c}
\gamma_{R e f} \\
\Delta T
\end{array}\right]
$$

and the output matrix,

$$
\mathbf{C}_{\bar{v}}=\left[\begin{array}{lllll}
1 & 0 & 0 & 0 & 0
\end{array}\right]
$$




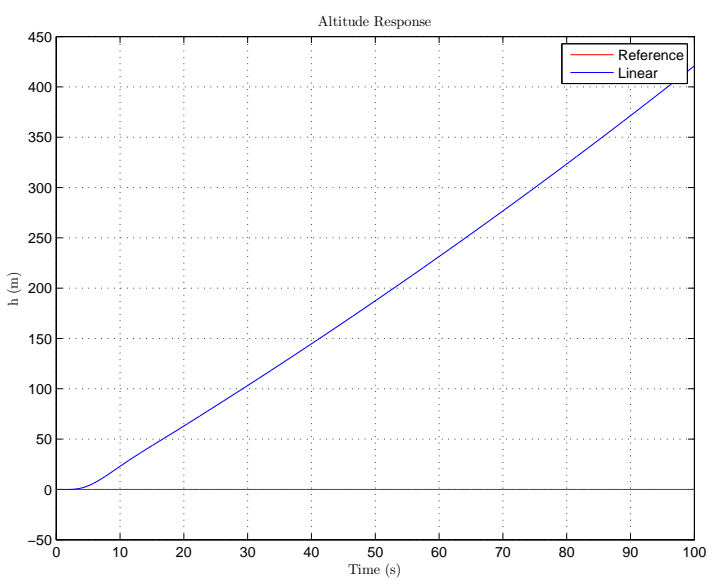

(a) Altitude response

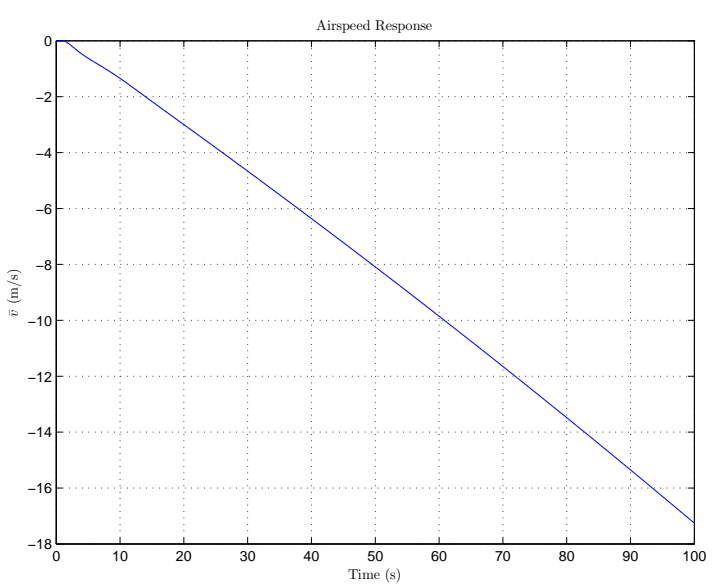

(c) Airspeed response

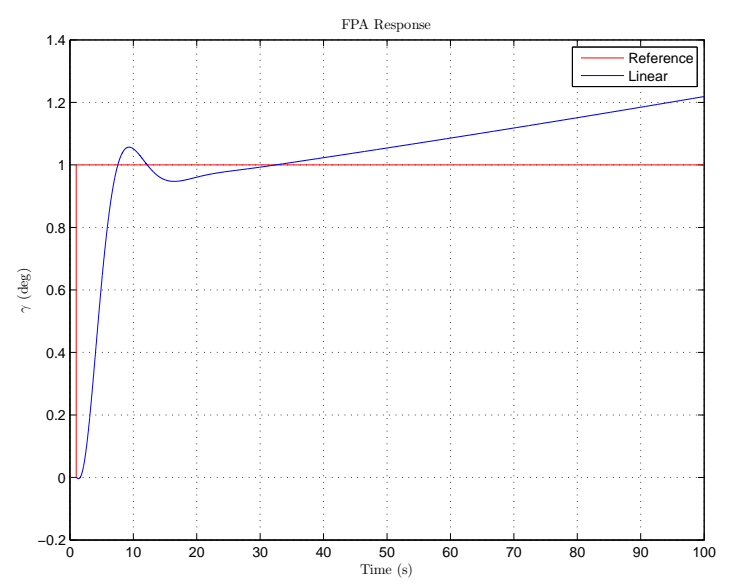

(b) FPA response

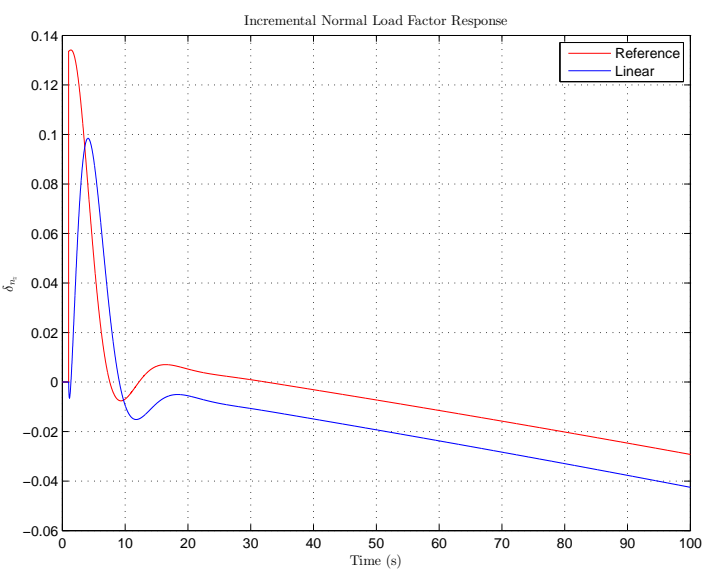

(d) Incremental Normal Load Factor response

Figure 5.23: Full-order longitudinal step response results for a $1^{\circ} \mathrm{FPA}$ command with the DQ law inner-loop controller and the FPA guidance controller.

extracts the airspeed from the FPA state vector $\boldsymbol{x}_{F P A}$. The autothrust controller is a classic PI controller that generates a thrust command $\Delta T$ proportional to the weighted sum of the airspeed error and the time integral of the airspeed error. The integral term in the PI controller makes the system type 1, which means that it should be able to follow a constant airspeed reference with zero error at steady state.

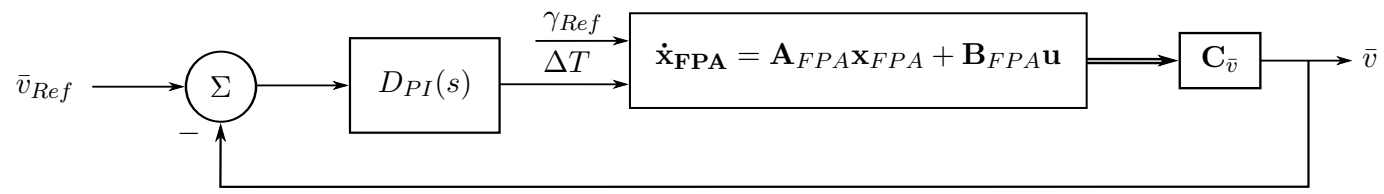

Figure 5.24: Autothrust controller architecture.

The transfer function from commanded thrust $\Delta T$ to the airspeed output $\bar{v}$ is obtained from the state space model through 


$$
\frac{\bar{v}(s)}{\Delta T(s)}=\mathbf{C}_{\bar{v}}\left(s I-\mathbf{A}_{F P A}\right)^{-1} \mathbf{B}_{\triangle T}
$$

where $\mathbf{B}_{\Delta T}$ is the second column of $\mathbf{B}_{F P A}$. Figure 5.25 shows the root locus of the autothrust controller with respect to an arbitrary feedback gain $K$.

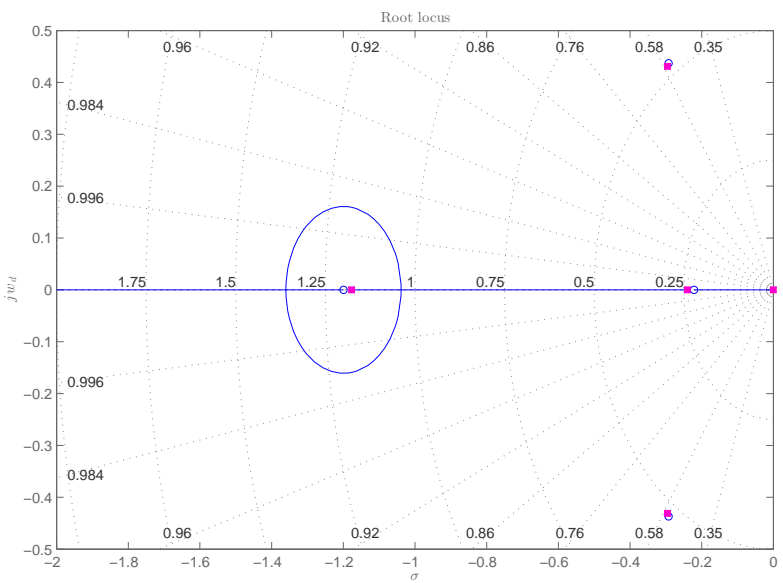

Figure 5.25: Root locus of autothrust controller with respect to an arbitrary feedback gain $K$.

\section{Specifications}

The specifications for the autothrust controller were chosen by using the A330 Simulink model at the same flight condition and evaluating the airspeed response. Different airspeed step responses were evaluated and are shown in Figure 5.26. Using the airspeed response of the A330 model as a guideline, the following specifications were selected: the airspeed should respond as a second order system with a $2 \%$ settling time of approximately 50s and a damping coefficient of 0.9 . Figure $5.27 \mathrm{a}$ shows the root locus for the PI controller that attempts to satisfy the desired specifications. The constraints are not fully achieved due to the slow mode pole added to the system after the PI design. The response is however considered acceptable for the current study.

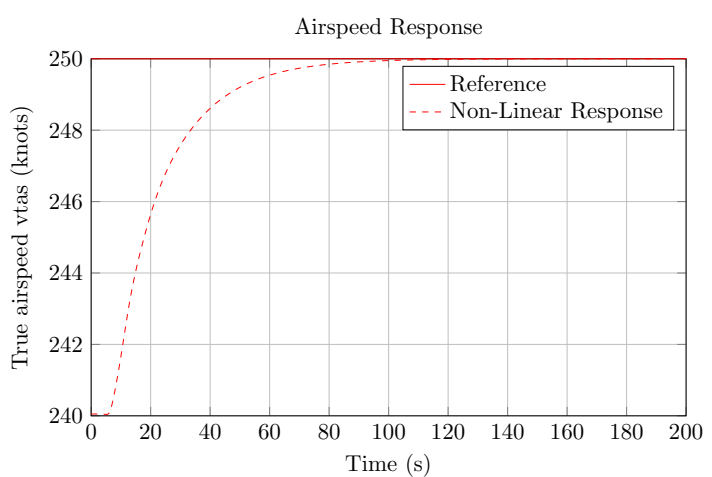

(a) 10 knots $(5 \mathrm{~m} / \mathrm{s})$ airspeed change

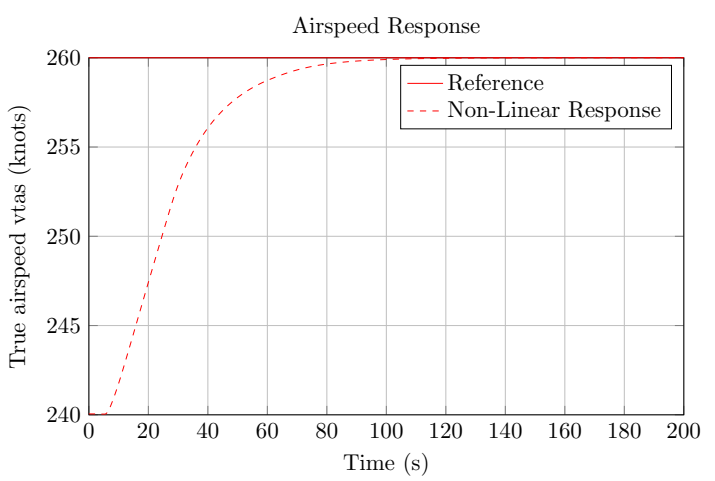

(b) 20 knots $(10 \mathrm{~m} / \mathrm{s})$ airspeed change

Figure 5.26: Evaluation of the A330 non-linear model response to commanded airspeeds. 


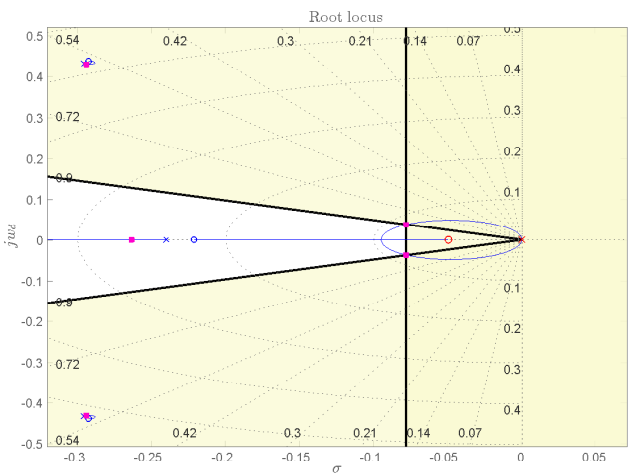

(a) Root locus plot

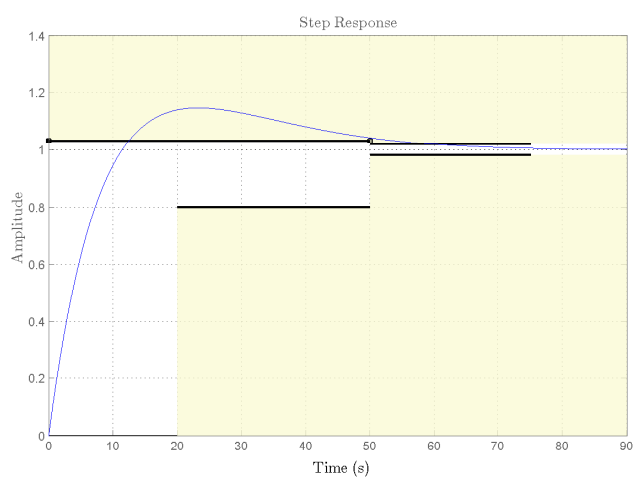

(b) Unit step response

Figure 5.27: Root locus of the autothrust controller for a designed proportional-integral controller that tries to meet the specifications. The step response is also shown.

\section{Results}

Figures $5.28 \mathrm{a}$ to $5.28 \mathrm{~d}$ show the closed-loop step response for a 5 and $10 \mathrm{~m} / \mathrm{s}$ airspeed step, as well as the corresponding control signal $\Delta T$ before and after actuator saturation. These results highlight two problems that arise when controlling a system with input saturation.

For a reference airspeed, the commanded control signal from the PI controller, indicated as a red line in Figures 5.28b and 5.28d, reaches a peak value far outside the range of the actuator. Over a short time interval, the controller will be operating in a non-linear region, where the control signal will have no effect on the system output, namely the airspeed. This condition is well known as integrator windup. The second problem that arises is illustrated in Figures $5.28 \mathrm{a}$ and $5.28 \mathrm{c}$ by the large overshoot. This is caused by the considerable delay before the PI controller output returns to within the actuator's range.

It is thus necessary to design a PI controller to account for the effect of actuator saturation to improve its performance, and by only allowing operation in the linear region. An anti-windup scheme, known as back-calculation, will be used to prevent integrator windup. Figures $5.28 \mathrm{e}$ to $5.28 \mathrm{~h}$ illustrate the results of simulating the autothrust controller with anti-windup activated. The control signal in Figures $5.28 \mathrm{f}$ and $5.28 \mathrm{~h}$ returns to the linear region and recovers from saturation faster than before anti-windup. The airspeed response also shows minimal overshoot and exhibits a dominant second-order response, settling in approximately 40 seconds for a $5 \mathrm{~m} / \mathrm{s}$ step and in approximately one minute for a $10 \mathrm{~m} / \mathrm{s}$ step in airspeed.

Figure 5.29 shows the actuator responses, the reference flight path angle and thrust command, for a commanded airspeed of $1 \mathrm{~m} / \mathrm{s}$. Clearly the actuators are well within its capabilities. There is however, a slight steady-state error in the flight path angle and the desired flight path angle. This is because the FPA controller was only a proportional controller. 


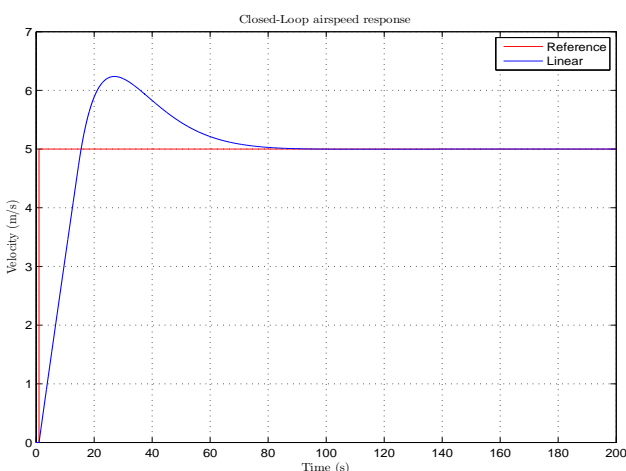

(a) $5 \mathrm{~m} / \mathrm{s}$ Airspeed step response with no antiwindup

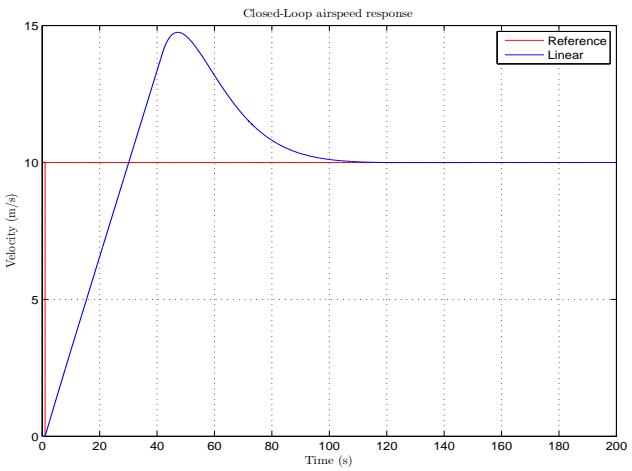

(c) $10 \mathrm{~m} / \mathrm{s}$ Airspeed step response with no anti-windup

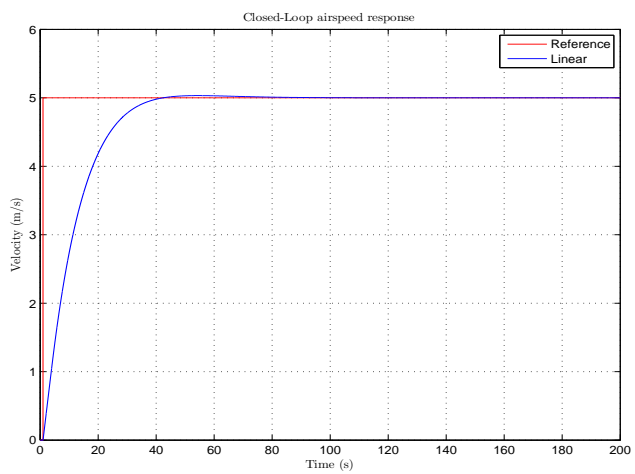

(e) $5 \mathrm{~m} / \mathrm{s}$ Airspeed step response with antiwindup

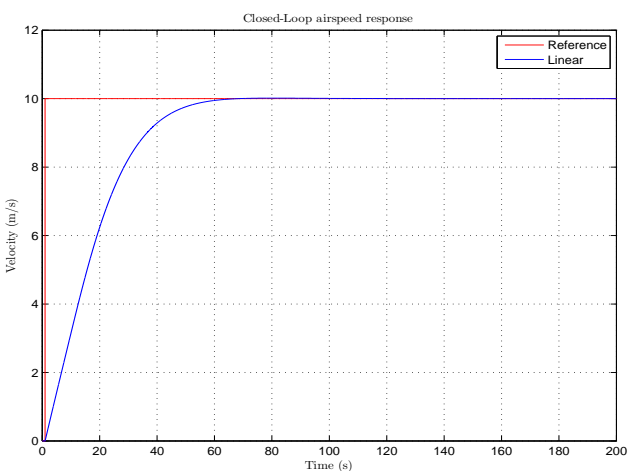

(g) $10 \mathrm{~m} / \mathrm{s}$ Airspeed step response with antiwindup

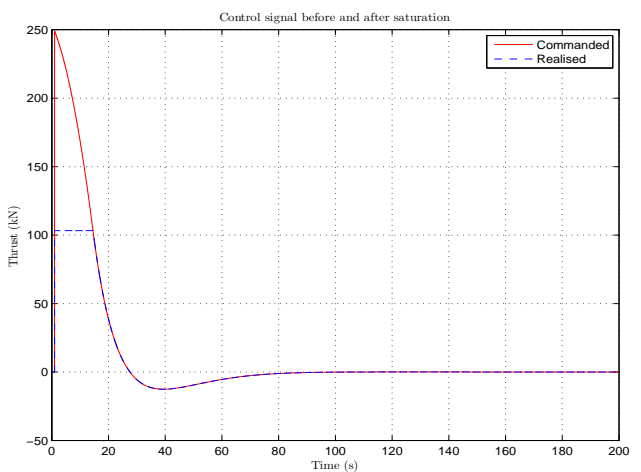

(b) Control signal after a $5 \mathrm{~m} / \mathrm{s}$ airspeed step with no anti-windup

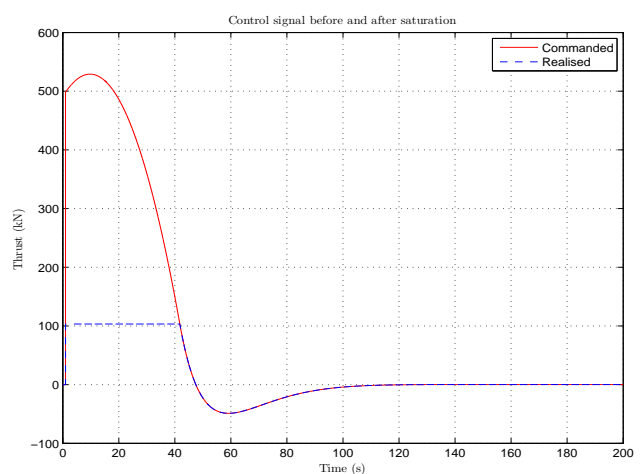

(d) Control signal after a $10 \mathrm{~m} / \mathrm{s}$ airspeed step with no anti-windup

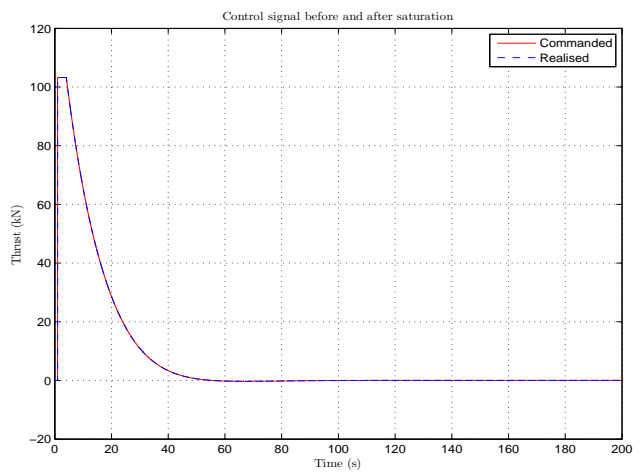

(f) Control signal after a $5 \mathrm{~m} / \mathrm{s}$ airspeed step with anti-windup

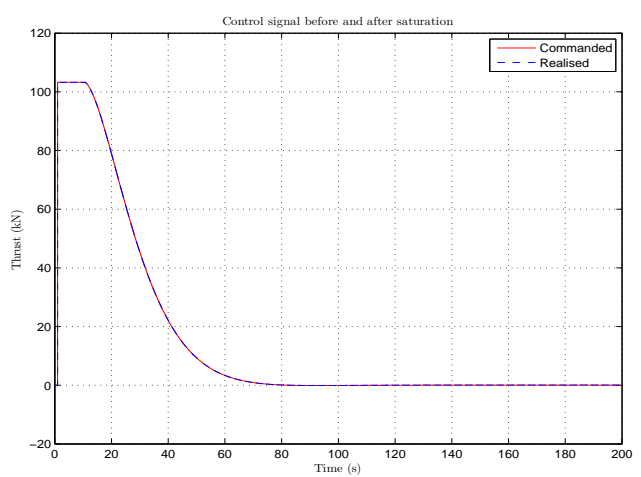

(h) Control signal after a $10 \mathrm{~m} / \mathrm{s}$ airspeed step with anti-windup

Figure 5.28: The closed-loop step response for a 5 and $10 \mathrm{~m} / \mathrm{s}$ airspeed step, as well as the corresponding control signal $\Delta T$ before and after actuator saturation for: (a)-(d) No anti-windup (e)-(h) Back-calculation anti-windup. 


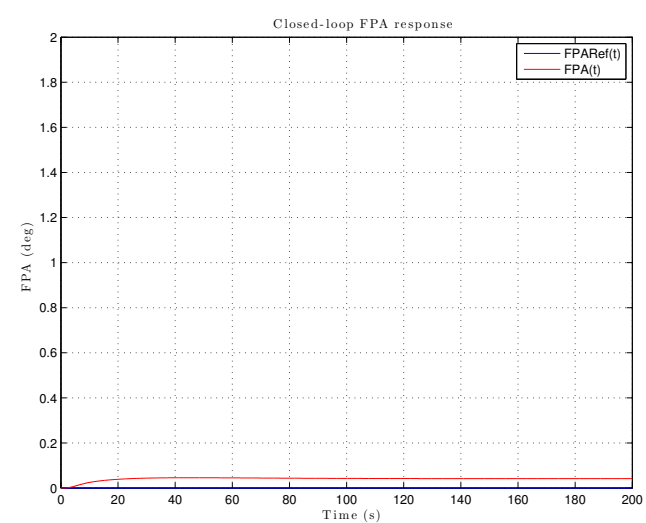

(a) FPA Response

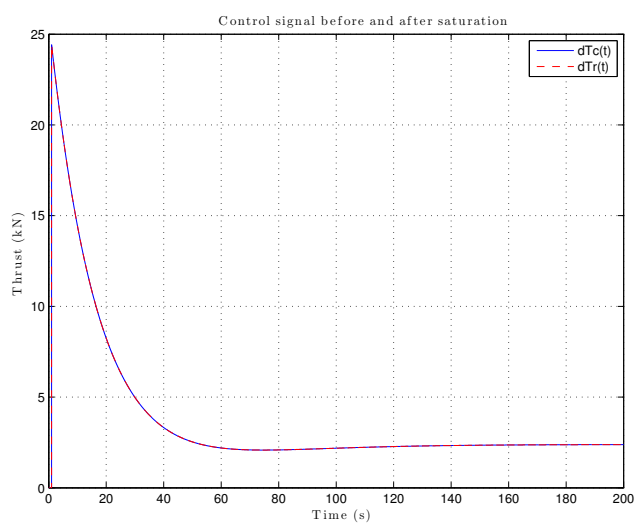

(b) Thrust Response

Figure 5.29: Actuator response to a commanded airspeed of $1 \mathrm{~m} / \mathrm{s}$.

\section{Closed-Loop Model}

The integrator dynamics can be written as,

$$
\dot{x}_{I_{A T}}=\bar{v}_{R e f}-\bar{v}
$$

where,

$$
x_{I_{A T}}=\int_{0}^{t}\left(\bar{v}_{R e f}-\bar{v}\right) d t
$$

The full-order FPA state vector $\boldsymbol{x}_{F P A}$ is augmented with the integrated error state $x_{I_{A T}}$ to give the augmented state space model,

$$
\left[\begin{array}{c}
\dot{\boldsymbol{x}}_{F P A} \\
\dot{x}_{I_{A T}}
\end{array}\right]=\left[\begin{array}{cc}
\boldsymbol{A}_{F P A} & \mathbf{0}_{5 \times 1} \\
-\mathbf{C}_{\bar{v}} & 0
\end{array}\right]\left[\begin{array}{c}
\boldsymbol{x}_{F P A} \\
x_{I_{A T}}
\end{array}\right]+\left[\begin{array}{cc}
\mathbf{B}_{\gamma_{R e f}} & \mathbf{B}_{\Delta T} \\
0 & 0
\end{array}\right]\left[\begin{array}{c}
\gamma_{R e f} \\
\Delta T
\end{array}\right]+\left[\begin{array}{c}
\mathbf{0}_{5 \times 1} \\
1
\end{array}\right] \bar{v}_{R e f}
$$

The control law is derived as follows,

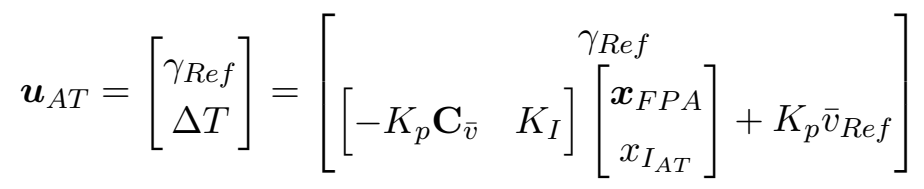

where $K_{p}$ and $K_{I}$ are the proportional and integral gains respectively, derived from the designed PI controller architecture $D_{P I}(s)$. Once an acceptable proportional and integral gain has been selected, the closed-loop state space model that encapsulates the longitudinal dynamics with the DQ law, the FPA controller and the autothrust controller can be formulated as follows,

$$
\begin{aligned}
\dot{\boldsymbol{x}}_{A T} & =\mathbf{A}_{A T} \boldsymbol{x}_{A T}+\mathbf{B}_{A T} \boldsymbol{u} \\
{\left[\begin{array}{c}
\dot{\boldsymbol{x}}_{F P A} \\
\dot{x}_{I_{A T}}
\end{array}\right] } & =\left[\begin{array}{cc}
\boldsymbol{A}_{F P A}-\boldsymbol{B}_{\Delta T} K_{p} \mathbf{C}_{\bar{v}} & \mathbf{B}_{\Delta T} K_{I} \\
-\mathbf{C}_{\bar{v}} & 0
\end{array}\right]\left[\begin{array}{c}
\boldsymbol{x}_{F P A} \\
x_{I_{A T}}
\end{array}\right]+\left[\begin{array}{cc}
\mathbf{B}_{\gamma_{R e f}} & \mathbf{B}_{\Delta T} K_{P} \\
0 & 1
\end{array}\right]\left[\begin{array}{c}
\gamma_{R e f} \\
\bar{v}_{R e f}
\end{array}\right]
\end{aligned}
$$


This closed-loop model will serve as the open-loop plant for the next controller. Figure 5.30 illustrates the new closed-loop poles after the PI autothrust controller, capable of maintaining a desired airspeed during level flight as well as recovering the airspeed when a longitudinal manoeuvre is executed.

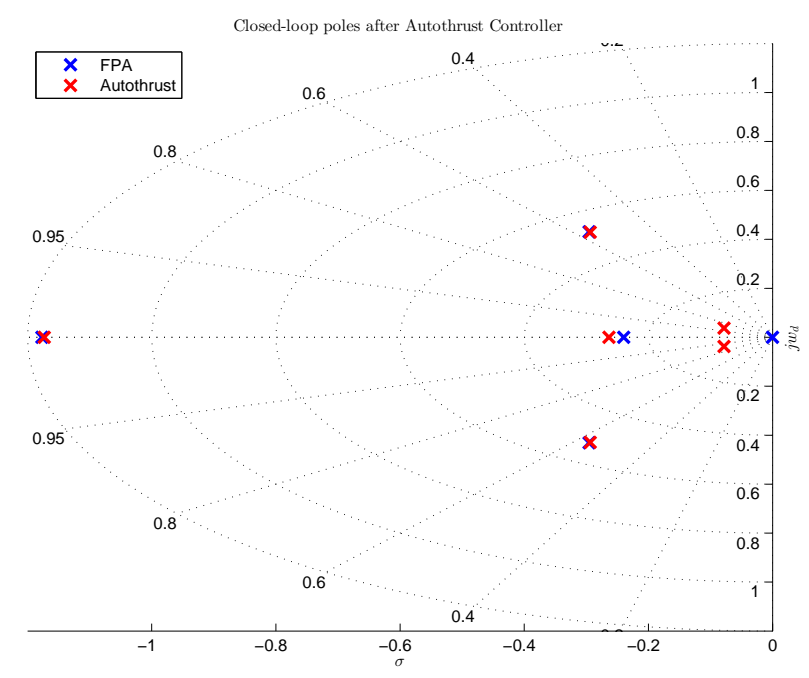

Figure 5.30: Closed-loop poles after adding the autothrust controller.

\subsubsection{Altitude-Hold Guidance: Altitude Controller}

The last longitudinal controller, an altitude controller, is now designed in this section, and forms the second part of the altitude-hold guidance strategy.

\section{Design}

The control architecture of the altitude controller is shown in the block diagram of Figure 5.31. The plant is the DQ law, FPA and autothrust controller with the longitudinal dynamics of the aircraft in state space form represented by Equation (5.50),

$$
\begin{aligned}
\dot{\boldsymbol{x}}_{A T} & =\boldsymbol{A}_{A T} \boldsymbol{x}_{A T}+\boldsymbol{B}_{A T} \boldsymbol{u} \\
y & =\dot{h}=\mathbf{C}_{\dot{h}} \boldsymbol{x}_{A T}
\end{aligned}
$$

where,

$$
\boldsymbol{u}=\left[\begin{array}{c}
\gamma_{R e f} \\
\bar{v}_{R e f}
\end{array}\right]
$$

and the output matrix,

$$
\mathbf{C}_{\dot{h}}=\left[\begin{array}{llllll}
0 & -\bar{V}_{T} & 0 & \bar{V}_{T} & 0 & 0
\end{array}\right]
$$

extracts the climb rate $\dot{h}$ from the autothrust state vector $\boldsymbol{x}_{A T}$ and feeds it to a natural integrator to become the altitude state $h$. The altitude is typically measured by a barometric pressure 
sensor or GPS. The altitude controller consists of a classic proportional controller that generates an FPA command $\gamma_{\text {Ref }}$ proportional to the altitude error.

The inverse of the DCM transformation matrix may be used to transform the velocity components in the body axes to the corresponding components in the inertial axes, as in Equation (3.10). The rate of change in height due to a perturbation in aircraft motion is then given by

$$
\dot{h}=-\dot{D}=U \sin \theta-V \cos \theta \sin \phi-W \cos \theta \cos \phi
$$

Through substitution of $(U, V, W)$ with the theory of Equation (4.1) and on the assumption that the perturbations are small, then $U_{T} \approx \bar{V}_{T}, W_{T} \approx 0$ and to a good approximation Equation (5.54) may be written,

$$
\dot{h}=\bar{V}_{T} \theta-w
$$

and using Equation 4.2a,

$$
\dot{h}=\bar{V}_{T}(\theta-\alpha)
$$

The natural integration from climb rate to altitude makes the system type 1, which means it should be able to follow a constant altitude reference with zero error at steady state. A saturation block is added to ensure that the controller does not command FPA signals that exceed the limitations of the aircraft, and that the aircraft maintains a constant FPA when large altitude step commands are issued.

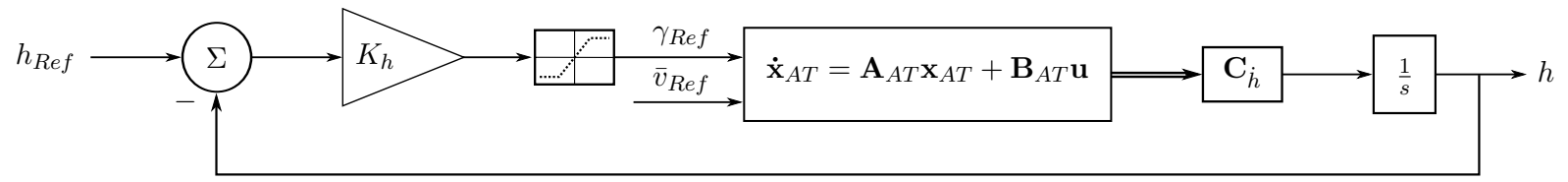

Figure 5.31: Altitude controller architecture.

The transfer function from the reference input $\gamma_{\text {Ref }}$ to the altitude output $h$ is obtained from the state space model through

$$
\frac{h(s)}{\gamma_{R e f}}=\frac{1}{s} \mathbf{C}_{\dot{h}}\left(s I-\mathbf{A}_{A T}\right)^{-1} \mathbf{B}_{\gamma_{R e f}}
$$

where $\mathbf{B}_{\gamma_{R e f}}$ is the first column of $\mathbf{B}_{A T}$. Figure 5.32 shows the root locus with respect to the altitude control gain. 


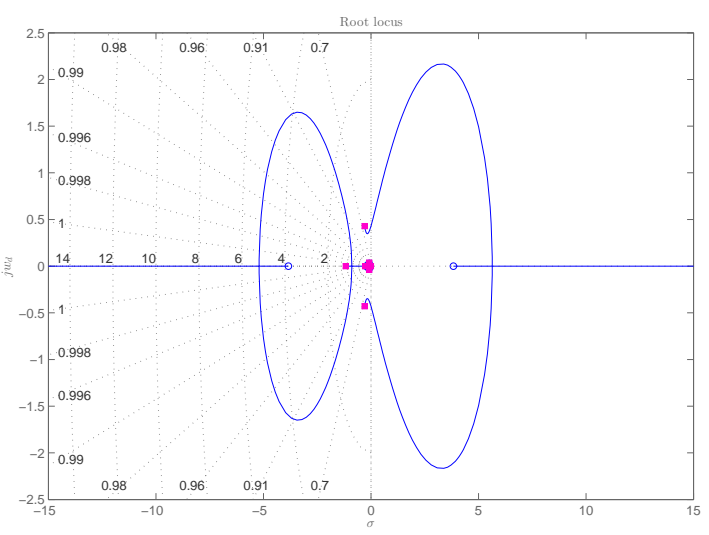

(a) Full view

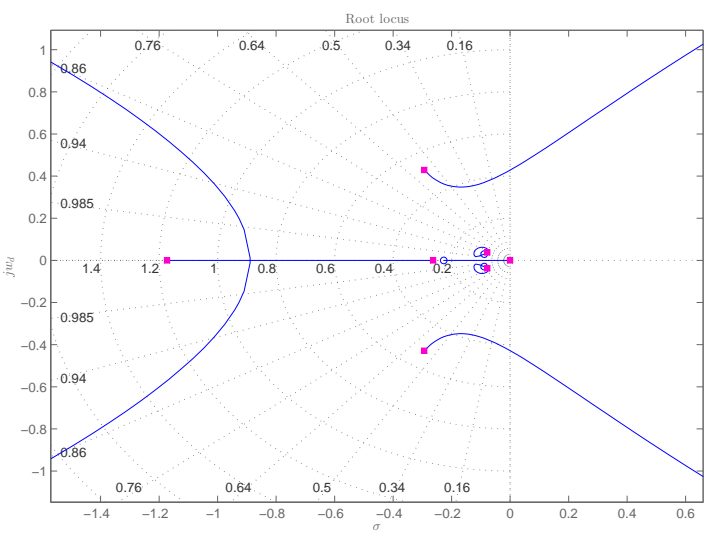

(b) Zoomed-in view

Figure 5.32: Root locus of altitude controller with respect to the altitude feedback gain $K_{h}$.

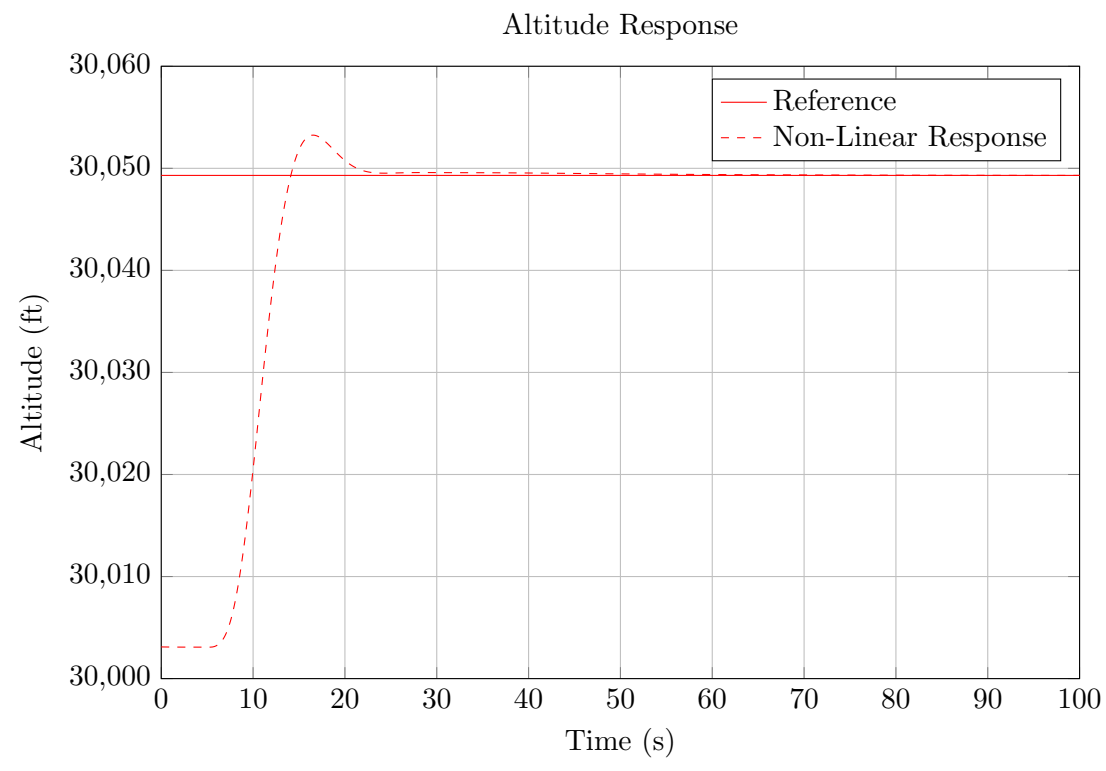

Figure 5.33: Altitude response of A330 non-linear model.

\section{Specifications}

Figure 5.33 shows the non-linear altitude response of the A330 aircraft model. Using the response as a guideline, the following specifications were selected: a second-order response with approximately $10-15 \%$ overshoot, peaking after approximately 10 seconds and settling with a $2 \%$ error bound after about 20 seconds. The FPA limits of the aircraft were chosen as $\pm 6^{\circ}$. Figure 5.34 shows the root locus and the step response that closely meet the desired specifications. 


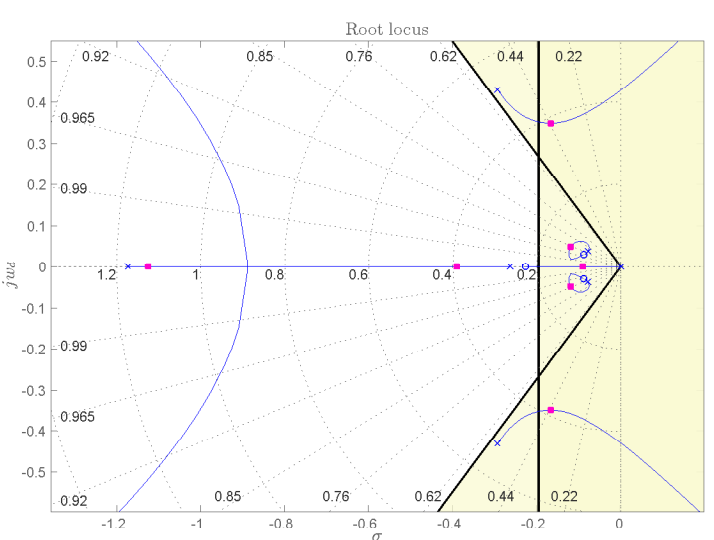

(a) Root locus

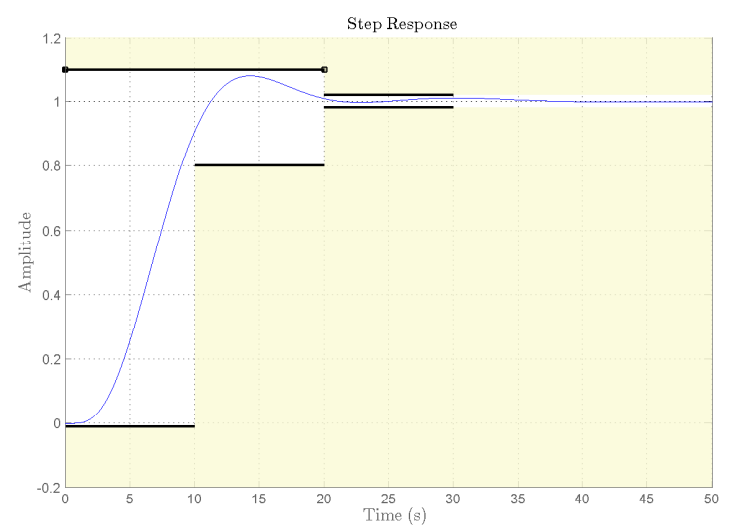

(b) Unit step response

Figure 5.34: Root locus and unit step response of altitude controller for a chosen proportional gain $K_{h}$. The controller was tuned to obtain the best possible response that is within the design requirements.

\section{Results}

Figure 5.35 shows the response results with the designed altitude controller. The altitude response to a $15 \mathrm{~m}$ (approximately $50 \mathrm{ft}$, as in Figure 5.33) altitude step is shown in Figure $5.35 \mathrm{a}$. The response exhibits a settling time of approximately 20s and overshoot of just under $10 \%$. The corresponding airspeed and FPA response plots are shown in Figures 5.35b and 5.35c. 


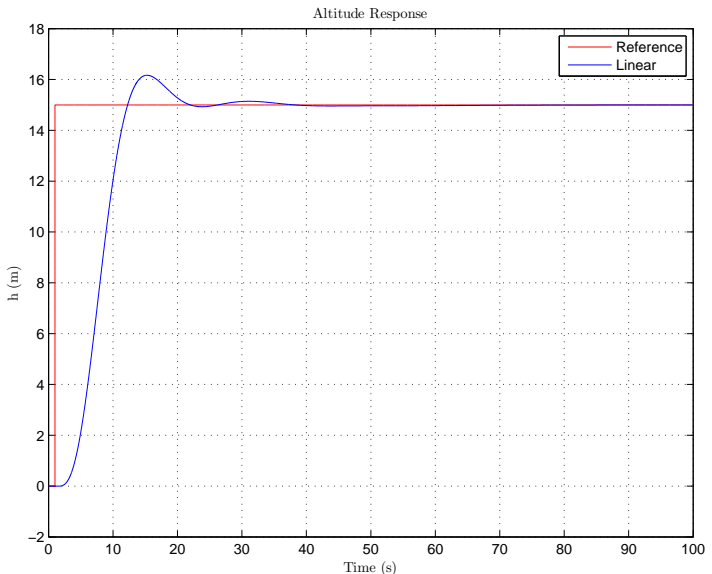

(a) Altitude response

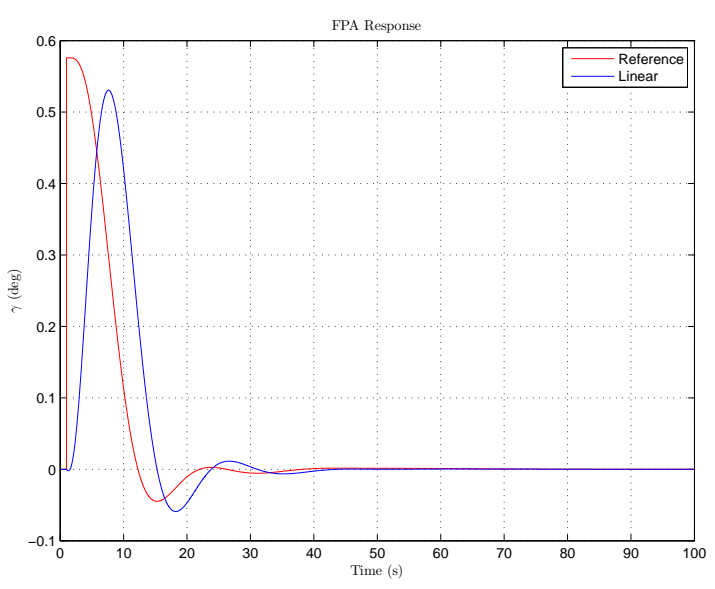

(c) Airspeed response

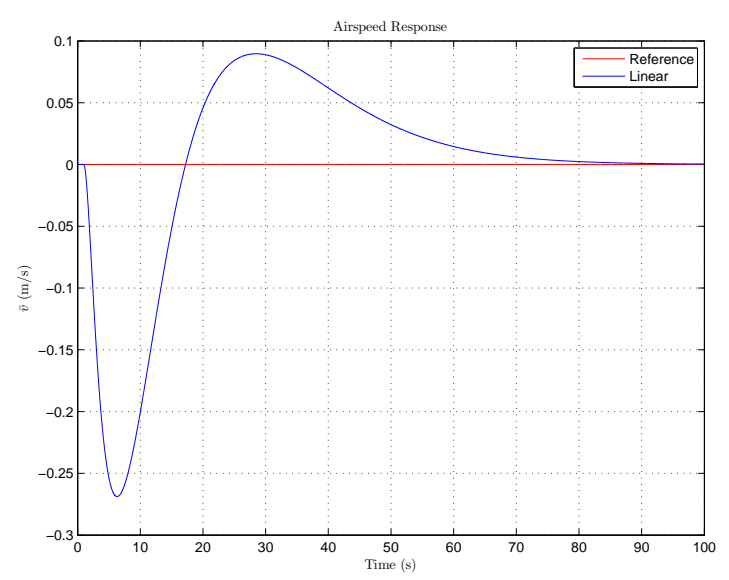

(b) FPA response

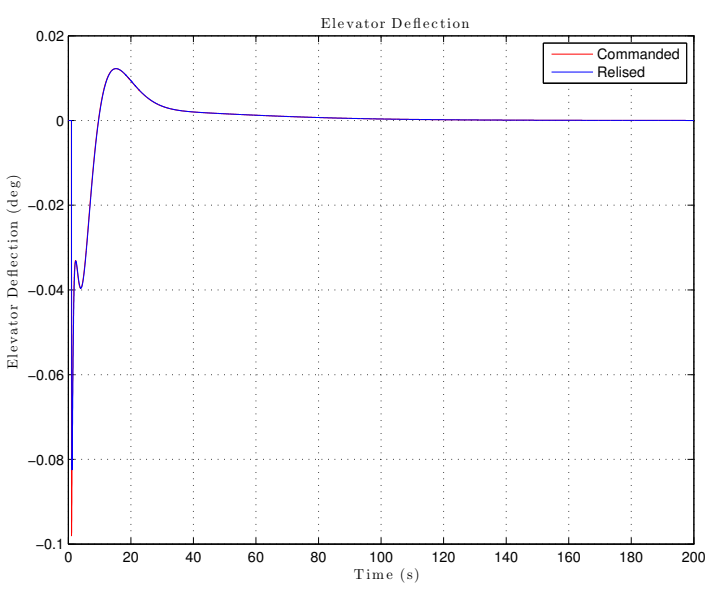

(d) Elevator response

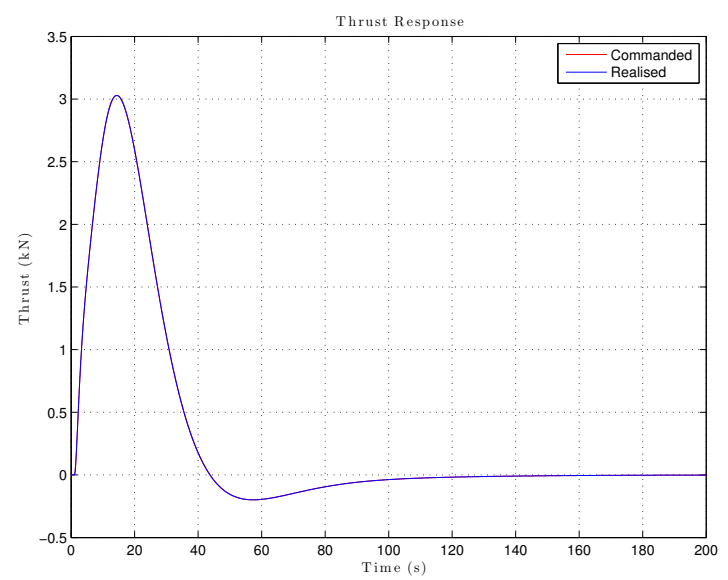

(e) Thrust response

Figure 5.35: Full-order longitudinal step response results for a $15 \mathrm{~m}$ altitude command. 


\section{Closed-Loop Model}

Following the design of the altitude controller, a closed-loop state space model that encapsulates the longitudinal dynamics, the DQ law, FPA, autothrust and altitude controller can be derived. The autothrust state space matrix is first augmented to include the height variable.

The autothrust state equation in Equation 5.50 is augmented to include the height variable by the inclusion of Equation (5.56),

$$
\left[\begin{array}{c}
\dot{\boldsymbol{x}}_{A T} \\
\dot{h}
\end{array}\right]=\left[\begin{array}{cc}
\boldsymbol{A}_{A T} & \mathbf{0}_{6 \times 1} \\
\mathbf{C}_{\dot{h}} & 0
\end{array}\right]\left[\begin{array}{c}
\boldsymbol{x}_{A T} \\
h
\end{array}\right]+\left[\begin{array}{cc}
\boldsymbol{B}_{\gamma_{R e f}} & \boldsymbol{B}_{\bar{v}_{R e f}} \\
0 & 0
\end{array}\right]\left[\begin{array}{c}
\gamma_{R e f} \\
\bar{v}_{R e f}
\end{array}\right]
$$

The control law is given as follows,

$$
\boldsymbol{u}=\left[\begin{array}{c}
K_{h}\left(h_{\text {Ref }}-h\right) \\
\bar{v}_{\text {Ref }}
\end{array}\right]
$$

Substitution of the control law into the augmented state equation gives the final closed-loop model as follows,

$$
\begin{aligned}
\dot{\boldsymbol{x}}_{A L T} & =\mathbf{A}_{A L T} \boldsymbol{x}_{A L T}+\mathbf{B}_{A L T} \boldsymbol{u} \\
{\left[\begin{array}{c}
\dot{\boldsymbol{x}}_{A T} \\
\dot{h}
\end{array}\right] } & =\left[\begin{array}{cc}
\boldsymbol{A}_{A T} & -\boldsymbol{B}_{\gamma_{R e f}} K_{h} \\
\mathbf{C}_{\dot{h}} & 0
\end{array}\right]\left[\begin{array}{c}
\boldsymbol{x}_{A T} \\
h
\end{array}\right]+\left[\begin{array}{cc}
\boldsymbol{B}_{\gamma_{R e f}} K_{h} & \boldsymbol{B}_{\bar{v}_{R e f}} \\
0 & 0
\end{array}\right]\left[\begin{array}{c}
h_{R e f} \\
\bar{v}_{R e f}
\end{array}\right]
\end{aligned}
$$

Figure 5.36 shows the final closed-loop poles after adding the altitude controller.

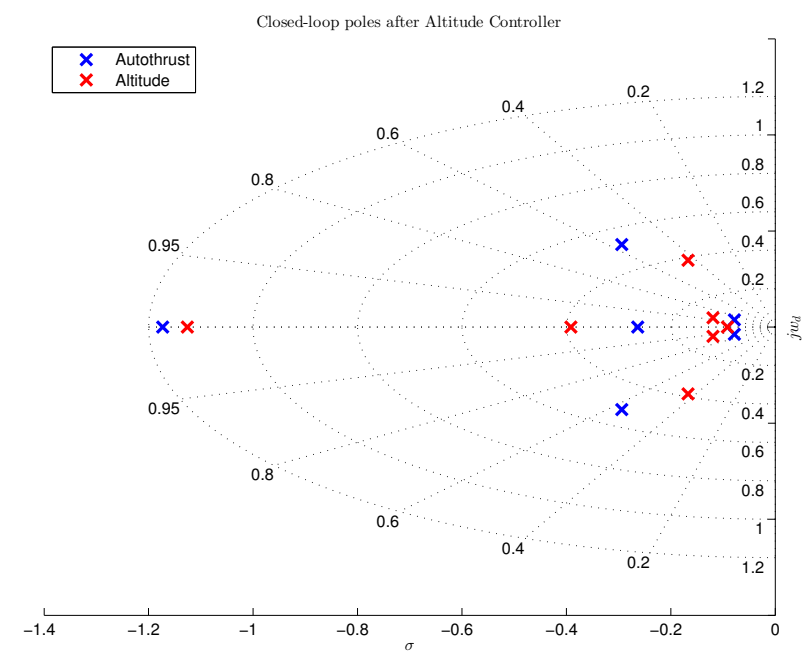

Figure 5.36: Closed-loop poles after adding the altitude controller. 


\subsection{Lateral Control Systems Design}

In this section, the lateral control depicted in Figure 5.5, namely the DP and DR laws as well as the Cross-Track Error guidance law, will be designed and verified in simulation. The linearised longitudinal state space model in Equation $4.25 \mathrm{~b}$ will be used with the following notation,

$$
\dot{\boldsymbol{x}}=\boldsymbol{A x}+\boldsymbol{B u}
$$

where,

$$
\begin{aligned}
& \boldsymbol{x}=\left[\begin{array}{llll}
v & p & r & \phi
\end{array}\right]^{T} \\
& \boldsymbol{u}=\left[\begin{array}{ll}
\delta_{a} & \delta_{r}
\end{array}\right]^{T} \\
& \boldsymbol{A}=\boldsymbol{A}_{\text {lat }} \\
& \boldsymbol{B}=\boldsymbol{B}_{l a t}=\left[\begin{array}{ll}
\mathbf{B}_{\delta_{a}} & \mathbf{B}_{\delta_{r}}
\end{array}\right]
\end{aligned}
$$

\subsubsection{DP and DR Law: A Roll and Sideslip Angle Controller}

A controller capable of regulating the roll and sideslip angles will be designed in this section, along with rudder turn coordination and a natural response for a commanded sideslip.

\section{Design}

The architecture of the DP and DR laws is shown in Figure 5.37. The plant for this design is the lateral dynamics,

$$
\dot{\boldsymbol{x}}=\boldsymbol{A} \boldsymbol{x}+\boldsymbol{B}_{\delta_{a}} \delta_{a}
$$

where the output matrix,

$$
\mathbf{C}_{\beta, \phi}=\left[\begin{array}{llll}
1 & 0 & 0 & 0 \\
0 & 0 & 0 & 1
\end{array}\right]
$$

is used to extract the sideslip and roll angles from the lateral state space matrix $\boldsymbol{x}$.

Full-state feedback is used to place the lateral poles and in such a way obtain a desired set of specifications. The rudder turn coordination controller is represented by the transfer function $H(s)_{R C C}$ and will be used to compute the amount of rudder required to produce a sideslip angle that minimises the adverse yaw effect of the aircraft. In the DP law, no turn coordination controller is added, but the law is designed to produce a natural response for a commanded sideslip by allowing a slight roll angle when commanding sideslip.

The full-state feedback control law is given by,

$$
\boldsymbol{u}=\mathbf{K}_{F S F} \boldsymbol{x}
$$



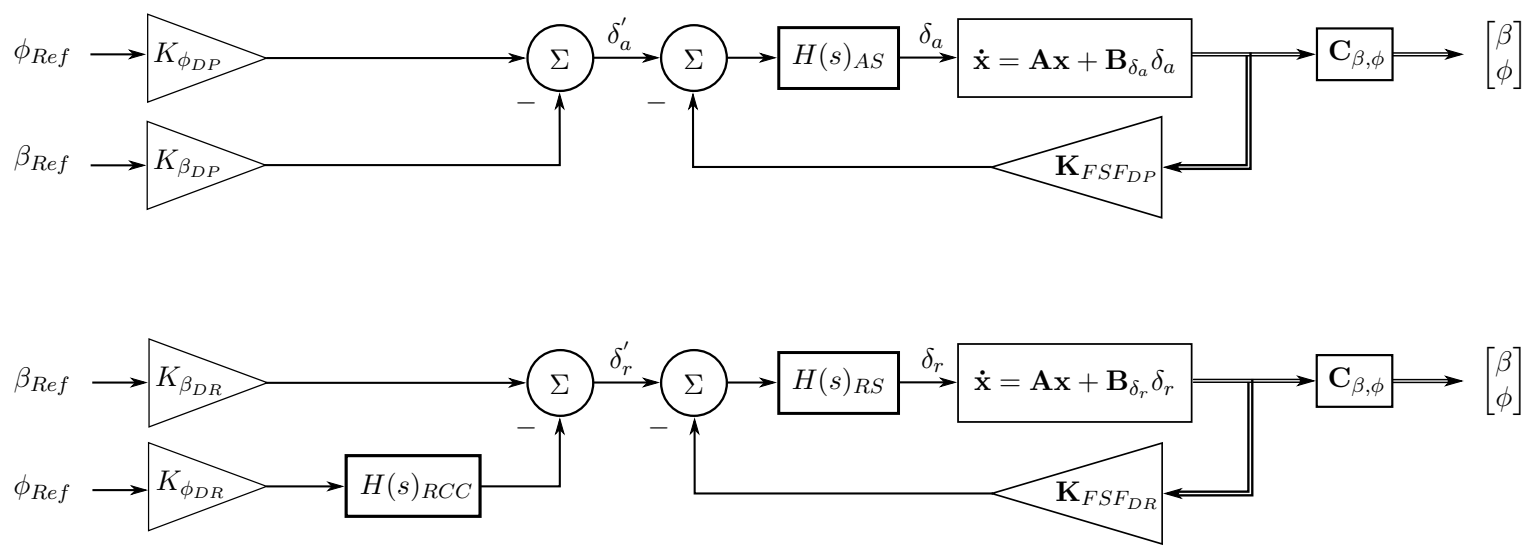

Figure 5.37: Full architecture of the DP (top) and DR (bottom) laws.

where,

$$
\mathbf{K}_{F S F}=\left[\begin{array}{l}
\mathbf{K}_{F S F_{D P}} \\
\mathbf{K}_{F S F_{D R}}
\end{array}\right]
$$

and is an optimal control gain calculated by an LQR optimisation algorithm that minimises the cost function,

$$
J=\int_{0}^{\infty}\left(\boldsymbol{x}^{T} \mathbf{Q} \boldsymbol{x}+\boldsymbol{u}^{T} \mathbf{R} \boldsymbol{u}\right) d t
$$

where the state weighting matrix $\mathbf{Q}$ and the control weighting matrix $\mathbf{R}$ are both selected by the designer. A reasonable choice for the weighting matrices is given by Bryson's rule [75], which recommends to select them as diagonals with,

$$
\begin{aligned}
\mathbf{Q}_{i i} & =\frac{1}{\text { max acceptable value of } \boldsymbol{x}_{i}^{2}} \\
\mathbf{R}_{j j} & =\frac{1}{\text { max acceptable value of } \boldsymbol{u}_{j}^{2}}
\end{aligned}
$$

where,

$$
\begin{aligned}
& i \in\{1,2,3,4\} \\
& j \in\{1,2\}
\end{aligned}
$$

The maximum acceptable values for the state and control variables were selected accordingly with the help of Hanke and Nordwall [10]. The weightings were then iteratively modified to achieve an acceptable trade-off between performance and control effort.

Turn Coordination Controller In aviation, a coordinated turn is defined as one where the CG of the aircraft experiences zero lateral acceleration - resulting in zero sideslip [59] 64]. In 
asymmetrical aircraft, however, the sideslip angle may not be exactly zero as desired. This could be as a result of asymmetric thrust or the effects of the angular momentum of spinning rotors [64]. Nevertheless, this coordination is known to be desirable for passenger comfort and allows pilots to function more effectively [64]. Moreover, by minimising the sideslip with turn coordination, maximum aerodynamic efficiency is obtained and undesirable aerodynamic loads on the structure are reduced to a minimum [64. Several methods of obtaining turn coordination exist (see Blakelock [59]) and in this study, a method that utilises a coordination computer is discussed and used in the DR law.

A rudder coordination controller (RCC) is added to the DR law in Figure 5.37 that computes the amount of rudder required to produce a sideslip angle that minimises the adverse yaw effect of the aircraft. Consider Figure 5.38, which replaces the state space plant models in Figure 5.37 with transfer functions from control input to sideslip output,

$$
\begin{aligned}
& H(s)_{\delta_{r} \rightarrow \beta}=\frac{\beta(s)}{\delta_{r}(s)} \\
& H(s)_{\delta_{a} \rightarrow \beta}=\frac{\beta(s)}{\delta_{a}(s)}
\end{aligned}
$$

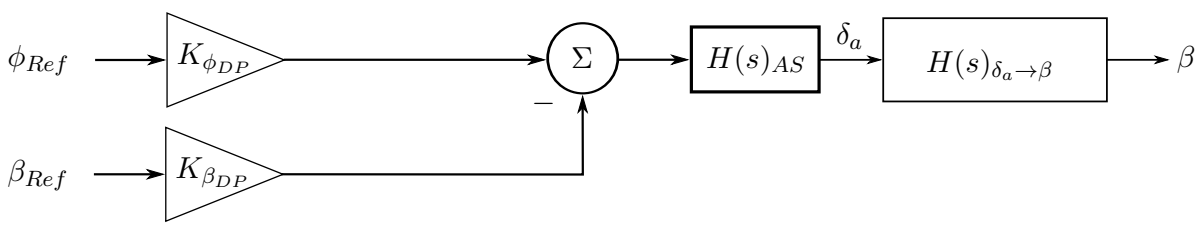

(a) DP law

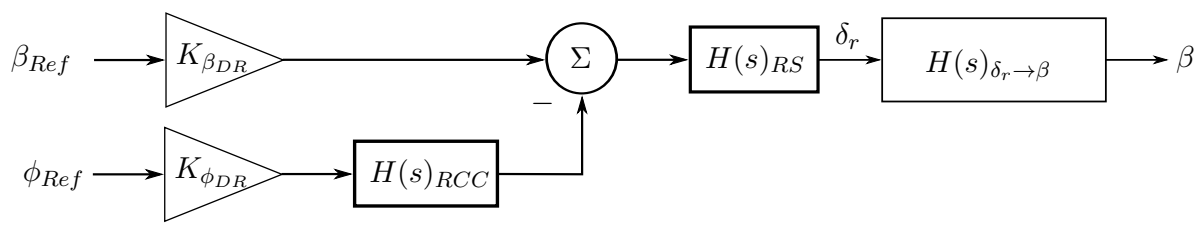

(b) DR law

Figure 5.38: Block diagram of the DP and DR laws with sideslip output and using a rudder turn coordination controller to achieve coordination.

The total sideslip experienced by the aircraft is equal to the sideslip resulting from the rudder and the aileron,

$$
\beta_{\text {Total }}=H(s)_{\delta_{r} \rightarrow \beta} \delta_{r}+H(s)_{\delta_{a} \rightarrow \beta} \delta_{a}
$$

For perfect coordination, the total sideslip must be zero and it follows from (5.71),

$$
\begin{aligned}
H(s)_{\delta_{r} \rightarrow \beta} \delta_{r} & =-H(s)_{\delta_{a} \rightarrow \beta} \delta_{a} \\
\frac{\delta_{r}}{\delta_{a}} & =\frac{-H(s)_{\delta_{a} \rightarrow \beta}}{H(s)_{\delta_{r} \rightarrow \beta}}
\end{aligned}
$$


Assuming now that for zero sideslip, a zero sideslip reference is applied to the controllers in Figure 5.38. The only non-zero input is then the roll angle reference $\phi_{\text {Ref }}$. It then follows from Figure $5.38 \mathrm{a}$ and $5.38 \mathrm{~b}$ that,

$$
\begin{aligned}
& \delta_{r}=-K_{\phi_{D R}} \phi_{R e f} H(s)_{R C C} H(s)_{R S} \\
& \delta_{a}=K_{\phi_{D P}} \phi_{\text {Ref }} H(s)_{A S}
\end{aligned}
$$

The negative term arises from the fact that for the same contribution that a positive aileron produces, negative rudder is required. Dividing the two equations above gives the following result,

$$
\frac{\delta_{r}}{\delta_{a}}=\frac{-K_{\phi_{D R}} \phi_{R e f} H(s)_{R C C} H(s)_{R S}}{K_{\phi_{D P}} \phi_{R e f} H(s)_{A S}} \approx-H(s)_{R C C}
$$

assuming that the aileron and rudder servo transfer functions as well as the the feed-forward gains are more or less the same. The transfer function for the RCC can now be obtained by equating Equations (5.72) and (5.74),

$$
H(s)_{R C C}=\frac{H(s)_{\delta_{a} \rightarrow \beta}}{H(s)_{\delta_{r} \rightarrow \beta}}
$$

\section{Specifications}

Figures $5.39 \mathrm{a}$ and $5.39 \mathrm{~b}$ show the non-linear response of the A330 aircraft model while using turn coordination. Using the response characteristics as a guide, as well as the proceedings of Holzapfel et al. [76], the specifications for the lateral DP and DR laws were selected as follows: the roll and sideslip response time should be quite fast - a peak time of less than 10 seconds is desired. The overshoot in roll angle is not well specified in the available literature [71] [72] but should intuitively remain reasonably low. Since an overshoot in a banking angle is not desirable for passenger comfort, it is chosen to limit the overshoot to less than 5\%. The steady-state error does not need to be zero. Using turn coordination, the sideslip needs to be minimised as much as possible.

The sideslip response was selected based on the response of an A330 at a similar flight condition. Flying with sideslip at the current flight condition of $40000 \mathrm{ft}$ and $0.8 \mathrm{M}$ is not desirable, and thus it will be kept very small. As such, the realised sideslip will achieve a final value of approximately half the commanded sideslip exhibiting an overshoot of approximately $20 \%$. Lastly, the steady-state ratio for a bank angle induced by a sideslip angle command should be,

$$
\frac{\phi_{s s}}{\beta_{s s}}=-5
$$




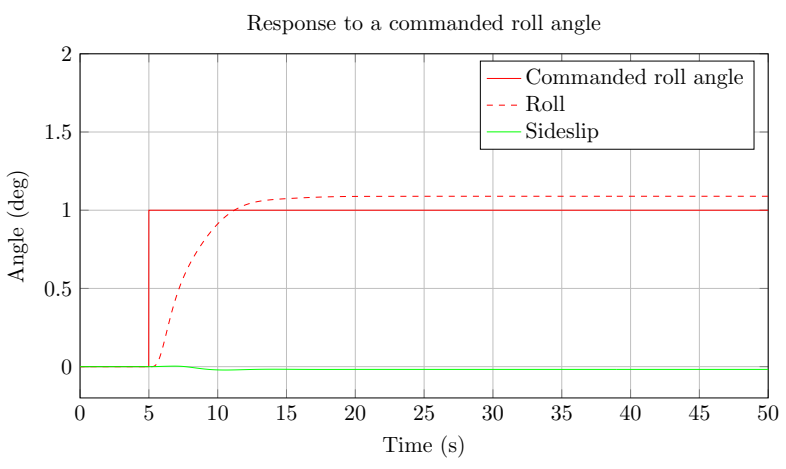

(a) Response to a roll angle reference

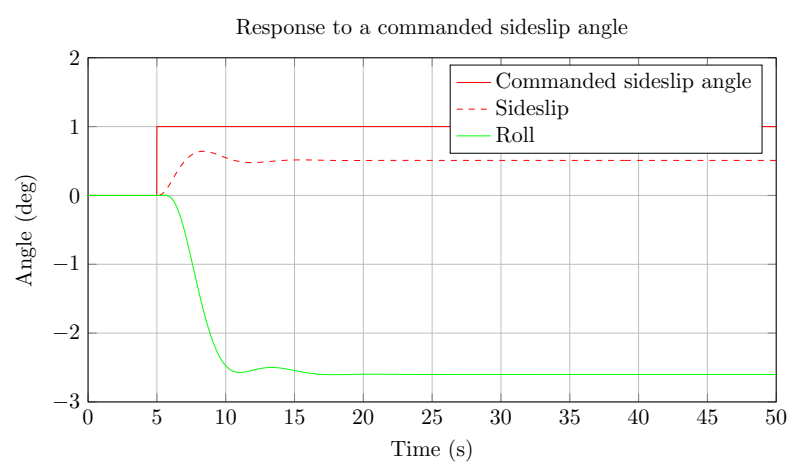

(b) Response to a sideslip angle reference

Figure 5.39: Non-linear roll and sideslip response in the A330 aircraft model for a commanded roll and sideslip angle.

\section{Results}

Figure 5.40 shows the new system poles after the LQR optimisation algorithm. The dominant poles are placed with the following natural frequency and damping characteristics,

$$
\begin{aligned}
w_{n} & =0.867 \mathrm{rad} / \mathrm{s} \\
\zeta & =0.786
\end{aligned}
$$

which translates into a dominant $2 \%$ settling time of approximately,

$$
t_{s_{2} \%}=\frac{4}{\zeta w_{n}} \approx 5.87 \mathrm{~s}
$$

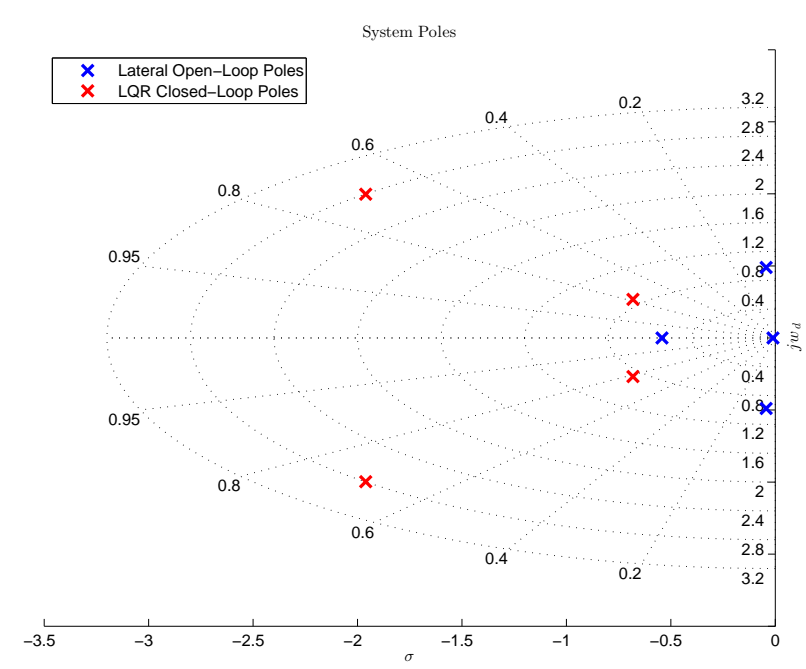

Figure 5.40: Closed-loop poles after full-state feedback using an LQR optimisation algorithm

Figure 5.41 shows the roll response to a commanded roll angle after applying full-state feedback (Figure 5.41a) and then after adding a feed-forward gain $K_{\phi_{D P}}$ to minimise the steady-state error 


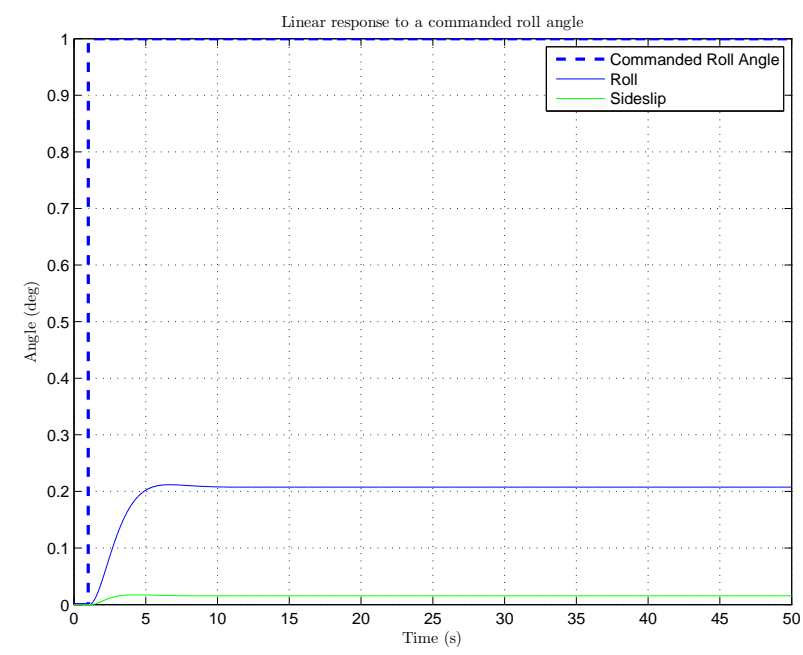

(a) No turn coordination and feed-forward

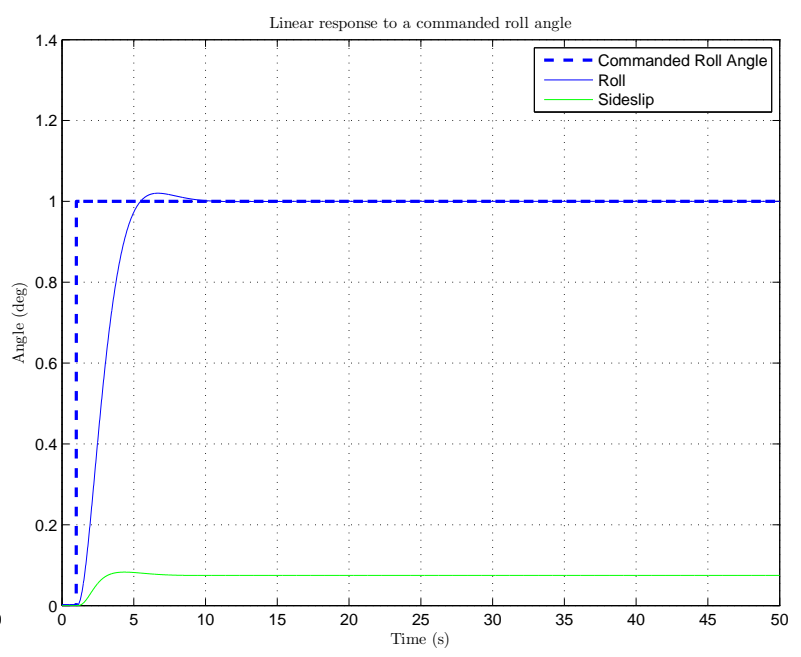

(b) No turn coordination but with feed-forward

Figure 5.41: Roll response of the linear aircraft model after full-state feedback but with no turn coordination.

in the roll angle (Figure 5.41b). Without a rudder turn coordination controller as discussed in the design, the sideslip is not minimised.

After applying the methods as discussed earlier, the full-order rudder coordination controller transfer function is obtained as a $7^{\text {th }}$ order polynomial. The bode plot for this transfer function is shown in Figure 5.42. The full-order transfer function is comparatively complex; however, it is well approximated with a first-order transfer function in the frequency range over which the control law operates. The reduced-order RCC bode plot is also illustrated in Figure 5.42 .

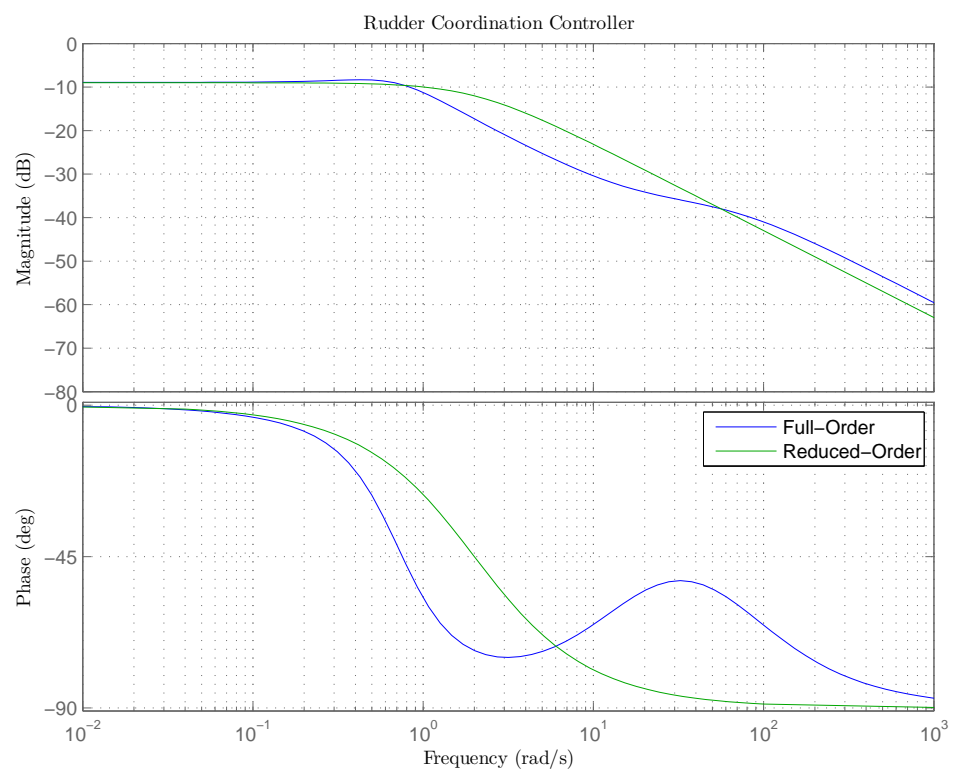

Figure 5.42: Bode plot of the reduced-order RCC.

After adding rudder turn coordination to the lateral DR control law, Figure 5.43a shows 
the roll response to a $1^{\circ}$ roll angle reference command. To reduce the steady-state error on the roll angle output, the feed-forward gain $K_{\phi_{D P}}$ was adapted and the resulting response is shown in Figure 5.43b. The steady-state error in the roll response of the aircraft is not ideal, but was made to agree with the response of the Airbus aircraft model. The amount of sideslip - not zero, but minimised - is better than without turn coordination, as shown in Figure 5.41b. The actuator responses are shown in Figure $5.43 \mathrm{c}$ and $5.43 \mathrm{~d}$ well within the capabilities of the ailerons and the rudder.

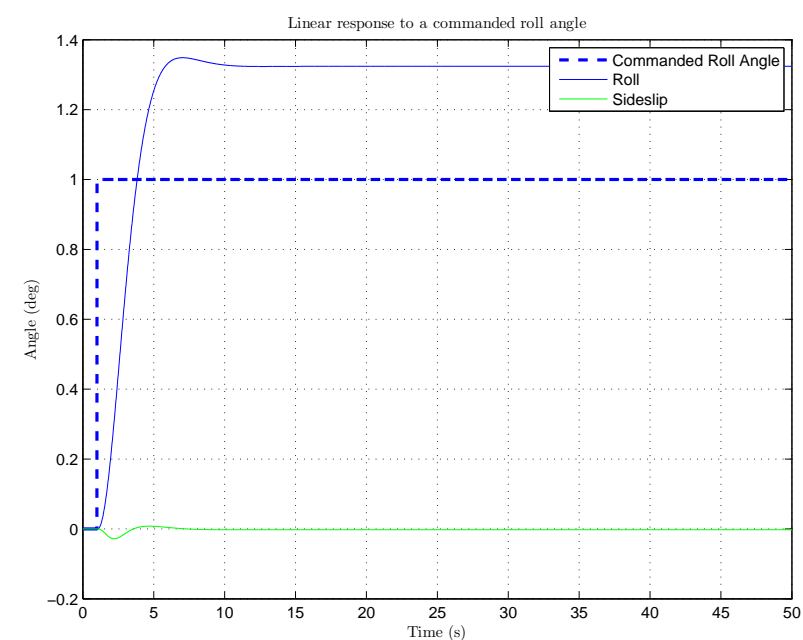

(a) Turn coordination

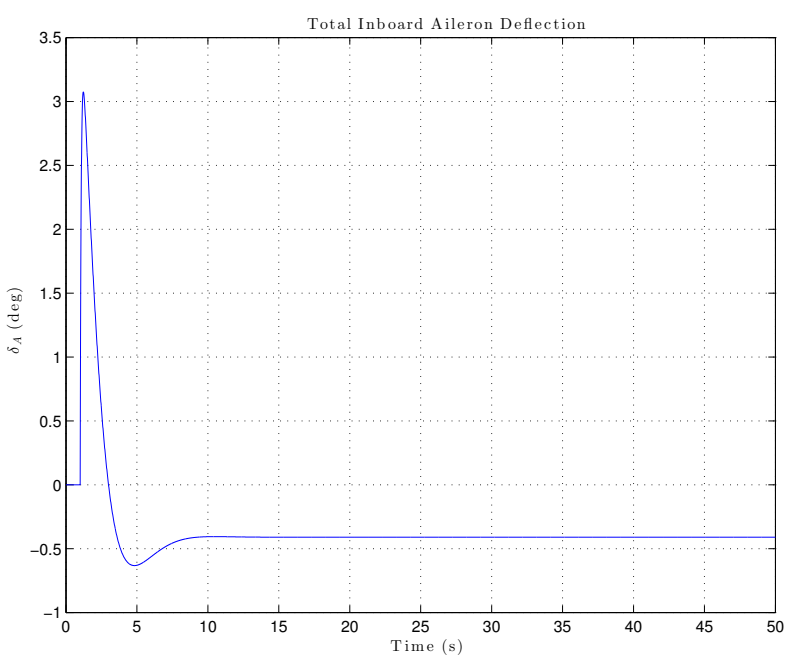

(c) Aileron Response

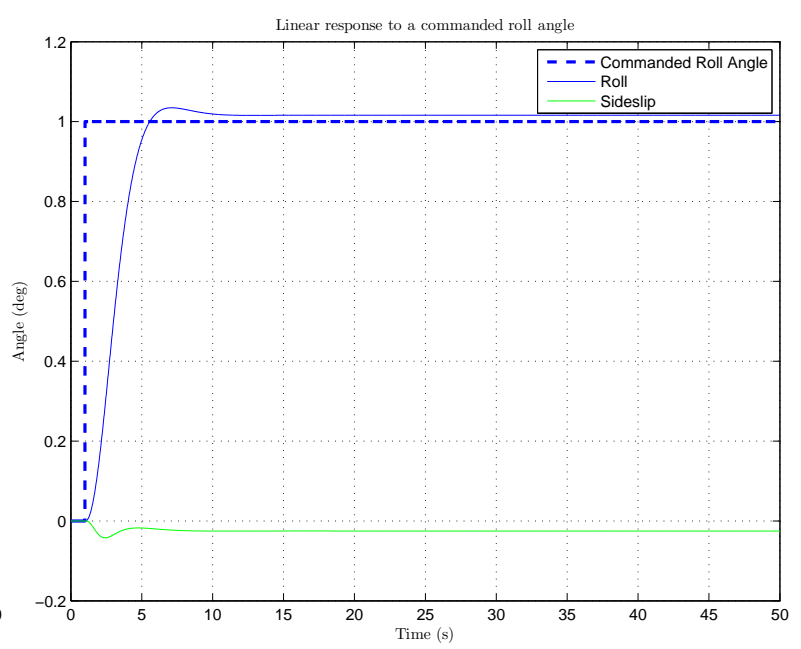

(b) Turn coordination and improved steady-state error

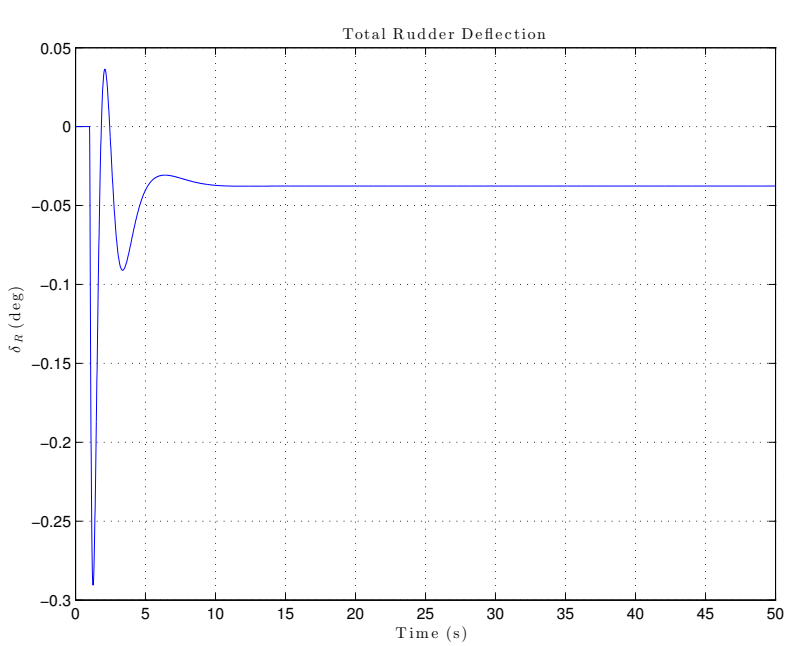

(d) Rudder Response

Figure 5.43: Roll response of the linear aircraft model after full-state feedback and a rudder turn coordination controller in the lateral DR law.

Finally, the initial sideslip response (Figure 5.44a), was adapted to obtain an improved sideslip response with a natural roll response (5.44b), similar to what a pilot would expect from an aircraft without fly-by-wire. The aileron and rudder deflections are also shown in Figure $5.44 \mathrm{c}$ and $5.44 \mathrm{~d}$ respectively. 


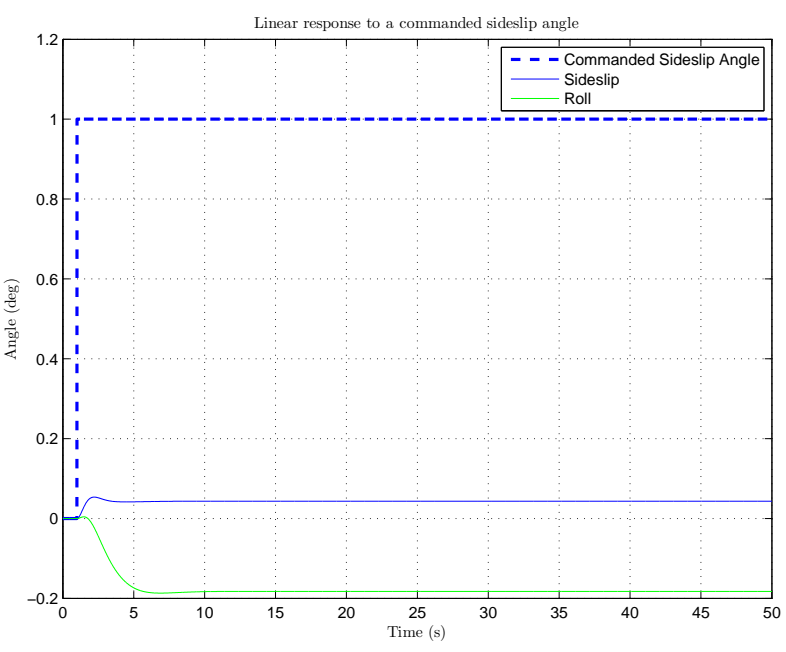

(a) Poor sideslip response

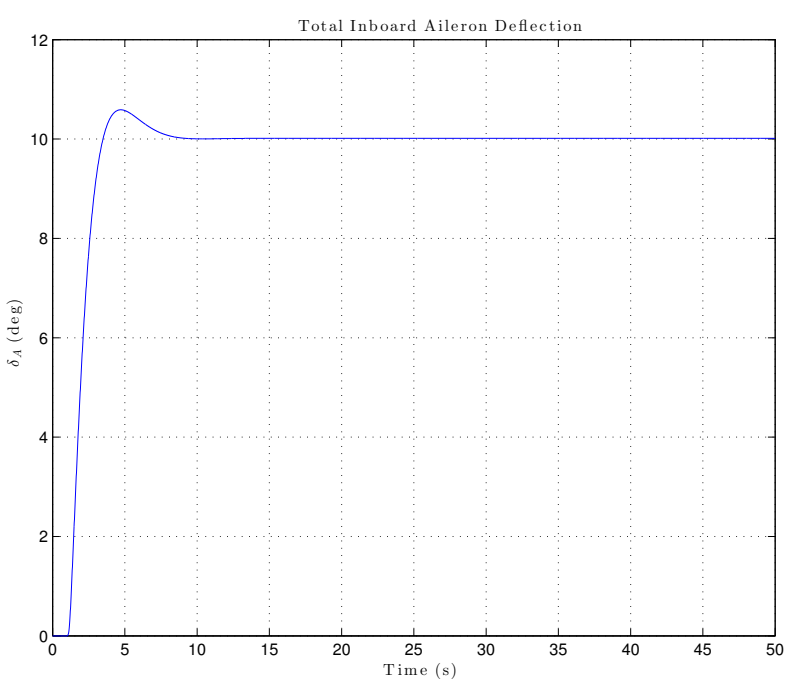

(c) Aileron Response

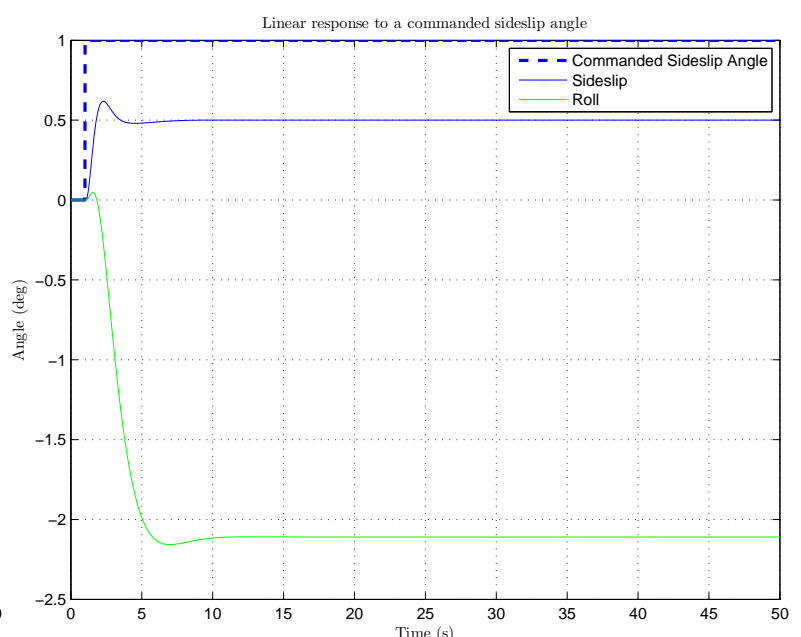

(b) Improved sideslip response with natural roll

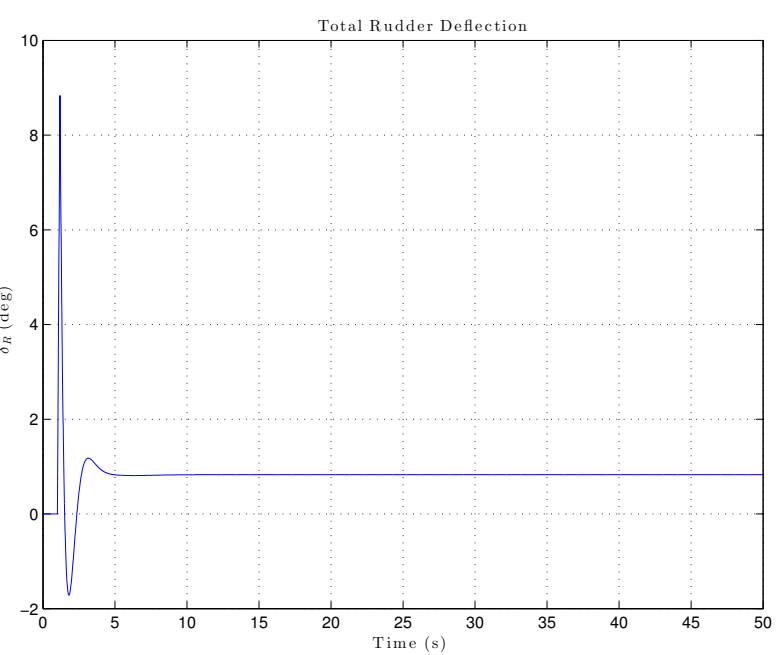

(d) Rudder Response

Figure 5.44: Sideslip response of the linear aircraft model after the full-state feedback and a steady-state ratio are applied to obtain a natural roll response during sideslip.

Overall, the desired specifications were satisfactorily achieved, with a fast roll and sideslip response, turn coordination to minimise the sideslip in a bank, and a more natural roll response during a commanded sideslip angle.

\section{Closed-Loop Model}

The open-loop plant in Figure 5.37, with the lateral dynamics given by Equation (5.63), is adapted to include the optimal full-state feedback gain,

$$
\dot{\boldsymbol{x}}=\boldsymbol{A}_{C L} \boldsymbol{x}+\boldsymbol{B}\left[\begin{array}{l}
\delta_{a}^{\prime} \\
\delta_{r}^{\prime}
\end{array}\right]
$$


where $\boldsymbol{A}_{C L}=\left[\boldsymbol{A}-\boldsymbol{B K}_{F S F}\right]$. The first-order RCC transfer function is now added to the system by first representing it as a single-input-single-output (SISO) state space model,

$$
\begin{aligned}
& \dot{x}_{R C C}=A_{R C C} x_{R C C}+B_{R C C} K_{\phi_{D R}} \phi_{R e f} \\
& y_{R C C}=C_{R C C} x_{R C C}+D_{R C C} K_{\phi_{D R}} \phi_{R e f}
\end{aligned}
$$

and then augmenting it into the full-order state space representation,

$$
\left[\begin{array}{c}
\dot{\boldsymbol{x}} \\
\dot{x}_{R C C}
\end{array}\right]=\left[\begin{array}{cc}
\boldsymbol{A}_{C L} & \mathbf{0}_{4 \times 1} \\
\mathbf{0}_{1 \times 4} & A_{R C C}
\end{array}\right]\left[\begin{array}{c}
\boldsymbol{x} \\
x_{R C C}
\end{array}\right]+\left[\begin{array}{cc}
\mathbf{B}_{\delta_{a}} & \mathbf{B}_{\delta_{r}} \\
0 & 0
\end{array}\right]\left[\begin{array}{c}
\delta_{a}^{\prime} \\
\delta_{r}^{\prime}
\end{array}\right]+\left[\begin{array}{cc}
\mathbf{0}_{4 \times 1} & \mathbf{0}_{4 \times 1} \\
B_{R C C} K_{\phi_{D R}} & 0
\end{array}\right]\left[\begin{array}{c}
\phi_{R e f} \\
\beta_{R e f}
\end{array}\right]
$$

Adding the feed-forward and turn coordination to the state space model to obtain the closed-loop model requires the following substitutions,

$$
\begin{aligned}
& \delta_{a}^{\prime}=K_{\phi_{D P}} \phi_{R e f}-K_{\beta_{D P}} \beta_{R e f} \\
& \delta_{r}^{\prime}=K_{\beta_{D R}} \beta_{R e f}-C_{R C C} x_{R C C}-D_{R C C} K_{\phi_{D R}} \phi_{R e f}
\end{aligned}
$$

After finding suitable values for the feed-forward gains and a sufficient reduced-order RCC, the closed-loop model that encapsulates the full-order lateral dynamics with the DPDR law can be expressed in state space form as,

$$
\begin{aligned}
\dot{\boldsymbol{x}}_{D P D R} & =\mathbf{A}_{D P D R} \boldsymbol{x}_{D P D R}+\mathbf{B}_{D P D R} \boldsymbol{u} \\
{\left[\begin{array}{c}
\dot{\boldsymbol{x}} \\
\dot{x}_{R C C}
\end{array}\right] } & =\left[\begin{array}{cc}
\boldsymbol{A}_{C L} & -\boldsymbol{B}_{\delta_{r}} C_{R C C} \\
\mathbf{0}_{1 \times 4} & A_{R C C}
\end{array}\right]\left[\begin{array}{c}
\boldsymbol{x} \\
x_{R C C}
\end{array}\right] \\
& +\left[\begin{array}{cc}
\boldsymbol{B}_{\delta_{a}} K_{\phi_{D P}}-\boldsymbol{B}_{\delta_{r}} D_{R C C} K_{\phi_{D R}} & B_{\delta_{r}} K_{\beta_{D R}}-\boldsymbol{B}_{\delta_{a}} K_{\beta_{D P}} \\
B_{R C C} K_{\phi_{D R}} & 0
\end{array}\right]\left[\begin{array}{c}
\phi_{R e f} \\
\beta_{R e f}
\end{array}\right]
\end{aligned}
$$

Figure 5.45 shows the new closed-loop poles after adding the DP and DR lateral laws. Notice that the real pole at $s=-2$ is the fast first-order RCC state.

\subsubsection{Cross-Track Error Guidance: A Lateral Guidance Controller}

Following the design of the inner-loop lateral laws that directly command an aileron and rudder deflection, a rudimentary guidance controller is required. This controller will aim to navigate the aircraft along a flight path plan consisting of a set of waypoints, defined as a series of straight-line segments. Before designing the guidance controller, we first define the guidance axis system.

\section{Guidance Axis System}

Consider Figure 5.46, which shows the aircraft flying at a constant heading relative to the straight-line segment between the source and destination waypoint. The right-handed orthogonal 


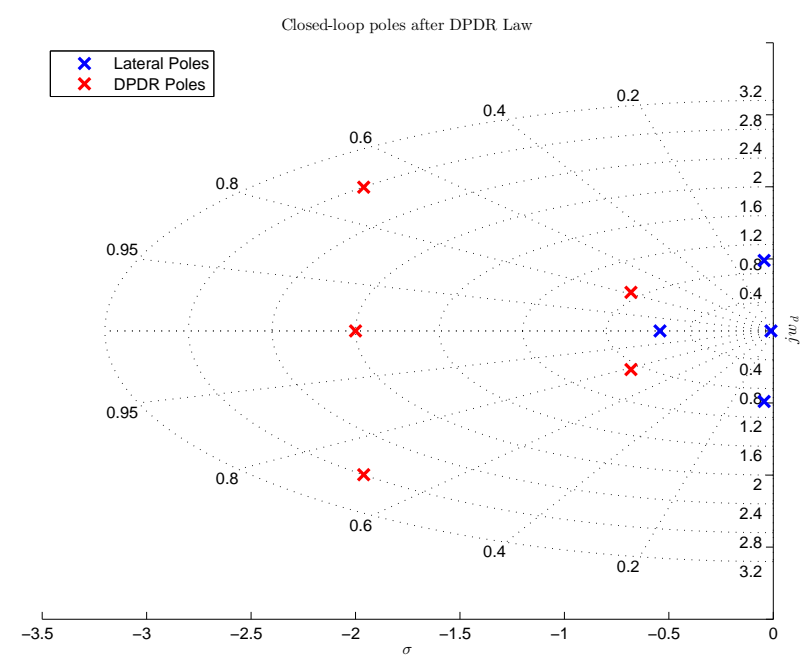

Figure 5.45: Closed-loop poles after adding the DP and DR law.

guidance axis system is placed with its origin at the source waypoint and its $z$-axis such that it coincides with the inertial down axis. The $x$-axis is parallel to the ground track, pointing in the direction of the destination waypoint, and the $y$-axis is perpendicular to the ground track. Given the source and destination waypoint, the track heading $\psi_{\text {Track }}$ and length of the ground track $L_{\text {Track }}$ are calculated from Figure 5.46 as,

$$
\begin{aligned}
\tan \psi_{\text {Track }} & =\frac{E_{\text {dest }}-E_{\text {src }}}{N_{\text {dest }}-N_{\text {src }}} \\
L_{\text {Track }} & =\sqrt{\left(N_{\text {dest }}-N_{\text {src }}\right)^{2}+\left(E_{\text {dest }}-E_{\text {src }}\right)^{2}}
\end{aligned}
$$

The aircraft's cross-track error $y$ and in-track distance $x$ are shown in Figure 5.46. The crosstrack error will be the controlled output of the guidance controller, and is the perpendicular distance from the aircraft's position to the ground track. The in-track distance is the distance of the aircraft's projection onto the track, and will determine whether a waypoint has been reached. This should trigger a waypoint scheduler to produce the next waypoint, if it exists.

To obtain the cross-track error and in-track distance, the aircraft's position is first translated and rotated from the inertial axes to the guidance axes,

$$
\left[\begin{array}{l}
x \\
y
\end{array}\right]=\left[\begin{array}{cc}
\cos \psi_{\text {Track }} & \sin \psi_{\text {Track }} \\
-\sin \psi_{\text {Track }} & \cos \psi_{\text {Track }}
\end{array}\right]\left[\begin{array}{c}
N_{L}-N_{\text {src }} \\
E_{L}-E_{\text {src }}
\end{array}\right]
$$

\section{Design}

The control architecture of the cross-track error guidance controller is shown in the block diagram of Figure 5.47. The plant is the DPDR law with the lateral dynamics of the aircraft in state space form represented by Equation (5.83), 


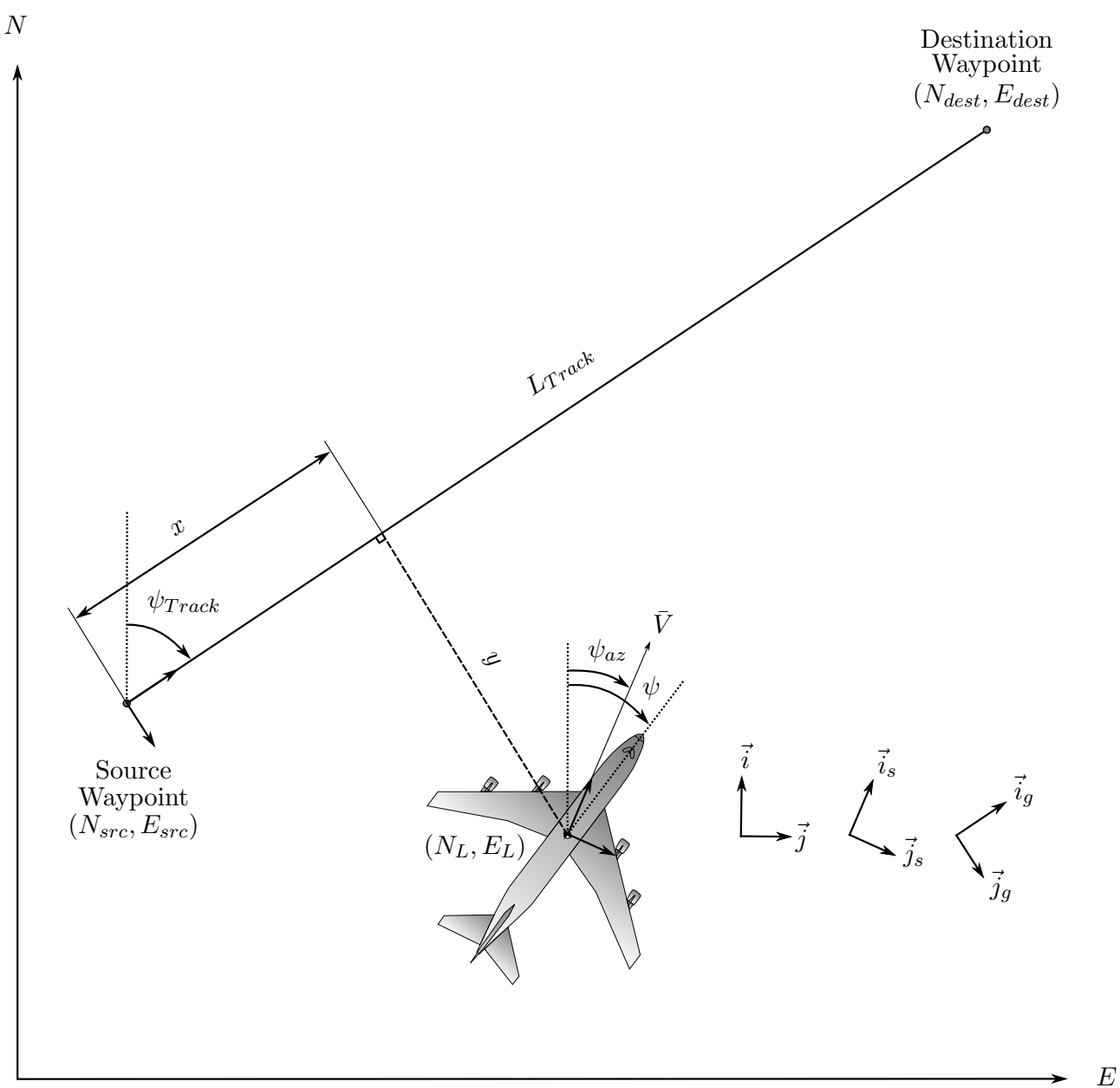

Figure 5.46: Illustration of cross-track error and in-track distance for a source and destination waypoint. The guidance axis system is also defined and illustrated together with the track heading and length of the ground track.

$$
\begin{aligned}
\dot{\boldsymbol{x}}_{D P D R} & =\boldsymbol{A}_{D P D R} \boldsymbol{x}_{D P D R}+\boldsymbol{B}_{D P D R} \boldsymbol{u} \\
y & =\dot{\psi}=\mathbf{C}_{\dot{\psi}} \boldsymbol{x}_{D P D R}
\end{aligned}
$$

where,

$$
\boldsymbol{u}=\left[\begin{array}{l}
\phi_{R e f} \\
\beta_{R e f}
\end{array}\right]
$$

and the output matrix,

$$
\mathbf{C}_{\dot{\psi}}=\left[\begin{array}{lllll}
0 & 0 & 0 & \frac{g}{V_{T}} & 0
\end{array}\right]
$$

extracts the heading rate $\dot{\psi}$ from the DPDR law state vector $\boldsymbol{x}_{D P D R}$ and feeds it to a natural integrator to become the heading angle $\psi$. Consider Figure 5.10, where the aircraft is illustrated during a steady coordinated turn. During the turn, the force vector $\vec{F}$ is responsible for countering the weight of the aircraft and thereby maintaining the nominal load factor. The centripetal acceleration during the turn can be written as follows, 


$$
a_{L}=g \tan \phi=\bar{V}_{T} \dot{\psi}
$$

and for small banking angles, $\tan \phi \approx \phi$ and the centripetal acceleration in Equation 5.89 is simplified to,

$$
\dot{\psi}=\frac{g}{\bar{V}_{T}} \phi
$$

The heading rate is typically measured with angular rate sensors in the $Z_{B}$-axis of the aircraft.

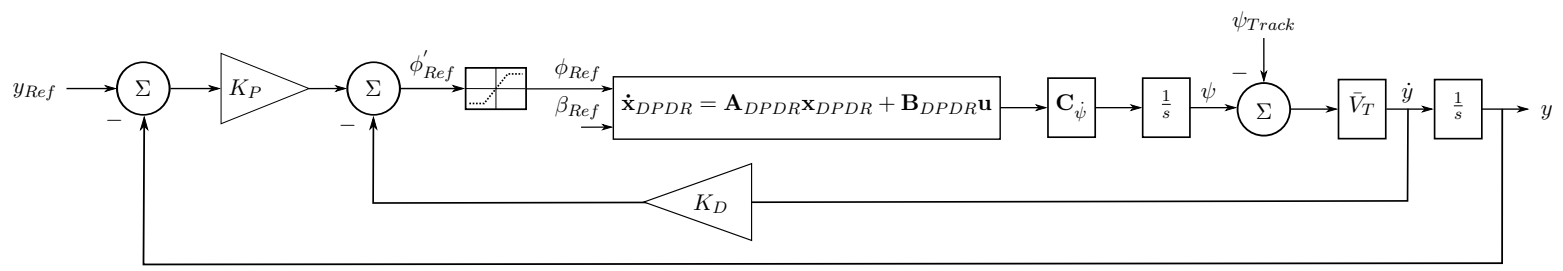

Figure 5.47: Full architecture of the cross-track error controller.

Consider Figure 5.46, which illustrates the rate of change of the cross-track error. The crosstrack error rate is the projection of the aircraft ground speed into the $\vec{j}_{g}$ axis, represented as follows,

$$
\dot{y}=\bar{V} \sin \left(\psi-\psi_{\text {Track }}\right)
$$

and for small heading angles relative to the ground track heading, Equation (5.91) may be simplified to

$$
\dot{y}=\bar{V}_{T}\left(\psi-\psi_{\text {Track }}\right)
$$

Equations (5.91) and (5.92) assumes that the sideslip angle is regulated to zero. Otherwise, the sideslip angle produces a side force which causes a lateral acceleration. With the rudder coordination controller active, the sideslip is negligibly small, and this lateral acceleration will thus be ignored.

The cross-track error controller consists of a two-loop closure design: a derivative feedback followed by a proportional feedback loop. The derivative loop consists of cross-track error rate $\dot{y}$ feedback, and the proportional feedback loop generates a roll angle reference proportional to the error of the cross-track error. The natural integration from cross-track error rate to cross-track error makes the system type 1, which means it should be able to follow a constant cross-track error reference with zero error at steady state. A saturation block is added to ensure that the controller does not command roll angle signals that exceed the limitations of the aircraft and that the aircraft maintains a constant roll angle when large cross-track error step commands are issued. The design of the cross-track error controller consists of determining appropriate values for the gains $K_{D}$ and $K_{P}$.

First the state space model of the DPDR law is augmented with the heading state, 


$$
\begin{aligned}
\dot{\boldsymbol{x}}_{H} & =\mathbf{A}_{H} \boldsymbol{x}_{H}+\mathbf{B}_{H} \boldsymbol{u} \\
{\left[\begin{array}{c}
\dot{\boldsymbol{x}}_{D P D R} \\
\dot{\psi}
\end{array}\right] } & =\left[\begin{array}{cc}
\boldsymbol{A}_{D P D R} & \mathbf{0}_{5 \times 1} \\
\mathbf{C}_{\dot{\psi}} & 0
\end{array}\right]\left[\begin{array}{c}
\boldsymbol{x}_{D P D R} \\
\psi
\end{array}\right]+\left[\begin{array}{c}
\boldsymbol{B}_{D P D R} \\
\mathbf{0}_{1 \times 2}
\end{array}\right]\left[\begin{array}{c}
\phi_{R e f} \\
\beta_{R e f}
\end{array}\right]
\end{aligned}
$$

where,

$$
C_{\dot{\psi}}=\left[\begin{array}{lll}
\mathbf{0}_{1 \times 3} & \frac{g}{V_{T}} & 0
\end{array}\right]
$$

and extracts the heading rate from the DPDR law state vector $\boldsymbol{x}_{D P D R}$. Next, the state space model in Equation (5.93) is augmented with the cross-track error state,

$$
\left[\begin{array}{c}
\dot{\boldsymbol{x}}_{H} \\
\dot{y}
\end{array}\right]=\left[\begin{array}{cc}
\boldsymbol{A}_{H} & \mathbf{0}_{6 \times 1} \\
\mathbf{C}_{\dot{y}} & 0
\end{array}\right]\left[\begin{array}{c}
\boldsymbol{x}_{H} \\
y
\end{array}\right]+\left[\begin{array}{c}
\boldsymbol{B}_{H} \\
\mathbf{0}_{1 \times 2}
\end{array}\right]\left[\begin{array}{c}
\phi_{\text {Ref }} \\
\beta_{\text {Ref }}
\end{array}\right]+\left[\begin{array}{c}
\mathbf{0}_{6 \times 1} \\
-\bar{V}_{T}
\end{array}\right] \psi_{\text {Track }}
$$

where,

$$
C_{\dot{y}}=\left[\begin{array}{ll}
\mathbf{0}_{1 \times 5} & \bar{V}_{T}
\end{array}\right]
$$

and extracts the rate of change of the cross-track error from the heading state vector $\boldsymbol{x}_{H}$.

Derivative Control Gain Concerning the first feedback loop gain, $K_{D}$, first obtain the transfer function from the roll angle reference to the rate of change of the cross-track error,

$$
\frac{\dot{y}(s)}{\phi_{R e f}(s)}=\mathbf{C}_{\dot{y}}\left(s I-\boldsymbol{A}_{H}\right)^{-1} \boldsymbol{B}_{H_{\phi_{R e f}}}
$$

where $\boldsymbol{B}_{H_{\phi_{R e f}}}$ is the first column of $\boldsymbol{B}_{H}$. Figure 5.48 shows the root locus with respect to a derivative feedback gain, with the architecture as shown in Figure 5.47.

After obtaining a suitable derivative gain, the closed-loop model after derivative feedback can be obtained as follows,

$$
\begin{aligned}
\dot{\boldsymbol{x}}_{C T R} & =\mathbf{A}_{C T R} \boldsymbol{x}_{C T R}+\mathbf{B}_{C T R} \boldsymbol{u}+\mathbf{N}_{C T R} \psi_{\text {Track }} \\
{\left[\begin{array}{c}
\dot{\boldsymbol{x}}_{H} \\
\dot{y}
\end{array}\right] } & =\left[\begin{array}{cc}
\boldsymbol{A}_{H}-\boldsymbol{B}_{H_{\phi_{R e f}}} K_{D} \mathbf{C}_{\dot{y}} & \mathbf{0}_{6 \times 1} \\
\mathbf{C}_{\dot{y}} & 0
\end{array}\right]\left[\begin{array}{c}
\boldsymbol{x}_{H} \\
y
\end{array}\right]+\left[\begin{array}{cc}
\boldsymbol{B}_{H_{\phi_{R e f}}} K_{D} & \boldsymbol{B}_{H_{\beta R e f}} \\
0 & 0
\end{array}\right]\left[\begin{array}{c}
\dot{y}_{R e f} \\
\beta_{R e f}
\end{array}\right] \\
& +\left[\begin{array}{c}
\boldsymbol{B}_{H_{\phi_{R e f}} K_{D}} \bar{V}_{T} \\
-\bar{V}_{T}
\end{array}\right] \psi_{\text {Track }}
\end{aligned}
$$




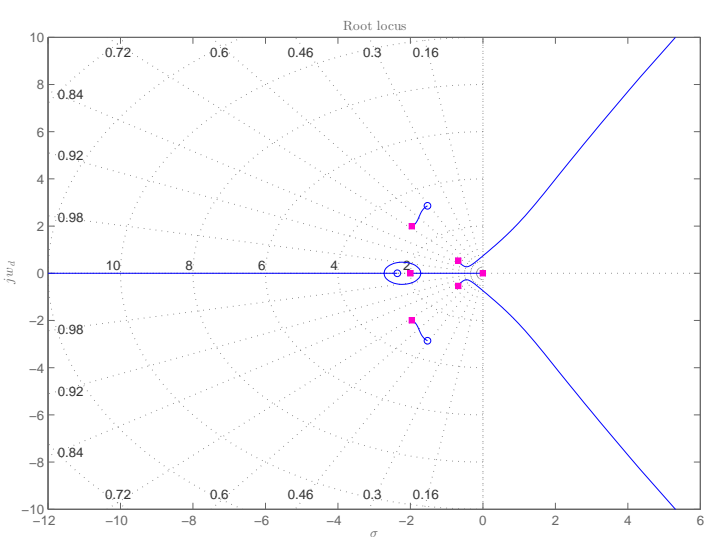

(a) Full view

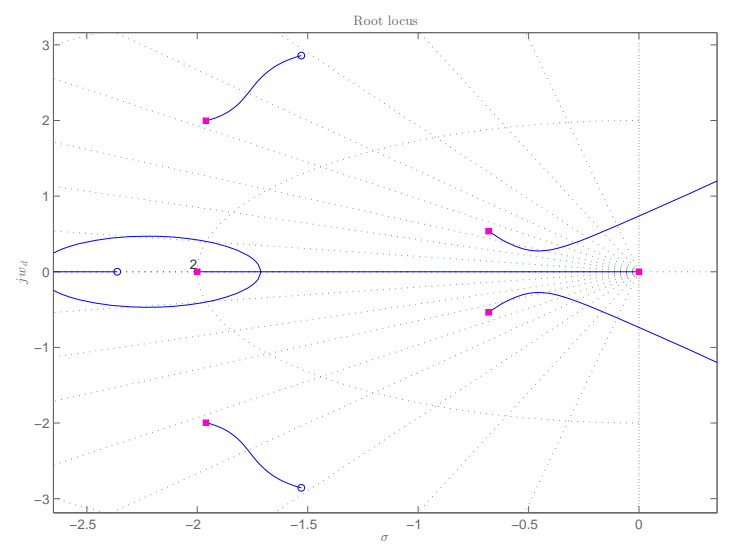

(b) Zoomed-in view

Figure 5.48: Root locus of derivative feedback loop with respect to a derivative feedback gain $K_{D}=0$.

Proportional Control Gain Concerning the second proportional gain in Figure 5.47, namely $K_{P}$, the transfer function from the cross-track error rate reference to the cross-track error is obtained as follows,

$$
\frac{y(s)}{\dot{y}_{R e f}(s)}=\mathbf{C}_{y}\left(s I-\boldsymbol{A}_{C T R}\right)^{-1} \boldsymbol{B}_{C T R_{\dot{y}_{R e f}}}
$$

where $\boldsymbol{B}_{C T R_{\dot{y}_{R e f}}}$ is the first column of $\boldsymbol{B}_{C T R}$. Figure 5.49 shows the root locus with respect to a proportional control gain, with the architecture as shown in Figure 5.47. This root locus was for a designed derivative feedback gain $K_{D}=0$.

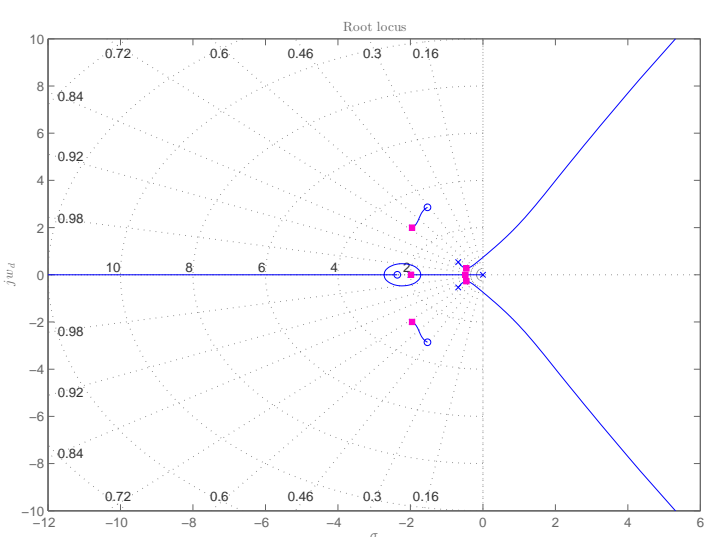

(a) Full view

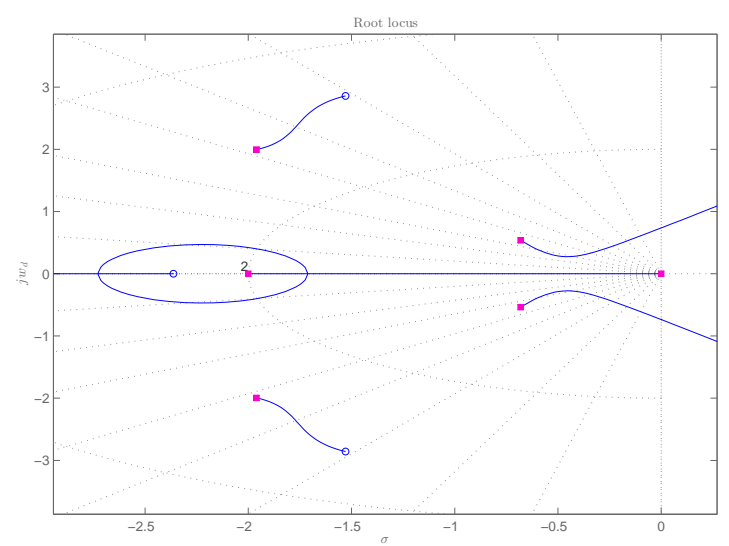

(b) Zoomed-in view

Figure 5.49: Root locus of proportional control loop using $K_{D}$ with respect to a proportional control gain $K_{P}=0$. 


\section{Specifications}

The specifications were selected based on a sense of what seems desired for lateral tracking performance. Since these specifications will determine where the desired closed-loop poles need to be for the proportional control loop, the control gain of the derivative feedback loop is firstly chosen such that the desired closed-loop poles for the cross-track controller gain be achieved with only the proportional gain $K_{P}$. Figure 5.50 shows the root locus and unit step response for the derivative loop with $K_{P}=0$. The specifications were then chosen for the cross-track controller (or the proportional control loop) as follows: minimal overshoot not more than $5 \%$, an $80 \%$ rise time of less than 30 seconds, and a $2 \%$ settling time of no more than a minute $\left(t_{2 \% s}<60 s\right)$. Figure 5.51 shows the root locus that meets these specifications and the unit step response of the cross-track controller.

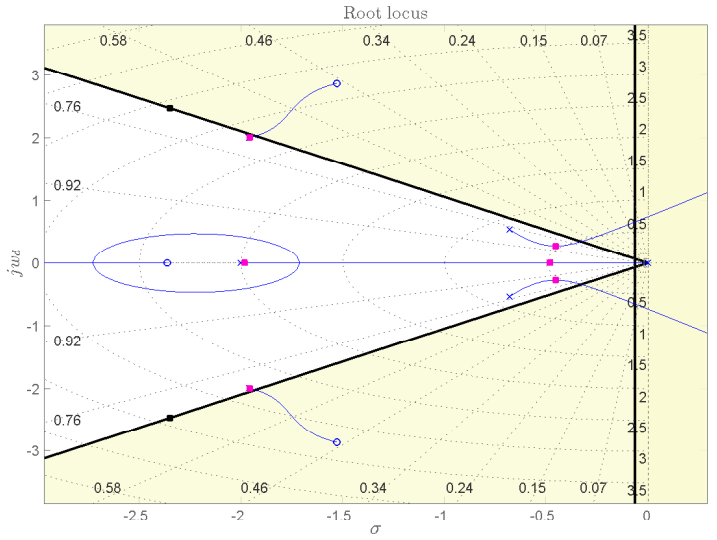

(a) Root locus

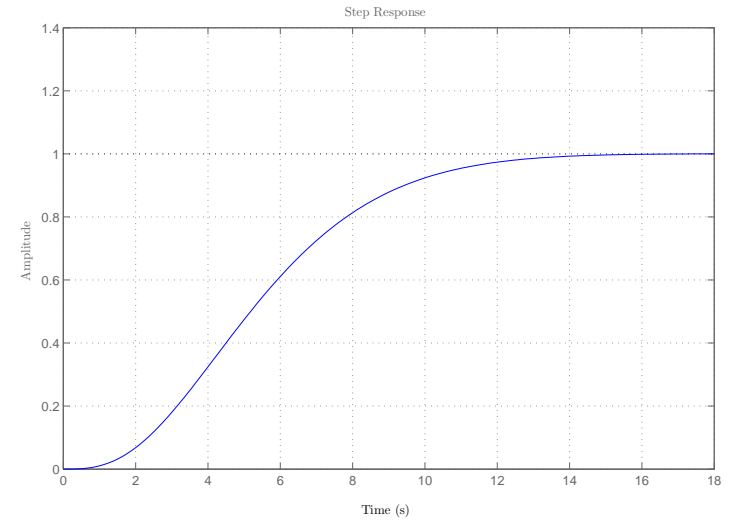

(b) Unit step response

Figure 5.50: Root locus and unit step response of derivative loop controller gain $K_{D}$ with the proportional gain $K_{P}=0$.

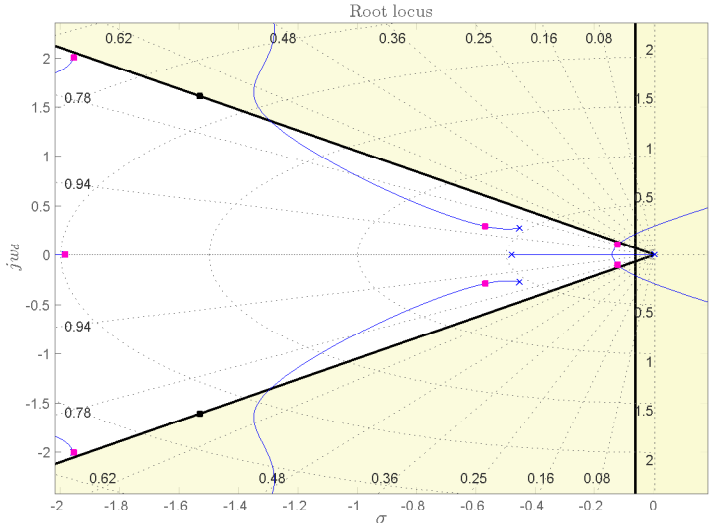

(a) Root locus

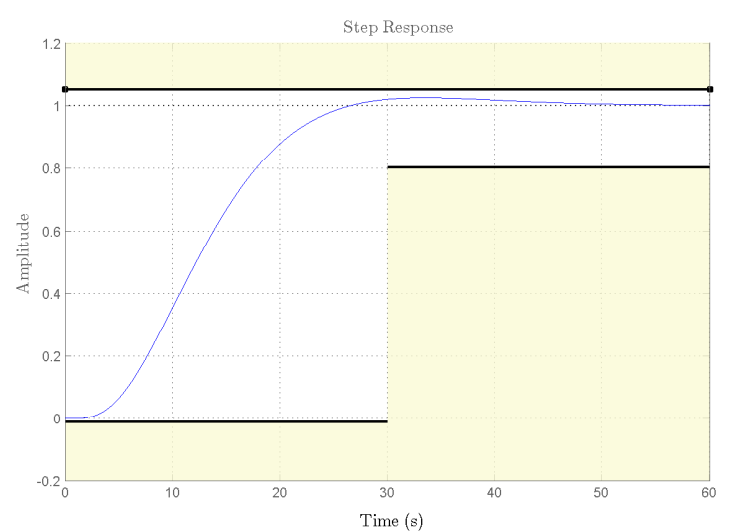

(b) Unit step response

Figure 5.51: Root locus and unit step response of cross-track controller for a designed proportional $K_{P}$ and derivative $K_{D}$ gain. 


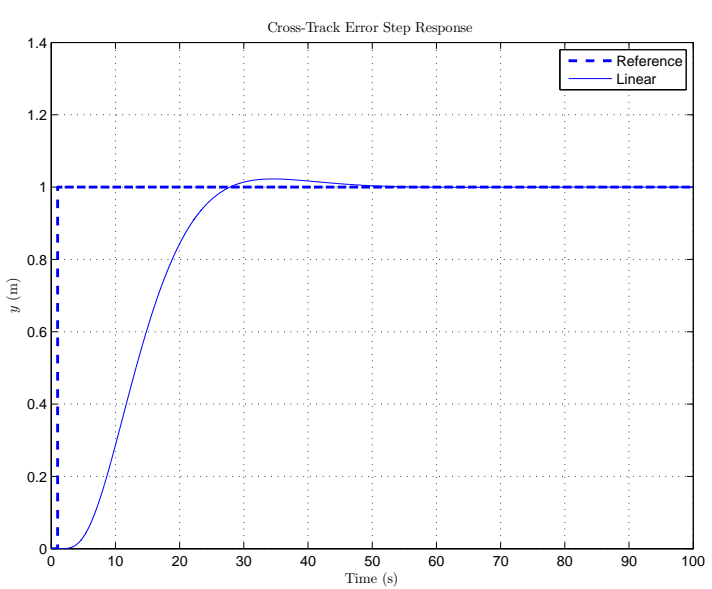

(a) Cross-Track error response

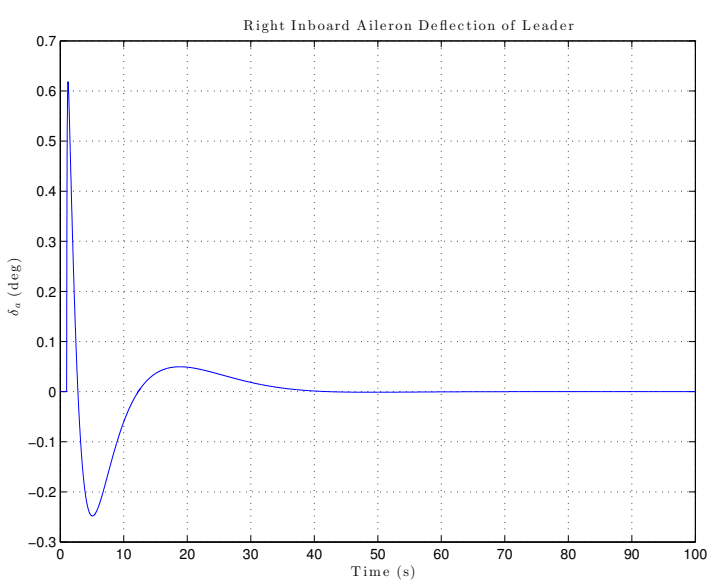

(c) Aileron response

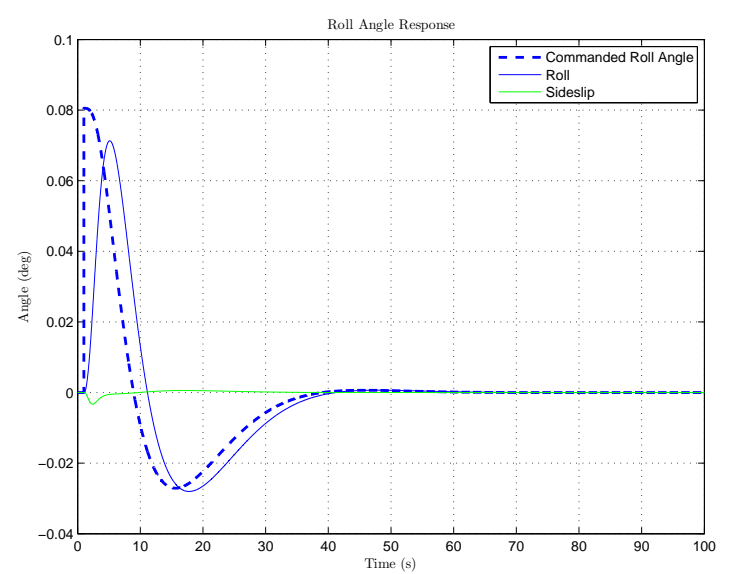

(b) Roll response

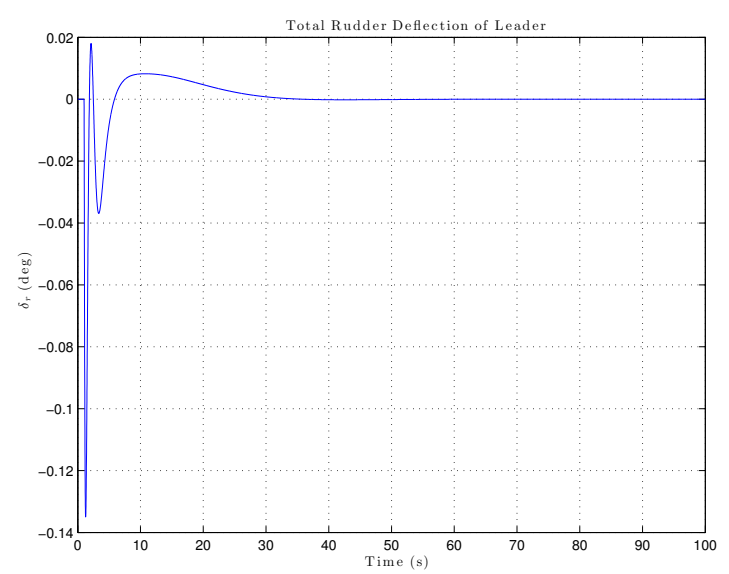

(d) Rudder response

Figure 5.52: Cross-track error response of the full-order linear aircraft model after adding the cross-track error guidance controller to the lateral control architecture.

\section{Results}

Figure 5.52 shows the unit step cross-track error response, as well as the corresponding roll response of the full-order linear aircraft model. The cross-track error response has a settling time of well under a minute. It also exhibits a small amount of overshoot, but less than 5\%, as desired. In the roll response, the DPDR law ensures that the roll angle is tracked with zero steady-state error, and minimises the sideslip during the roll. 


\section{Closed-Loop Model}

After obtaining a suitable proportional and derivative gain, the closed-loop model that encapsulates the lateral dynamics, the DPDR law and the designed cross-track controller can be obtained as follows,

$$
\begin{aligned}
\dot{\boldsymbol{x}}_{C T} & =\mathbf{A}_{C T} \boldsymbol{x}_{C T}+\mathbf{B}_{C T} \boldsymbol{u}+\mathbf{N}_{C T} \psi_{\text {Track }} \\
& =\left[\boldsymbol{A}_{C T R}-\boldsymbol{B}_{C T R_{\dot{y}_{R e f}}} K_{P} \mathbf{C}_{y}\right]\left[\begin{array}{c}
\boldsymbol{x}_{H} \\
y
\end{array}\right]+\left[\boldsymbol{B}_{C T R_{\dot{y}_{R e f}} K_{P}} \boldsymbol{B}_{C T R_{\beta R e f}}\right]\left[\begin{array}{c}
y_{R e f} \\
\beta_{R e f}
\end{array}\right] \\
& +\mathbf{N}_{C T R} \psi_{\text {Track }}
\end{aligned}
$$

Figure 5.53 shows the new closed-loop poles after adding the cross-track error controller.

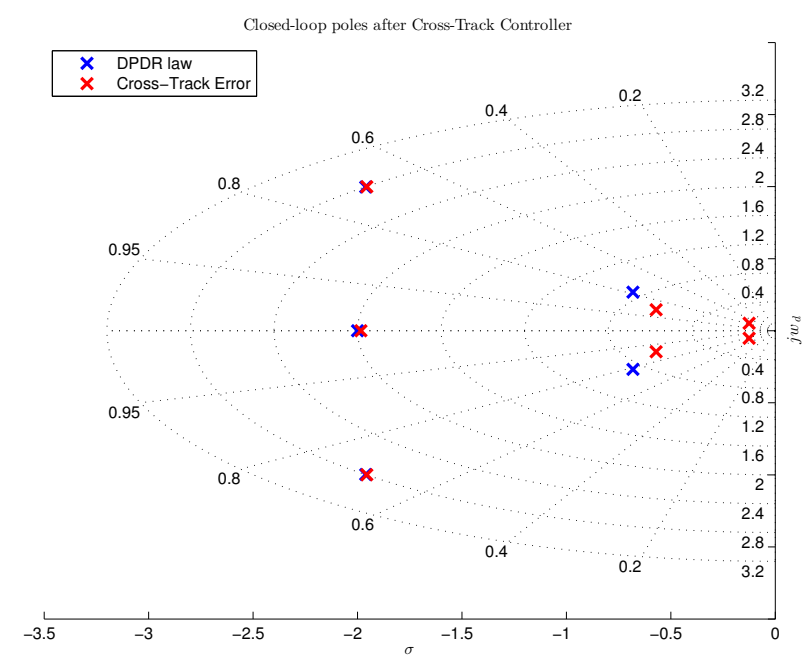

Figure 5.53: Closed-loop poles after adding the cross-track error guidance controller.

\subsection{Conventional Flight Control Simulations}

All of the designed longitudinal and lateral controllers will now be implemented in an integrated formation flight simulation model, as illustrated in Figure 3.20. In this section, a discussion is given for various simulation results using the designed conventional fly-by-wire flight control systems, as well as the conventional guidance laws on an isolated aircraft. No wind is assumed and only relevant results are illustrated in this section. Appendix E documents all the results for longitudinal and lateral manoeuvres 1 .

\subsubsection{Longitudinal Controller Performance}

A $5 \mathrm{~m}$ altitude step command and a $5 \mathrm{~m} / \mathrm{s}$ airspeed step command was executed to evaluate the longitudinal controller performance.

\footnotetext{
${ }^{1}$ Including the response of the actuators.
} 


\section{Altitude Step}

For a $5 \mathrm{~m}$ altitude step command, all linear and non-linear longitudinal responses match closely. Figure 5.54 shows a comparison of the altitude step response of the linear model and the output of the full non-linear simulation. The non-linear model agrees closely with the linear model, exhibiting time characteristics as summarised in Table 5.1. It exhibits a second-order response with overshoot slightly less than designed for, peaking after 10 seconds and settling with a $2 \%$ error bound just under 20 seconds.

Table 5.1: Comparison of altitude response specifications.

\begin{tabular}{lrrr}
\hline Specification & Desired & Linear & Non-Linear \\
\hline Peak Time (s) & 10 & 14.4 & 14.5 \\
\% Overshoot & $10-15$ & 9.14 & 6.76 \\
2\% Settling Time (s) & 20 & 19.44 & 19.19 \\
\hline
\end{tabular}

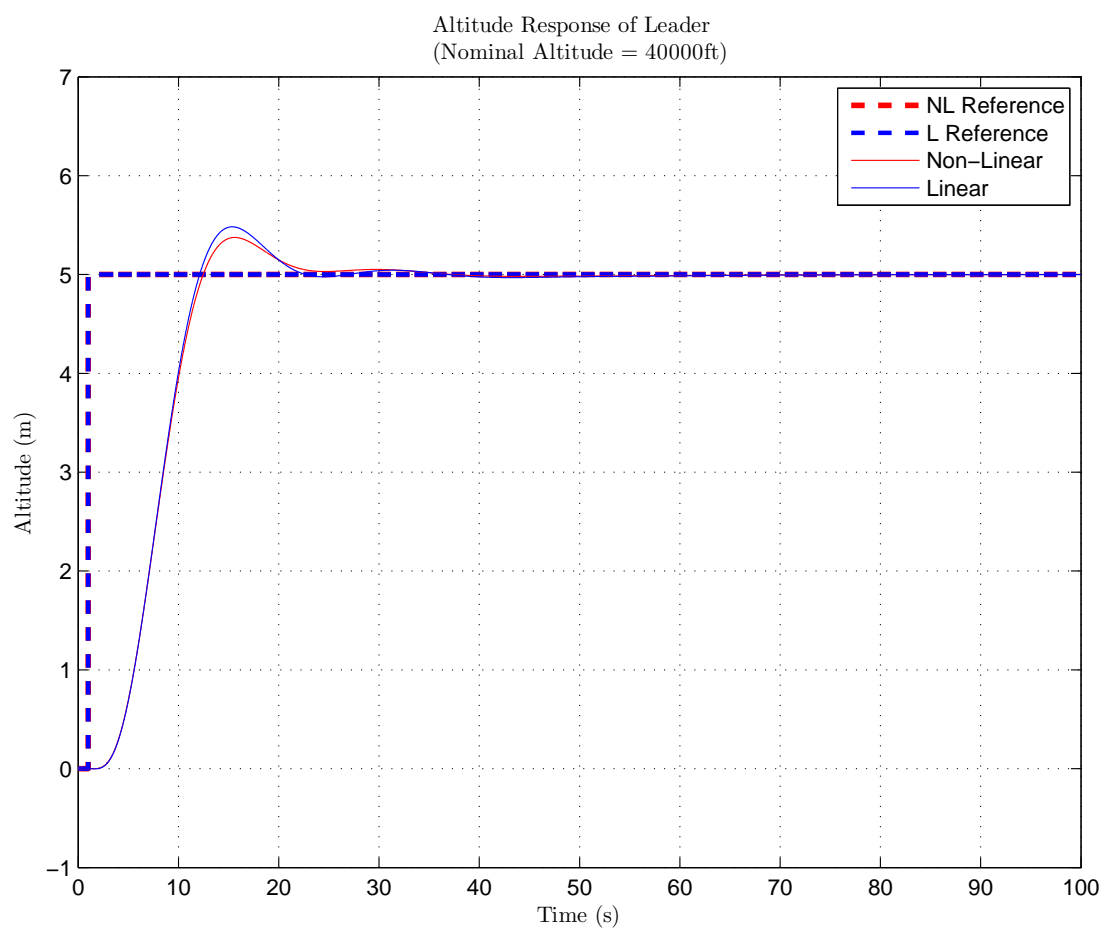

Figure 5.54: Comparison of the altitude step response of the linear model and the output of the full non-linear simulation.

\section{Airspeed Step}

For a $5 \mathrm{~m} / \mathrm{s}$ airspeed step command, all but the altitude and climb rate linear and non-linear longitudinal responses, illustrated in Figure E.2, match closely. Figure 5.55 shows the airspeed step response and Table 5.2 summarises the response characteristics. The non-linear model 
agrees closely with the linear model and exhibits a very well-damped response with a $2 \%$ settling time of approximately $40 \mathrm{~s}$, less than desired. It is noted here that although the response might be overdamped, the slightly faster response is advantageous for formation flight, increasing the chances of effectively maintaining the desired axial separation between the aircraft.

Table 5.2: Comparison of airspeed response specifications.

\begin{tabular}{lrrr}
\hline Specification & Desired & Linear & Non-Linear \\
\hline Damping $(\zeta)$ & 0.9 & 1 & 1 \\
$2 \%$ Settling Time $(\mathrm{s})$ & 50 & 42.23 & 36.85 \\
\hline
\end{tabular}

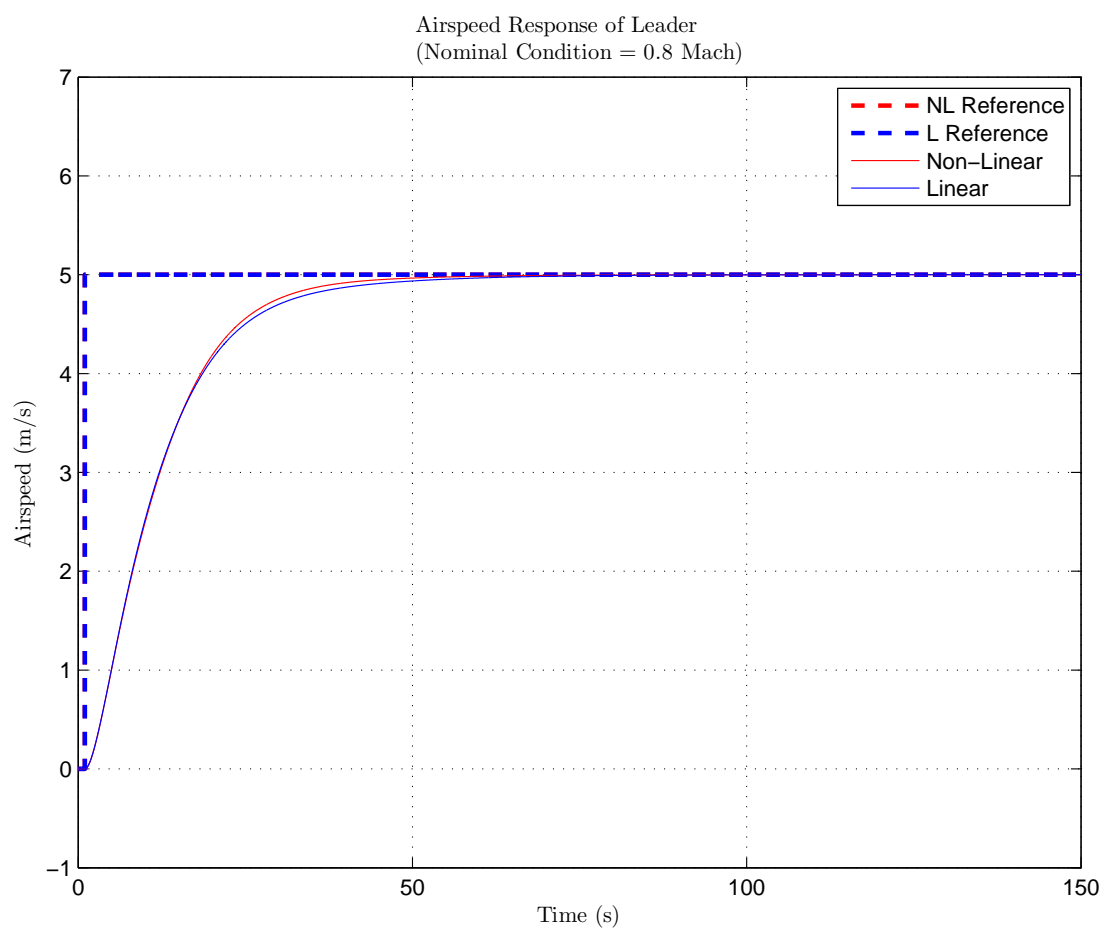

Figure 5.55: Comparison of the airspeed step response of the linear model and the output of the full non-linear simulation.

An unexpected result is illustrated in Figure 5.56a, showing that for a commanded airspeed, the linear model exhibits a steady-state altitude error after achieving the desired airspeed. The non-linear model behaves as expected, though: initially increasing with increasing airspeed, but eventually returning to the desired altitude reference. The altitude steady-state error in the linear model is caused by the steady-state error in the climb rate response. Consider Figure 5.56b the commanded climb rate remains non-zero - trying to retain the desired altitude - but the measured linear climb rate returns to zero after the response transients. The solution to this undesired response in the linear model remains unknown, but it is expected to be a result of unaccounted coupling between airspeed and climb rate. 


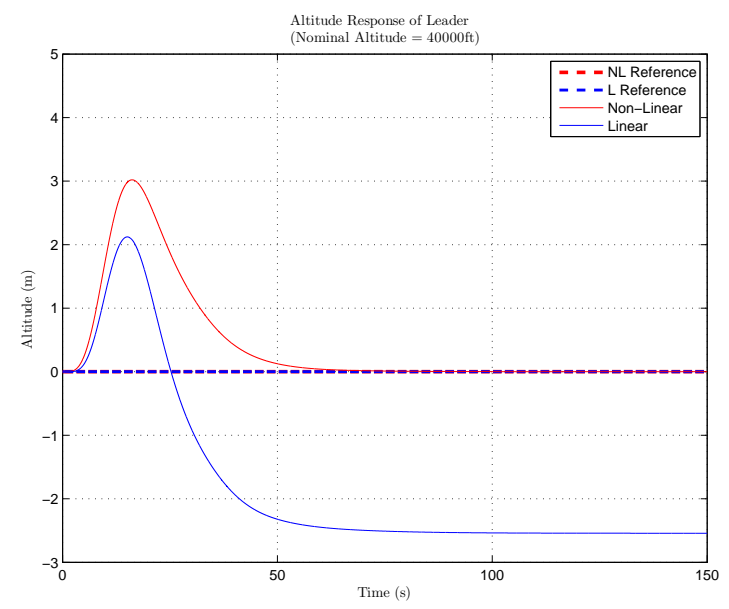

(a) Altitude response

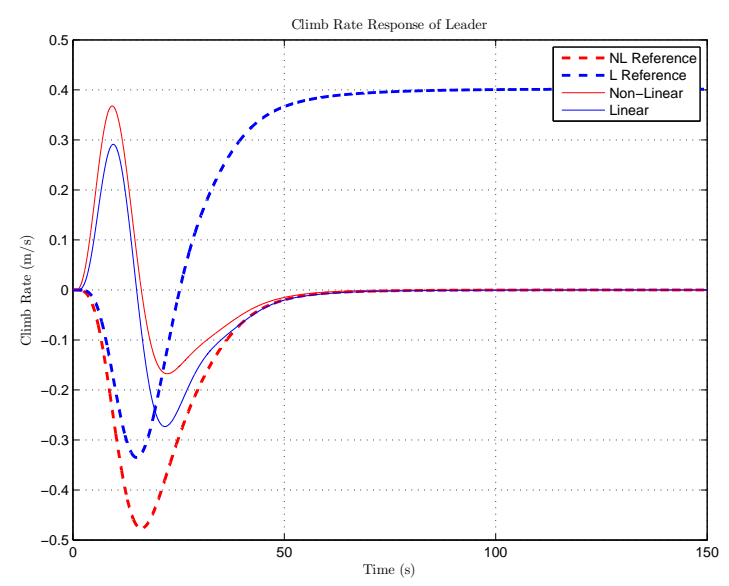

(b) Climb rate response

Figure 5.56: Unexpected response in linear model for an airspeed step command, showing how the linear model exhibits a steady-state error in the altitude and climb rate response when an airspeed step is commanded.

\subsubsection{Lateral Controller Performance}

A $5^{\circ}$ roll angle step command, a $1^{\circ}$ sideslip angle step command, and a $5 \mathrm{~m}$ commanded crosstrack error was exectured to evaluate the lateral controller performance.

\section{Roll and Sideslip Angle Steps}

Figure 5.57 shows the lateral response of the linear and non-linear model, with only the DPDR law active for lateral-directional control. For a $5^{\circ}$ roll angle step command, the linear and non-linear responses match very well, and the response characteristics are summarised in Table 5.3. The peak time for the roll response is well below 10 seconds, with a very small amount of overshoot. The steady-state error in the linear and non-linear model responses is very small (approximately 2\%). As desired, the turn co-ordination controller minimised the amount of sideslip during a commanded roll angle to a negligibly small value.

Table 5.3: Comparison of roll response specifications.

\begin{tabular}{lrrr}
\hline Specification & Desired & Linear & Non-Linear \\
\hline Peak Time (s) & $<10$ & 6.15 & 6.15 \\
\% Overshoot & $<5$ & 1.94 & 1.94 \\
Sideslip (deg) & 0 & -0.116 & -0.116 \\
\hline
\end{tabular}

Regarding the sideslip response in Figure $5.57 \mathrm{~b}$, for a $1^{\circ}$ sideslip angle step command, the linear and non-linear responses match very well. The response characteristics are summarised in Table 5.4. The realised sideslip is only half of the commanded sideslip, exhibiting an overshoot of roughly $20 \%$. Exhibiting a natural response during a sideslip command, the achieved steadystate ratio for a bank angle induced by a sideslip angle command is, 


$$
\frac{\phi_{s s}}{\beta_{s s}} \approx \frac{-2.1}{0.5}=-4.2
$$

Table 5.4: Comparison of sideslip response specifications.

\begin{tabular}{lrrr}
\hline Specification & Desired & Linear & Non-Linear \\
\hline Peak Time (s) & $<10$ & 1.3 & 1.3 \\
$\%$ Overshoot & 20 & 23.64 & 23.64 \\
$\phi_{s s} / \beta_{\text {ss }}$ & -5 & -4.2 & -4.2 \\
\hline
\end{tabular}

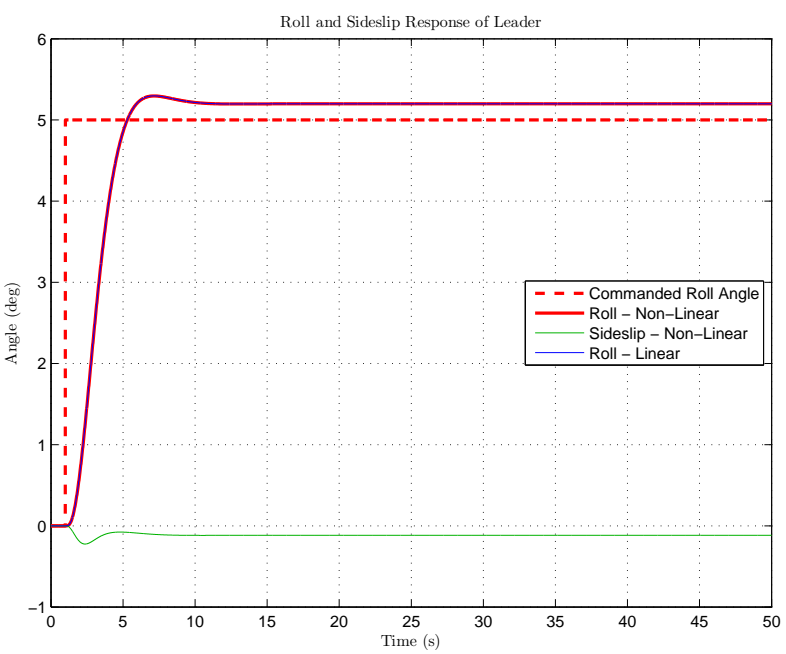

(a) Lateral Response for a commanded roll

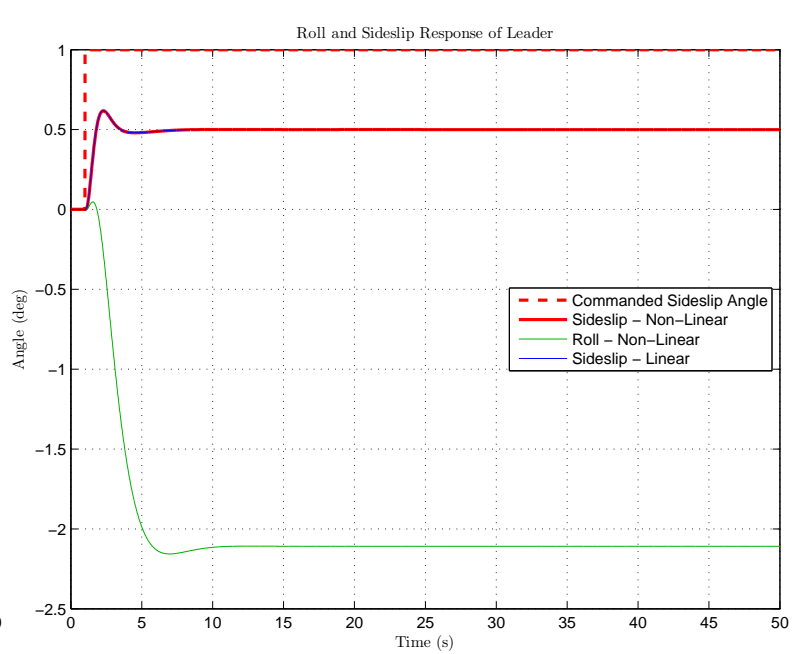

(b) Lateral Response for a commanded sideslip

Figure 5.57: The lateral response of the linear and non-linear model with only the DPDR law active for lateral-directional control. As shown, the linear and non-linear models share the same response with good characteristics.

\section{Cross-Track Error Step}

Figure $5.58 \mathrm{a}$ shows the step response of the cross-track error for a $5 \mathrm{~m}$ commanded tracking error. A comparison of the specifications for the desired and realised characteristics is given in Table 5.5. The linear model response characteristics are well within the desired specifications, but the non-linear model exhibits a larger overshoot (almost 10\%). In an attempt to decrease the overshoot to below $5 \%$, a rate limiter can be added to the reference input of the cross-track guidance controller. Although the overshoot will improve, the rise and settling time may increase substantially. An acceptable trade-off between overshoot and response time is selected and the focus is given to reducing the overshoot. As such, the response after adding a rate limiter is illustrated in Figure $5.58 \mathrm{~b}$. 
Table 5.5: Comparison of cross-track error tracking response specifications.

\begin{tabular}{lrrrr}
\hline Specification & Desired & Linear & Non-Linear & Rate Limited \\
\hline \% Overshoot & $<5 \%$ & 2.26 & 8.98 & 3.32 \\
80\% Rise Time (s) & $<30$ & 17.84 & 19.9 & 52.43 \\
2\% Settling Time (s) & $<60$ & 36.93 & 54.3 & 86.29 \\
\hline
\end{tabular}

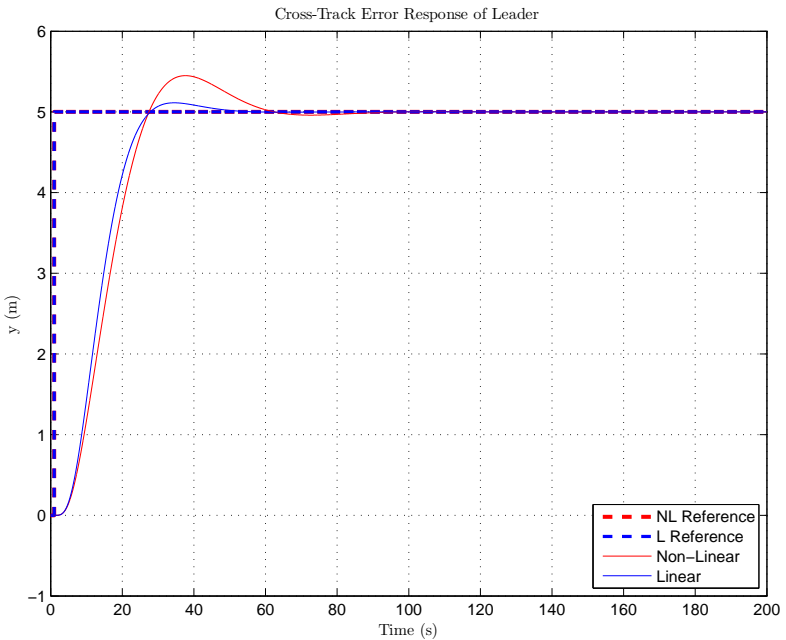

(a) No rate limiter at input

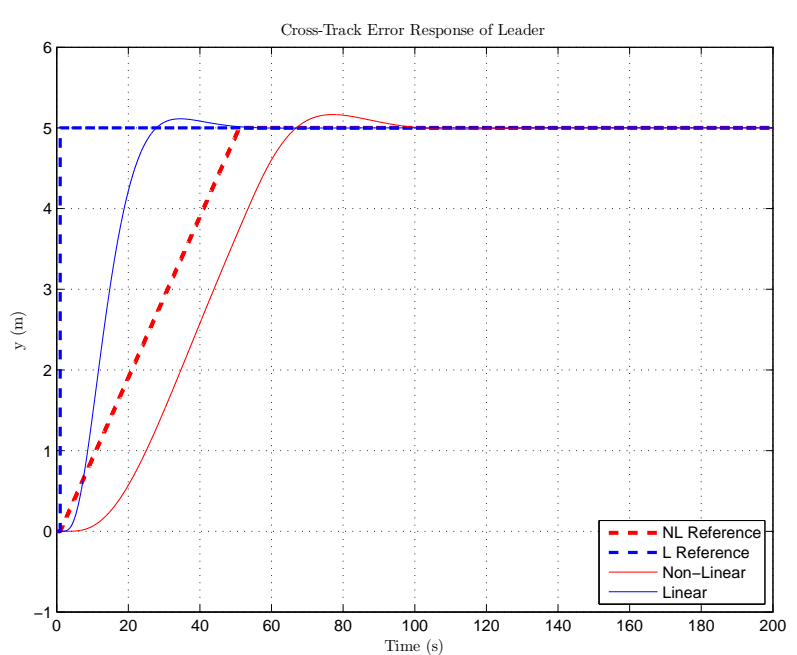

(b) With rate limiter at input

Figure 5.58: Lateral tracking response for a $5 \mathrm{~m}$ step command.

\subsection{Conclusions}

In this chapter, a representative conventional fly-by-wire architecture and a set of guidance laws were designed, implemented and verified in an integrated simulation. Longitudinal manoeuvres are controlled using: a DQ law with incremental normal load factor control; an autothrust controller to command a thrust based on airspeed; and finally the altitude-hold guidance law to obtain a desired altitude with FPA or CR specific control. For lateral-directional control, there was a DPDR law that controls the roll $\phi$ and sideslip angle $\beta$ of the aircraft, using rudder turn co-ordination during roll; and ensuring a natural roll response during sideslip, enclosed by a rudimentary cross-track error guidance law to navigate along a set of waypoints. The stability and response characteristics of the representative fly-by-wire system as well as the set of guidance laws were evaluated for a conventional isolated aircraft in simulation. Specifications were reasonably achieved with a good match between the linear and non-linear model responses.

With the conventional fly-by-wire architecture in place, it was shown by Büchner that no changes need to be made to the inner-loop controllers, and only minor changes to the outer-loop guidance laws are required for formation flight aircraft [4]. The next chapter focuses on the design, implementation and verification of the extended formation flight specific guidance laws. 


\section{Chapter 6}

\section{Extended Formation Flight Control Systems Design}

In this chapter, the flight control system architecture will be extended for a formation follower aircraft. The inner-loop fly-by-wire flight control architecture needs to remain representative of that used in modern-day transport aircraft. As such, the inner-loop flight control architecture remains unchanged. At this point, the goal is not to produce an optimal controller for passenger comfort, but instead to create a system that will be successful in ensuring that the follower maintains the formation separations with acceptable performance.

This chapter starts with an overview of the extended formation flight control architecture. This is followed by the design of three formation-specific guidance controllers: an axial guidance controller, required to maintain the desired geometric longitudinal separation $\xi$ by commanding a desired airspeed; a vertical guidance controller, required to maintain the desired geometric vertical separation $\zeta$; and lastly a lateral guidance controller, required to maintain the desired geometric lateral separation $\eta$. To evaluate the performance of the extended formation flight guidance laws, extended simulations were performed at different levels of turbulence intensity and geometric lateral separations. The feasible and most practical regions of geometric separation are investigated. The ability to maintain the separations after the leader performs longitudinal manoeuvres is also investigated. Finally, the formation-hold performance of the controllers is evaluated in simulation.

\subsection{Extended Formation Flight Control Architecture}

Figure 6.1 illustrates the flight control architecture for extended formation flight control. The inner-loop flight controllers are the conventional fly-by-wire flight controllers in the Normal law configuration. The fly-by-wire architecture was not redesigned for the follower aircraft. To command the fly-by-wire flight control, a set of formation-extended guidance laws is required, used to guide the follower aircraft by maintaining the relative geometric separation between the two formation aircraft. 


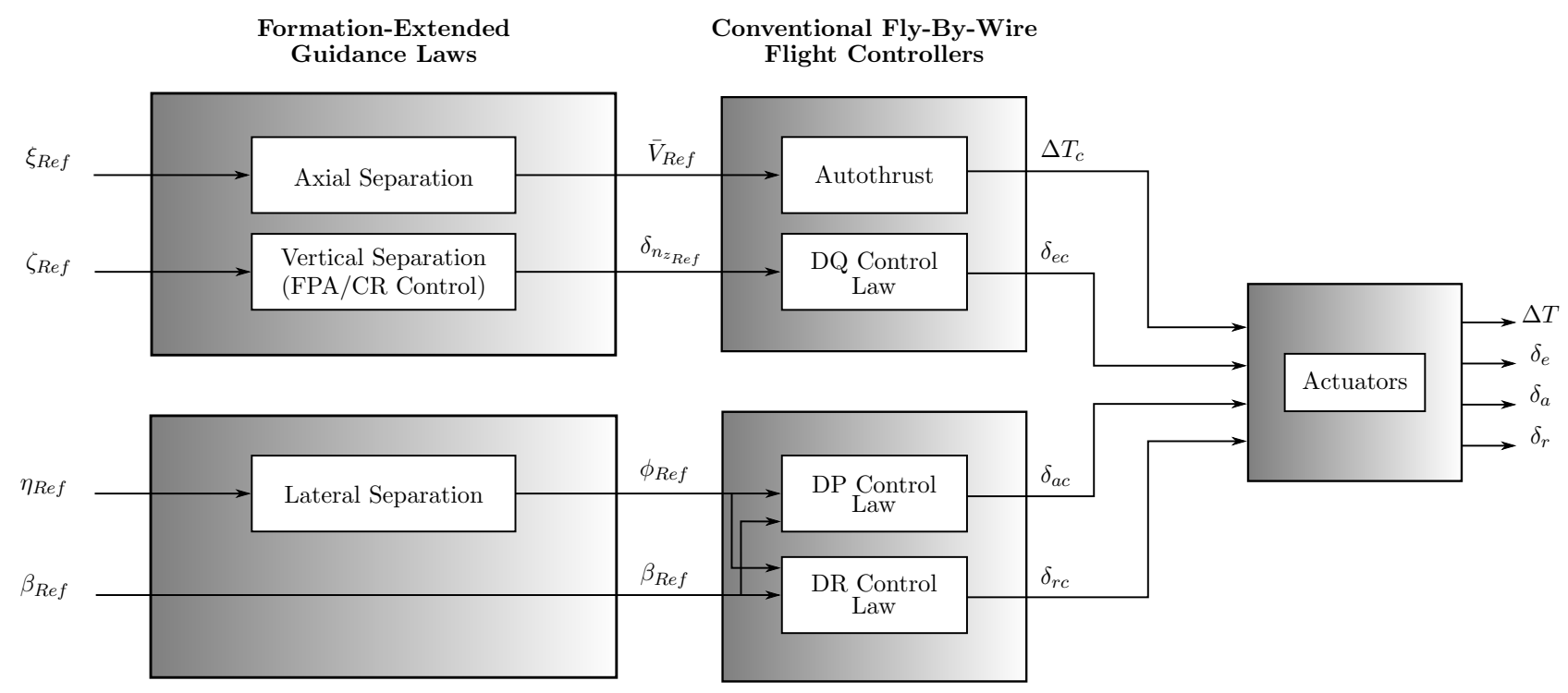

Figure 6.1: Overview of the extended formation flight control architecture.

\subsubsection{Formation-Extended Guidance Laws}

Regarding the longitudinal guidance laws, an axial separation controller is added to maintain the desired longitudinal in-track distance between the aircraft. A vertical separation controller is required to maintain the differential altitude between the aircraft. For the lateral-directional guidance laws, a lateral separation controller is added, which is used to maintain the geometric lateral separation between the aircraft during formation flight.

\subsection{Longitudinal Formation Guidance}

In this section, the longitudinal guidance depicted in Figure 6.1, namely the axial and vertical separation guidance laws, will be designed, implemented and verified in simulations.

\subsubsection{Axial Guidance Controller}

An autothrust controller, capable of maintaining or recovering to a desired airspeed, has been designed in Chapter 5 and is used by both the leader and follower aircraft. The axial controller, which is sometimes referred to as the longitudinal guidance controller, is illustrated in Figure 6.2. It is designed specifically for the follower aircraft as an extra outer-loop controller, required to maintain the desired geometric geometric separation $\xi$ by commanding a desired airspeed.

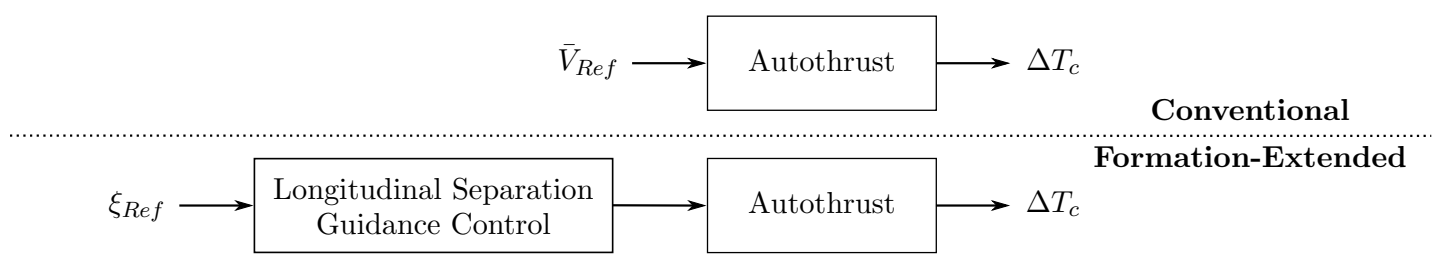

Figure 6.2: Illustration of the architectural change required from the conventional autothrust controller to the formation-extended axial separation and autothrust controller. 


\section{Design}

The architecture of the longitudinal axial separation controller is shown in Figure 6.3. The plant is the DQ law, FPA or CR and autothrust controller with the longitudinal dynamics of the aircraft, represented in state space form by Equation (5.50),

$$
\begin{aligned}
\dot{\boldsymbol{x}}_{A T} & =\boldsymbol{A}_{A T} \boldsymbol{x}_{A T}+\boldsymbol{B}_{A T} \boldsymbol{u} \\
y & =\bar{v}=\mathbf{C}_{\bar{v}} \boldsymbol{x}_{A T}
\end{aligned}
$$

where,

$$
\boldsymbol{u}=\left[\begin{array}{l}
\gamma_{R e f} \\
\bar{v}_{R e f}
\end{array}\right]
$$

and the output matrix,

$$
\mathbf{C}_{\bar{v}}=\left[\begin{array}{llllll}
1 & 0 & 0 & 0 & 0 & 0
\end{array}\right]
$$

extracts the airspeed $\bar{v}$ from the autothrust state vector $\boldsymbol{x}_{A T}$. The airspeed is naturally integrated to get the in-track distance $x$. Considering Figure 5.46, the rate of change of the in-track distance is the projection of the aircraft ground speed into the in-track axis, represented by,

$$
\dot{x}=\bar{V} \cos \left(\psi-\psi_{\text {Track }}\right)
$$

For small heading angles relative to the ground track heading, Equation (6.4) is simplified to,

$$
\dot{x}=\bar{V}
$$

Now considering the small disturbance theory, Equation (6.5) may be written as follows,

$$
\dot{x}=\Delta \bar{V}+\bar{V}_{T}
$$

where the trim airspeed $\bar{V}_{T}$ is chosen as the measured velocity of the leader aircraft.

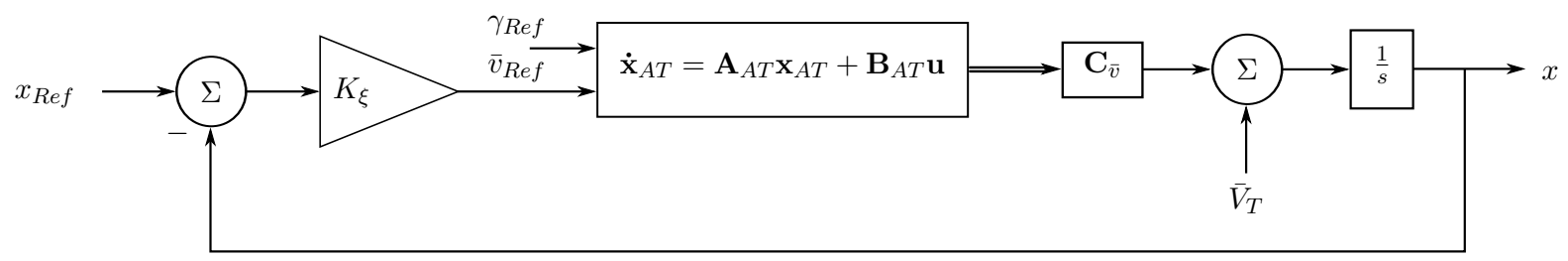

Figure 6.3: Longitudinal/axial separation controller architecture.

The axial controller consists of a classic proportional controller that generates a reference airspeed proportional to the in-track perturbation distance error. The natural integration from airspeed to in-track distance makes the system type 1, which means it should be able to follow a constant in-track perturbation distance reference with zero error at steady state. If there is an offset in the measured velocity of the leader aircraft, then using a proportional controller for 
the axial separation controller will result in a non-zero steady state error in axial separation. It is assumed that there are no measurement offsets.

The transfer function from the reference input $\bar{v}_{R e f}$ to the in-track distance output $x$ is obtained from the state space model through,

$$
\frac{x(s)}{\bar{v}_{R e f}}=\frac{1}{s} \mathbf{C}_{\bar{v}}\left(s I-\mathbf{A}_{A T}\right)^{-1} \mathbf{B}_{\bar{v}_{R e f}}
$$

where $\mathbf{B}_{\bar{v}_{R e f}}$ is the second column of $\mathbf{B}_{A T}$. Figure 6.4 shows the root locus with respect to the in-track proportional gain.

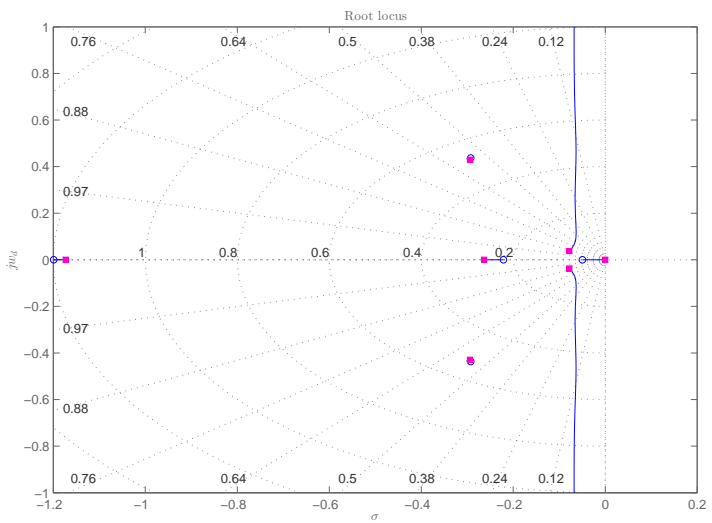

(a) Full view

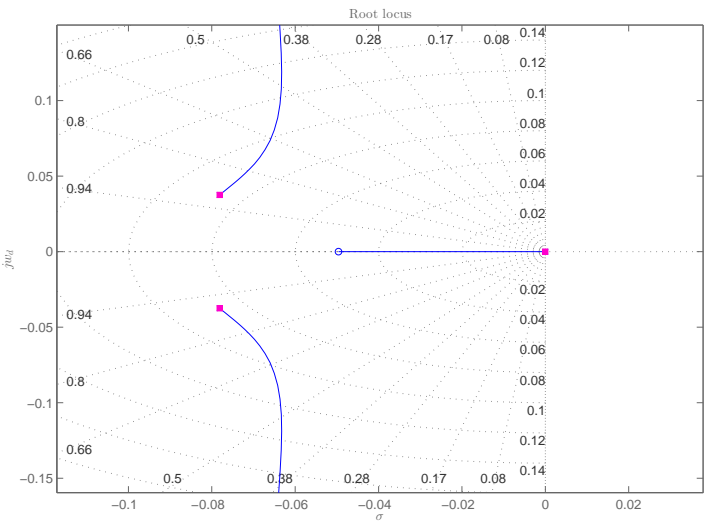

(b) Zoomed-in view

Figure 6.4: Root locus of full-order axial controller for an arbitrary proportional gain $K_{\xi}$.

\section{Specifications}

For the longitudinal separation controller, it was decided to only ensure zero steady-state error and minimal overshoot in the response. The settling time was not a constraint in this study, since the objective of this thesis lies in optimising for passenger comfort in formation flight - assuming that the formation-hold guidance laws are sufficient to maintain the geometric separation desired to reasonable accuracy. The specifications for the longitudinal separation controller are then selected to produce a second-order response, with zero steady-state error tracking for a constant reference input and minimal overshoot (less than 10\%). Figure 6.5 shows the root locus and unit step response that closely meet the desired specifications.

\section{Results}

Figure 6.6 shows the step response results with the designed axial controller in simulation. The in-track distance response, shown in Figure 6.6a, exhibits an overshoot of approximately $4.6 \%$ and achieves a zero steady-state error after approximately two minutes. 


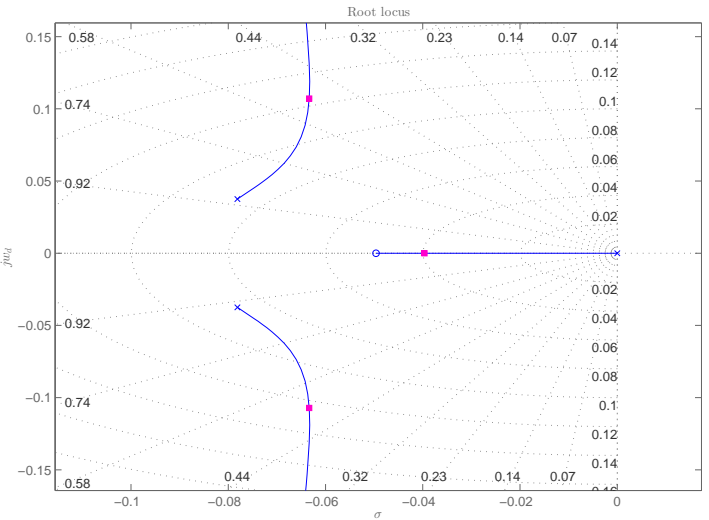

(a) Root locus

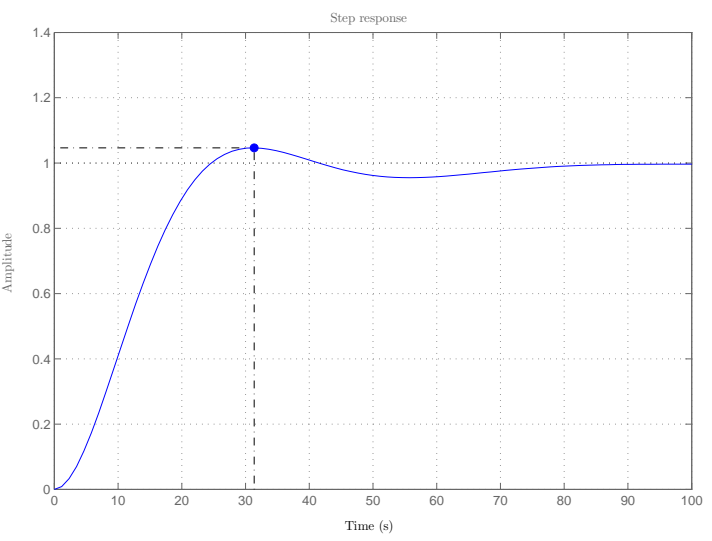

(b) Unit step response

Figure 6.5: Root locus and unit step response of axial separation guidance controller for a chosen proportional gain $K_{\xi}$. The controller was tuned to obtain the best possible response within the design requirements.

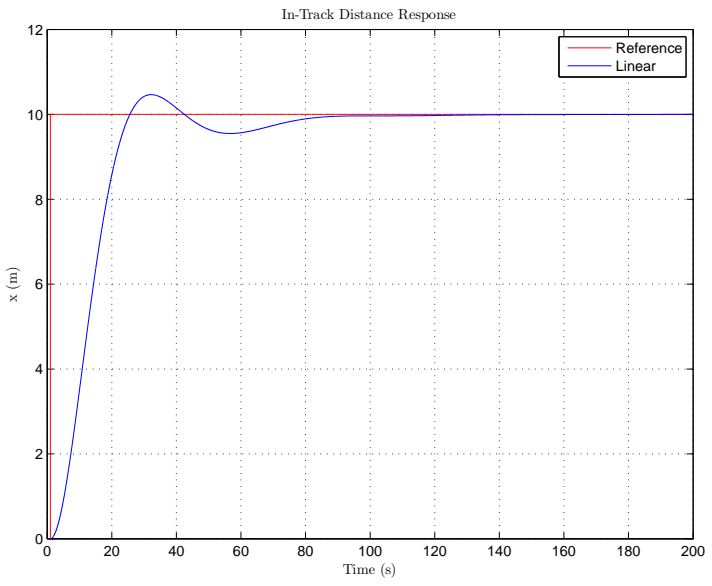

(a) In-track distance response

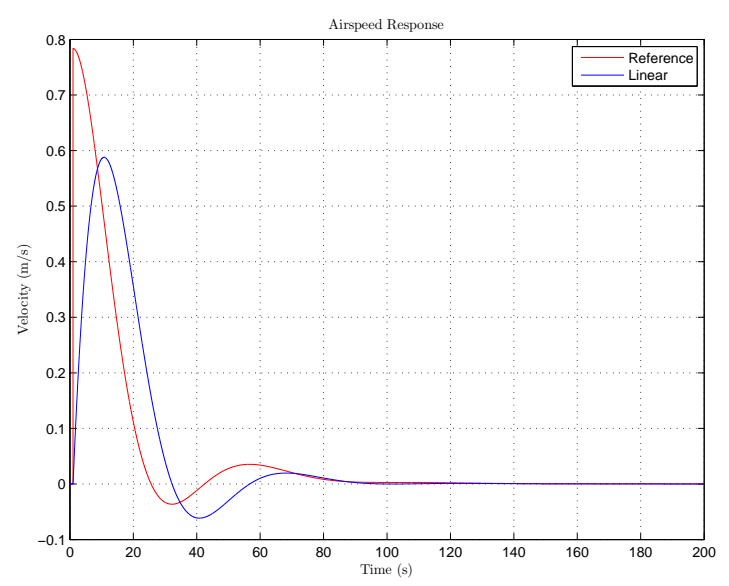

(b) Airspeed response

Figure 6.6: Full-order axial step response results for a $10 \mathrm{~m}$ in-track distance separation command.

\section{Closed-Loop Model}

Following the design of the longitudinal separation controller, a closed-loop state space model that encapsulates the longitudinal dynamics, the DQ law, FPA, autothrust and longitudinal separation controller can be derived.

The autothrust state equation $(5.50)$ is augmented to include the axial separation state variable by the inclusion of Equation 6.6,

$$
\left[\begin{array}{c}
\dot{\boldsymbol{x}}_{A T} \\
\dot{x}
\end{array}\right]=\left[\begin{array}{cc}
\boldsymbol{A}_{A T} & \mathbf{0}_{6 \times 1} \\
\mathbf{C}_{\bar{v}} & 0
\end{array}\right]\left[\begin{array}{c}
\boldsymbol{x}_{A T} \\
x
\end{array}\right]+\left[\begin{array}{cc}
\boldsymbol{B}_{\gamma_{R e f}} & \boldsymbol{B}_{\bar{v}_{R e f}} \\
0 & 0
\end{array}\right]\left[\begin{array}{c}
\gamma_{R e f} \\
\bar{v}_{R e f}
\end{array}\right]+\left[\begin{array}{c}
\mathbf{0}_{6 \times 1} \\
1
\end{array}\right] \bar{V}_{T}
$$

The control law is given as follows, 


$$
\boldsymbol{u}=\left[\begin{array}{c}
\gamma_{R e f} \\
K_{\xi}\left(x_{R e f}-x\right)
\end{array}\right]
$$

Substitution of the control law into the augmented state equation gives the final closed-loop model as follows,

$$
\begin{aligned}
\dot{\boldsymbol{x}}_{A S} & =\mathbf{A}_{A S} \boldsymbol{x}_{A S}+\mathbf{B}_{A S} \boldsymbol{u}+\mathbf{N}_{A S} \bar{V}_{T} \\
{\left[\begin{array}{c}
\dot{\boldsymbol{x}}_{A T} \\
\dot{x}
\end{array}\right] } & =\left[\begin{array}{cc}
\boldsymbol{A}_{A T} & -\boldsymbol{B}_{\bar{v}_{R e f}} K_{\xi} \\
\mathbf{C}_{\bar{v}} & 0
\end{array}\right]\left[\begin{array}{c}
\boldsymbol{x}_{A T} \\
h
\end{array}\right]+\left[\begin{array}{cc}
\boldsymbol{B}_{\gamma_{R e f}} & \boldsymbol{B}_{\bar{v}_{R e f}} K_{\xi} \\
0 & 0
\end{array}\right]\left[\begin{array}{c}
\gamma_{R e f} \\
\bar{v}_{R e f}
\end{array}\right]+\left[\begin{array}{c}
\mathbf{0}_{6 \times 1} \\
1
\end{array}\right] \bar{V}_{T}
\end{aligned}
$$

This closed-loop model will serve as the open-loop plant for the next controller: the vertical separation guidance controller. Figure 6.7 illustrates the new closed-loop poles after adding the axial separation guidance controller. The additional guidance controller added a slow mode since the autothrust state equation was augmented to include the axial separation state variable.

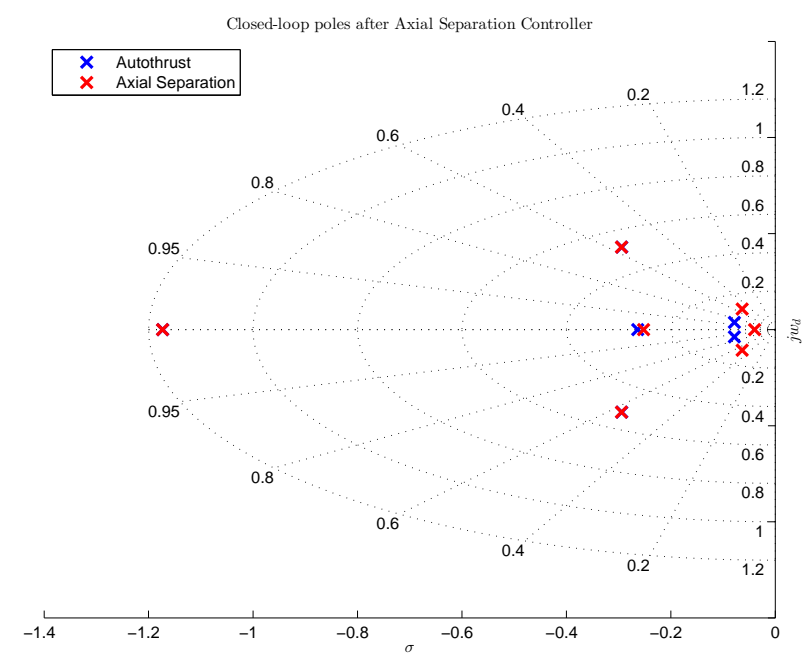

Figure 6.7: Closed-loop poles after adding the axial separation controller.

\subsubsection{Vertical Guidance Controller}

The vertical guidance controller is illustrated in Figure 6.8. The input of the vertical separation controller is the desired geometric vertical separation, in wingspans, unlike the conventional controller, which uses the altitude of the aircraft. Furthermore, with the addition of the longitudinal separation controller, the open-loop plant for the vertical guidance controller is not the same as that for the altitude controller. However, it can be seen from the results of the design to follow that the poles with the most dominant effect on the response are similar to those with the altitude-hold law for the conventional control design (see Figure 5.36 in Chapter 5). 


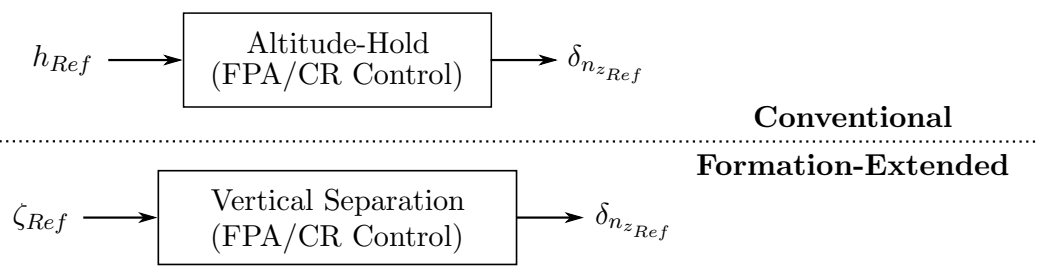

Figure 6.8: Illustration of the architectural change required from the conventional altitude-hold guidance controller to the formation-extended vertical separation controller.

\section{Design}

The architecture of the vertical separation controller is shown in Figure 6.9. The plant is the DQ law, FPA or CR, autothrust and axial separation controller with the longitudinal dynamics of the aircraft, represented in state space form by Equation 6.10,

$$
\begin{aligned}
\dot{\boldsymbol{x}}_{A S} & =\boldsymbol{A}_{A S} \boldsymbol{x}_{A S}+\boldsymbol{B}_{A S} \boldsymbol{u}+\mathbf{N}_{A S} \bar{V}_{T} \\
y & =\dot{\zeta}=\mathbf{C}_{\dot{\zeta}} \boldsymbol{x}_{A S}
\end{aligned}
$$

where,

$$
\boldsymbol{u}=\left[\begin{array}{l}
\gamma_{R e f} \\
x_{R e f}
\end{array}\right]
$$

and the output matrix,

$$
\mathbf{C}_{\dot{\zeta}}=\left[\begin{array}{lllllll}
0 & -\bar{V}_{T} / b & 0 & \bar{V}_{T} / b & 0 & 0 & 0
\end{array}\right]
$$

extracts the climb rate normalised to wingspan, and also the vertical separation rate $\dot{\zeta}$ from the axial separation state vector $\boldsymbol{x}_{A S}$. The vertical separation rate is fed into a natural integrator to become the vertical separation state $\zeta$. The vertical separation between the aircraft will require the knowledge of both aircraft's altitude. These measurements can be made with barometric pressure sensors or GPS/DGPS data. The vertical separation controller consists of a classic proportional gain that generates an FPA command $\gamma_{\text {Ref }}$ proportional to the separation error.

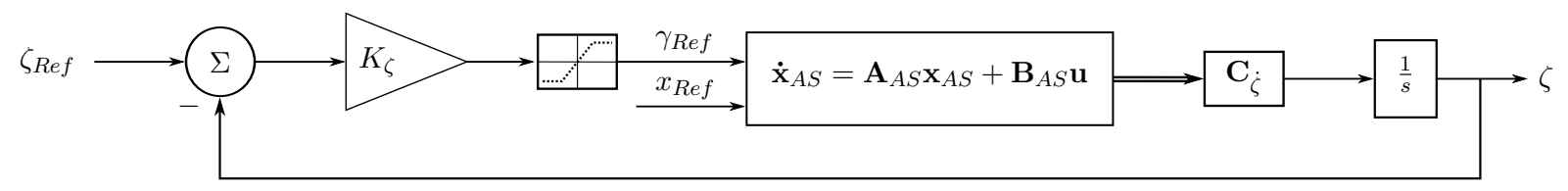

Figure 6.9: Vertical separation controller architecture.

The vertical separation rate may be written as,

$$
\dot{\zeta}=\frac{\bar{V}_{T}}{b}(\theta-\alpha)
$$

The natural integration from separation rate to vertical separation makes the system type 1 , which means it should be able to maintain a constant geometric vertical separation with zero 
error at steady state. A saturation block is added to ensure that the controller does not command FPA signals that exceed the limitations of the aircraft, and that the aircraft maintains a constant FPA when large vertical separation step commands are issued.

The transfer function from the reference input $\gamma_{\text {Ref }}$ to the vertical separation output $\zeta$ is obtained from the state space model through,

$$
\frac{\zeta(s)}{\gamma_{R e f}}=\frac{1}{s} \mathbf{C}_{\dot{\zeta}}\left(s I-\mathbf{A}_{A S}\right)^{-1} \mathbf{B}_{\gamma_{R e f}}
$$

where $\mathbf{B}_{\gamma_{R e f}}$ is the first column of $\mathbf{B}_{A S}$. Figure 6.10 shows the root locus with respect to the altitude control gain. A right-half plane (RHP) zero is found at approximately $3.5 \mathrm{rad} / \mathrm{s}$ and $4 \mathrm{rad} / \mathrm{s}$. This RHP zero seems to indicate that if you give a positive flight path angle input, then the vertical separation first goes down, and then goes up. Recall, that the inner-loop DQ Law has a non-minimum phase effect in the incremental load factor response which manifests itself here as well.

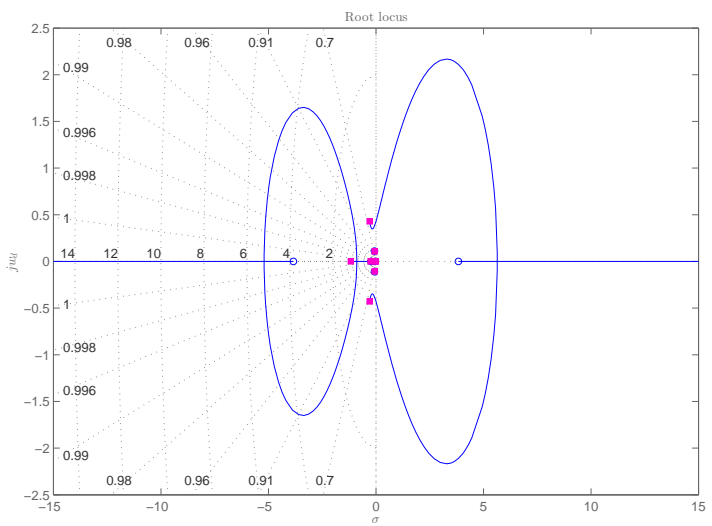

(a) Full view

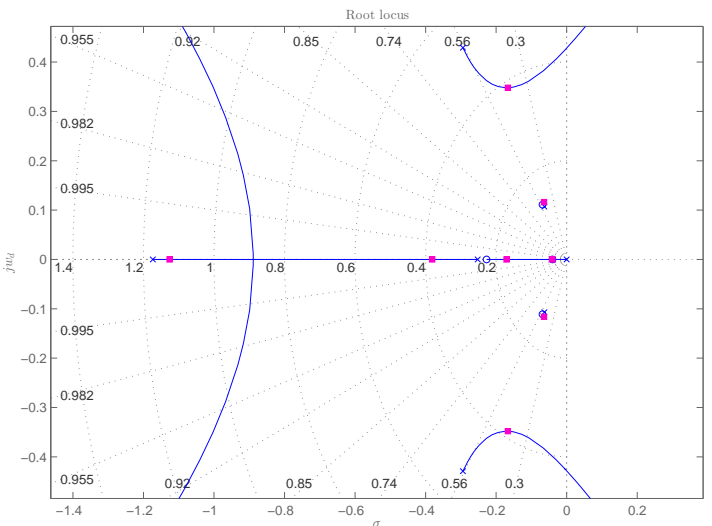

(b) Zoomed-in view

Figure 6.10: Root locus of vertical separation controller with respect to the proportional gain $K_{\zeta}$

\section{Specifications}

With the vertical separation controller similar in design to the altitude controller, as explained before, the same specifications will be selected: a second-order response with approximately 10-15\% overshoot, peaking after approximately 10 seconds and settling with a $2 \%$ error bound after about 20 seconds. The FPA limits of the aircraft were chosen as $\pm 6^{\circ}$. Figure 6.11 shows the root locus and the step response that closely meet the desired specifications.

\section{Results}

Figure 6.12 shows the response results with the designed vertical separation controller. The unit step response is shown in Figure 6.12a. The response exhibits a settling time of approximately 


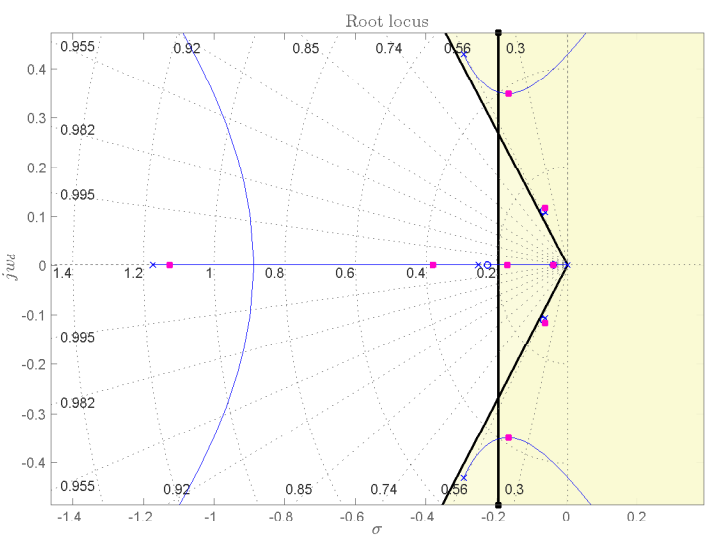

(a) Root locus

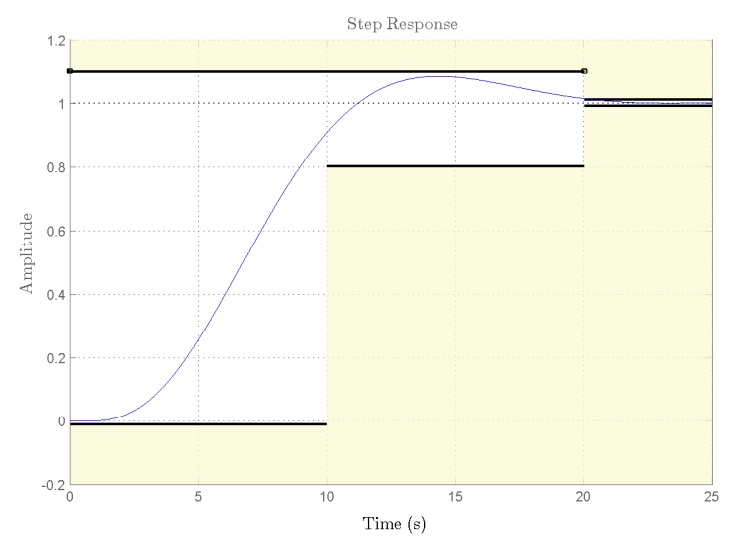

(b) Unit step response

Figure 6.11: Root locus and unit step response of vertical separation controller for a chosen proportional gain $K_{\zeta}$. The controller was tuned to obtain the best possible response within the design requirements.

20s and overshoot of just under 10\%. The corresponding in-track distance and FPA response plots are shown in Figures $6.12 \mathrm{c}$ and $6.12 \mathrm{~b}$. The results of the vertical separation controller are very similar to those of the altitude controller designed in Chapter 5 .

\section{Closed-Loop Model}

Following the design of the vertical separation controller, the last controller in the longitudinal control architecture, a closed-loop state space model that encapsulates the longitudinal dynamics, the DQ law, FPA, autothrust, axial and vertical separation controller, can be derived.

The axial separation state equation in Equation 6.10 is augmented to include the vertical separation variable by the inclusion of Equation (6.14),

$$
\left[\begin{array}{c}
\dot{\boldsymbol{x}}_{A S} \\
\dot{\zeta}
\end{array}\right]=\left[\begin{array}{cc}
\boldsymbol{A}_{A S} & \mathbf{0}_{7 \times 1} \\
\mathbf{C}_{\dot{\zeta}} & 0
\end{array}\right]\left[\begin{array}{c}
\boldsymbol{x}_{A S} \\
\zeta
\end{array}\right]+\left[\begin{array}{cc}
\boldsymbol{B}_{\gamma_{R e f}} & \boldsymbol{B}_{x_{R e f}} \\
0 & 0
\end{array}\right]\left[\begin{array}{c}
\gamma_{R e f} \\
x_{R e f}
\end{array}\right]+\left[\begin{array}{c}
\mathbf{N}_{A S} \\
0
\end{array}\right] \bar{V}_{T}
$$

The control law is given as follows,

$$
\boldsymbol{u}=\left[\begin{array}{c}
K_{\zeta}\left(\zeta_{\text {Ref }}-\zeta\right) \\
x_{\text {Ref }}
\end{array}\right]
$$

Substitution of the control law into the augmented state equation gives the final closed-loop model as follows,

$$
\begin{aligned}
\dot{\boldsymbol{x}}_{V S} & =\mathbf{A}_{V S} \boldsymbol{x}_{V S}+\mathbf{B}_{V S} \boldsymbol{u}+\mathbf{N}_{V S} \bar{V}_{T} \\
{\left[\begin{array}{c}
\dot{\boldsymbol{x}}_{A S} \\
\dot{\zeta}
\end{array}\right] } & =\left[\begin{array}{cc}
\boldsymbol{A}_{A S} & -\boldsymbol{B}_{\gamma_{R e f}} K_{\zeta} \\
\mathbf{C}_{\dot{\zeta}} & 0
\end{array}\right]\left[\begin{array}{c}
\boldsymbol{x}_{A S} \\
\zeta
\end{array}\right]+\left[\begin{array}{cc}
\boldsymbol{B}_{\gamma_{R e f}} K_{\zeta} & \boldsymbol{B}_{x_{R e f}} \\
0 & 0
\end{array}\right]\left[\begin{array}{c}
\zeta_{R e f} \\
x_{R e f}
\end{array}\right]+\left[\begin{array}{c}
\mathbf{N}_{A S} \\
0
\end{array}\right] \bar{V}_{T}
\end{aligned}
$$




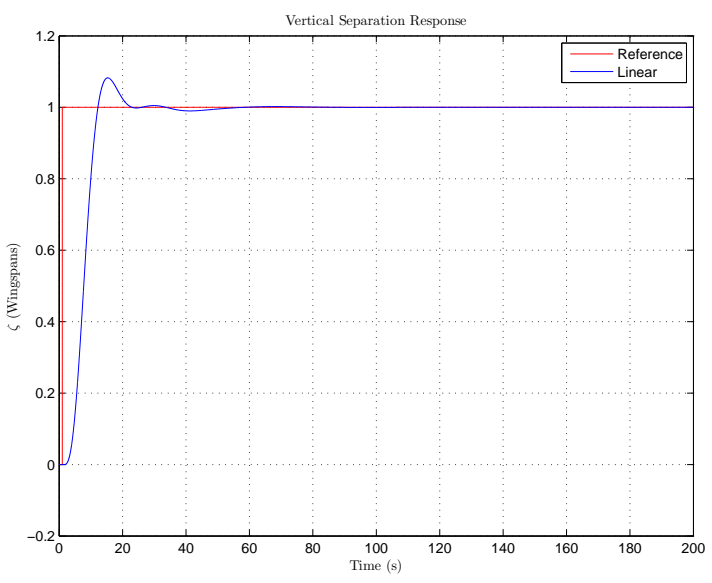

(a) Vertical separation response

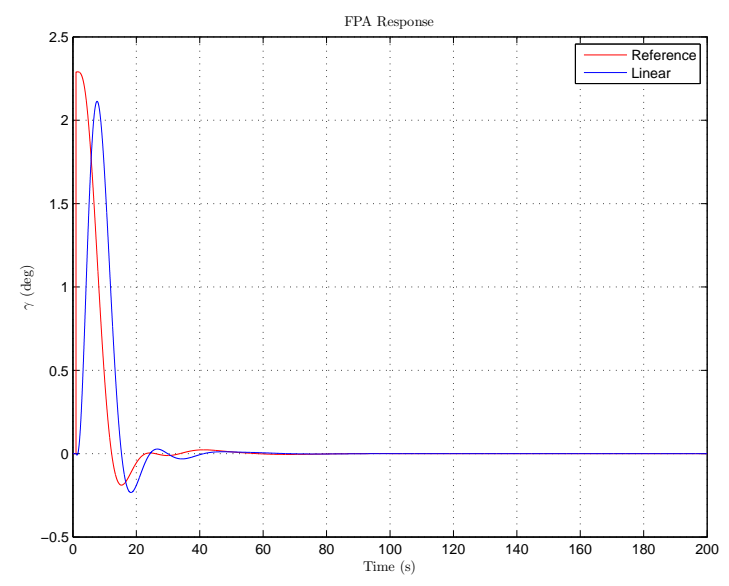

(b) FPA response

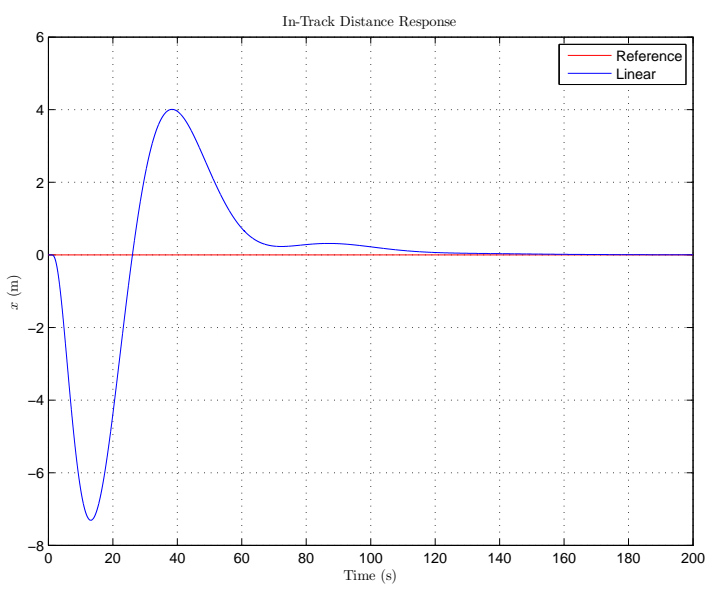

(c) In-track distance response

Figure 6.12: Full-order longitudinal unit step response results for a commanded vertical separation.

Figure 6.13 shows the final closed-loop poles after adding the altitude controller.

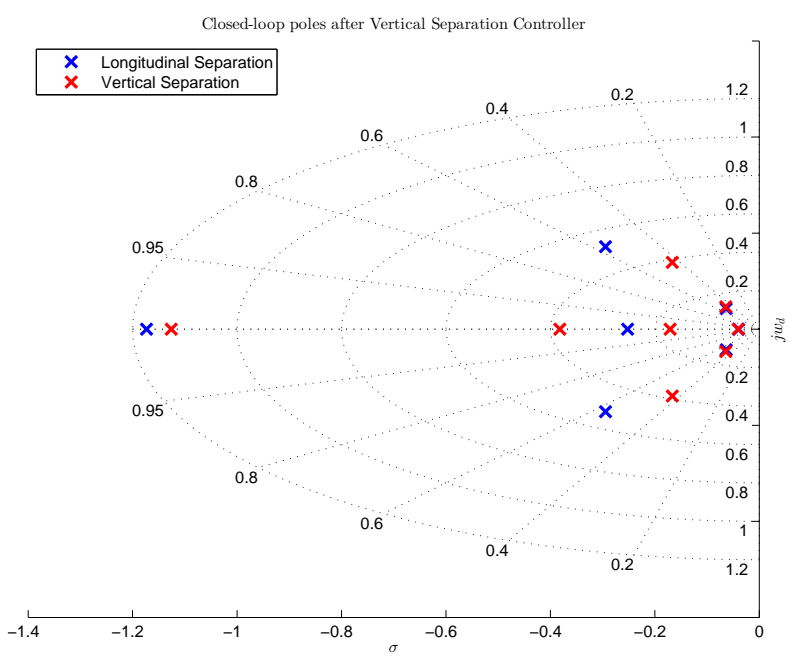

Figure 6.13: Closed-loop poles after adding the vertical separation controller 


\subsection{Lateral Formation Guidance}

In this section, the lateral guidance depicted in Figure 6.1, namely the lateral separation guidance law, will be designed, implemented and verified in simulations.

\subsubsection{Lateral Guidance Controller}

In Chapter 5, a lateral guidance controller, namely a cross-track error controller, was designed, implemented and verified in simulation. This guidance law ensured that a desired cross-track error is achieved as desired within a reasonable amount of time and with good accuracy. The cross-track error is the perpendicular distance from the aircraft's position to the ground track the straight-line segment connecting two waypoints.

The lateral guidance controller for the follower aircraft is illustrated in Figure 6.14, and is required to maintain the geometric lateral separation between the aircraft in formation. It is adapted from the conventional cross-track error controller by remapping the cross-track error variables to lateral separation. It is also necessary to perform the appropriate unit conversion from wingspan to metres before reaching the controller gains, i.e. the same gains used in the cross-track error controller.

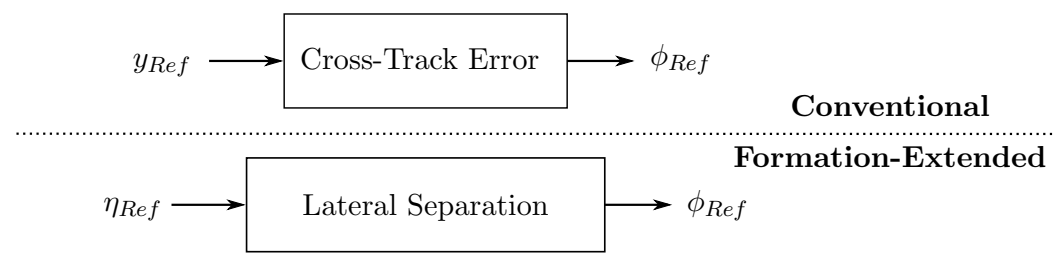

Figure 6.14: Illustration of the architectural change required from the conventional cross-track error controller to the formation-extended lateral separation controller.

Since the design process was very similar to that of the cross-track error controller, the complete redesign process is not shown here. Instead, a brief overview of the design is given to show the similarity in the designs.

\section{Design}

The control architecture of the lateral separation guidance controller is shown in the block diagram of Figure 6.15. The plant is the DPDR law with the lateral dynamics of the aircraft in state space form represented by Equation (5.83),

$$
\begin{aligned}
\dot{\boldsymbol{x}}_{D P D R} & =\boldsymbol{A}_{D P D R} \boldsymbol{x}_{D P D R}+\boldsymbol{B}_{D P D R} \boldsymbol{u} \\
y & =\dot{\psi}=\mathbf{C}_{\dot{\psi}} \boldsymbol{x}_{D P D R}
\end{aligned}
$$

where,

$$
\boldsymbol{u}=\left[\begin{array}{l}
\phi_{R e f} \\
\beta_{R e f}
\end{array}\right]
$$


and the output matrix,

$$
\mathbf{C}_{\dot{\psi}}=\left[\begin{array}{lllll}
0 & 0 & 0 & \frac{g}{V_{T}} & 0
\end{array}\right]
$$

extracts the heading rate $\dot{\psi}$ from the DPDR law state vector $\boldsymbol{x}_{D P D R}$. The heading rate is calculated with Equation (5.90). Regarding the lateral separation rate, consider Figure 3.9 and notice that it is the projection of the aircraft ground speed into the $\vec{j}_{s}$ axis, represented as follows,

$$
\Delta \dot{y}=\bar{V} \sin \left(\psi-\psi_{a z}\right)
$$

and for small heading angles relative to the flight path heading, Equation $\sqrt{6.22}$ ) may be simplified to,

$$
\Delta \dot{y}=\bar{V}_{T}\left(\psi-\psi_{a z}\right)
$$

The lateral separation controller consists of a two-loop closure design, just as was the case with the cross-track error controller: a derivative feedback followed by a proportional feedback loop. The derivative loop consists of lateral separation rate $\Delta \dot{y}$ feedback, and the proportional feedback loop generates a roll angle reference proportional to the error of the lateral separation. The natural integration makes the system type 1, which means it should be able to follow a constant lateral separation reference with zero error at steady state. A saturation block is added to ensure that the controller does not command roll angle signals that exceed the limitations of the aircraft. It also ensures that the aircraft maintains a constant roll angle when large step commands are issued.

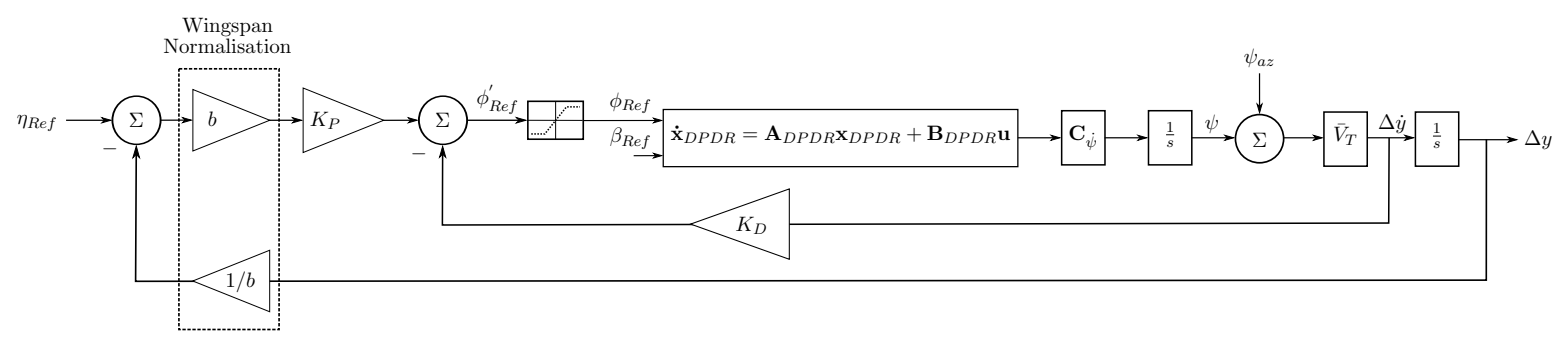

Figure 6.15: Lateral separation controller architecture.

The design of the lateral separation controller consists of determining appropriate values for the derivative and proportional gains. Due to the similarity in the design, the gains calculated for the cross-track error controller will be used in the formation guidance controller. The specifications and results are the same as in Chapter 5 .

\subsection{Extended Formation Flight Simulations}

Before measuring and evaluating passenger comfort in formation flight, the flight control performance needs to be investigated within the relevant feasible formation flight regions (see Section 
3.5.2. As such, extended non-linear simulations were performed to evaluate the performance of the formation guidance laws. In all these simulations, the two aircraft were initialised at a nominal altitude of $40000 \mathrm{ft}$ with a flying speed of $236 \mathrm{~m} / \mathrm{s}$. Various geometric separations within the feasible ranges were investigated, with the focus on lateral separation variation. To increase fidelity, various levels of turbulence intensity were added to the simulation.

\subsubsection{Performance of Formation Guidance Laws}

The simulation settings used for these simulations are summarised in Table 6.1, and Appendix $\mathrm{G}$ tries to provide some of the simulation results in a concise manner. The relevant results will be shown and discussed in this chapter.

Table 6.1: Simulation settings used to evaluate the formation guidance performance.

\begin{tabular}{|c|c|c|}
\hline \multirow{2}{*}{ Simulation Time (s) } & \multicolumn{2}{|c|}{ Description of aircraft commands } \\
\hline & Leader & Follower \\
\hline $0-T_{N}($ User-specified $)$ & $\begin{array}{l}\text { Straight and level flight at } \\
\text { nominal condition, allowing } \\
\text { longitudinal step commands. }\end{array}$ & $\begin{array}{l}\text { Obtain and maintain the fol- } \\
\text { lowing geometric separations } \\
\text { during formation flight: } \\
\eta=\text { User-specified } \\
\zeta=0 \\
\xi=-10\end{array}$ \\
\hline
\end{tabular}

\section{Lateral Separation Tracking Errors}

Consider the simulation in light turbulence where the desired lateral separation is selected as $\eta=1.1$ wingspans. Initialising the aircraft at a lateral separation of 2 wingspans, the follower is immediately commanded to move to the desired lateral separation. Figure 6.16 clearly shows that the controller architecture in Figure 6.15, which was designed to obtain and maintain a desired lateral separation, is unsuccessful in doing so. A deeper investigation revealed the following reasoning:

The lateral DP and DR inner-loop fly-by-wire flight control laws were designed as full-state feedback controllers with feed-forward for turn co-ordination and a natural roll response during sideslip. No integral control was added to the system. As a result, the control laws are not robust against any roll angle disturbances, such as the disturbance on roll angle due to the trailing vortex interaction. The effect is a steady-state error on the lateral separation, as shown in Figure 6.16a. Two solutions are proposed to resolve this steady-state error:

1 Add integral control to the DP and DR laws

2 Add a limited integrator with an integral control gain to the lateral separation guidance law 


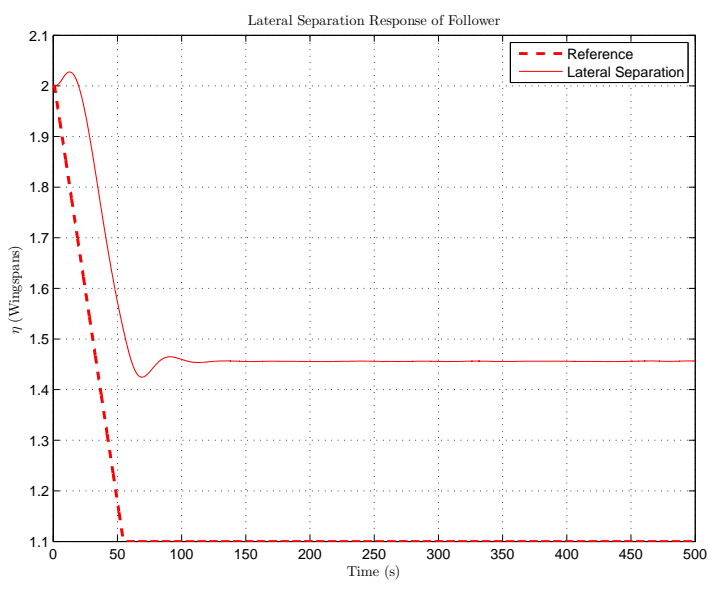

(a) Lateral separation tracking response

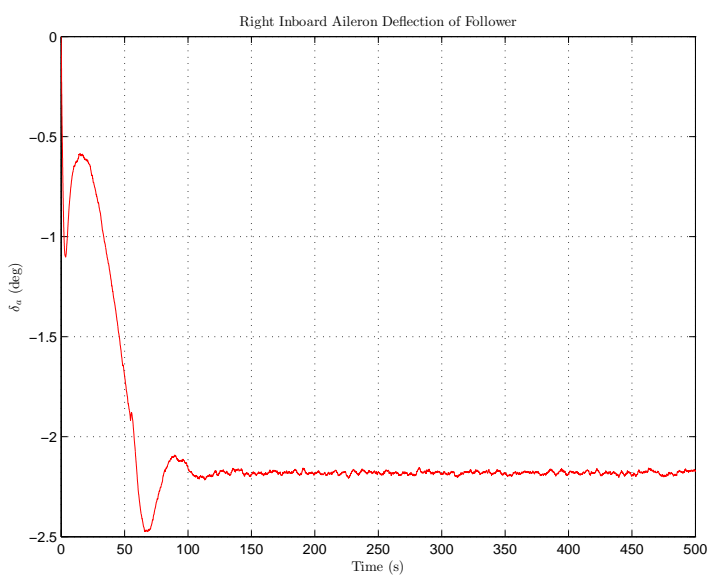

(c) Aileron response

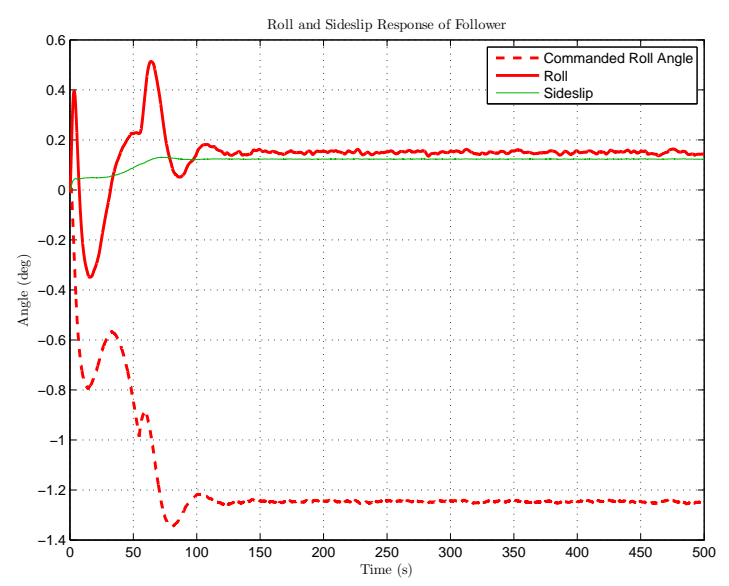

(b) Roll response

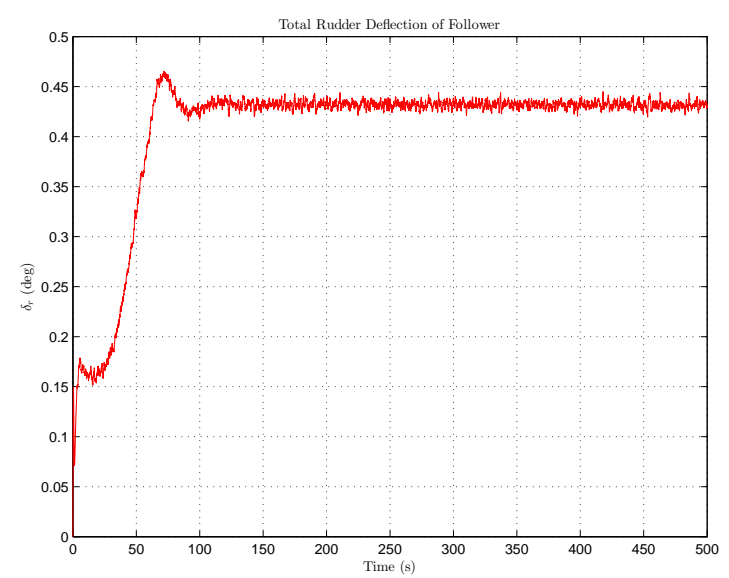

(d) Rudder response

Figure 6.16: Lateral response results in light turbulence for a commanded lateral separation of 1.1 wingspans. These results clearly illustrate a steady-state error in the lateral separation response, indicating the need for integral correction.

The first solution would be the ideal solution. It fixes the source of the problem by commanding enough aileron deflection for a given roll angle, thereby ensuring zero steady-state error in the roll response. However, in an attempt to minimise the architectural changes to the conventional fly-by-wire flight controls, the second solution is implemented. This means that the roll angle reference is changed so that the induced roll and sideslip force the lateral separation to achieve zero steady-state error. The architectural change to the lateral separation controller is illustrated in Figure 6.17. The integrator is limited to minimise its contribution to the transient response, but is selected so that the steady-state error is reduced to zero.

Using a trial and error approach, the limits of the integrator and integral gain were selected. The final results, superimposed onto the results without the limited integrator, are shown in Figure 6.18. The lateral separation eventually reaches the desired value. As can be seen from Figures 6.18b, 6.18c and 6.18d, a blend of aileron and rudder deflection is used, which is slightly more than without the limited integrator. The net effect is a larger sideslip, slightly larger roll angle and ultimately the desired lateral separation. Illustrated in Figure 6.18a are also the 


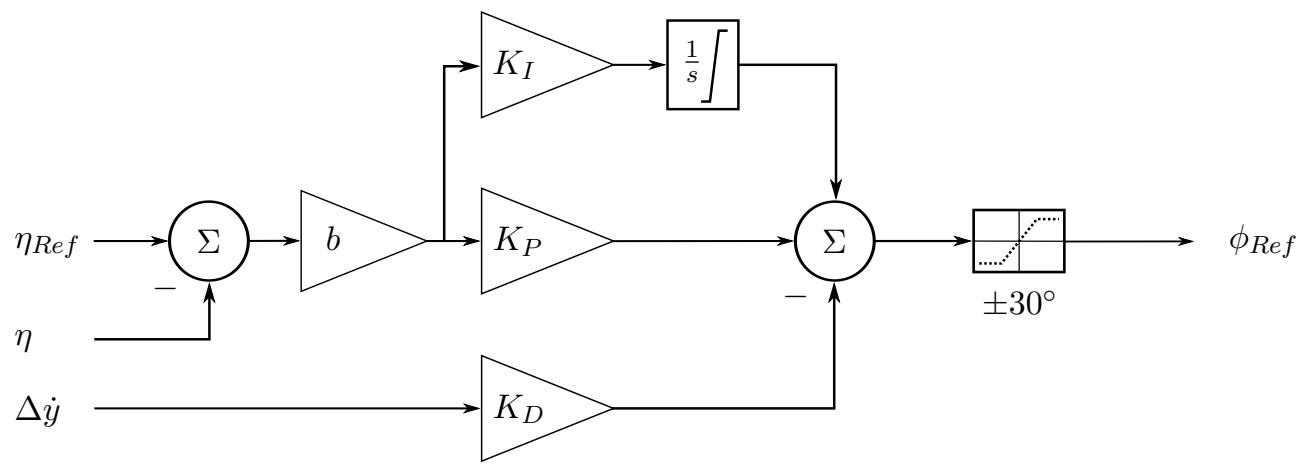

Figure 6.17: Revised lateral separation controller architecture.

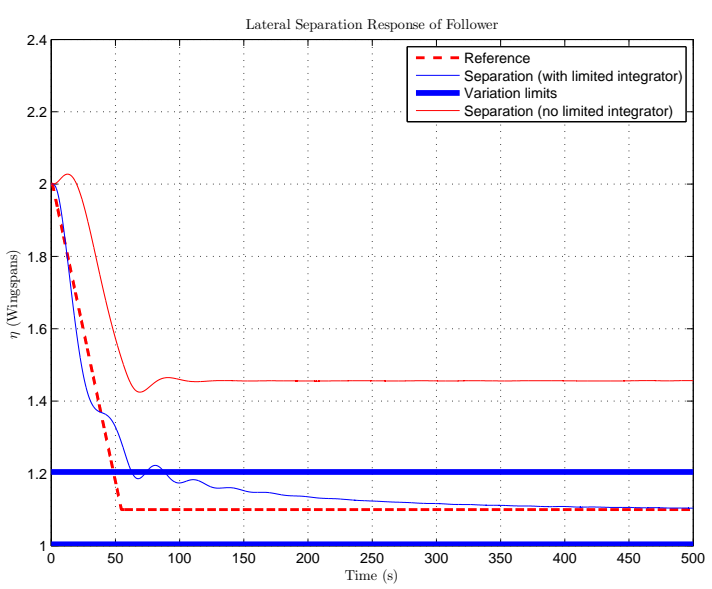

(a) Lateral separation tracking response

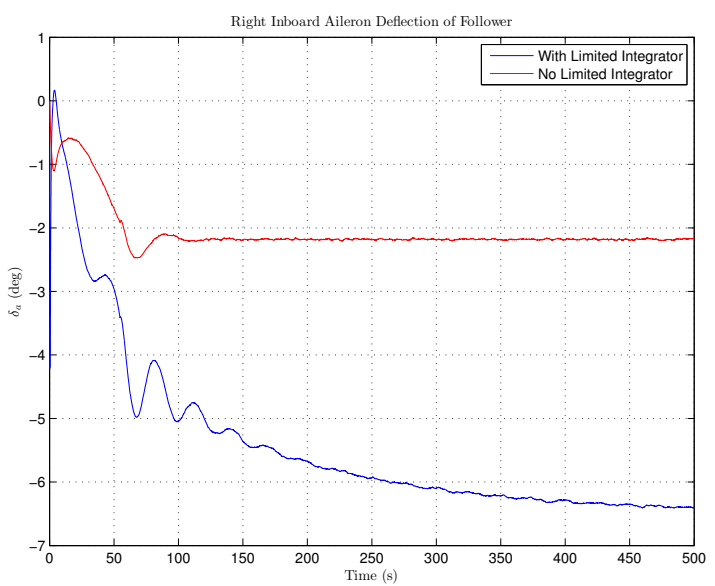

(c) Aileron response

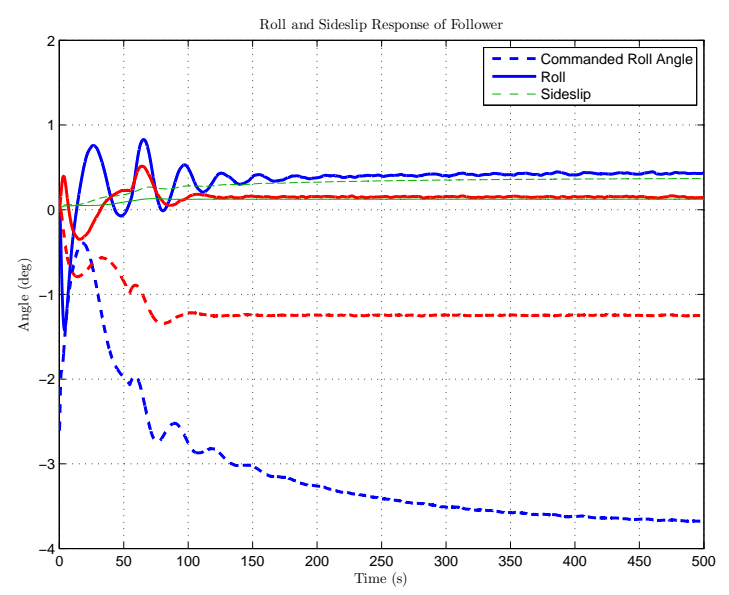

(b) Roll response

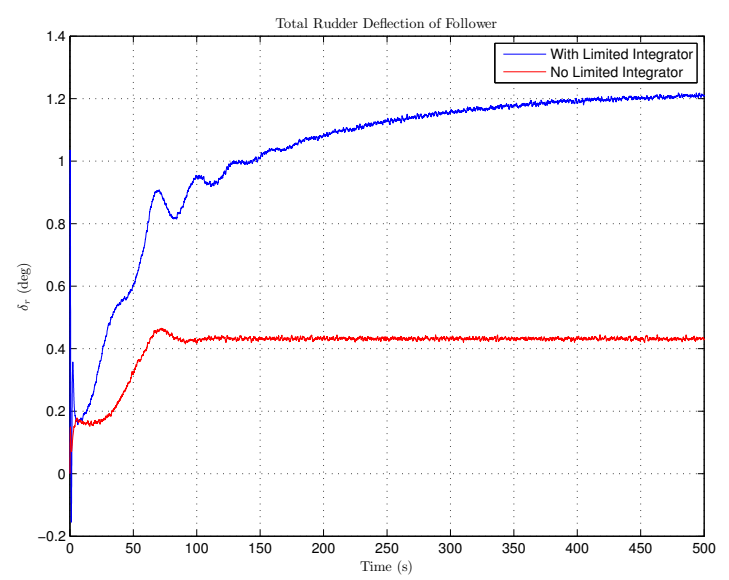

(d) Rudder response

Figure 6.18: Lateral response results in light turbulence after adding a limited integrator and integral gain to the lateral separation architecture. The results are superimposed onto the same results without the architectural change. In Figure 6.18b, the legend is only showing entries of the new response results. 
allowable variation bounds on lateral separation according to Table 3.3 . After approximately 100 seconds, the lateral separation remains well within the boundaries for the rest of the simulation.

\section{Vertical, Axial and Lateral Separation Tracking Performance}

Consider the simulation where the trailing aircraft is initialised at a lateral separation of 2 wingspans, and the vertical and longitudinal separations are initialised as in Table 3.3. The desired lateral, vertical and longitudinal separations are given in Table 3.3 . During the simulation, the leader performs longitudinal manoeuvres in varying levels of turbulence intensity. Table 6.2 gives a summary of the step commands given over the entire simulation period.

Table 6.2: Simulation settings used to evaluate the formation guidance performance.

\begin{tabular}{cll}
\hline \multirow{2}{*}{ Simulation Time (s) } & \multicolumn{1}{c}{ Description of aircraft commands } \\
\cline { 2 - 3 } & \multicolumn{1}{c}{ Leader } & \multicolumn{1}{c}{ Follower } \\
\hline 0 & $\begin{array}{l}\text { Straight and level flight at nominal } \\
\text { condition }\end{array}$ & $\begin{array}{l}\text { Obtain and maintain desired } \\
\text { separations }\end{array}$ \\
250 & $10 \mathrm{~m}$ altitude step & \\
500 & $5 \mathrm{~m} / \mathrm{s}$ airspeed step \\
700 & 0.5 wingspans cross-track error step \\
\hline
\end{tabular}

Figure 6.19 illustrates the NED position of the aircraft during formation and in light atmospheric turbulence. The follower is required to maintain the desired separations $\eta=1.3, \zeta=0$ and $\xi=-10$.

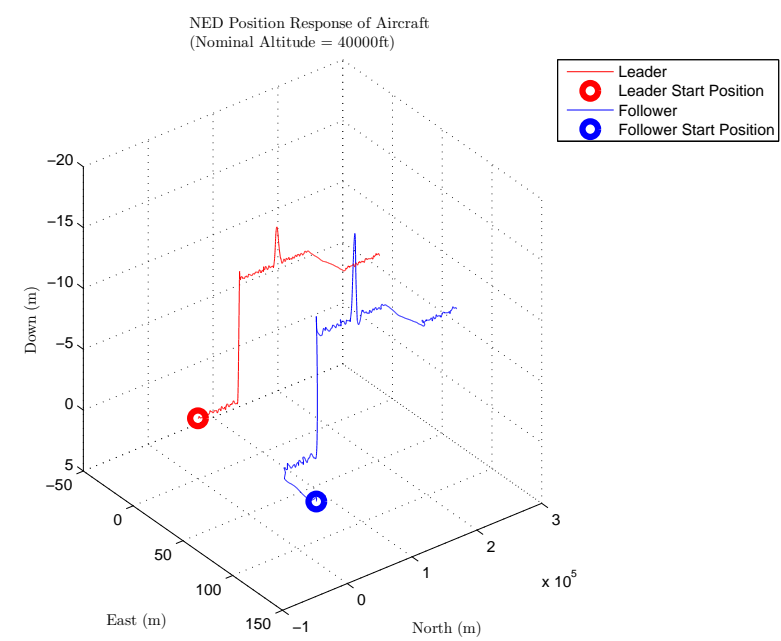

(a) NED in light turbulence

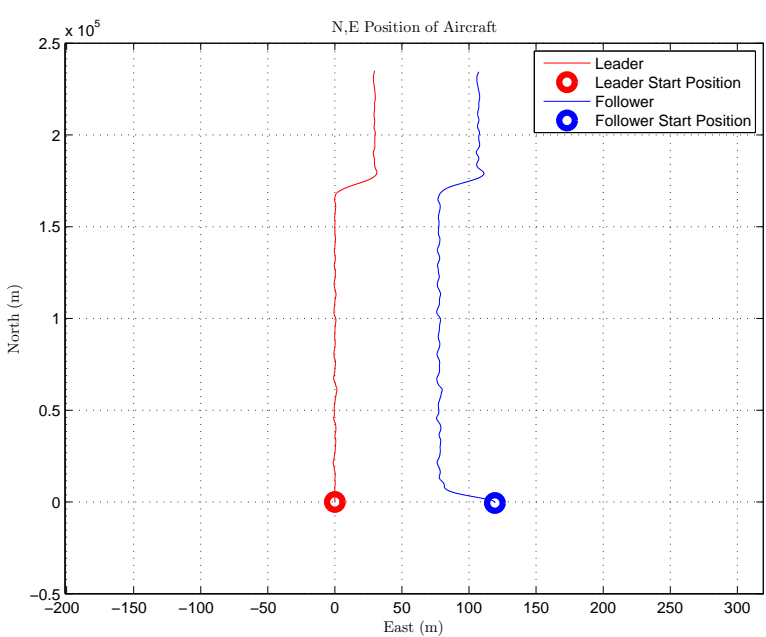

(b) NE in light turbulence

Figure 6.19: Position response of aircraft during formation flight at $\eta=1.3, \zeta=0$ and $\xi=-10$ in moderate turbulence while the leader aircraft performs longitudinal and lateral manoeuvres. 
Figure 6.20 shows the leader and follower responses after the commands in Table 6.2 were executed in light turbulence. Also illustrated is the measured geometric vertical, axial and lateral separation during the simulation. In light turbulence, the tracking performance of the follower aircraft is quite satisfactory.

Concerning the altitude step at $250 \mathrm{~s}$, the follower successfully follows suit, and the vertical separation is recovered to zero while peaking slightly over the allowable variation. During the altitude step, negligible changes occured in the airspeed and lateral tracking response of the leader and follower.

A few seconds later, an airspeed command to the leader is executed and as expected, the follower closely follows pursuit by changing its airspeed and recovering the desired axial separation. The axial separation remains well within the allowable region and is maintained at approximately -10 wingspans. During the airspeed step, no changes are recorded in the lateral tracking response. A notable change in altitude is seen by the leader, however, and as a result, the follower initially also increases altitude. Nevertheless, with the guidance laws in place, both aircraft recover the reference altitude and the vertical separation remains just within the allowable variation boundaries. This indicates that a leader in a formation flight scenario should be careful not to make large airspeed changes. During cruise conditions, on the other hand, aircraft travel at a relatively constant airspeed and thus should not experience any large airspeed changes.

Lastly, the leader aircraft increases its cross-track error from the straight path by about half a wingspan. As this occurs, the follower reacts accordingly to maintain the desired lateral separation. During the cross-track step, negligible changes occurred in the altitude and airspeed response of the leader and follower. All separations remained well within the boundaries of allowable variation.

For higher levels of turbulence intensity, the performance of the formation guidance laws degrade - particularly the performance of the lateral separation guidance. The results are shown in Appendix G. In moderate turbulence, the follower maintains the desired vertical and axial separations throughout the simulation, with the vertical separation peaking a few times just outside of the allowable variation boundaries. The lateral separation, however, is found outside of the allowable variation boundaries more often. This is not desired when the aircraft is at a lateral separation closer than 1.3 wingspans, since it might get pulled into the vortex, directly behind the leader. Due to the high induced forces and moments at lateral separations less than one wingspan, the follower aircraft can get pushed out very aggressively. This does not happen in the case of moderate turbulence at a lateral separation of 1.3 wingspans, but it is exactly what happens in the case of severe turbulence. As shown in Figures G.1e, G.1f and G.4, the follower aircraft is found to the left of the leader's East position. Particularly at 500 seconds, the follower is pushed out very aggressively and far to the left of the vortex. 


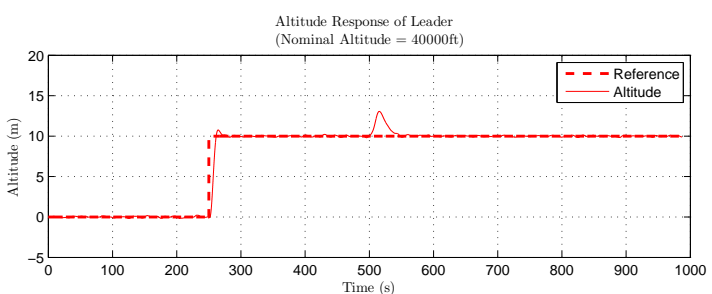

Altitude Response of Follower
(Nominal Altitude $=40000 \mathrm{ft}$ )

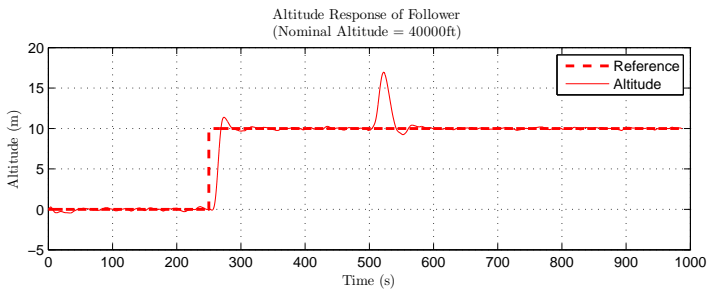

(a) Altitude response

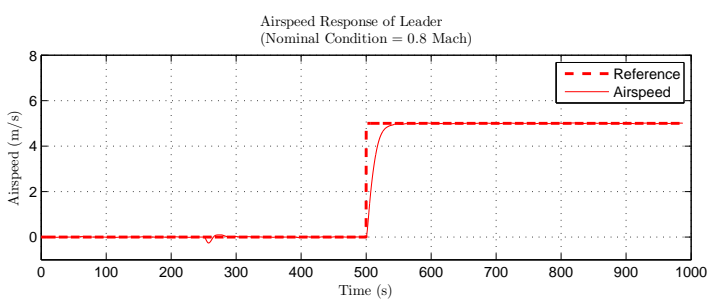

Airspeed Response of Follower
(Nominal Condition $=0.8$ Mach)

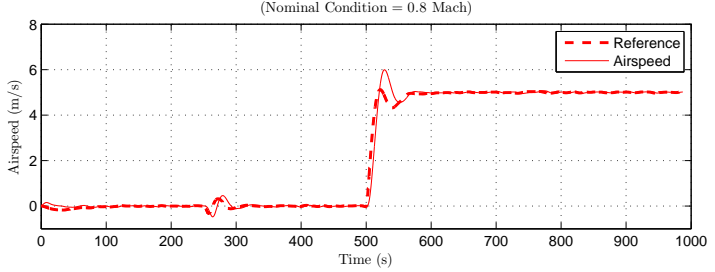

(c) Airspeed response
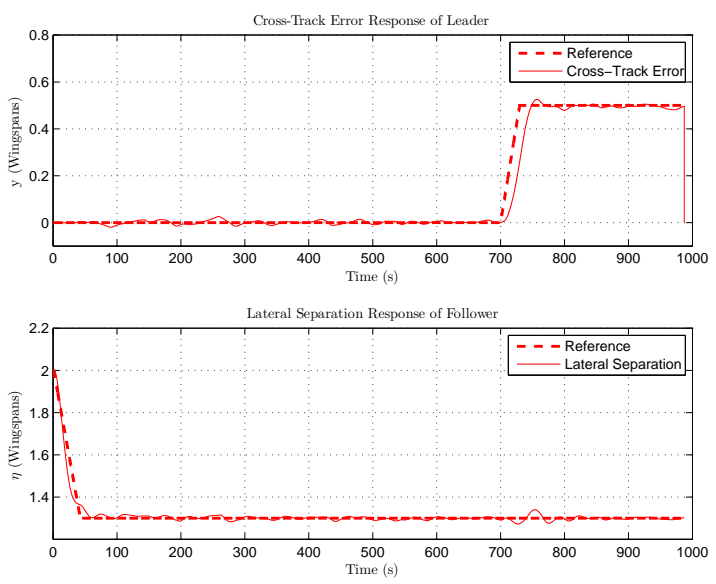

(e) Tracking response

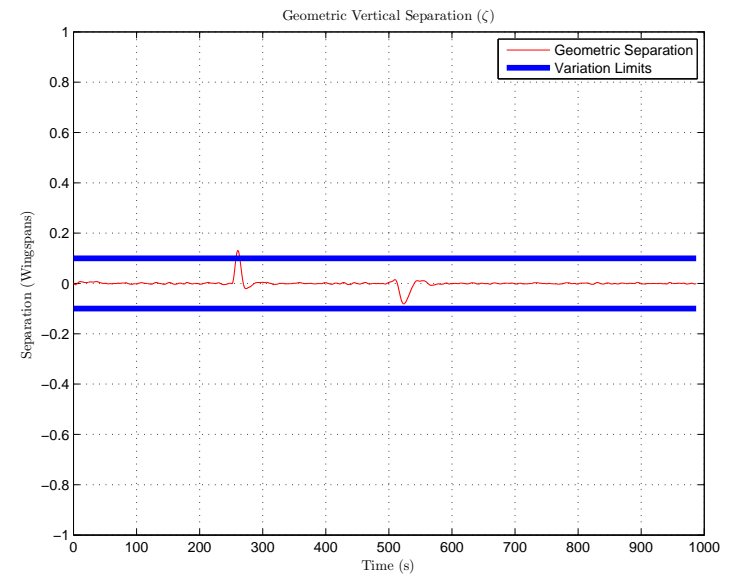

(b) Geometric vertical separation

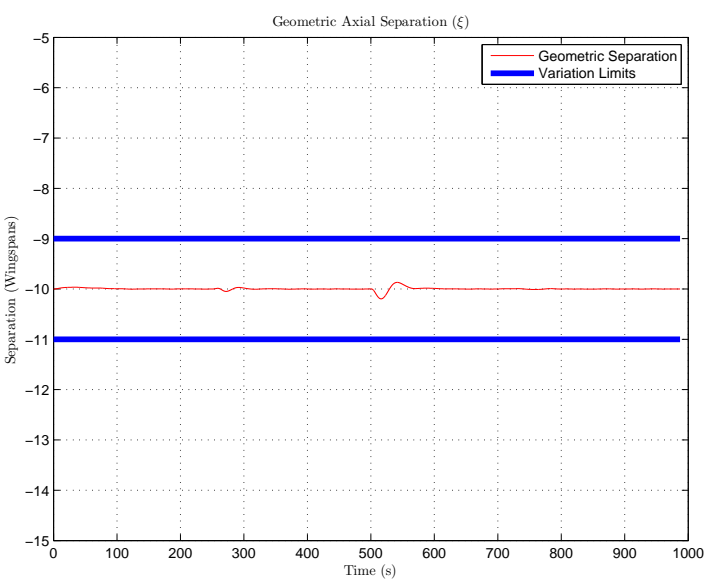

(d) Geometric axial separation

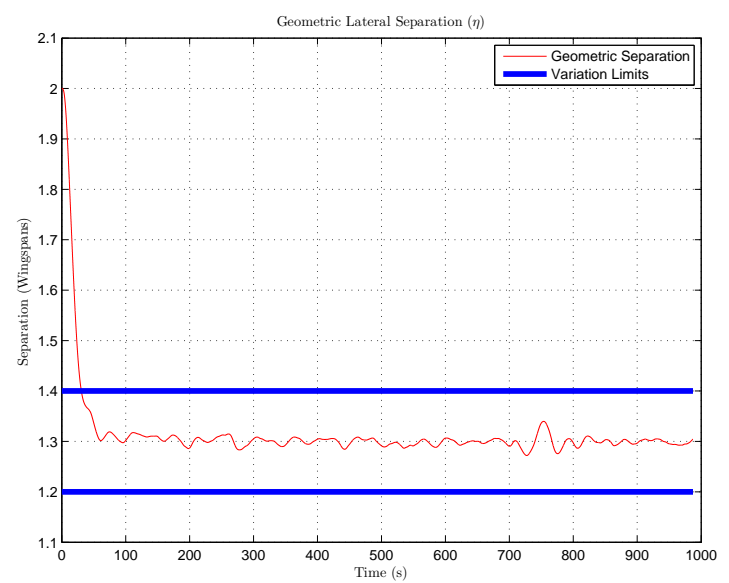

(f) Geometric lateral separation

Figure 6.20: Longitudinal and lateral response results during formation flight in light turbulence. The leader aircraft performed manoeuvres according to Table 6.2. 


\section{Formation-Hold Performance}

To evaluate how well the formation guidance laws hold the desired separations during turbulence, the following simulation exercise was performed: the trailing aircraft is initialised at any lateral separation in the range $1.0 \leqslant \eta \leqslant 1.5$ from the trailing vortex. The leader aircraft is commanded to fly along a straight path with no flight path heading changes, and the follower is commanded to maintain the desired geometric separations. The simulations were performed over an extended period of 30 minutes and at various turbulence intensities. All results are concisely summarised in Appendix G. For each level of turbulence intensity, the lateral separation is plotted against the axial and vertical separation over the entire simulation period. Also indicated in the plots as thick blue boundaries are the allowable separation variations, as summarised in Table 3.3 . Finally, indicated in the bottom left of the each plot, is the number of times the aircraft happened to be outside of the allowable separation boundaries.

Figure 6.21 shows the results of the formation-hold capabilities at light, moderate and severe turbulence conditions for a desired lateral separation of 1.3 wingspans. The results show much variation in geometric separation is present as the turbulence intensity increases and, as expected, the hold performance degrades as the intensity increases. The best formation-hold performance is achieved at light turbulence conditions - at least for decreasing lateral separations of up to 1.1 wingspans. 


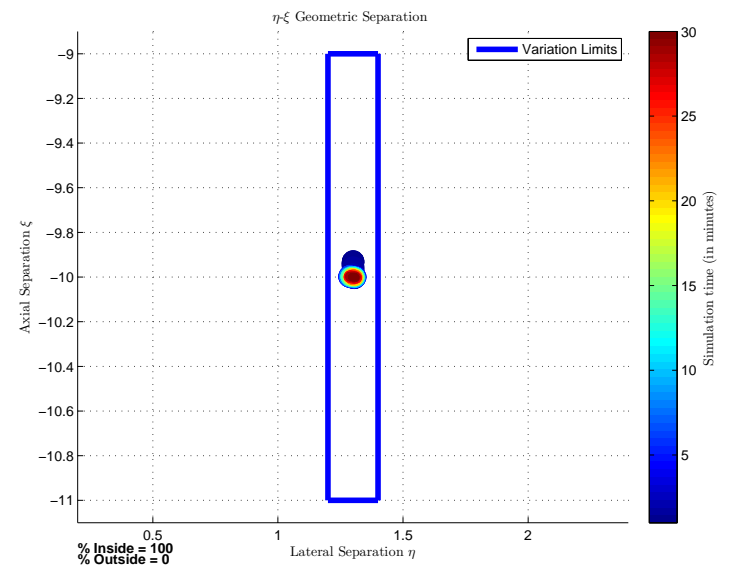

(a) Lateral vs axial separation (Light turbulence)

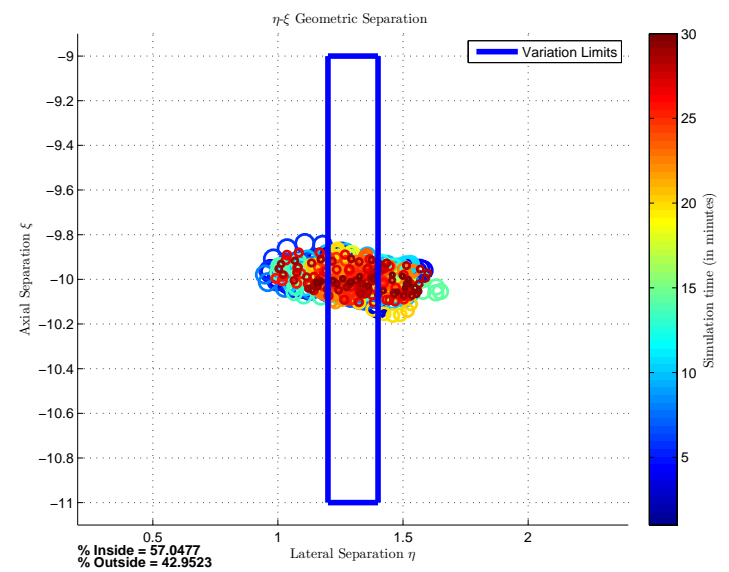

(c) Lateral vs axial separation (Moderate turbulence)

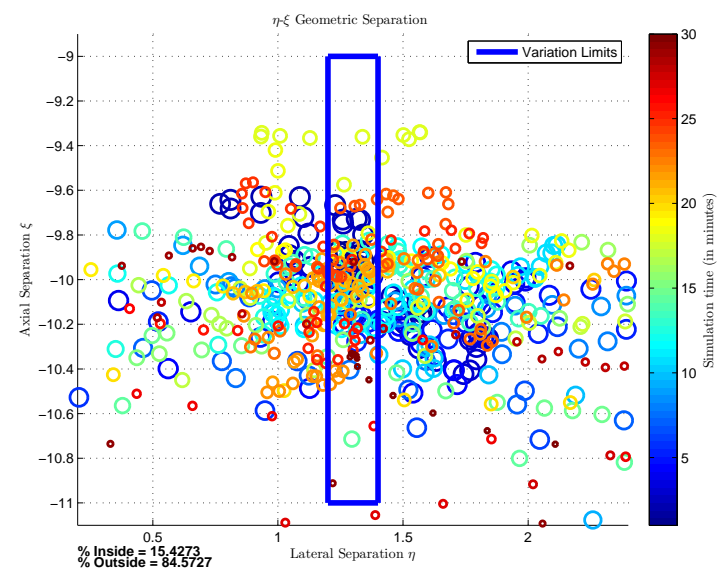

(e) Lateral vs axial separation (Severe turbulence)

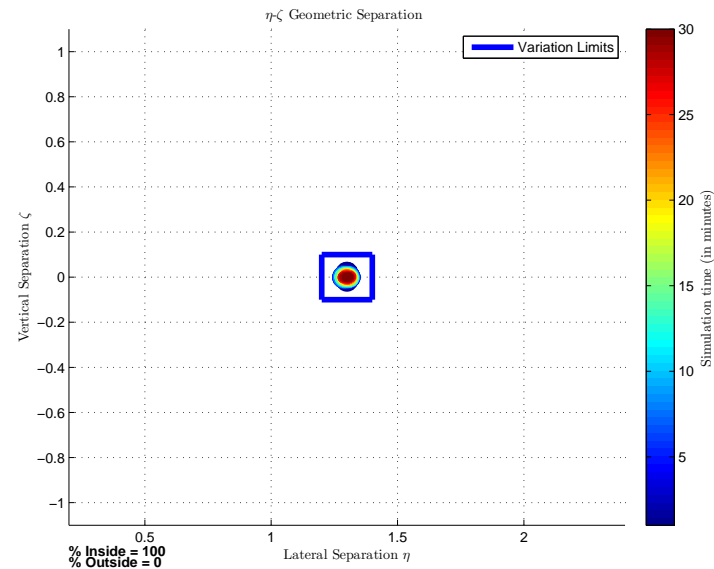

(b) Lateral vs vertical separation (Light turbulence)

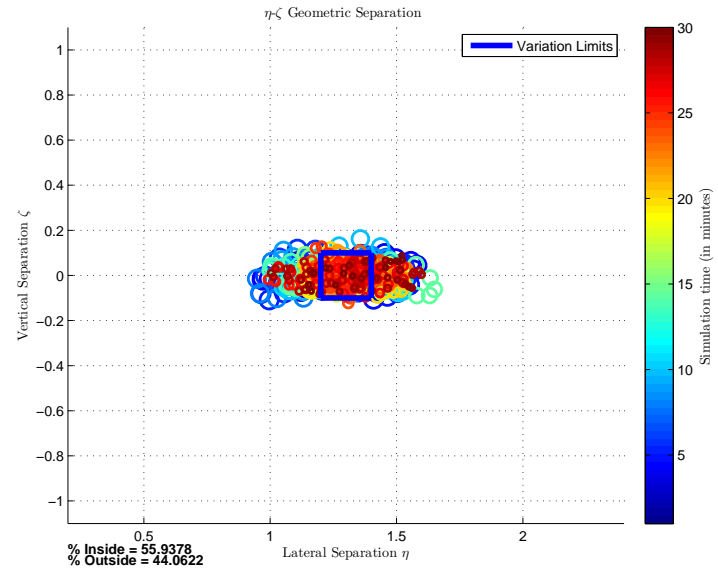

(d) Lateral vs vertical separation (Moderate turbulence)

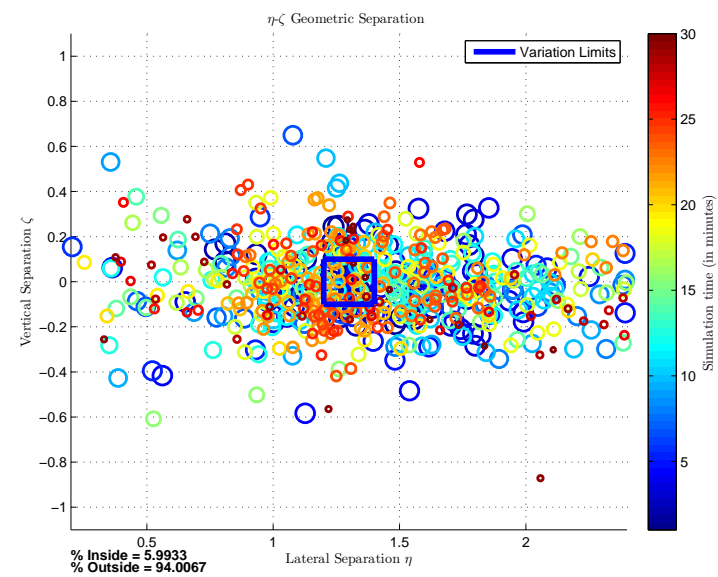

(f) Lateral vs vertical separation (Severe turbulence)

Figure 6.21: Geometric separations during formation flight in varying levels of turbulence intensity for a desired lateral separation of 1.3 wingspans. 
Consider the results for light turbulence and a desired lateral separation of 1 wingspan, conveniently illustrated here in Figure 6.22. Compared to the results for light turbulence at lateral separations larger than 1 wingspan, where the separations remained within the boundaries throughout the simulation, the recorded number of times that the separations were within the boundaries dropped to about $60 \%$. The reason for such a different response in geometric separation can be explained by way of another simulation.

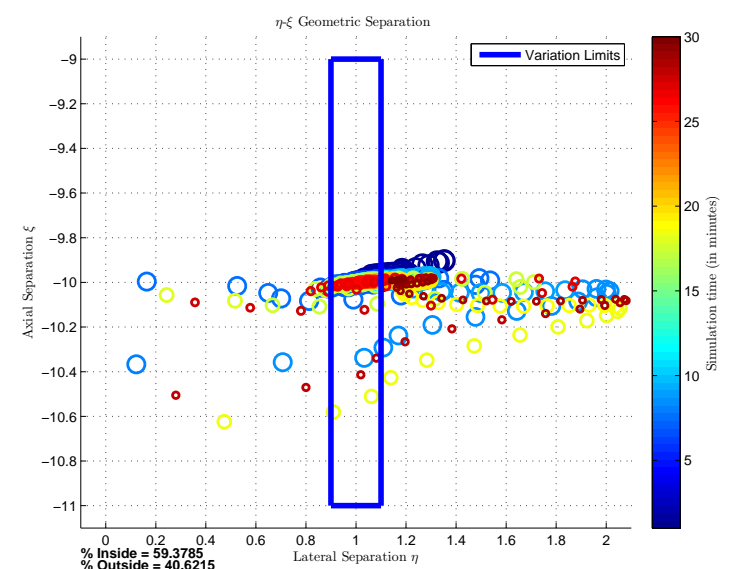

(a) Lateral vs axial separation (Light turbulence)

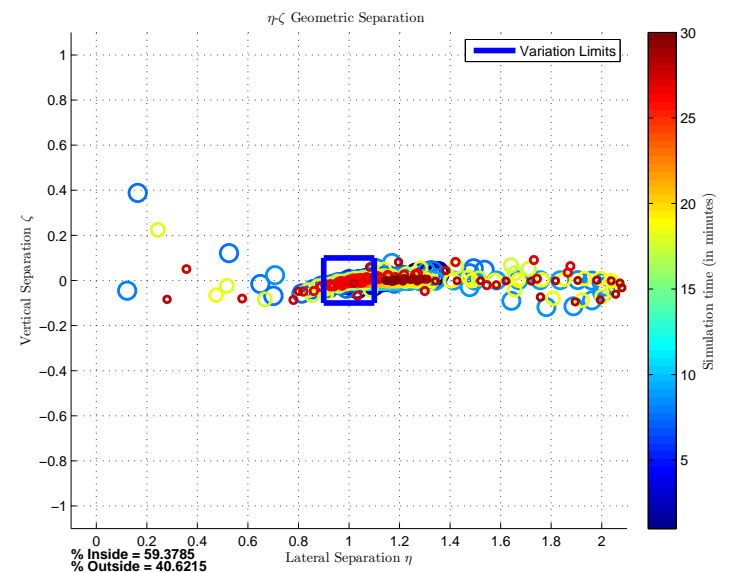

(b) Lateral vs vertical separation (Light turbulence)

Figure 6.22: Formation-hold performance at desired geometric separations $\eta=1.0, \zeta=0$ and $\xi=-10$ wingspans during formation flight in light turbulence only.

Consider a simulation of light turbulence where the lateral separation is decreased from 1.3 to 1 wingspan while maintaining the axial and vertical separation, as summarised in Table 6.1. The results in Figure 6.23 highlight a very important limitation: for lateral separations less than 1.1 wingspan, the designed flight control architecture is unable to reach a steady-state value, resulting in unstable oscillatory behaviour. The actuators do not show any saturation during this oscillatory behaviour. When the follower gets pushed through and past the wake at roughly $30 \mathrm{~min}$, the guidance laws try again to move the aircraft to 1 wingspan. The same oscillatory behaviour will occur. As a result, the lower bound for any lateral separations in further simulations is changed from 1 wingspan, as in Table 3.3 , to 1.1 wingspans. 


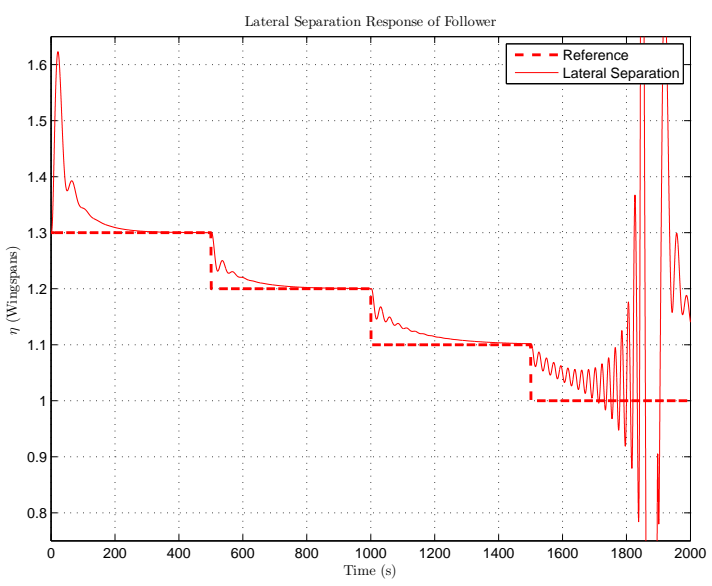

(a) Lateral separation response

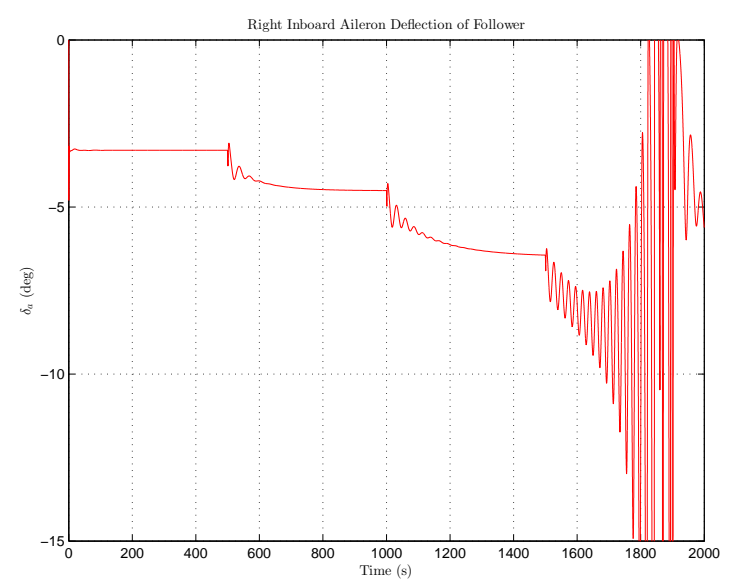

(b) Aileron response

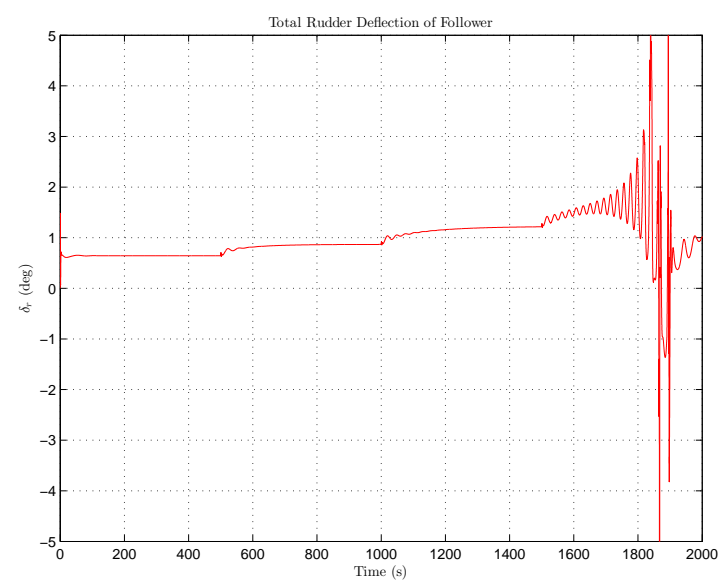

(c) Rudder response

Figure 6.23: Aircraft response in light turbulence as lateral separation is decreased from $\eta=1.3$ to $\eta=1$ wingspan. The axial and vertical separations are maintained at -10 and 0 wingspans respectively.

In the study by Büchner [4], numerous non-linear simulations were also performed to assess the formation-hold performance - similar to the experiments done here. Figures 6.24a and $6.24 \mathrm{~b}$ show the tracking performance of the follower aircraft in moderate and severe turbulence respectively. The separation performances were much better than expected [4], staying below 0.05 wingspans for moderate turbulence, and less than 0.25 wingspans for severe turbulence. These results do seem an order of magnitude better than the formation-hold performance of the results in the current study. However, upon closer inspection of how Büchner generated atmospheric turubulence, method inaccuracies were found to be possible, and this makes it hard to compare the results of the two studies.

In conclusion, it is deduced that formation flight is not at all feasible in severe turbulence. As pointed out by Büchner [4], this high level of turbulence is rarely encountered in flight, but in such a case formation flight should be disengaged. With regard to moderate turbulence, at lateral separations closer than 1.3 wingspans the aircraft comes too close to the trailing vortex, 


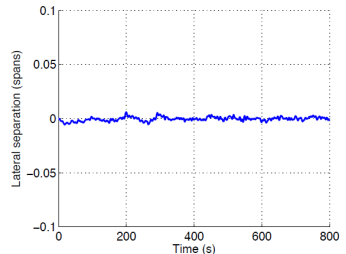

(a) $\eta$-tracking

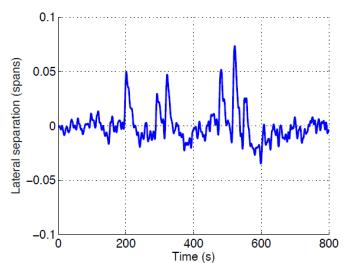

(a) $\eta$-tracking

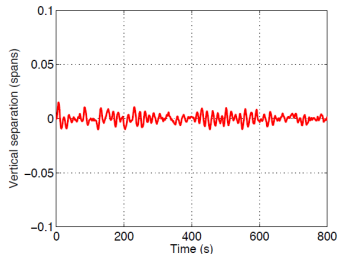

(b) $\zeta$-tracking

(a) Moderate turbulence

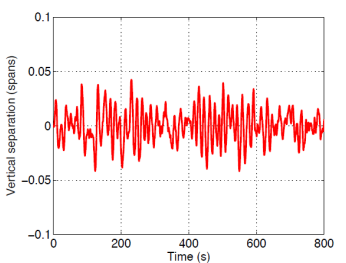

(b) $\zeta$-tracking

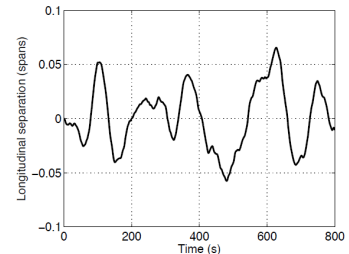

(c) $\xi$-tracking

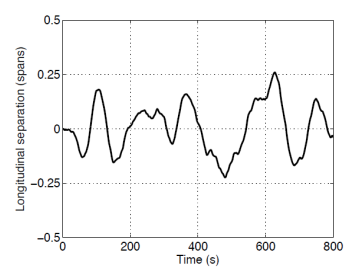

(c) $\xi$-tracking

(b) Severe turbulence

Figure 6.24: Formation-hold error of follower aircraft in moderate and severe turbulence, illustrating the formation-hold performance as found by Büchner (from Büchner [4]).

getting 'sucked into' the vortex. Happening multiple times over the entire simulation period, the trailing aircraft goes far to the left of the leader aircraft, resulting in large separation errors. The formation flight guidance laws eventually guide the aircraft back to the correct separations, but this process is not without aggressiveness, making it unfavourable for passenger comfort. Regarding light turbulence, the formation guidance laws have no problem in maintaining a lateral separation larger than one wingspan.

Table 6.3 summarises the results in Appendix $\mathrm{G}$ that are used to evaluate the formationhold performance. It depicts the performance during different levels of turbulence intensity and at various lateral separations in terms of three performance categories: good, average and poor. The performance is good if all separations remain within all boundaries for $100 \%$ of the simulation period; average if it they remain within for at least $50 \%$ of the simulation; and poor if they are outside of the boundaries for more than $50 \%$ of the simulation.

Table 6.3: Summary of formation-hold performance during different levels of turbulence intensity and at various lateral separaions.

\begin{tabular}{ccccccc}
\hline \multicolumn{2}{c}{ Desired Separations } & & \multicolumn{3}{c}{ Turbulence Intensity } \\
\cline { 1 - 2 } \cline { 5 - 6 }$\eta$ & $\zeta$ & $\xi$ & & Light & Moderate & Severe \\
\hline 1.5 & 0 & -10 & & Good & Average & Poor \\
1.4 & 0 & -10 & & Good & Average & Poor \\
1.3 & 0 & -10 & & Good & Average & Poor \\
1.2 & 0 & -10 & & Good & Average & Poor \\
1.1 & 0 & -10 & & Good & Poor & Poor \\
1 & 0 & -10 & & Average & Poor & Poor \\
\hline
\end{tabular}




\subsection{Conclusions}

In this chapter, the extended formation flight control architecture has been proposed, designed and verified in simulation. The flight control architecture consists of a set of conventional flyby-wire inner-loop controllers - left unchanged from the design in Chapter 5. The outer-loop guidance laws were designed as an extension of the conventional guidance laws: an axial guidance controller, required to maintain the desired geometric longitudinal separation $\xi$ by commanding a desired airspeed; a vertical guidance controller, required to maintain the desired geometric vertical separation $\zeta$; and, lastly, a lateral guidance controller, required to maintain the desired geometric lateral separation $\eta$.

To evaluate the performance of the extended formation flight guidance laws, extended simulations were preformed at different levels of turbulence intensity and geometric lateral separations. An investigation into other studies showed that the optimum longitudinal and vertical separations to use for the current study were at -10 and 0 wingspans respectively. In light turbulence simulations, the formation guidance laws were successfully able to maintain the desired geometric separations and retain the separations after the leader aircraft performs longitudinal manoeuvres.

The guidance laws also showed acceptable formation-hold performance in light turbulence conditions. However, it was shown that the guidance laws were unable to maintain the desired separations for lateral separations closer than 1.1 wingspans, at which point oscillatory and unstable behaviour results. Furthermore, the formation-hold performance degrades very quickly as the level of turbulence intensity increases to moderate and severe turbulence.

The formation flight simulation model is now ready to be used for a passenger comfort evaluation during formation flight and in atmospheric turbulence. The following chapter will investigate the levels of comfort experienced at different seating locations by measurement and evaluation using the method described in Chapter 3. The results of this chapter will be used to determine the feasible formation flight scenarios in which to consider, measure and evaluate passenger comfort. 


\section{Chapter 7}

\section{Passenger Comfort}

The developed formation flight model illustrated in Figure 3.6 will be used in dynamic simulations to produce typical vibrations during formation flight in atmospheric turbulence. These accelerations will be measured and used to evaluate passenger comfort in a formation aircraft compared to an isolated aircraft. This chapter starts by outlining the case for investigation, defining the passenger comfort criteria for the evaluation of comfort, and then discusses important post-simulation settings. A discussion of the most important results are then provided followed by a conclusion of the results.

\subsection{Rigid Body Aircraft with Seat Transmissibility}

For the evaluation of comfort, the translational and rotational accelerations at the feet are combined with the accelerations at the seat-surface of a seated passenger. In a study performed by Leatherwood at the Langley Research Center [77, the ratio of peak seat accelerations to peak input accelerations at the floor was combined for discrete frequencies to produce vertical and horizontal transmissibility ratios. Vertical transmissibility data showed that the accelerations experienced at the seat-surface differ by a significant margin from the accelerations at the floor. Lateral transmissibility data proved to be less accurate, since it was more difficult to measure. Nevertheless, the most important observation made was the fact that the transmissibility ratios for different seats peak in a frequency range considered to be critical comfort frequencies.

Assuming a rigid body aircraft, the time series acceleration data are measured at the feet and seat-surface, and the accelerations at the seat-surface are measured with vertical or lateral transmissibility ratios. Figure 7.1 illustrates the vertical and lateral transmissibility ratios, reproduced from the data measured by Leatherwood [77] and linearly interpolated with data points of a finer sampling over the frequency range 1 to $50 \mathrm{~Hz}$. Notice that the ratio peaks at approximately $5 \mathrm{~Hz}$ for vertical transmissibility and $2 \mathrm{~Hz}$ for lateral transmissibility. 


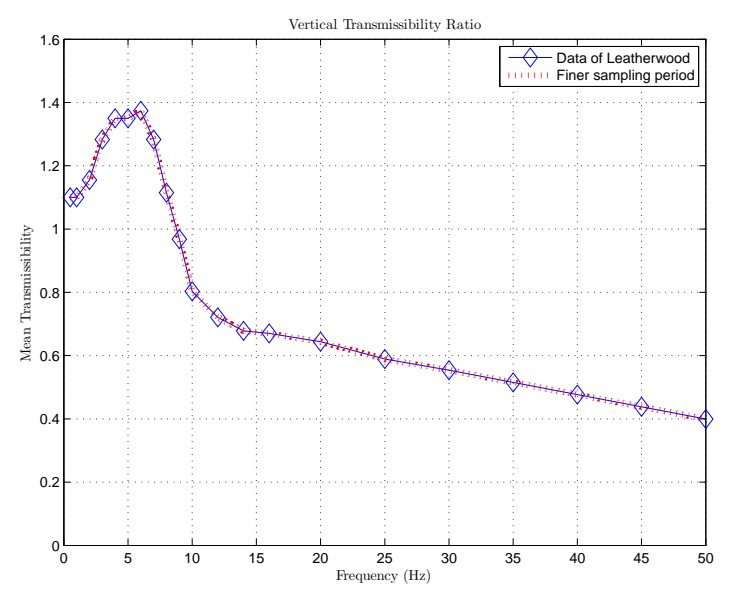

(a) Vertical

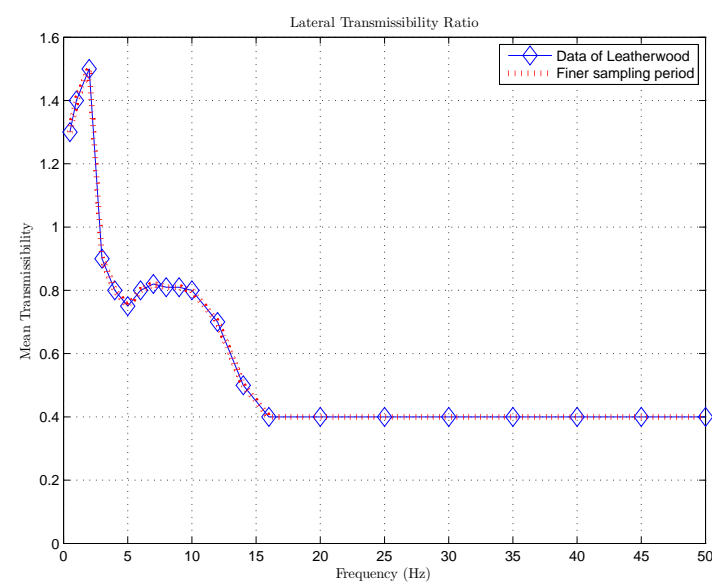

(b) Lateral

Figure 7.1: Vertical and lateral transmissibility ratios, used to obtain time series acceleration at the seat-surface from accelerations at the feet of a seated passenger.

\subsection{Passenger Comfort Criteria}

In Chapter 3, the method to measure the vibrations experienced by a seated passenger was outlined. In this section, the evaluation of these vibrations will be explained, where the primary focus is on vibrational comfort, recognised as the predominant influencing factor [53]. The effect of the vibrations on levels of comfort and motion sickness incidence is also defined. Figure 7.2 illustrates the different axis systems for a passenger in the seated position, repeated from Chapter 3 for the convenience of the reader. The notation described in Table 7.1 will be used in the evaluation method.

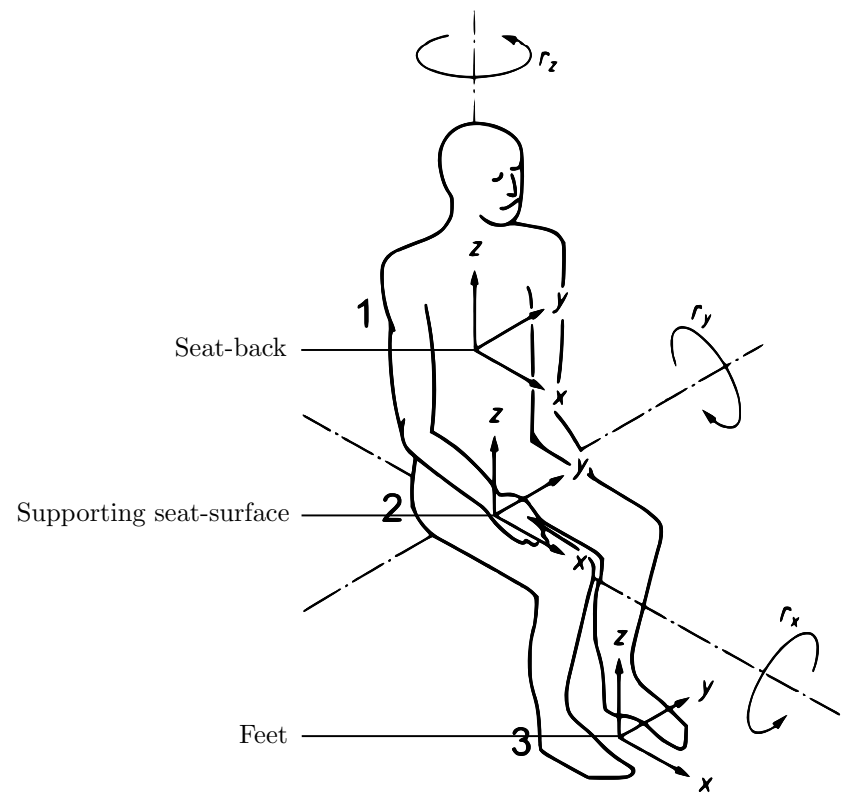

Figure 7.2: Axis systems for a passenger in the seated position (from ISO 2631-1 [53]). 
Table 7.1: Standard notation used in the seated passenger axis.

\begin{tabular}{|c|c|}
\hline Symbol & Description \\
\hline$a$ & $\begin{array}{l}\text { Translational or rotational root mean square (RMS) vibrational accel- } \\
\text { eration, measured in metres per second squared or radians per second } \\
\text { squared respectively }\end{array}$ \\
\hline$W$ & Frequency weighting \\
\hline$H(s)$ & Transfer functions for the different frequency weightings \\
\hline Subscripts & Description \\
\hline$c, d, e, f, j, k$ & $\begin{array}{l}\text { Type of frequency weighting recommended for the evaluation of the ef- } \\
\text { fect of vibrations on health, comfort, perception and motion sickness }\end{array}$ \\
\hline$p$ & Arbitrary point in fuselage \\
\hline$w$ & Frequency-weighted value \\
\hline$s$ & Frequency-weighted value at the seat-surface \\
\hline$f$ & Frequency-weighted value at the feet \\
\hline$v$ & Total frequency-weighted value \\
\hline
\end{tabular}

\subsubsection{Vibration Evaluation Method}

Figure 7.3 shows a flow diagram of the vibration evaluation method known as the Basic Evaluation Method. In order to determine passenger comfort, the frequency-weighted RMS accelerations will be required to evaluate the vibration and associated comfort levels in accordance with ISO 2631-1 [53]. To obtain frequency-weighted accelerations, the power spectral density estimations for the accelerations need to be calculated and weighted with frequency weighting filters.

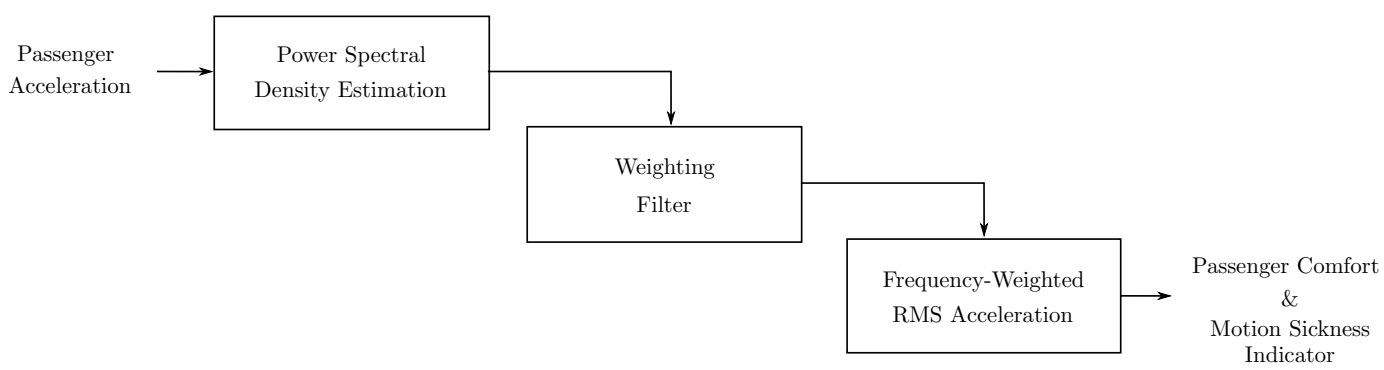

Figure 7.3: Basic Evaluation Method for vibration evaluation.

\section{Frequency-Dependent Acceleration Spectra}

The Welch method, named after P.D. Welch [78, is a well known approach to spectral density approximation. The power of a signal is estimated at different frequencies by finding the average spectral density of overlapping segments of window-modified periodograms [79]. Appendix I provides an overview of the Welch method and how the method can be implemented in practice.

In MATLAB, pwelch.m is a function from the Signal Processing toolbox that implements the Welch method (see Appendix I for details). Using $N$ samples for the length of the DFT 
and a Hamming window with $50 \%$ overlap, the frequency-dependent acceleration spectra can be calculated for real-valued time series acceleration data.

After obtaining the accelerations as frequency-dependent, the standard provides weighting filters which are to be used before the comfort rating can be obtained and evaluated.

\section{Weighting Filters and Frequency-Weighted Acceleration Spectra}

For a seated passenger in an aircraft, the manner in which the random vibration experienced in both isolated and formation flight affects health, comfort, perception and motion sickness is dependent on the vibration frequency content. To this end, different frequency weightings are required for the different axes of vibration. Regarding vibrational comfort, Table 7.2 indicates the different weighting filters and appropriate weighting factors $k$ to be applied for a seated passenger, as illustrated in Figure 7.2 , in any $x, y, z$ translational or rotational direction for comfort evaluation. The weighting factors that are used with the weighting filter for rotational accelerations $\left(W_{e}\right)$ are provided in units of $\mathrm{m} / \mathrm{rad}$. For the evaluation of motion sickness, a single frequency weighting filter $W_{f}$ is recommended and applied to $z$-axis translational vibrations.

Table 7.2: Guide for the application of frequency-weighting curves for weightings $W_{w}$ in the seated position (ISO 2631-1 [53]). These weightings are used for the prediction of the effects of vibration on levels of comfort.

\begin{tabular}{lccccccc}
\hline & & \multicolumn{2}{c}{ Supporting Seat-Surface } & & \multicolumn{2}{c}{ Feet } \\
Vibrations & Axes & Weighting & Factor $(k)$ & & Weighting & Factor $(k)$ \\
\hline \multirow{2}{*}{ Translational } & $x$-axis & $W_{d}$ & 1 & & $W_{k}$ & 0.25 \\
vibrations & $y$-axis & $W_{d}$ & 1 & & $W_{k}$ & 0.25 \\
& $z$-axis & $W_{k}$ & 1 & & $W_{k}$ & 0.4 \\
\hline \multirow{2}{*}{ Rotational } & $r_{x}$-axis & $W_{e}$ & 0.63 & & - & - \\
vibrations & $r_{y}$-axis & $W_{e}$ & 0.4 & & - & - \\
& $r_{z}$-axis & $W_{e}$ & 0.2 & & - & - \\
\hline
\end{tabular}

The transfer functions for each filter described in Table 7.2 are expressed as a product of several factors that make several transfer functions. Using Annexure A of ISO 2631-1 [53], which describes the mathematical definition of the frequency weightings, the total transfer function for any weighting is expressed as follows,

$$
W_{w}(s)=H_{h}(s) H_{l}(s) H_{t}(s) H_{s}(s)
$$


where the subscript $w$ is the type of weighting $(k, d, f, e), H_{h}$ is a high-pass filter, $H_{l}$ is a low-pass filter $H_{t}$ is an acceleration-velocity transition filter ${ }^{2}$ and $H_{s}$ is an upward step filter $\square^{3}$. Figures $7.4 \mathrm{a}$ and $7.4 \mathrm{~b}$ illustrate the weightings required for the evaluation of comfort and motion sickness [53.

After selecting the appropriate weighting filter, the weighting is applied to the power spectral density estimation of the acceleration as follows,

$$
P_{y y_{w}}[i]=P_{y y}[i] \cdot\left|W_{w}[i]\right|^{2}
$$

where $P_{y y_{w}}$ represents the frequency-weighted power spectral density for any linear or rotational direction, and $P_{y y}$ represents the power spectral density before frequency weighting for any linear or rotational direction. The square in Equation $(7.2)$ is necessary, since the weightings provided in the ISO 2631-1 standard are listed to be applied to units of acceleration and not to units of acceleration squared.

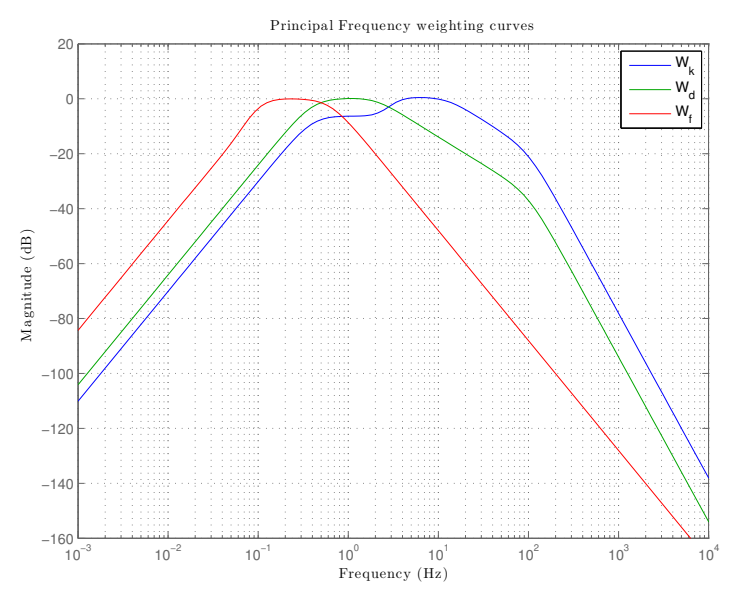

(a) Principal weightings

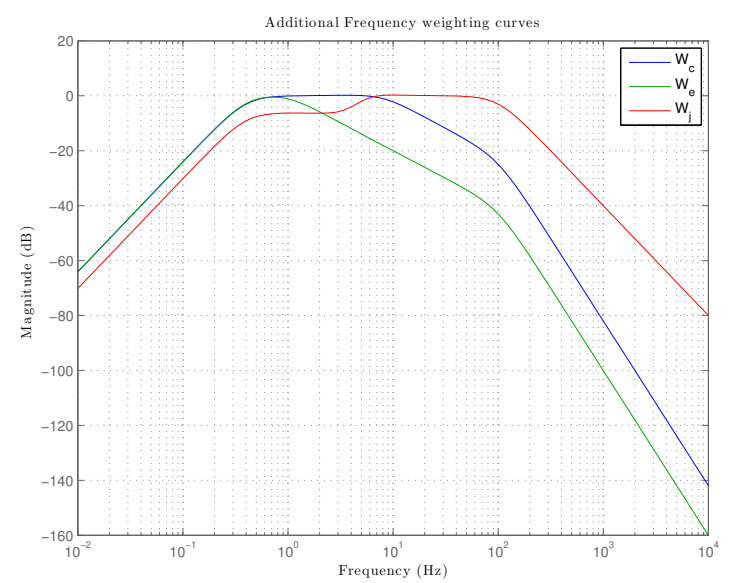

(b) Additional weightings

Figure 7.4: Frequency weighting curves for principal and additional weightings. In the seated position, $W_{k}$ is applied to vertical accelerations, $W_{d}$ is applied to axial and lateral accelerations and $W_{c}$ is applied to axial accelerations at the seat-back (only). These are all used for health, comfort and perception evaluation. $W_{f}$ is applied to vertical accelerations and is used for motion sickness evaluation. $W_{e}$ is applied to rotational accelerations in all three axes and is used for comfort and perception evaluation. The weighting $W_{j}$, not used in this thesis, is used for a vertical recumbent position. (Reproduced from ISO 2631-1 [53])

After obtaining the frequency-weighted acceleration spectra, the frequency-weighted RMS accelerations will be required to evaluate the vibration and associated comfort levels in accordance with ISO 2631-1 [53].

\footnotetext{
${ }^{1}$ The product $H_{h} H_{l}$ represents the band-limiting transfer function; it is a two-pole filter with Butterworth characteristics, $Q_{1}=Q_{2}=1 / \sqrt{2}[53]$.

${ }^{2}$ Proportionality to acceleration at lower frequencies, proportionality to velocity at higher frequencies [53].

${ }^{3}$ Steepness approximately $6 \mathrm{~dB}$ per octave, proportionality to jerk [53].
} 


\section{Overall Frequency-Weighted RMS Acceleration}

In Clause 6 of the ISO standard, it states that the frequency-weighted RMS acceleration $a_{w}$ for any $x, y, z$ axes shall be determined by weighting and appropriate addition of the acceleration spectra,

$$
a_{w}=\sqrt{\sum_{i}\left(W_{w}[i] a[i]\right)^{2}}
$$

where,

$W_{w}[i]$ is the weighting magnitude for the $i^{\text {th }}$ frequency bin

$a[i]$ is the RMS acceleration for the $i^{\text {th }}$ frequency bin

Since the weighting has already been applied to the power spectral density as in Equation 7.2 , Equation (7.3) can be re-written as follows,

$$
a_{w}=\sqrt{\sum_{i} P_{y y_{w}}[i] s_{n}}
$$

to obtain the frequency-weighted RMS acceleration from the power spectral density estimation of the acceleration. The term $s_{n}$ is the required scaling to read the RMS signal value from the power spectral density produced with pwelch.m (see Appendix I for details).

\subsubsection{Effect of Vibrations on Levels of Comfort}

At any seating location, an overall frequency-weighted RMS acceleration (translational or rotational) at a single point $p$ is computed from the RMS values of the weighted accelerations in each direction,

$$
a_{p}=\sqrt{k_{x}^{2} a_{w_{x}}^{2}+k_{y}^{2} a_{w_{y}}^{2}+k_{z}^{2} a_{w_{z}}^{2}}
$$

where,

$$
\begin{aligned}
& a_{w_{x, y, z}} \text { are the RMS values of the weighted translational or rotational accelerations } \\
& k_{x, y, z} \text { are the respective weighting factors }
\end{aligned}
$$

The overall frequency-weighted RMS accelerations at the feet $\left(a_{f}\right)$ and supporting seat-surface $\left(a_{s}\right)$ are then obtained using Equation (7.5). The weighting factors $k$ for the rotational accelerations are provided in units of $\mathrm{m} / \mathrm{rad}$, which converts the accelerations to units of linear acceleration. In the ISO 2631-1 standard, only translational accelerations are considered at the feet, whereas both translational and rotational accelerations are considered at the seat-surface [53. The translational and rotational accelerations at the seat-surface are combined via an additional root sum of square to obtain the total vibration at the seat-surface, 


$$
\begin{aligned}
& a_{s}=\sqrt{a_{s_{t}}^{2}+a_{s_{r}}^{2}} \\
& a_{f}=a_{f_{t}}
\end{aligned}
$$

where $a_{()_{t}}$ denote the translational accelerations and $a_{()_{r}}$ denote the rotational accelerations, each obtained using Equation (7.5) appropriately. The total frequency-weighted RMS vibration $\left(a_{v}\right)$ at any seating location, which includes the vibrations at the supporting seat-surface and the feet, can be computed from the RMS values of the weighted acceleration at each point,

$$
a_{v}=\sqrt{a_{s}^{2}+a_{f}^{2}}
$$

Finally, the standard gives approximate indications of the likely reactions to various magnitudes of total frequency-weighted RMS accelerations, listed in Table 7.3. Using this guide, a level of comfort can be associated with the seating location under investigation.

Table 7.3: Approximate indications of likely reactions to various magnitudes of the total vibration (from ISO 2631-1 53]).

\begin{tabular}{cl}
\hline Vibration magnitude $\left(\mathrm{m} / \mathrm{s}^{2}\right)$ & Comfort indication \\
\hline Less than 0.315 & Not uncomfortable \\
$0.315-0.63$ & A little uncomfortable \\
$0.5-1$ & Fairly uncomfortable \\
$0.8-1.6$ & Uncomfortable \\
$1.25-2.5$ & Very uncomfortable \\
Larger than 2 & Extremely uncomfortable \\
\hline
\end{tabular}

\subsubsection{Effect of Vibrations on the Incidence of Motion Sickness}

For frequencies below $0.5 \mathrm{~Hz}$, the ISO standard proposes to calculate a Motion Sickness Dose Value (MSDV) defined as [53],

$$
M S D V_{z}=\sqrt{\int_{0}^{T} a_{w_{z}}^{2}(t) d t}
$$

where,

$a_{w_{z}}$ is the frequency-weighted acceleration in the $z$-axis at the seat-surface

$T$ is the total period during which motion occurs

In the application of Equation (7.8), the ISO standard suggests that it be used primarily to indicate motion sickness in ships or other sea vessels. However, there is some evidence to suggest that the use of this method could be applicable to motion in aircraft [11]. Furthermore, although the standard suggests that motion sickness incidence should be evaluated in the vertical axis, 
it further notes that should enough data be available and evidence be shown that the effects of other directions become apparent, the procedure can be extended for all directions [53]. In the study by Kubica and Madelaine [11], who evaluated the comfort improvement posed by an integrated control law design, the method was also applied to the lateral axis such that a MSDV can also be defined for the longitudinal and lateral axes,

$$
M S D V_{x, y}=\sqrt{\int_{0}^{T} a_{w_{x, y}}^{2}(t) d t}
$$

where,

$a_{w_{x, y}}$ is the frequency-weighted acceleration in the $x$ - or $y$-axis at the seat-surface

These dose values will then be used to determine the incidence of motion sickness for each translational axis of a seated passenger. Higher dose values indicate a greater incidence of motion sickness. Many factors may affect the susceptibility of an individual to motion sickness, but a percentage of ill passengers can be approximated by,

$$
P I P=K_{m} \times M S D V
$$

where $K_{m}$ is an ill constant. This constant may vary according to the current formation scenario and the group of people that are exposed to the motion. Assuming a mixed population of male and female adults unadapted to the motion, the ISO 2631-1 standard recommends [53],

$$
K_{m}=1 / 3
$$

which was also used by Kubica and Madelaine [11].

\subsection{Development of Simulations for Ride Comfort Evaluation}

An overview of the various considerations given to the simulation setup is presented in the following section.

\subsubsection{Simulation Resolution}

The ISO 2631-1 standard provides guidance methods for the effects of whole-body vibration on health, comfort, perception and motion sickness over a frequency range of 0.1 to $80 \mathrm{~Hz}$. Houbolt 80] presented results that showed that von Kármán spectra agree well with recorded flight data up to frequencies of $10^{3} \mathrm{~Hz}$. In a recent study by Bizinos [3], an evaluation of passenger comfort was done based on extended simulations of 30 minutes and aliasing avoided up to $50 \mathrm{~Hz}$. Closely following on the procedure used by Bizinos, in the current study extended simulations of 30 minutes at different levels of turbulence intensity are performed with a sampling period of $0.01 \mathrm{~s}$. Using the sampling theorem, the Nyquist frequency with this sampling period is also $50 \mathrm{~Hz}$, ensuring that no aliasing occurs up to this frequency. The dynamics of the system do not contain frequencies higher than $50 \mathrm{~Hz}$, and was checked by considering: the natural frequency 
of the fastest pole in the flight control systems; the natural frequency of the fastest pole in the natural dynamics of the aircraft; the cutoff frequency of the turbulence model forming filters; and the cutoff frequency of the seat transmissibility data. This simulation setup should provide reasonable computational speed, with reliable average vibration values in the entire frequency range of interest.

\subsubsection{Seating Locations}

Figure 7.5 illustrates the international all-economy seating arrangement in the Boeing 747-100, reprinted and adapted from a document produced by the Boeing Commercial Airplane Company [81]. In the study by Bizinos [3], several seating locations were investigated in this arrangement with respect to a seating location near the CG - denoted as A. Seating locations B-E are considered as the maximum seating displacements from the CG. A worst-case seating location F was selected by the author based on the comfort results produced during the study.

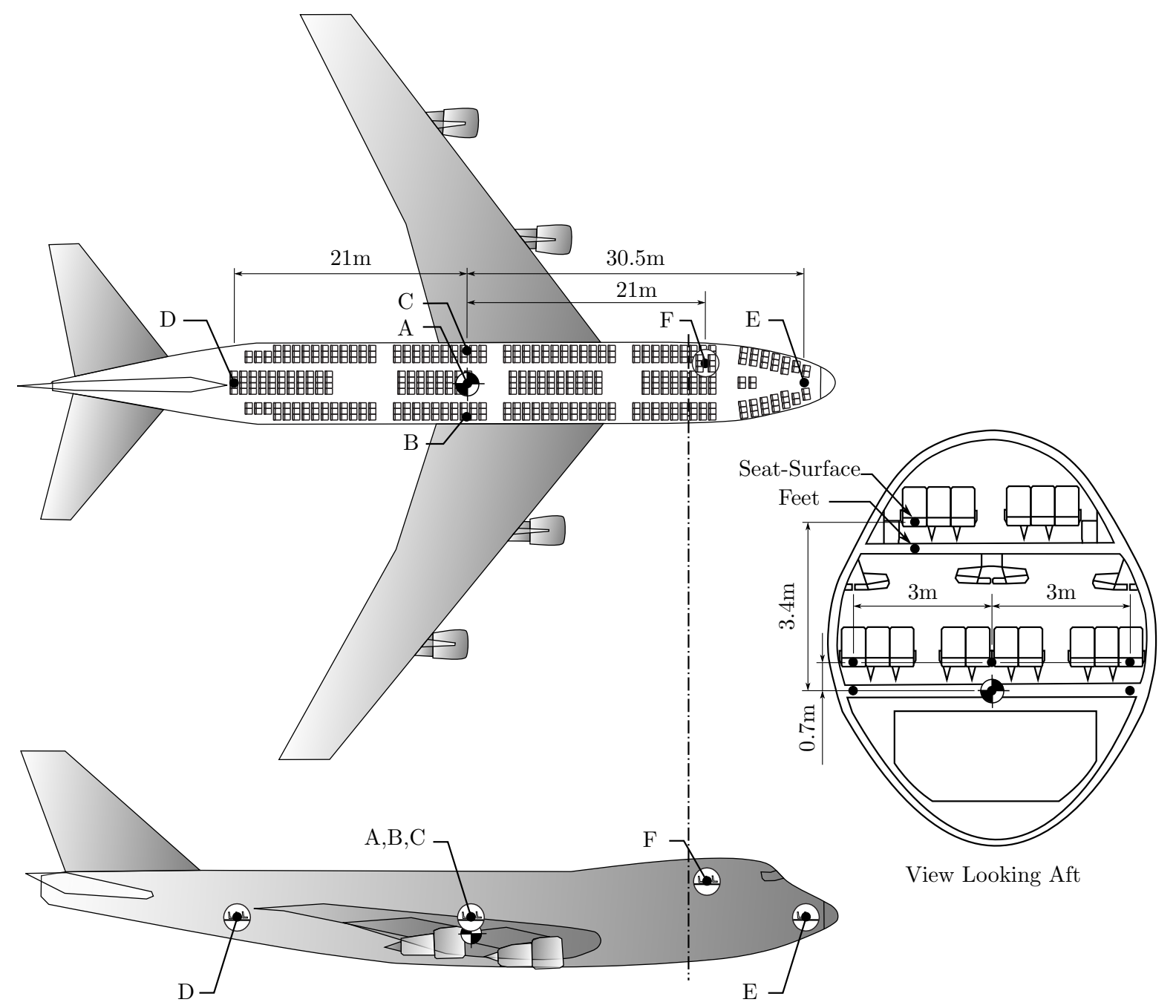

Figure 7.5: Seating locations selected for the evaluation of passenger comfort and motion sickness during formation flight. The interior seating arrangement was selected as the international alleconomy arrangement (adapted from Bizinos [3] and Boeing document D6-58326 [81]). 
Table 7.4: Seating location coordinates from the CG in an international all-economy B747-100.

\begin{tabular}{cccccccc}
\hline Location & \multicolumn{4}{c}{ Coordinates from CG $(m)$} \\
\cline { 2 - 3 } \cline { 7 - 8 } & \multicolumn{3}{c}{ Seat-surface } & & \multicolumn{3}{c}{ Feet } \\
\cline { 2 - 3 } \cline { 6 - 7 } & $x$ & $y$ & $z$ & & $x$ & $y$ & $z$ \\
\hline A & 0 & 0 & -0.7 & & 0 & 0 & 0 \\
B & 0 & 3 & -0.7 & & 0 & 3 & 0 \\
C & 0 & -3 & -0.7 & & 0 & -3 & 0 \\
D & -21 & 0 & -0.7 & & -21 & 0 & 0 \\
E & 30.5 & 0 & -0.7 & & 30.5 & 0 & 0 \\
F & 21 & -1.7 & -3.4 & & 21 & -1.7 & -2.7 \\
\hline
\end{tabular}

Table 7.4 summarises the seat location coordinates considered in this study, used also by Bizinos, and verified to have reasonable agreement with Boeing document D6-58326 [81].

\subsubsection{Feasible Geometric Separations for Passenger Comfort Evaluation}

In Section 6.4 of Chapter 6, it was concluded that acceptable formation-hold performance was only achieved at lateral separations larger than 1 wingspan. Moreover, good formation-hold performance is strongly related to the type of turbulence intensity. It was revealed that the formation-hold guidance laws struggled at all lateral separations investigated during severe turbulence. In moderate turbulence, the guidance laws successfully maintained the desired separations of up to $\eta=1.2$ wingspans of lateral separation. Separations closer to the wake caused the follower to get pulled in and pushed out of the vortex rather aggressively. Considering the safety and comfort of passengers during formation flight, a scenario where the aircraft is aggressively pulled into and pushed out of the trailing wake vortex is deemed unacceptable. As such, these scenarios will be omitted from the evaluation of passenger comfort, and it is assumed these scenarios will be avoided in future formation flight. Table 7.5 summarises the feasible formation flight scenarios for passenger comfort evaluation at the separations listed in Table 3.3. An "unacceptable" rating implies that the formation flight scenario will be omitted from the passenger comfort evaluation, while "acceptable" ratings will be included.

Table 7.5: Summary of feasible formation flight regions for passenger comfort evaluation.

\begin{tabular}{cccc}
\hline Lateral Separation & \multicolumn{3}{c}{ Turbulence Intensity } \\
\cline { 2 - 4 }$\eta$ & Light & Moderate & Severe \\
\hline 1.5 & Acceptable & Acceptable & Unacceptable \\
1.4 & Acceptable & Acceptable & Unacceptable \\
1.3 & Acceptable & Acceptable & Unacceptable \\
1.2 & Acceptable & Acceptable & Unacceptable \\
1.1 & Acceptable & Unacceptable & Unacceptable \\
1 & Unacceptable & Unacceptable & Unacceptable \\
\hline
\end{tabular}




\subsection{Results and Discussion}

The vibrations on-board a leader and follower aircraft were measured at various locations in an international all-economy seating arrangement. Using the overall frequency-weighted RMS accelerations at the feet and seat-surface, a total RMS acceleration magnitude was computed and used to determine the comfort level according to ISO 2631-1. The accelerations at the seatsurface were obtained from the feet accelerations with vertical and lateral transmissibility ratios. The PIP values were also computed for the whole duration of the motion in the simulation.

Results showed that the vibration magnitudes do not differ much between aircraft in isolated flight and formation flight under the same conditions. A clear increase in discomfort from light to moderate turbulence was found. For moderate turbulence, the acceleration magnitudes were found to be above the perception threshold, but remained well within the not uncomfortable region suggested by the ISO 2631-1 standard. It was concluded for both aircraft that a seating location at the front of the aircraft is more comfortable than one at the back of the aircraft.

It was further concluded that formation flight at the lateral separations investigated produce motion sickness incidence levels almost no different to those in isolated flight. The results showed that the PIP levels in isolated and formation flight are very low, peaking at approximately $0.01 \%$ in light turbulence and approximately $0.25 \%$ in moderate turbulence.

In the rest of this section, a detailed discussion of the most important results is given. The focus is placed particularly on acceleration data measured, power spectral densities, weighted power spectral densities, and lastly an evaluation of the effects of vibrations on levels of comfort and motion sickness incidence.

\subsubsection{Measured Acceleration Data}

Figure 7.6 shows the measured linear accelerations in a leader aircraft at the feet of location A near the $\mathrm{CG}$ of the aircraft. The measured time series acceleration data are very similar in shape and form for any seating location and at different turbulence intensities, making it difficult to make any conclusive notes on passenger comfort. However, the general motion time histories in an isolated aircraft were compared with the available data found in related literature [49] [51] 52 ] to verify the validity of the range of the acceleration data measured in the current study.

McKenzie et al. [52] suggest a typical relative vertical acceleration of $0.2 \mathrm{~m} / \mathrm{s}^{2}$ for large jet transport aircraft cruising at $0.82 \mathrm{M}$ at $35000 \mathrm{ft}$. Stephens [49] reported peak acceleration ranges of a Boeing 747 during cruise of $0.03 \mathrm{~g}-0.15 \mathrm{~g}\left(0.29 \mathrm{~m} / \mathrm{s}^{2}-1.47 \mathrm{~m} / \mathrm{s}^{2}\right)$ for vertical and $0.01 \mathrm{~g}-0.17 \mathrm{~g}\left(0.1 \mathrm{~m} / \mathrm{s}^{2}-1.67 \mathrm{~m} / \mathrm{s}^{2}\right)$ for lateral peak accelerations (see Figure 7.7 ). Richards et al. [51] illustrated typical motion time histories produced by portable ride quality measuring apparatus during turbulent conditions. A typical motion history of 2 minutes indicated absolute longitudinal and lateral accelerations ranges of less than $0.25 \mathrm{~g}\left(2.45 \mathrm{~m} / \mathrm{s}^{2}\right)$ and vertical accelerations of less than $0.5 \mathrm{~g}\left(4.9 \mathrm{~m} / \mathrm{s}^{2}\right)$. 


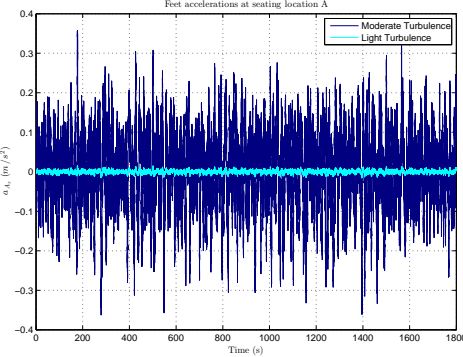

(a) Axial accelerations

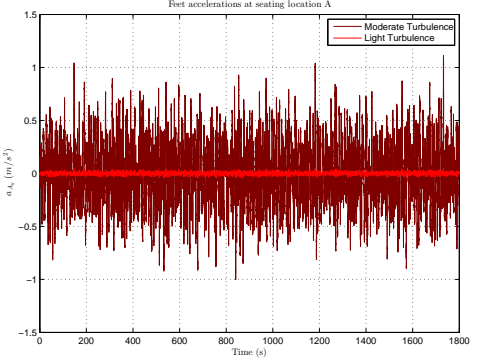

(b) Lateral accelerations

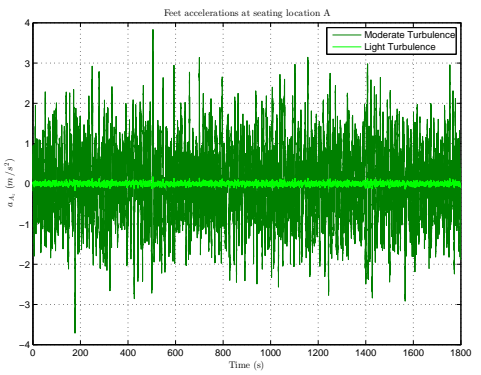

(c) Vertical accelerations

Figure 7.6: Measured time series acceleration data in a leader aircraft at the feet of a passenger seated at location A. Simulations were performed in light and moderate turbulence conditions.

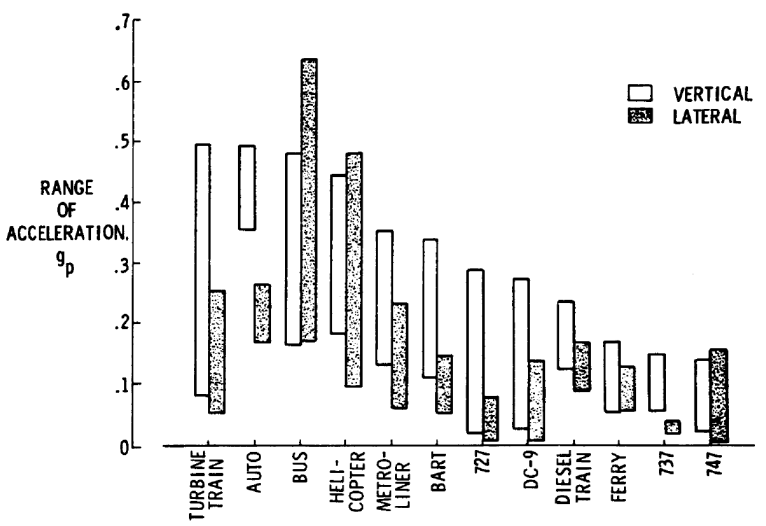

Figure 7.7: Comparative data indicating the range of peak accelerations obtained on a number of vehicles during cruise. An indication is given for the Boeing 747 during cruise of $0.03 \mathrm{~g}-0.15 \mathrm{~g}$ $\left(0.29 \mathrm{~m} / \mathrm{s}^{2}-1.47 \mathrm{~m} / \mathrm{s}^{2}\right)$ for vertical and $0.01 \mathrm{~g}-0.17 \mathrm{~g}\left(0.1 \mathrm{~m} / \mathrm{s}^{2}-1.67 \mathrm{~m} / \mathrm{s}^{2}\right)$ for lateral peak accelerations. (from Stephens [49])

In summary, the linear time series acceleration data shown in Figure 7.6 provide realistic accelerations as experienced in an isolated aircraft during cruise conditions. To the knowledge of the author of the current study, no conclusive literature has been found to indicate the typical time series acceleration data measured in a formation aircraft, but it is not expected to differ significantly from the data found and illustrated in Figure 7.6 .

\subsubsection{Power Spectral Densities}

In the study by Bizinos [3], the author decided to investigate four lateral separations, $\eta=0.7$, $0.76,0.9$, and 1.2. Assuming longitudinal geometric separation of 10 wingspans, $\xi=-10$, and zero geometric vertical separation, $\zeta=0$, the simulations were performed in nominal cruising conditions of $\bar{V}=236 \mathrm{~m} / \mathrm{s}$ at $40000 \mathrm{ft}$. A clear increase in acceleration magnitudes was found for longitudinal, vertical, roll and yaw acceleration magnitudes at lateral separations $\eta=0.7$ and 0.76 over a frequency range of 0.01 to $50 \mathrm{~Hz}$. The increase of the same acceleration magnitudes was significantly reduced at lateral separations $\eta=0.9$ and 1.2 wingspans. In the discussion of the results, high roll instability was reported during formation flight at $\eta=0.7$ and 0.76 . 
The current study has revealed, as explained in Section 6.4.1 of Chapter 6, that the designed formation guidance laws are unable to maintain the desired separations $(\xi=-10, \zeta=0$ and $\eta)$ as the follower comes closer than $\eta=1.1$ wingspans. Thus, although Bizinos [3] produced results for lateral separations closer than $\eta=1.1$ wingspans, the possibility of the flight controllers being able to maintain the separations and hold the aircraft in formation is practically unfeasible if not impossible with the current flight control architecture and wake model.

In summary, this means only one of the four lateral separations considered by Bizinos [3], namely $\eta=1.2$ wingspans, will be valid for comparison with the results produced in the current study. The acceleration power spectral densities for both the leader and the follower aircraft during formation flight are concisely and comparatively summarised in Appendix J. The results were produced from simulations in light and moderate turbulence at different acceptable lateral separations, as summarised in Table 7.5. All simulations assume nominal cruising conditions of $\bar{V}=236 \mathrm{~m} / \mathrm{s}$ at $40000 \mathrm{ft}$. A detailed discussion of the results and important observations is given in the next section where some of the related literature [3] [48] is also used to validate the accuracy of the results.

\section{Leader Acceleration Spectra near the CG}

For the leader aircraft in isolation, Figure 7.8 illustrates the linear acceleration spectra at the feet and seat-surface near the CG in moderate turbulence. The effect of the seat transmissibility ratios can clearly be seen. The axial and lateral seat-surface accelerations in Figures $7.8 \mathrm{a}$ and $7.8 \mathrm{~b}$ are amplified at frequencies around $2 \mathrm{~Hz}$ and attenuated after approximately $10 \mathrm{~Hz}$. The vertical seat-surface acceleration spectra in Figure $7.8 \mathrm{c}$ is amplified at frequencies closer to $5 \mathrm{~Hz}$. The general vibratory responses in Figure 7.8 for acceleration spectra at the feet and seat-surface are similar. As such, assuming that the seat-surface accelerations will contribute the most to passenger comfort (see weighting factors in Table 7.2), only the results at the seat-surface will be used in the discussions to follow.

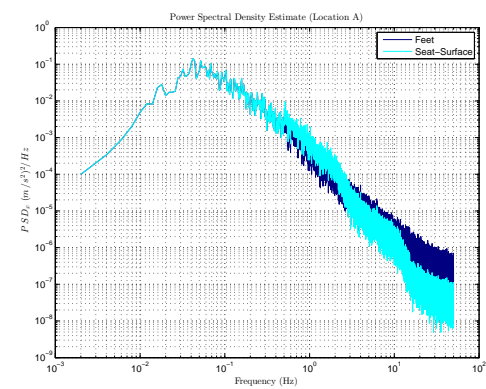

(a) Axial acceleration spectra

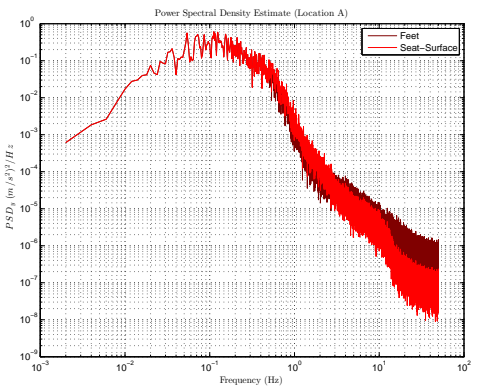

(b) Lateral acceleration spectra

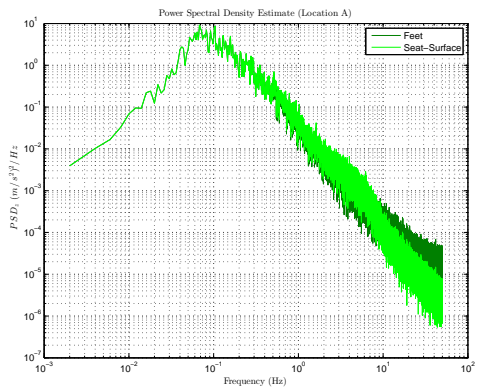

(c) Vertical acceleration spectra

Figure 7.8: Linear acceleration spectra measured at the feet and seat-surface at location A near the CG. Simulation performed in moderate turbulence.

Figure 7.9 illustrates the measured linear $(7.9 \mathrm{a}-7.9 \mathrm{c})$ and rotational $(7.9 \mathrm{~d}-\mathrm{7.9f})$ acceleration 
spectra at the seat-surface of location A near the CG. The measured data represents typical acceleration magnitudes over linearly scaled frequencies of up to $50 \mathrm{~Hz}$ during light and moderate turbulence. In all cases, the general vibratory response of the aircraft is of a similar nature in all three directions - both translational and rotational. A clear increase is found from light to moderate turbulence, and the highest vibration magnitudes are found to be in the linear vertical axis. The energy is concentrated at very low frequencies between $0 \mathrm{~Hz}$ and $1 \mathrm{~Hz}$, similar to what was observed in other literature studies [47] [49] [50], emphasising the need to pay special attention to motion sickness incidence.

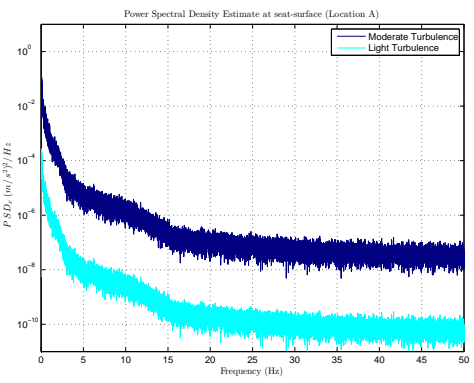

(a) Axial acceleration spectra

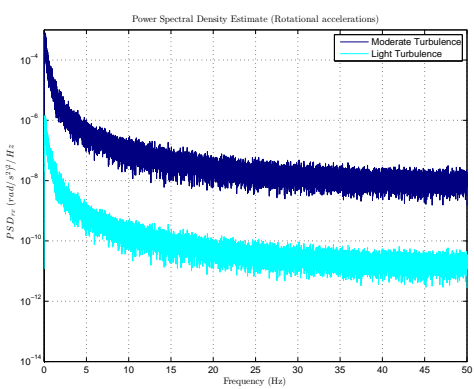

(d) Roll acceleration spectra

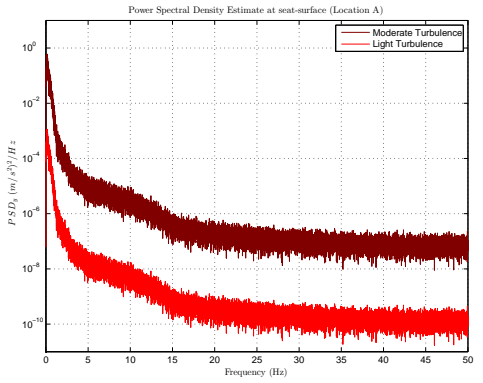

(b) Lateral acceleration spectra

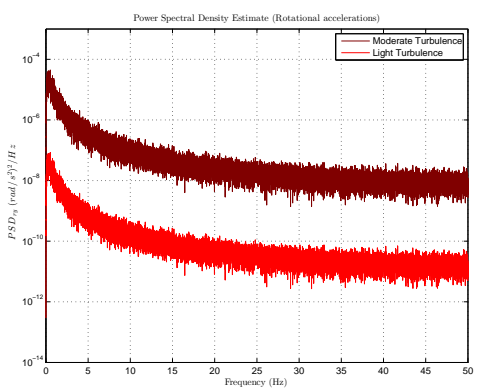

(e) Pitch acceleration spectra

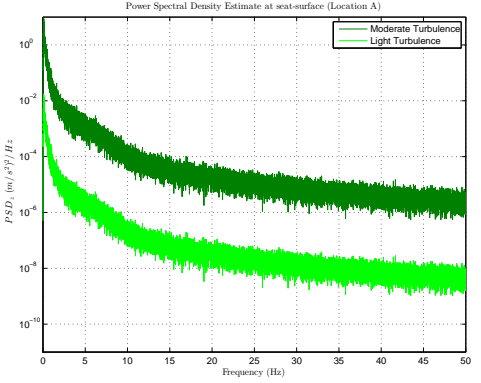

(c) Vertical acceleration spectra

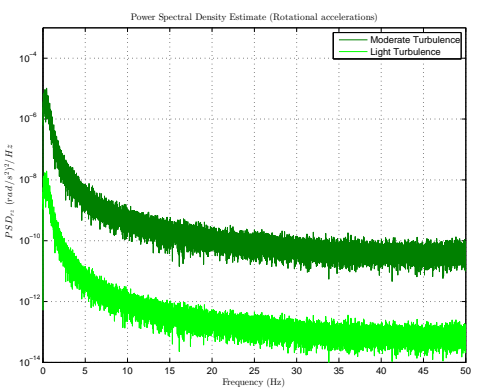

(f) Yaw acceleration spectra

Figure 7.9: Measured (a)-(c) linear and (d)-(f) rotational acceleration spectra at the seat-surface of location A near the CG.

In a study by Stephens [49], various vibration and noise data were measured for different air and surface vehicles. The vibration and noise measurements presented were sampled at selected intervals for rides of approximately two minutes in duration. Concerning conventional transport aircraft, Figure 7.10 illustrates the typical range of vibration magnitudes (in g) experienced in the vertical and lateral directions during moderate turbulence 7.10a), and also the range of vertical vibrations during light and moderate turbulence $7.10 \mathrm{~b}$. Recorded data represents floor vibrations near the CG. It is noticed that this data is very similar in vibration magnitudes and shape to that produced in this current study as in Figure 7.9.

Concerning the acceleration power spectral densities for a follower aircraft near the CG as well as fore and aft of the fuselage, a discussion and comparison of the results are given next, comparing the results to the findings of Bizinos [3]. 


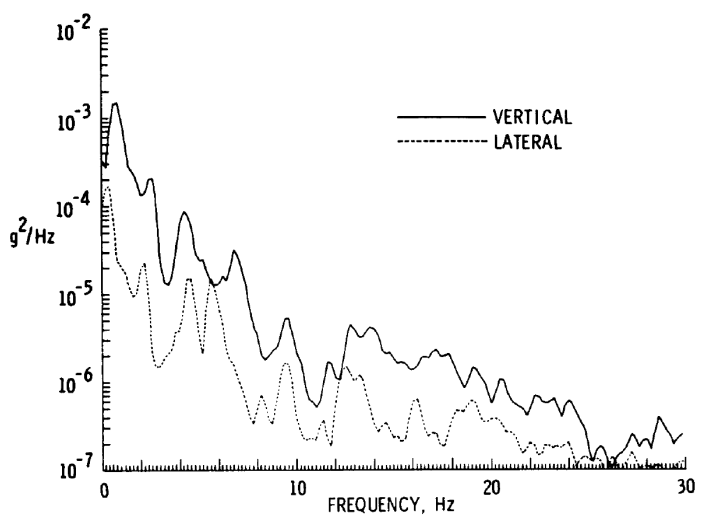

(a) Range of accelerations in moderate (rough) turbulence conditions

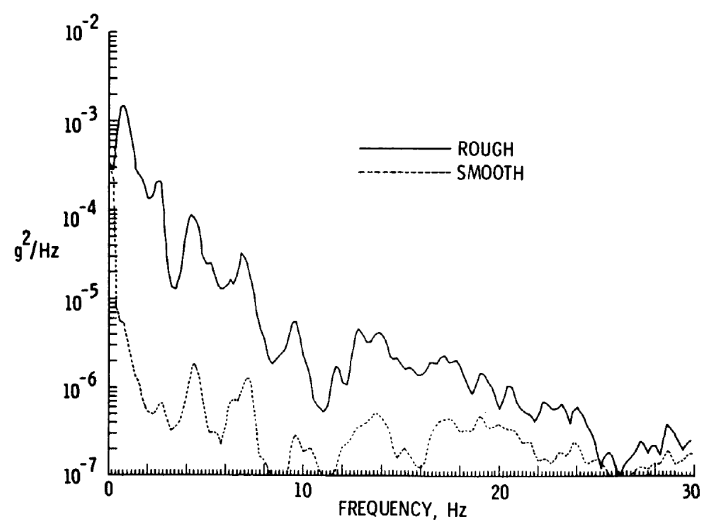

(b) Range of vertical accelerations in light (smooth) and moderate (rough) turbulence conditions

Figure 7.10: Typical range of vibration magnitudes experienced in the vertical and lateral directions during light and moderate turbulence (from Stephens [49]).

\section{Follower Acceleration Spectra near the CG}

A brief scan of the results for location A near the CG in Appendix J (use TableJ.1) indicates one very important finding: that the accelerations experienced in formation are not very different from those experienced in isolation under the same turbulence conditions. Similar to the leader aircraft in isolation, for a lateral separation of $\eta=1.5$ wingspans, Figure 7.11 shows that the general vibratory response of the follower aircraft near the CG is of a similar nature in all three directions, both translational and rotational. The highest linear vibration magnitudes are found to be in the vertical axis, whereas the highest rotational vibration magnitudes are found to be in the roll and yaw axes.

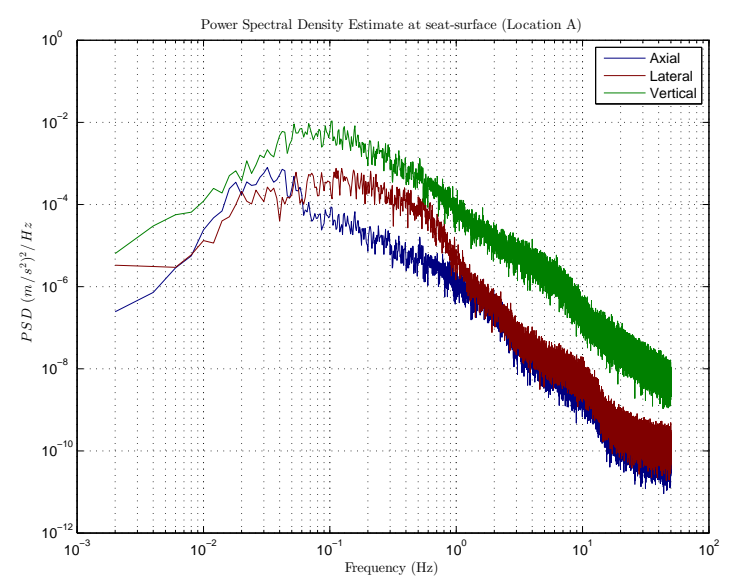

(a) Linear acceleration spectra at CG

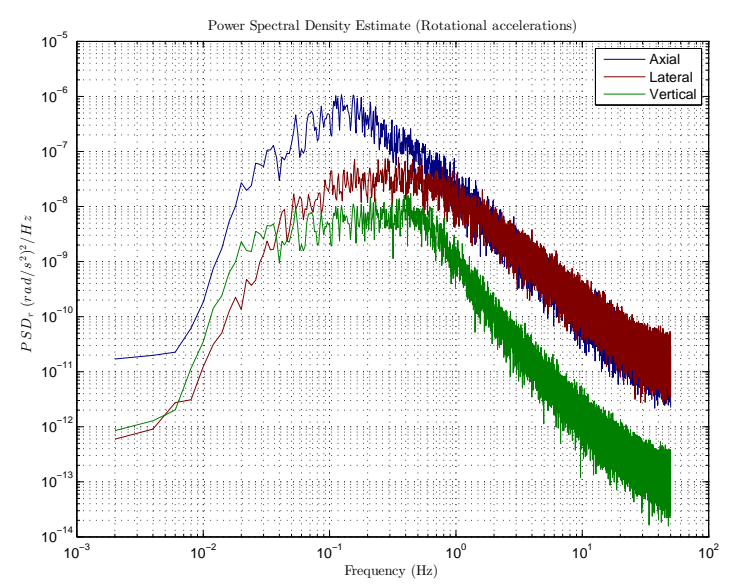

(b) Rotational acceleration spectra at CG

Figure 7.11: Linear and rotational acceleration spectra in formation during light turbulence and at a lateral separation of $\eta=1.5$.

Figure 7.12 illustrates the linear and rotational acceleration spectra at location A near the CG. Simulations were performed in turbulence and at different lateral separations. The linear 

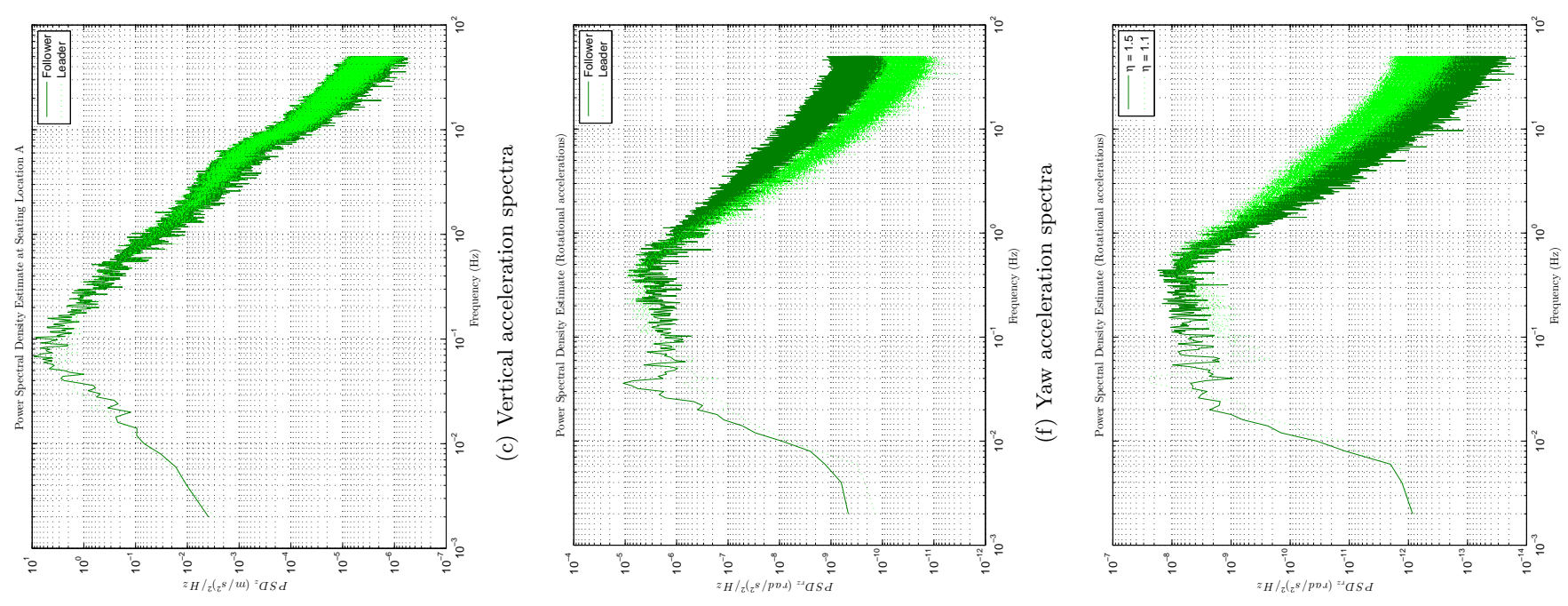

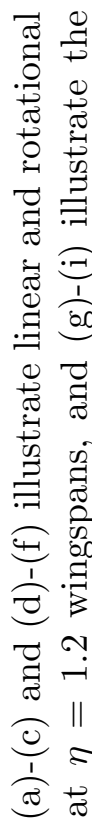
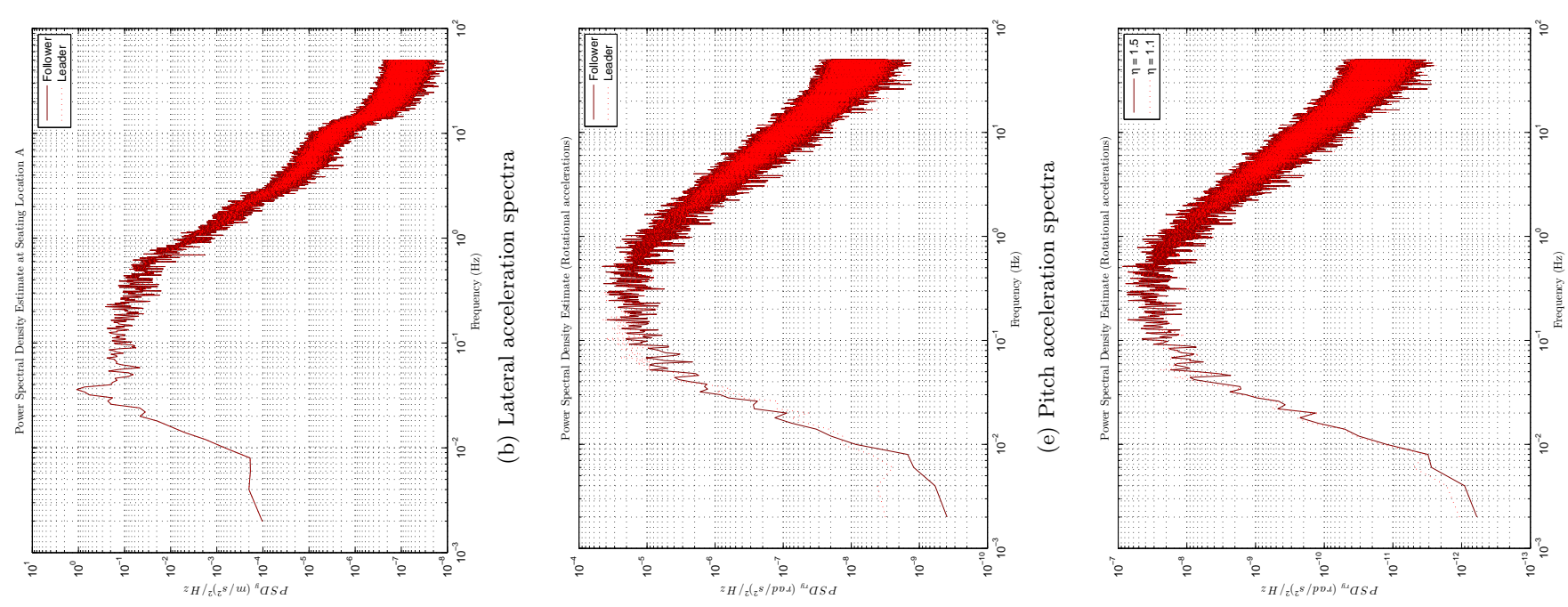

نु

은

สี

苞焉

苋

莺

造

है

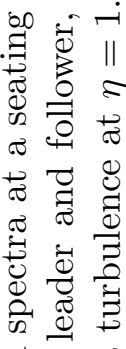

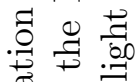

훙
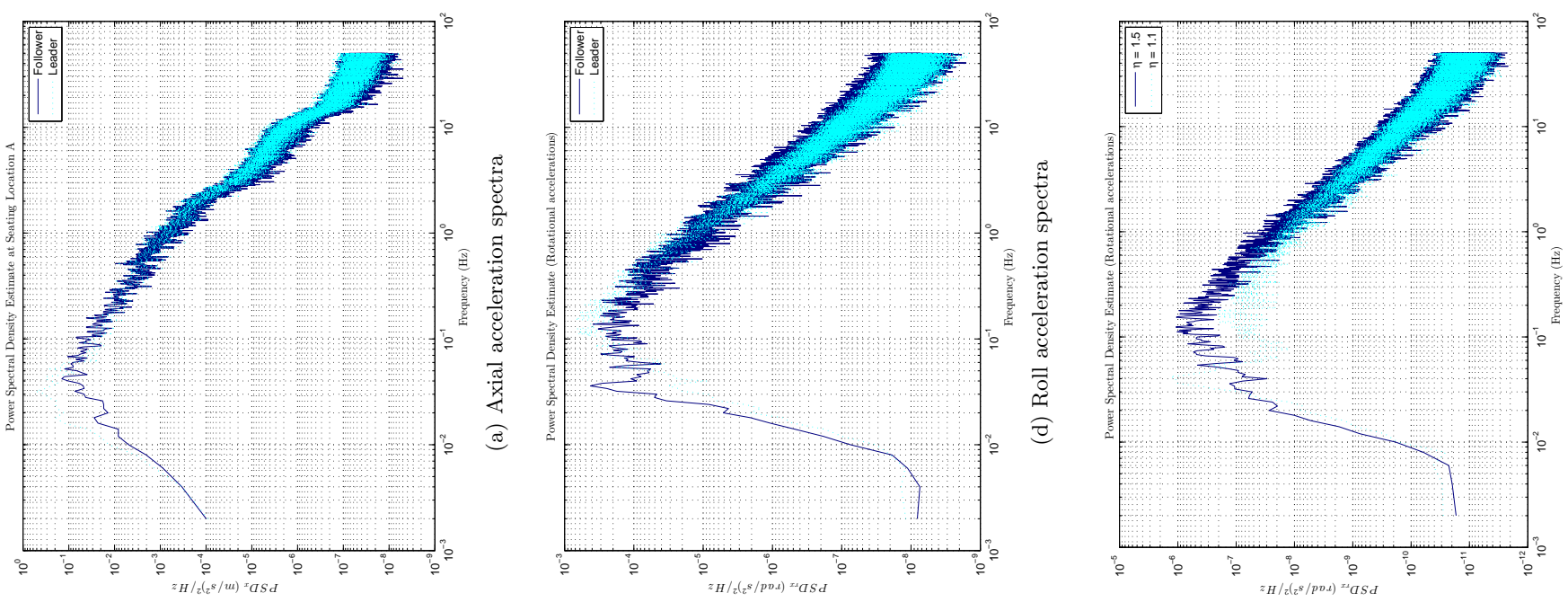

.

졍 $\rightarrow$

胥

苦

인

ఫ్ర

เ

Ð

龶 की.

긍

N

$\stackrel{0}{0}$

300 
acceleration spectra show a negligible difference in moderate turbulence (Figure $7.12 \mathrm{a}$ to $7.12 \mathrm{c}$ ), and also show a negligible difference as the follower moves closer to the trailing vortex in light turbulence (Figure J.9). Concerning the rotational acceleration spectra, pitch acceleration magnitudes also show negligible differences (Figures 7.12e and 7.12h). However, a clear increase in yaw acceleration magnitudes is found in a follower aircraft, illustrated in Figure 7.12f, emphasised by the results shown in Figure 7.12i as the follower moves closer to the trailing vortex. All of these observations are in line with similar trends noted by Bizinos [3] at the same lateral separations.

\section{Follower Acceleration Spectra Offset from CG}

Figure 7.13 illustrates the linear acceleration spectra at the seat-surface in a follower aircraft for different seating locations. The rotational spectra offset from the CG remains the same due to a rigid-body assumption. The results are in all cases compared to a location A near the CG. Simulations were performed in moderate turbulence and at a desired lateral separation of $\eta=1.2$ wingspans.

First consider seating locations B and C, as seen in Figures 7.13a to 7.13c - C being on the same side as the lead aircraft in a right echelon formation. Very little difference is found in all three translational acceleration magnitudes between the different seating locations in the follower aircraft. While Bizinos [3] illustrated that vertical and longitudinal accelerations increased at seating location $\mathrm{C}$ and decreased at location $\mathrm{B}$ with respect to seating location $\mathrm{A}$, the results in the current study show negligible changes in acceleration magnitudes when moving to the left or right of the $\mathrm{CG}$ in a follower aircraft.

Now consider seating locations D and E, as seen in Figures 7.13d to 7.13f, at the aft and fore of the aircraft fuselage respectively. Unlike B and C, seating locations longitudinally displaced from the $\mathrm{CG}$ have a more prominent effect on the lateral and vertical acceleration magnitudes. No difference in the longitudinal acceleration spectra is found between these seating locations. In the lateral axis, larger acceleration magnitudes are experienced at D compared to A. The opposite occurs at E, where smaller acceleration magnitudes are produced. In the vertical axis, the same pattern results: higher acceleration magnitudes at location $\mathrm{D}$, and smaller acceleration magnitudes at location E. Although the results for the leader are not produced here, these trends are true for both aircraft, with a seemingly larger change in the vibratory response of the follower in formation.

In the study by Bizinos [3], it was found for an isolated aircraft that a seat at the front of the aircraft will result in a more comfortable flight than a seat at the rear of the aircraft. It was further found that the opposite happens in formation flight, where larger acceleration magnitudes are found at the front of the fuselage compared to the rear of the aircraft. Choosing to investigate a worst seating location $\mathrm{F}$, it was suggested that for an aircraft in a right echelon formation, the front and port side of the aircraft are most uncomfortable.

The current study produced a different result for an aircraft in a right echelon formation. Similar to the leader aircraft, a seat in the aft of a follower aircraft will be more uncomfortable than a seat in the front. Moreover, consider Figures 7.13g to 7.13i, illustrating the linear 

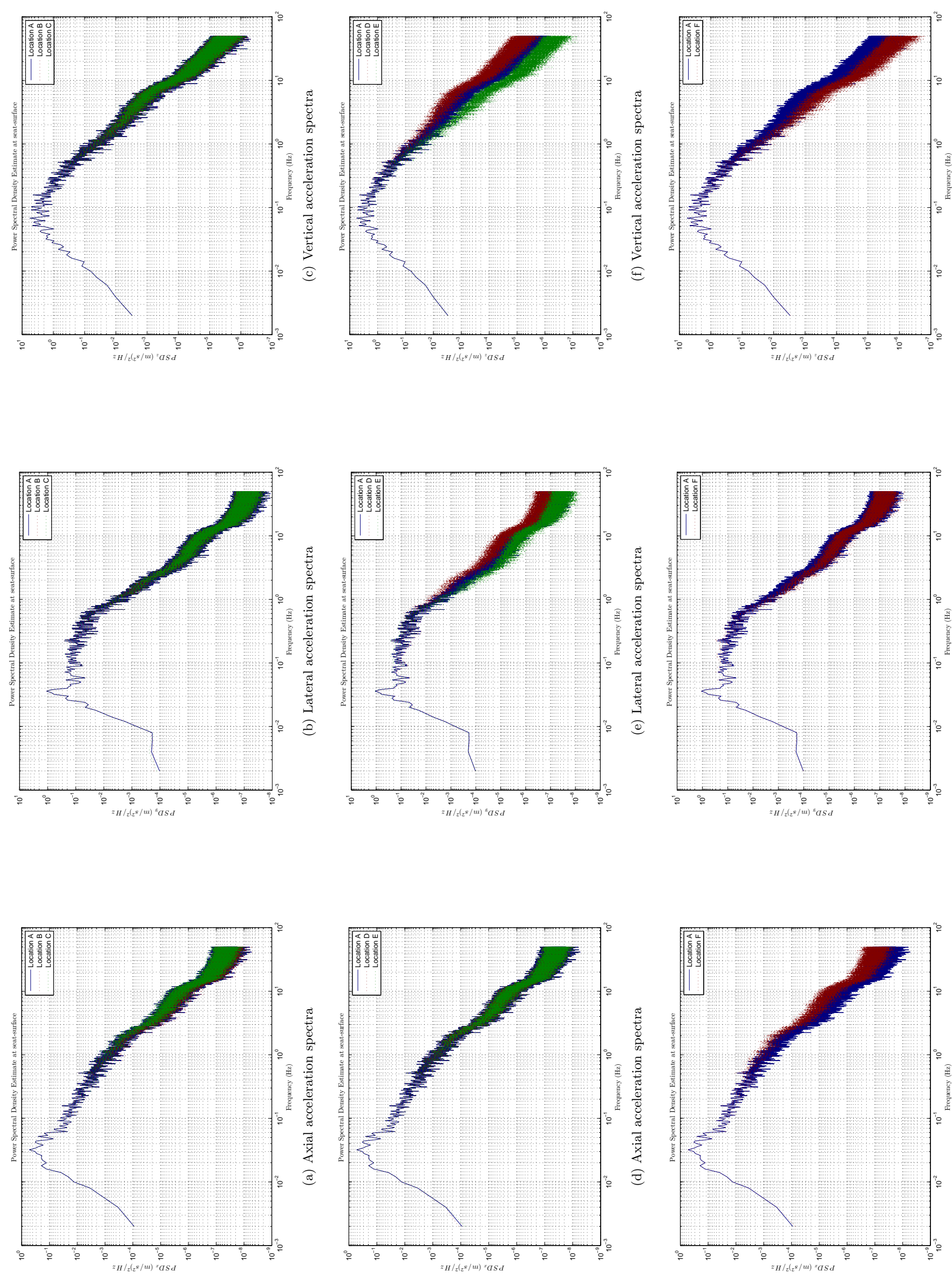

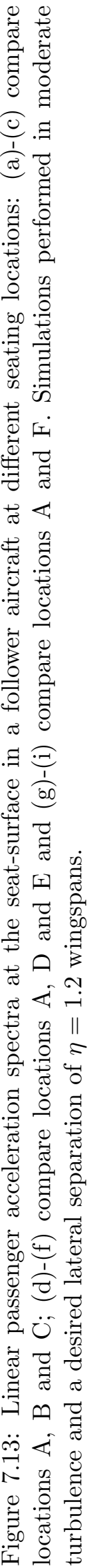


vibration magnitudes at seating location $\mathrm{F}$ in relation to $\mathrm{A}$. While longitudinal accelerations show a slight increase, vertical vibration magnitudes show a decrease in formation flight. Keeping in mind the guidance of the ISO 2631-1 standard, the weighting for vertical vibrations exhibits slightly stronger amplification than the weighting for longitudinal and lateral accelerations at frequencies larger than $5 \mathrm{~Hz}$. It is then concluded, unlike the conclusion made by Bizinos [3], that the port side of the aircraft and a seating location displaced higher up the vertical axis will be more comfortable than a seating location A near the CG.

\subsubsection{Weighted Power Spectral Densities}

The weighted linear acceleration spectra at the feet and seat-surface, as well as the weighted rotational spectra at the seat-surface, follow the prescribed method from Section 7.2.1. To illustrate the result of the weighting filters, Figure 7.14 shows the weighted spectral density estimations of the linear and rotational accelerations at seating location A for the vibrational comfort frequency range 0.5 to $50 \mathrm{~Hz}$. Figure 7.15 shows the weighted spectral density estimation of the linear vertical accelerations at seating location A for the motion sickness frequency range 0.1 to $0.5 \mathrm{~Hz}$.

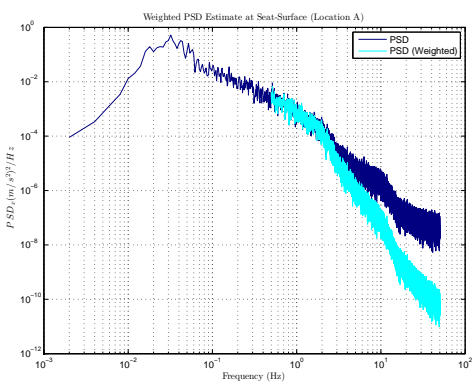

(a) Axial acceleration spectra

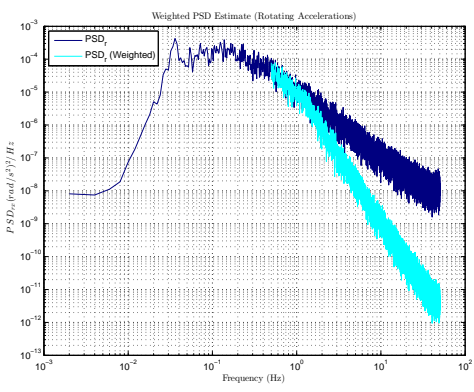

(d) Axial acceleration spectra

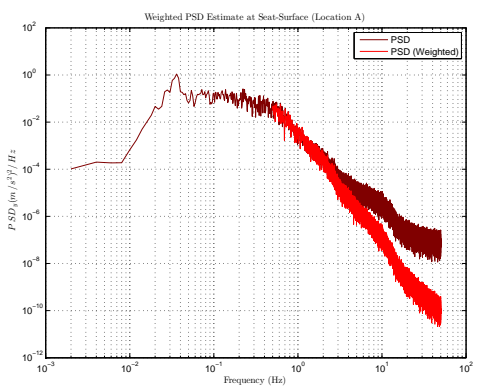

(b) Lateral acceleration spectra

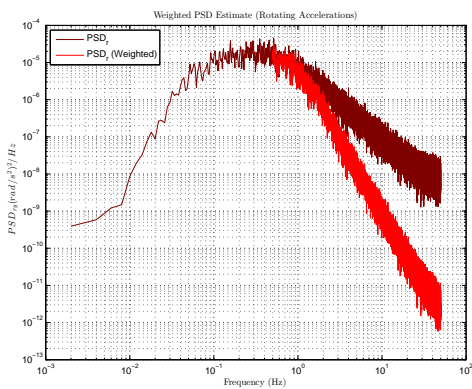

(e) Lateral acceleration spectra

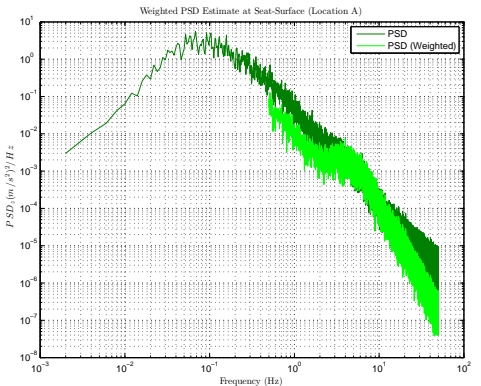

(c) Vertical acceleration spectra

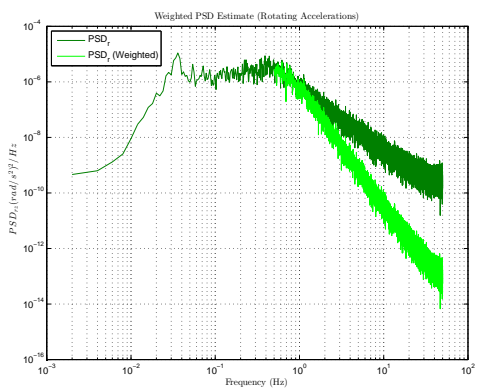

(f) Vertical acceleration spectra

Figure 7.14: Weighted spectral density estimations of the linear and rotational accelerations for the frequency range $0.5-50 \mathrm{~Hz}$, used in the evaluation of vibrational comfort. Simulation performed in moderate turbulence and a desired lateral separation of $\eta=1.2$ wingspans. 


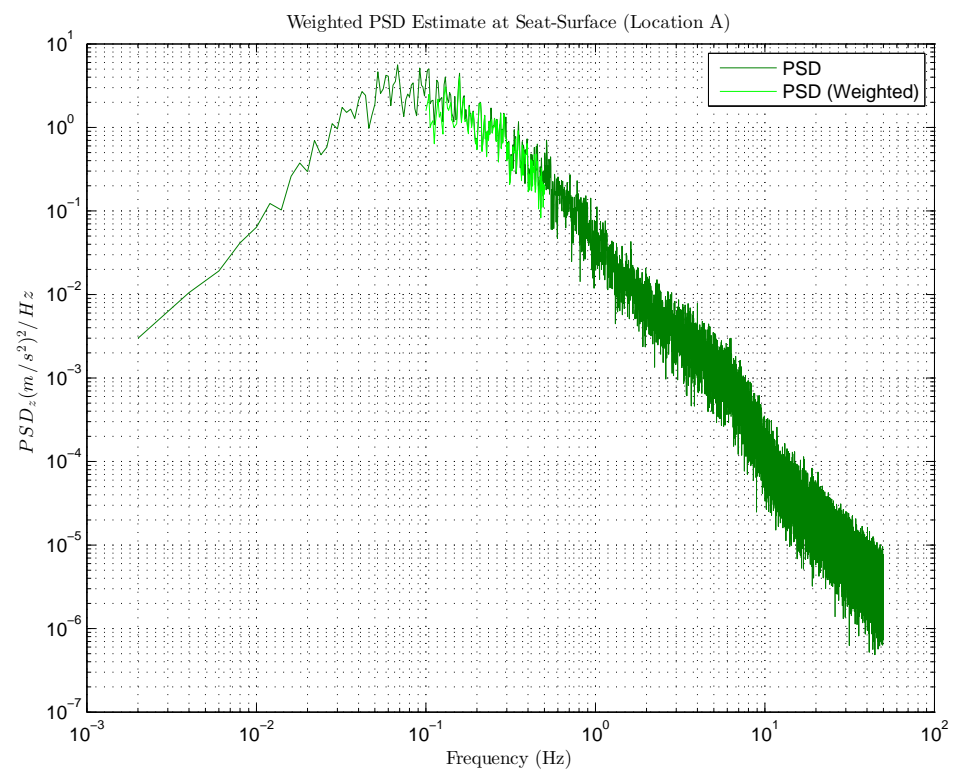

Figure 7.15: Weighted spectral density estimation of the linear vertical accelerations for the frequency range $0.1-0.5 \mathrm{~Hz}$, used in the evaluation of motion sickness. Simulation performed in moderate turbulence and a desired lateral separation of $\eta=1.2$ wingspans.

\subsubsection{Evaluating the Effect of Vibrations on Levels of Comfort}

Figure 7.16 shows the comfort along the fuselage of a follower aircraft during formation flight. Simulations were performed in light and moderate turbulence at different lateral separations. In Figure 7.16b, as a benchmark for comparison in moderate turbulence, the comfort in a leader aircraft in light turbulence is also shown. A brief scan of the results reveals an important finding: the vibration magnitudes do not differ significantly between an aircraft in isolated flight and the follower aircraft in formation flight under the same conditions.

As expected, a clear increase in discomfort is found from light to moderate turbulence for both the leader and follower aircraft. According to ISO 2631-1 [53], the median perception threshold is considered approximately $0.015 \mathrm{~m} / \mathrm{s}^{2}$, decreasing only slightly with increasing vibration durations. In light turbulence, the computed acceleration levels are considered sufficiently small to assume that no discomfort will be experienced by most of the passengers. For aircraft in moderate turbulence, however, the acceleration magnitudes are found to be above the perception threshold at any seating location, but remain well within the not uncomfortable region suggested by the ISO 2631-1 standard. From Figure 7.16b it is also evident that in both a leader and follower aircraft - flying at any lateral separation shown in the plot - a seating location at the front of the aircraft (E and F) would seem more comfortable than one aft of the aircraft (D).

Kubica et al. [11 presented results for comfort in realistic vertical turbulence. The results showed that comfort levels were better at the front of the fuselage compared to a location near the rear of the fuselage, but the best results were near the CG of the aircraft. Vertical weighted acceleration magnitudes ranged from $0.1-0.4 \mathrm{~m} / \mathrm{s}^{2}$ (not uncomfortable to a little uncomfortable). Similar ranges of vibration magnitudes were found in the current study, but showed that a 


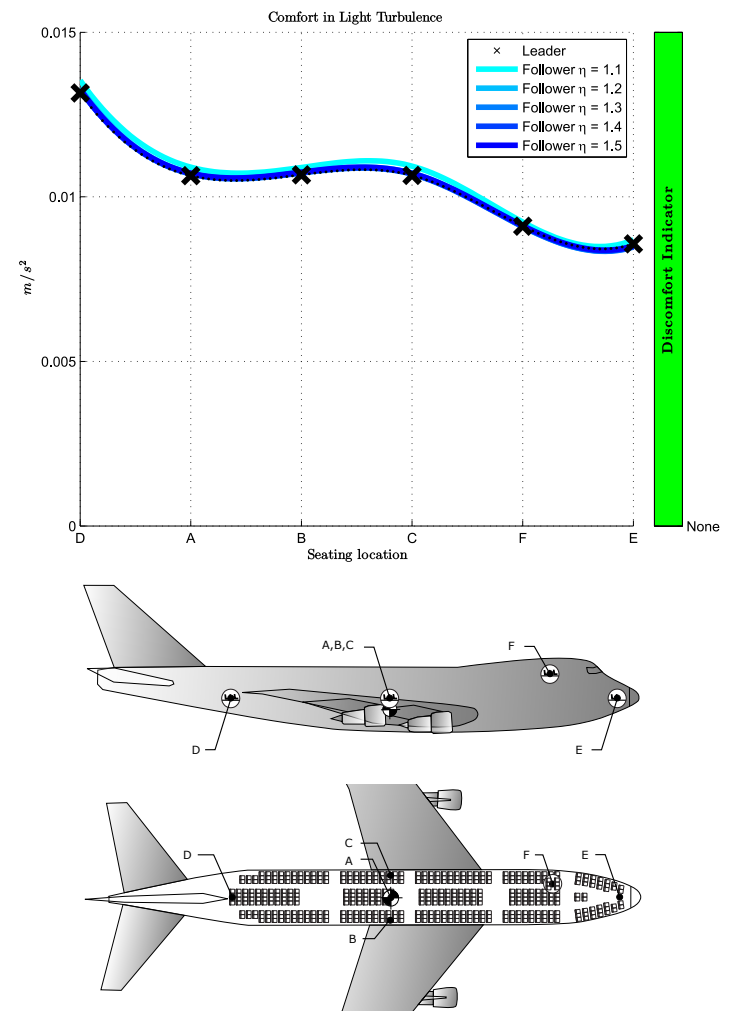

(a) Comfort in light turbulence
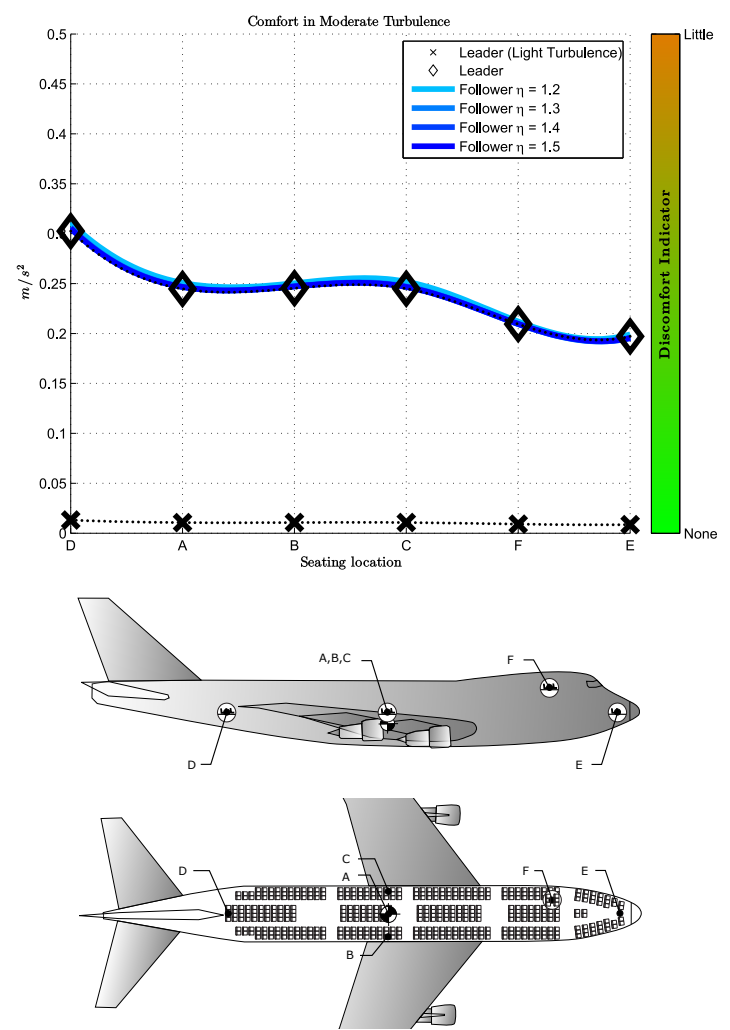

(b) Comfort in moderate turbulence

Figure 7.16: Levels of comfort in a leader and follower along the aircraft fuselage. Simulations were performed in light and moderate turbulence for lateral separations of up to $\eta=1.1$ wingspans.

seating location at the front is the most comfortable and not a location near the CG. This can be explained by noting that the study by Kubica et al. [11] considered bending modes of the aircraft, whereas the current study assumes a rigid body aircraft.

Figure 7.17 shows a comparison between the results of the current study with those of Bizinos [3]. Comparisons were done in relation to a seating location A near the CG. Small differences are seen in the results of locations A, B and C. Considering moderate turbulence, the acceleration magnitudes found in the current study remain constant at approximately $0.25 \mathrm{~m} / \mathrm{s}^{2}$ (not uncomfortable), and the level of comfort for seats laterally displaced from the CG remain the same. For seats longitudinally displaced from the CG, the differences are more apparent. The acceleration magnitudes in moderate turbulence at location $\mathrm{D}$ are in the same order of magnitude as those predicted by Bizinos at approximately $0.3 \mathrm{~m} / \mathrm{s}^{2}$ (not uncomfortable). At $\mathrm{E}$, the acceleration magnitudes are approximately $0.2 \mathrm{~m} / \mathrm{s}^{2}$ (not uncomfortable), more than predicted by Bizinos. Figure 7.17 also shows, as has been previously discussed, that a seating location $\mathrm{D}$ aft of the aircraft is likely more uncomfortable than a location $\mathrm{E}$ at the front of the aircraft. 

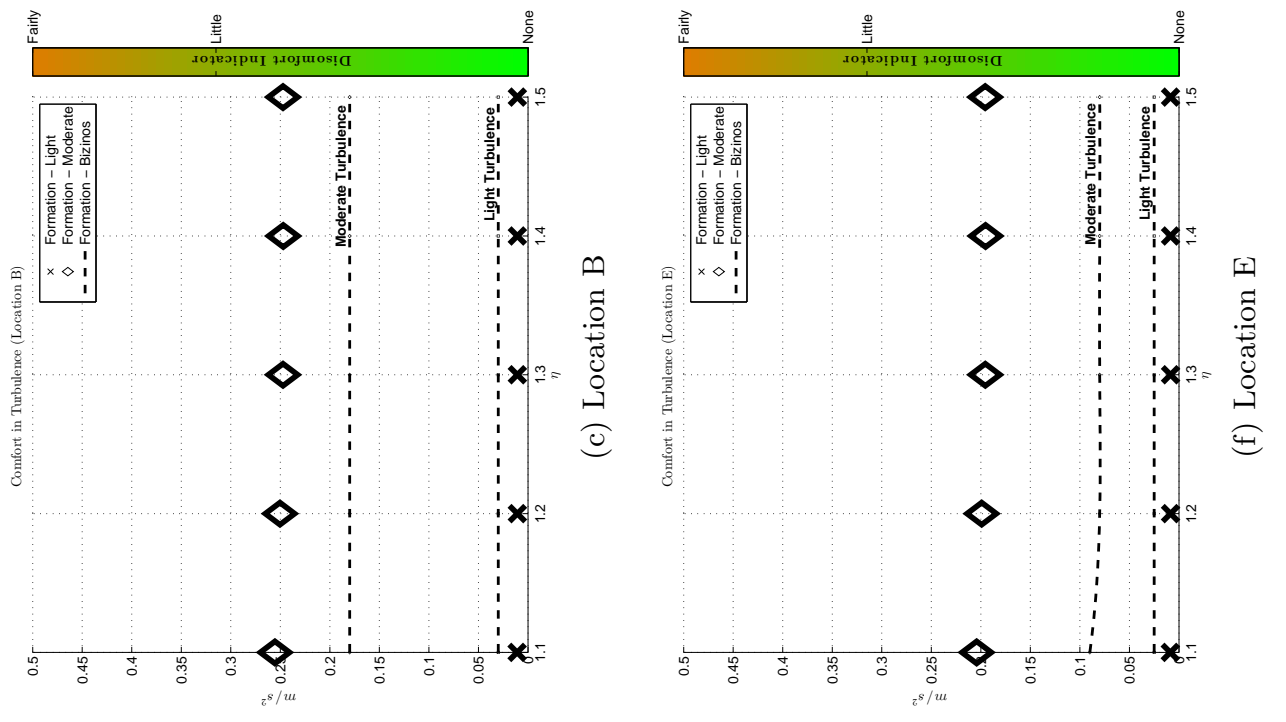

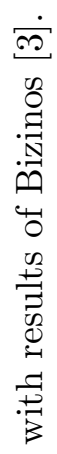
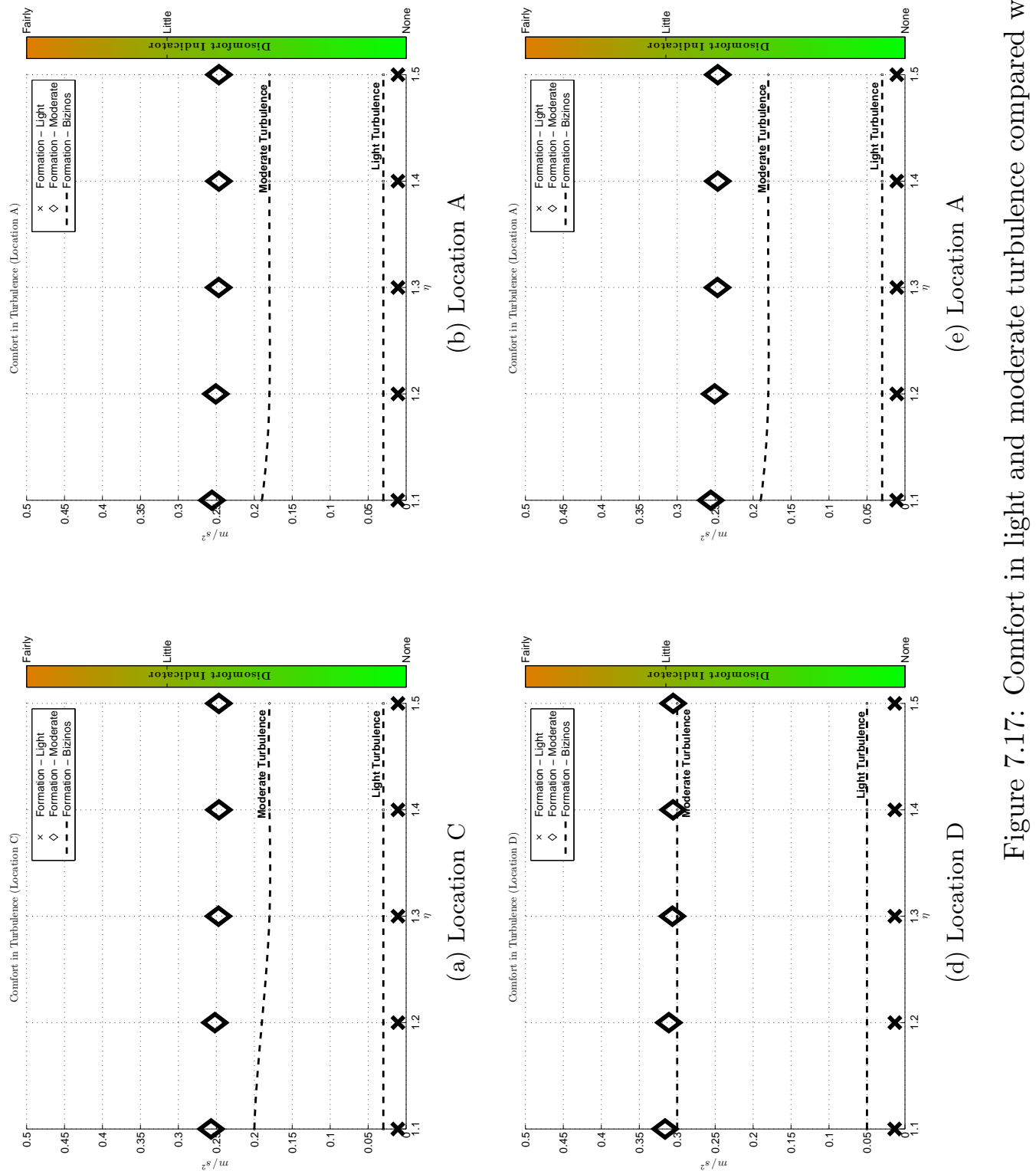


\subsubsection{Evaluating the Effect of Vibrations on Motion Sickness Incidence}

Figure 7.18 illustrates the PIP results in the vertical and lateral axes of a leader and follower aircraft for simulations in moderate turbulence. The rest of the results are summarised in Appendix $\mathrm{K}$ where, for light turbulence, a zoomed-in view of the results is given.
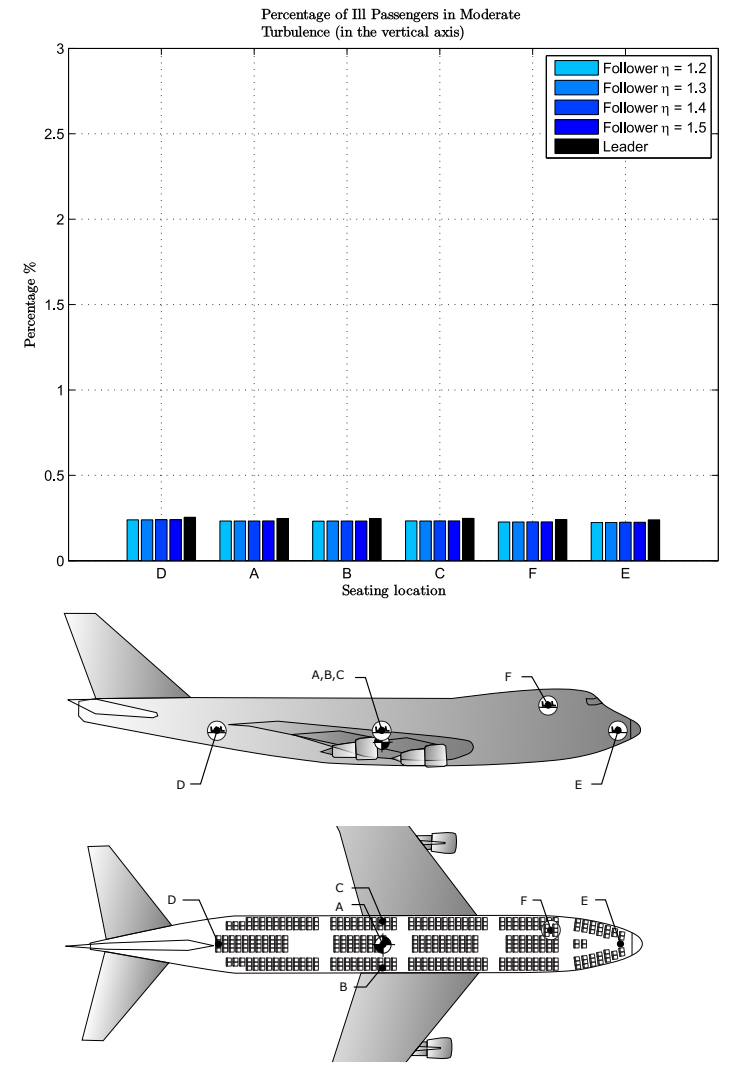

(a) Vertical axis
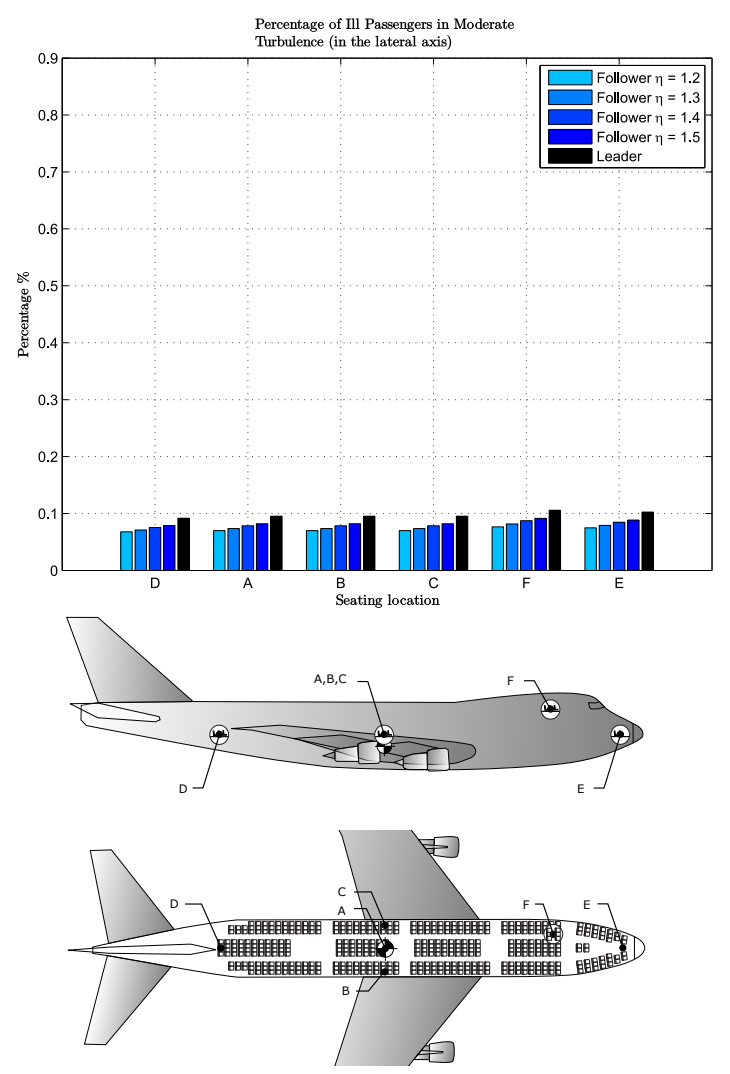

(b) Lateral axis

Figure 7.18: Percentage of ill passengers in the vertical and lateral axes of a leader and follower aircraft. Simulations were performed in moderate turbulence and lateral separations of up to $\eta=1.2$ wingspans.

The results show a clear increase from light turbulence (Figure K.2 to moderate turbulence (Figure K.3 in all axes of acceleration. PIP levels in light turbulence are negligibly small, peaking at approximately $0.01 \%$ in the vertical axis and much less in the other axes. Figures $7.18 \mathrm{a}$ and $7.18 \mathrm{~b}$ show the largest PIP values in moderate turbulence.

In the study by Kubica et al. [11], simulations were performed in strong turbulence in order to evaluate the comfort improvement posed by an active control law strategy. It was found that PIP levels progressively increase as the seating location moves from the front to the aft of the aircraft. The PIP levels range between $1.5 \%$ and $2 \%$ in the vertical axis, and between $0.1 \%$ and $0.15 \%$ in the lateral axis when active control law techniques are used.

Using the results of the study by Kubica et al. [11] as a benchmark, the vertical axis PIP levels in Figure $7.18 \mathrm{a}$ are considerably lower, ranging between $0.24 \%$ and $0.26 \%$. This large 
difference could be due to the consideration of aircraft flexibility by the authors, whereas in the current study a rigid body aircraft is assumed. Furthermore, it is unclear what level of turbulence was used by Kubica et al. [11] in their simulations. Assuming that the authors used severe turbulence, the considerably lower PIP levels in the results produced in Figure 7.18 are expected. For lateral axis accelerations, the PIP levels in Figure 7.18b agree more closely with Kubica et al., ranging between $0.09 \%$ and $0.11 \%$.

Considering the PIP levels in a follower aircraft at any lateral separation indicated, the PIP levels are less than those in a leader. Furthermore, it would seem that the PIP levels gradually decrease at any seating location of the follower as the aircraft moves closer to the wake flow field. Longitudinal axis accelerations show negligible change between leader and follower (see Figures $\mathrm{K.2 \textrm {c }}$ and $\mathrm{K.3 \textrm {c }}$. Although these trends are found, it is noted that the PIP levels in turbulence are quite low - less than $0.25 \%$ in moderate turbulence and less than $0.01 \%$ in light turbulence. The variations in PIP levels are even smaller, varying by approximately $\pm 0.01 \%$ in moderate turbulence and negligibly small in light turbulence.

In conclusion, the PIP levels in isolated and formation flight are very low. Peaking at approximately $0.01 \%$ in light turbulence and approximately $0.25 \%$ in moderate turbulence, the results indicate that the chances of having ill passengers in a leader or follower aircraft are in the same order of magnitude. This makes motion sickness incidence during formation flight at the lateral separations under investigation almost no different to motion sickness incidence during isolated flight.

\subsection{Conclusions}

The vibrations on-board a leader and follower aircraft were measured at various locations in an international all-economy seating arrangement. Using the overall frequency-weighted RMS accelerations at the feet and seat-surface, a total RMS acceleration magnitude was computed and used to determine the comfort level according to ISO 2631-1. The accelerations at the seat-surface were obtained from the feet accelerations with vertical and lateral transmissibility ratios.

The results showed a clear increase in discomfort from light to moderate turbulence. For moderate turbulence, the acceleration magnitudes were found to be above the perception threshold, but remained well within the not uncomfortable region suggested by the ISO 2631-1 standard. It was concluded for both aircraft that a seating location at the front of the aircraft is more comfortable than one at the back of the aircraft. More importantly, that the vibration magnitudes do not differ between aircraft in isolated flight and aircraft in formation flight under the same conditions.

The effect of vibrations on motion sickness incidence was also investigated and reported in this chapter. The results showed that the PIP levels in isolated and formation flight are very low, peaking at approximately $0.01 \%$ in light turbulence and approximately $0.25 \%$ in moderate turbulence. It was concluded that formation flight at the lateral separations investigated produce PIP levels almost no different to those in isolated flight. 


\section{Chapter 8}

\section{Conclusions, Recommendations and Future Work}

The final chapter will provide an overview of the project with the achieved objectives, a summary of the important results, discovered limitations, and recommendations for future work.

\subsection{Summary and Conclusions}

At the start of this project, the first research objective was addressed by performing an extensive literature study, developing the understanding required to address the rest of the objectives. A theoretical model of a Boeing B747-100 aircraft was implemented for aircraft during formation flight because of the availability of flight data in the open domain and the aircraft's popularity in the research industry. The required mathematical modelling used to describe each aircraft as a rigid body was briefly discussed, focusing on the aerodynamic modelling and the definition of aircraft separation variables. In a study by Bizinos [3], the author evaluated the levels of comfort experienced in formation flight during atmospheric turbulence. A clear increase in acceleration magnitudes was found for longitudinal, vertical, roll and yaw acceleration magnitudes. Significant increases in discomfort were found in a follower aircraft flying close to the trailing vortex of a leader - especially for seats longitudinally displaced from the aircraft centre of gravity. A shortcoming in the study by Bizinos [3] was the practical simulation of dynamic aircraft motion. The main contribution of the current study lies in the evaluation of passenger comfort during formation flight after taking the aircraft dynamics, atmospheric turbulence and effects of the flight control system into account. The comfort criteria used to measure and evaluate the effects of vibration on comfort levels and motion sickness incidence were also defined. At this stage, research objectives (2) and (4) had been achieved.

The non-linear 6DOF equations of motion of a time-variant aircraft model were used to derive and develop a linear aircraft model, linearised about a nominal flight condition (called the linearisation point), assuming a rigid body, constant mass, and a flat, non-rotating Earth. The responses of the non-linear and linearised model matched well during the initial part of the response, and the responses eventually diverged due to coupled longitudinal and lateral 
dynamics in the non-linear model, while in the linear model they are uncoupled.

During the current study, an A330 aircraft model was used to reverse engineer the conventional fly-by-wire architecture used in modern day transport aircraft. With limited access to proprietary information on the fly-by-wire system, a more representative fly-by-wire architecture was designed for the open domain Boeing 747-100 than the one used by Büchner [4], such that the aircraft produced similar responses to an A330 aircraft under the same flight conditions. Outer-loop guidance laws were also added to maintain a desired altitude and flight path. The design of the complete control architecture was executed with a focus on straight and level flight, allowing reasonable manoeuvres away from the current flight path. The longitudinal and lateral controller performances were good for an isolated aircraft, showing a good match between the linear and non-linear models for different responses, and the desired specifications were adequately met. At this point, research objective (3) had been addressed.

The same inner-loop fly-by-wire architecture was used in a follower aircraft, and three formation-specifc guidance laws were designed to maintain the desired geometric axial, vertical and lateral separation. Extended simulations were performed at different levels of turbulence intensity and lateral geometric separations, achieving research objectives (5) and (6). Dynamic simulations showed that the extended formation guidance laws were unable to maintain formation for desired lateral separations less than $\eta=1.1$ wingspans (outside the outer region). This led to an investigation into the wake model being used, uncovering the first limitation in this thesis. Used to model the interaction forces and moments between a follower and the trailing vortices of a leader aircraft, the wake model was compared to various other sources of available literature, with the conclusion that the peaks of the current wake model are unrealistically high for lateral separations close to the optimum separation. However, although an improved wake interaction model would allow for a more complete investigation of the interaction problem, this improved model is not expected to result in interaction forces significantly larger or smaller than those predicted by the current wake model at wingspans larger than $\eta=1$. It was thus decided to keep the lateral separation limited to no closer than one wingspan. This meant that although Bizinos [3] had performed a passenger comfort analysis for lateral separations less than $\eta=1$ wingspan, the results at these separations could not be compared to the results of the current study.

It was concluded from dynamic simulation results in the current study that the formationhold performance degrades very quickly with increasing turbulence. Showing the best results in light turbulence, the guidance laws maintained desired lateral separations of $\eta=1.1$ and larger (inside the outer region) at the desired vertical $(\zeta=0)$ and axial $(\xi=-10)$ separations. In moderate turbulence and at lateral separations closer than $\eta=1.2$ wingspans (just inside the outer region), the aircraft comes too close to the trailing vortex, getting pulled into and pushed out of the vortex. The trailing aircraft goes far to the left of the leader aircraft, resulting in large separation errors. The formation flight guidance laws eventually guide the aircraft back towards the correct separations, but this process is repeated and is not without aggressiveness, making it unacceptable for passenger comfort. For separations even closer to the trailing vortex in moderate turbulence, and for all cases of severe turbulence, this aggressive behaviour happens 
multiple times over the entire simulation period. As a result, the sandwich region, defined by Büchner [4] as a tightly bounded trimmable region between two un-trimmable regions at approximately 0.7 wingspans lateral separation, was not considered in this study. Feasible formation flight scenarios were introduced for passenger comfort evaluation, limiting the lateral geometric separations during formation flight to between $\eta=1.1$ and $\eta=1.5$ wingspans in light turbulence and $\eta=1.2$ and $\eta=1.5$ wingspans in moderate turbulence. The vertical and axial separation was limited to $\zeta=0$ and $\xi=-10$ wingspans respectively.

To successfully assess passenger comfort with suitable comfort criteria, and complete research objective (8), the vibrations on board a leader and follower aircraft were measured at various locations in an international all-economy seating arrangement. In the study by Bizinos [3], it was found for an isolated aircraft that a seat at the front of the aircraft will result in a more comfortable flight for the passenger than a seat at the rear of the aircraft. It was further found that the opposite happens in formation flight, where larger acceleration magnitudes are found at the front of the fuselage compared to the rear of the aircraft. Choosing to investigate a worst seating location F, it was suggested that for an aircraft in a right echelon formation, the front and port side of the aircraft are most uncomfortable. The current study suggests a different result for an aircraft in a right echelon formation. Similar to the leader aircraft, a seat in the aft of a follower aircraft will be more uncomfortable than a seat in the front. Moreover, it is concluded in the current study that the port side of the aircraft and a seating location displaced higher up the vertical axis will be slightly more comfortable - contrary to Bizinos' conclusions.

To measure the vibrations experienced by seated passengers in the current study, addressing research objective (7), the accelerations at the seat-surface were obtained from the feet accelerations with vertical and lateral transmissibility ratios. Combining the overall frequency-weighted RMS accelerations at the feet and seat-surface, a total RMS acceleration magnitude was computed and used to determine the comfort level according to the ISO 2631-1 standard. Also reported in this study and not investigated by Bizinos [3] is the incidence of motion sickness as a percentage of ill passengers (PIP); calculated with the overall frequency-weighted RMS accelerations at the seat-surface. Results showed that the vibration magnitudes do not differ much between aircraft in isolated flight and formation flight under the same conditions; a desired result if formation flight with passenger aircraft is to be considered.

A clear increase in passenger discomfort from light to moderate turbulence was found. For moderate turbulence, the acceleration magnitudes were found to be above the perception threshold, but remained well within the not uncomfortable region suggested by the ISO 2631-1 standard. It was concluded for both aircraft that a seating location at the front of the aircraft is more comfortable than one at the back of the aircraft. More importantly, that the vibration magnitudes do not differ between aircraft in isolated flight and aircraft in formation flight under the same conditions. It was also concluded that formation flight at the lateral separations investigated produce motion sickness incidence levels almost no different to those in isolated flight. Results showed that the PIP levels in isolated and formation flight are very low, peaking at approximately $0.01 \%$ in light turbulence and approximately $0.25 \%$ in moderate turbulence. This addressed research objective (8), the last research objective of the current study. 
Finally, since the vibration magnitudes do not differ between an aircraft in isolated flight and aircraft in formation flight under the same conditions, the ride comfort between the aircraft will not show significant differences, and passenger comfort in the follower aircraft is similar to passenger comfort in the leader aircraft. Furthermore, the PIP levels in isolated and formation flight are very low, making the chances of having ill passengers in isolated and formation flight unlikely. This means that flight control for optimal ride comfort, initially proposed because a large increase in discomfort was expected in regions of feasible formation flight, is no longer necessary and was not further pursued in the current study.

\subsection{Recommendations and Future Work}

Much work has been covered in this study, and although all the research objectives have been achieved, there still remains room for expansion or improvement. This section outlines some of the recommendations for future work.

1. The first recommendation for future work would be to obtain a more realistic wake model that would allow the aircraft to move closer to the trailing vortex of the leader aircraft in simulation. The current interaction model used in this study did not model compressibility effects. This meant that a large component of what can contribute to passenger discomfort (things such as buffet, flutter, flow separation, noise) was not considered in this study. Investigation into the use of more sophisticated wake models can be pursued to fill this gap and further collaboration with colleagues at UCT could produce improved wake models.

2. In this thesis, the type of formation was limited to a two aircraft in right echelon formation. The inverted- $V$ formation offers some unique characteristics that could be investigated in a future study. In an inverted- $\mathrm{V}$ formation, almost all the drag savings are realised by one aircraft [1]. Furthermore, the inverted-V formation is known to reduce the amount of control surface deflection required to trim the aircraft in roll [1]. This could not only reduce the drag but also give more control availability with which to combat aeroelastic effects and further improve ride comfort at smaller lateral separations than those achieved in this thesis.

3. The redesign of the inner-loop fly-by-wire flight control laws for formation flight situations could be investigated in future work. In the current study, the lateral DP and DR innerloop fly-by-wire flight control laws were designed as full-state feedback controllers with feed-forward for turn coordination and a natural roll response during sideslip. No integral control was added to the system. As a result, the control laws were not robust against any roll angle disturbances, such as the disturbance on roll angle due to the trailing vortex interaction. The effect was a steady-state error on the lateral separation for separation commands too close to the trailing vortex. The redesign of the DP and DR laws would fix the source of the problem by commanding enough aileron deflection for a given roll angle, thereby ensuring zero steady-state error in the roll response. 
4. Comfort levels and PIP results show that there is little to no difference between isolated and formation flight aircraft, a desired result for passenger aircraft. However, this result is based on the assumption of a rigid body aircraft. The development of an aircraft model that includes aeroelasticity, particularly wing aeroelasticity, is recommended for future work. Literature studies to consult as a starting point are available [82] 83]. Nguyen et al. 84 presented an integrated flight dynamics modelling method for flexible aircraft, capturing, amongst others, the coupled effects due to aeroelasticity. For an accurate passenger comfort analysis during formation flight, the dynamic aeroelasticity problem is of most interest, describing the transient responses of a wing structure subject to gust and instantaneous control surface deflections. Two types of elastic modes are present in symmetric aircraft such as the B747-100 aircraft, namely symmetric and anti-symmetric modes, illustrated in Figure 8.1. Symmetric modes are those where the structural deflection of both wings are in the same sense. With accurate structural stiffness and damping data, these modes will not produce any differences in vibrations between isolated and formation flight under the same flight conditions and at the lateral separations investigated in this study. As such, it is expected that comfort levels will not differ enough between aircraft in formation from the results already produced in this thesis to motivate the need for including aeroelasticity. It is the anti-symmetric modes that are of importance in a future study, where the structural deflections of the wings are in the opposite sense. It is expected that these modes will introduce differential vibration effects between aircraft in formation and will show larger differences in passenger comfort between aircraft in formation.

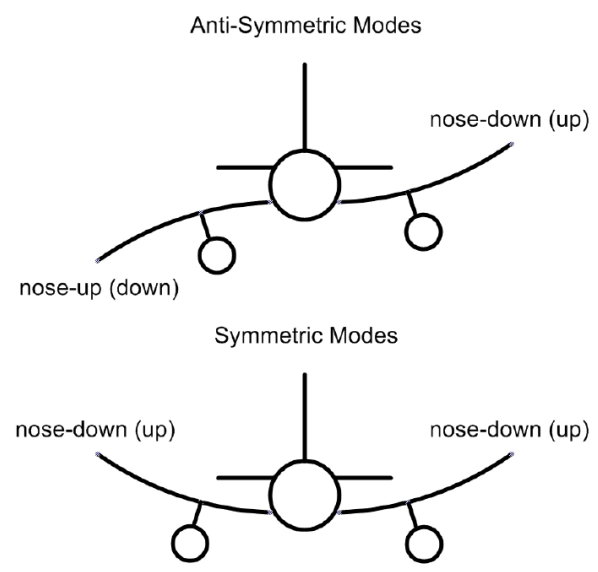

Figure 8.1: Illustration of symmetric and anti-symmetric elastic modes (from Nguyen et al. 84]).

5. A literature survey on seat modelling revealed that there is an evident need for good models of seat-occupant systems through which the effects of vibrations and seat properties on the dynamic response can be directly evaluated. It is recommended for a start to obtain more recent seat transmissibility data. Further future work could entail developing an accurate seat model where various literature studies can be consulted as a starting point. 
Teng et al. [85] presented a seated human body vibration model using the multi-body dynamics method. Joshi et al. [86] presented a non-linear planar seat-occupant model which incorporates the non-linear viscoelastic behaviour of seating foam. 
Appendices 


\section{Appendix A}

\section{Conventional Aerodynamic Model}

In Chapter 3, the conventional aircraft model that describes the equations of motion of the aircraft given the forces and moments that act on it was derived. In this Appendix, the aerodynamic model equations are listed, linearised about a general condition and termed the linearisation point. The chosen condition is for a clean aircraft in straight and level flight at $40000 \mathrm{ft}$, summarised in Table A.1. The angle of attack is with respect to the FRL. The value for all aerodynamic coefficients and derivatives were obtained from Bizinos [3], who scaled the values directly from plots by Heffley and Jewel [9], and computed the remaining values for which no data was provided. The aerodynamic coefficients at the linearisation point are summarised in Table A.2 and the longitudinal and lateral aerodynamic derivatives are summarised in Table A.3.

Table A.1: Summary of aerodynamic equation variables at the linearisation point for a straight and level flight condition at an altitude of $40000 \mathrm{ft}$.

\begin{tabular}{lcr}
\hline Variable Description & Symbol & Value \\
\hline Mach Number & $M_{l p}$ & 0.8 \\
Angle of Attack & $\alpha_{l p}$ & $4.6^{\circ}$ \\
Sideslip & $\beta_{l p}$ & $0^{\circ}$ \\
Angular Velocities & $P_{l p}, Q_{l p}, R_{l p}$ & $0^{\circ} / \mathrm{s}$ \\
Control Surface Deflections & $\delta_{A_{l p}}, \delta_{E_{l p}}, \delta_{R_{l p}}$ & $0^{\circ}$ \\
\hline
\end{tabular}

Table A.2: Summary of non-dimensional aerodynamic coefficients at the linearisation point for a straight and level flight condition at an altitude of $40000 \mathrm{ft}$.

\begin{tabular}{lcr}
\hline Variable Description & Symbol & Value \\
\hline Lift Coefficient & $C_{L_{l p}}$ & 0.66 \\
Drag Coefficient & $C_{D_{l p}}$ & 0.0415 \\
Pitching Moment Coefficient & $C_{m_{l p}}$ & 0 \\
\hline
\end{tabular}



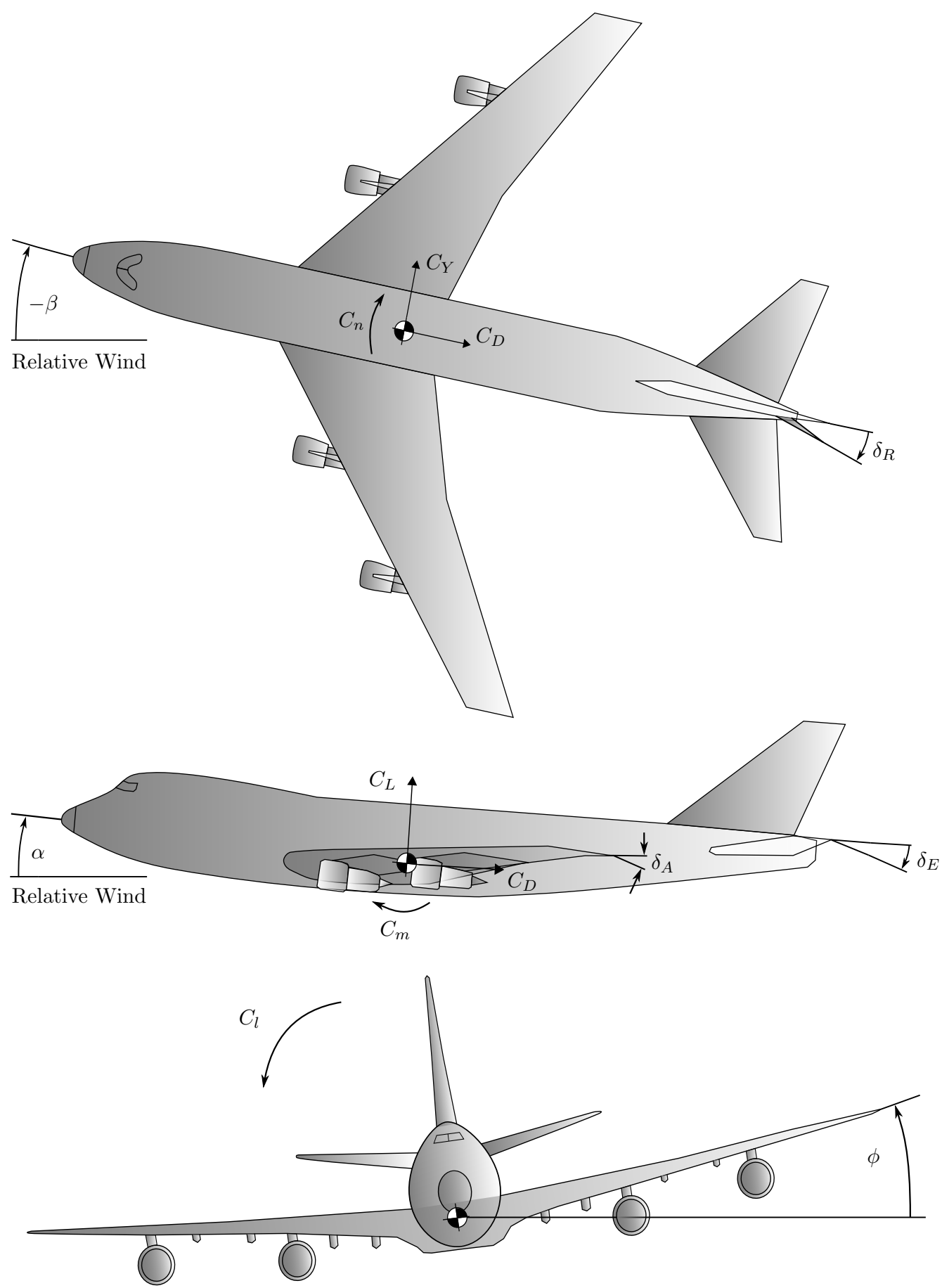

Figure A.1: Aerodynamic sign conventions (stability axis) 
Table A.3: Summary of the longitudinal and lateral aerodynamic stability and control derivatives at the linearisation point. The derivatives are categorised in terms of the six longitudinal and lateral forces and moments in the stability axis.

\begin{tabular}{|c|c|c|c|c|c|}
\hline \multicolumn{6}{|c|}{ Longitudinal Derivatives } \\
\hline \multicolumn{2}{|c|}{ Lift Force } & \multicolumn{2}{|c|}{ Drag Force } & \multicolumn{2}{|c|}{ Pitching Moment } \\
\hline$C_{L_{M}}$ & 0.205 & $C_{D_{M}}$ & 0.0275 & $C_{m_{M}}$ & 0.166 \\
\hline$C_{L_{\alpha}}$ & 4.92 & $C_{D_{\alpha}}$ & 0.425 & $C_{m_{\alpha}}$ & -1.033 \\
\hline$C_{L_{Q}}$ & 6 & $C_{D_{Q}}$ & 0 & $C_{m_{Q}}$ & -24 \\
\hline$C_{L_{n z}}^{q}$ & 0 & $C_{D_{n z}}$ & 0 & $C_{m_{n z}}$ & 0 \\
\hline$C_{L_{\delta_{E}}}$ & 0.367 & $C_{D_{\delta_{E}}}$ & 0 & $C_{m_{\delta_{E}}}$ & -1.45 \\
\hline \multicolumn{6}{|c|}{ Lateral Derivatives } \\
\hline \multicolumn{2}{|c|}{ Side Force } & \multicolumn{2}{|c|}{ Rolling Moment } & \multicolumn{2}{|c|}{ Yawing Moment } \\
\hline$C_{Y_{\beta}}$ & -0.88 & $C_{l_{\beta}}$ & -0.277 & $C_{n_{\beta}}$ & 0.195 \\
\hline$C_{Y_{P}}$ & 0 & $C_{l_{P}}$ & -0.334 & $C_{n_{P}}$ & -0.0415 \\
\hline$C_{Y_{R}}$ & 0 & $C_{l_{R}}$ & 0.3 & $C_{n_{R}}$ & -0.327 \\
\hline$C_{Y_{\delta}}$ & 0 & $C_{l_{\delta}}$ & 0.0137 & $C_{n_{\delta}}$ & 0.0002 \\
\hline$C_{Y_{\delta_{R}}}$ & 0.1157 & $C_{l_{\delta_{R}}}$ & 0.007 & $C_{n_{\delta_{R}}^{A}}^{A_{1}}$ & -0.1256 \\
\hline
\end{tabular}

The full aerodynamic coefficients $\left(C_{(.)_{S}}\right)$ were expanded at the linearisation point in the stability axis and are shown in Equations (3.15a) to $3.15 \mathrm{~h}$ ) on the following page. The terms on the right-hand side of these aerodynamic equations of the form,

$$
C_{A_{B}} \equiv n \frac{\partial C_{A}}{\partial B}
$$

are the non-dimensional aerodynamic stability and control derivatives. The term $n$ is the appropriate normalising coefficient for the term $B$. For the incidence and control deflection angles, this normalisation is unity while for the pitch rate $Q$ it is $\bar{c} / 2 \bar{V}$ and for the roll and yaw rates it is $b / 2 \bar{V}$. The term $M$ is the Mach number to take into account the compressibility effect at large Mach numbers, and is defined as $\frac{\bar{V}}{V_{S}}$. 


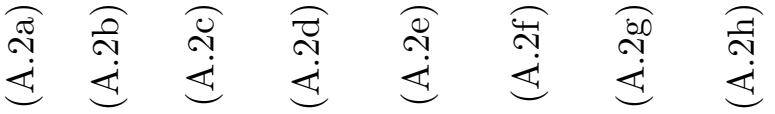

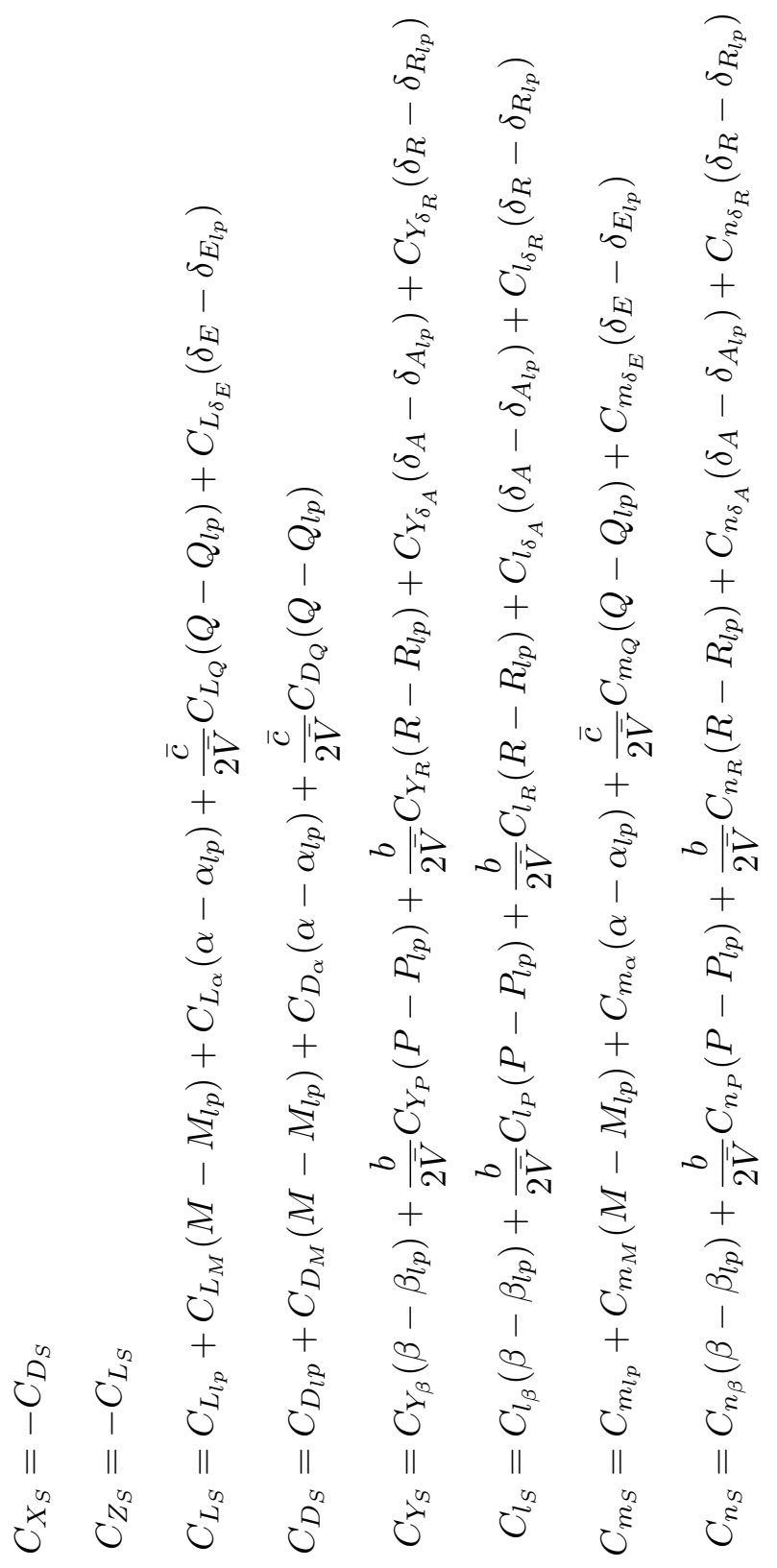




\section{Appendix B}

\section{Conventional Linear Model Partial Derivatives}

In Chapter 4, the expanded state space matrices contained partial derivatives of state variables with respect to the state and control variables. The partial derivatives of the linear longitudinal and lateral models are defined in this appendix.

\section{B.1 Longitudinal Partial Derivatives}

In Chapter 4, the longitudinal dynamics of Equation 4.25a were expanded as partial derivatives to obtain the linearised longitudinal model,

$$
\left[\begin{array}{c}
\dot{\bar{v}} \\
\dot{\alpha} \\
\dot{q} \\
\dot{\theta}
\end{array}\right]=\left[\begin{array}{cccc}
\frac{\partial \dot{U}}{\partial U} & \bar{V}_{T} \frac{\partial \dot{U}}{\partial W} & \frac{\partial \dot{U}}{\partial Q} & \frac{\partial \dot{U}}{\partial \Theta} \\
\frac{1}{V_{T}} \frac{\partial \dot{W}}{\partial U} & \frac{\partial \dot{W}}{\partial W} & \frac{1}{V_{T}} \frac{\partial \dot{W}}{\partial Q} & \frac{1}{V_{T}} \frac{\partial \dot{W}}{\partial \Theta} \\
\frac{\partial \dot{Q}}{\partial U} & \bar{V}_{T} \frac{\partial \dot{Q}}{\partial W} & \frac{\partial \dot{Q}}{\partial Q} & \frac{\partial \dot{Q}}{\partial \Theta} \\
\frac{\partial \dot{\Theta}}{\partial U} & \bar{V}_{T} \frac{\partial \dot{\Theta}}{\partial W} & \frac{\partial \dot{\Theta}}{\partial Q} & \frac{\partial \dot{\Theta}}{\partial \Theta}
\end{array}\right]\left[\begin{array}{c}
\bar{v} \\
\alpha \\
q \\
\theta
\end{array}\right]+\left[\begin{array}{cc}
\frac{\partial \dot{U}}{\partial \delta_{E}} & \frac{\partial \dot{U}}{\partial \Delta T} \\
\frac{1}{V_{T}} \frac{\partial W}{\partial \delta_{E}} & \frac{1}{V_{T}} \frac{\partial \dot{W}}{\partial \Delta T} \\
\frac{\partial \dot{Q}}{\partial \delta_{E}} & \frac{\partial \dot{Q}}{\partial \Delta T} \\
\frac{\partial \dot{\Theta}}{\partial \delta_{E}} & \frac{\partial \dot{\Theta}}{\partial \Delta T}
\end{array}\right]\left[\begin{array}{c}
\delta_{e} \\
\Delta T
\end{array}\right]
$$

In the next section follow the translational acceleration, pitch acceleration and pitch attitude rate derivatives. 


\section{B.1.1 Translational Acceleration Derivatives}

$$
\begin{aligned}
& \frac{\partial \dot{U}}{\partial U}=\frac{\rho S \bar{V}_{T}}{m} C_{X_{T}}+\frac{q_{T} S}{m}\left[\frac{C_{L_{M}}}{\bar{V}_{S}} \sin \alpha_{T}+\frac{-C_{D} M}{\bar{V}_{S}} \cos \alpha_{T}+\right. \\
& \left.\left(\frac{W_{T}}{U_{T}^{2}+W_{T}^{2}}\right)\left[\left(-C_{L_{\alpha}}+C_{X S_{T}}\right) \sin \alpha_{T}+\left(C_{D_{\alpha}}+C_{Z S_{T}}\right) \cos \alpha_{T}\right]\right] \\
& \frac{\partial \dot{U}}{\partial W}=\frac{q_{T} S}{m}\left[\left(C_{L_{\alpha}}+C_{D_{T}}\right) \alpha_{T}+\left(-C_{D_{\alpha}}+C_{L_{T}}\right)\right] \\
& \frac{\partial \dot{U}}{\partial Q}=-\bar{V}_{T} \alpha_{T} \\
& \frac{\partial \dot{U}}{\partial \Theta}=-g \cos \Theta_{T} \\
& \frac{\partial \dot{U}}{\partial \delta_{E}}=\frac{q_{T} S \sin \alpha_{T}}{m} C_{L_{\delta_{E}}} \\
& \frac{\partial \dot{U}}{\partial \Delta T}=\frac{1}{m} \\
& \frac{\partial \dot{W}}{\partial U}=\frac{\rho S}{m} C_{Z_{T}}+\frac{q_{T} S}{m \bar{V}_{T}}\left[\frac{-C_{L_{M}}}{\bar{V}_{S}} \cos \alpha_{T}+\frac{-C_{D} M}{\bar{V}_{S}} \sin \alpha_{T}\right. \\
& \left.+\left(\frac{W_{T}}{U_{T}^{2}+W_{T}^{2}}\right)\left[\left(C_{D_{\alpha}}+C_{Z S_{T}}\right) \sin \alpha_{T}+\left(C_{L_{\alpha}}-C_{X S_{T}}\right) \cos \alpha_{T}\right]\right] \\
& \frac{\partial \dot{W}}{\partial W}=\frac{q_{T} S}{m \bar{V}_{T}}\left[\left(-C_{D_{\alpha}}+C_{L_{T}}\right) \alpha_{T}+\left(-C_{L_{\alpha}}-C_{D_{T}}\right)\right] \\
& \frac{\partial \dot{W}}{\partial Q}=1-\frac{q_{T} S \bar{c}}{2 m \bar{V}_{T}^{2}}\left(C_{D_{Q}} \sin \alpha_{T}+C_{L_{Q}} \cos \alpha_{T}\right) \\
& \frac{\partial \dot{W}}{\partial \Theta}=-\frac{g}{\bar{V}_{T}} \cos \Phi_{T} \sin \Theta_{T} \\
& \frac{\partial \dot{W}}{\partial \delta_{E}}=-\frac{q_{T} S \cos \alpha_{T}}{m \bar{V}_{T}} C_{L_{\delta_{E}}} \\
& \frac{\partial \dot{W}}{\partial \Delta T}=0
\end{aligned}
$$




\section{B.1.2 Pitch Acceleration Derivatives}

$$
\begin{aligned}
\frac{\partial \dot{Q}}{\partial U} & =\frac{S \bar{c} \rho \bar{V}_{T}}{I_{y y}} C_{m_{T}}+\frac{q_{T} S \bar{c}}{I_{y y}}\left[\frac{C_{m_{M}}}{\bar{V}_{S}}-\frac{W_{T} C_{m_{\alpha}}}{U_{T}^{2}+W_{T}^{2}}\right] \\
\frac{\partial \dot{Q}}{\partial W} & =\frac{q_{T} S \bar{c}}{I_{y y}} C_{m_{\alpha}} \\
\frac{\partial \dot{Q}}{\partial Q} & =\frac{q_{T} S \bar{c}^{2}}{2 I_{y y} \bar{V}_{T}} C_{m_{Q}} \\
\frac{\partial \dot{Q}}{\partial \Theta} & =0 \\
\frac{\partial \dot{Q}}{\partial \delta_{E}} & =\frac{q_{T} S \bar{c}}{I_{y y}} C_{m_{\delta_{E}}} \\
\frac{\partial \dot{Q}}{\partial \Delta T} & =0
\end{aligned}
$$

\section{B.1.3 Pitch Attitude Rate Derivatives}

$$
\begin{gathered}
\frac{\partial \dot{Q}}{\partial U}=0 \\
\frac{\partial \dot{Q}}{\partial W}=0 \\
\frac{\partial \dot{Q}}{\partial Q}=1 \\
\frac{\partial \dot{Q}}{\partial \Theta}=0 \\
\frac{\partial \dot{Q}}{\partial \delta_{E}}=0 \\
\frac{\partial \dot{Q}}{\partial \Delta T}=0
\end{gathered}
$$

\section{B.2 Lateral Partial Derivatives}

In Chapter 4, the lateral dynamics of Equation 4.25b were expanded as partial derivatives to obtain the linearised lateral model,

$$
\left[\begin{array}{c}
\dot{\beta} \\
\dot{p} \\
\dot{r} \\
\dot{\phi}
\end{array}\right]=\left[\begin{array}{cccc}
\frac{\partial \dot{V}}{\partial V} & \frac{1}{\bar{V}_{T}} \frac{\partial \dot{V}}{\partial P} & \frac{1}{V_{T}} \frac{\partial \dot{V}}{\partial R} & \frac{1}{V_{T}} \frac{\partial \dot{V}}{\partial \Phi} \\
\bar{V}_{T} \frac{\partial \dot{P}}{\partial V} & \frac{\partial \dot{P}}{\partial P} & \frac{\partial \dot{P}}{\partial R} & \frac{\partial \dot{P}}{\partial \Phi} \\
\bar{V}_{T} \frac{\partial \dot{R}}{\partial V} & \frac{\partial \dot{R}}{\partial P} & \frac{\partial \dot{R}}{\partial R} & \frac{\partial \dot{R}}{\partial \Phi} \\
\bar{V}_{T} \frac{\partial \dot{\Phi}}{\partial V} & \frac{\partial \dot{\Phi}}{\partial P} & \frac{\partial \dot{\Phi}}{\partial R} & \frac{\partial \dot{\Phi}}{\partial \Phi}
\end{array}\right]\left[\begin{array}{l}
\beta \\
p \\
r \\
\phi
\end{array}\right]+\left[\begin{array}{cc}
\frac{1}{V_{T}} \frac{\partial \dot{V}}{\partial \delta_{A}} & \frac{1}{V_{T}} \frac{\partial \dot{V}}{\partial \delta_{R}} \\
\frac{\partial \dot{P}}{\partial \delta_{A}} & \frac{\partial \dot{P}}{\partial \delta_{R}} \\
\frac{\partial \dot{R}}{\partial \delta_{A}} & \frac{\partial \dot{R}}{\partial \delta_{R}} \\
\frac{\partial \dot{\Phi}}{\partial \delta_{A}} & \frac{\partial \dot{\Phi}}{\partial \delta_{R}}
\end{array}\right]\left[\begin{array}{c}
\delta_{a} \\
\delta_{r}
\end{array}\right]
$$


In the next section follow the translational, roll, yaw acceleration and roll attitude rate derivatives.

\section{B.2.1 Translational Acceleration Derivatives}

$$
\begin{aligned}
& \frac{\partial \dot{V}}{\partial V}=\frac{q_{T} S}{m \bar{V}_{T}} C_{Y_{\beta}} \\
& \frac{\partial \dot{V}}{\partial P}=\frac{q_{T} S b}{2 m \bar{V}_{T}^{2}} C_{Y_{P}}+\alpha_{T} \\
& \frac{\partial \dot{V}}{\partial R}=\frac{q_{T} S b}{2 m \bar{V}_{T}^{2}} C_{Y_{R}}-1 \\
& \frac{\partial \dot{V}}{\partial \Phi}=\frac{g}{\bar{V}_{T}} \cos \Theta_{T} \cos \Phi_{T} \\
& \frac{\partial \dot{V}}{\partial \delta_{A}}=\frac{q_{T} S}{m \bar{V}_{T}} C_{y_{\delta_{A}}} \\
& \frac{\partial \dot{V}}{\partial \delta_{R}}=\frac{q_{T} S}{m \bar{V}_{T}} C_{y_{\delta_{R}}}
\end{aligned}
$$

\section{B.2.2 Roll Acceleration Derivatives}

$$
\begin{aligned}
& \frac{\partial \dot{P}}{\partial V}=\frac{q_{T} S b}{I_{x x}}\left(C_{l_{\beta}} \cos \alpha_{T}-C_{n_{\beta}} \sin \alpha_{T}\right) \\
& \frac{\partial \dot{P}}{\partial P}=\frac{q_{T} S b^{2}}{2 I_{x x} \bar{V}_{T}}\left(C_{l_{P}} \cos \alpha_{T}-C_{n_{P}} \sin \alpha_{T}\right) \\
& \frac{\partial \dot{P}}{\partial R}=\frac{q_{T} S b^{2}}{2 I_{x x} \bar{V}_{T}}\left(C_{l_{R}} \cos \alpha_{T}-C_{n_{R}} \sin \alpha_{T}\right) \\
& \frac{\partial \dot{P}}{\partial \Phi}=0 \\
& \frac{\partial \dot{P}}{\partial \delta_{A}}=\frac{q_{T} S b}{I_{x x}}\left(C_{l_{\delta_{A}}} \cos \alpha_{T}-C_{n_{\delta_{A}}} \sin \alpha_{T}\right) \\
& \frac{\partial \dot{P}}{\partial \delta_{R}}=\frac{q_{T} S b}{I_{x x}}\left(C_{l_{\delta_{R}}} \cos \alpha_{T}-C_{n_{\delta_{R}}} \sin \alpha_{T}\right)
\end{aligned}
$$




\section{B.2.3 Yaw Acceleration Derivatives}

$$
\begin{aligned}
& \frac{\partial \dot{R}}{\partial V}=\frac{q_{T} S b}{I_{z z}}\left(C_{l_{\beta}} \sin \alpha_{T}+C_{n_{\beta}} \cos \alpha_{T}\right) \\
& \frac{\partial \dot{R}}{\partial P}=\frac{q_{T} S b^{2}}{2 \bar{V}_{T} I_{z z}}\left(C_{l_{P}} \sin \alpha_{T}+C_{n_{P}} \cos \alpha_{T}\right) \\
& \frac{\partial \dot{R}}{\partial R}=\frac{q_{T} S b^{2}}{2 \bar{V}_{T} I_{z z}}\left(C_{l_{R}} \sin \alpha_{T}+C_{n_{R}} \cos \alpha_{T}\right) \\
& \frac{\partial \dot{R}}{\partial \Phi}=0 \\
& \frac{\partial \dot{R}}{\partial \delta_{A}}=\frac{q_{T} S b}{I_{z z}}\left(C_{l_{\delta_{A}}} \sin \alpha_{T}+C_{n_{\delta_{A}}} \cos \alpha_{T}\right) \\
& \frac{\partial \dot{R}}{\partial \delta_{R}}=\frac{q_{T} S b}{I_{z z}}\left(C_{l_{\delta_{R}}} \sin \alpha_{T}+C_{n_{\delta_{R}}} \cos \alpha_{T}\right)
\end{aligned}
$$

\section{B.2.4 Roll Attitude Rate Derivatives}

$$
\begin{aligned}
\frac{\partial \dot{\Phi}}{\partial V} & =0 \\
\frac{\partial \dot{\Phi}}{\partial P} & =1 \\
\frac{\partial \dot{\Phi}}{\partial R} & =\tan \Theta_{T} \\
\frac{\partial \dot{\Phi}}{\partial \Phi} & =0 \\
\frac{\partial \dot{\Phi}}{\partial \delta_{A}} & =0 \\
\frac{\partial \dot{\Phi}}{\partial \delta_{R}} & =0
\end{aligned}
$$




\section{Appendix C}

\section{Linear Incremental Normal Load Factor Partial Derivatives}

In Chapter 5, the incremental load factor was approximately linearised as a first-order polynomial,

$$
\Delta \delta_{n_{z}} \approx \mathbf{C}_{\delta n_{z}} \Delta \boldsymbol{x}+D_{\delta n_{z}} \Delta u+N_{\delta n_{z}} \Delta v
$$

where

$$
\begin{aligned}
\mathbf{C}_{\delta n_{z}}=\left.\frac{\partial f}{\partial \boldsymbol{x}}\right|_{T}=\left[\begin{array}{llll}
\frac{\partial \delta_{n_{z}}}{\partial \bar{V}} & \frac{\partial \delta_{n_{z}}}{\partial \alpha} & \frac{\partial \delta_{n_{z}}}{\partial Q} & \frac{\partial \delta_{n_{z}}}{\partial \Theta}
\end{array}\right]_{T} \\
D_{\delta n_{z}}=\left.\frac{\partial f}{\partial u}\right|_{T}=\left.\frac{\partial \delta_{n_{z}}}{\partial \delta_{E}}\right|_{T} \\
N_{\delta n_{z}}=\left.\frac{\partial f}{\partial v}\right|_{T}=\left.\frac{\partial \delta_{n_{z}}}{\partial \Phi}\right|_{T}
\end{aligned}
$$

In this appendix, the partial derivatives of the linear incremental normal load factor are defined.

$$
\begin{aligned}
\frac{\partial \delta_{n_{z}}}{\partial \bar{V}} & =\frac{S}{m g}\left[\rho \bar{V}_{T} C_{L_{T}}+\frac{q_{T} C_{L_{M}}}{\bar{V}_{S}}-\frac{q_{T} \bar{c}}{2 \bar{V}_{T}^{2}} C_{L_{Q}} Q_{T}\right] \\
\frac{\partial \delta_{n_{z}}}{\partial \alpha} & =\frac{q_{T} S}{m g} C_{L_{\alpha}} \\
\frac{\partial \delta_{n_{z}}}{\partial Q} & =\frac{q_{T} S}{m g} \frac{\bar{c}}{2 \bar{V}_{T}} C_{L_{Q}} \\
\frac{\partial \delta_{n_{z}}}{\partial \Theta} & =\sin \Theta_{T} \cos \Phi_{T} \\
\frac{\partial \delta_{n_{z}}}{\partial \Phi} & =-\sin \Phi_{T}-\tan \Phi_{T} \sec \Phi_{T}+\cos \Theta_{T} \sin \Phi_{T} \\
\frac{\partial \delta_{n_{z}}}{\partial \delta_{E}} & =\frac{q_{T} S}{m g} C_{L_{\delta_{E}}}
\end{aligned}
$$




\section{Appendix D}

\section{Conventional Aircraft Response Transfer Functions}

In the following Appendix, the transfer functions for a conventional aircraft that completely describe the linear dynamic response to a control input are summarised. All transfer functions are conveniently written with individual numerator terms with a common denominator,

$$
G_{u}^{x}(s)=\frac{N_{u}^{x}(s)}{D_{u}(s)}=\frac{a s^{3}+b s^{2}+c s+d}{e s^{4}+f s^{3}+g s^{2}+h s+i}
$$

where $u$ is the control input, $x$ is the state variable for the specific transfer function $G(s)$ and the terms $N$ and $D$ represent the numerator and denominator terms respectively. The common denominator represents the characteristic polynomial, which in turn describes the stability characteristics of the aircraft. The numerator, however, plays no role in the stability, but only determines the individual responses. [58] 

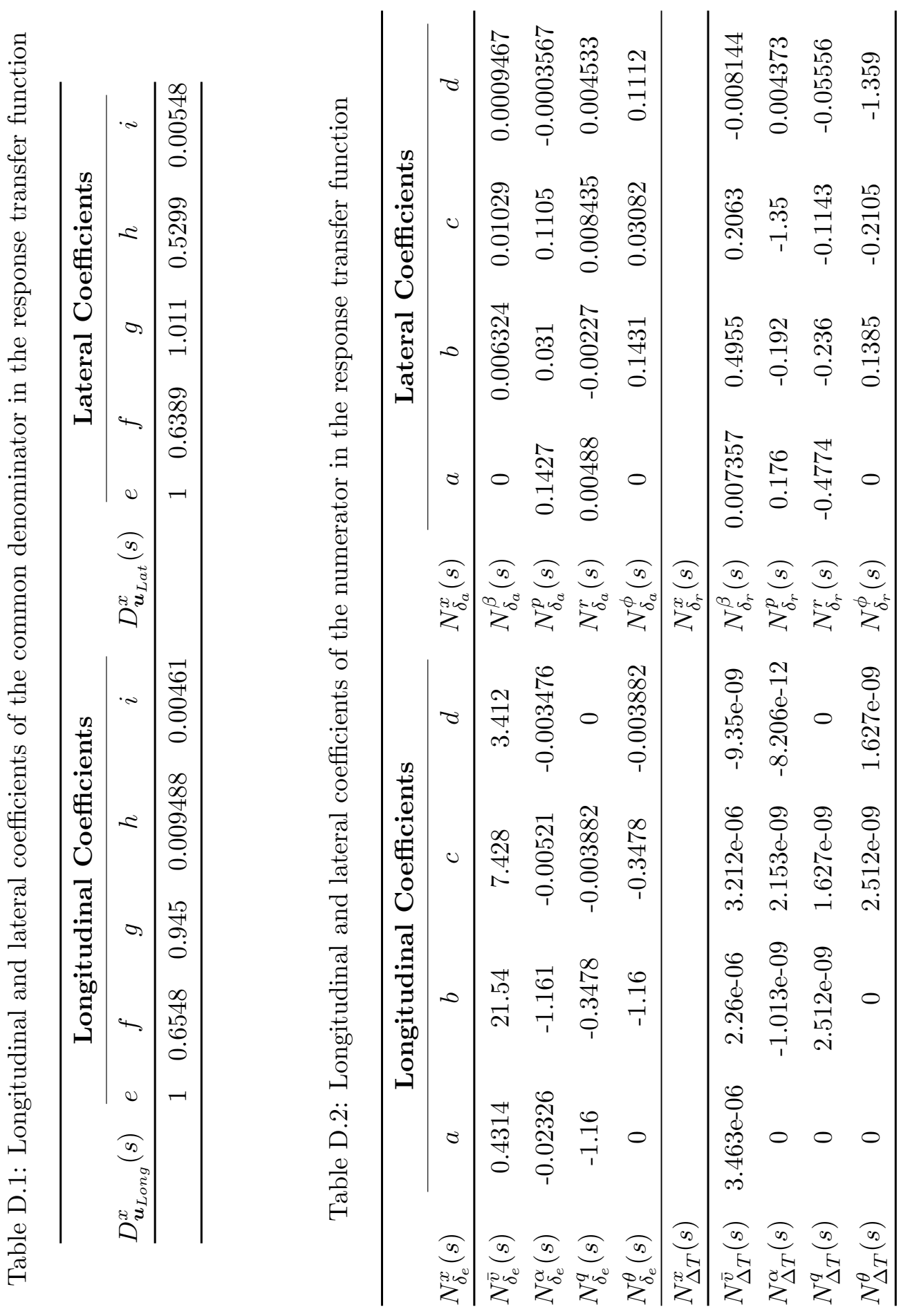


\section{Appendix E}

\section{Conventional Flight Control Simulation Results}

A discussion of the results of the conventional flight control laws is given in Chapter 5. In this appendix the conventional flight control simulation results are concisely summarised. Regarding the longitudinal controller performance, Table E.1 provides a figure reference guide to the longitudinal response for a $5 \mathrm{~m}$ altitude step command and a $5 \mathrm{~m} / \mathrm{s}$ airspeed step command.

Table E.1: Reference guide for longitudinal response simulation results

\begin{tabular}{|c|c|c|c|c|}
\hline \multirow{7}{*}{$\begin{array}{ll}4 & 0 \\
0 & 0 \\
0 & 0 \\
0 & 0 \\
\lambda_{1} & 0\end{array}$} & Step Command: & Altitude E.1 & Airspeed & E.2 \\
\hline & Altitude response & E.1a & E.2a & \\
\hline & Airspeed response & E.1b & E.2b & \\
\hline & Climb rate response & E.1c & E.2c & \\
\hline & Load factor response & E.1d & E.2d & \\
\hline & Elevator response & E.1e & E.2e & \\
\hline & Thrust response & E.1f & $\overline{\mathrm{E} .2 \mathrm{f}}$ & \\
\hline
\end{tabular}

Regarding the lateral controller performance, Table E.2 provides a figure reference guide to the lateral responses to: a $5^{\circ}$ roll angle step command, a $1^{\circ}$ sideslip angle step command and finally a $5 \mathrm{~m}$ step command.

Table E.2: Reference guide for lateral response simulation results

\begin{tabular}{|c|c|c|c|c|}
\hline \multirow{6}{*}{ 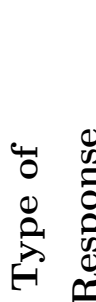 } & Step Command: & Roll Angle E.3 & Sideslip Angle E.4 & Cross-Track Error E.5 \\
\hline & NE position response & E.3a & E.4a & E.5a \\
\hline & Tracking response & - & - & E.5b \\
\hline & Roll angle response & E.3b & E.4b & E.5c \\
\hline & Aileron response & E.3c & E.4c & E.5a \\
\hline & Rudder response & E.3d & E.4d & E.5e \\
\hline
\end{tabular}




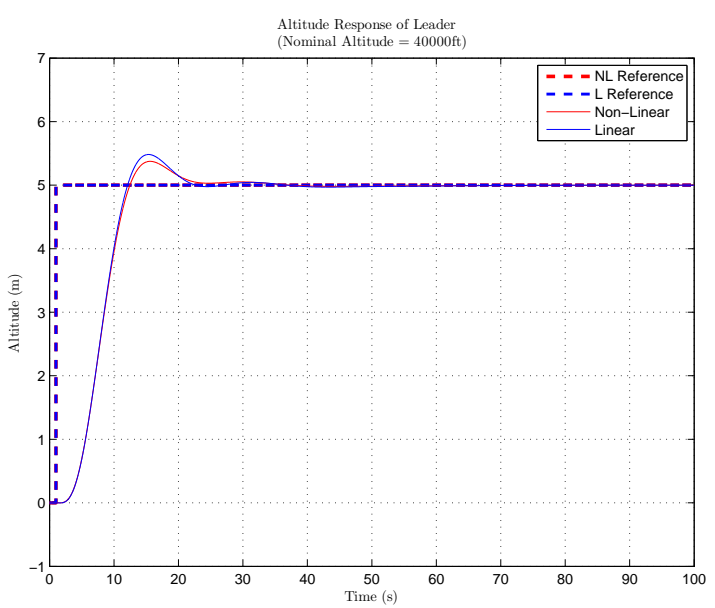

(a) Altitude Response

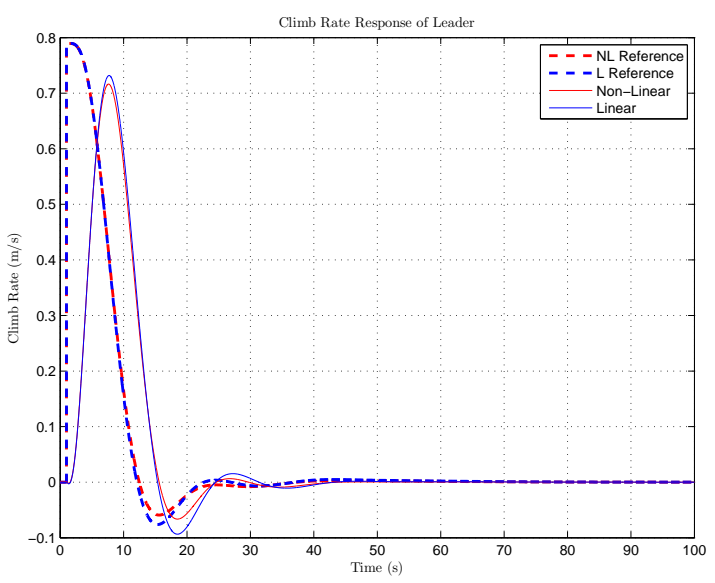

(c) Climb Rate Response

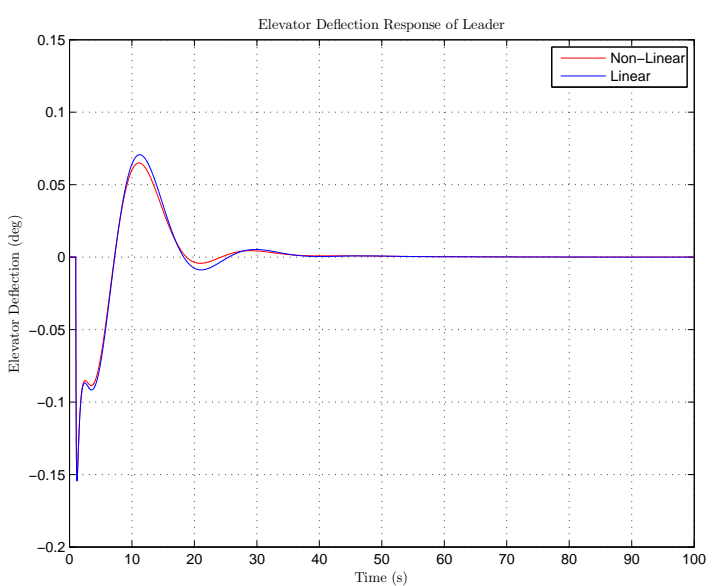

(e) Elevator Deflection Response

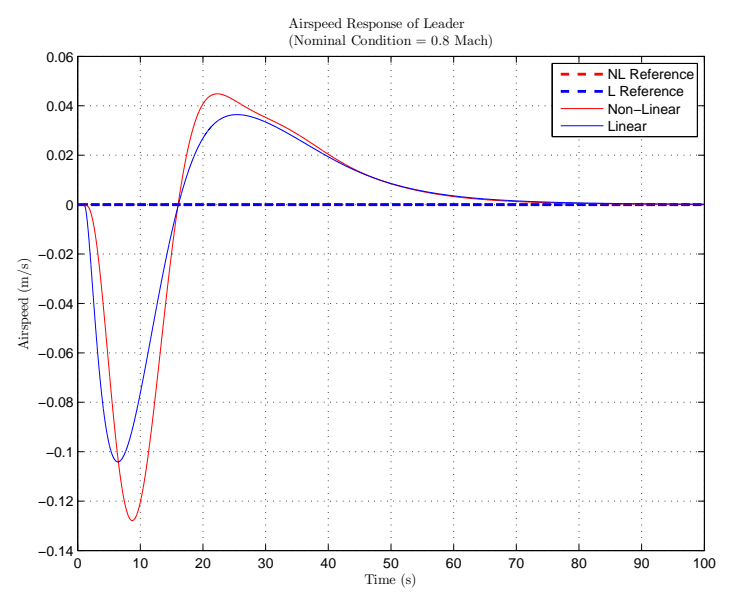

(b) Airspeed Response

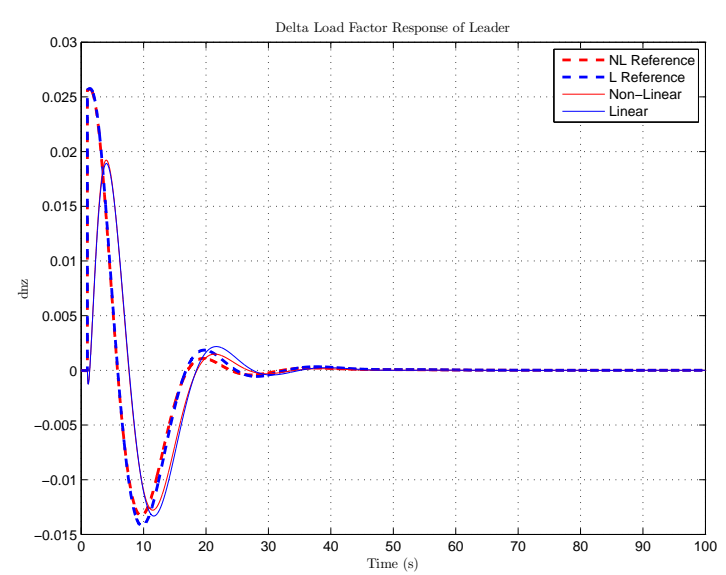

(d) Incremental Normal Load Factor Response

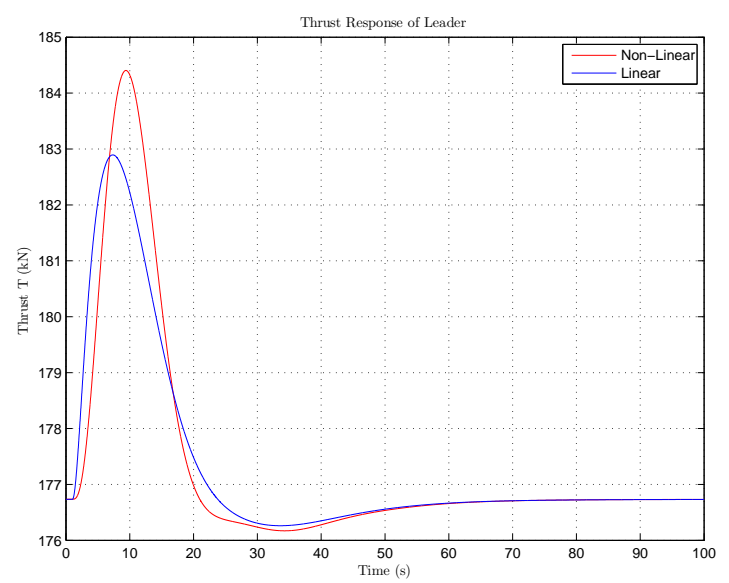

(f) Thrust Response

Figure E.1: Longitudinal response results for a $5 \mathrm{~m}$ altitude step command 


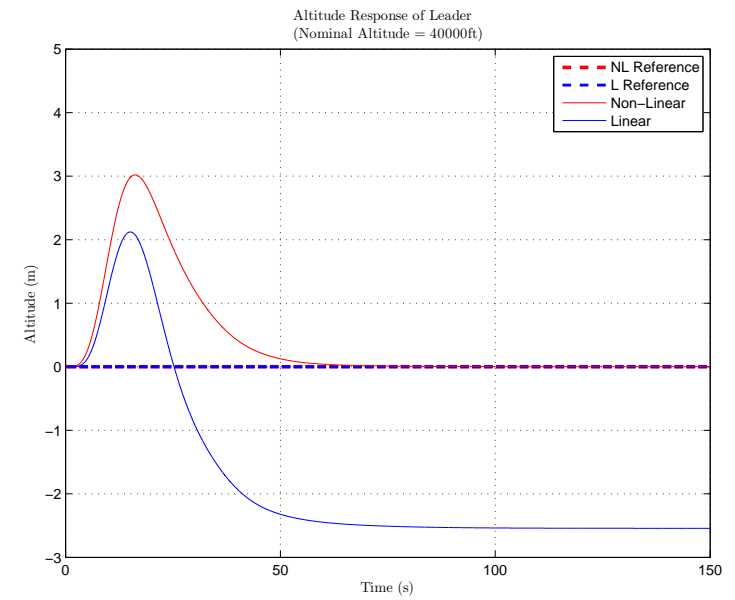

(a) Altitude Response

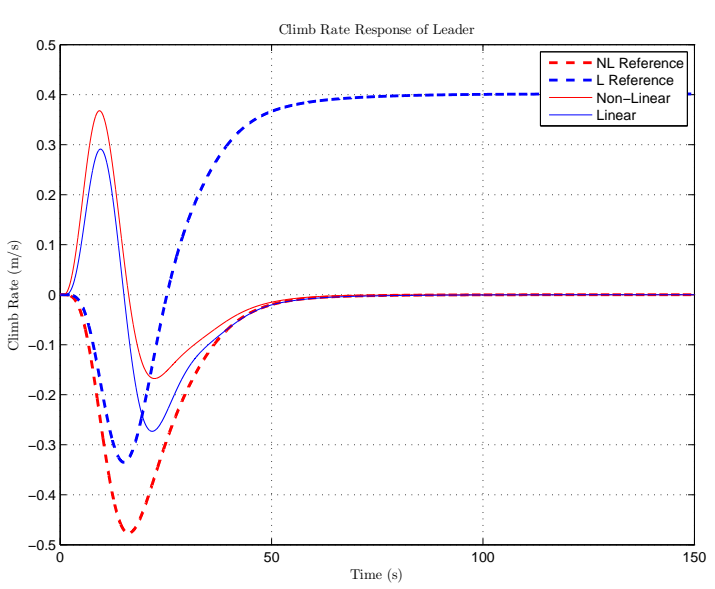

(c) Climb Rate Response

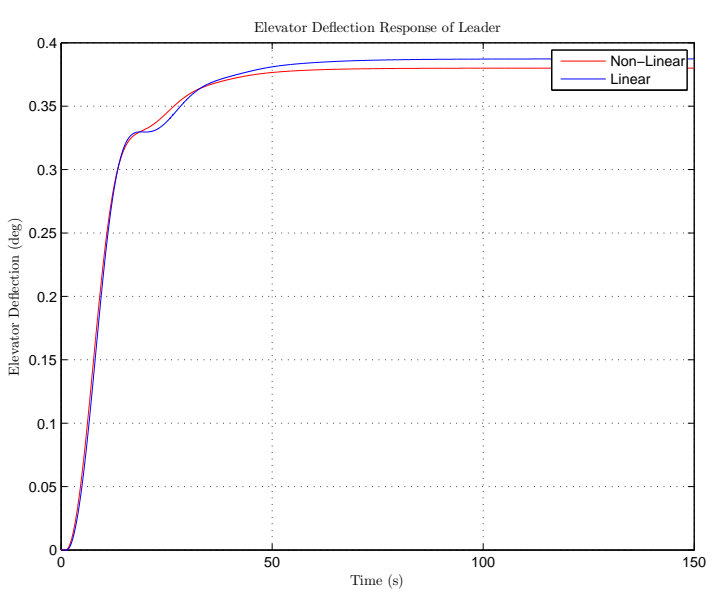

(e) Elevator Deflection Response

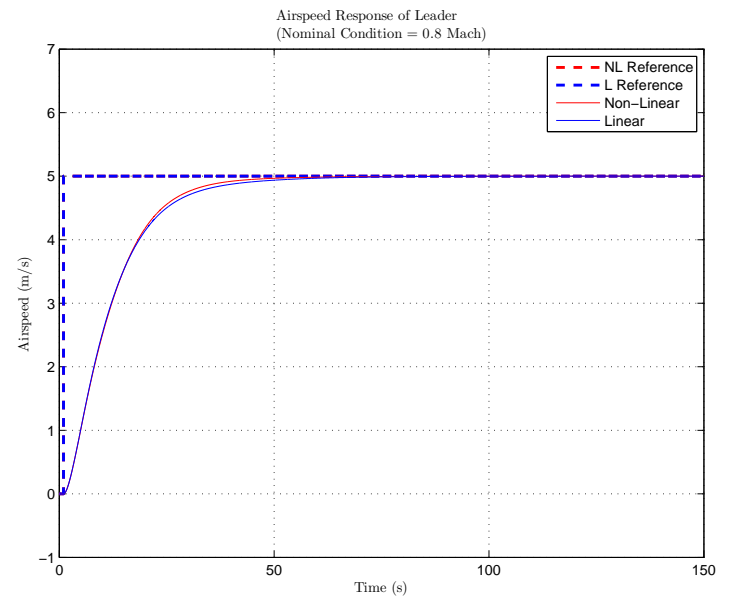

(b) Airspeed Response

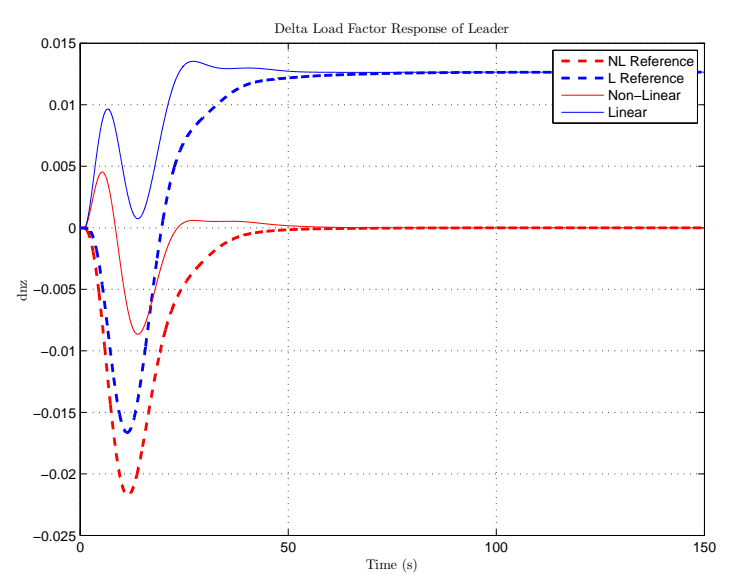

(d) Incremental Normal Load Factor Response

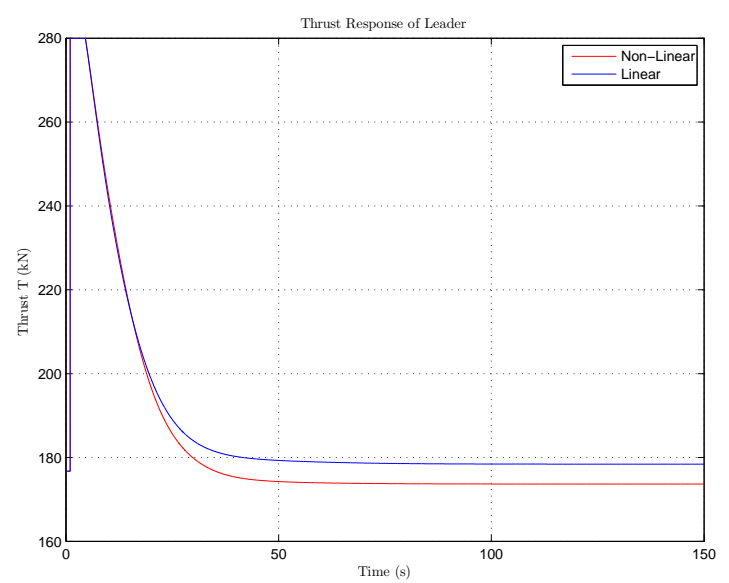

(f) Thrust Response

Figure E.2: Longitudinal response results for a $5 \mathrm{~m} / \mathrm{s}$ airspeed step command 


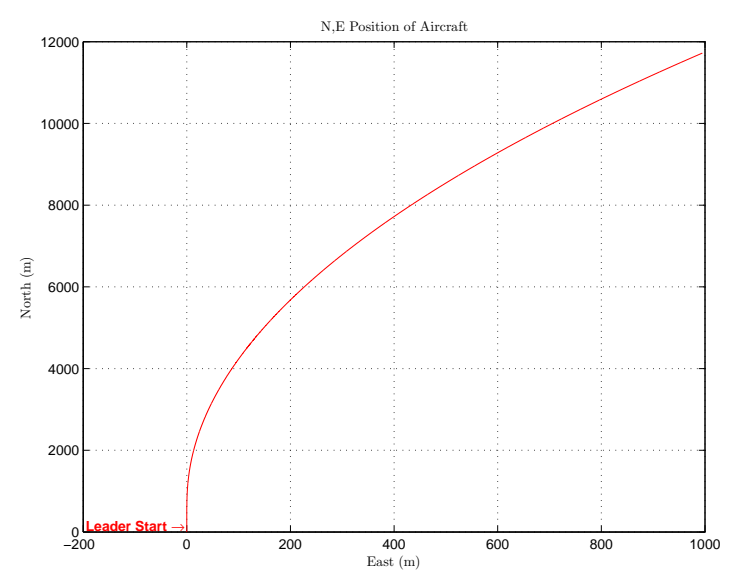

(a) North-East Position Response

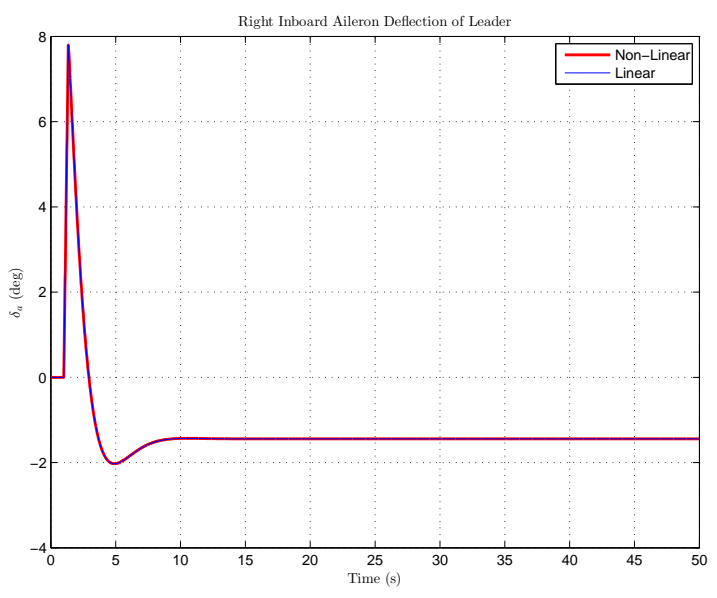

(c) Aileron Response

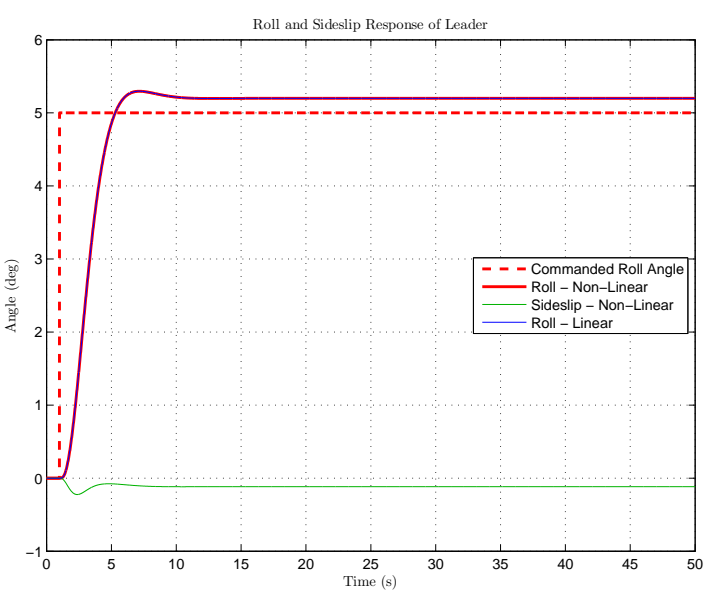

(b) Roll Response

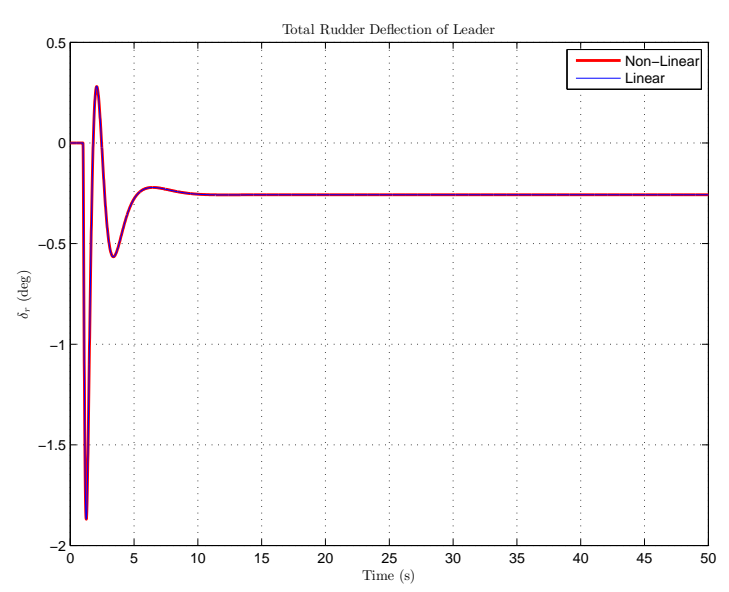

(d) Rudder Response

Figure E.3: Lateral response results for a $5^{\circ}$ roll reference command 


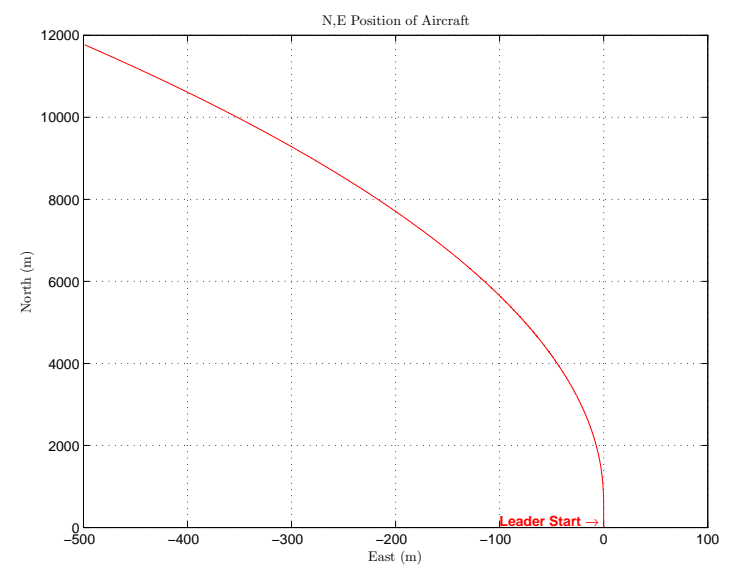

(a) North-East Position Response

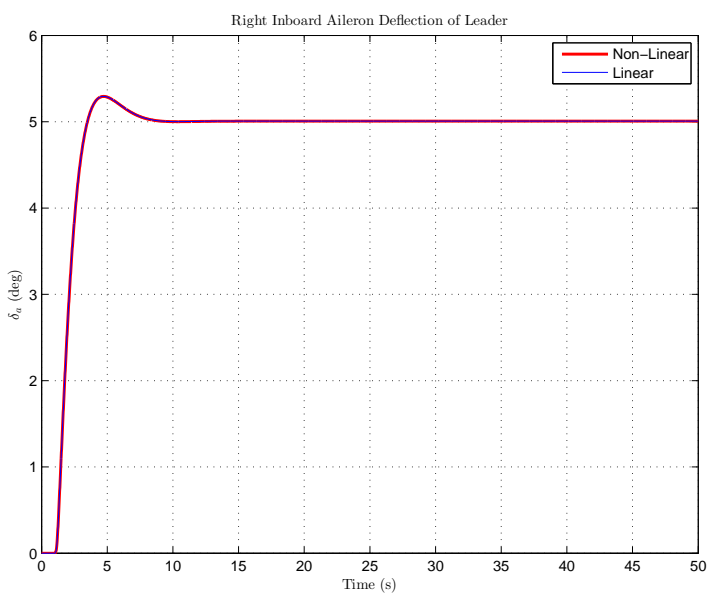

(c) Aileron Response

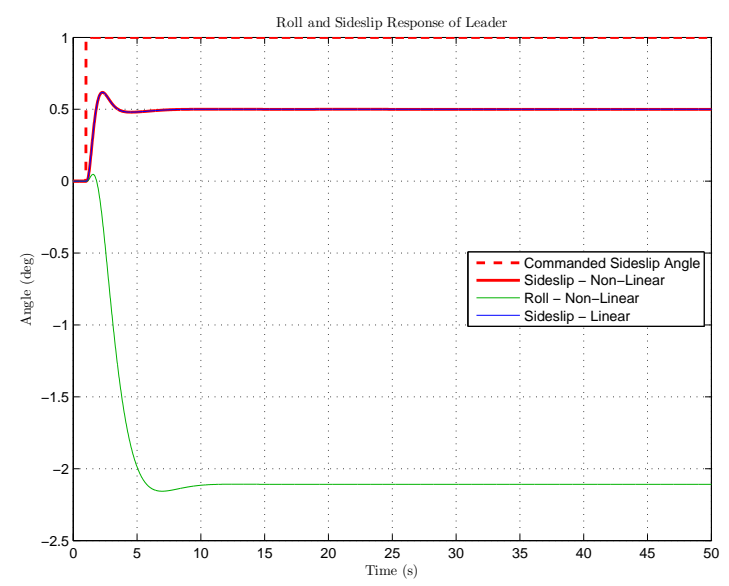

(b) Roll Response

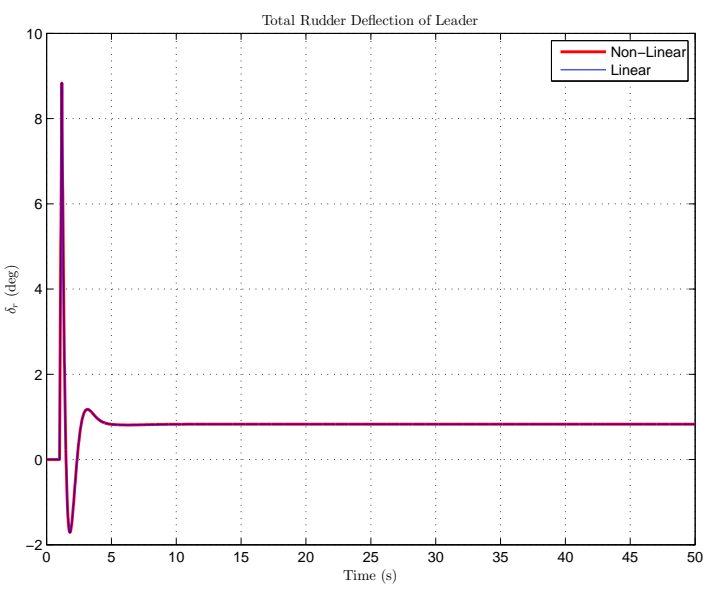

(d) Rudder Response

Figure E.4: Lateral response results for a $1^{\circ}$ sideslip reference command 


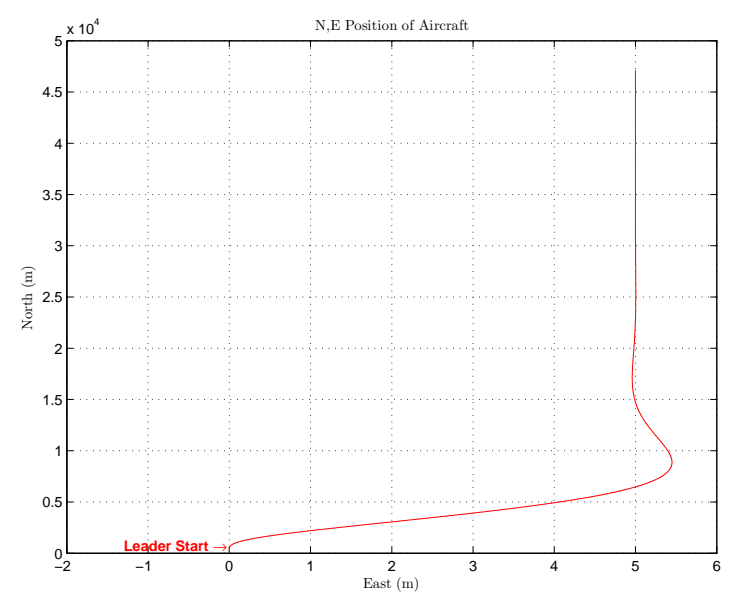

(a) North-East Position Response

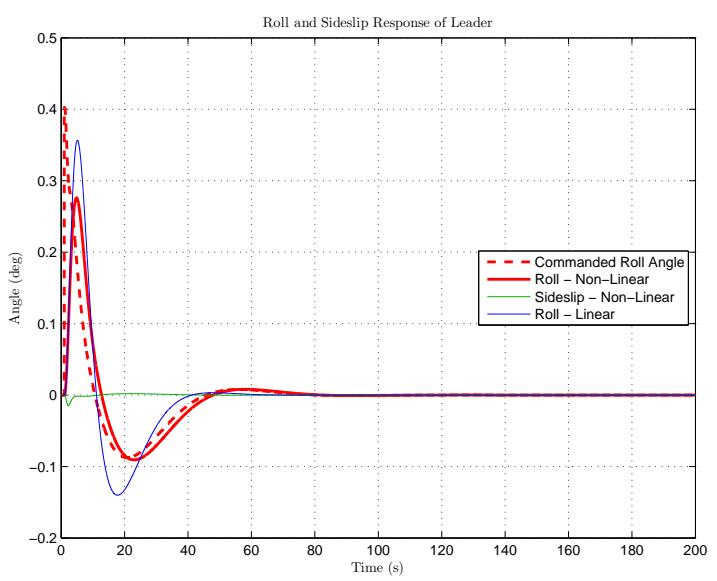

(c) Roll Response

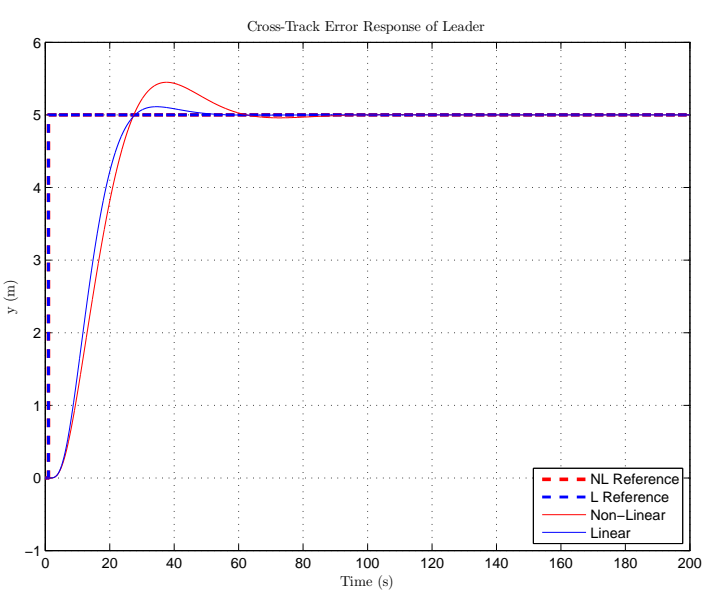

(b) Tracking Response

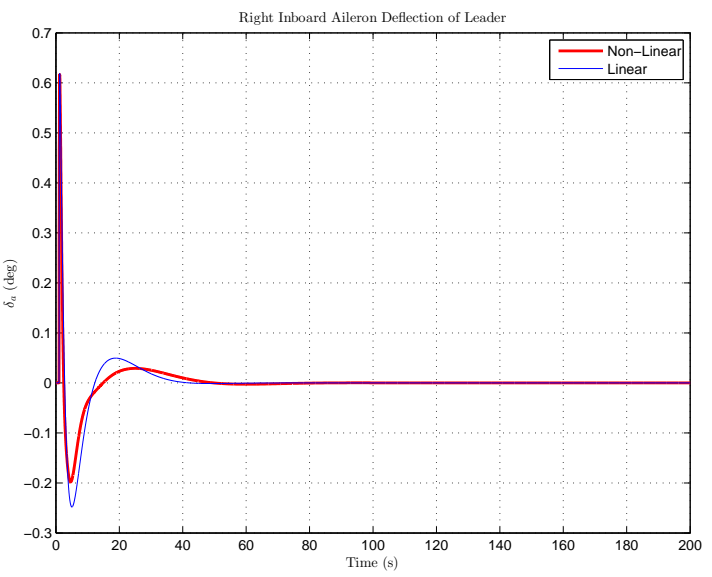

(d) Aileron Response

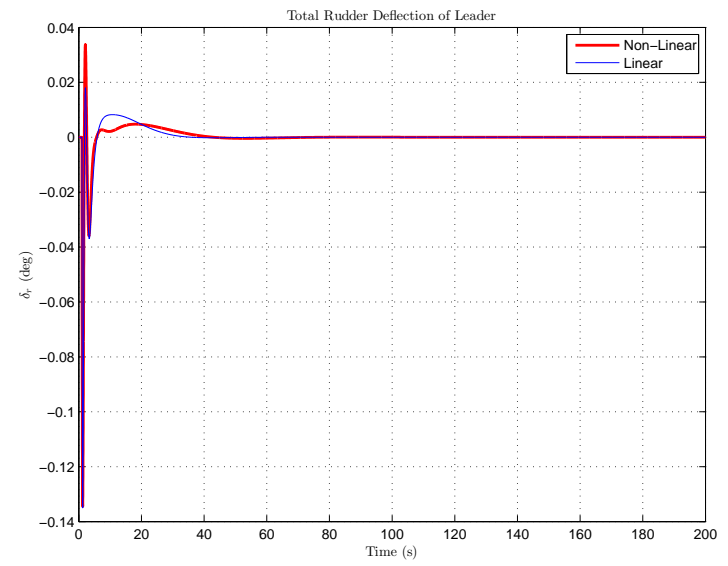

(e) Rudder Response

Figure E.5: Lateral response results for a $5 \mathrm{~m}$ cross-track error step command 


\section{Appendix F}

\section{Wake Interaction Model}

The model used in this thesis to describe the aerodynamic interaction effects of the trailing vortices of the leader on the follower aircraft, is the same model derived by N. Bizinos at the University of Cape Town [3] [56] and used by D. Büchner at Stellenbosch University [87] [4]. In this appendix, the equations of the different influence factors are given, followed by a discussion of the current wake model. With this currently being the only model available at our disposal, it was again used in this thesis but with some restrictions applied to its usage.

\section{F.1 Influence Parameters}

The formation-induced coefficients in Chapter 3 are proportional to certain dimensionless parameters, known as influence factors. These influence parameters, reproduced from Bizinos [3] in Equations (F.1a) to (F.1d are: $\sigma$ (influencing lift, side force, drag and yawing moment) and $\tau$ (influencing rolling and yawing moment). Assumptions made by the author in the approximated horseshoe vortex model included [3]: (1) the induced lift, drag and rolling moment are caused by the wing, (2) the induced side force is caused by the tailfin, (3) the induced yawing moment is caused by both the wing and the tailfin, (4) and the induced pitching moment is estimated by considering the change in downwash at the tailplane due to the formation induced downwash at the wing.

The resulting formation aerodynamic coefficients are implemented as detailed in Chapter 3 and are illustrated in Figure 3.15, reproduced here in Figure F.1. These formation-induced coefficients are shown for various lateral separations $\eta$, while assuming zero vertical separation $\zeta=0$ and an absolute axial separation of $\xi=10$ wingspans. 

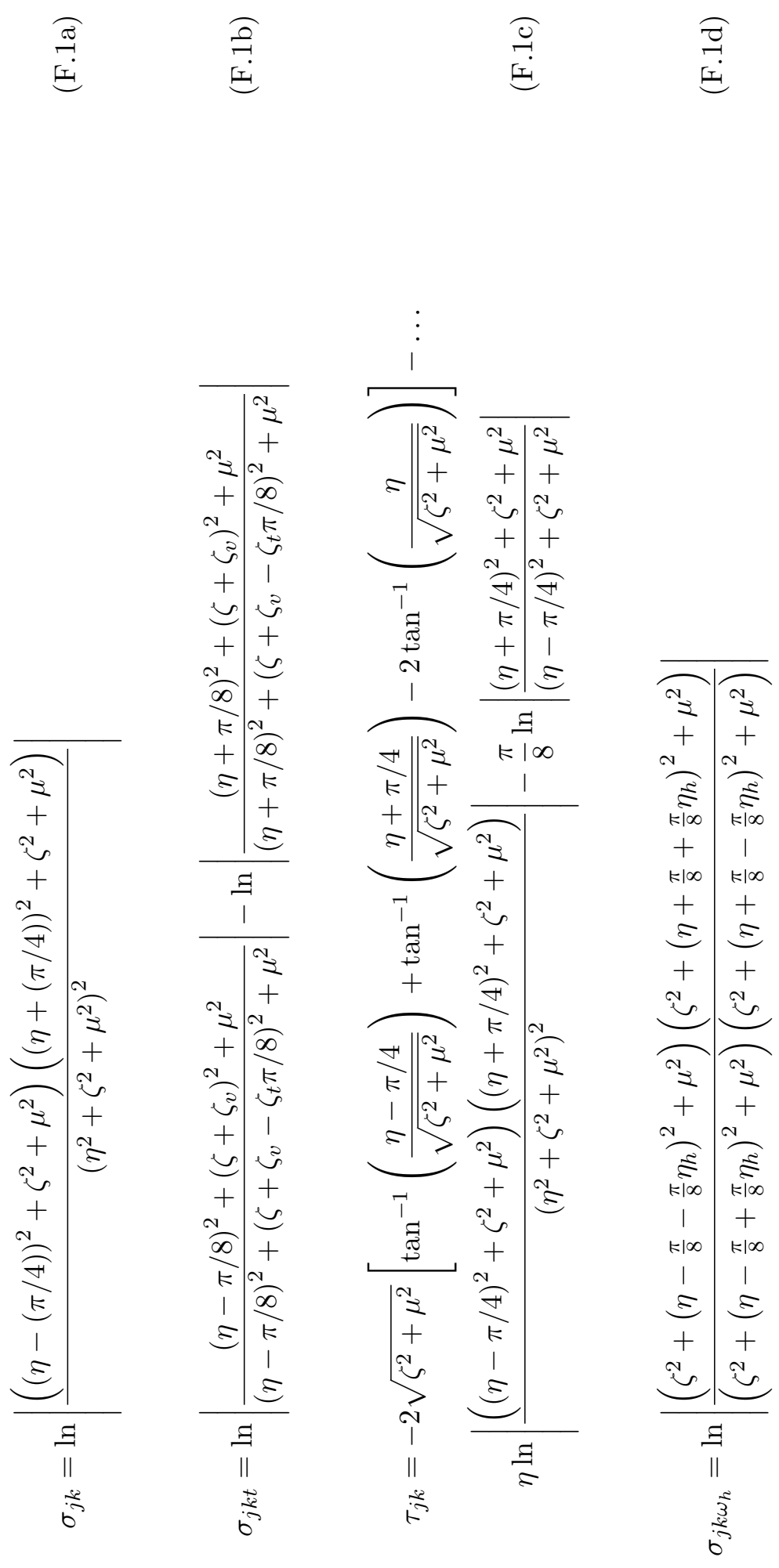

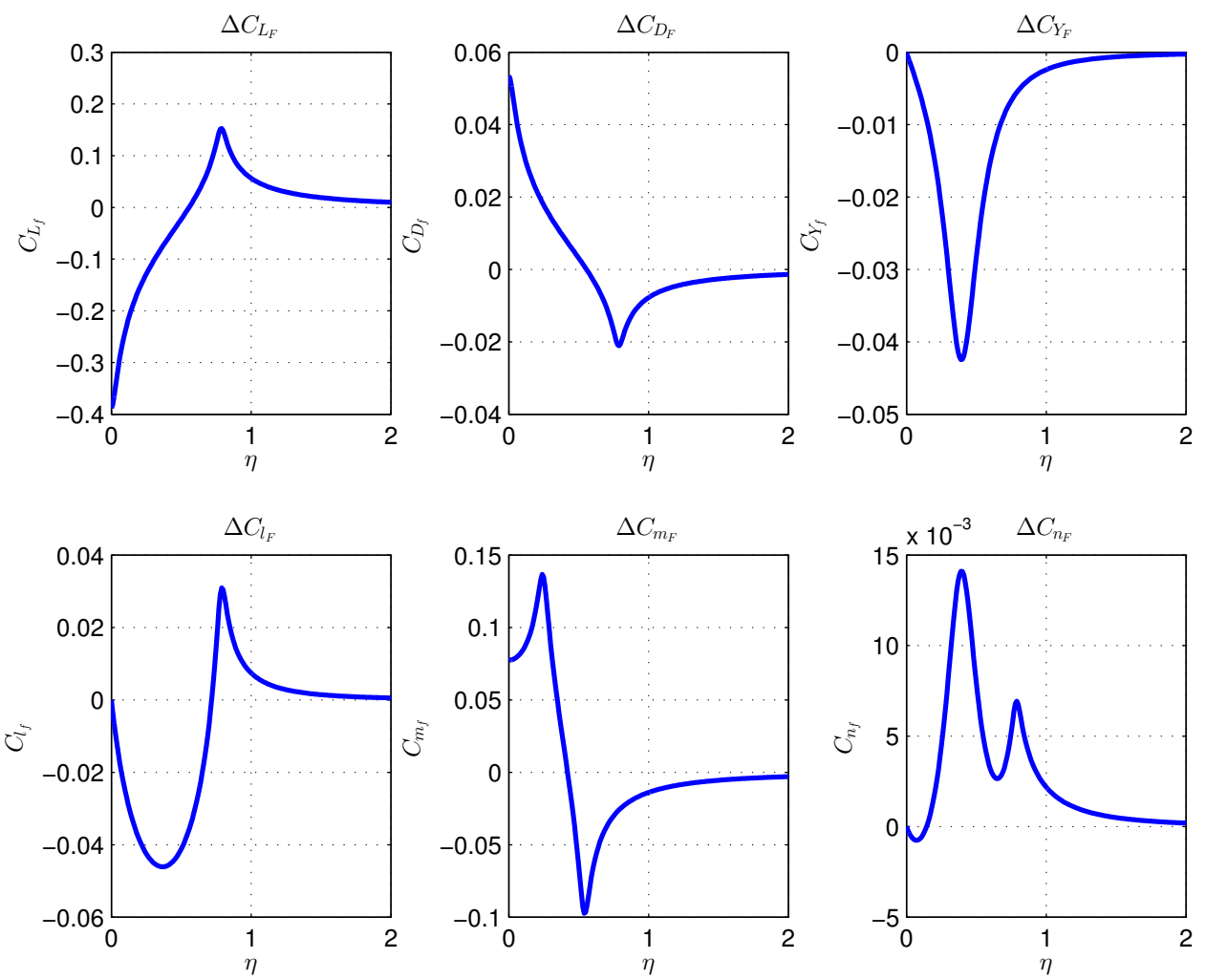

Figure F.1: Formation-induced aerodynamic coefficients as a function of lateral separation and at $\xi=-10$ and $\zeta=0$.

\section{F.2 Wake Model Inaccuracies}

In the study by Bizinos [3] [56], a short analysis was done on the accuracy of the approximated wake model used. Figure F.2 illustrates and compares induced forces and moments in formation due to the interaction of the trailing vortices with the follower aircraft. The approximated method, which assumes numerical integration over a rectangular wing, is compared to the results of numerical integration over an elliptical wing. It is clear from these plots that for most of the induced coefficients the approximated method is much more exaggerated at the peaks.

At the time of the current study, the author found limited experimental data on formation flight for performance benefit. A number of wind tunnel experiments were performed, but these tests were focused on aerial refuelling and separations that were not in the same range to that which is of interest in the current study. Moreover, the models used in these tests - F-18 models, tailless delta wing models and similar models - are not representative of typical large transport aircraft models. Figure F.3 illustrates the comparison of the approximate method, numerical integration over an elliptical wing as well as flight test data, experimental data and data from vortex lattice methods. 


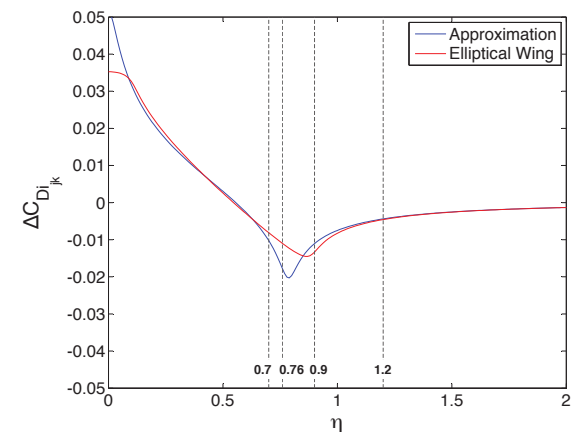

(a) $\Delta C_{D}$

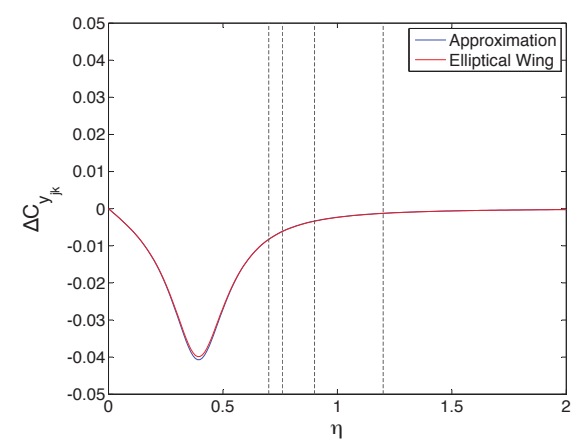

(b) $\Delta \mathrm{C}_{\mathrm{S}}$

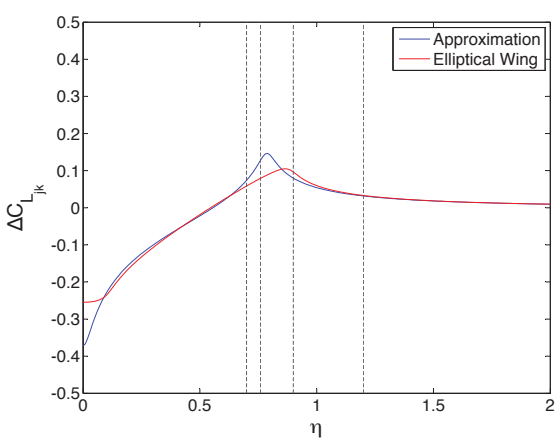

(c) $\Delta \mathrm{C}_{\mathrm{L}}$

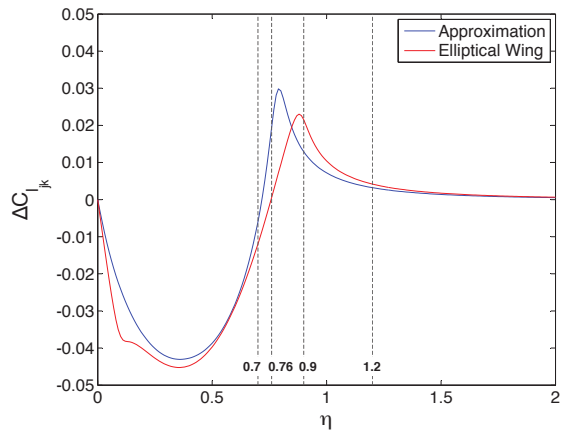

(d) $\Delta C_{1}$

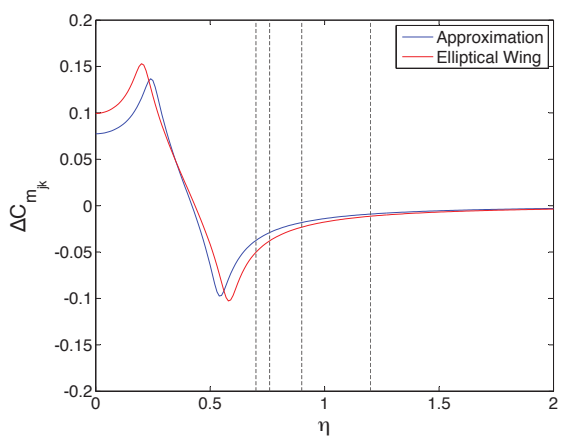

(e) $\Delta \mathrm{C}_{\mathrm{m}}$

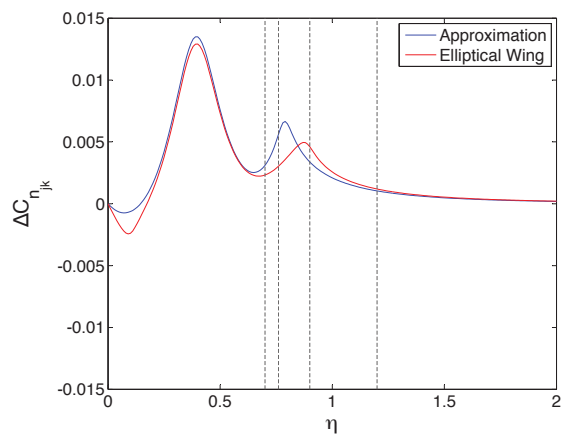

(f) $\Delta C_{n}$

Figure F.2: Comparison of the approximated method, which assumes numerical integration over a rectangular wing, to the results of numerical integration over an elliptical wing. The plots clearly show an exaggeration of the induced coefficients at the peaks when using the approximated method. (from Bizinos [3]) 


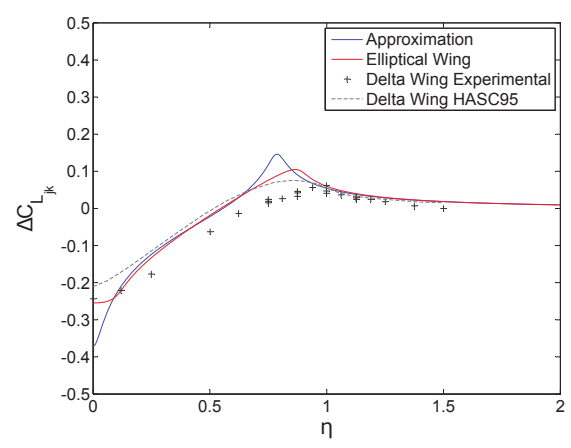

(a) $\Delta \mathrm{C}_{\mathrm{L}}$

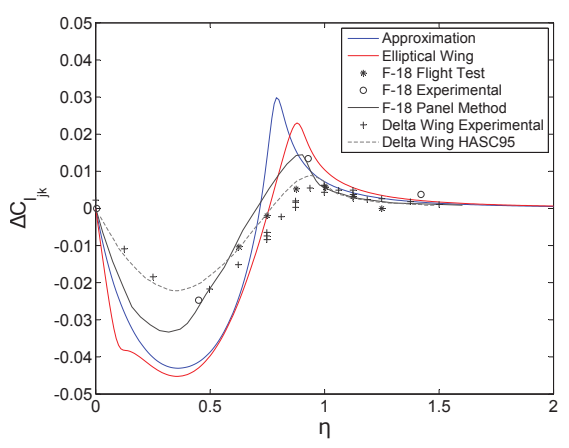

(b) $\Delta \mathrm{C}_{1}$

Figure F.3: Induced lift and roll coefficients comparison after using the approximate method, numerical integration over an elliptical wing together with flight test data, experimental data and data from vortex lattice methods.

\section{F.3 Feasible Regions}

In the study by Büchner, an extensive analysis was performed to determine feasible regions for formation flight. The conclusion drawn by the author was that two feasible regions exist for formation flight: a higher-benefit but less safe "sandwich" region, and a lower-benefit but safer "outer" region. As illustrated in Figure 2.16, the sandwich region is tightly bounded between two untrimmable regions at approximately 0.7 wingspans of lateral separation. The outer region lies at lateral separations of larger than 1.1 wingspans.

In a study between NASA and academics from Stanford University [88], an extensive analysis of extended formation flight for tens of wingspans was performed. The results showed that for subsonic flight conditions, the optimal vortex position occurs at the wingtip and migrates to about $10 \%$ overlap from the wingtip when the wing is roll-trimmed through aileron deflection. This means that the optimum should be close to about 0.9 wingspans lateral separation, as illustrated in Figure 3.17, which shows the optimum peak for flight test data, experimental data and the results with an elliptical wing.

\section{F.4 Conclusion}

After the consideration of various sources of information, although an improved wake interaction model would allow a more complete investigation of the wake interaction problem, the model is not expected to result in interaction forces significantly larger or smaller than those predicted by the current model at wingspans larger than one. The unrealistic peaks of the current model will be in close agreement to the forces predicted at 0.9 wingspans. It has thus been decided to keep the lateral separation limited to no closer than one wingspan. 


\section{Appendix G}

\section{Extended Formation Flight Simulations}

In the current study, extended non-linear simulations were performed to evaluate the formation guidance and formation-hold performance of the follower aircraft. These non-linear simulations were performed as multiple straight and level flight simulations at a nominal altitude of $40000 \mathrm{ft}$ and $0.8 \mathrm{M}$. Various lateral separations were investigated, together with various levels of turbulence intensity being added to the simulation.

\section{G.1 Geometric Separation Tracking Performance Simulations}

To evaluate the tracking performance of the formation guidance laws, the follower is required to obtain and maintain a set of desired lateral, vertical and longitudinal separations, given in Table 3.3, and during the simulation, the leader will perform longitudinal manoeuvres. Table 6.2 gives a summary of the step commands given over the entire simulation period. In this appendix, only the simulation results for a desired lateral separation for 1.3 wingspans in varying levels of turbulence intensity will be shown. The simulation results are of a similar nature at different lateral separations. Results for different lateral separations are given in the next section. Table G.1 provides a reference for the different simulation results.

Table G.1: Reference guide for separation tracking performance simulations at different separations and varying levels of turbulence intensity.

\begin{tabular}{|c|c|c|c|c|c|c|}
\hline \multirow[t]{2}{*}{ Turbulence Intensity } & \multicolumn{3}{|c|}{ Desired Separations } & \multicolumn{3}{|c|}{ Figures } \\
\hline & $\eta$ & $\zeta$ & $\xi$ & & & \\
\hline Light & 1.3 & 0 & -10 & G.1a & G.1b, & G.2 \\
\hline Moderate & 1.3 & 0 & -10 & G.1c, & G.1d, & G.3 \\
\hline Severe & 1.3 & 0 & -10 & G.1e, & G.1f & G.4 \\
\hline
\end{tabular}




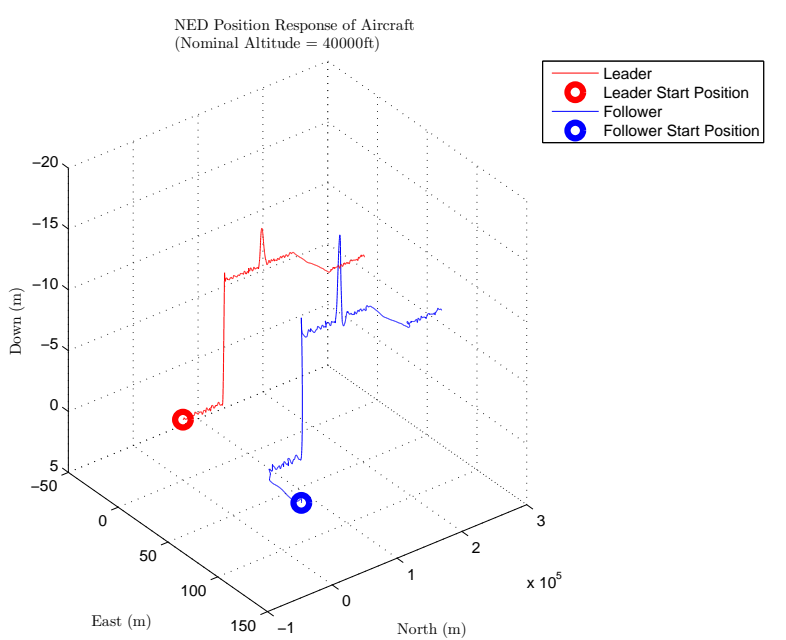

(a) NED in light turbulence

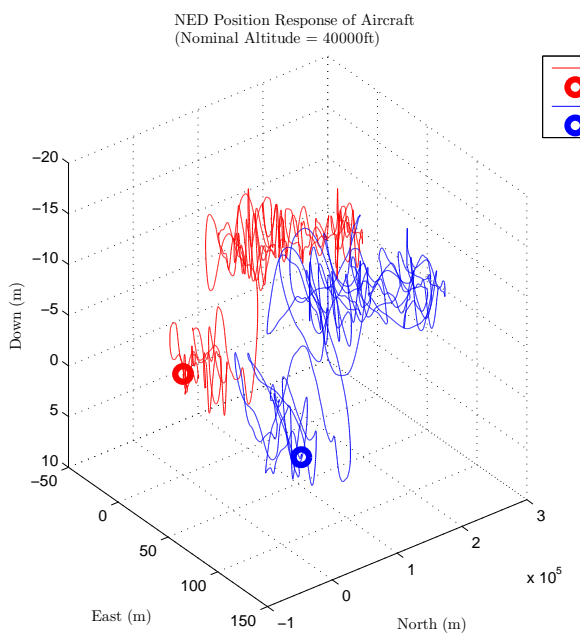

(c) NED in moderate turbulence

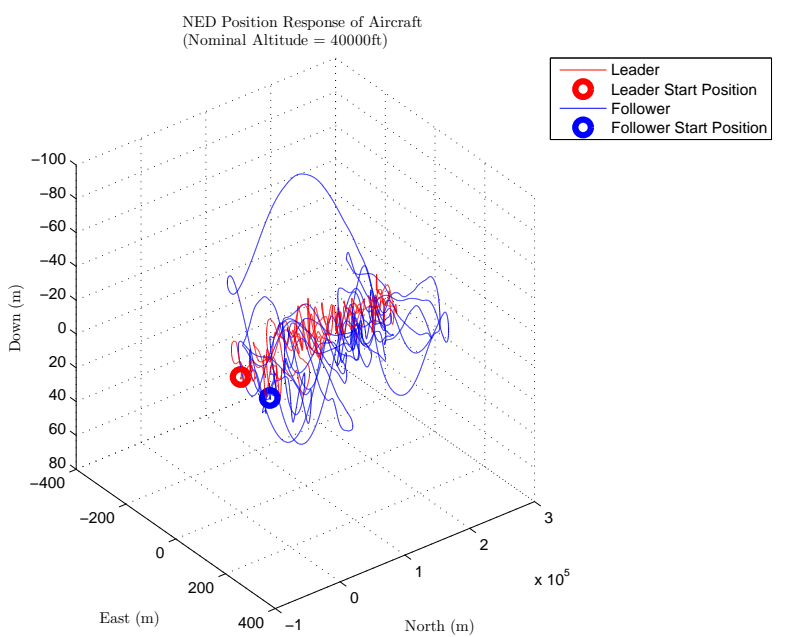

(e) NED in severe turbulence

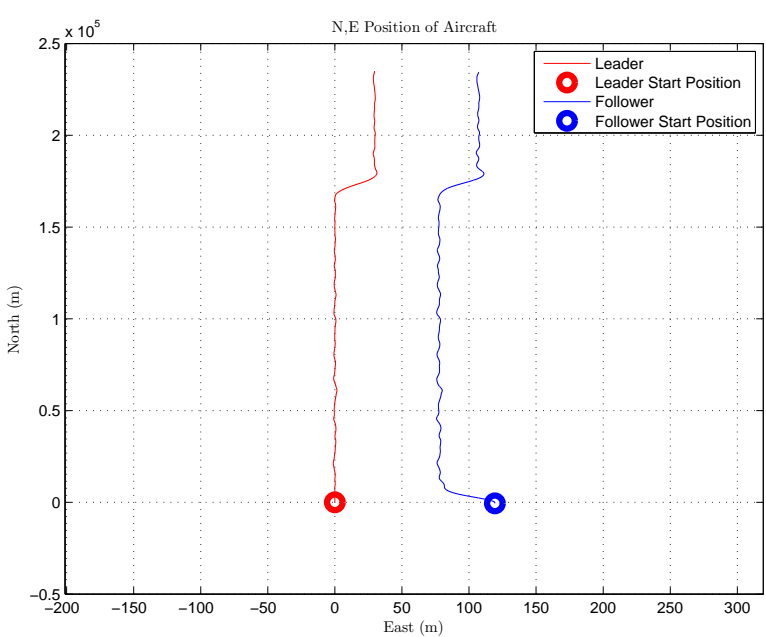

(b) NE in light turbulence

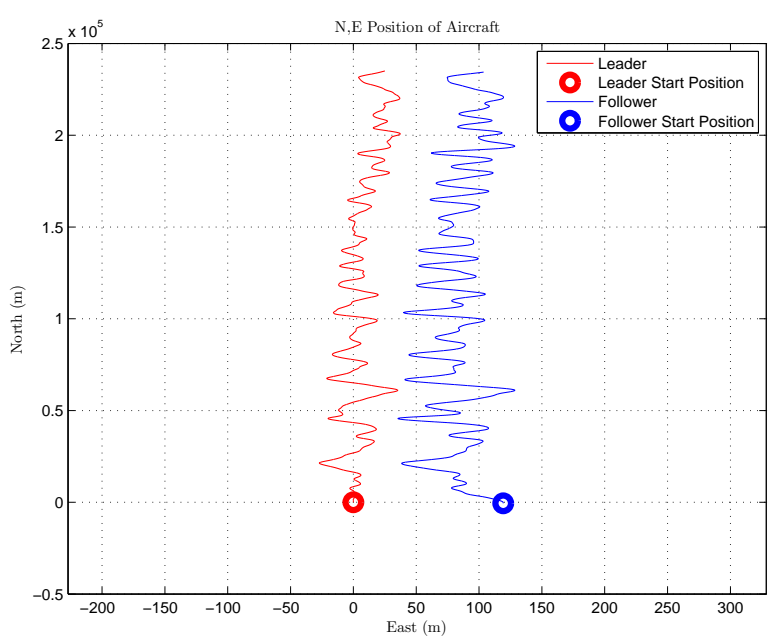

(d) NE in moderate turbulence

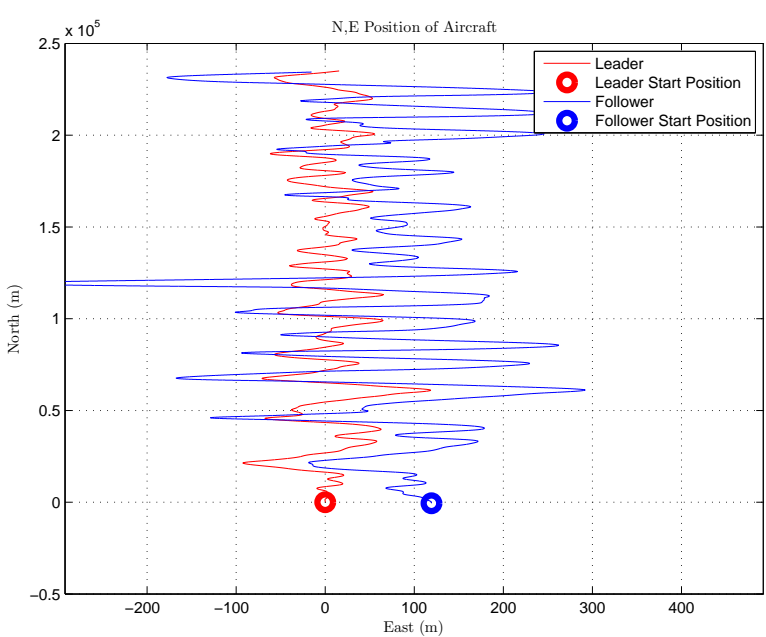

(f) NE in severe turbulence

Figure G.1: Position response of aircraft during formation flight at $\eta=1.3, \zeta=0$ and $\xi=-10$ in varying levels of turbulence intensity while the leader aircraft performs longitudinal and lateral manoeuvres. 


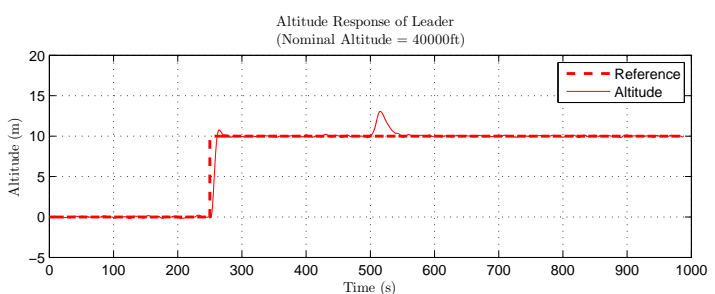

Altitude Response of Follower

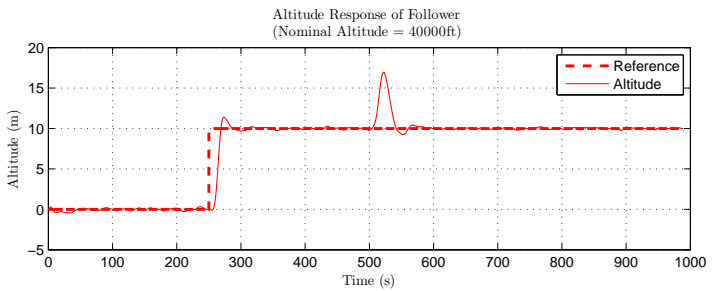

(a) Altitude response

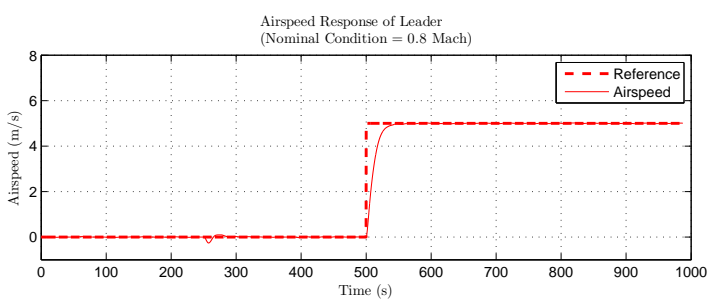

Airspeed Response of Follower
(Nominal Condition $=0.8$ Mach

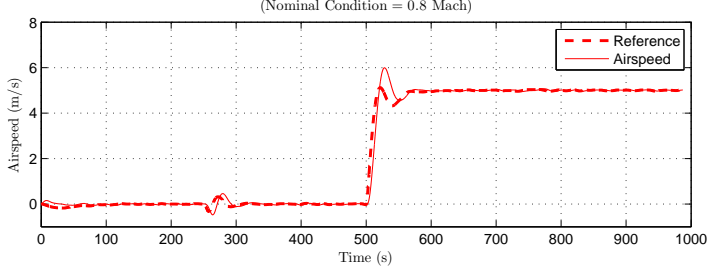

(c) Airspeed response
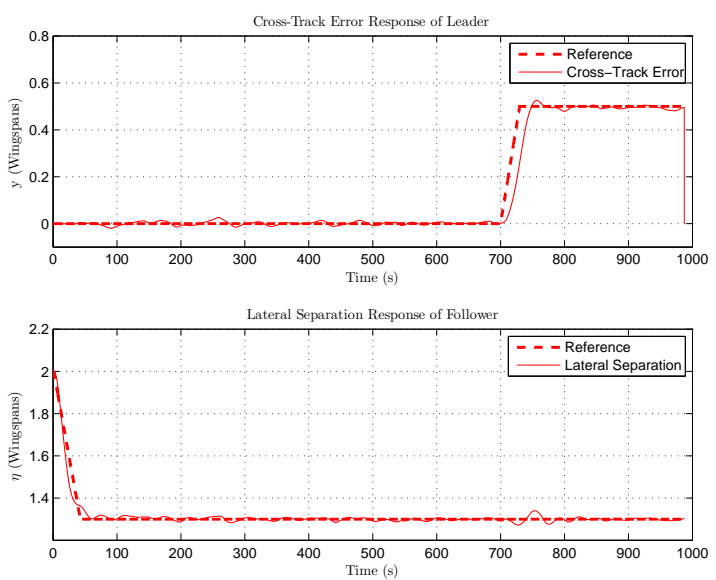

(e) Tracking response

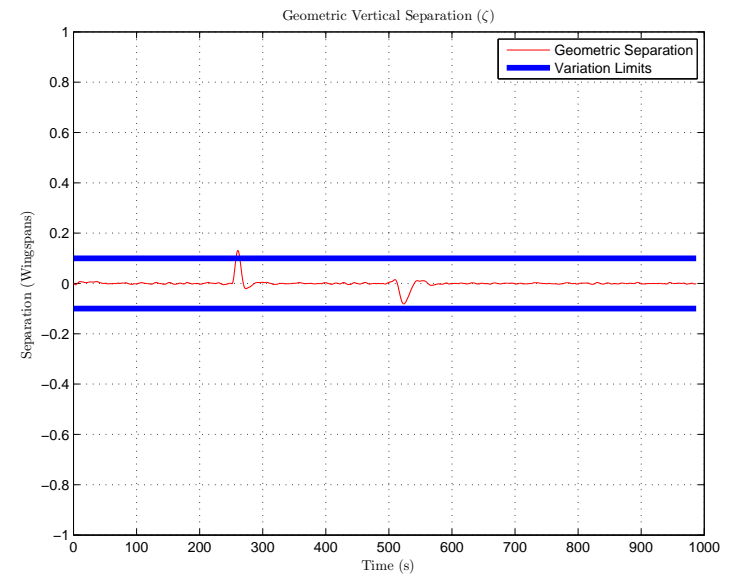

(b) Geometric vertical separation

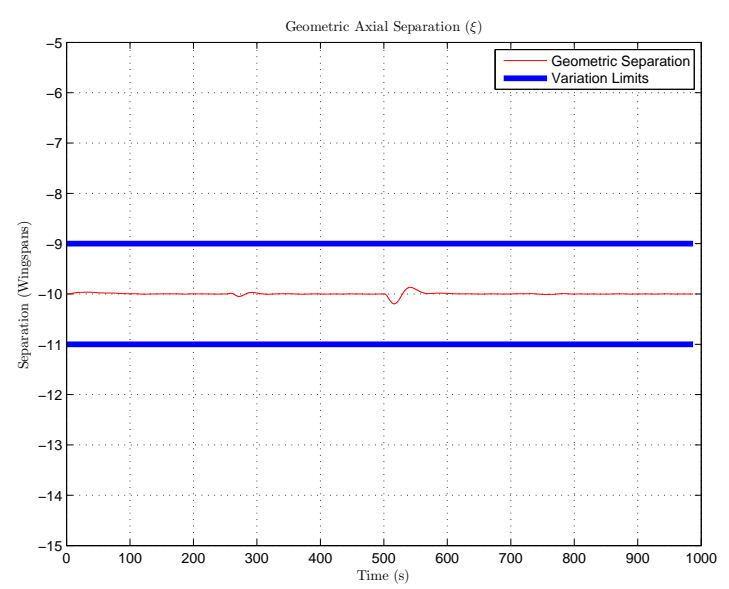

(d) Geometric axial separation

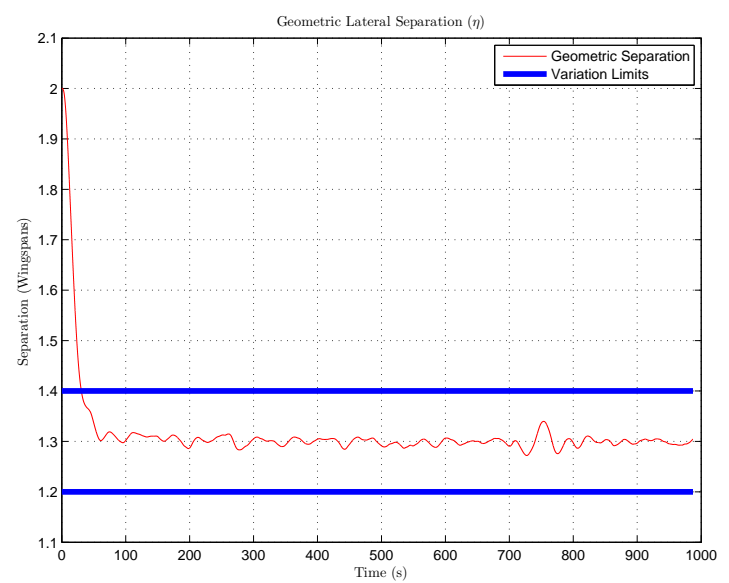

(f) Geometric lateral separation

Figure G.2: Longitudinal and lateral response during formation at $\eta=1.3, \zeta=0$ and $\xi=-10$ wingspans in light turbulence. 


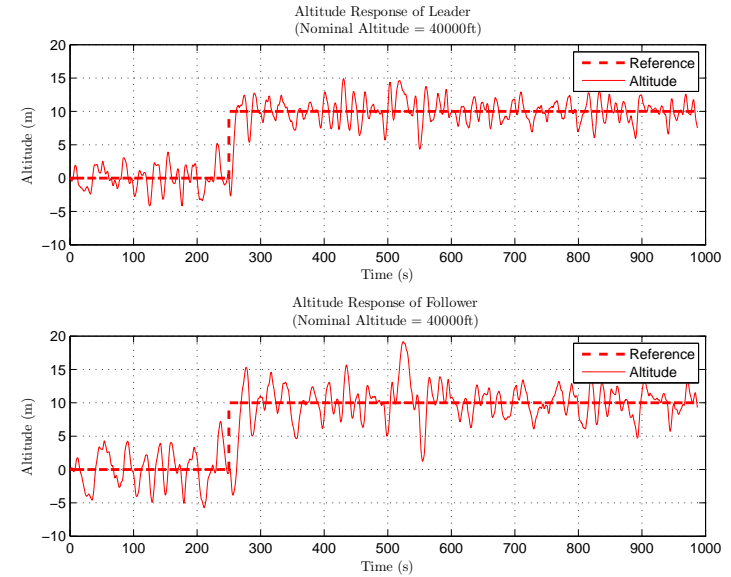

(a) Altitude response

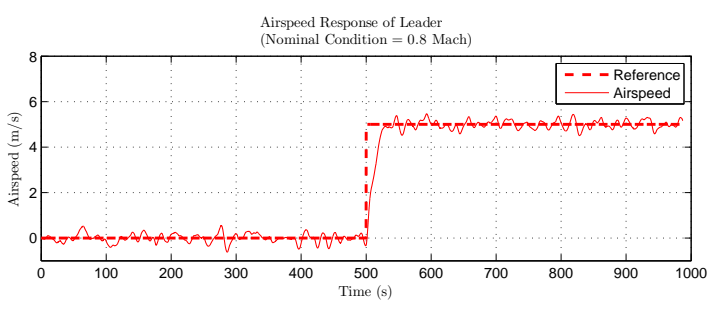

Airspeed Response of Follower
(Nominal Condition $=0.8$ Mach)

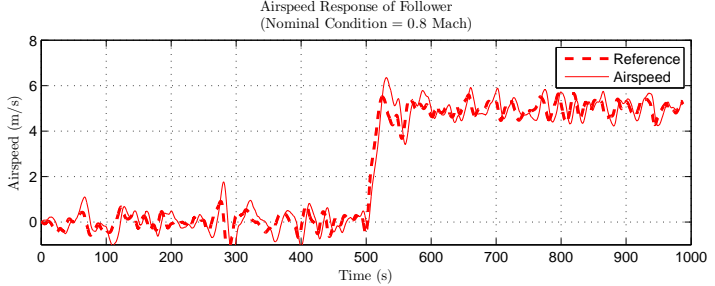

(c) Airspeed response
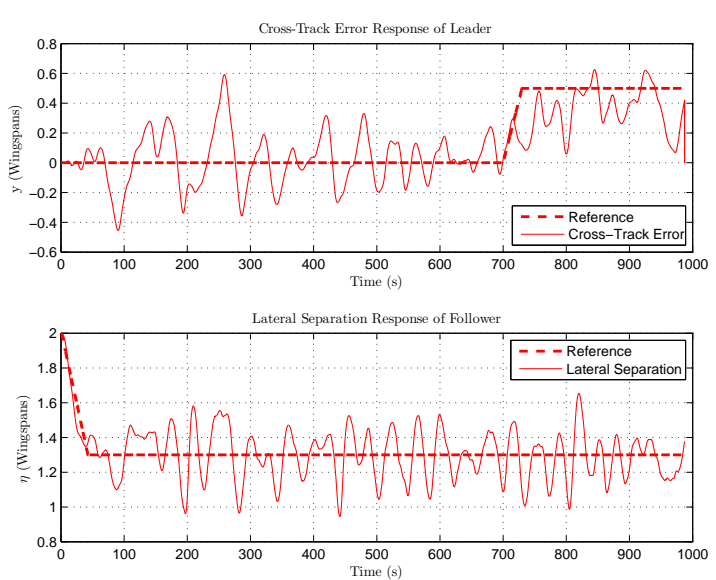

(e) Tracking response

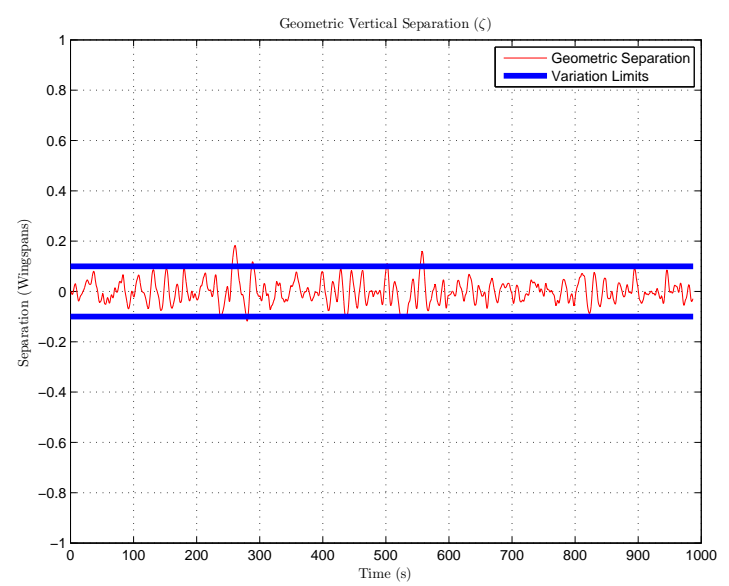

(b) Geometric vertical separation

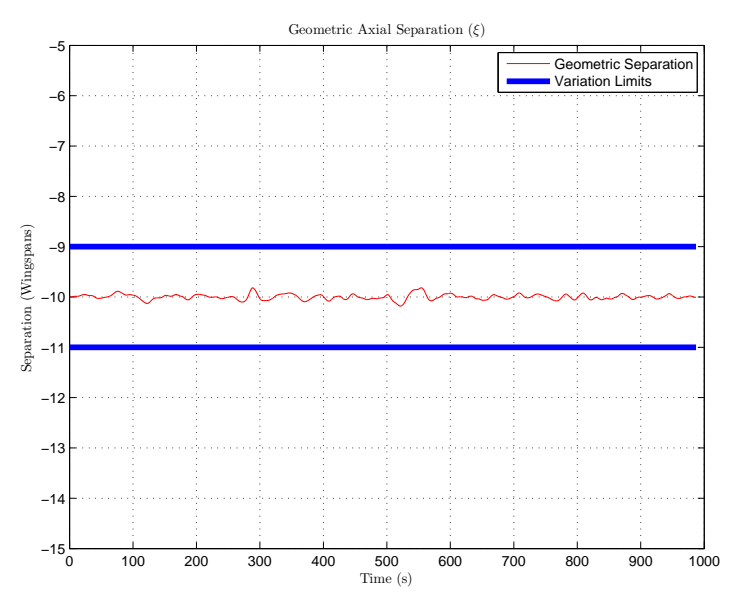

(d) Geometric axial separation

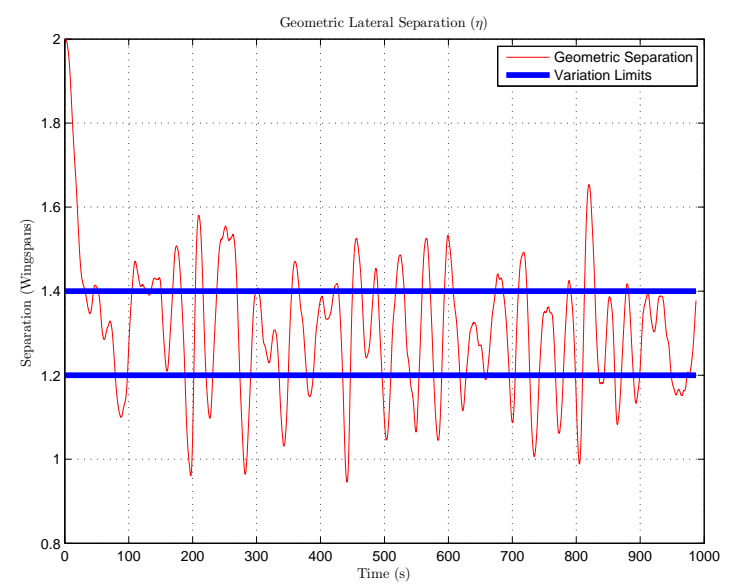

(f) Geometric lateral separation

Figure G.3: Longitudinal and lateral response during formation at $\eta=1.3, \zeta=0$ and $\xi=-10$ wingspans in moderate turbulence. 

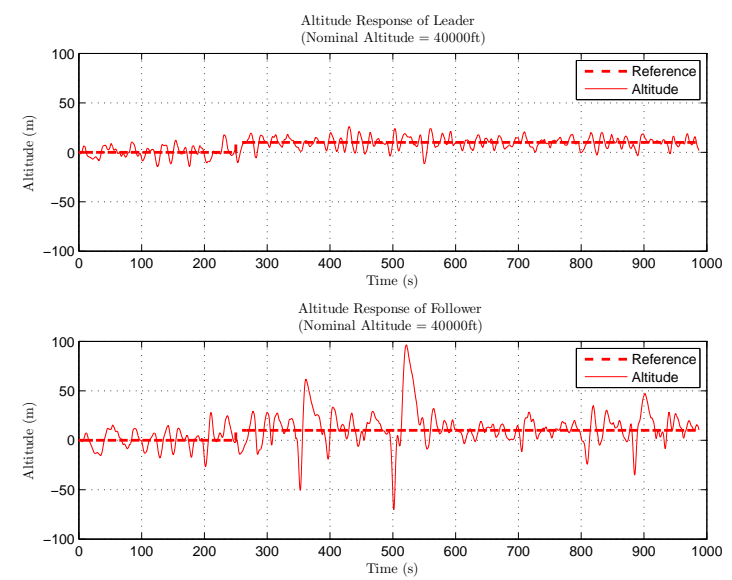

(a) Altitude response

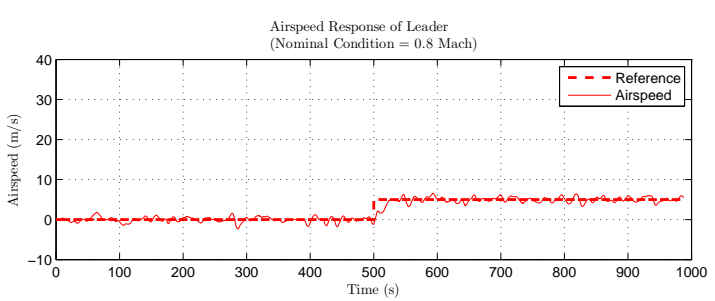

Airspeed Response of Follower
(Nominal Condition $=0.8$ Ma

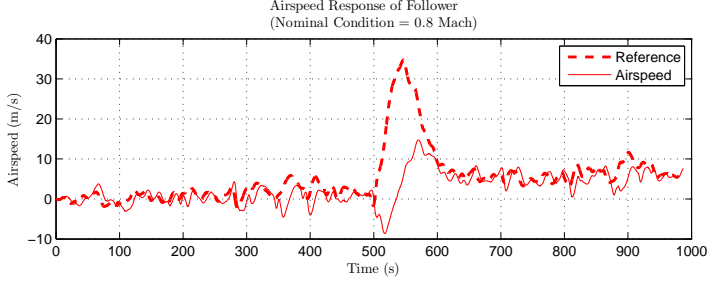

(c) Airspeed response
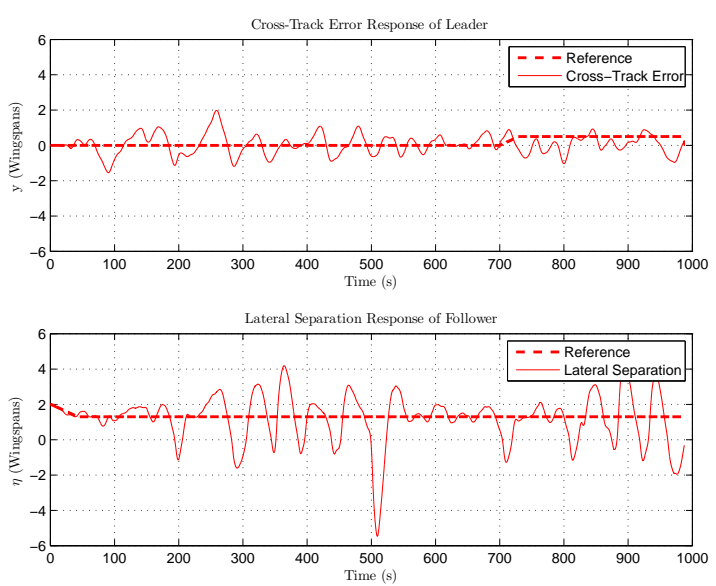

(e) Tracking response

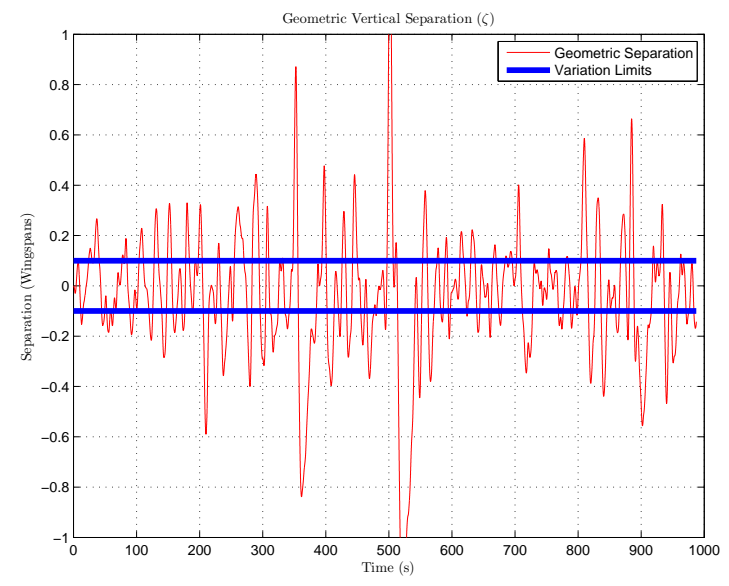

(b) Geometric vertical separation

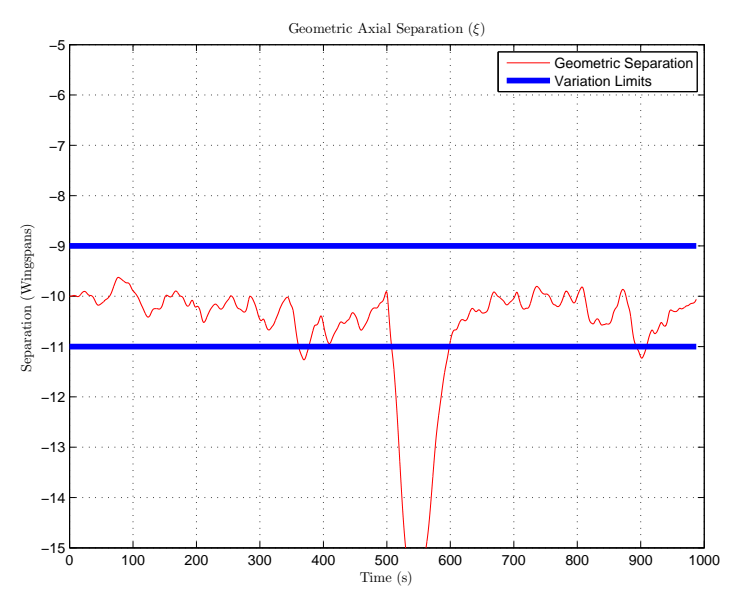

(d) Geometric axial separation

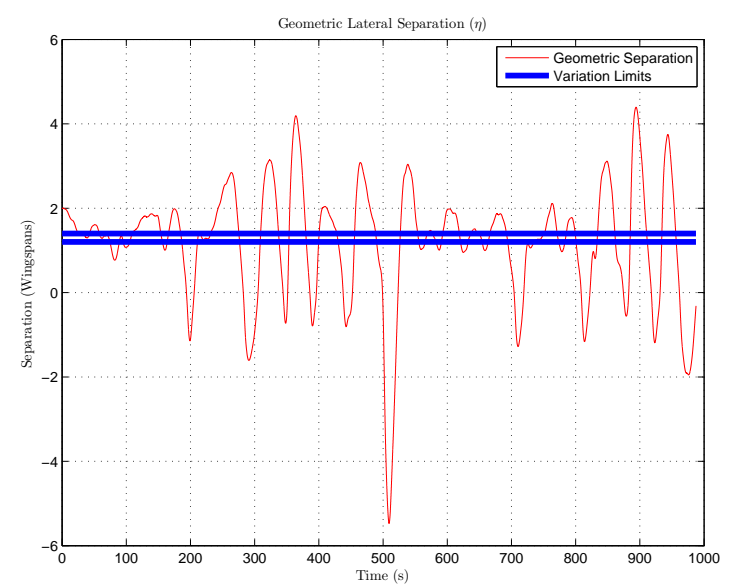

(f) Geometric lateral separation

Figure G.4: Longitudinal and lateral response during formation at $\eta=1.3, \zeta=0$ and $\xi=-10$ wingspans in severe turbulence. 


\section{G.2 Formation-Hold Performance Simulations}

To evaluate how well the formation guidance laws hold the desired separations during turbulence, the following simulation exercise was performed: The trailing aircraft is initialised at any lateral separation in the range $1.0 \leqslant \eta \leqslant 1.5$ from the trailing vortex. The leader aircraft is commanded to fly along a straight path with no flight path heading changes, and the follower is required to maintain the desired geometric separations. The simulations were performed over an extended period of 30 minutes and at various turbulence intensities.

For each level of turbulence intensity, the lateral separation is plotted against the axial and vertical separations over the entire simulation period. Also indicated in the plots as thick blue boundaries are the allowable separation variations, as summarised in Table 3.3 . Finally, in the bottom left of the each plot is an indication of how many times the aircraft happened to be outside of the allowable separations boundaries. Table G.2 provides a reference for the different simulation results.

Table G.2: Reference guide for formation-hold performance simulations at different separations and varying levels of turbulence intensity

\begin{tabular}{|c|c|c|c|c|c|c|}
\hline \multirow[t]{2}{*}{ Turbulence Intensity } & \multicolumn{3}{|c|}{ Desired Separations } & \multicolumn{3}{|c|}{ Figures } \\
\hline & $\eta$ & $\zeta$ & $\xi$ & & & \\
\hline \multirow{6}{*}{ Light } & 1.5 & 0 & -10 & G.6a & G.6b, G.5a & G.5b \\
\hline & 1.4 & 0 & -10 & G.8a & G.8b, G.7a, & G.7b \\
\hline & 1.3 & 0 & -10 & G.10a & G.10b $\mathrm{G} .9 \mathrm{a}$ & G.9b \\
\hline & 1.2 & 0 & -10 & G.12a & G.12b G.11a & G.11b \\
\hline & 1.1 & 0 & -10 & G.14a & G.14b, G.13a & G.13b \\
\hline & 1.0 & 0 & -10 & G.16a, & G.16b, G.15a & G.15b \\
\hline \multirow{6}{*}{ Moderate } & 1.5 & 0 & -10 & G.6c & G.6d, G.5c, & $\mathrm{G} .5 \mathrm{~d}$ \\
\hline & 1.4 & 0 & -10 & $\overline{\mathrm{G} .8 \mathrm{c}}$ & G.8d, G.7c, & G.7d \\
\hline & 1.3 & 0 & -10 & G.10c & G.10d G.9c & G.9d \\
\hline & 1.2 & 0 & -10 & G.12c & $\mathrm{G.12 \textrm {d }}$ G.11c & $\mathrm{G} .11 \mathrm{~d}$ \\
\hline & 1.1 & 0 & -10 & G.14c & $\overline{\mathrm{G} .14 \mathrm{~d}}, \mathrm{G} .13 \mathrm{c}$ & $\overline{G .13 d}$ \\
\hline & 1.0 & 0 & -10 & G.16c & $\overline{\mathrm{G} .16 \mathrm{~d}}, \mathrm{G} .15 \mathrm{c}$ & $\mathrm{G} .15 \mathrm{~d}$ \\
\hline \multirow{6}{*}{ Severe } & 1.5 & 0 & -10 & G.6e & G.6f, G.5e & G.5f \\
\hline & 1.4 & 0 & -10 & $\overline{\mathrm{G} .8 \mathrm{e}}$ & G.8f, $\mathrm{G.7e}$ & G.7f \\
\hline & 1.3 & 0 & -10 & G.10e & G.10f G.9e & G.9f \\
\hline & 1.2 & 0 & -10 & G.12e & G.12f G.11e & G.11f \\
\hline & 1.1 & 0 & -10 & G.14e, & G.14f & G.13f \\
\hline & 1.0 & 0 & -10 & G.16e, & G.16f G.15e & G.15f \\
\hline
\end{tabular}




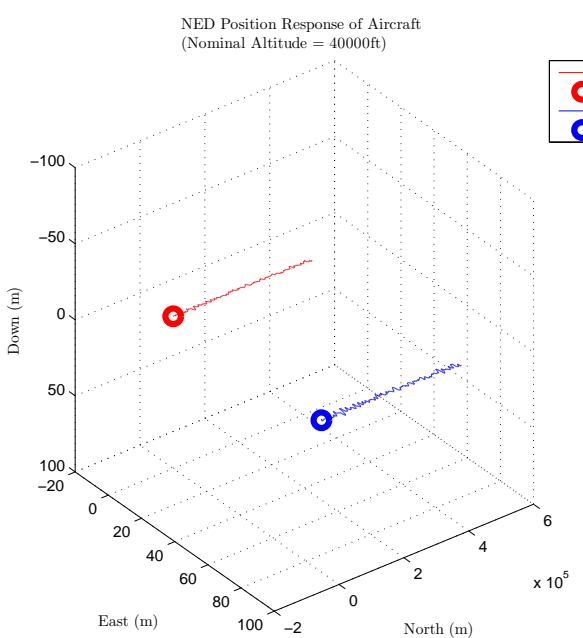

(a) NED in light turbulence

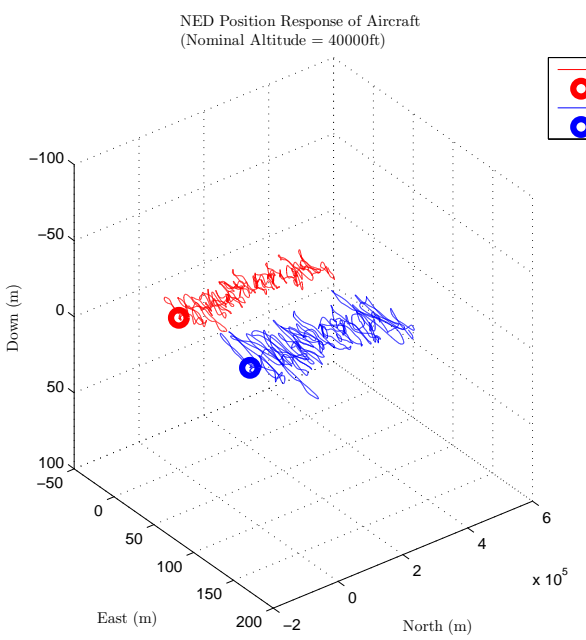

(c) NED in moderate turbulence

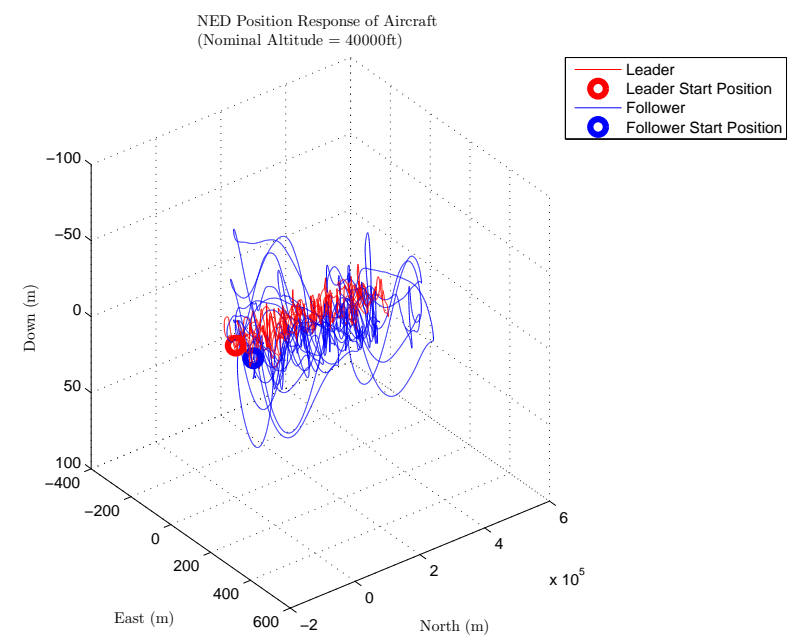

(e) NED in severe turbulence

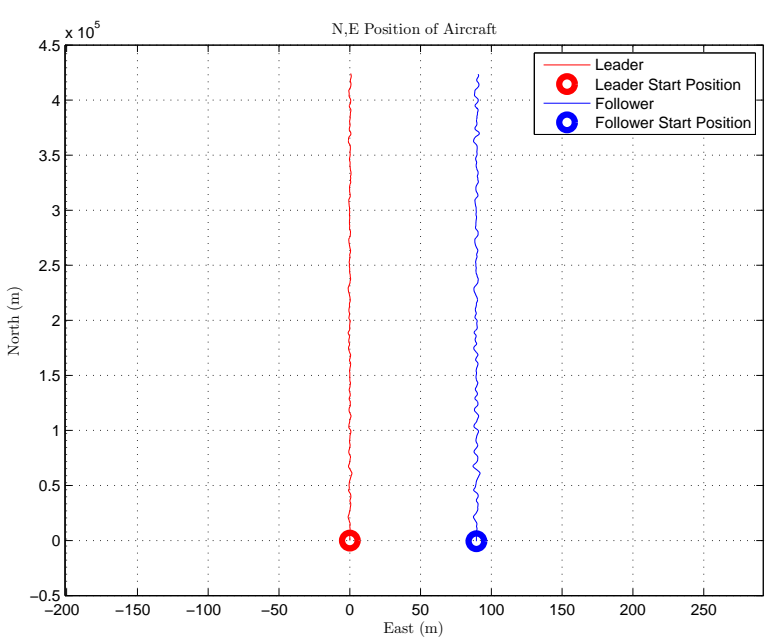

(b) NE in light turbulence

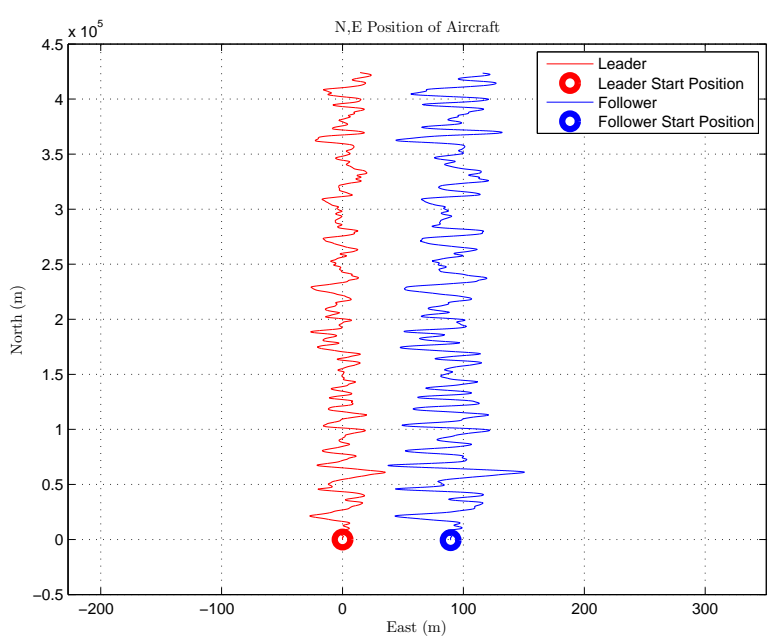

(d) NE in moderate turbulence

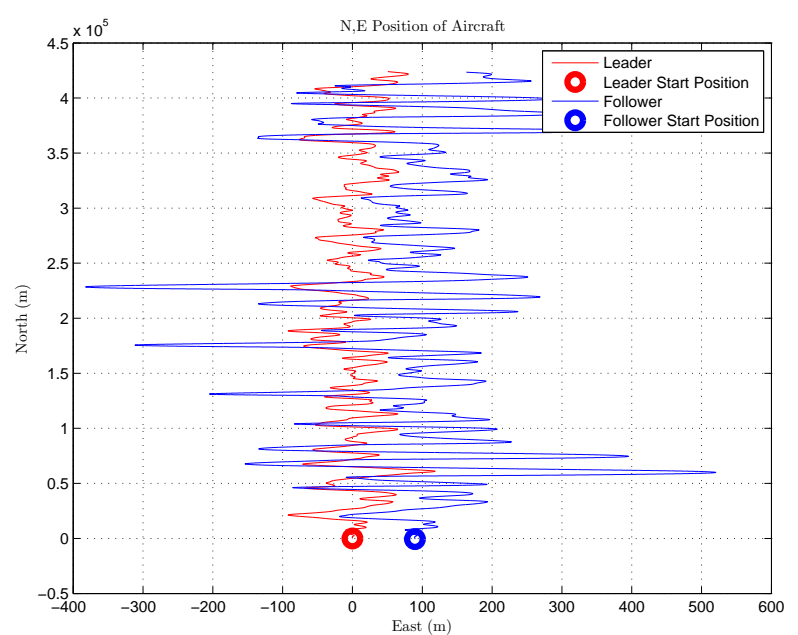

(f) NE in severe turbulence

Figure G.5: Position response of aircraft during formation flight at $\eta=1.5, \zeta=0$ and $\xi=-10$ in varying levels of turbulence intensity. 


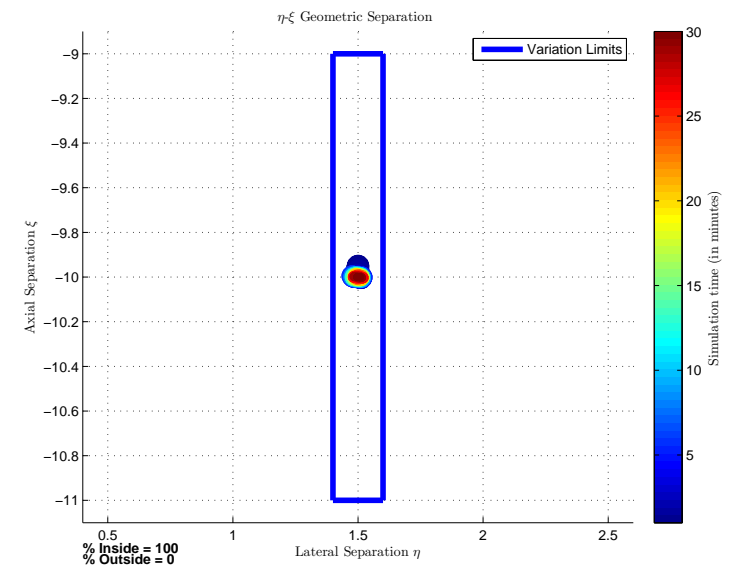

(a) Lateral vs axial separation (Light turbulence)

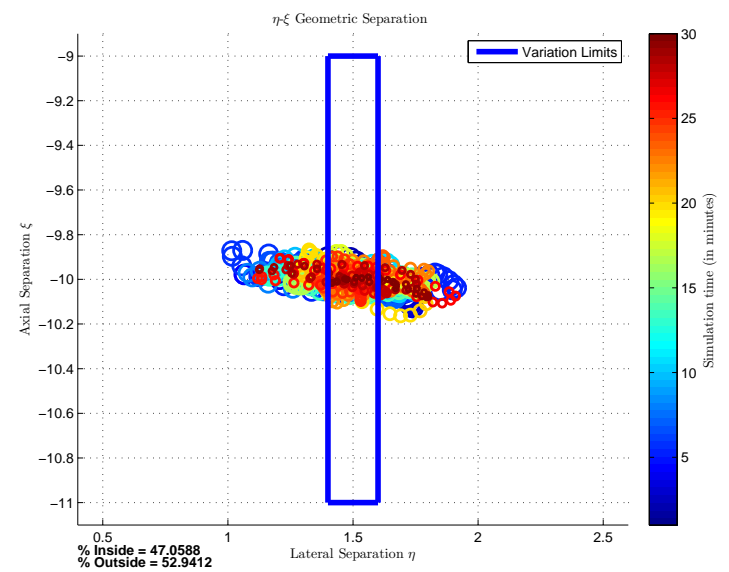

(c) Lateral vs axial separation (Moderate turbulence)

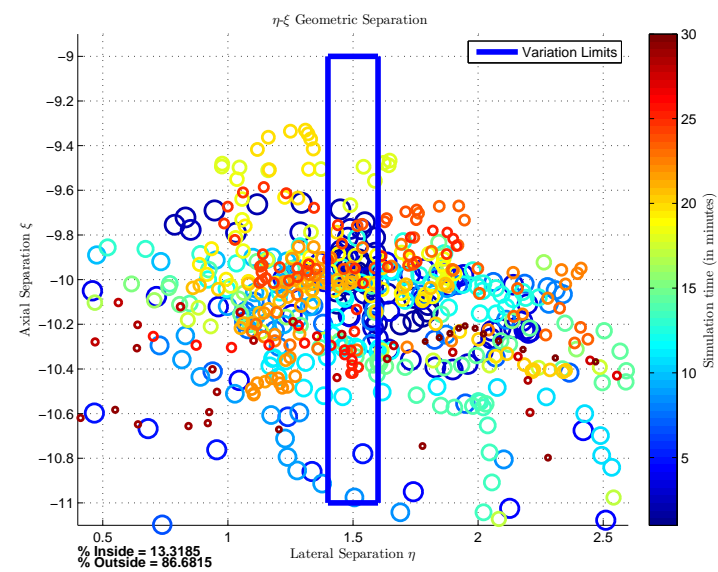

(e) Lateral vs axial separation (Severe turbulence)

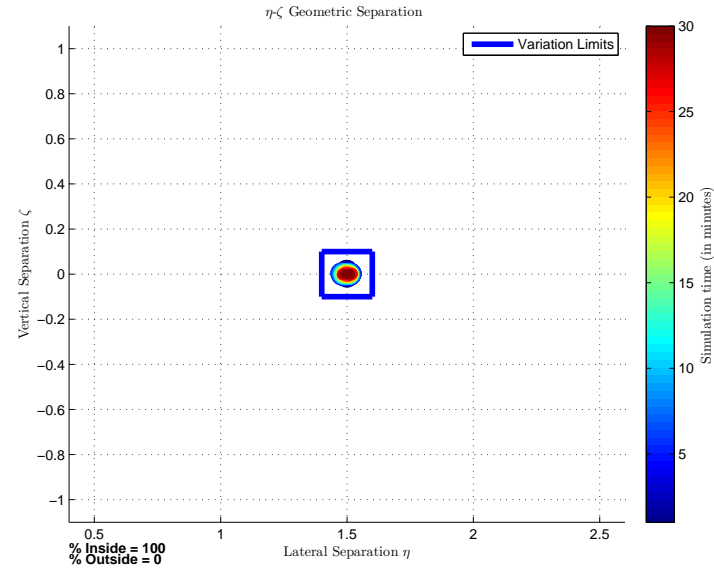

(b) Lateral vs vertical separation (Light turbulence)

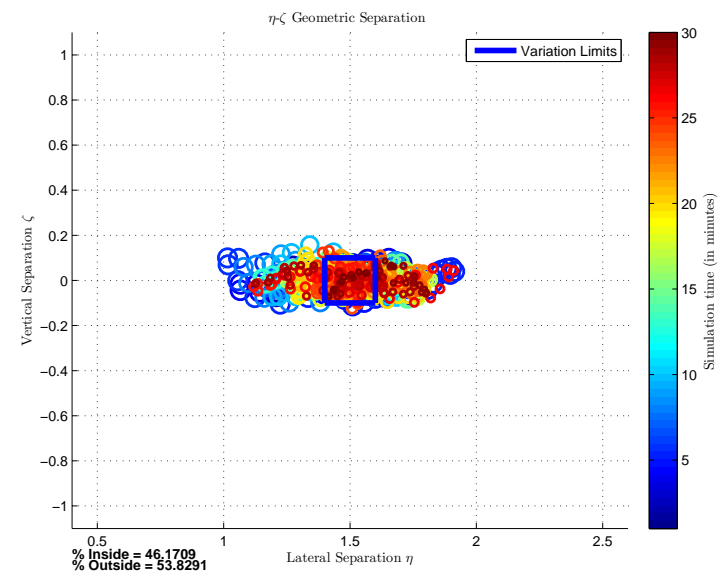

(d) Lateral vs vertical separation (Moderate turbulence)

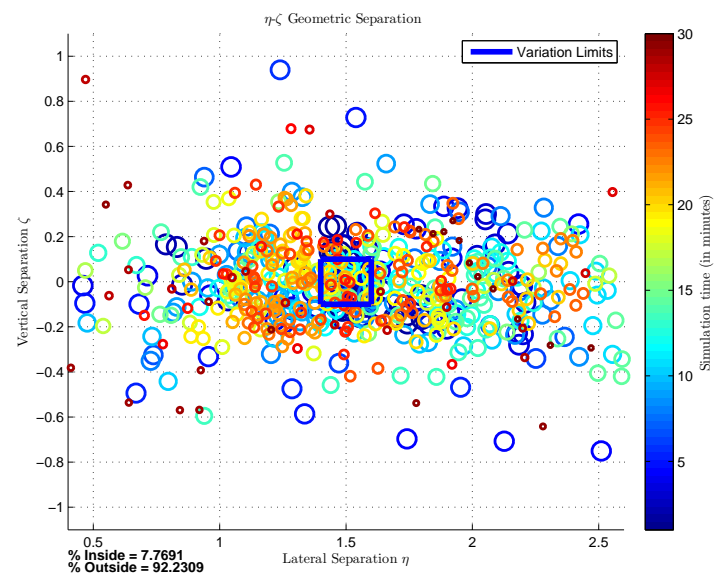

(f) Lateral vs vertical separation (Severe turbulence)

Figure G.6: Formation-hold performance at desired geometric separations $\eta=1.5, \zeta=0$ and $\xi=-10$ wingspans during formation flight in varying levels of turbulence 


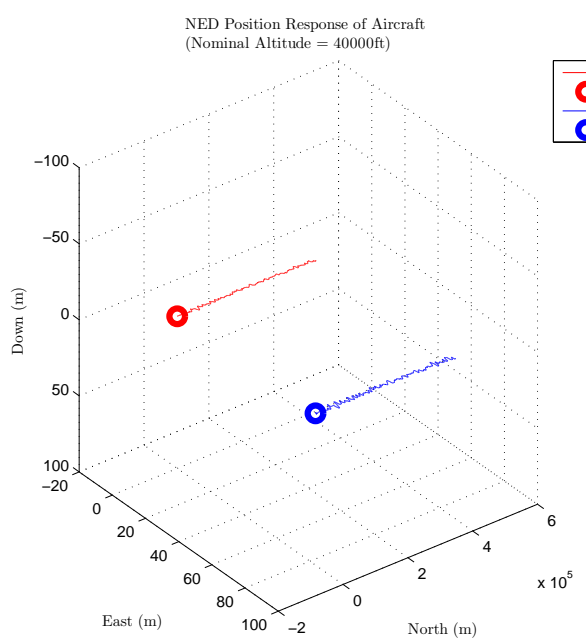

(a) NED in light turbulence

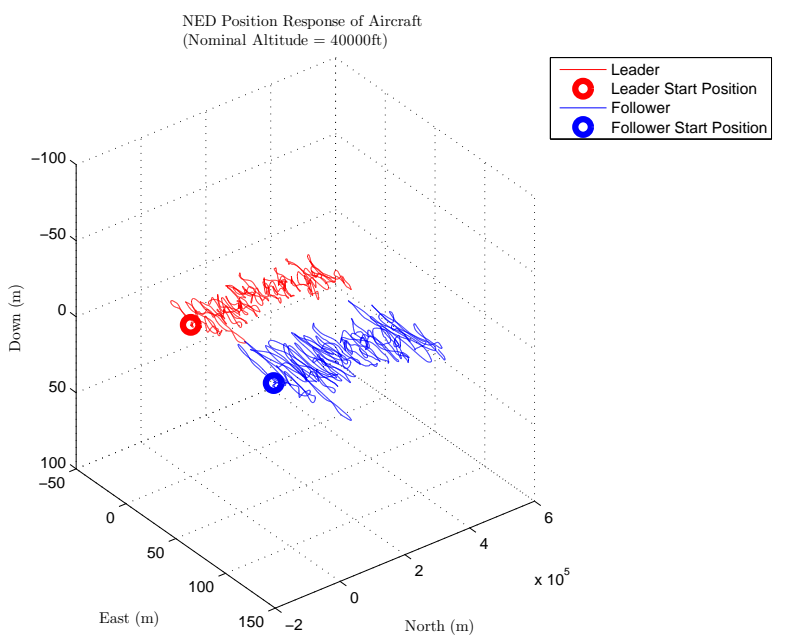

(c) NED in moderate turbulence

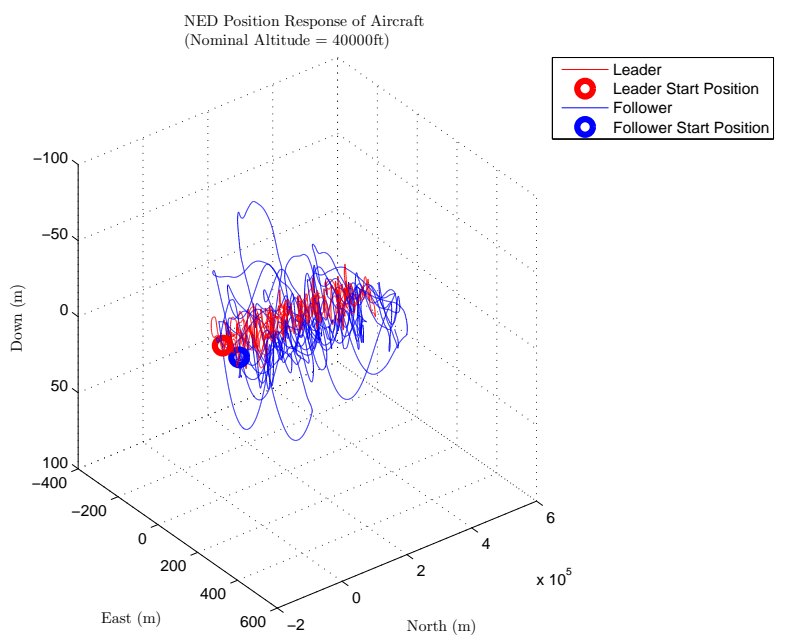

(e) NED in severe turbulence

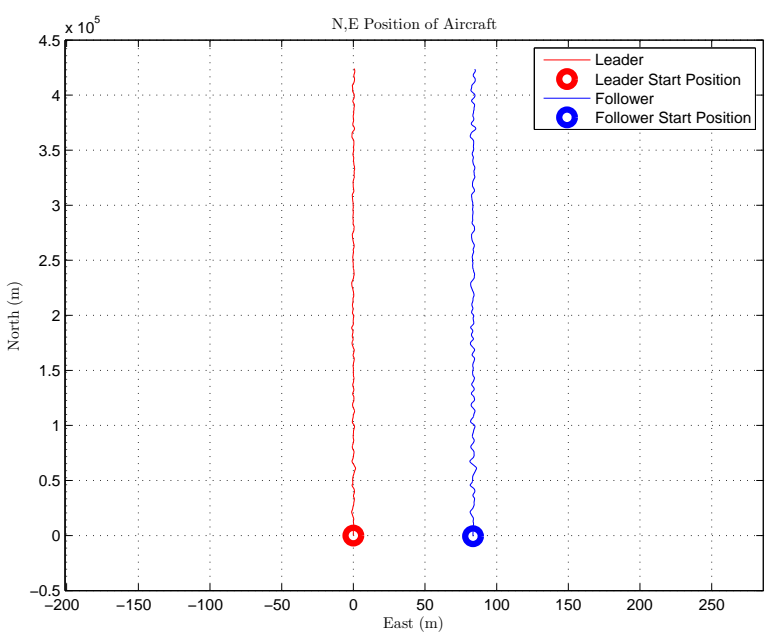

(b) NE in light turbulence

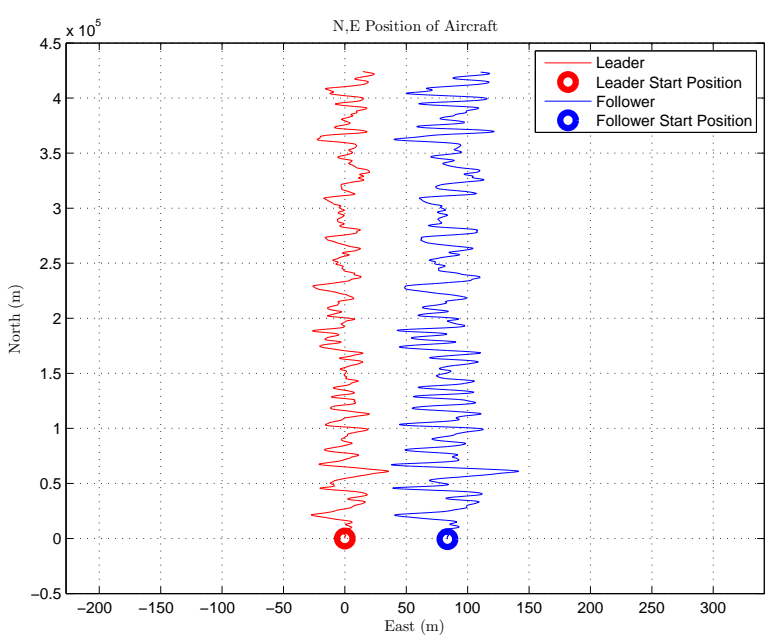

(d) NE in moderate turbulence

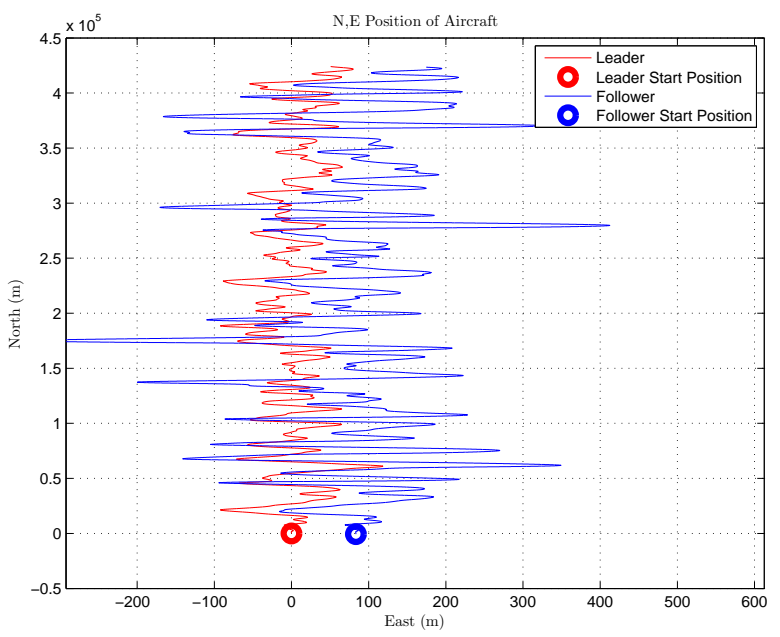

(f) NE in severe turbulence

Figure G.7: Position response of aircraft during formation flight at $\eta=1.4, \zeta=0$ and $\xi=-10$ in varying levels of turbulence intensity. 


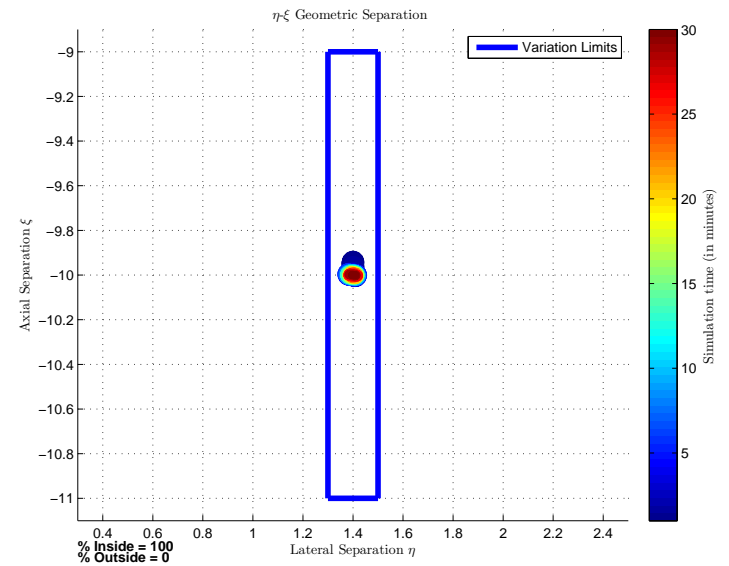

(a) Lateral vs axial separation (Light turbulence)

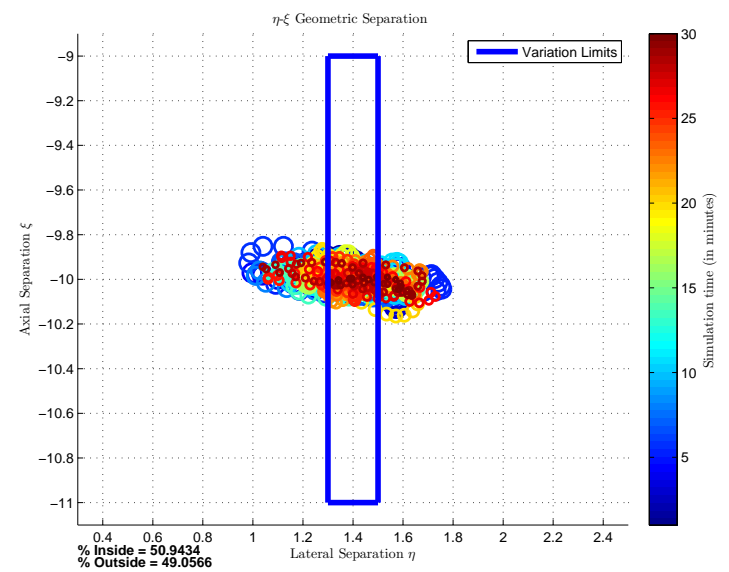

(c) Lateral vs axial separation (Moderate turbulence)

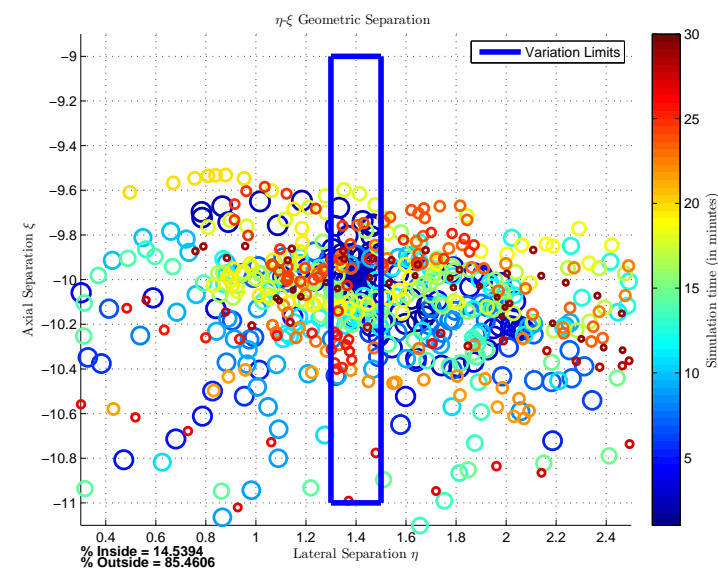

(e) Lateral vs axial separation (Severe turbulence)

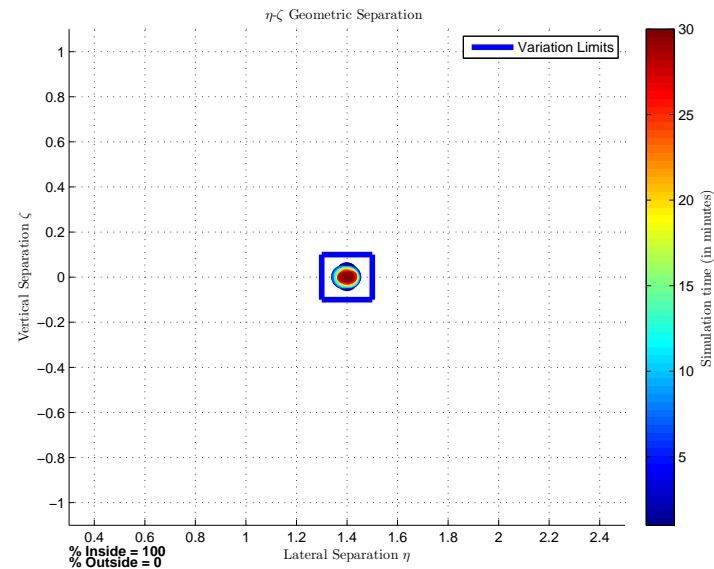

(b) Lateral vs vertical separation (Light turbulence)

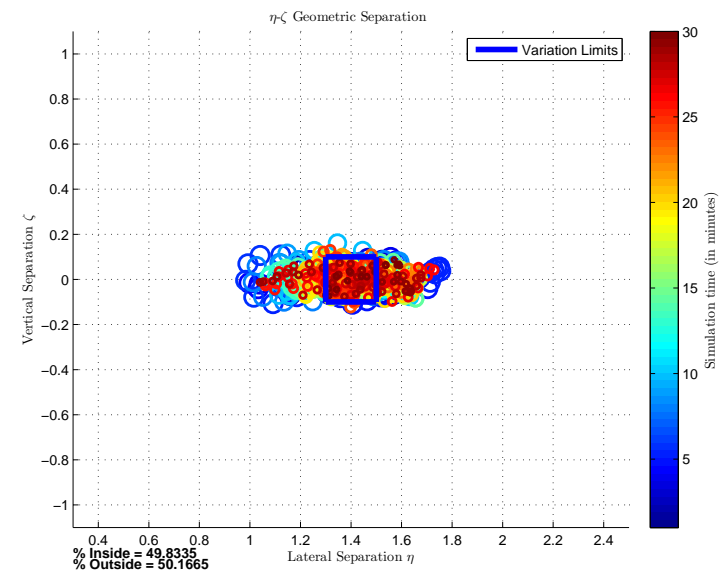

(d) Lateral vs vertical separation (Moderate turbulence)

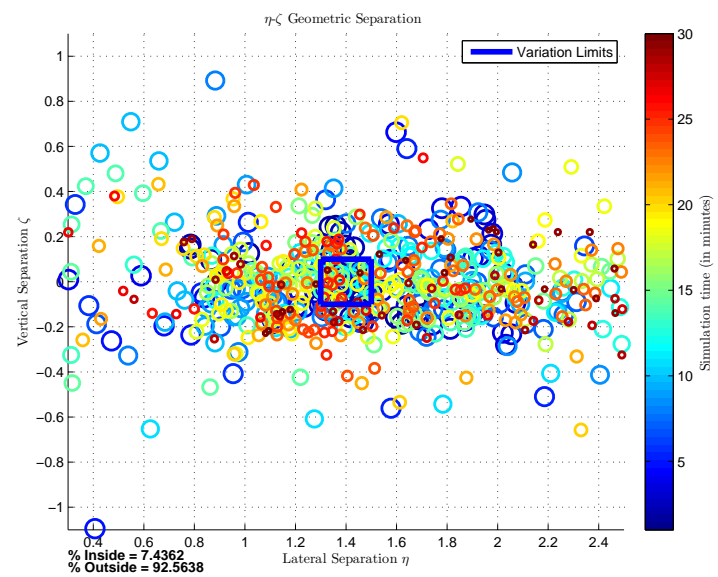

(f) Lateral vs vertical separation (Severe turbulence)

Figure G.8: Formation-hold performance at desired geometric separations $\eta=1.4, \zeta=0$ and $\xi=-10$ wingspans during formation flight in varying levels of turbulence 


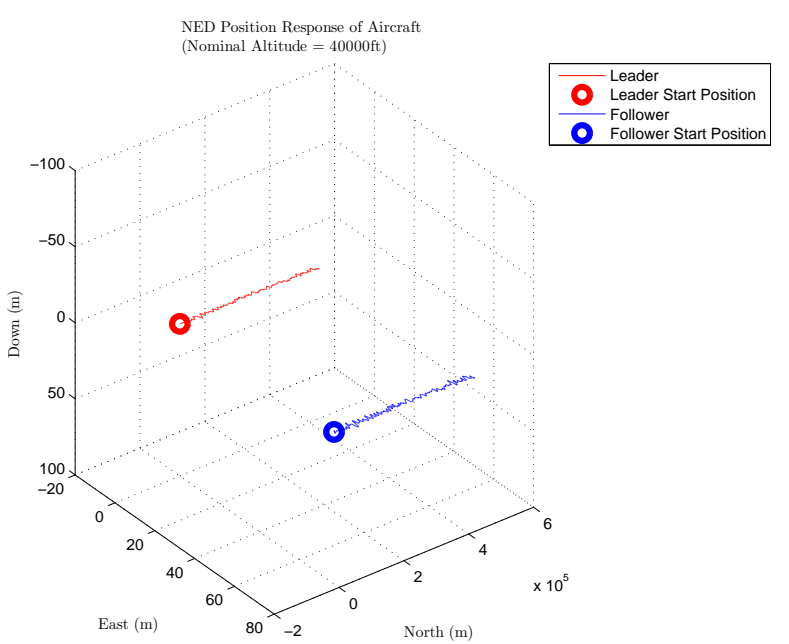

(a) NED in light turbulence

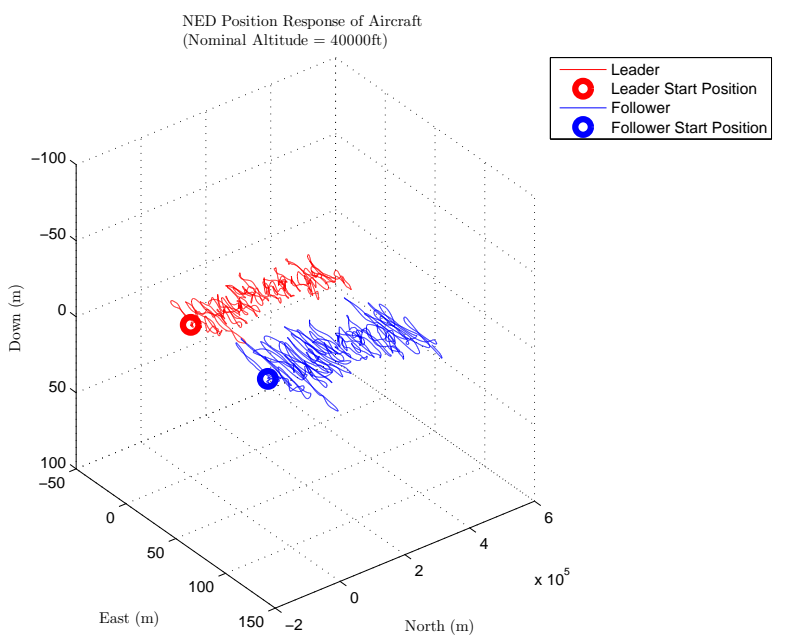

(c) NED in moderate turbulence

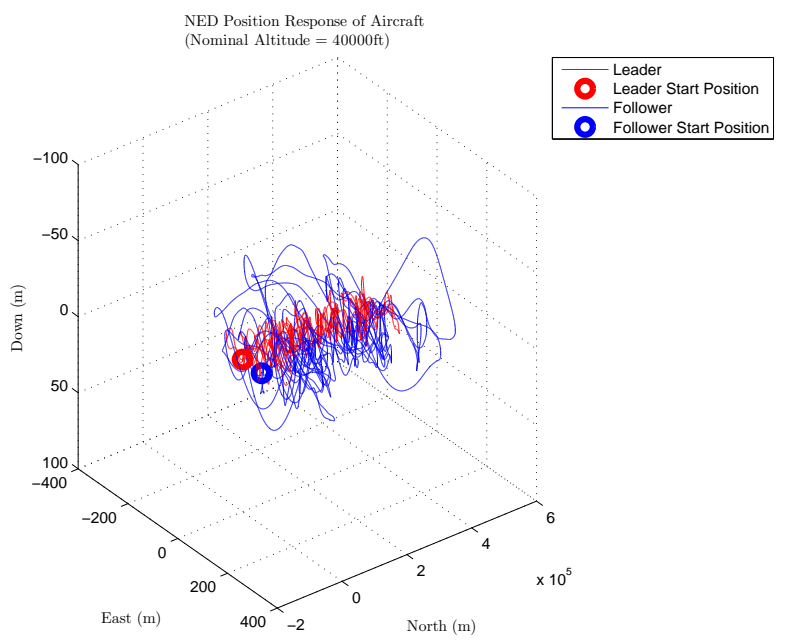

(e) NED in severe turbulence

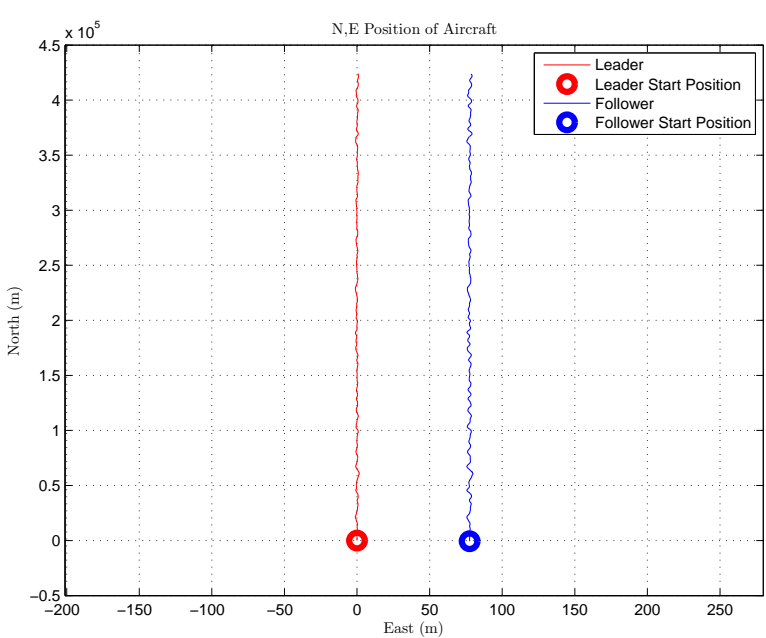

(b) NE in light turbulence

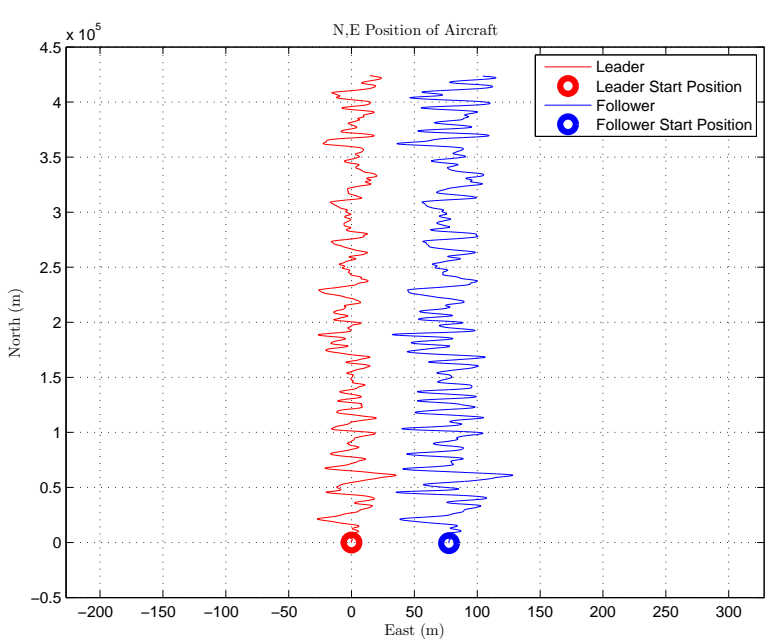

(d) NE in moderate turbulence

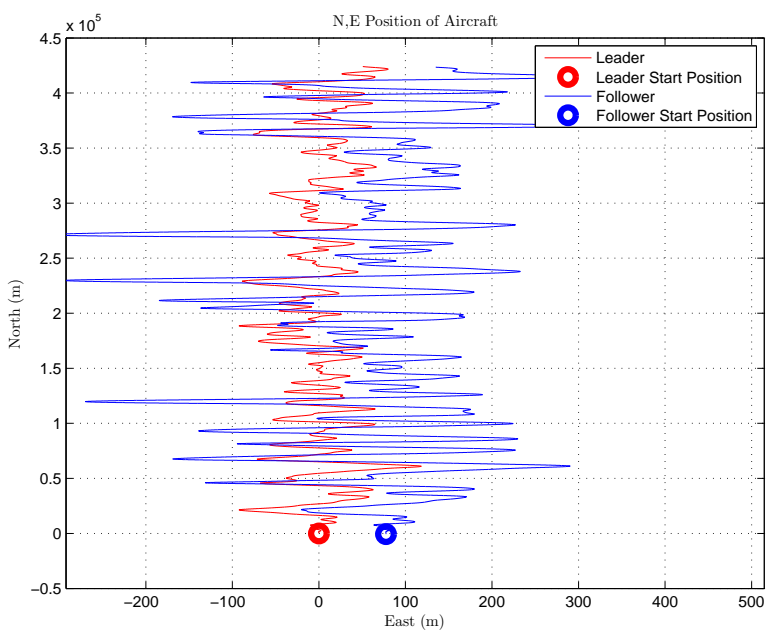

(f) NE in severe turbulence

Figure G.9: Position response of aircraft during formation flight at $\eta=1.3, \zeta=0$ and $\xi=-10$ in varying levels of turbulence intensity. 


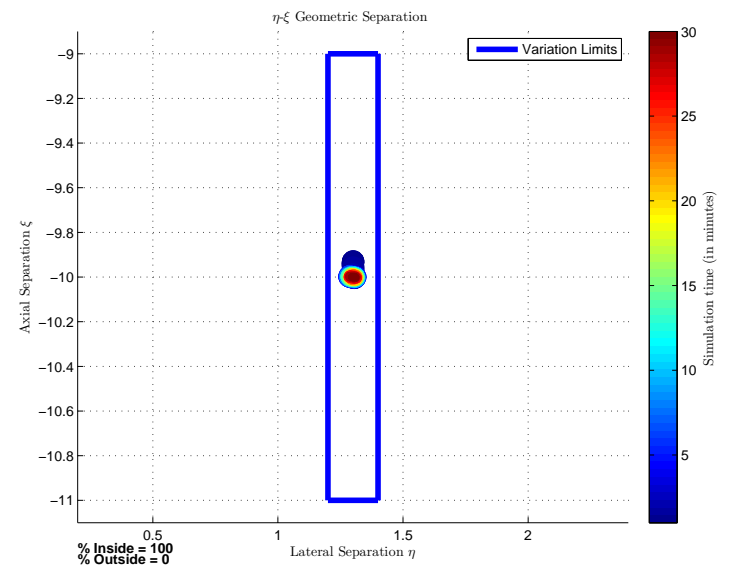

(a) Lateral vs axial separation (Light turbulence)

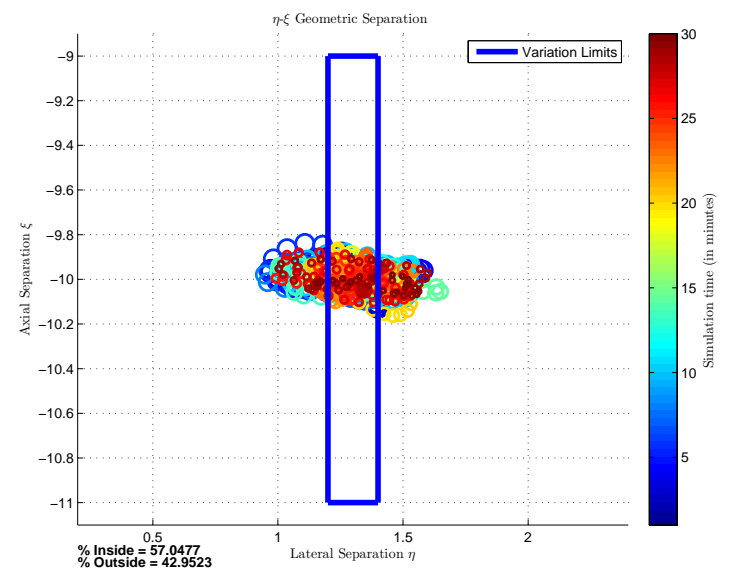

(c) Lateral vs axial separation (Moderate turbulence)

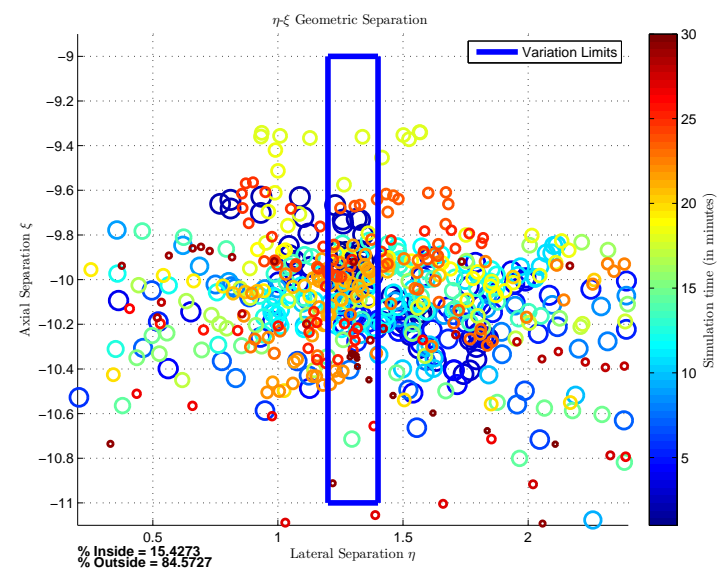

(e) Lateral vs axial separation (Severe turbulence)

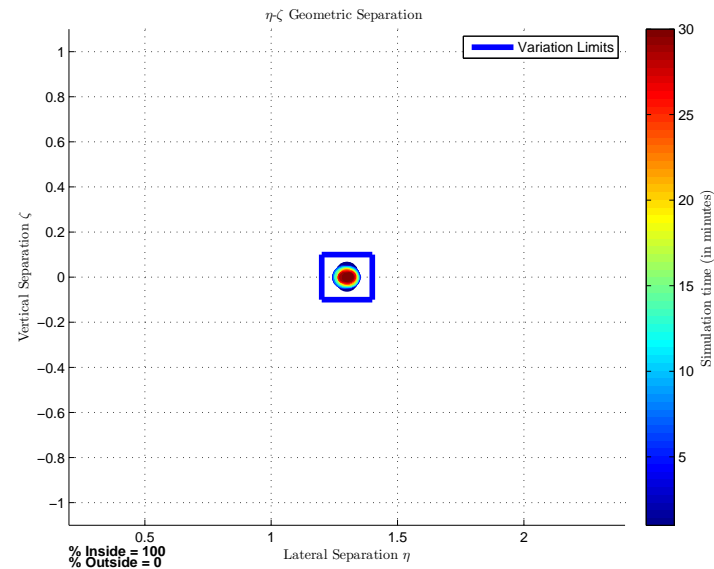

(b) Lateral vs vertical separation (Light turbulence)

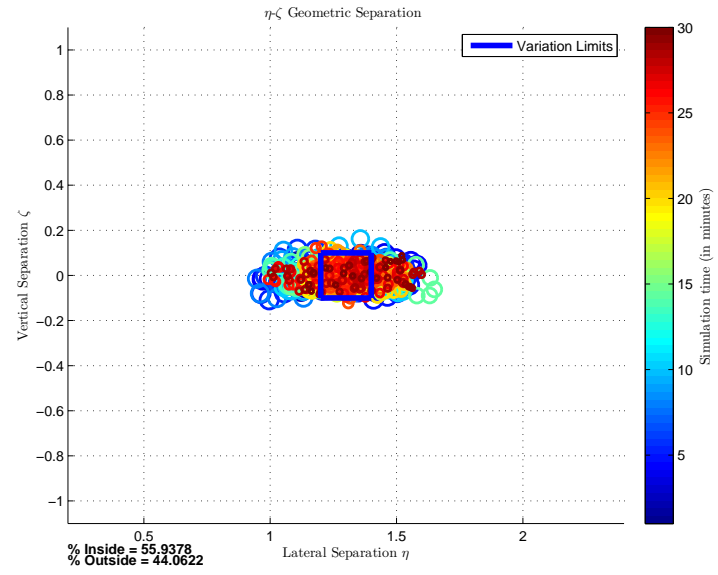

(d) Lateral vs vertical separation (Moderate turbulence)

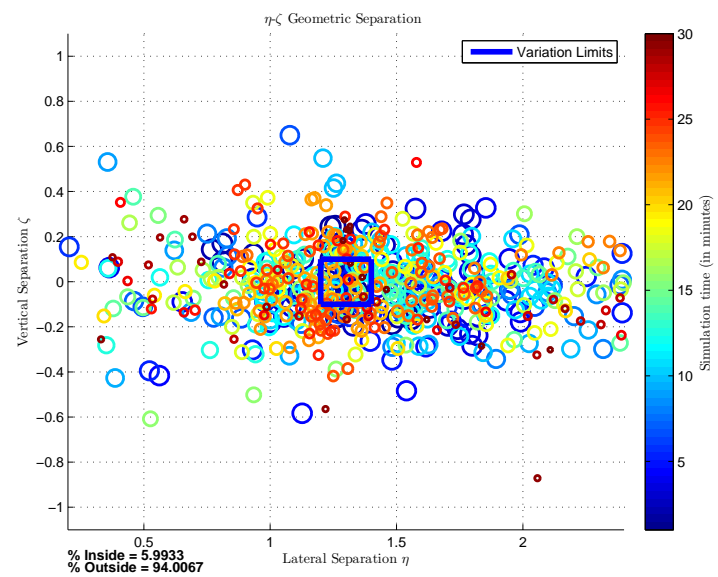

(f) Lateral vs vertical separation (Severe turbulence)

Figure G.10: Formation-hold performance at desired geometric separations $\eta=1.3, \zeta=0$ and $\xi=-10$ wingspans during formation flight in varying levels of turbulence 


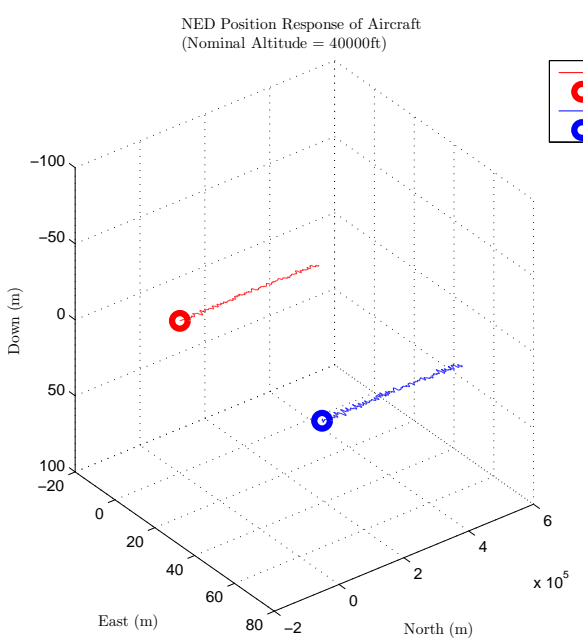

(a) NED in light turbulence

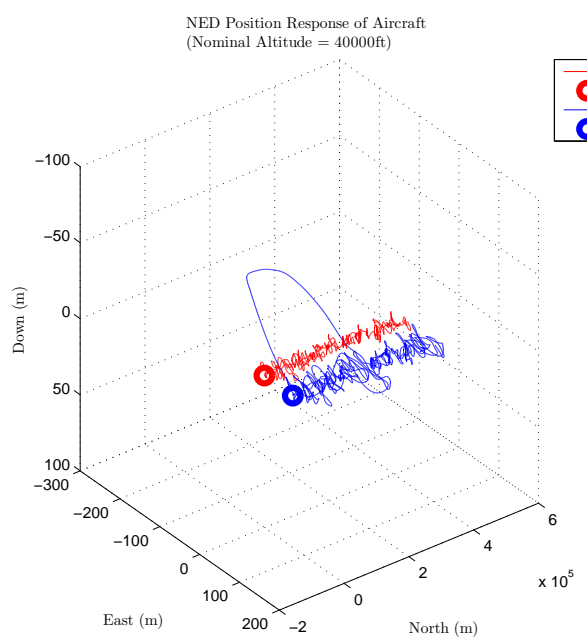

(c) NED in moderate turbulence

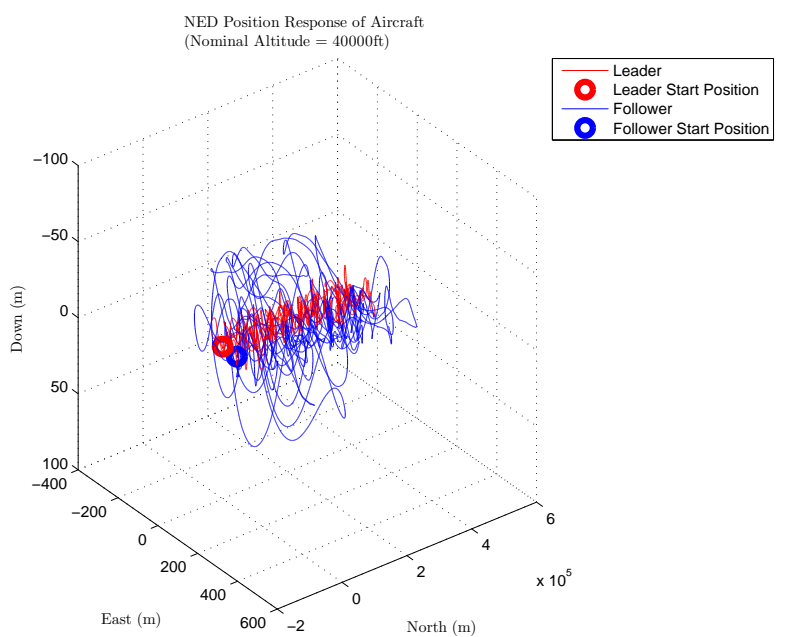

(e) NED in severe turbulence

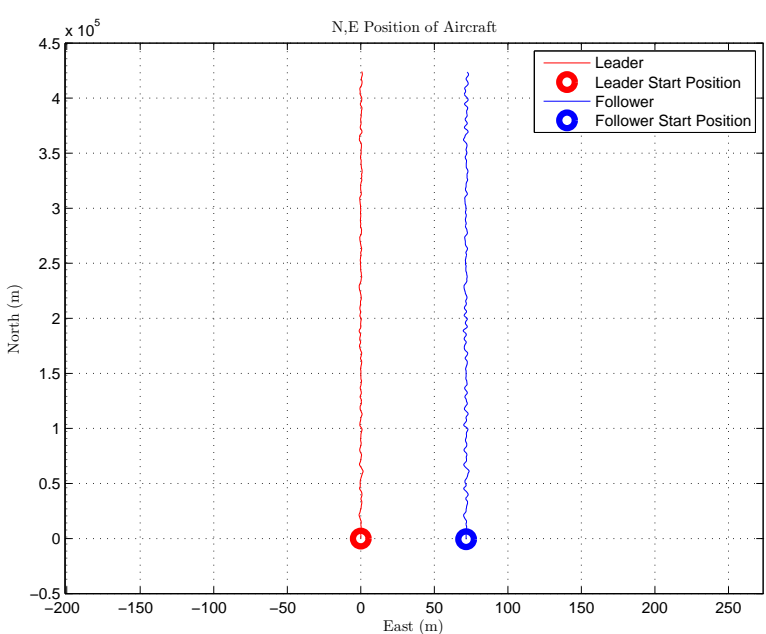

(b) NE in light turbulence

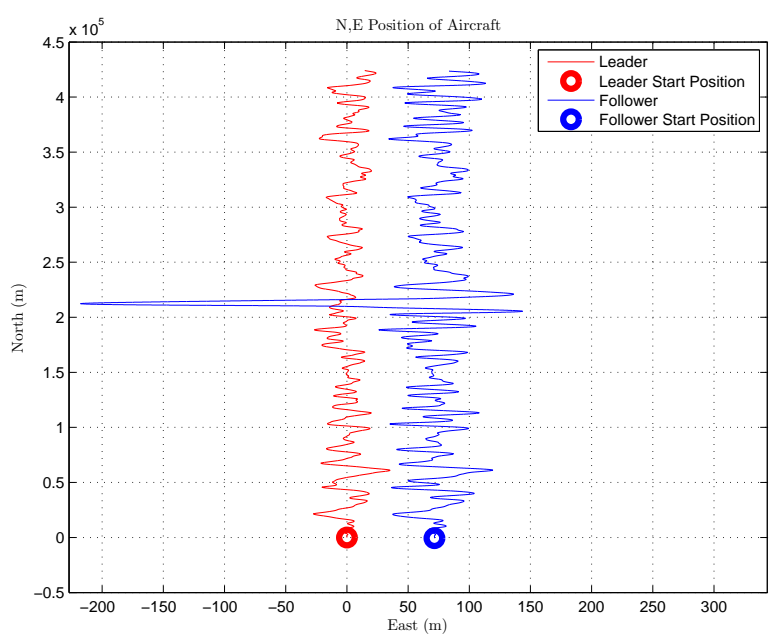

(d) NE in moderate turbulence

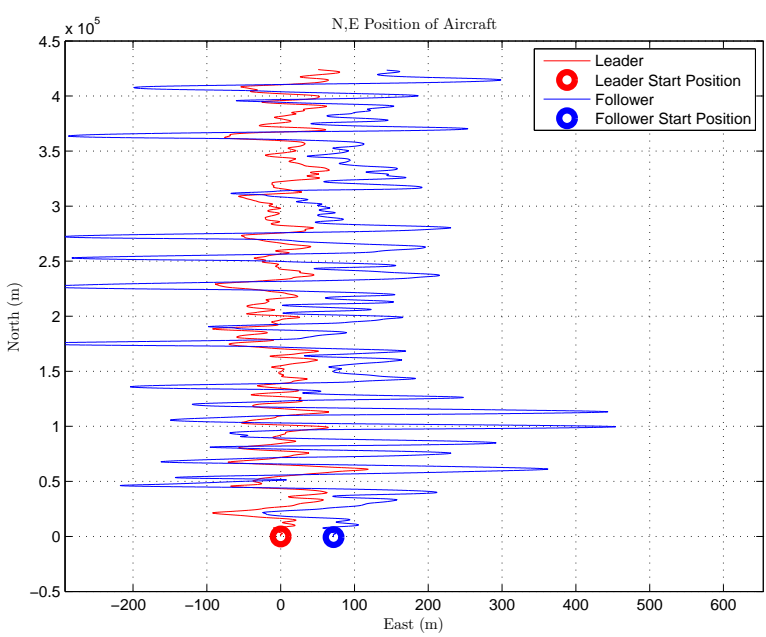

(f) NE in severe turbulence

Figure G.11: Position response of aircraft during formation flight at $\eta=1.2, \zeta=0$ and $\xi=-10$ in varying levels of turbulence intensity. 


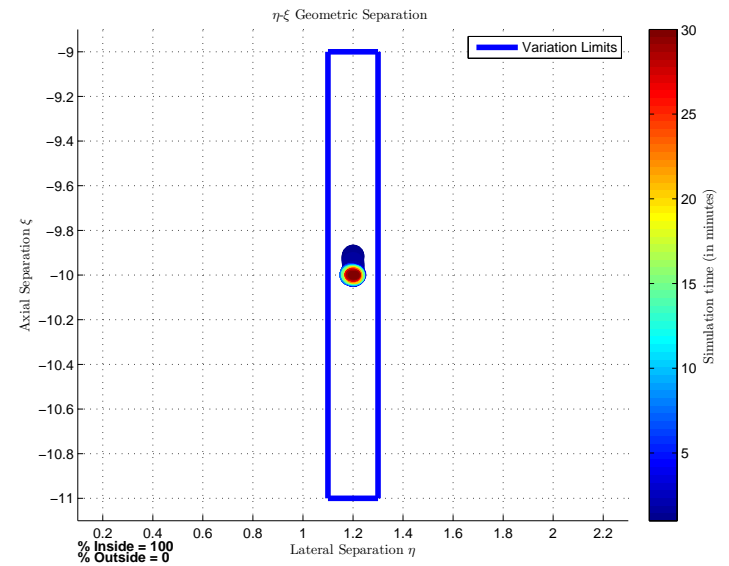

(a) Lateral vs axial separation (Light turbulence)

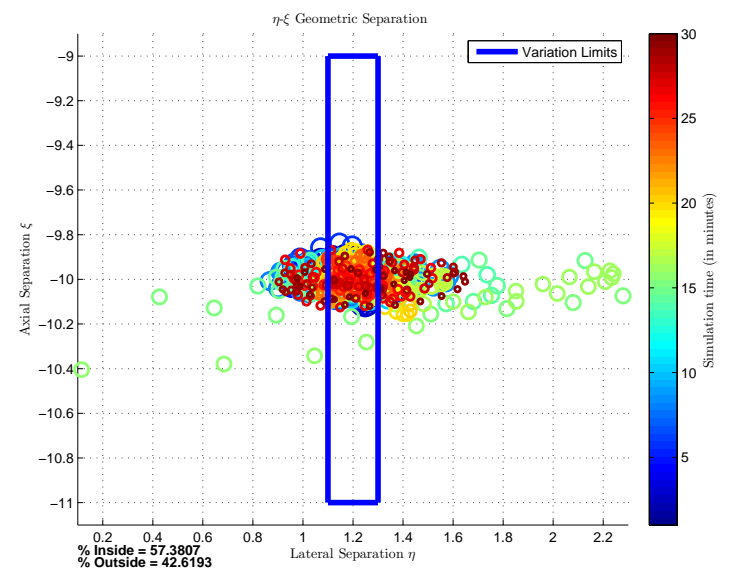

(c) Lateral vs axial separation (Moderate turbulence)

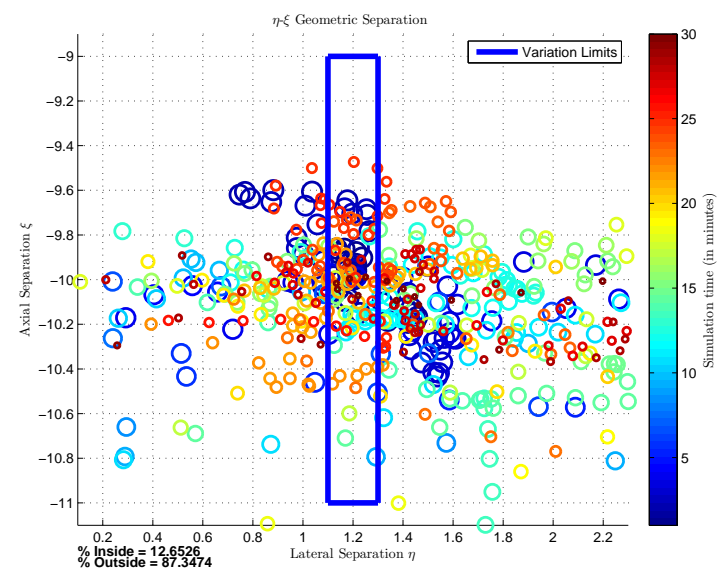

(e) Lateral vs axial separation (Severe turbulence)

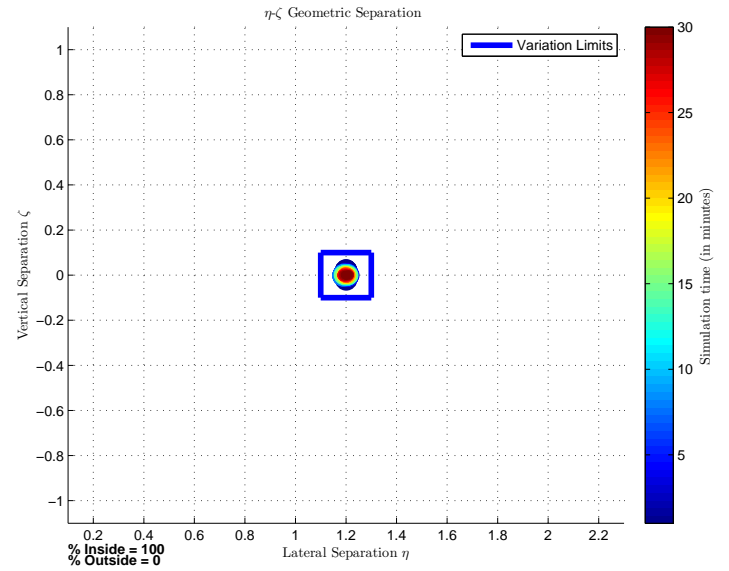

(b) Lateral vs vertical separation (Light turbulence)

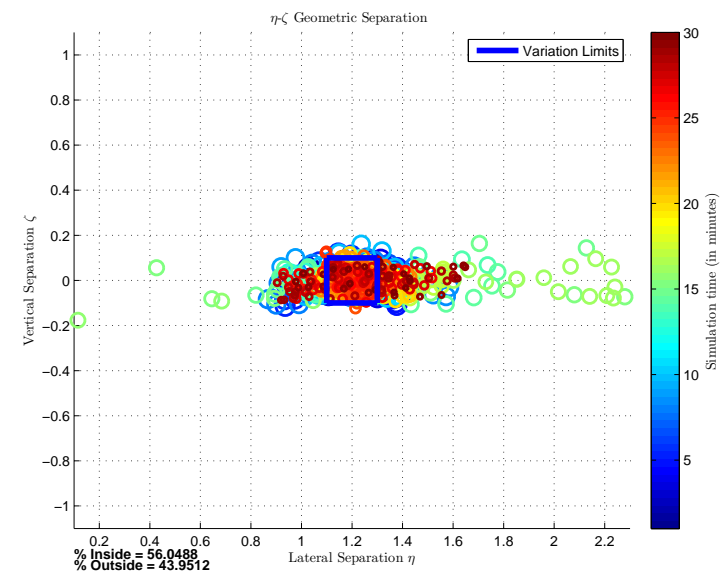

(d) Lateral vs vertical separation (Moderate turbulence)

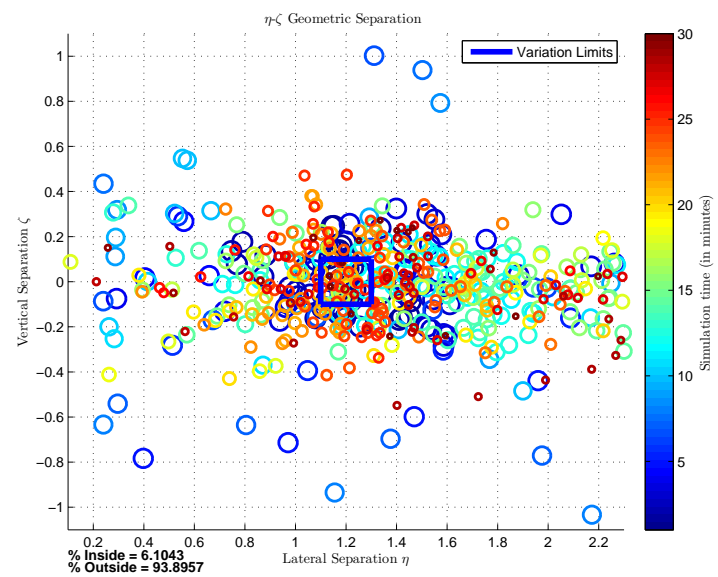

(f) Lateral vs vertical separation (Severe turbulence)

Figure G.12: Formation-hold performance at desired geometric separations $\eta=1.2, \zeta=0$ and $\xi=-10$ wingspans during formation flight in varying levels of turbulence 


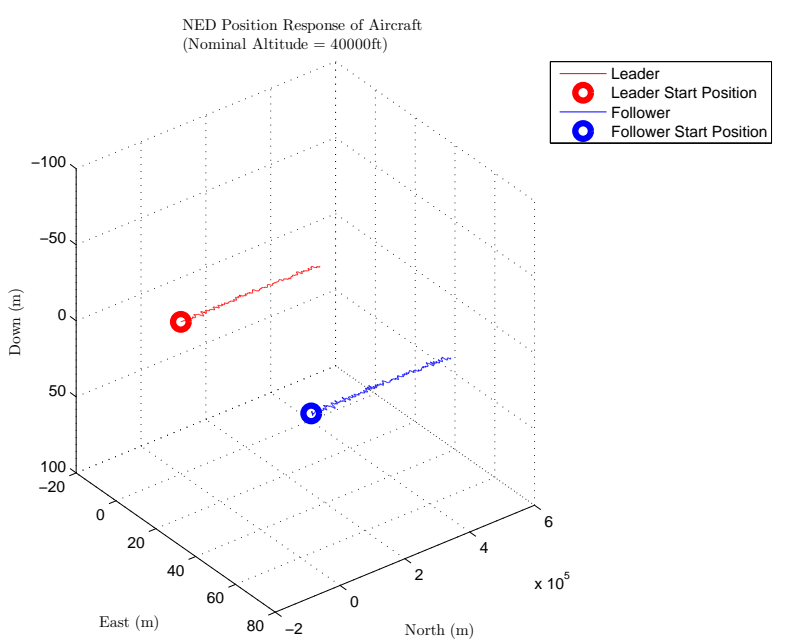

(a) NED in light turbulence

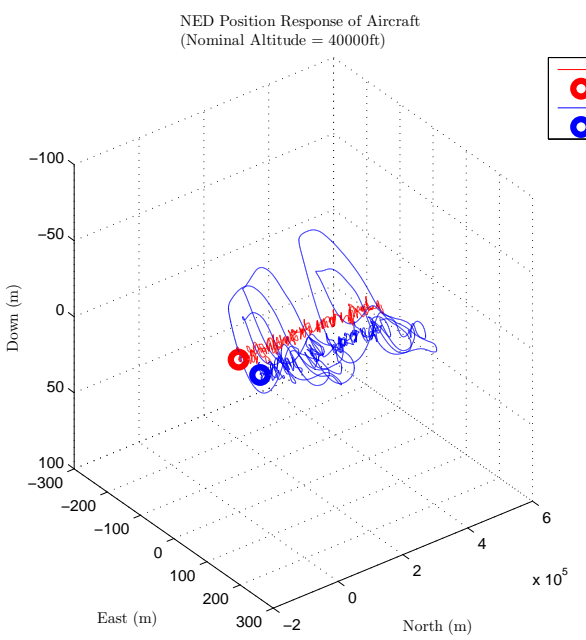

(c) NED in moderate turbulence

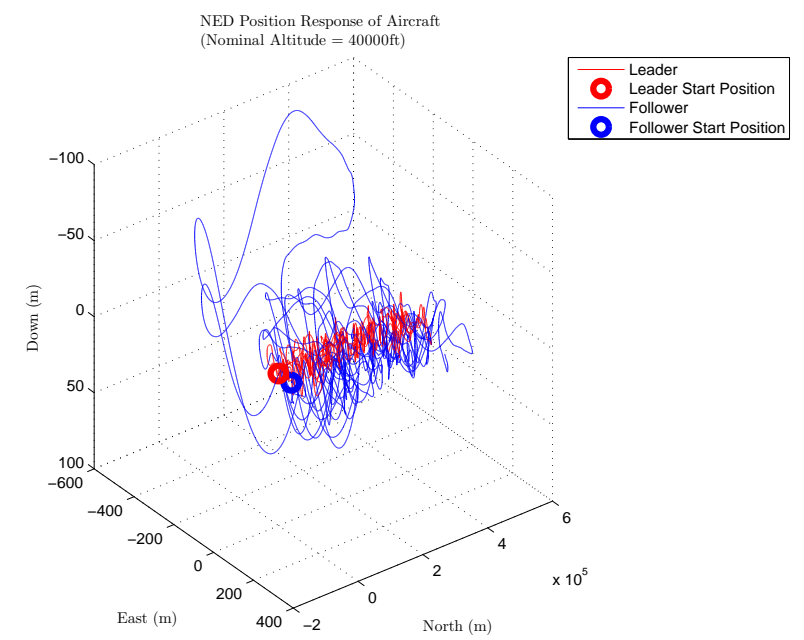

(e) NED in severe turbulence

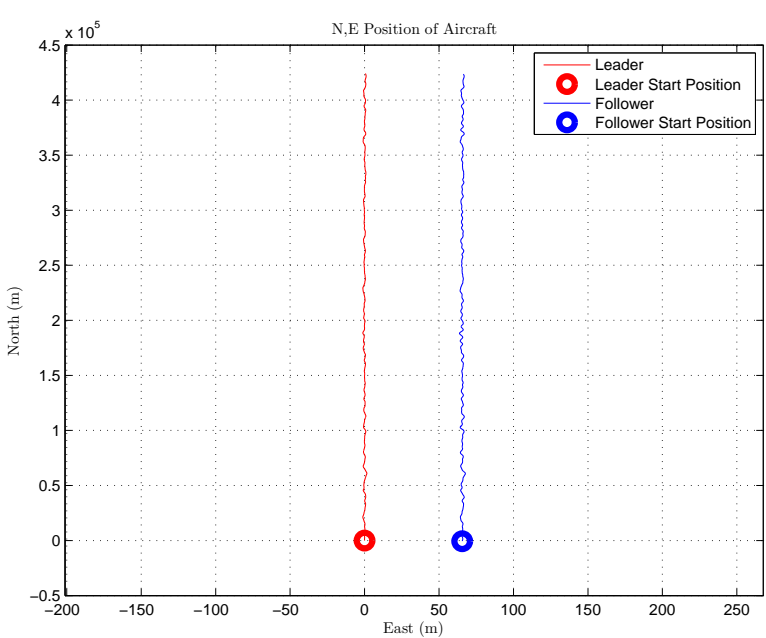

(b) NE in light turbulence

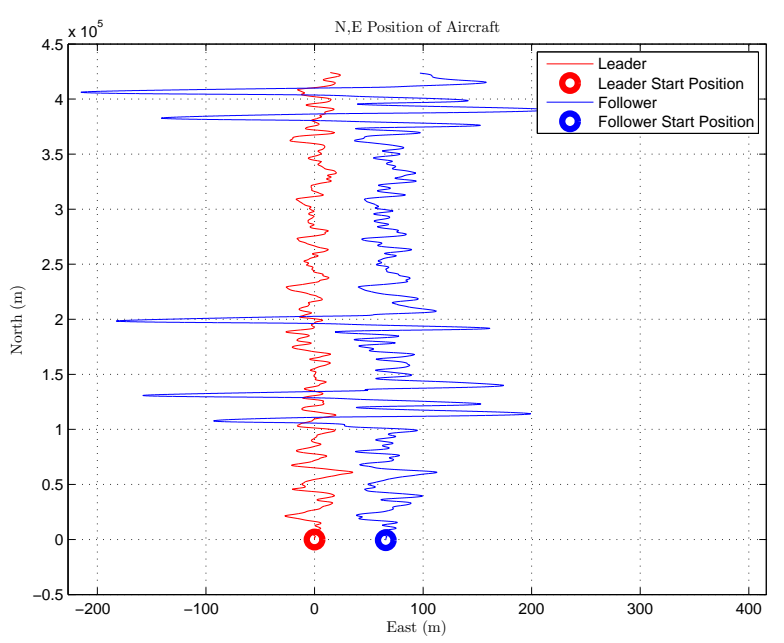

(d) NE in moderate turbulence

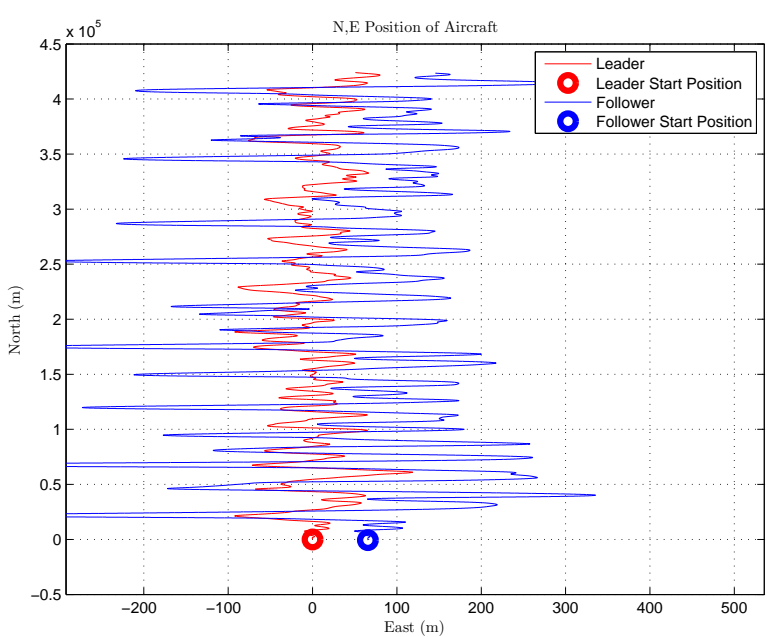

(f) NE in severe turbulence

Figure G.13: Position response of aircraft during formation flight at $\eta=1.1, \zeta=0$ and $\xi=-10$ in varying levels of turbulence intensity. 


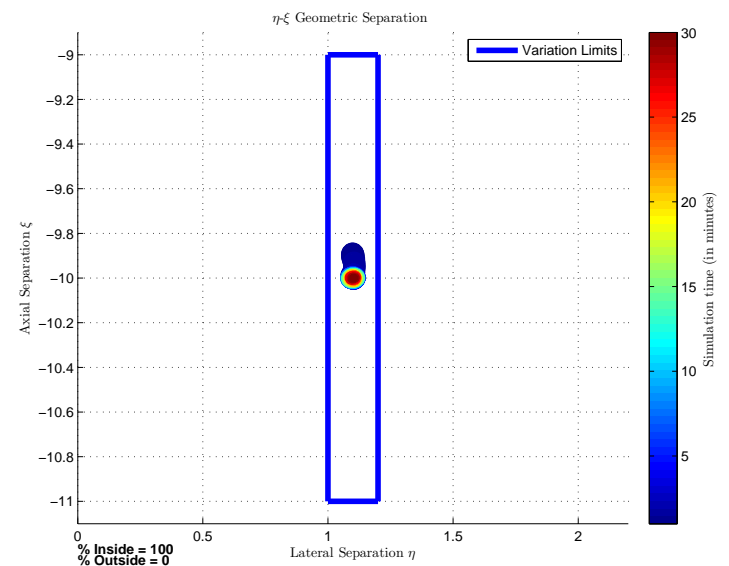

(a) Lateral vs axial separation (Light turbulence)

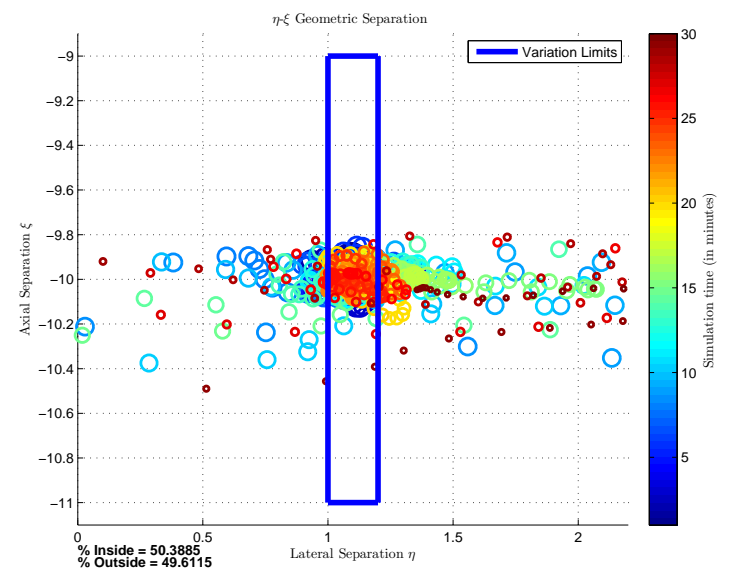

(c) Lateral vs axial separation (Moderate turbulence)

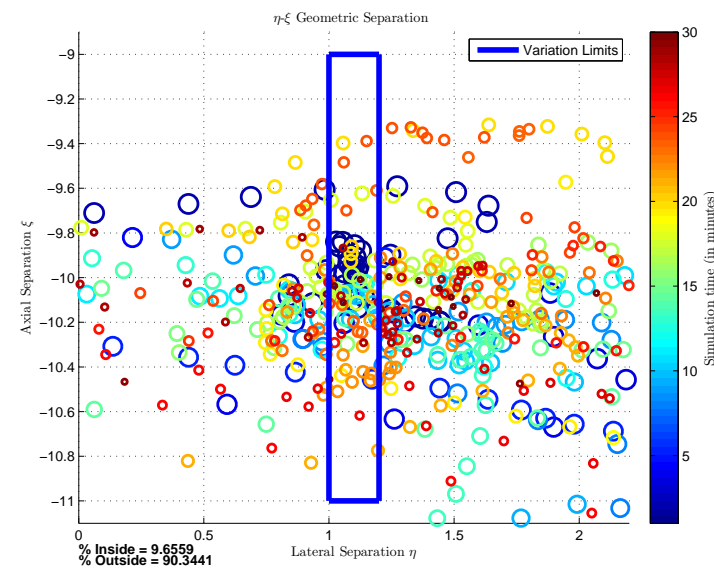

(e) Lateral vs axial separation (Severe turbulence)

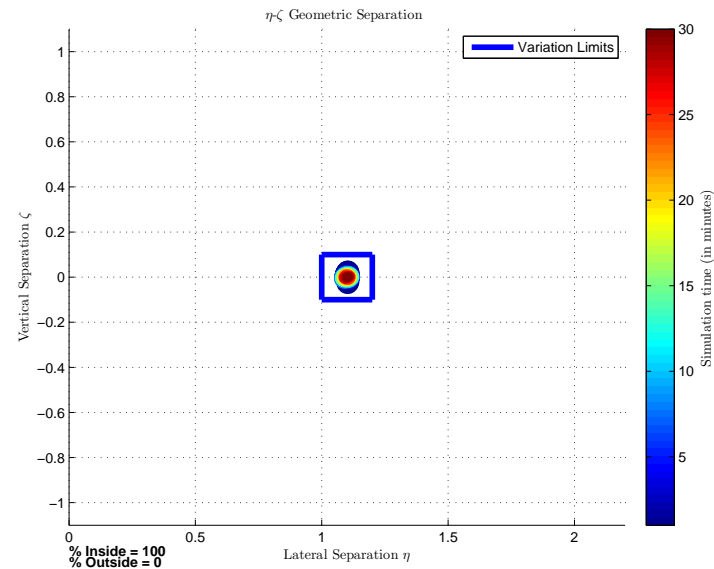

(b) Lateral vs vertical separation (Light turbulence)

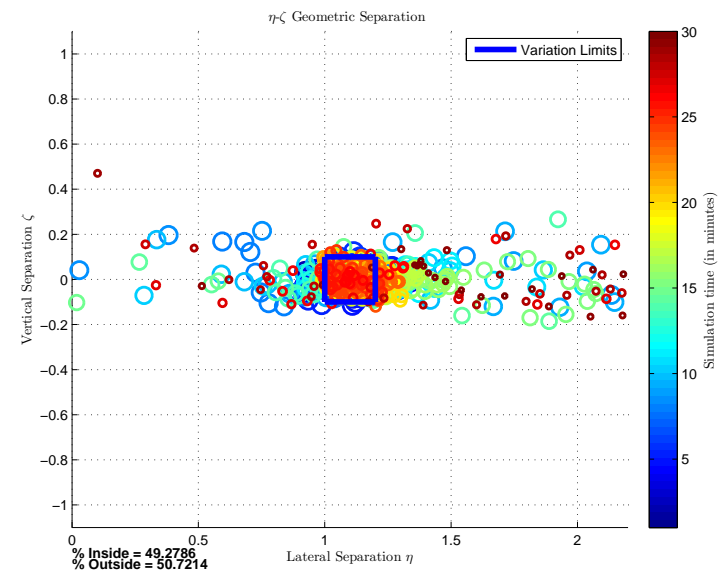

(d) Lateral vs vertical separation (Moderate turbulence)

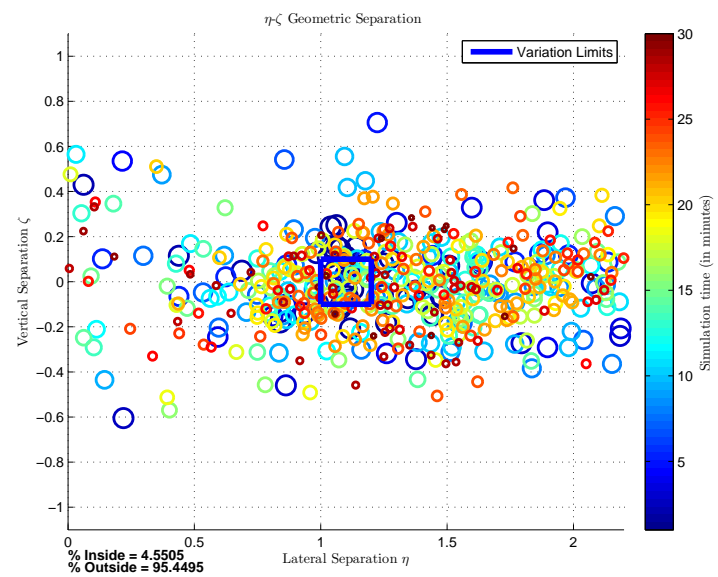

(f) Lateral vs vertical separation (Severe turbulence)

Figure G.14: Formation-hold performance at desired geometric separations $\eta=1.1, \zeta=0$ and $\xi=-10$ wingspans during formation flight 


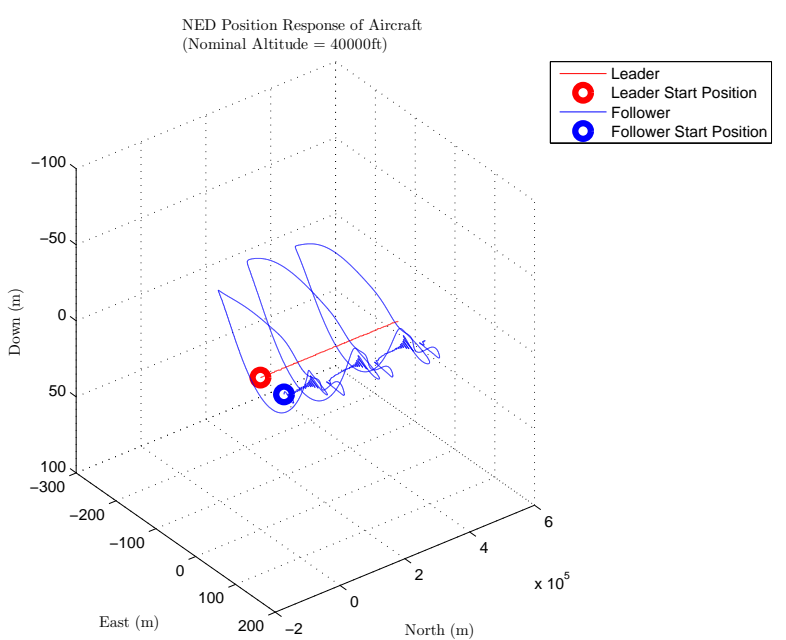

(a) NED in light turbulence

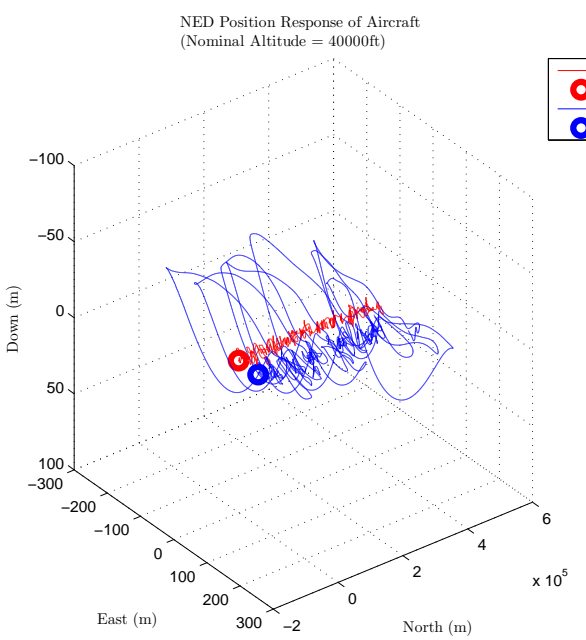

(c) NED in moderate turbulence

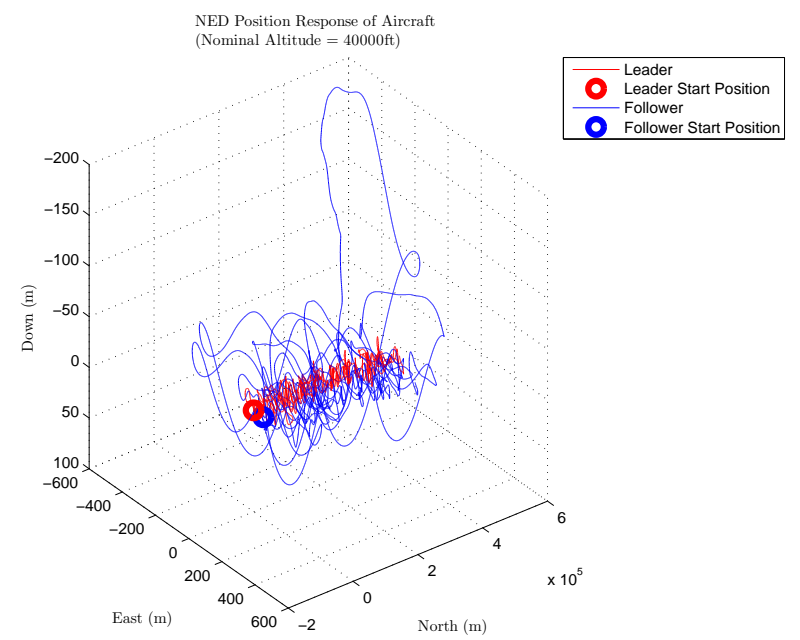

(e) NED in severe turbulence

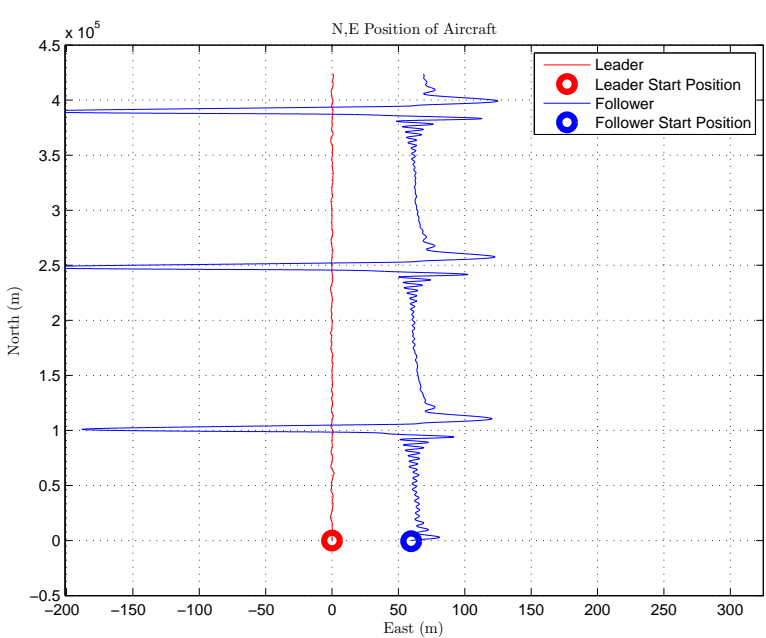

(b) NE in light turbulence

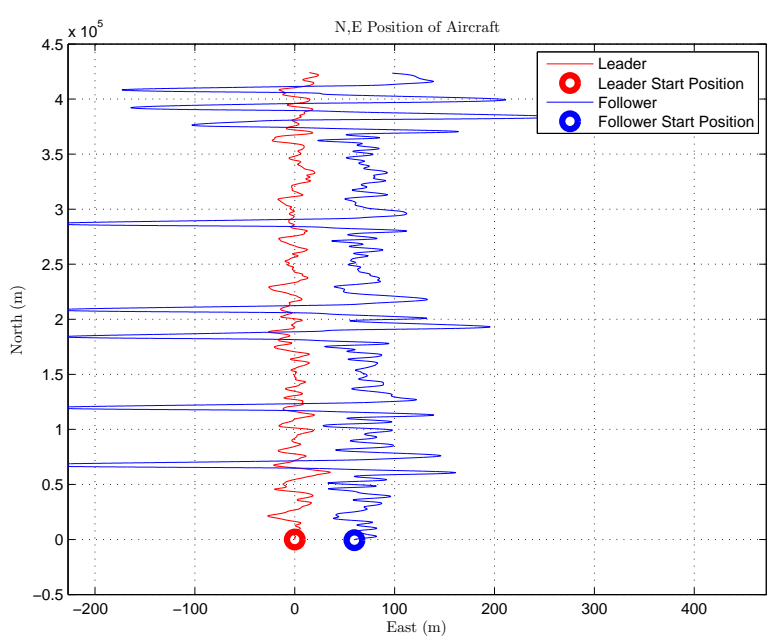

(d) NE in moderate turbulence

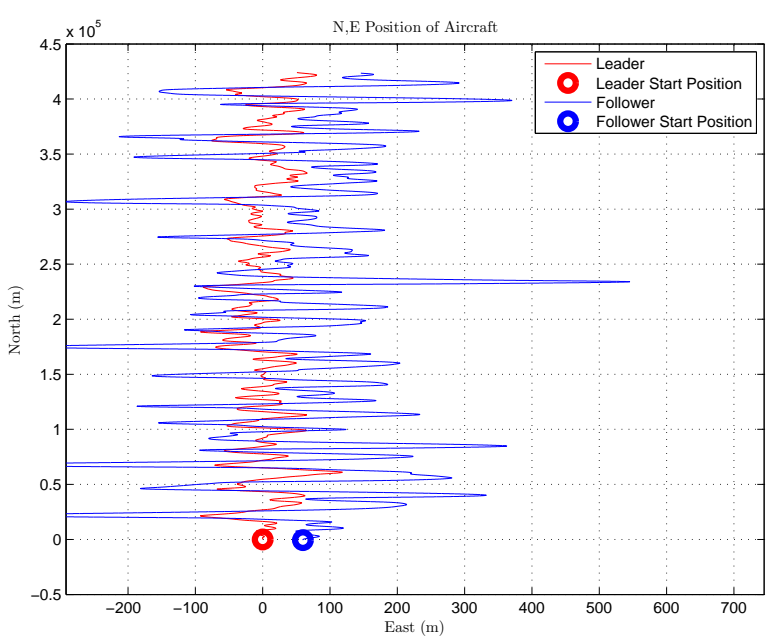

(f) NE in severe turbulence

Figure G.15: Position response of aircraft during formation flight at $\eta=1.0, \zeta=0$ and $\xi=-10$ in varying levels of turbulence intensity. 


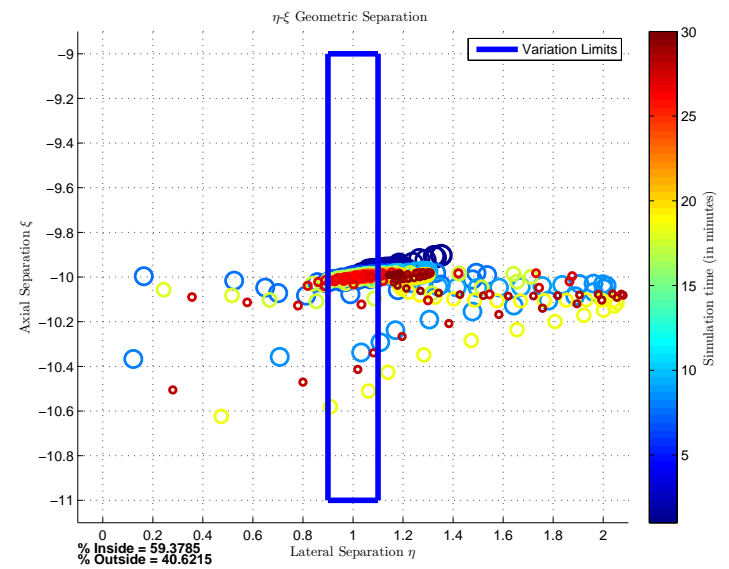

(a) Lateral vs axial separation (Light turbulence)

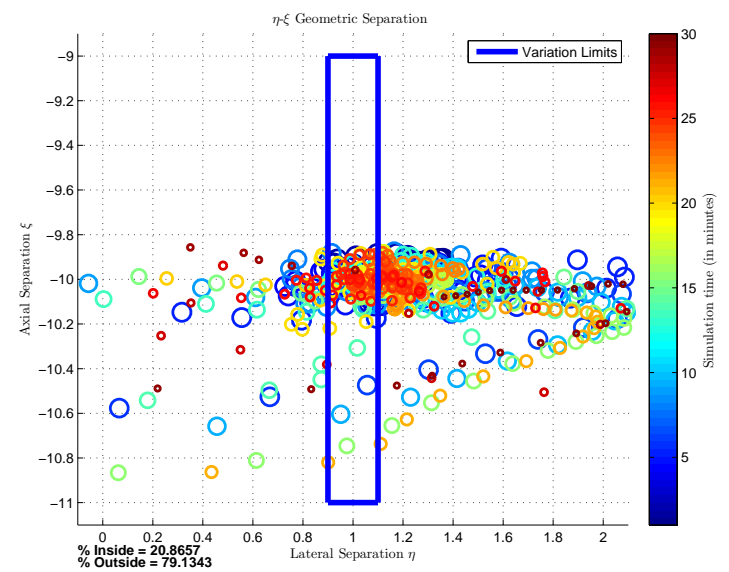

(c) Lateral vs axial separation (Moderate turbulence)

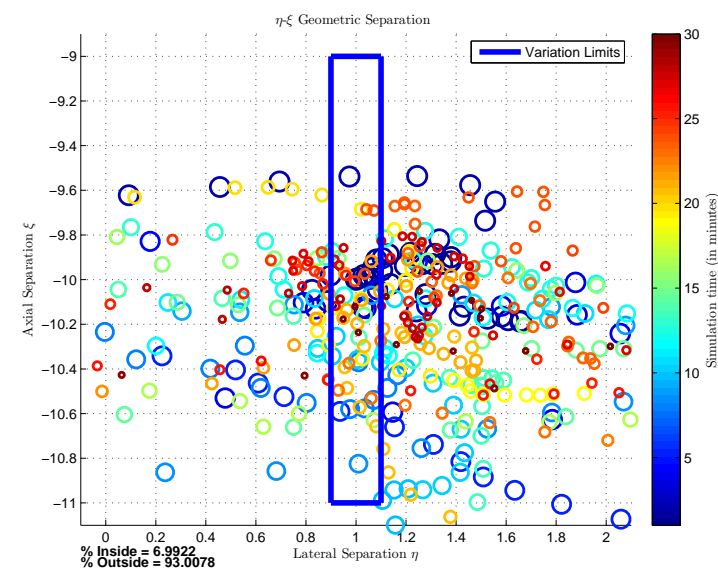

(e) Lateral vs axial separation (Severe turbulence)

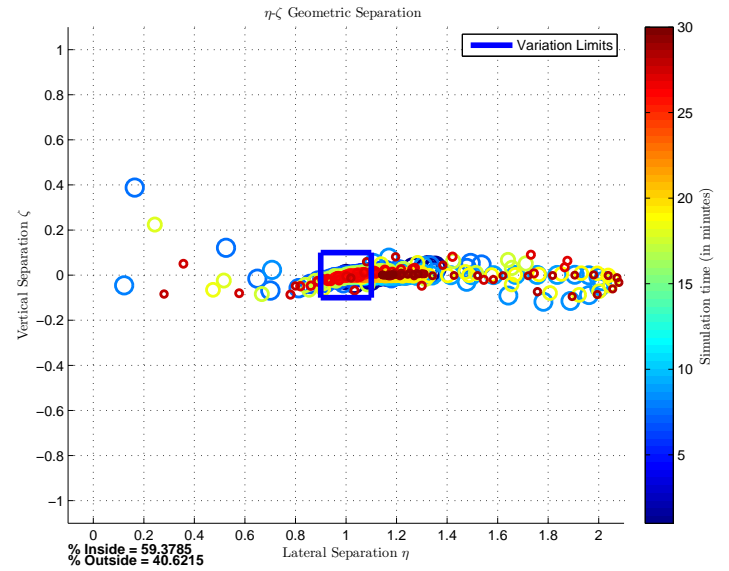

(b) Lateral vs vertical separation (Light turbulence)

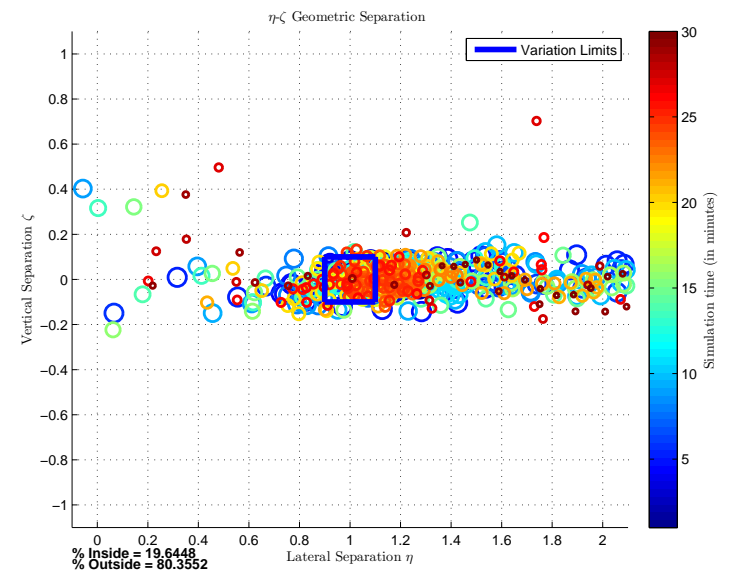

(d) Lateral vs vertical separation (Moderate turbulence)

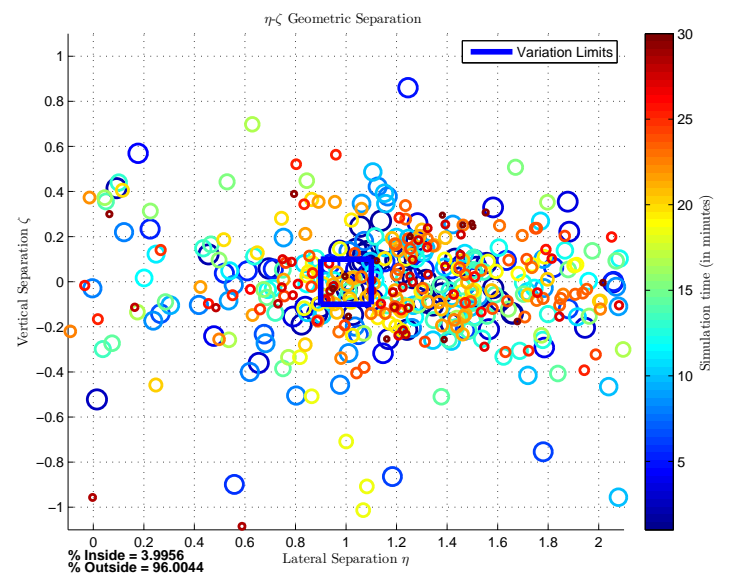

(f) Lateral vs vertical separation (Severe turbulence)

Figure G.16: Formation-hold performance at desired geometric separations $\eta=1.0, \zeta=0$ and $\xi=-10$ wingspans during formation flight 


\section{Appendix $\mathbf{H}$}

\section{Von Kármán Atmospheric Turbulence}

The wind model in Figure 3.6 adds atmospheric turbulence to the extended formation flight simulations, generating the wind as stationary, homogeneous, isotropic, Gaussian turbulence. This disturbance is realised by passing band-limited white noise through forming filters to achieve either the Dryden or von Kármán spectral forms. The US Department of Defense military handbook MIL-STD-1797A $\rrbracket^{1}$ recommends the use of a von Kármán form to generate continuous gusts and is preferred over the Dryden turbulence model. The Aerospace Blockset in MATLAB includes a continuous von Kármán wind turbulence model [89]. Under the research collaboration agreement between Stellenbosch University and the University of Cape Town, the wind model in this Blockset was provided by UCT and used to generate the turbulence for the extended formation flight simulations.

The military references describe turbulence as a stochastic process defined by velocity spectra. An aircraft flying at an airspeed $V$ through a frozen turbulence field with a spatial frequency of $\Omega$ radians per meter will experience a circular frequency of $\omega=V \times \Omega$. Table H.1 displays the spectra forms used for implementation. In this table, $b$ is the aircraft's wingspan, $\sigma$ are the turbulence intensities and $L$ are the turbulence scale lengths. Finally, to generate a signal with the correct characteristics, a unit variance band-limited white noise signal is passed through forming filters. The filter forms are derived as approximations from the von Kármán velocity spectra. The implemented filter forms are shown in Table H.1

The turbulence scale lengths and intensities found in the velocity spectra forming filter transfer functions in Table H.1 are strong functions of altitude. Three distinct regions are defined and used to specify these variables:

\section{Low altitudes (Less than 1000 feet)}

\footnotetext{
${ }^{1}$ MIL-STD-1797A has been redesignated as a handbook, and is to be used for guidance purposes only. This document is no longer to be cited as a requirement.
} 
2. High altitudes (Larger than 2000 feet)

3. Altitudes between low and high altitudes (1000 feet $<$ Altitude $<2000$ feet)

In the current study, a nominal flight altitude of 40000 feet is selected and thus the second region, namely high altitudes, will be used to specify the turbulence scale lengths and intensities. For high altitudes above 2000 feet, the scale lengths are defined as

$$
L_{u}=2 L_{v}=2 L_{w}=2500 \mathrm{ft}
$$

and the turbulence intensities are obtained from a lookup table, shown in Figure H.1 and summarised in Table H.2.

Table H.1: The component spectra functions according to the military handbook MIL-STD1797A and used to describe the velocity spectra. Also shown are the forming filter transfer functions, approximations of the von Kármán velocity spectra.

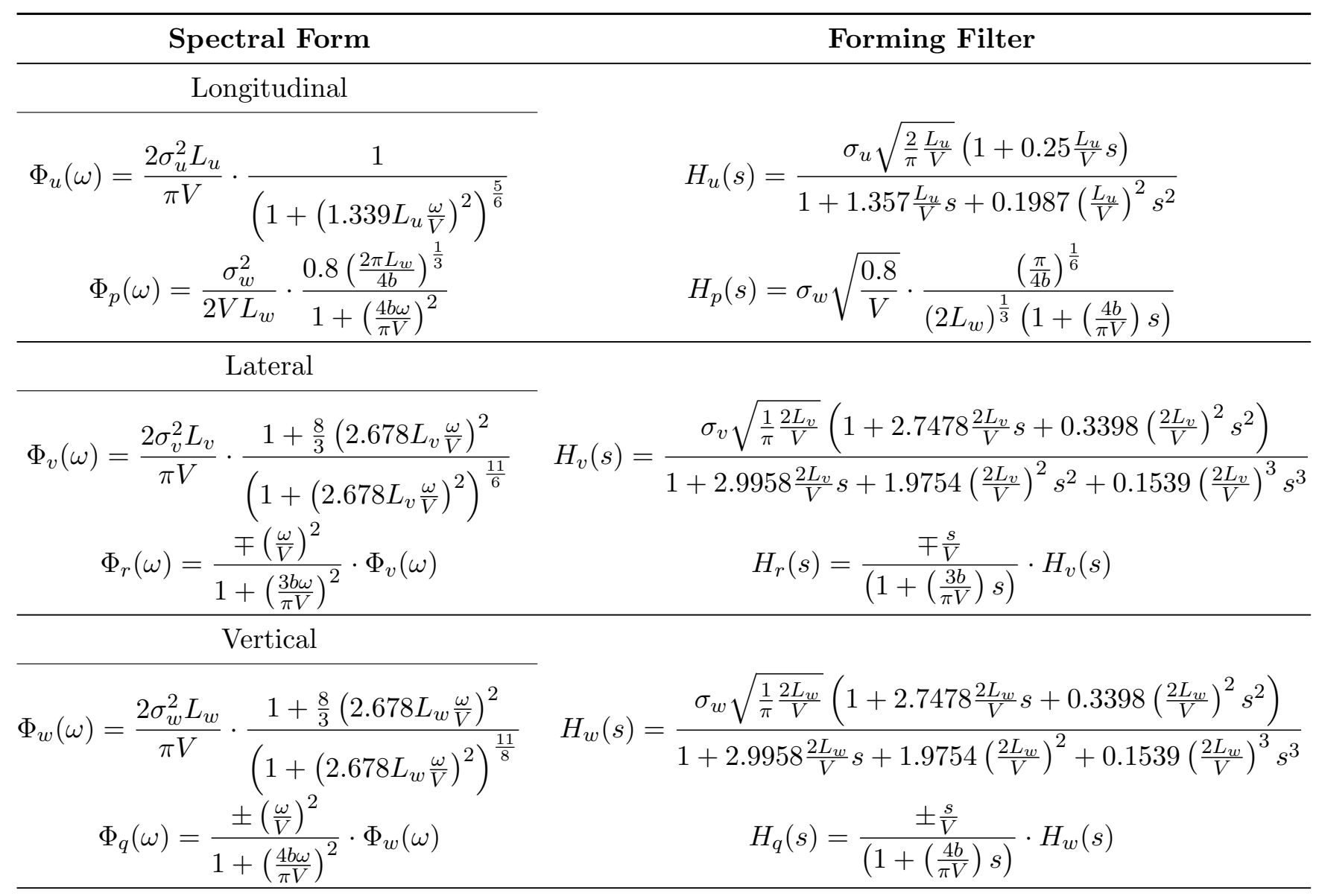


Table H.2: Turbulence severities and corresponding contour

\begin{tabular}{cc}
\hline Turbulence Severity & Contour \\
\hline \multirow{2}{*}{ Light } & $2 \times 10^{-1}$ \\
& $10^{-1}$ \\
& $10^{-2}$ \\
\hline \multirow{2}{*}{ Moderate } & $10^{-3}$ \\
& $10^{-4}$ \\
\hline \multirow{2}{*}{ Severe } & $10^{-5}$ \\
& $10^{-6}$ \\
\hline
\end{tabular}

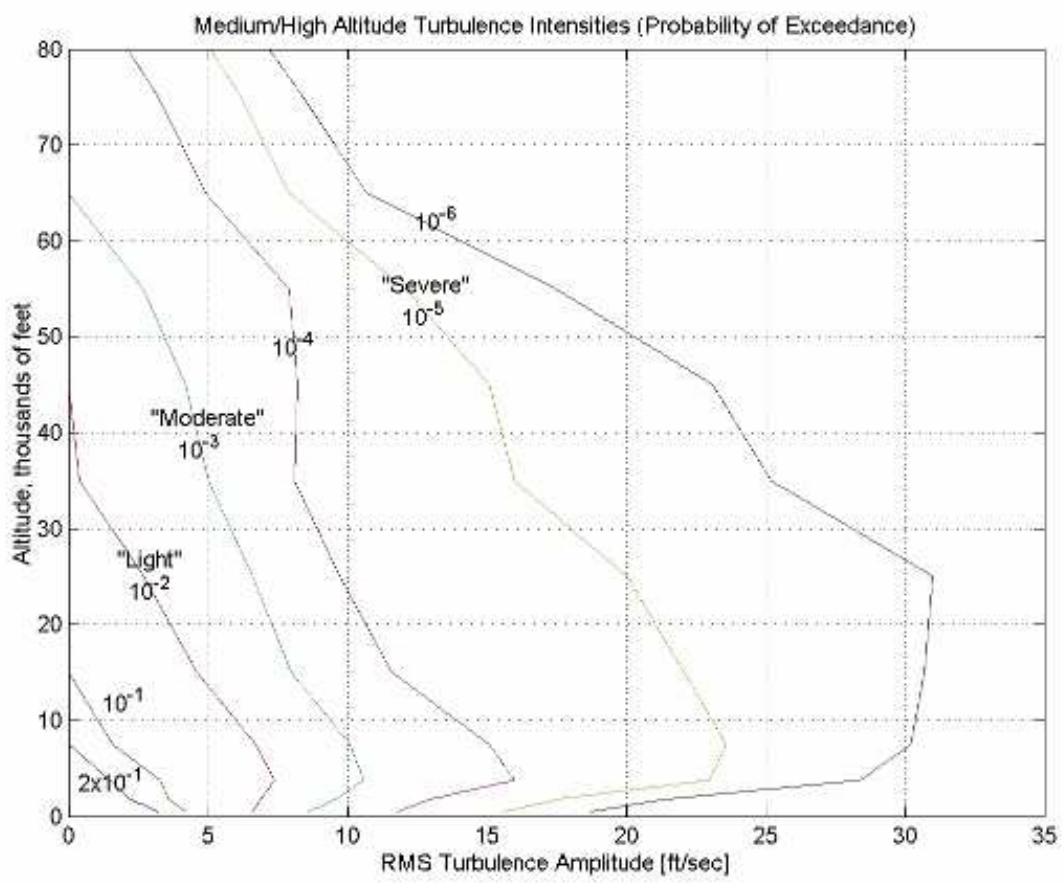

Figure H.1: Lookup table used for turbulence intensities [89] 


\section{Appendix I}

\section{Spectral Density Estimation}

In physics, a signal is usually a wave, such as an electromagnetic wave, a random vibration, or an acoustic wave. The signal wave experienced by a passenger on board a transport aircraft is considered a random vibration, and the power spectral density (PSD) can be used to describe the signal power distribution over a range of frequencies. Mathematically, it describes how the variance of the data is distributed over the frequency domain. In this appendix, the Welch method, a well-known method used to estimate the spectral density function of input data, is discussed.

\section{I.1 The Welch Method}

The Welch method, named after P.D. Welch [78, is a well-known approach to spectral density approximation. The power of a signal is estimated at different frequencies by finding the average spectral density of overlapping segments of window-modified periodograms 79. The process, which follows from available literature studies produced by Heinzel et al. [79] and Schmid [90], is also used by Bizinos in a study of passenger comfort during formation flight within atmospheric turbulence [3].

With the Welch method, a time series of random vibration data is divided into overlapping segments and a window function is applied to the segments. The Discrete Fourier Transform (DFT) is applied to each window-modified segment, and the resulting outputs are scaled and averaged to represent the normalised power spectral density of the entire signal.

\section{I.1.1 FFT and Windowing}

The DFT takes a vector of $N$ real or complex numbers $x[i]$ and transforms it into a vector of $N$ complex numbers $Y[i](i=0,1, \ldots, N-1)$. Many common definitions for the implementation of a DFT can be used, but the most practical implementation in software consists of using the Fast Fourier Transform (FFT) algorithm.

Although the input signal $x[i]$ of the general DFT can contain complex numbers, the input signal is almost always real. The output signal $Y[i]$ then obeys the following relationship, 


$$
Y[N-i]=Y[i]^{*}
$$

where $*$ denotes the complex conjugate. As a consequence, the upper half of the output signal $Y[N / 2], \ldots, Y[N-1]$ is redundant and never computed by a FFT algorithm.

The DFT implicitly assumes periodicity [79] [90] [91, and if the input signal is not as such the resulting output will contain much spectral leakage - i.e. power spread all across the spectrum. The remedy for avoiding spectral leakage as much as possible is by windowing the input signal. Windowing a time series input signal is simply a term used to describe the process whereby the input signal $x[i]$ is multiplied by a window function $w[i]$ before the FFT algorithm is executed.

Although the process of windowing might seem simple, its effects on the resulting interpretation of the transformed signal are manifold. In order to correctly interpret the results from a transformed signal that is multiplied by a windowing function, normalisation or correction factors are required [90]. The first correction factor is defined as,

$$
C G=\frac{1}{N} \sum_{i=0}^{N-1} w[i]
$$

and is termed the coherent gain of the window [90]. The second important correction factor is defined as,

$$
N G=\frac{1}{N} \sum_{i=0}^{N-1} w[i]^{2}
$$

and is termed the noise gain of the window [90]. Table I.1 lists the approximate values of the correction factors for three different window types [90]. These correction factors are only accurate for sufficiently large window lengths. The use of these correction factors is explained in the next section.

Table I.1: Correction factors for three different window types (from Schmid [90])

\begin{tabular}{lll}
\hline Window Function & CG & NG \\
\hline Rectangular & 1.000 & 1.0000 \\
Hanning & 0.500 & 0.3750 \\
Hamming & 0.540 & 0.3974 \\
\hline
\end{tabular}

\section{I.1.2 Normalised Power Spectral Density}

After applying a real-to-complex FFT algorithm to each window-modified segment of the time series acceleration data, a complex vector $Y[i]$ of length $N / 2+1$ is scaled and averaged to obtain a normalised power spectral density. The type of normalisation is dependent on the spectral density is to be interpreted. Since the pwelch.m function, available in the MATLAB Signal Processing Toolbox, is used in this thesis, its type of normalisation is pursued. For details of other normalisation types, consult Schmid [90]. 
The pwelch.m function in MATLAB normalises the resulting power spectral density such that the noise power spectral density can be read directly off the plot. After applying the FFT transform to each window-modified segment of the time series data, a complex vector,

$$
Y[i]=\frac{1}{N} \mathcal{F}\{x[i] w[i]\}
$$

of length $N / 2$ results. The appropriate normalised one-sided power spectral density is then given by,

$$
P_{y y}[i]= \begin{cases}\frac{Y[i] Y[i]]^{*}}{N G f_{R e s}} & i=0 \\ \frac{2 Y[i] Y[i]^{*}}{N G f_{\text {Res }}} & i>0\end{cases}
$$

where

$$
f_{\text {Res }}=f_{s} / N
$$

and is the frequency resolution of each frequency bin. The normalisation just described is not, however, what is desired for this thesis. It is desired to be able to read the RMS value of a signal from the power spectral density of translational or rotational acceleration. Using the derivation and explanation of Schmid [90, the power spectral density in Equation (I.5) is first scaled by the factor,

$$
s_{n}=\frac{N G f_{\text {Res }}}{C G^{2}}
$$

where the coherent and noise gains of the window are defined in Equations (I.2) and (I.3) respectively. The RMS value of the input signal $x[i]$ is then,

$$
x=\sqrt{\sum_{i} P_{y y}[i] s_{n}}
$$

In summary, the Welch method can be considered as a five step process to obtain the estimated power spectral density of a time series:

1. Obtain time series of the data

2. Divide the time series into overlapping segments

3. Apply a window function to the segments

4. Apply the DFT to each window-modified segment by using a FFT algorithm

5. Scale and average the resulting outputs to obtain the normalised power spectral density of the entire signal 


\section{I.2 Implementation of the Welch Method in MATLAB}

Implementation of the Welch method can easily be done in MATLAB with the pwelch.m function, provided by the Signal Processing Toolbox. This function conveniently computes the Power Spectral Density (PSD) estimate, $P x x$, of a discrete-time input signal vector $X$, using Welch's averaged, modified periodogram method. While this function is easy to use, the understanding and choice of its input parameters are essential for a correct interpretation of its results. According to the MATLAB online documentation, consider the function as follows,

$[\operatorname{Pxx}, f]=\operatorname{pwelch}(x$, nwin, noverlap, nfft,fs $)$

where

$\mathrm{x}$ is the input signal vector.

nwin is specified as a vector or an integer. If a vector, this vector describes the window function. If an integer, it describes the length of the default Hamming window.

noverlap is the number of overlapped samples, specified as a positive integer smaller than the length of nwin. If noverlap is unspecified or empty, a value is used to obtain the default $50 \%$ overlap between segments.

$\mathrm{nfft}$ is the length of the DFT, specified as a positive integer.

fs is the sampling frequency.

\section{I.2.1 Frequency Resolution and Length of the DFT}

Assuming the input signal is available, the first consideration to make is the frequency resolution $f_{\text {Res }}$ and length $N$ of the DFT. Heinzel et al. [79] suggest choosing a typical frequency resolution between $f_{s} / 100$ and $f_{s} / 100000$. Since the input signal will be real-valued acceleration data, the FFT algorithm used will not compute the redundant upper half of the output signal. After applying the FFT algorithm to each window-modified segment of the real-valued time series acceleration data $x_{k}$, an output signal $y_{m}$ of length $(N / 2+1)$ is produced. Arbitrarily choosing,

$$
(N / 2+1)=50000
$$

the length of the DFT and the frequency resolution can be defined. The frequency range of the power spectral is computed according to,

$$
f_{m}=m f_{\text {Res }}=\left[\begin{array}{ll}
f_{m_{\text {min }}} & f_{m_{\max }}
\end{array}\right]
$$

where $m=0,1, \ldots, N / 2$ and the minimum and maximum are,

$$
\begin{aligned}
& f_{m_{\text {min }}}=0 \\
& f_{m_{\text {max }}}=\frac{N}{2} f_{\text {Res }}=\frac{f_{s}}{2}
\end{aligned}
$$




\section{I.2.2 Window Function}

After defining the frequency resolution and length of the DFT, an appropriate window function needs to be selected. Heinzel et al. [79] suggest to first determine whether a flat-top window is necessary. Flat-top windows are considered as those functions that are as flat as possible in the frequency domain. These types of windows are essential if the exact amplitude of a sinusoidal component in the input signal is required, such as those required in spectrum analysers [79. In this current study, however, this will not be neccessary, and other windows with smaller bandwidths are deemed acceptable.

Consider Figure I.1, a comparison in the time and frequency domains between the Hanning and Hamming window functions. The Hanning window is a commonly used window function, and a standard used in many commercial spectrum analysers [79. The Hamming window, similar in shape to the Hanning window, is constructed as a weighted sum of a constant term and some cosine terms. With low spectral leakage (but slightly more than the Hanning window), it is the simplest window function used by Bizinos [3], and also the default window used by MATLAB. 79
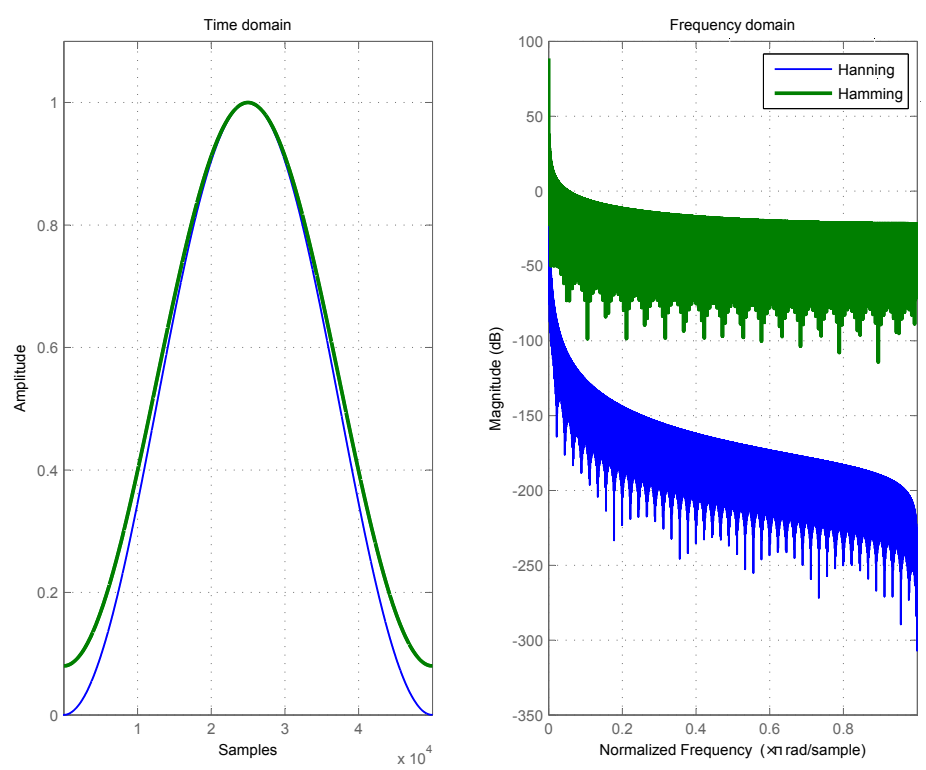

Figure I.1: A comparison between an arbitrary length Hanning and Hamming window in the time and frequency domains.

\section{I.3 Overlapping}

A common property of window functions is at its ends, where it typically tends to be very small or zero. Overlapping is necessary to avoid losing a significant portion of the data stream in the analysis after the window function is applied. The amount of overlapping is very dependent on the application and type of window being used. Heinzel et al. [79] recommend about 50\% 
overlapping for relatively wide windows in the time domain (such as the Hanning and Hamming windows). 


\section{Appendix J}

\section{Acceleration Spectral Densities}

The power spectral densities for both the leader and follower aircraft during formation flight are concisely summarised here. The general vibratory responses in Figure 7.8 for acceleration spectra at the feet and seat-surface are similar. As such, assuming that the seat-surface accelerations will contribute the most to passenger comfort, only the linear and rotational acceleration spectra at the seat-surface for different locations are shown in this appendix. Simulations were performed in light and moderate turbulence and decreasing geometric lateral separations. Table J.1 provides a reference guide for the different formation flight scenarios.

Table J.1: Reference guide for linear and rotational acceleration spectra estimations at different separations and varying levels of turbulence intensity

\begin{tabular}{|c|c|c|c|c|}
\hline \multirow[t]{2}{*}{ Turbulence Intensity } & \multicolumn{3}{|c|}{ Desired Separations } & \multirow[t]{2}{*}{ Figures } \\
\hline & $\eta$ & $\zeta$ & $\xi$ & \\
\hline \multirow{5}{*}{ Light } & 1.5 & 0 & -10 & \begin{tabular}{|l|l|}
$\mathrm{J} .1$ & $\mathrm{~J} .10 \mathrm{a}$ \\
\end{tabular} \\
\hline & 1.4 & 0 & -10 & \begin{tabular}{|l|l|}
$J .3$ & $\mathrm{~J} .10 \mathrm{~b}$ \\
\end{tabular} \\
\hline & 1.3 & 0 & -10 & \begin{tabular}{|l|l|}
$J .5$ & $\mathrm{~J} .10 \mathrm{c}$ \\
\end{tabular} \\
\hline & 1.2 & 0 & -10 & \begin{tabular}{|l|l}
$\mathrm{J} .7$ & $\mathrm{~J} .10 \mathrm{~d}$ \\
\end{tabular} \\
\hline & 1.1 & 0 & -10 & \begin{tabular}{|l|l|}
$J .9$ & $\mathrm{~J} .10 \mathrm{e}$ \\
\end{tabular} \\
\hline \multirow{4}{*}{ Moderate } & 1.5 & 0 & -10 & \begin{tabular}{|l|l|} 
J.2 & J.11a \\
\end{tabular} \\
\hline & 1.4 & 0 & -10 & $\mathrm{~J} .4 \mathrm{~J} .11 \mathrm{~b}$ \\
\hline & 1.3 & 0 & -10 & \begin{tabular}{|l|l|}
$\mathrm{J} .6$ & $\mathrm{~J} .11 \mathrm{C}$ \\
\end{tabular} \\
\hline & 1.2 & 0 & -10 & $\overline{\bar{J} .8} \overline{\bar{J} .11 \mathrm{~d}}$ \\
\hline
\end{tabular}



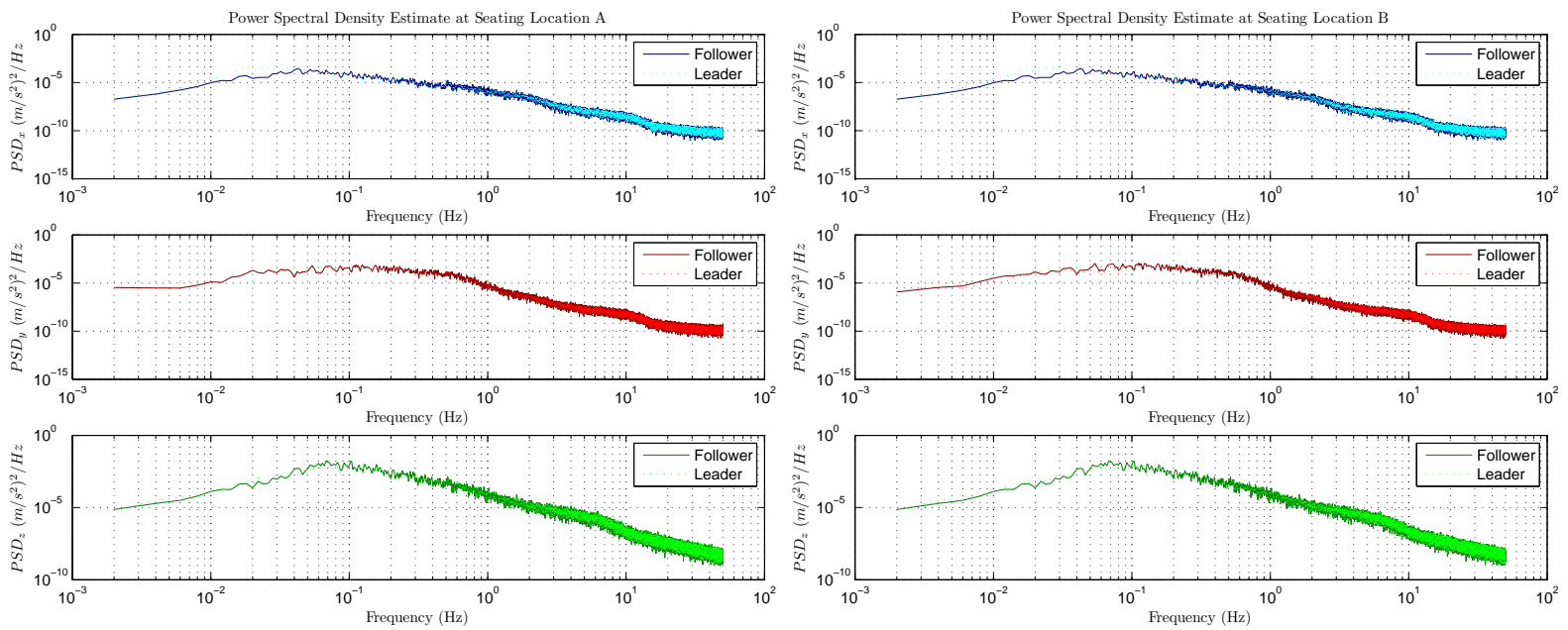

(a) Seating location A

(b) Seating location B
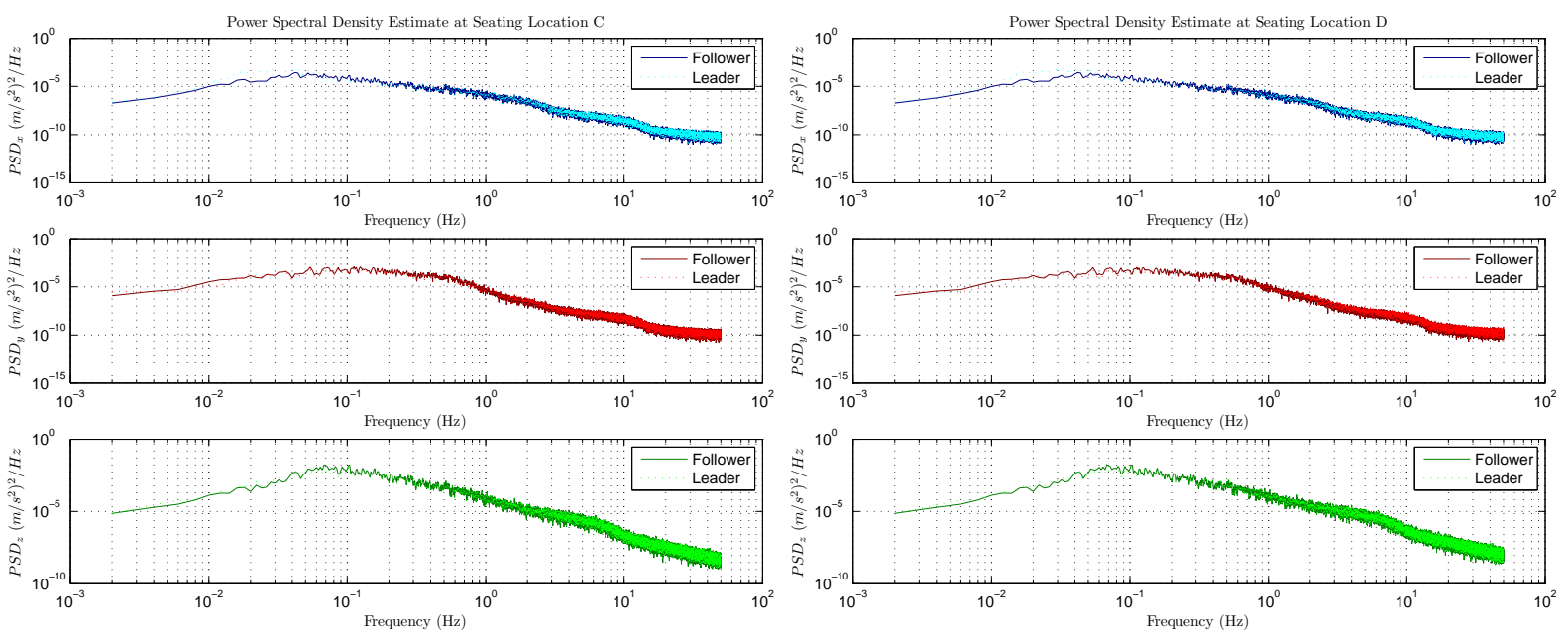

(c) Seating location $\mathrm{C}$

(d) Seating location D
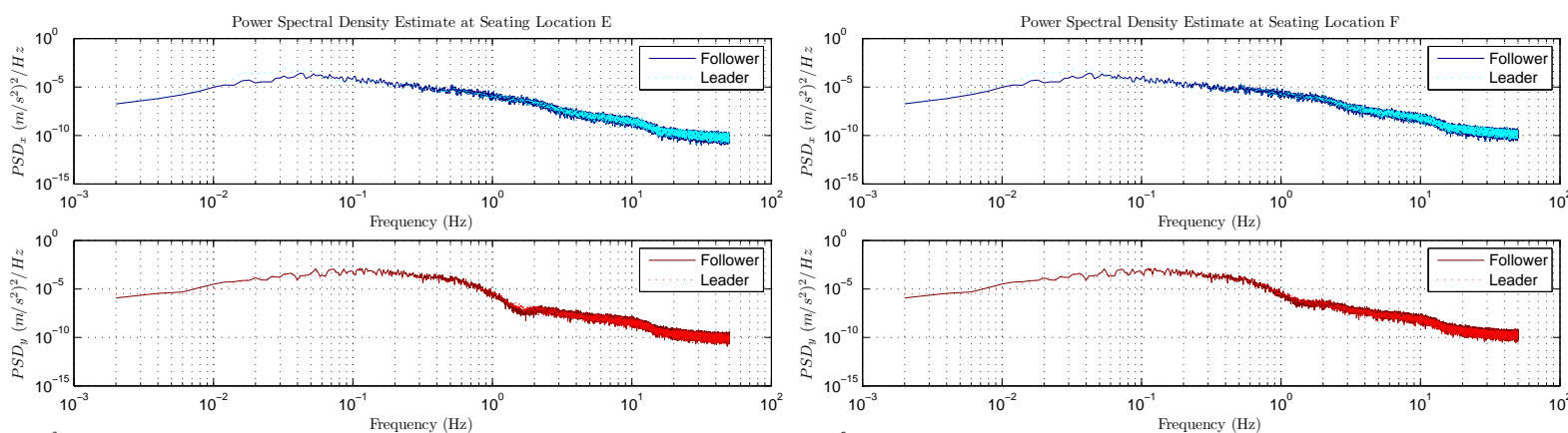

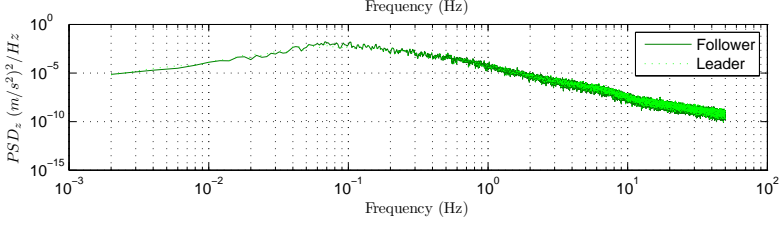

(e) Seating location E

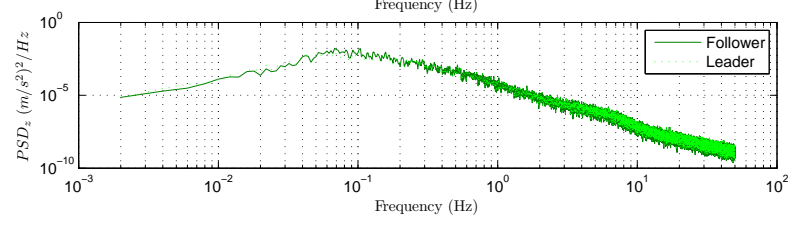

(f) Seating location F

Figure J.1: Linear acceleration power spectral densities of a leader and follower aircraft in light turbulence where $\eta=1.5$ 

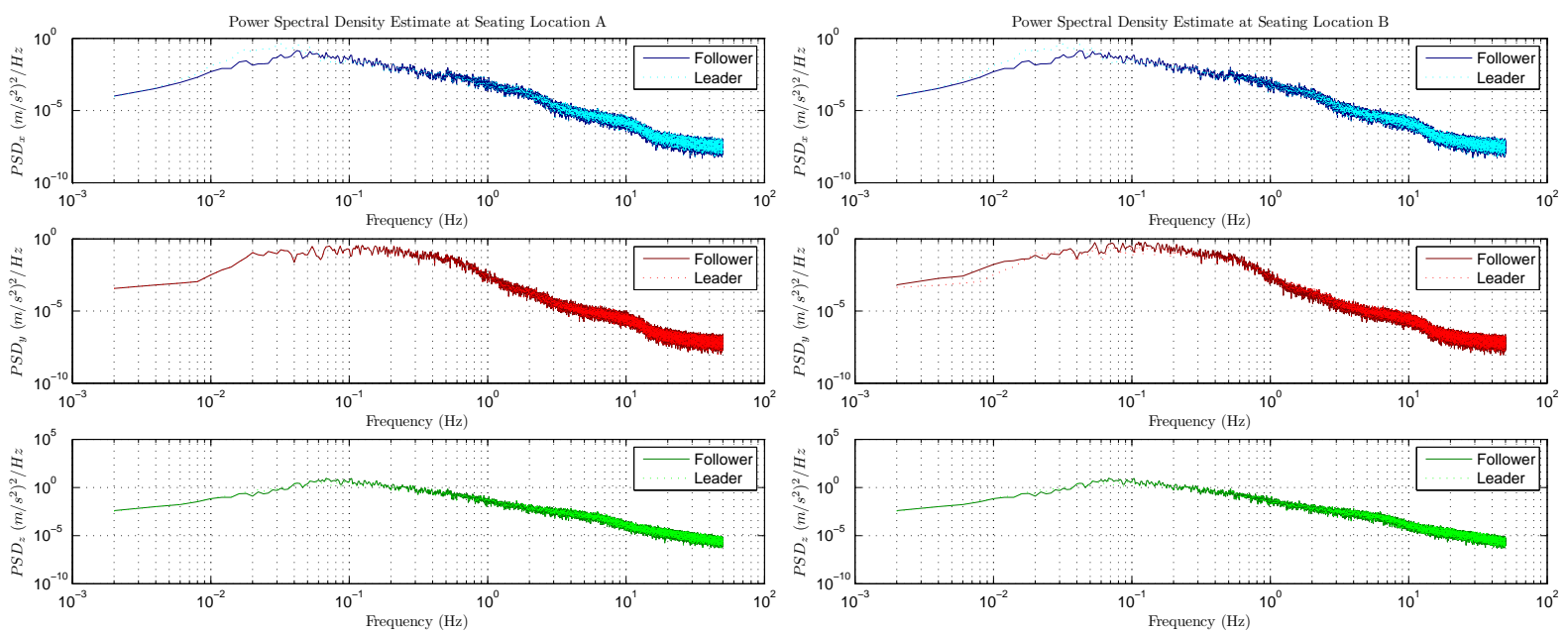

(a) Seating location A

(b) Seating location B
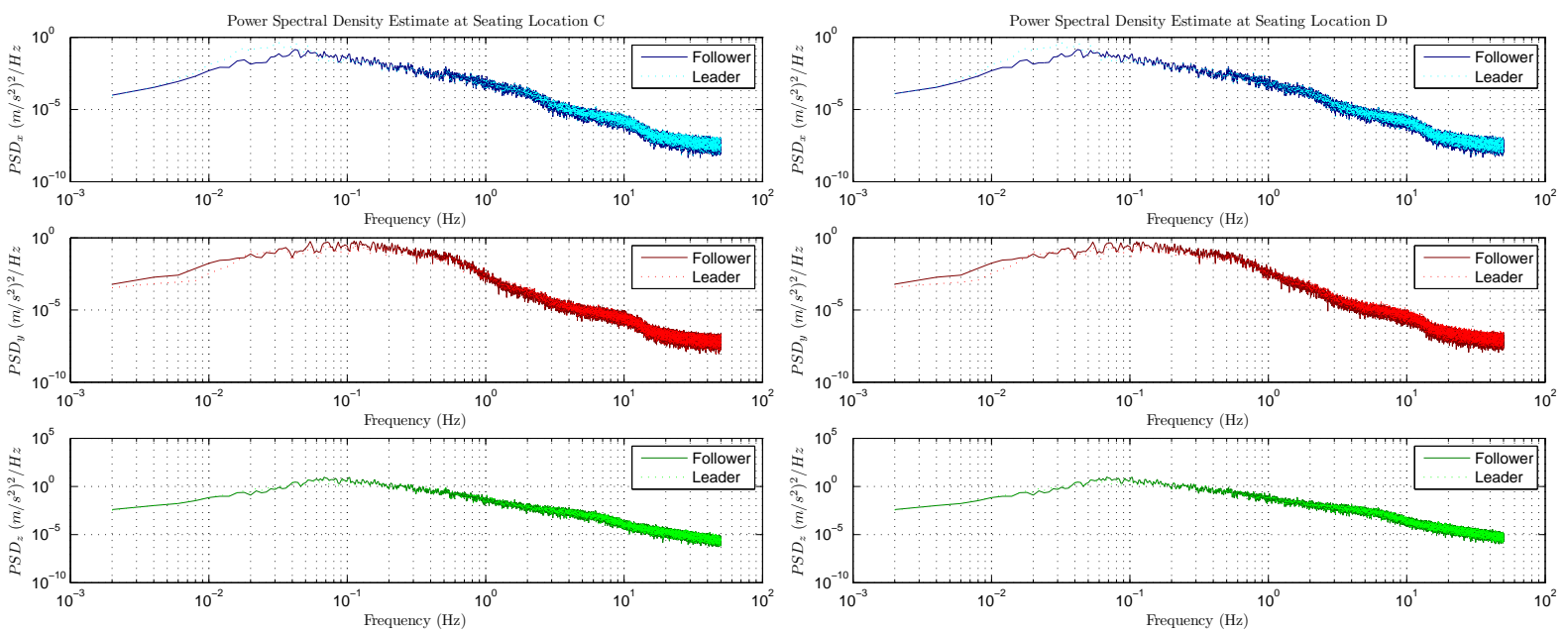

(c) Seating location $\mathrm{C}$

(d) Seating location D
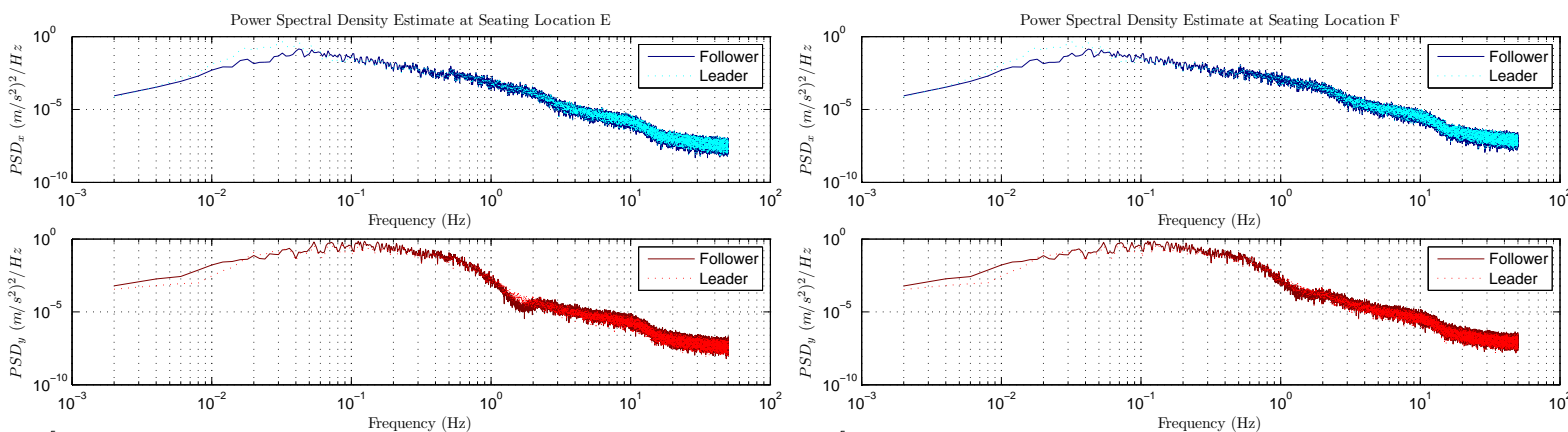

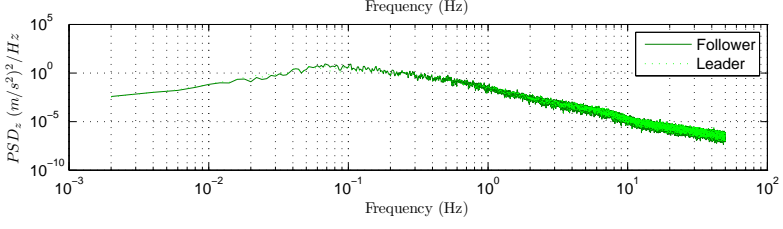

(e) Seating location E

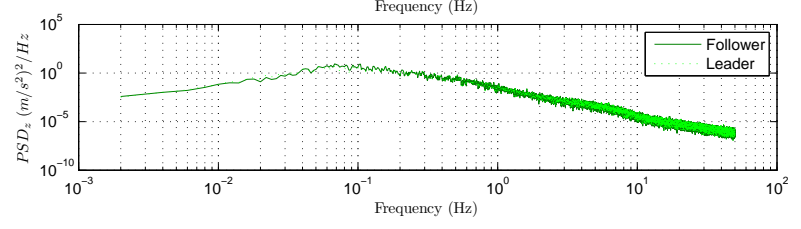

(f) Seating location F

Figure J.2: Linear acceleration power spectral densities of a leader and follower aircraft in moderate turbulence where $\eta=1.5$ 

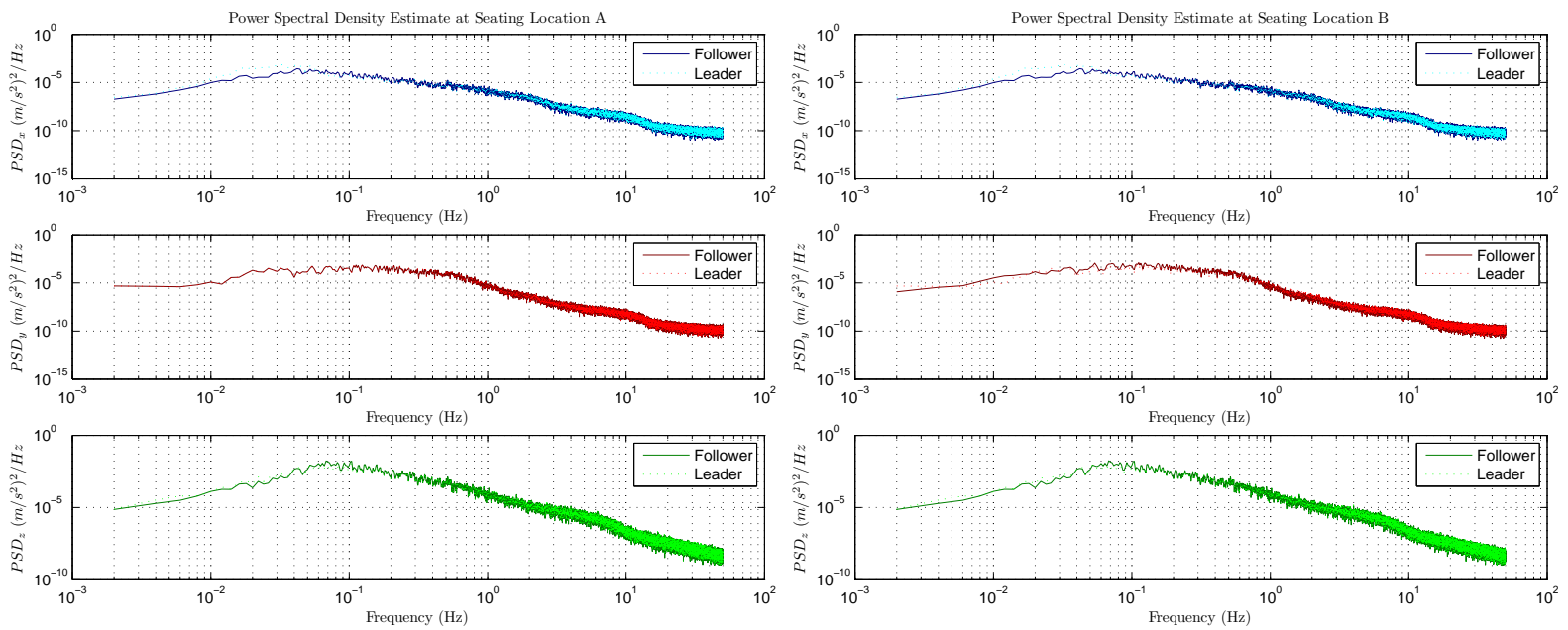

(a) Seating location A

(b) Seating location B
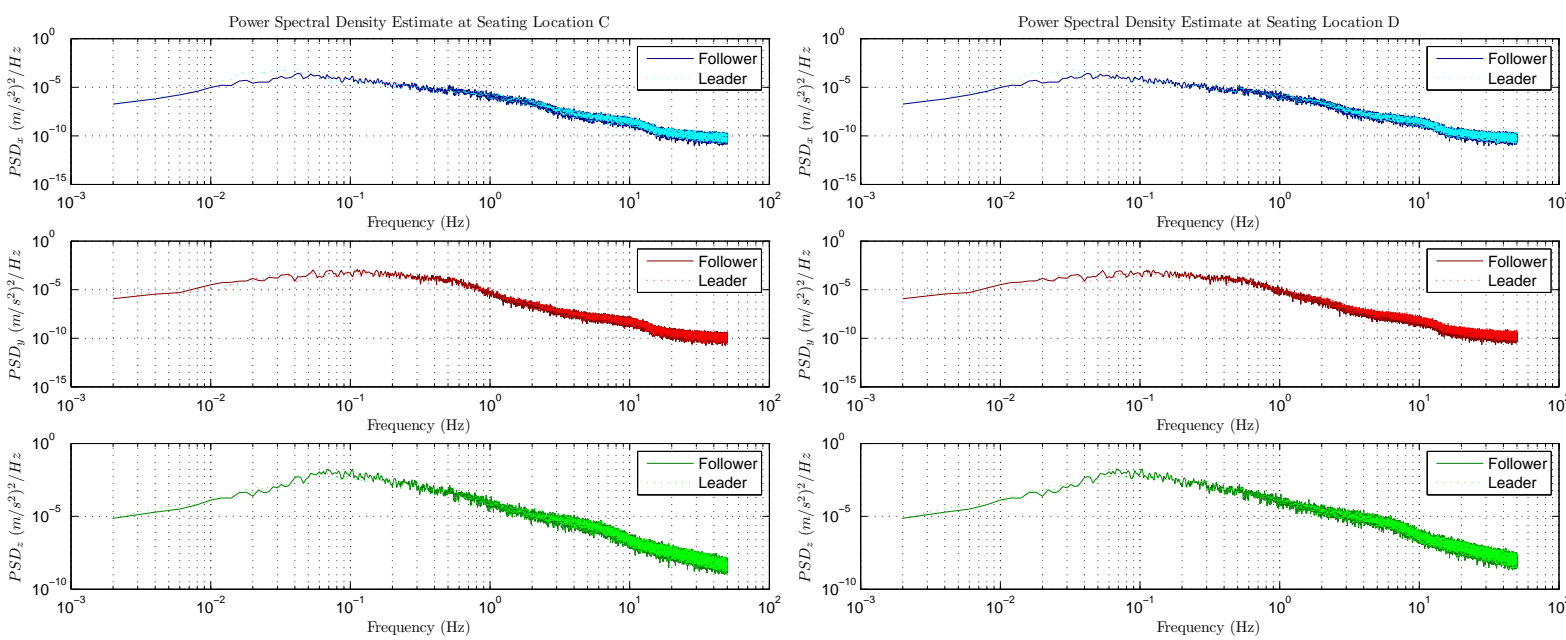

(c) Seating location $\mathrm{C}$

(d) Seating location D
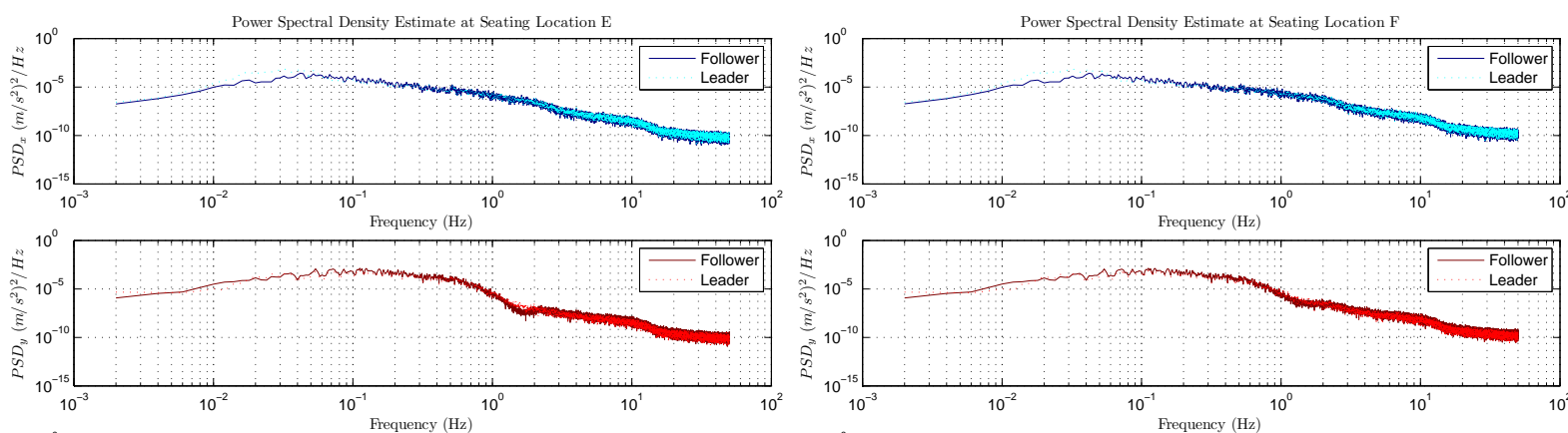

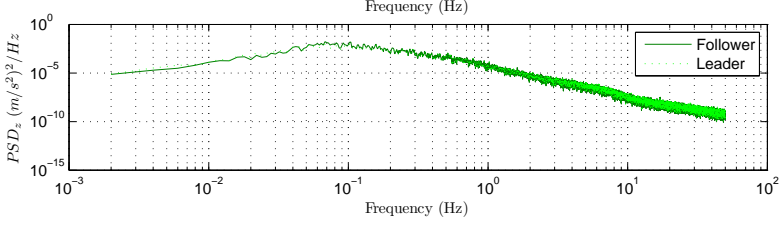

(e) Seating location E

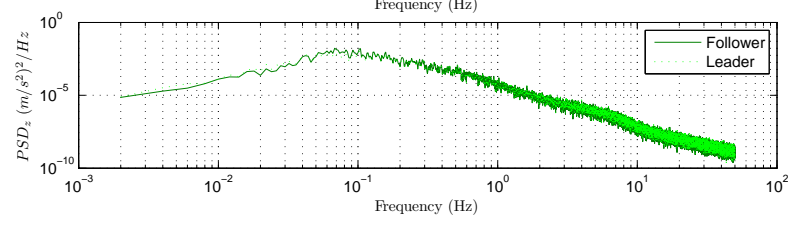

(f) Seating location F

Figure J.3: Linear acceleration power spectral densities of a leader and follower aircraft in light turbulence where $\eta=1.4$ 

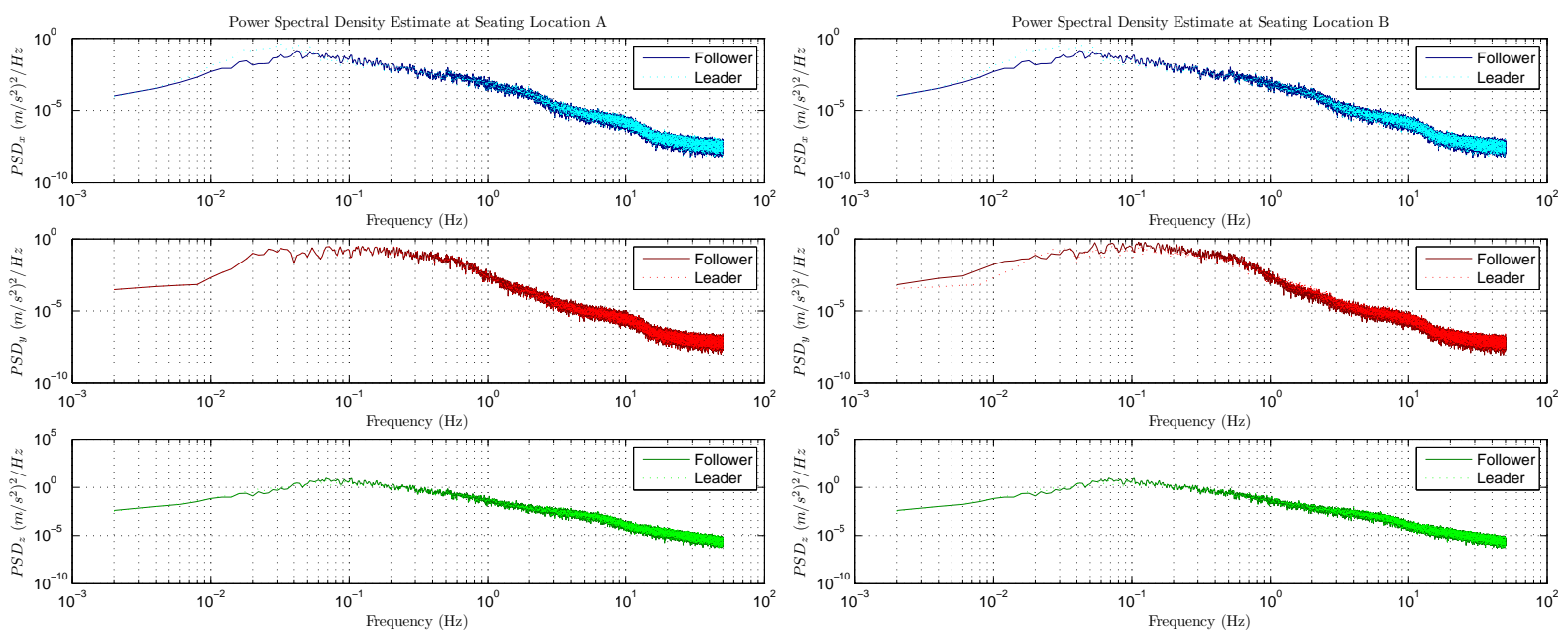

(a) Seating location A

(b) Seating location B
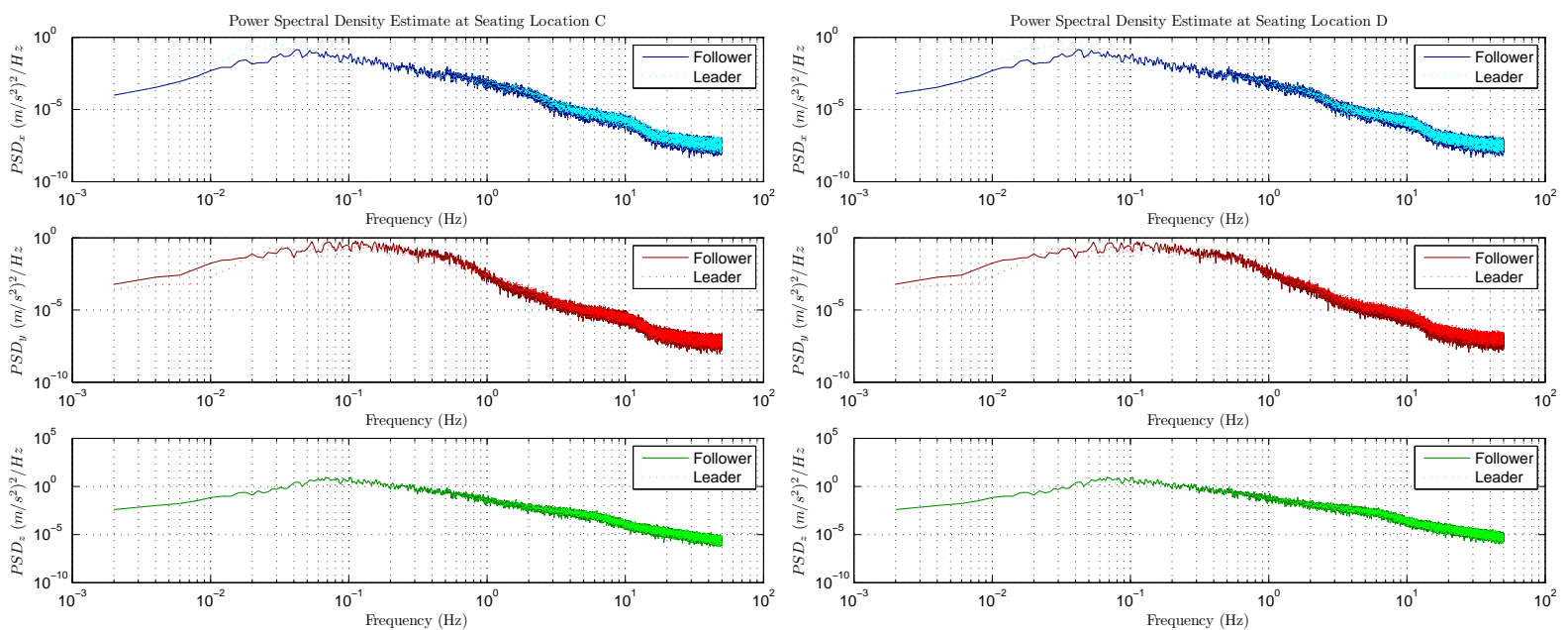

(c) Seating location $\mathrm{C}$

(d) Seating location D
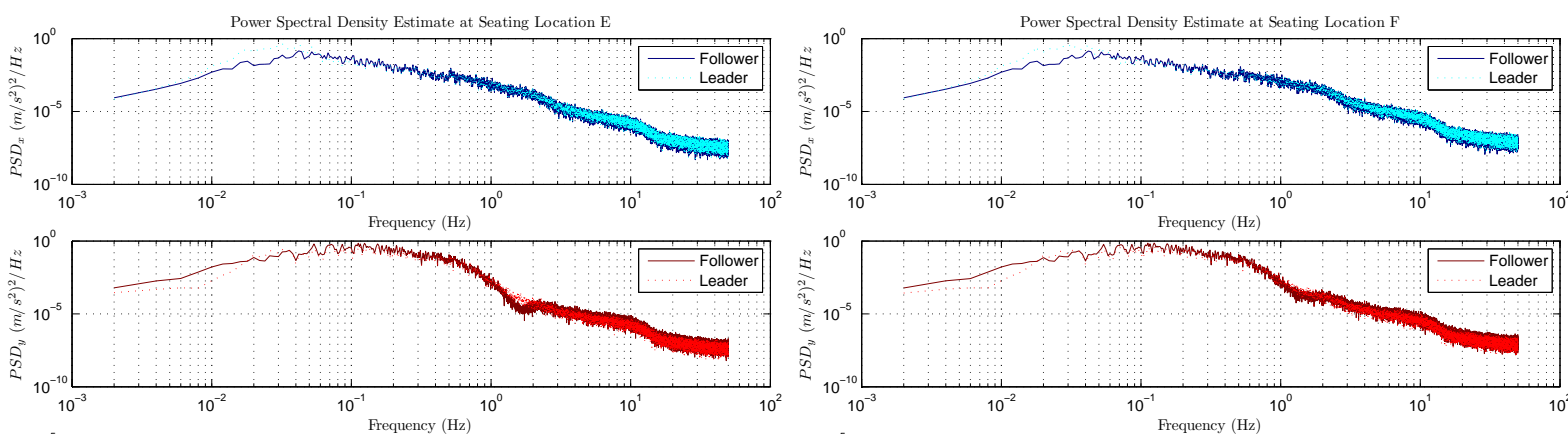

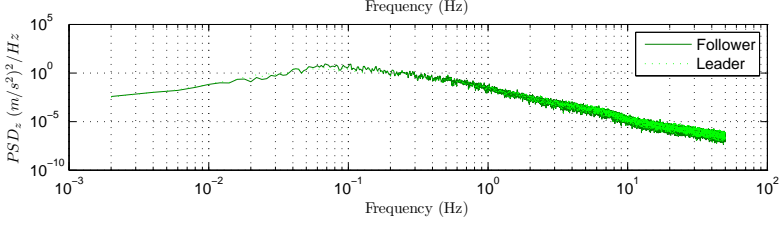

(e) Seating location E

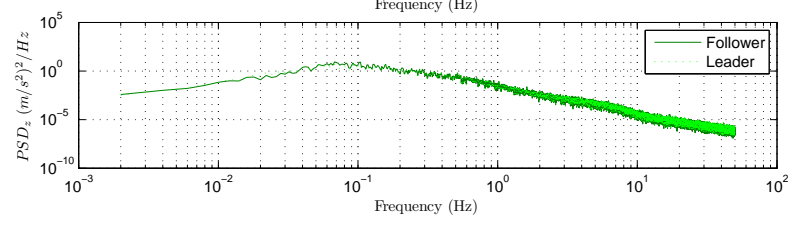

(f) Seating location F

Figure J.4: Linear acceleration power spectral densities of a leader and follower aircraft in moderate turbulence where $\eta=1.4$ 

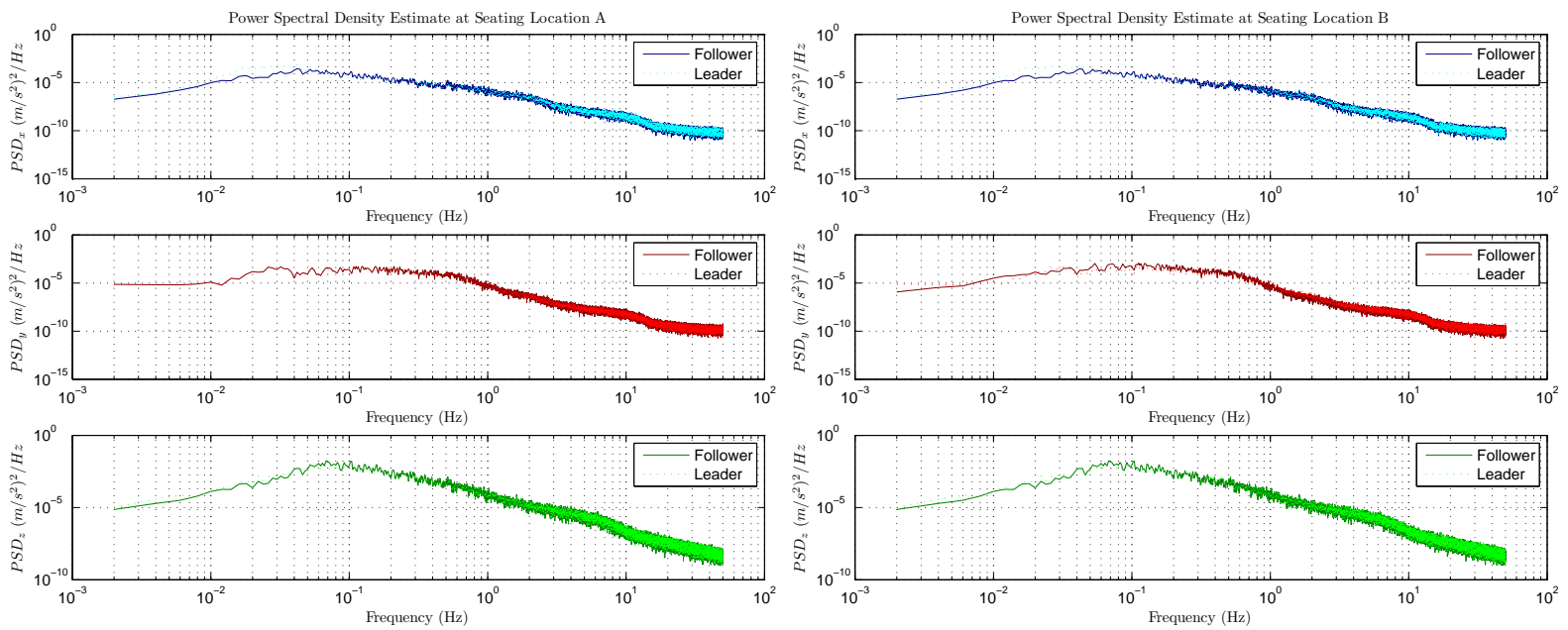

(a) Seating location A

(b) Seating location B
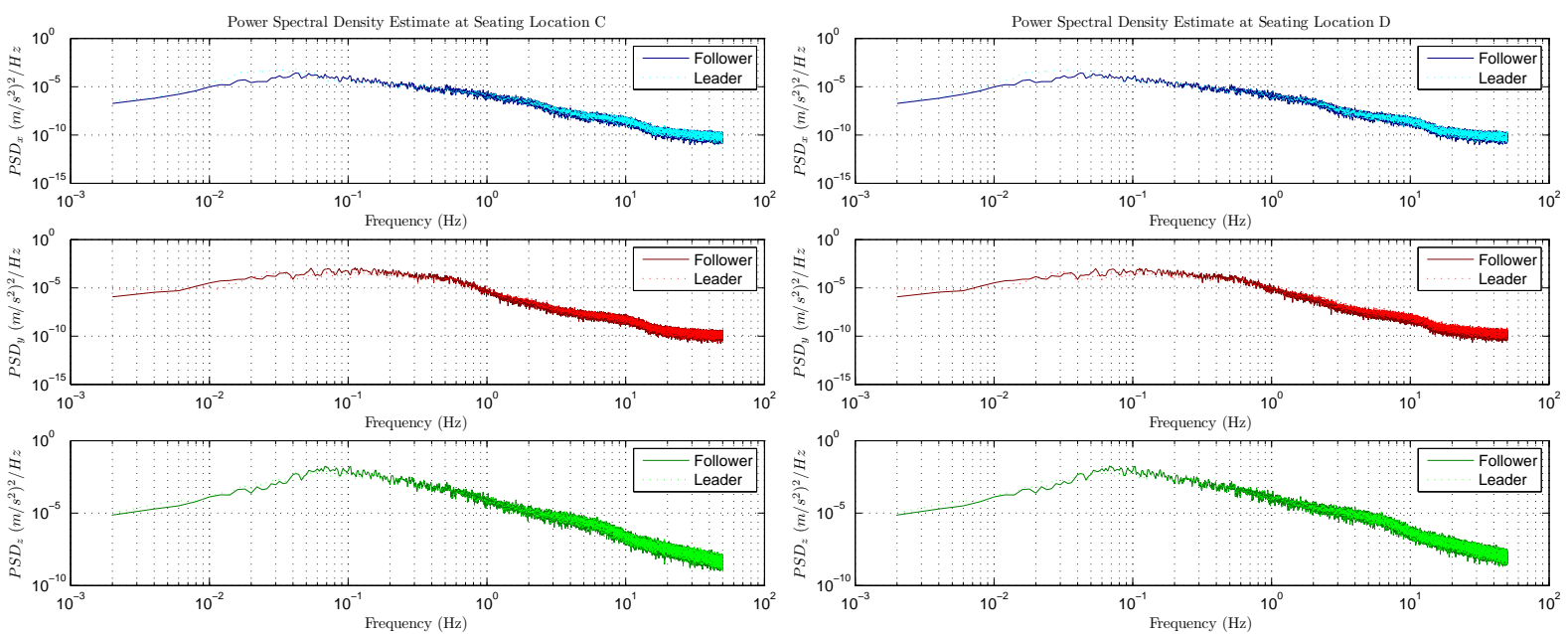

(c) Seating location $\mathrm{C}$

(d) Seating location D
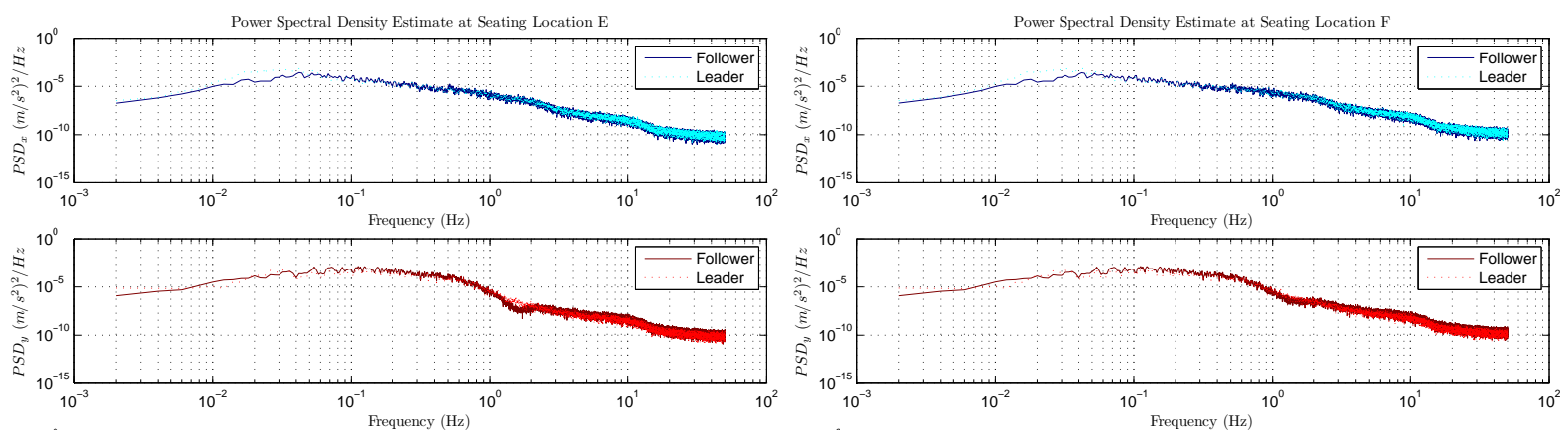

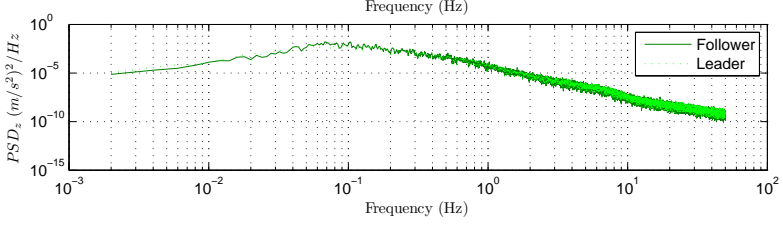

(e) Seating location E

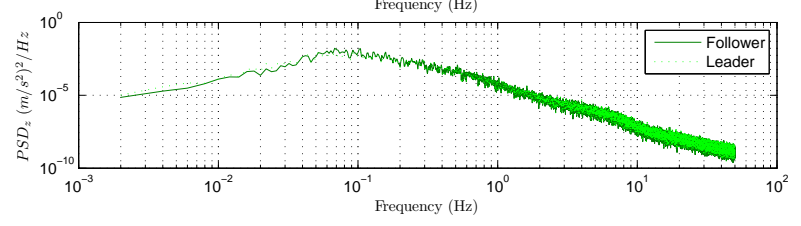

(f) Seating location F

Figure J.5: Linear acceleration power spectral densities of a leader and follower aircraft in light turbulence where $\eta=1.3$ 

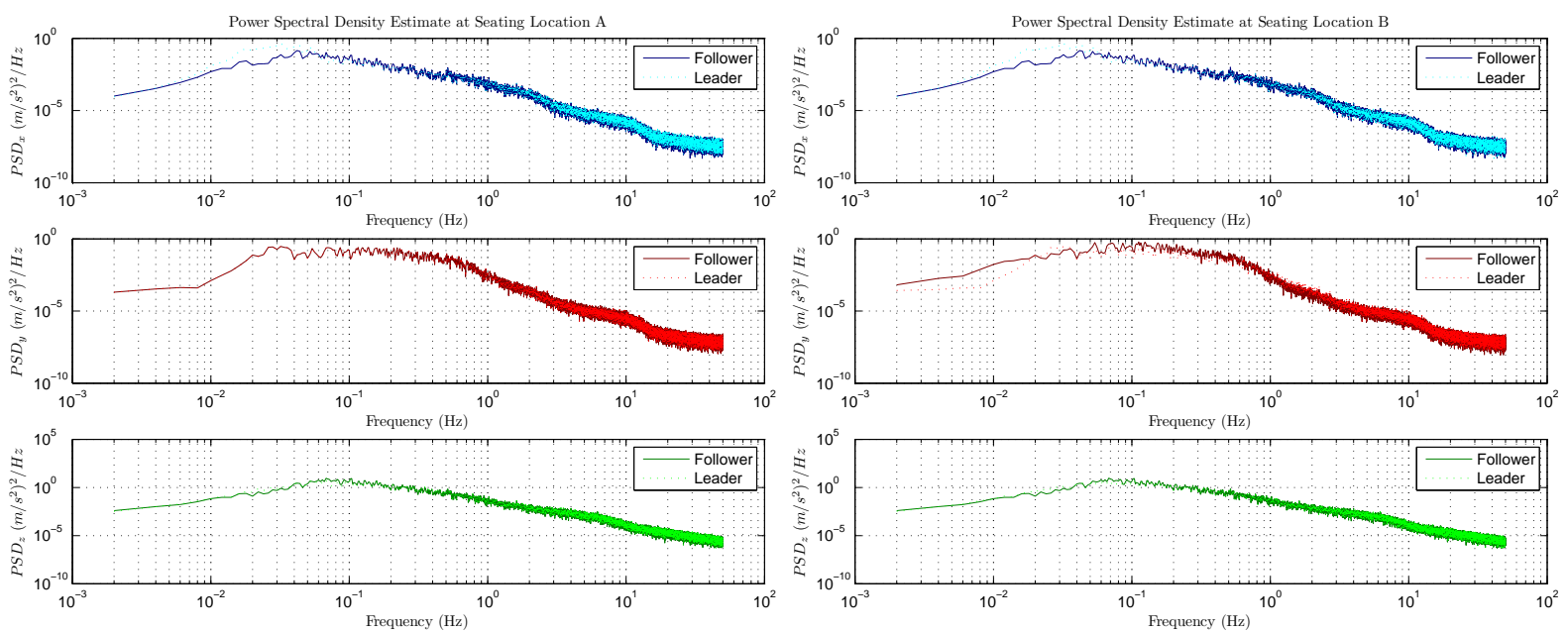

(a) Seating location A

(b) Seating location B
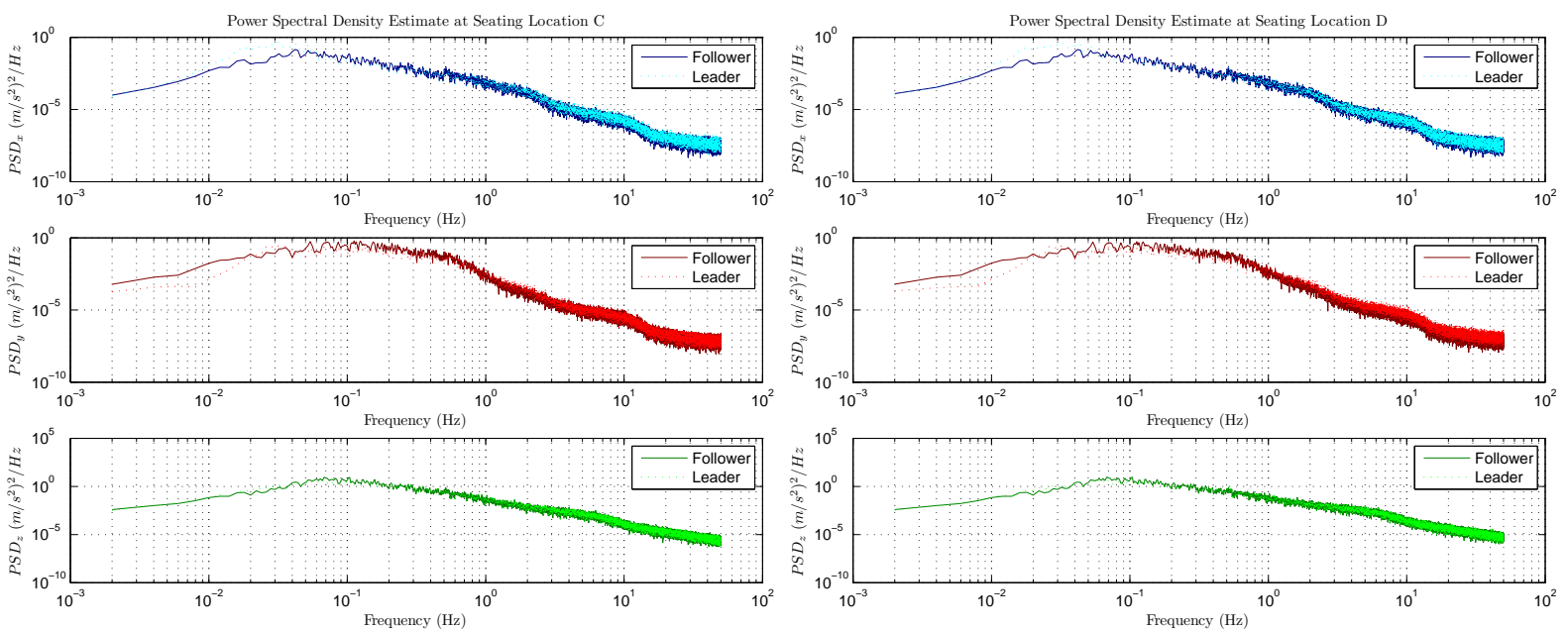

(c) Seating location $\mathrm{C}$

(d) Seating location D
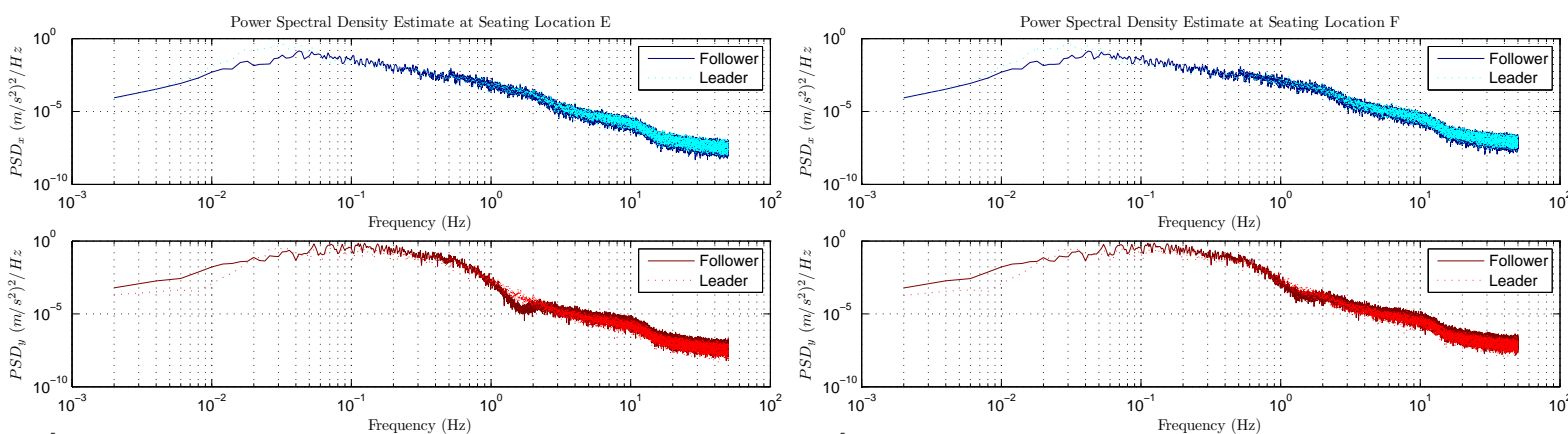

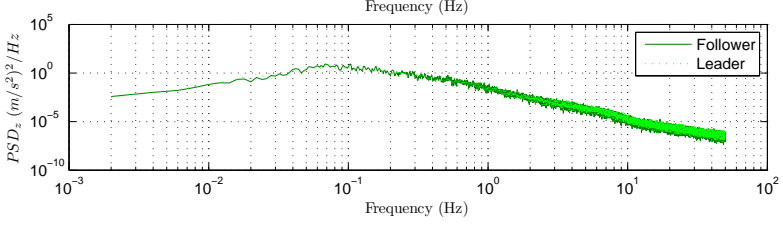

(e) Seating location E

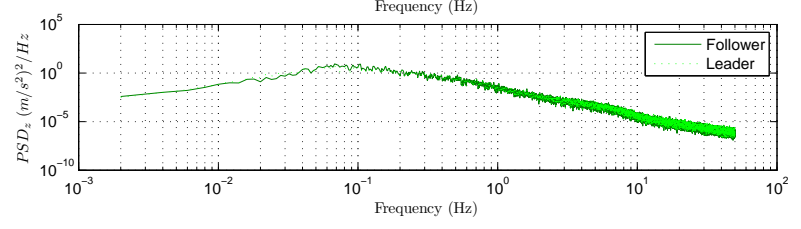

(f) Seating location F

Figure J.6: Linear acceleration power spectral densities of a leader and follower aircraft in moderate turbulence where $\eta=1.3$ 

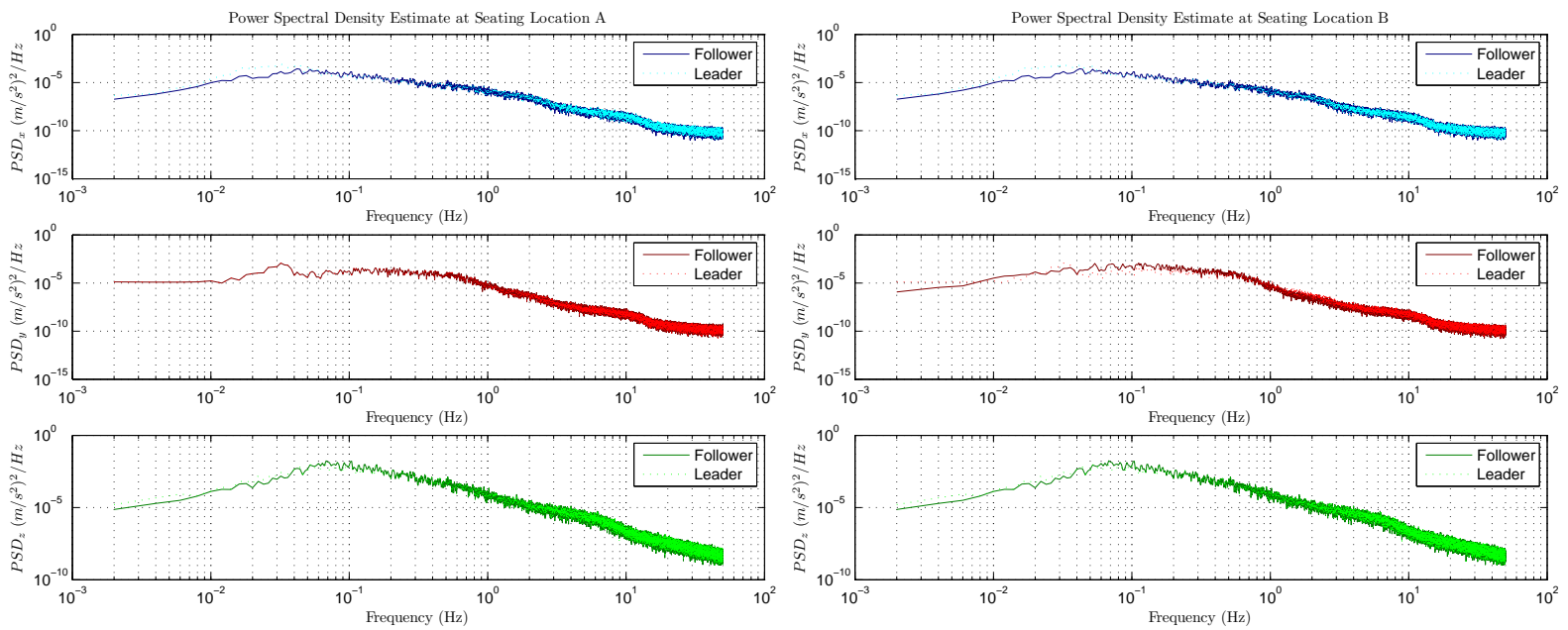

(a) Seating location A

(b) Seating location B
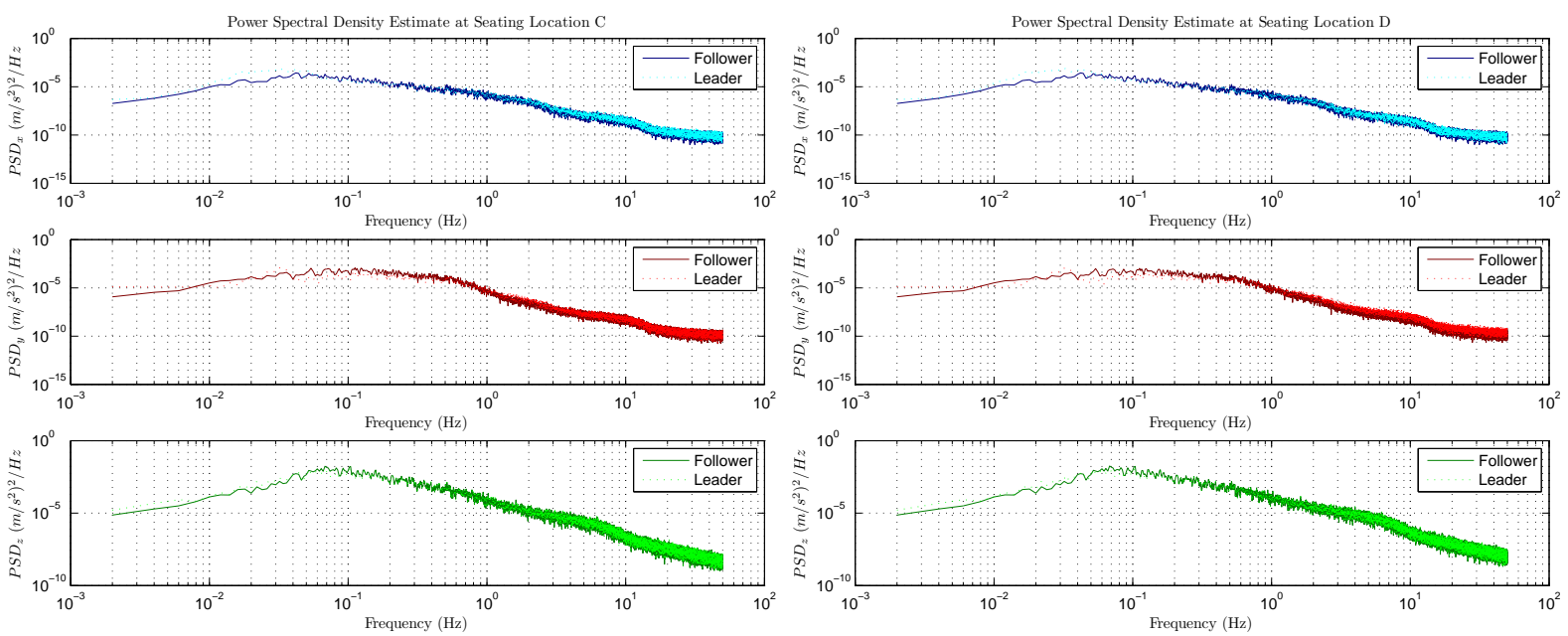

(c) Seating location $\mathrm{C}$

(d) Seating location D
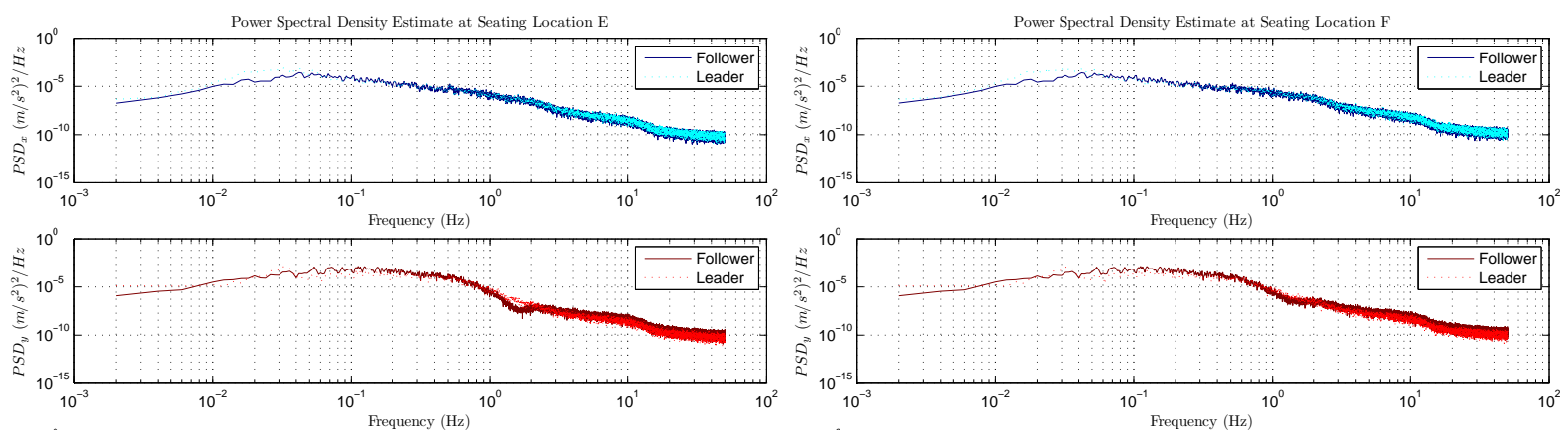

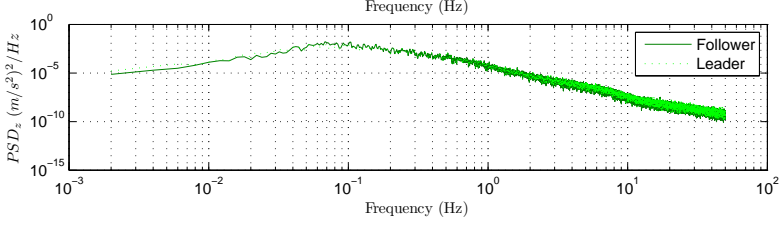

(e) Seating location E

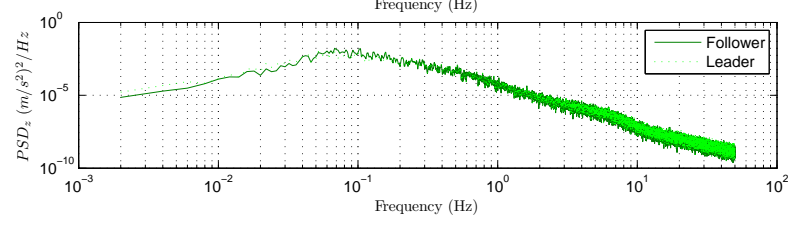

(f) Seating location F

Figure J.7: Linear acceleration power spectral densities of a leader and follower aircraft in light turbulence where $\eta=1.2$ 

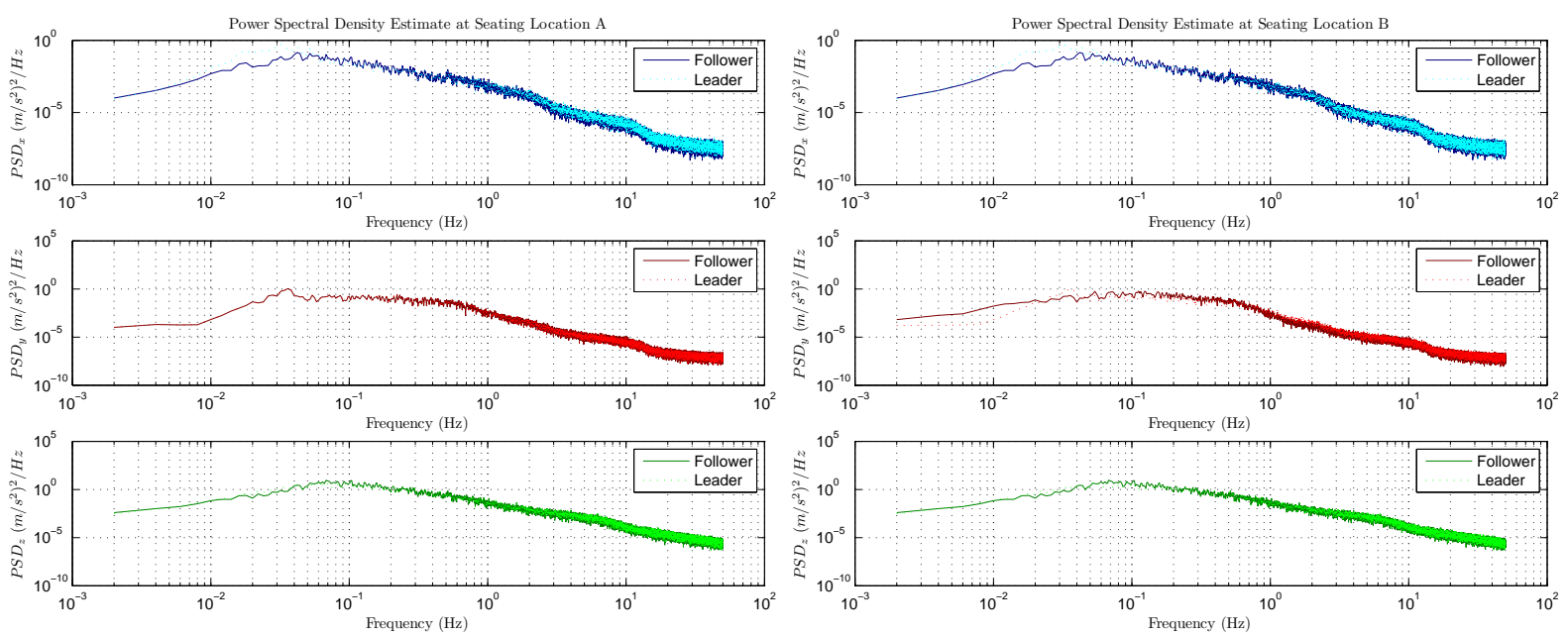

(a) Seating location A

(b) Seating location B
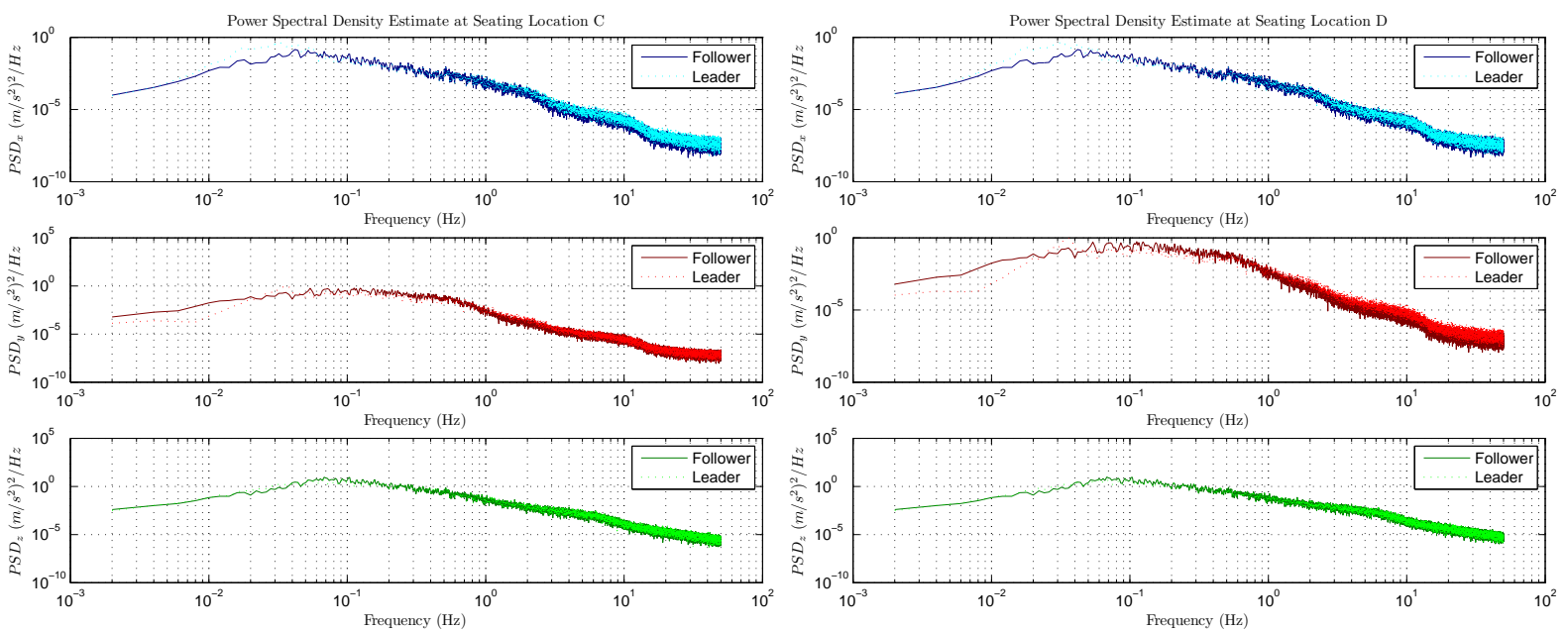

(c) Seating location $\mathrm{C}$

(d) Seating location D
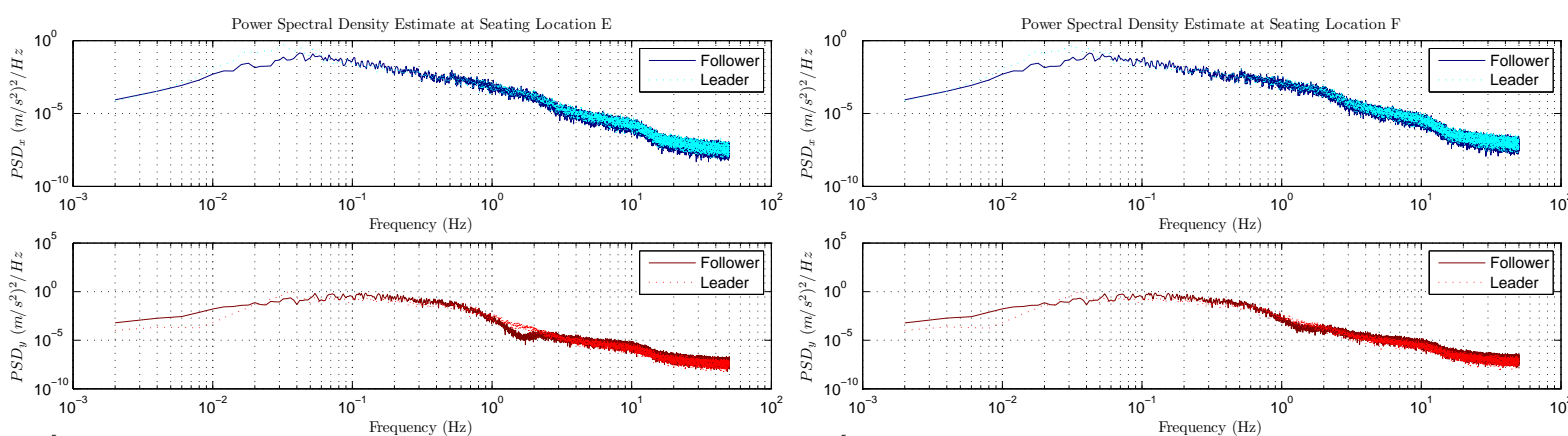

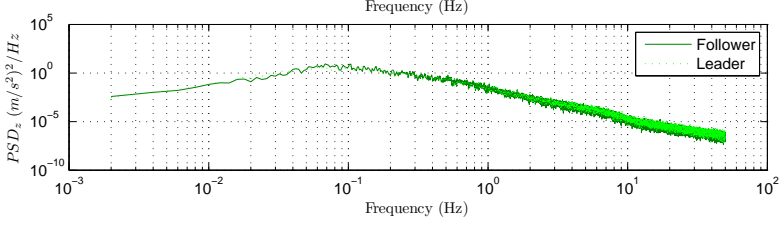

(e) Seating location E

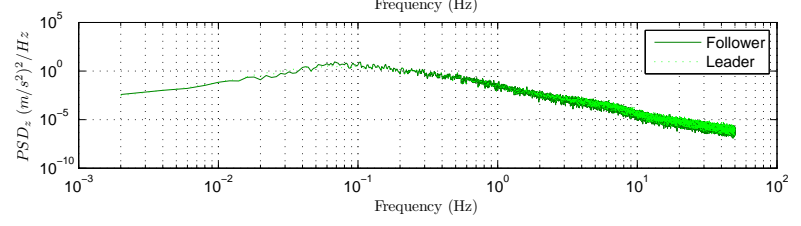

(f) Seating location F

Figure J.8: Linear acceleration power spectral densities of a leader and follower aircraft in moderate turbulence where $\eta=1.2$ 

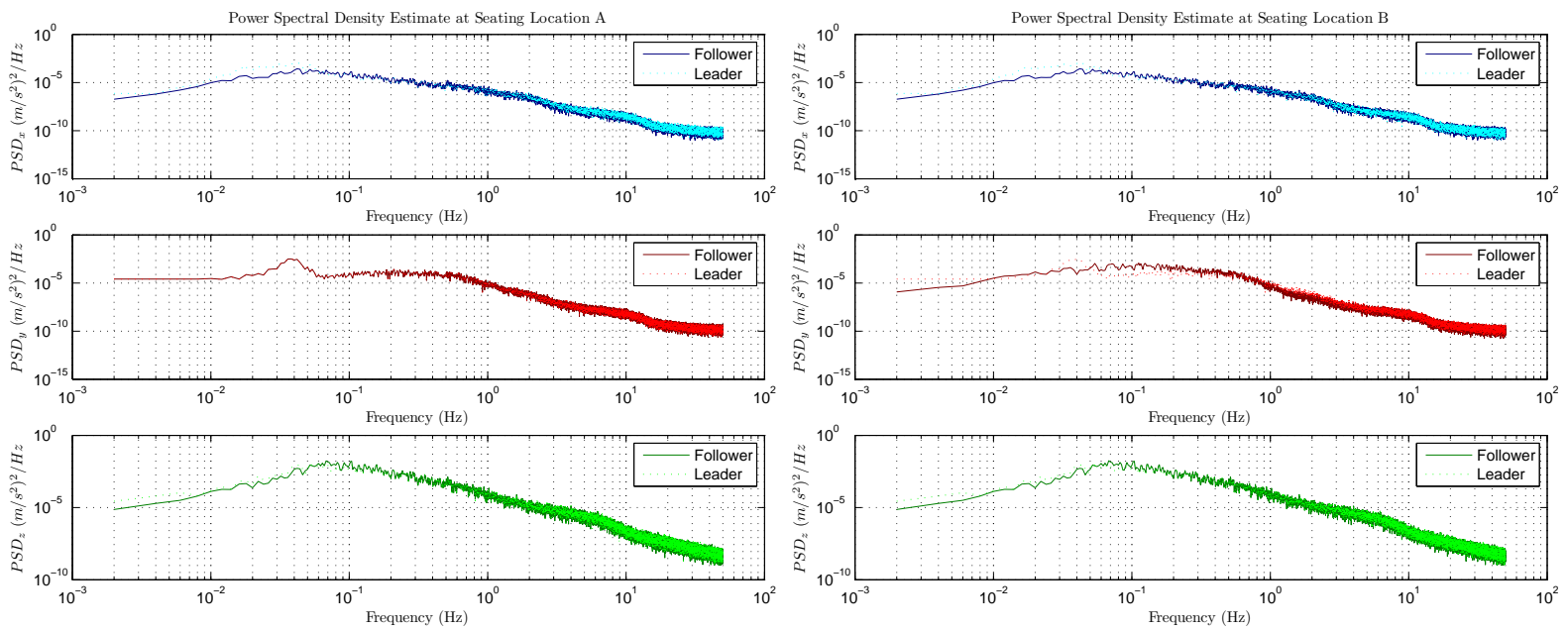

(a) Seating location A

(b) Seating location B
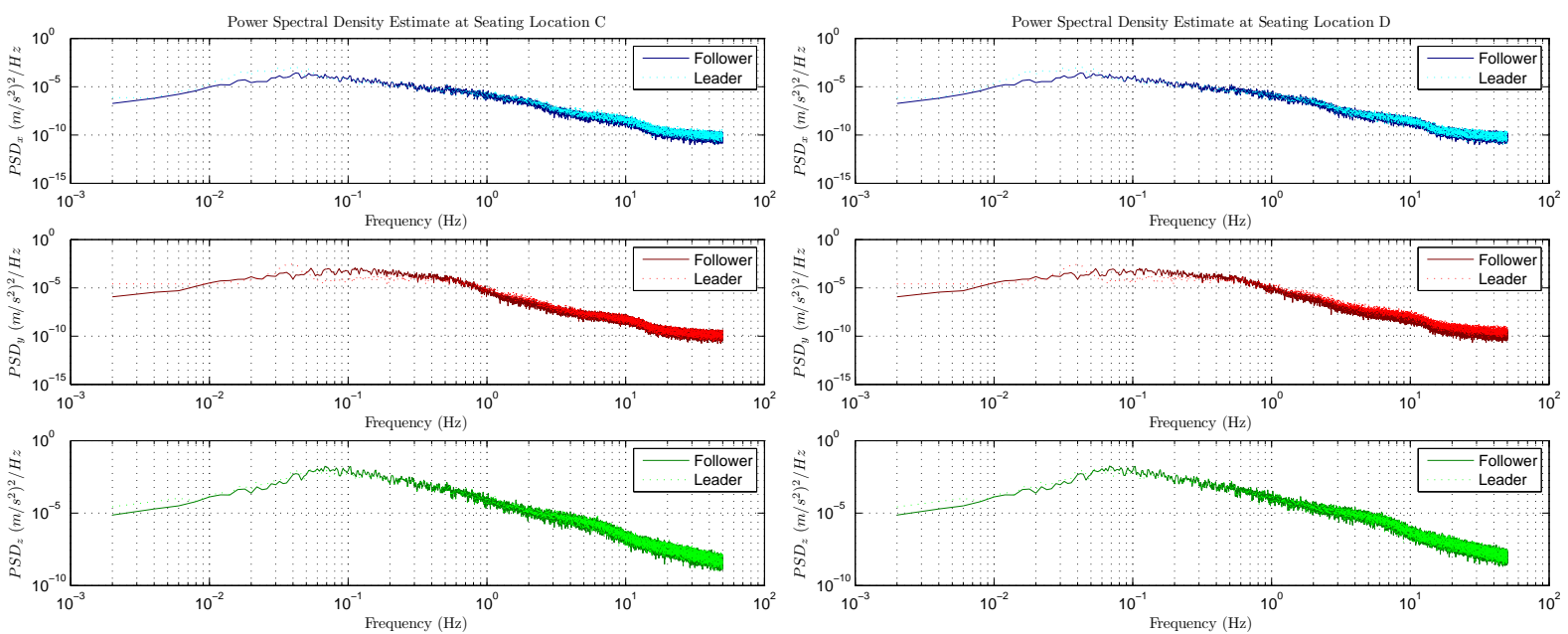

(c) Seating location $\mathrm{C}$

(d) Seating location D
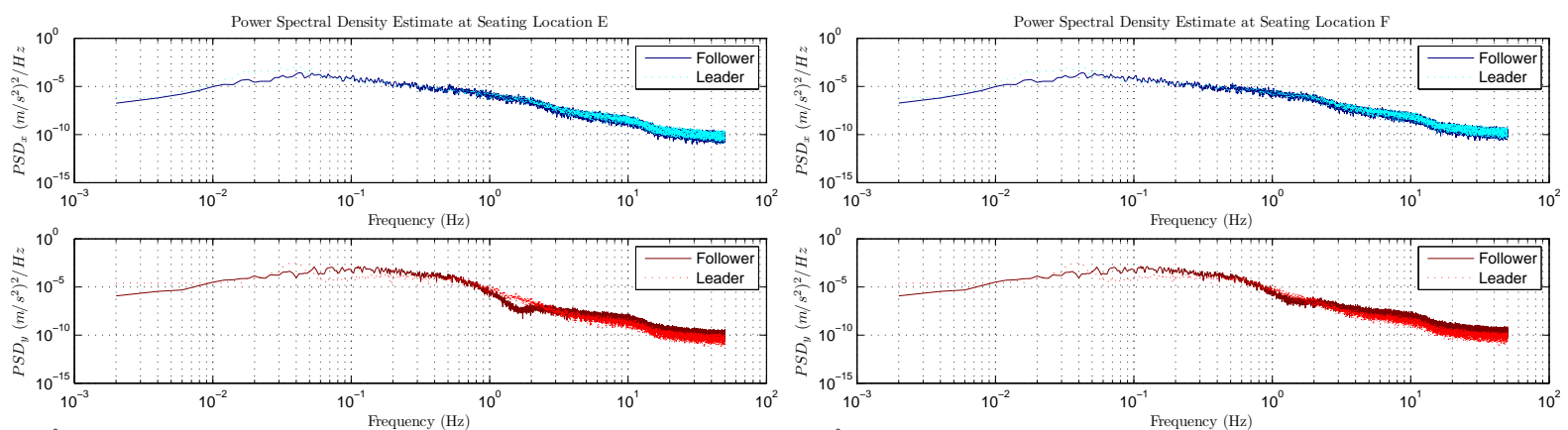

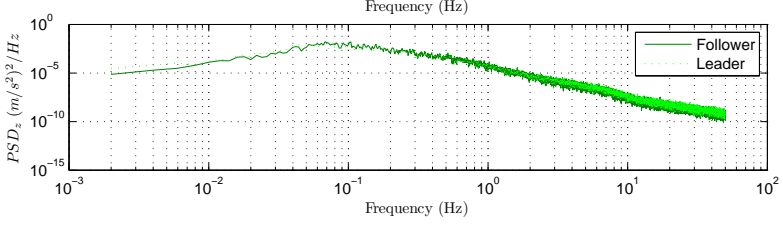

(e) Seating location E

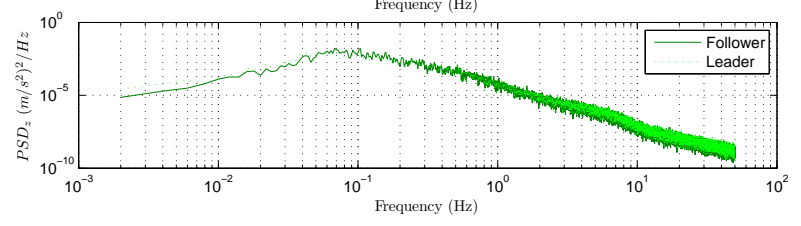

(f) Seating location F

Figure J.9: Linear acceleration power spectral densities of a leader and follower aircraft in light turbulence where $\eta=1.1$ 

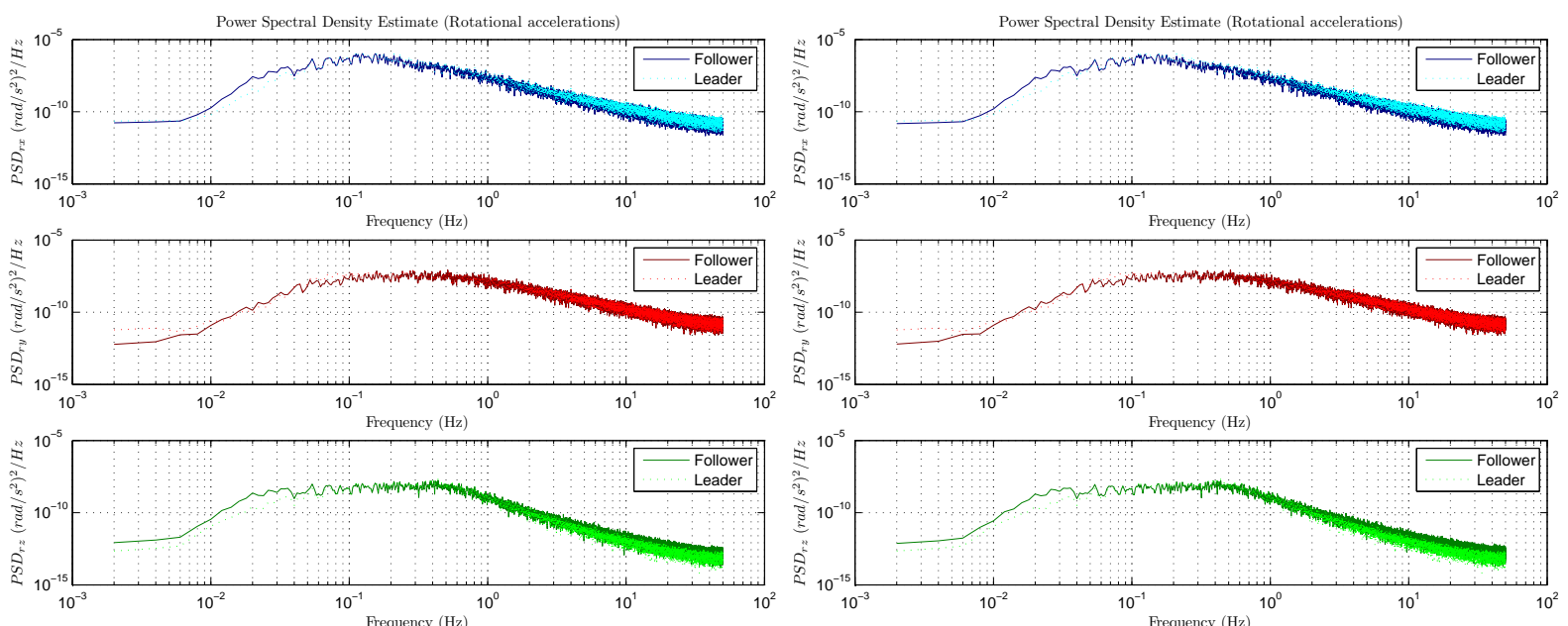

(a) $\eta=1.5$

(b) $\eta=1.4$
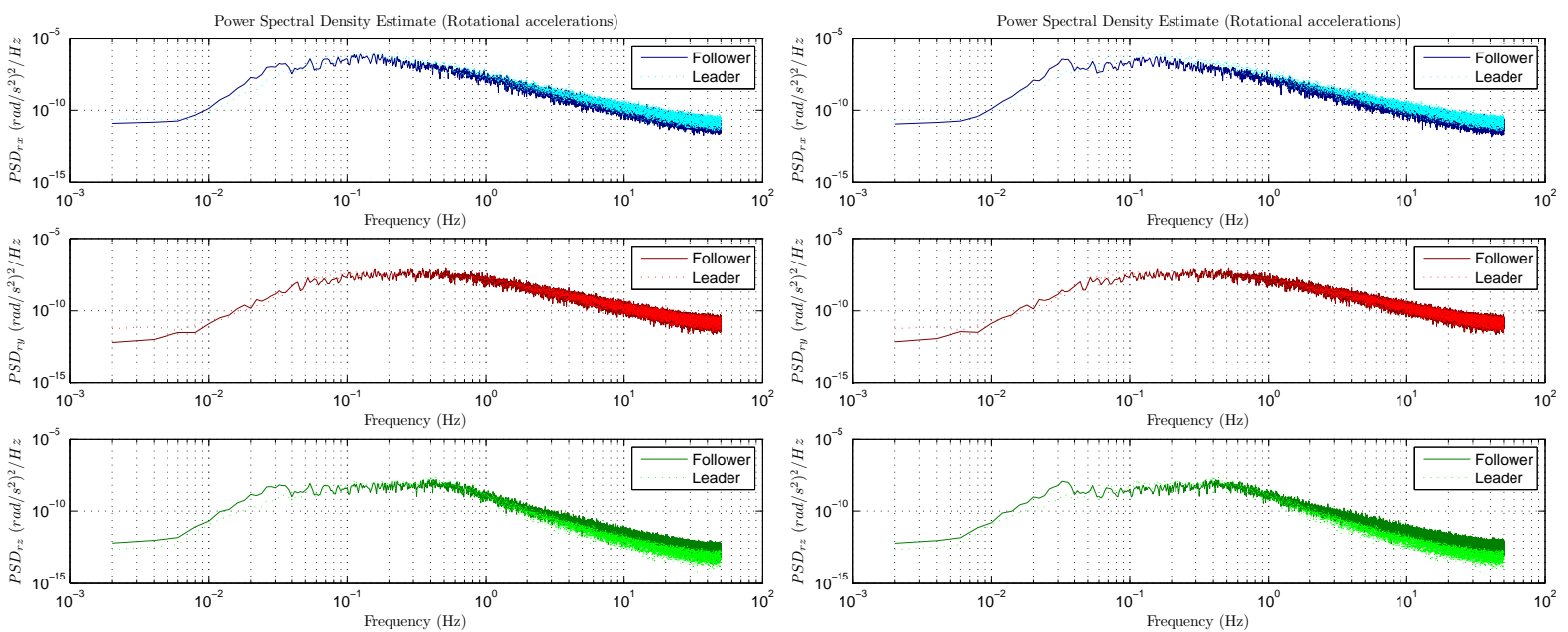

(c) $\eta=1.3$

(d) $\eta=1.2$
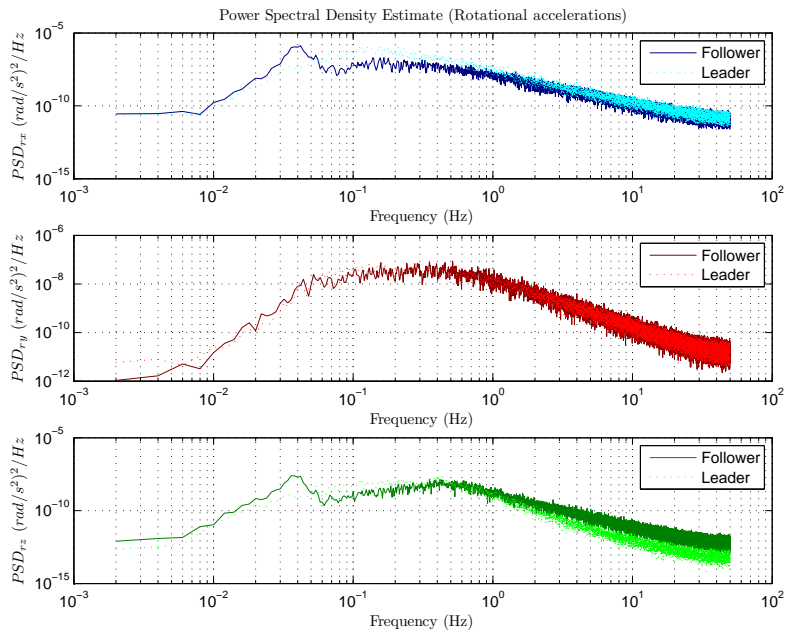

(e) $\eta=1.1$

Figure J.10: Rotational acceleration power spectral densities of a leader and follower aircraft at any seating location in light turbulence 

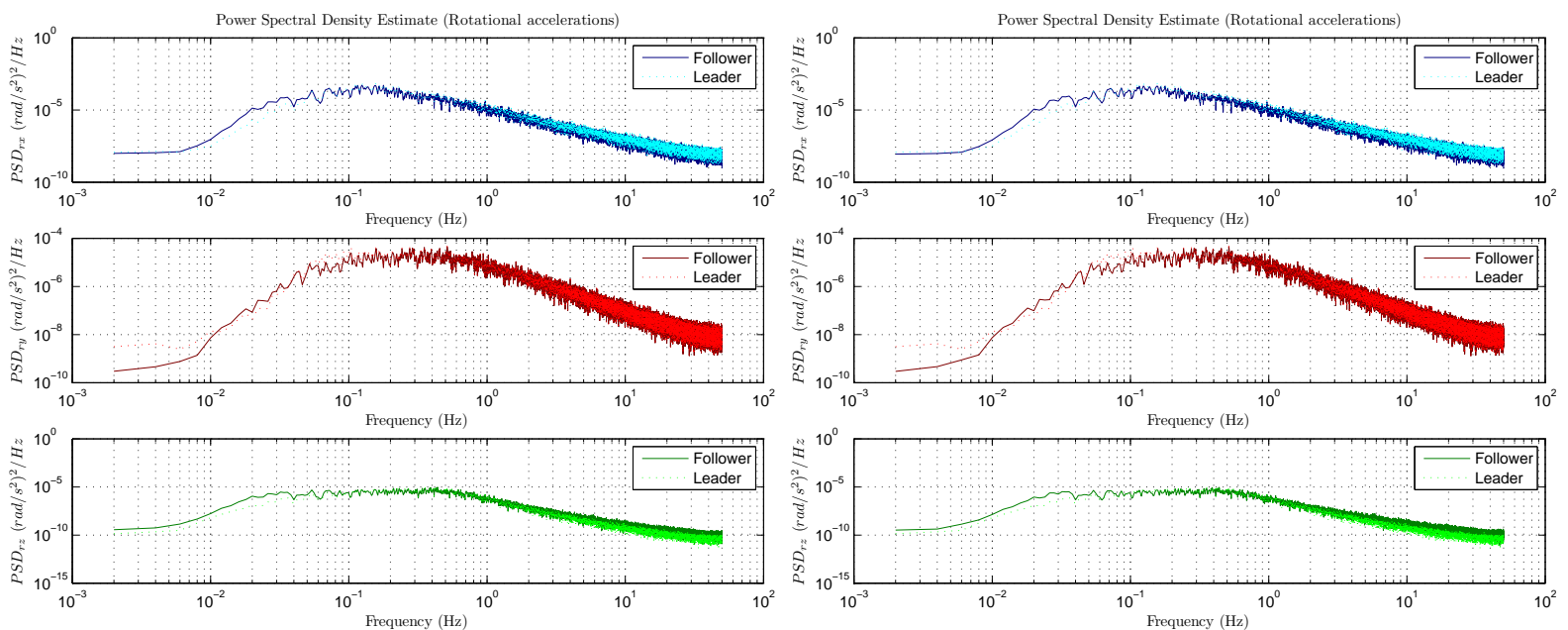

(a) $\eta=1.5$

(b) $\eta=1.4$
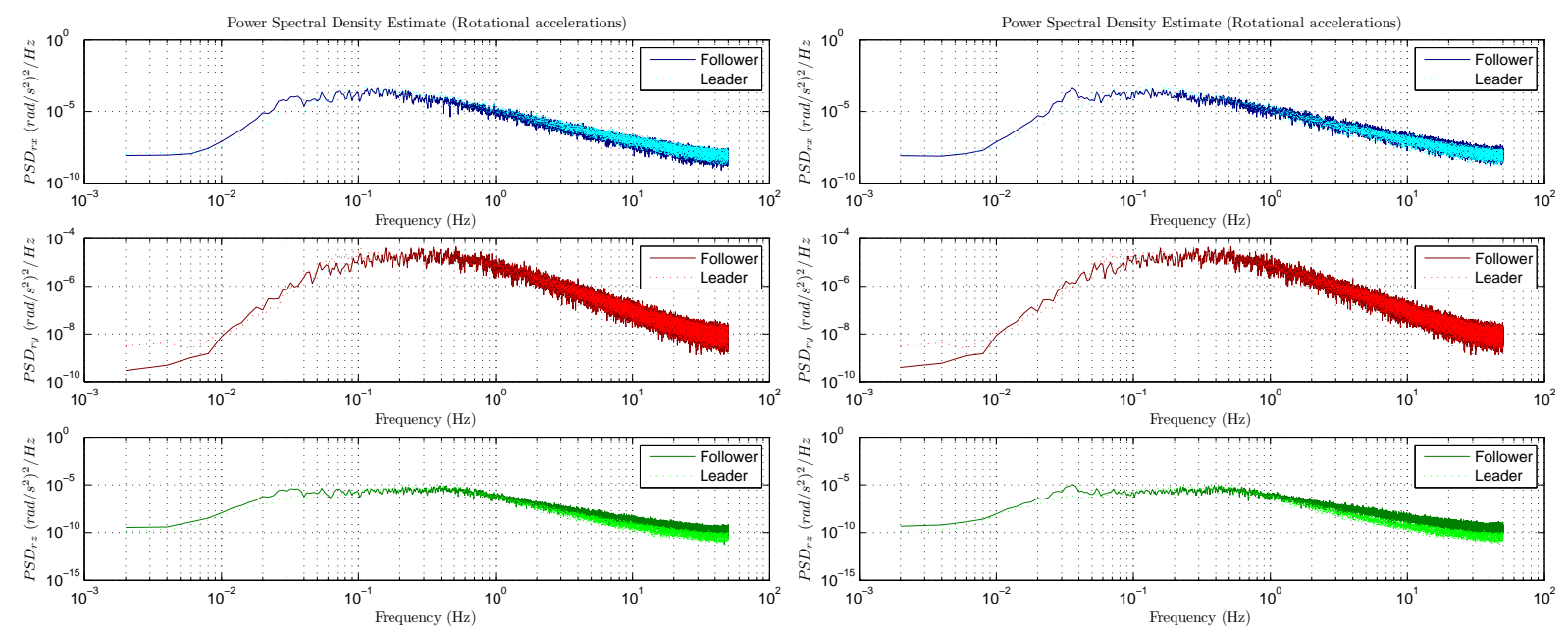

(c) $\eta=1.3$

(d) $\eta=1.2$

Figure J.11: Rotational acceleration power spectral densities of a leader and follower aircraft at any seating location in light turbulence 


\section{Appendix K}

\section{Comfort and Motion Sickness}

The vibrational comfort and motion sickness incidence results for both the leader and follower aircraft during formation flight in light and moderate turbulence intensities are concisely summarised here. Concerning vibrational comfort, the total passenger RMS acceleration magnitudes were used in accordance with the ISO 2631-1 guidance to likely reactions to various magnitudes of overall vibration values. In the same manner, the motion sickness and percentage of ill passengers were evaluated according to the prescribed method in ISO 2631-1. The application of these methods is described in Chapter 3. Concerning vibrational comfort and motion sickness incidence for a rigid body aircraft, Table K.1 provides a reference guide for the different formation flight scenarios.

Table K.1: Reference guide for case 1 vibrational comfort and PIP results at different separations and varying levels of turbulence intensity

\begin{tabular}{|c|c|c|c|c|c|}
\hline \multirow{2}{*}{$\begin{array}{c}\text { Turbulence } \\
\text { Intensity }\end{array}$} & \multicolumn{3}{|c|}{ Desired Separations } & \multicolumn{2}{|c|}{ Figures } \\
\hline & $\eta$ & $\zeta$ & $\xi$ & Vibrational Comfort & Motion Sickness \\
\hline \multirow{5}{*}{ Light } & 1.5 & 0 & -10 & K.1a & K.2 \\
\hline & 1.4 & 0 & -10 & K.1a & $\overline{\mathrm{K} .2}$ \\
\hline & 1.3 & 0 & -10 & $\overline{\text { K.1a }}$ & $\overline{\bar{K} .2}$ \\
\hline & 1.2 & 0 & -10 & K.1a & $\overline{\mathrm{K} .2}$ \\
\hline & 1.1 & 0 & -10 & $\overline{\mathrm{K} .1 \mathrm{a}}$ & $\overline{\mathrm{K} .2}$ \\
\hline \multirow{4}{*}{ Moderate } & 1.5 & 0 & -10 & K.1b & K.3 \\
\hline & 1.4 & 0 & -10 & $\overline{\mathrm{K} .1 \mathrm{~b}}$ & $\overline{\bar{K} .3}$ \\
\hline & 1.3 & 0 & -10 & $\overline{\mathrm{K} .1 \mathrm{~b}}$ & $\overline{\mathrm{K} .3}$ \\
\hline & 1.2 & 0 & -10 & $\overline{\mathrm{K} .1 \mathrm{~b}}$ & $\overline{\mathrm{K} .3}$ \\
\hline
\end{tabular}



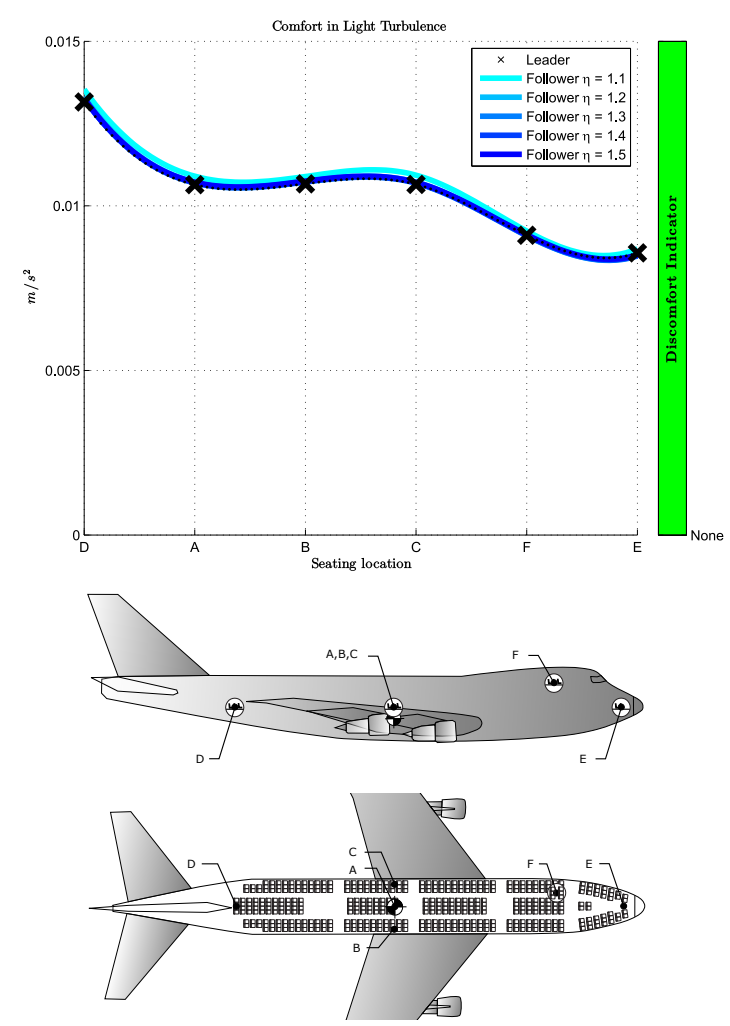

(a) Light Turbulence
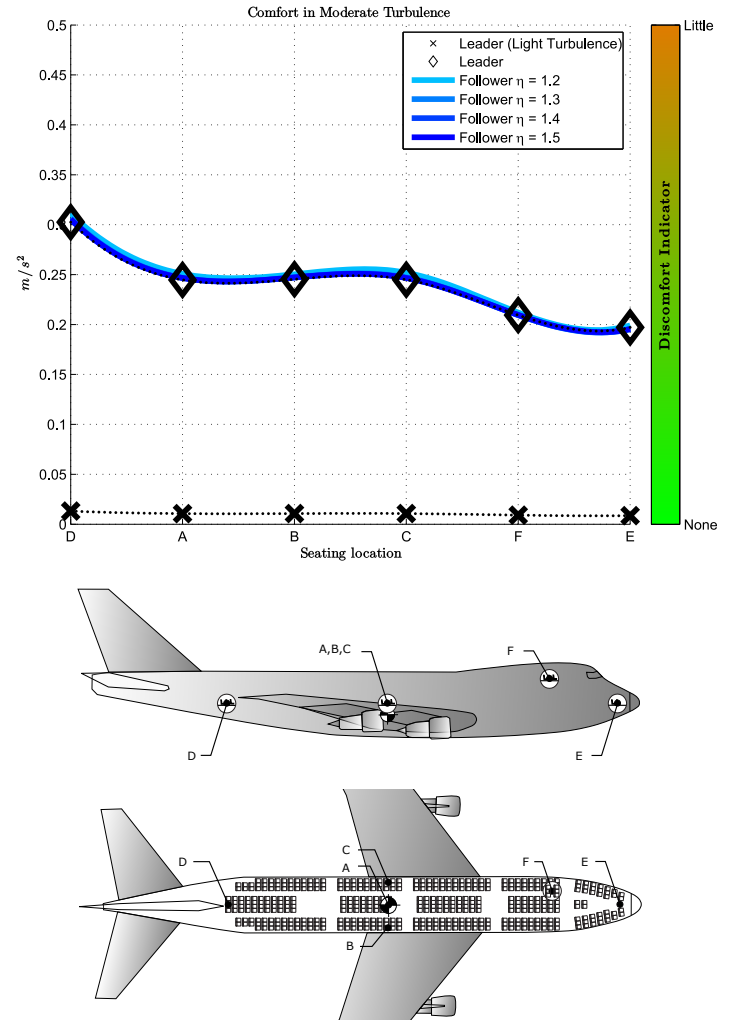

(b) Moderate Turbulence

Figure K.1: Levels of comfort in a leader and follower along the aircraft fuselage. Simulations were performed in light and moderate turbulence for lateral separations of up to $\eta=1.1$ wingspans. 

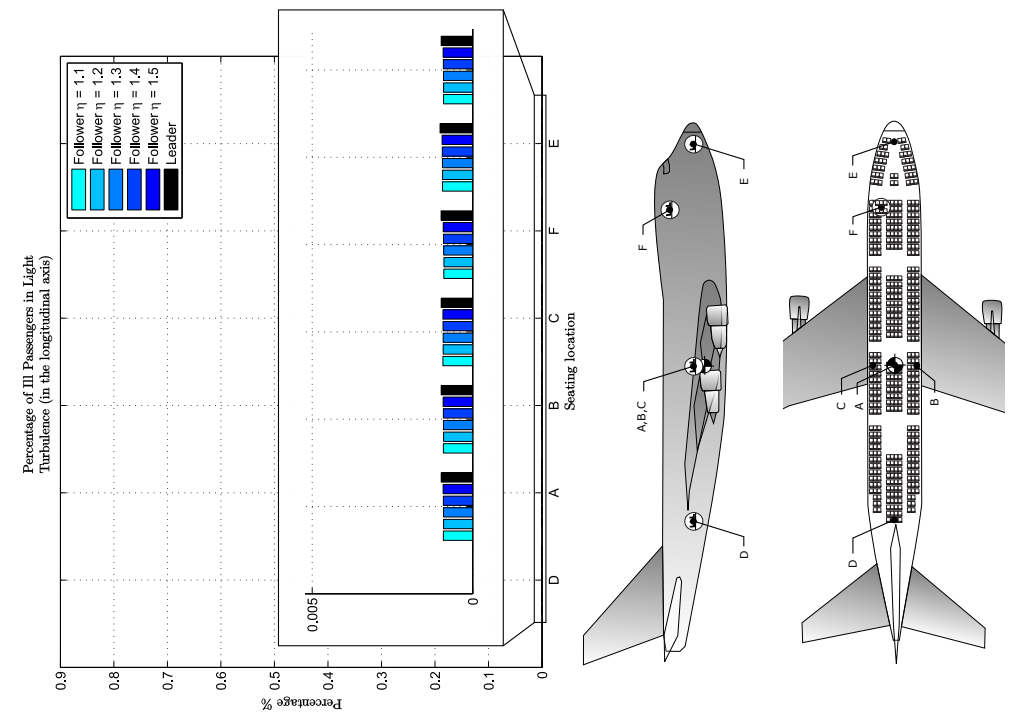

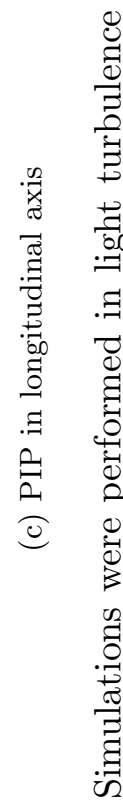
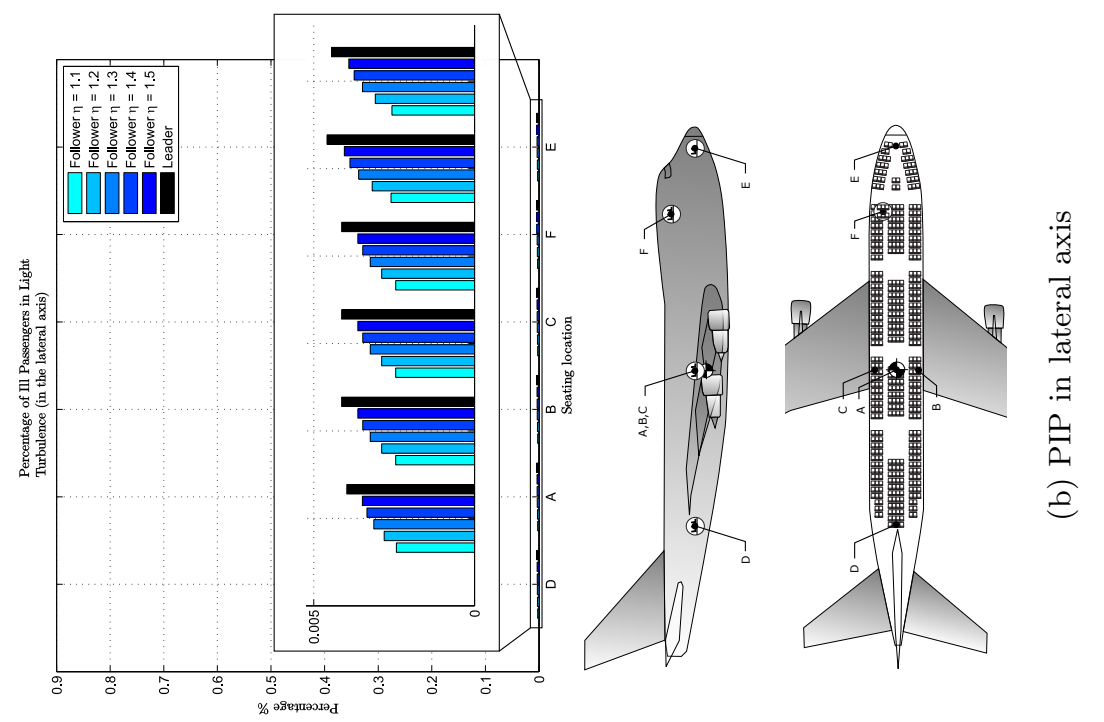

03
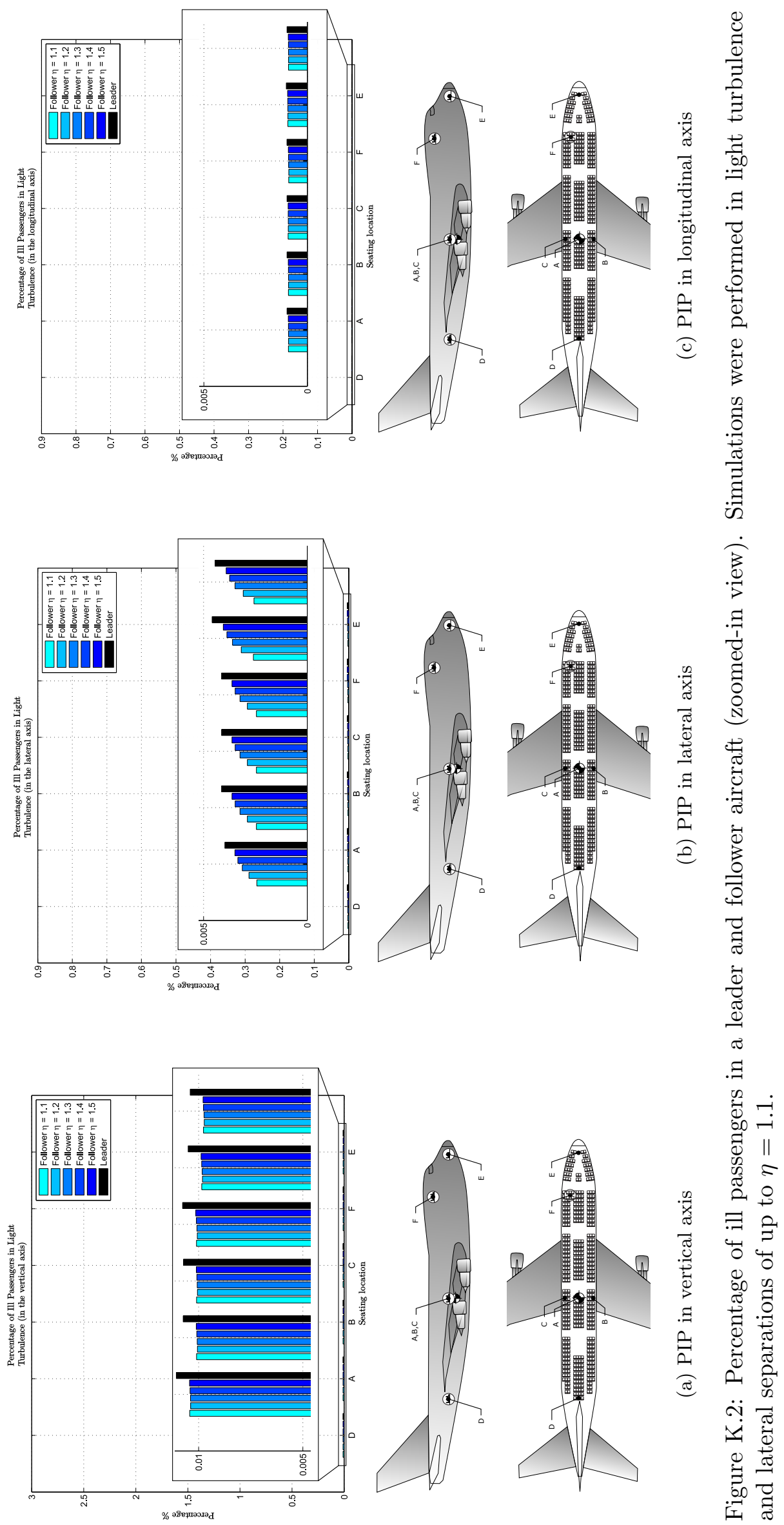

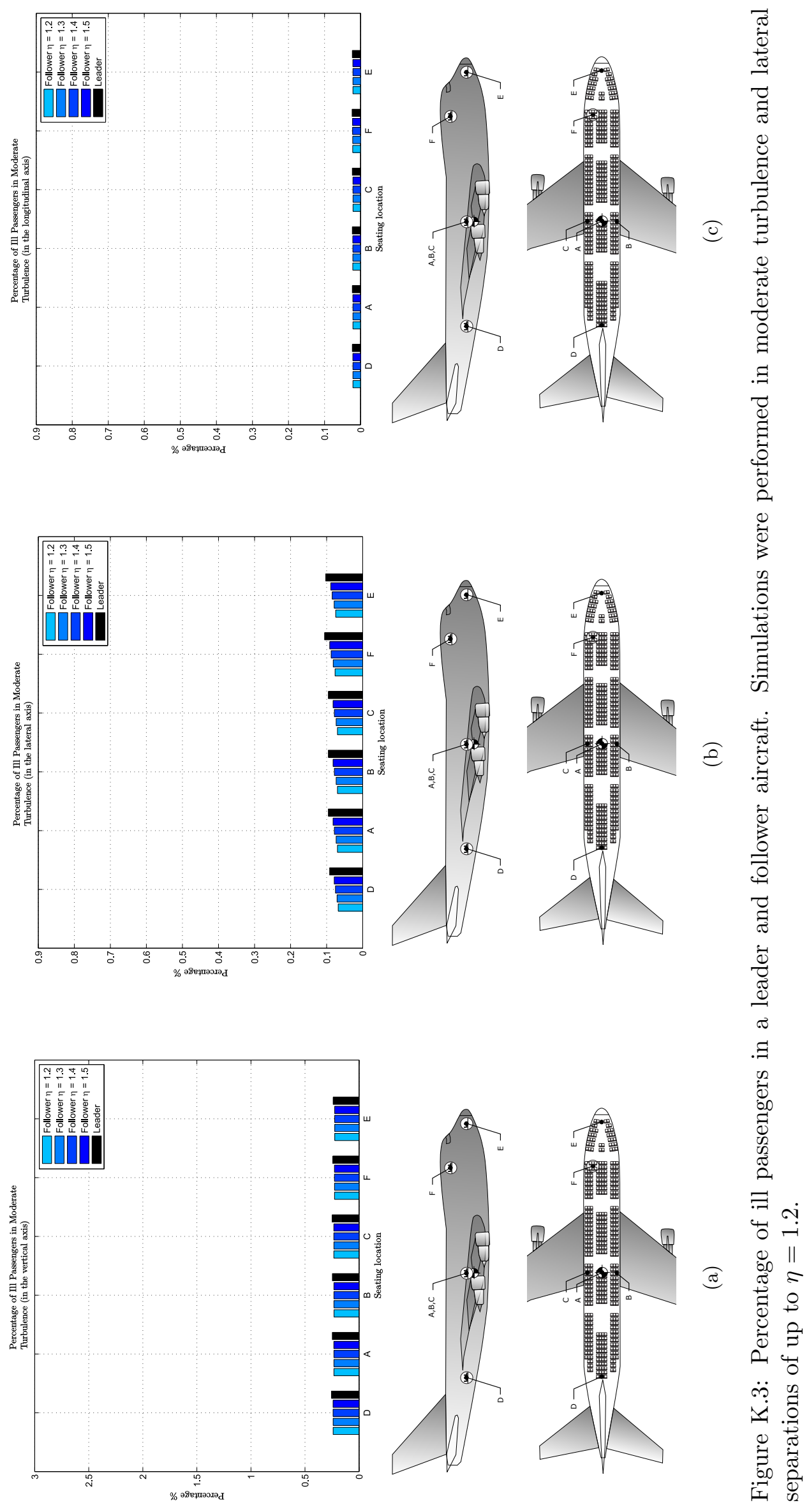


\section{Bibliography}

[1] S. Andrew Ning, Aircraft Drag Reduction Through Extended Formation Flight. Stanford University, 2011.

$[2]$ G. Bower, T. Flanzer, and I. Kroo, "Formation Geometries and Route Optimization for Commercial Formation Flight," in 27th AIAA Applied Aerodynamics Conference, Fluid Dynamics and Co-located Conferences, American Institute of Aeronautics and Astronautics, jun 2009 .

[3] N. Bizinos, Passenger Comfort during Formation Flight within Atmospheric Turbulence. Masters of science in mechanical engineering, University of Cape Town, 2012.

[4] D. Büchner, Automatic Control of Commercial Airliners in Formation Flight. Masters thesis, Stellenbosch University, 2015.

[5] D. J. Osbourne, F. Leal, R. Saran, P. Shipley, and T. Stewart, Person-Centred Ergonomics: A Brantonian View Of Human Factors. Taylor \& Francis, 2003.

[6] N. Kirk and E. Corlett, "Passenger comfort - An overview," The ergonomics of passenger comfort, no. September 1978, pp. 131-136, 1979.

[7] L. G. Richards, I. D. Jacobson, and a. R. Kuhlthau, "What the passenger contributes to passenger comfort.," Applied ergonomics, vol. 9, no. September, pp. 137-142, 1978.

[8] D. S. Sanders, The effects of atmospheric turbulence on fuel consumption in extended formation flight. Masters thesis, University of Cape Town, 2014.

[9] R. K. Heffley and W. F. Jewell, "Aircraft Handling Qualities Data," tech. rep., Systems Technology, Inc., Hawthorne, 1972.

[10] C. R. Hanke and D. R. Nordwall, "The Simulation of A Jumbo Jet Transport Aircraft Volume II: Modeling Data," tech. rep., The Boeing Company, Kansas, 1970.

[11] F. Kubica and B. Madelaine, "Passenger Comfort Improvement by Integrated Control Law Design," 1999.

[12] C. Breitsamter, "Wake vortex characteristics of transport aircraft," Progress in Aerospace Sciences, vol. 47, pp. 89-134, feb 2011. 
[13] L. Jacquin, D. Fabre, P. Geffroy, and E. Coustols, "The properties of a transport aircraft wake in the extended near field - An experimental study," in 39th Aerospace Sciences Meeting and Exhibit, Aerospace Sciences Meetings, American Institute of Aeronautics and Astronautics, jan 2001.

[14] P. R. Spalart, "Airplane Trailing Vortices," Annual Review of Fluid Mechanics, vol. 30, pp. 107-138, jan 1998.

[15] W. J. Devenport, M. C. Rife, S. I. Liapis, and G. J. Follin, "The structure and development of a wing-tip vortex," Journal of Fluid Mechanics, vol. 312, p. 67, apr 2006.

[16] P. P. Wegener, What Makes Airplanes Fly?: History, Science, and Applications of Aerodynamics. Springer New York, 2012.

[17] S. E. Widnall, "The Structure and Dynamics of Vortex Filaments," Annual Review of Fluid Mechanics, vol. 7, pp. 141-165, jan 1975.

[18] L. Jacquin, D. Fabre, D. Sipp, V. Theofilis, and H. Vollmers, "Instability and unsteadiness of aircraft wake vortices," Aerospace Science and Technology, vol. 7, pp. 577-593, dec 2003.

[19] F. Holzäpfel, T. Gerz, and R. Baumann, "The turbulent decay of trailing vortex pairs in stably stratified environments," Aerospace Science and Technology, vol. 5, no. 2, pp. 95-108, 2001.

[20] P. B. S. Lissaman and C. A. Shollenberger, "Formation Flight of Birds," Science, vol. 168, pp. 1003-1005, 1970.

[21] D. Hummel, "Aerodynamic aspects of formation flight in birds," Journal of Theoretical Biology, vol. 104, no. 3, pp. 321-347, 1983.

[22] W. Blake and D. Multhopp, "Design, performance and modeling considerations for close formation flight," in 23rd Atmospheric Flight Mechanics Conference, Guidance, Navigation, and Control and Co-located Conferences, American Institute of Aeronautics and Astronautics, aug 1998.

[23] D. Gingras, "Experimental investigation of a multi-aircraft formation," 17th Applied Aerodynamics Conference, jun 1999.

[24] W. B. Blake and D. R. Gingras, "Comparison of Predicted and Measured Formation Flight Interference Effects," Journal of Aircraft, vol. 41, no. 2, pp. 201-207, 2004.

[25] J. Myatt and W. Blake, "Aerodynamic database issues for modeling close formation flight," Modeling and Simulation Technologies Conference and Exhibit, aug 1999.

[26] S. A. Ning, T. C. Flanzer, and I. M. Kroo, "Aerodynamic Performance of Extended Formation Flight," Journal of Aircraft, vol. 48, pp. 855-865, may 2011. 
[27] J. E. Kless, M. J. Aftosmis, S. A. Ning, and M. Nemec, "Inviscid Analysis of ExtendedFormation Flight," AIAA Journal, vol. 51, pp. 1703-1715, jun 2013.

[28] J. Hallock, "Proceedings of the Aircraft Wake Vortices Conference," 1977.

[29] S. F. Hoerner, Fluid-dynamic drag: practical information on aerodynamic drag and hydrodynamic resistance. Hoerner Fluid Dynamics, 1965.

[30] M. Kshatriya and R. W. Blake, "Theoretical model of the optimum flock size of birds flying in formation," Journal of Theoretical Biology, vol. 157, no. 2, pp. 135-174, 1992.

[31] A. Filippone, "Heuristic optimization applied to an intrinsically difficult problem - Birds formation flight," in 34th Aerospace Sciences Meeting and Exhibit, Aerospace Sciences Meetings, American Institute of Aeronautics and Astronautics, jan 1996.

[32] E. H. Hoganson, A study of aerodynamic interference effects during aerial refuelling. Master's thesis, US Air Force Institute of Technology, 1983.

[33] C. B. Millikan, Aerodynamics of the Airplane. Galcit aeronautical series, John Wiley \& Sons, 1941.

[34] B. W. McCormick, Aerodynamics, Aeronautics, and Flight Mechanics. Wiley, 1994.

[35] L. Garodz, "Federal Aviation Administration full-scale aircraft vortex wake turbulence flight test investigations - Past, present, future," 9th Aerospace Sciences Meeting, jan 1971.

[36] D. J. Oborne, "Techniques available for the assessment of passenger comfort.," Applied ergonomics, vol. 9, pp. 45-9, mar 1978.

[37] C. C. Smith, D. Y. McGehee, and A. J. Healey, "The Prediction of Passenger Riding Comfort from Acceleration Data," tech. rep., Department of Transportation Office of University Research Washington, D. C. 20590, 1976.

[38] A. J. Healey, Passenger Response to Random Vibration in Transportation Vehicles - A Literature Review. Texas: University of Texas, Council for Advanced Transportation Studies, 1975.

[39] J. Quehl, Comfort studies on aircraft interior sound and vibration. Dissertation, Universitat Oldenburg zur Erlangung des Grades, 1971.

[40] D. G. Stephens, Development and Application of Ride Quality Criteria. National Aeronautics and Space Administration, 1974.

[41] R. N. Janeway and S. of Automotive Engineers, Vehicle Vibration Limits to Fit the Passenger. S.A.E. Special publications dept, Society of Automotive Engineers, 1948.

[42] R. A. Lee and F. Pradko, Analytical Analysis of Human Vibration. Society of Automotive Engineers, 1968. 
[43] A. Butkunas, Power Spectral Density and Ride Evaluation. Society of Automotive Engineers, 1966.

[44] S. S. Stevens, "On the Psychophysical Law," The Psychological Review, vol. 64, no. 3, pp. 153-181, 1957.

[45] K. Hiramatsu and M. J. Griffin, "Predicting the subjective response to nonsteady vibration based on the summation of subjective magnitude.," Journal of the Acoustical Society of America, vol. 76, pp. 1080-1089, 984.

[46] H. V. Howarth and M. J. Griffin, "The frequency dependence of subjective reaction to vertical and horizontal whole-body vibration at low magnitudes," Journal of the Acoustical Society of America, vol. 83, pp. 1406-1413, 1988.

[47] I. D. Jacobson, A. R. Kuhlthau, and L. G. Richards, "Application of Ride Quality Technology to Predict Ride Satisfaction for Commuter-Type Aircraft," in The 1975 Ride Quality Symposium, pp. 45-64, National Aeronautics and Space Administration, 1975.

[48] NASA, "1975 Ride Quality Symposium," in National Aeronautics and Space Administration, (Williamsburg), National Aeronautics and Space Administration, reproduced by National Technical Information Service, 1975.

[49] D. G. Stephens, "Review of measured vibration and noise environments experienced by passengers in aircraft and in ground transportation systems," in The 1975 Ride Quality Symposium, pp. 65-85, National Aeronautics and Space Administration, 1975.

[50] T. Wolf, T. Rezek, and S. Gee, "Passenger Ride Quality Response to an Airborne Simulator Environment," in The 1975 Ride Quality Symposium, National Aeronautics and Space Administration, 1975 .

[51] L. G. Richards, A. R. Kuhlthau, and I. D. Jacobson, "Passenger Ride Quality Determined from Commercial Airline Flights," in The 1975 Ride Quality Symposium1, pp. 409-436, National Aeronautics and Space Administration, 1975.

[52] J. McKenzie and S. H. Brumaghim, "Review of Ride Quality Technology Needs of Industry and User Groups," in The 1975 Ride Quality Symposium, pp. 5-30, National Aeronautics and Space Administration, 1975.

[53] ISO2631-1, Mechanical vibration and shock - Evaluation of human exposure to whole body vibration. BSI Standards, 1997.

[54] C. L. Garrec and F. Kubica, "In-Flight Structural Modes Identification for Comfort Improvement by Flight Control Laws," Journal of Aircraft, vol. 42, pp. 90-92, jan 2005.

[55] F. Kubica, "New Flight Control Laws for Large Capacity Aircraft: Experimentation on Airbus A340," in 21st International Council of the Aeronautical Sciences (ICAS), ICAS and AIAA, 1998. 
[56] N. Bizinos and C. Redelinghuys, "Tentative Study of Passenger Comfort During Formation Flight Within Atmospheric Turbulence," Journal of Aircraft, vol. 50, pp. 886-900, may 2013.

[57] I. K. Peddle and J. A. Engelbrecht, "Advanced Automation 833 - Introductory course to aircraft dynamics," 2013.

[58] M. V. Cook, Flight Dynamics Principles. Elsevier, second edi ed., 2007.

[59] J. H. Blakelock, Automatic Control of Aircraft and Missiles. A Wiley-Interscience publication, Wiley, 1991.

[60] B. Etkin and L. D. Reid, Dynamics of Flight: Stability and Control. Wiley, 3rd editio ed., 1995.

[61] K. Kosar, S. Durmaz, and E. M. Jafarov, "LONGITUDINAL DYNAMICS ANALYSIS of BOEING 747-400," in Proceedings of the 9th WSEAS International Conference on Automatic Control, Modeling and Simulation, (Istanbul), pp. 82-87, 2007.

[62] I. K. Peddle, Autonomous Flight of a Model Aircraft. PhD thesis, Stellenbosch University, 2005.

[63] R. S. Shevell, Fundamentals of Flight. Prentice Hall, 1989.

[64] B. L. Stevens and F. L. Lewis, Aircraft Control and Simulation. Wiley, 2003.

[65] M. Brodecki, K. Subbarao, and Q. P. Chu, "Formation Flight Control System for InFlight Sweet Spot Estimation," in 51st AIAA Aerospace Sciences Meeting including the New Horizons Forum and Aerospace Exposition, Aerospace Sciences Meetings, American Institute of Aeronautics and Astronautics, jan 2013.

[66] J. L. Meriam and L. G. Kraige, Engineering Mechanics: Dynamics. Engineering Mechanics, John Wiley \& Sons, seventh ed ed., 2012.

[67] L. J. Clancy, Aerodynamics. Pitman Aeronautical Engineering Series, New York: John Wiley \& Sons, 1975.

[68] D. T. McRuer, D. Graham, and I. Ashkenas, Aircraft Dynamics and Automatic Control. Princeton Legacy Library, Princeton University Press, 2014.

[69] I. Northrop Aircraft, Dynamics of the Airframe. Flight control and fire control system manuals, United States: Bureau of Aeronautics, Navy Department, 1952.

[70] J. A. Thelander, Aircraft Motion Analysis. [U.S. Air Force. Flight Dynamics Laboratiry] Technical documentary report FDL-TDR-64-70, Air Force Flight Dynamics Laboratory, Research and Technology Division, Air Force Systems Command, 1965. 
[71] C. Favre, "Fly-by-wire for commercial aircraft - The Airbus Experience," International Journal of Control, vol. 59, no. 1, pp. 139-157, 1994.

[72] Z. Kun, W. Lixin, and T. Xiangsheng, "Flying qualities reduction of fly-by-wire commercial aircraft with reconfiguration flight control laws," Procedia Engineering, vol. 17, pp. 179-196, jan 2011.

[73] P. Traverse, I. Lacaze, and J. Souyris, "Airbus fly-by-wire: a process toward total dependability," International Congress of the Aeronautical Sciences, pp. 1-10, 2006.

[74] E. Field, "The application of a $C^{*}$ flight control law to large civil transport aircraft," 1993.

[75] A. E. Bryson, Control of Spacecraft and Aircraft. Princeton University Press, 1994.

[76] F. Holzapfel, M. Heller, M. Weingartner, G. Sachs, and O. da Costa, "Development of control laws for the simulation of a new transport aircraft," Proceedings of the Institution of Mechanical Engineers, Part G: Journal of Aerospace Engineering, vol. 223, pp. 141-156, 2009 .

[77] J. D. Leatherwood, U. S. N. Aeronautics, S. Administration, and L. R. Center, Vibrations Transmitted to Human Subjects Through Passenger Seats and Considerations of Passenger Comfort. NASA technical note, National Aeronautics and Space Administration, 1975.

[78] P. D. Welch, "The use of fast Fourier transform for the estimation of power spectra: A method based on time averaging over short, modified periodograms," Audio and Electroacoustics, IEEE Transactions on, vol. 15, no. 2, pp. 70-73, 1967.

[79] G. Heinzel, A. Rüdiger, and R. Schilling, "Spectrum and spectral density estimation by the Discrete Fourier transform (DFT), including a comprehensive list of window functions and some new flat-top windows," tech. rep., Max-Planck-Institut fur Gravitationsphysik (Albert-Einstein-Institut), 2002.

[80] J. C. Houbolt, "Atmospheric Turbulence," AIAA Journal, vol. 11, pp. 421-437, apr 1973.

[81] "747 Airplane Characteristics - Airport Planning," tech. rep., Boeing Commercial Airplane Company, 1984.

[82] T. H. G. Megson, Introduction to Aircraft Structural Analysis. Elsevier Science, 2013.

[83] J. R. Wright and J. E. Cooper, Introduction to Aircraft Aeroelasticity and Loads. Aerospace Series, Wiley, 2014.

[84] N. Nguyen and I. Tuzcu, "Flight Dynamics of Flexible Aircraft with Aeroelastic and Inertial Force Interactions," in AIAA Atmospheric Flight Mechanics Conference, Guidance, Navigation, and Control and Co-located Conferences, American Institute of Aeronautics and Astronautics, aug 2009. 
[85] T.-L. Teng, F.-a. Chang, and C.-P. Peng, "Analysis of human body response to vibration using multi-body dynamics method," Proceedings of the Institution of Mechanical Engineers, Part K: Journal of Multi-body Dynamics, vol. 220, no. 3, pp. 191-202, 2006.

[86] G. Joshi, A. K. Bajaj, and P. Davies, "Whole-body vibratory response study using a nonlinear multi-body model of seat-occupant system with viscoelastic flexible polyurethane foam.," Industrial health, vol. 48, no. 5, pp. 663-674, 2010.

[87] D. Büchner, J. A. A. Engelbrecht, J. L. Adams, and C. Redelinghuys, "Towards Automatic Flight Control for Commercial Airliners in Formation Flight," in Proceedings of the 19th IFAC World Congress (B. Edward, ed.), pp. 12188-12194, aug 2014.

[88] J. Kless, M. J. Aftosmis, S. A. Ning, and M. Nemec, "Inviscid Analysis of Extended Formation Flight," Seventh International Conference on Computational Fluid Dynamics, pp. 1-22, 2012.

[89] MathWorks, "Von Kármán Wind Turbulence Model (Continuous)," 2015.

[90] H. Schmid, "How to use the FFT and Matlabs pwelch function for signal and noise simulations and measurements," no. August, pp. 1-13, 2012.

[91] B. P. Lathi and Z. Ding, Modern Digital and Analog Communication Systems. Oxford series in electrical and computer engineering, Oxford University Press, 2009. 